\section{The First Census Optical Character Recognition System Conference}

R. Allen Wilkinson Jon Geist

Stanley Janet

Patrick J. Grother

Christopher J. C. Burges

Robert Creecy

Bob Hammond

Jonathan J. Hull

Norman J. Larsen

Thomas P. Vogl

Charles L. Wilson

U.S. DEPARTMENT OF COMMERCE Technology Administration

Natıonal Institute of Standards and Technology

Computer Systems Laboratory Advanced Systems Division

Image Recognition Group

Gavthersburg. MD 20899

QC

100

.456

$+4912$

NLT 



\title{
NISTIR 4912
}

\section{The First Census Optical} Character Recognition System Conference

\author{
R. Allen Wilkinson \\ Jon Geist \\ Stanley Janet \\ Patrick J. Grother \\ Christopher J. C. Burges \\ Robert Creecy \\ Bob Hammond \\ Jonathan J. Hull \\ Norman J. Larsen \\ Thomas P. Vogl \\ Chartes $L$ Wilson
}

\section{U.S. DEPARTMENT OF COMMERCE}

Technology Administration

National Institute of Standards

and Technology

Computer Systems Laboratory

Advanced Systems Division

Image Recognition Group

Galthersburg. MD 20899

August 1992

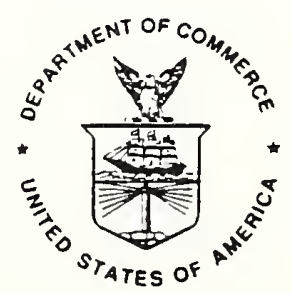

U.S. DEPARTMENT OF COMMERCE Barbara Hackman Franklin, Secretary

TECHNOLOGY ADMINISTRATION

Robert M. White, Under Secretary for Technology

NATIONAL INSTITUTE OF STANDARDS

AND TECHNOLOGY

John W. Lyons, Director 



\title{
The First Census Optical Character Recognition Systems Conference
}

\author{
R. Allen Wilkinson, Jon Geist, Stanley Janet, Patrick J. Grother, \\ Christopher J. C. Burges, Robert Creecy, Bob Hammond, \\ Jonathan J. Hull, Norman W. Larsen, Thomas P. Vogl, \\ and Charles L. Wilson
}

********* DISCLAIMER

The U.S. Bureau of the Census (Census) and the National Institute of Standards and Technology (NIST) sponsored this Conference as part of ongoing research into machine recognition of hand-print. The Conference and related exercises focused on a single step in the process: machine recognition of individual (or segmented) characters without context. With the single variable nature of this study, no valid comparisons can be made regarding cost or performance of systems designed to process entire forms or documents. Further, the efforts of the participants in conducting the tests were not proctored or monitored in any way by Census or NIST.

While some test results from this Conference may appear in marketing literature, potential buyers must beware! Census and NIST can make only one recommendation to potential buyers: use your own application-specific data to thoroughly test the performance of any system (or component) in a realistic setting.

Also, reference is made to some commercial products at various points in this report. Such reference constitutes neither endorsement by Census or NIST, nor implication that the product so referenced is the best for the particular application. 


\section{Contents}

1 Executive Summary 1

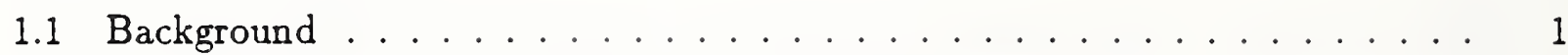

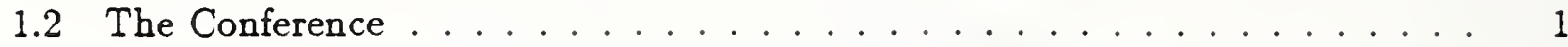

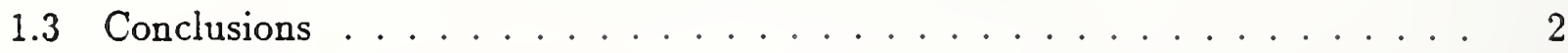

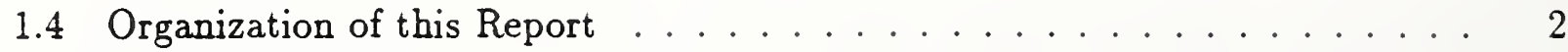

2 Introduction $\quad 4$

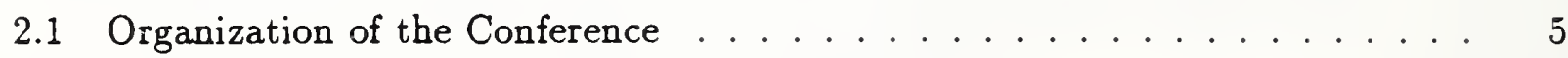

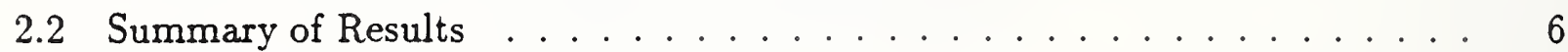

2.3 Differences between Test and Training Databases . . . . . . . . . . . 10

2.4 Error Rates as a Function of Rejection Rates . . . . . . . . . . . . . . . . . 11

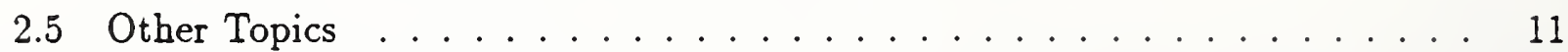

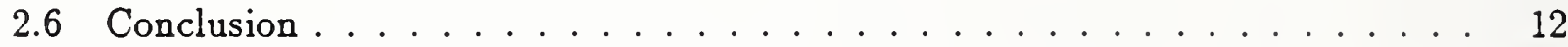

3 Cross Validation Studies $r$

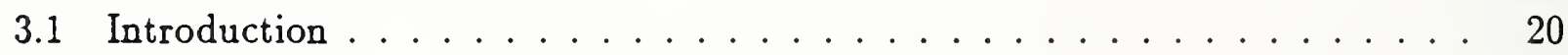

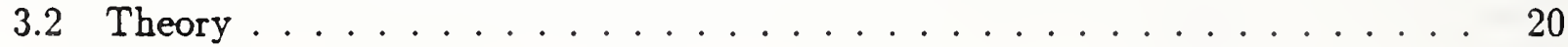

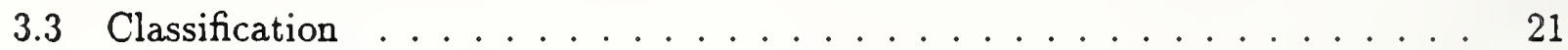

3.4 Cross Validation Results ...................... . . 23

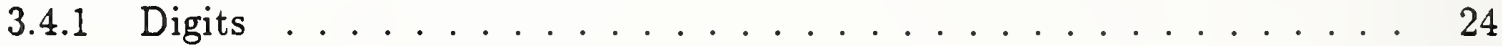

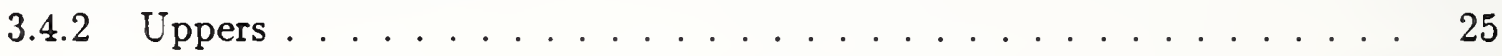

3.4 Lowers ........................ . . . 26

3.5 Caveats ............................. 27

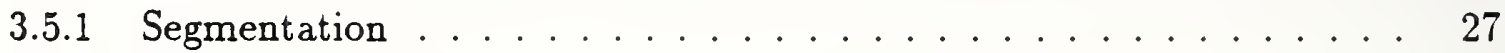

3.5.2 Classifier Dependence . . . . . . . . . . . . . . . . 28

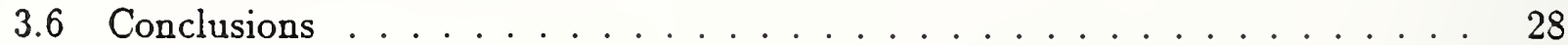

4 System Error Rates Versus Rejection Rates $\quad 30$

4.1 Theory .............................. 30

4.2 Fit of Model to Experimental Data . . . . . . . . . . . . . . . 31

4.3 Conclusion ........................... . . 31

5 Types of Algorithms Used $\quad 36$ 
5.1 Rule-based versus Machine learning . . . . . . . . . . . . . . 36

5.2 Statistical Rules versus Mathematical Rules . . . . . . . . . . . . . . 37

5.3 Linear versus Non-linear Methods . . . . . . . . . . . . . . . . . . 37

5.4 Statistical and Neural Methods . . . . . . . . . . . . . . . . . . 38

5.5 Role of Learning and Rules in Feature Extraction and Classification . . . . . 38

$\begin{array}{lll}6 & \text { System Speed } & \mathbf{4 0}\end{array}$

$\begin{array}{lr}\text { A Issues Raised by Participants } & \mathbf{5 0}\end{array}$

B NIST Perspective on Perceived Problems

B.1 Perceived Problem $1 \ldots \ldots \ldots \ldots \ldots$

B.1.1 Proposed Solutions . . . . . . . . . . . . . . . . 52

B.1.2 Discussion . . . . . . . . . . . . . . . . . . . 52

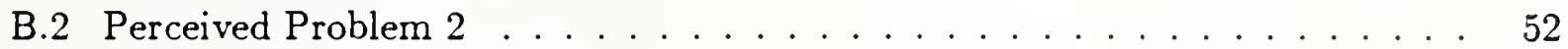

B.2.1 Proposed Solutions . . . . . . . . . . . . . . . 52

B.2.2 Discussion . . . . . . . . . . . . . . . . . . 53

B.3 Perceived Problem $3 \ldots \ldots \ldots \ldots \ldots \ldots$

B.3.1 Proposed Solutions . . . . . . . . . . . . . . . . . . 53

B.3.2 Discussion . . . . . . . . . . . . . . . . . . . . . . 53

B.4 Perceived Problem $4 \ldots \ldots \ldots \ldots \ldots \ldots$

B.4.1 Proposed Solutions . . . . . . . . . . . . . . . . 53

B.4.2 Discussion . . . . . . . . . . . . . . . . . . 54

B.5 Perceived Problem $5 \ldots \ldots \ldots \ldots \ldots \ldots$

B.5.1 Proposed Solutions . . . . . . . . . . . . . . . 54

B.5.2 Discussion . . . . . . . . . . . . . . . . 55

B.6 Perceived Problem $6 \ldots \ldots \ldots \ldots \ldots \ldots \ldots \ldots$

B.6.1 Proposed Solutions . . . . . . . . . . . . . . . . 55

B.6.2 Discussion . . . . . . . . . . . . . . . . . . . 55

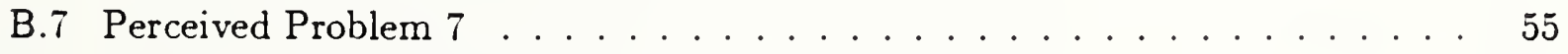

B.7.1 Proposed Solutions . . . . . . . . . . . . . . 56

B.7.2 Discussion . . . . . . . . . . . . . . . . 56

$\begin{array}{ll}\text { C The Call for Participation } & 57\end{array}$ 
D Instructions to Participants

E System Summaries For Results Submitted On Time

F System Summaries For Late Submitted Results 


\section{List of Figures}

1 Error rate versus rejection rate for all systems providing confidence data with their classifications for the digit test.

2 Error rate versus rejection rate for all systems providing rejection data with their classifications for the digit test. . . . . . . . . . . . . 14

3 Error rate versus rejection rate for all systems providing confidence data with their classifications for the upper case test. . . . . . . . . . . . 15

4 Error rate versus rejection rate for all systems providing rejection data with their classifications for the upper case test. . . . . . . . . . . . 16

5 Error rate versus rejection rate for all systems providing confidence data with their classifications for the lower case test. . . . . . . . . . . . .

6 Error rate versus rejection rate for all systems providing rejection data with their classifications for the lower case test. . . . . . . . . . . . 18

7 A typical filled-out sample form . . . . . . . . . . . . . . 19

8 Eigenvalue vs Index for SD3 and TD1. From top: digits, uppers and lowers. All writers were used. . . . . . . . . . . . . . . . . 29

9 Types of methods used for feature extraction and classification. . . . . . . 39

10 Data flow in a complete recognition system. . . . . . . . . . . . . . 42

11 Error rate versus rejection rate for AEG . . . . . . . . . . . . . 75

12 Error rate per writer of AEG . . . . . . . . . . . . . . 75

13 AEG - digit correlation . . . . . . . . . . . . . . . 76

14 AEG - upper case correlation . . . . . . . . . . . . . 77

15 AEG - lower case correlation . . . . . . . . . . . . . . 78

16 Error rate versus rejection rate for ASOL . . . . . . . . . . . . . 81

17 Error rate per writer of ASOL . . . . . . . . . . . . . 81

18 ASOL - digit correlation ........................ 82

19 ASOL - upper case correlation . . . . . . . . . . . . . . 83

20 ASOL - lower case correlation . . . . . . . . . . . . . . . 84

21 Error rate versus rejection rate for ATT_1 . . . . . . . . . . . . 87

22 Error rate per writer of ATT_1 . . . . . . . . . . . . . . 87

23 ATT_1 - digit correlation . . . . . . . . . . . . . 88

24 ATT_1 - upper case correlation . . . . . . . . . . . . . 89

25 ATT_1 - lower case correlation . . . . . . . . . . . . . 90

26 Error rate versus rejection rate for $\mathrm{ATT}_{2} \ldots \ldots \ldots 3$ 
27 Error rate per writer of $\operatorname{ATT} 2 \ldots \ldots \ldots \ldots . \ldots \ldots$

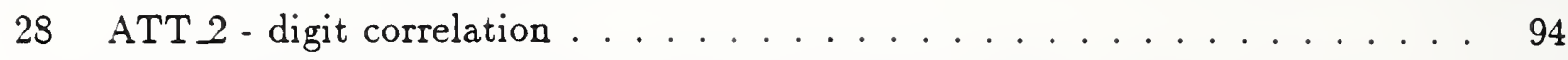

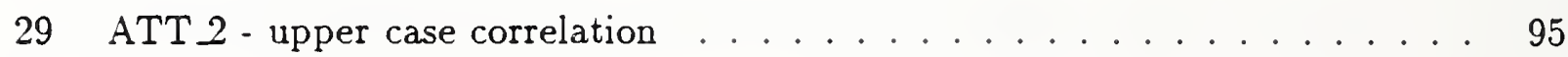

30 ATT 2 - lower case correlation . . . . . . . . . . . . . . . 96

31 Error rate versus rejection rate for ATT_3 . . . . . . . . . . . . . . 99

32 Error rate per writer of ATT_3 . . . . . . . . . . . . . . . . . 99

33 ATT_3 - digit correlation . . . . . . . . . . . . . . . . 100

34 ATT_3 - upper case correlation . . . . . . . . . . . . . . . 101

35 ATT_3 - lower case correlation . . . . . . . . . . . . . . . . . 102

36 Error rate versus rejection rate for ATT $4 \ldots \ldots \ldots$. . . . . . . . . 105

37 Error rate per writer of ATT_4 . . . . . . . . . . . . . . 105

38 ATT_4-digit correlation . . . . . . . . . . . . 106

39 ATT_4 - upper case correlation . . . . . . . . . . . . . 107

40 ATT_4 - lower case correlation . . . . . . . . . . . . . . . . . . 108

41 Error rate versus rejection rate for $\mathrm{COMCOM} \ldots \ldots \ldots \ldots . \ldots 111$

42 Error rate per writer of COMCOM . . . . . . . . . . . . 111

43 COMCOM - digit correlation . . . . . . . . . . . . . 112

44 COMCOM - upper case correlation . . . . . . . . . . . . . 113

45 COMCOM - lower case correlation . . . . . . . . . . . . . . . 114

46 Error rate versus rejection rate for ELSAGB_1 . . . . . . . . . . . . 117

47 Error rate per writer of ELSAGB_1 . . . . . . . . . . . . . 117

48 ELSAGB_1 - digit correlation . . . . . . . . . . . . . . 118

49 ELSAGB_1 - upper case correlation . . . . . . . . . . . . . . . . 119

50 ELSAGB_1 - lower case correlation . . . . . . . . . . . . . . 120

51 Error rate versus rejection rate for ELSAGB $2 \ldots \ldots \ldots . . \ldots . . \ldots 123$

52 Error rate per writer of ELSAGB 2 . . . . . . . . . . . . . . 123

53 ELSAGB_2 - digit correlation . . . . . . . . . . . . . . 124

54 ELSAGB_2 - upper case correlation . . . . . . . . . . . 125

55 ELSAGB_2 - lower case correlation . . . . . . . . . . . . 126

56 Error rate versus rejection rate for ELSAGB_3 . . . . . . . . . . . . . 129

57 Error rate per writer of ELSAGB_3 . . . . . . . . . . . . . . . . 129

58 ELSAGB_3 - digit correlation . . . . . . . . . . . . . . 130

59 ELSAGB_3 - upper case correlation . . . . . . . . . . . . . 131 
ELSAGB_3 - lower case correlation

62 Error rate per writer of ERIM_1 . . . . . . . . . . . . . . 135

63 ERIM_1 - digit correlation . . . . . . . . . . . . . . 136

64 ERIM_1 - upper case correlation . . . . . . . . . . . . . . . . 137

65 ERIM_1 - lower case correlation . . . . . . . . . . . . . . . 138

66 Error rate versus rejection rate for ERIM $2 \ldots \ldots \ldots \ldots$. . . . . . . . 141

67 Error rate per writer of ERIM_2 . . . . . . . . . . . . . . . 141

68 ERIM_2 - digit correlation . . . . . . . . . . . . . . . . . . . 142

69 ERIM_2 - upper case correlation . . . . . . . . . . . . . . . . . 143

70 ERIM_2 - lower case correlation . . . . . . . . . . . . . . . . . . . 144

71 Error rate versus rejection rate for GMD_1 . . . . . . . . . . . . . . 147

72 Error rate per writer of GMD_1 . . . . . . . . . . . . . . . . 147

73 GMD_1 - digit correlation . . . . . . . . . . . . . . . . . . . 148

74 GMD_1 - upper case correlation . . . . . . . . . . . . . . . . . 149

75 GMD_1 - lower case correlation . . . . . . . . . . . . . . 150

76 Error rate versus rejection rate for GMD_2 . . . . . . . . . . . . 153

77 Error rate per writer of GMD_2 . . . . . . . . . . . . . . . 153

78 GMD_2 - digit correlation . . . . . . . . . . . . . . . . . . 154

79 GMD_2 - upper case correlation . . . . . . . . . . . . . . . . . 155

80 GMD_2 - lower case correlation . . . . . . . . . . . . . . 156

81 Error rate versus rejection rate for GMD_3 . . . . . . . . . . . . . . 159

82 Error rate per writer of GMD_3 . . . . . . . . . . . . . . . . . . 159

83 GMD_3 - digit correlation . . . . . . . . . . . . . . . . 160

84 GMD_3 - upper case correlation . . . . . . . . . . . . . . . 161

85 GMD_3 - lower case correlation . . . . . . . . . . . . . . . . . 162

86 Error rate versus rejection rate for GMD $4 \ldots \ldots \ldots$

87 Error rate per writer of GMD_4 . . . . . . . . . . . . . . 165

88 GMD_4 - digit correlation . . . . . . . . . . . . . . . . 166

89 GMD_4 - upper case correlation . . . . . . . . . . . . . . 167

90 GMD_4 - lower case correlation . . . . . . . . . . . . . . . . . . 168

91 Error rate versus rejection rate for GTESS_1 . . . . . . . . . . . . . 171

92 Error rate per writer of GTESS_1 . . . . . . . . . . . . . . 171 
93 GTESS_1 - digit correlation

94 GTESS_1 - upper case correlation

95 GTESS_1 - lower case correlation

96 Error rate versus rejection rate for GTESS_2

97 Error rate per writer of GTESS_2 177

98 GTESS_2 - digit correlation 178

99 GTESS_2 - upper case correlation 179

100 GTESS_2 - lower case correlation 180

101 Error rate versus rejection rate for HUGHES_1 183

102 Error rate per writer of HUGHES_1 183

103 HUGHES_1 - digit correlation 184

104 HUGHES_1 - upper case correlation 185

105 HUGHES_1 - lower case correlation 186

106 Error rate versus rejection rate for HUGHES_2 189

107 Error rate per writer of HUGHES_2 . 189

108 HUGHES_2 - digit correlation 190

109 HUGHES_2 - upper case correlation 191

110 HUGHES_2 - lower case correlation 192

111 Error rate versus rejection rate for IBM . 195

112 Error rate per writer of IBM . 195

113 IBM - digit correlation 196

114 IBM - upper case correlation . 197

115 IBM - lower case correlation 198

116 Error rate versus rejection rate for IFAX 201

117 Error rate per writer of IFAX 201

118 IFAX - digit correlation 202

119 IFAX - upper case correlation 203

120 IFAX - lower case correlation 204

121 Error rate versus rejection rate for KAMAN_1 207

122 Error rate per writer of KAMAN_1 207

123 KAMAN_1 - digit correlation 208

124 KAMAN_1 - upper case correlation 209

125 KAMAN_1 - lower case correlation 210 
126 Error rate versus rejection rate for KAMAN $2 \ldots \ldots \ldots$

127 Error rate per writer of KAMAN $2 \ldots \ldots \ldots . \ldots . \ldots . \ldots . \ldots 213$

128 KAMAN2 - digit correlation . . . . . . . . . . . . . . 214

129 KAMAN2 - upper case correlation . . . . . . . . . . . . 215

130 KAMAN 2 - lower case correlation . . . . . . . . . . . . 216

131 Error rate versus rejection rate for KAMAN_3 . . . . . . . . . . . . 219

132 Error rate per writer of KAMAN_3 . . . . . . . . . . . . . . . . 219

133 KAMAN_3 - digit correlation . . . . . . . . . . . . . 220

134 KAMAN_3 - upper case correlation . . . . . . . . . . . . . . 221

135 KAMAN_3 - lower case correlation . . . . . . . . . . . . . 222

136 Error rate versus rejection rate for KAMAN_4 . . . . . . . . . . . 225

137 Error rate per writer of KAMAN_4 . . . . . . . . . . . . 225

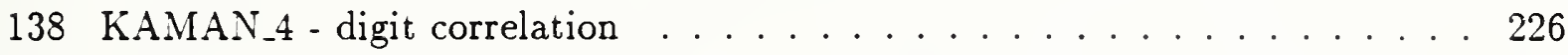

139 KAMAN_4 - upper case correlation . . . . . . . . . . . . 227

140 KAMAN_4 - lower case correlation . . . . . . . . . . . . 228

141 Error rate versus rejection rate for KAMAN_5 . . . . . . . . . . . . 231

142 Error rate per writer of KAMAN_5 . . . . . . . . . . . . 231

143 KAMAN_5 - digit correlation . . . . . . . . . . . . . . 232

144 KAMAN_5 - upper case correlation . . . . . . . . . . . . . 233

145 KAMAN_5 - lower case correlation . . . . . . . . . . . . . 234

146 Error rate versus rejection rate for KODAK_1 . . . . . . . . . . 237

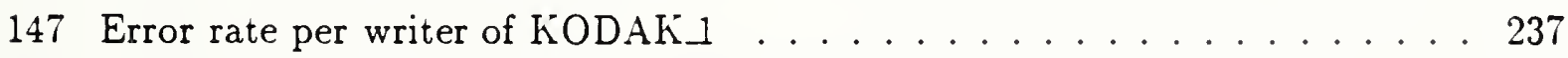

148 KODAK_1 - digit correlation . . . . . . . . . . . . . . 238

149 KODAK 1 - upper case correlation . . . . . . . . . . . . . 239

150 KODAK_1 - lower case correlation . . . . . . . . . . . . . . 240

151 Error rate versus rejection rate for $\operatorname{KODAK}_{2} \ldots \ldots \ldots . \ldots 243$

152 Error rate per writer of KODAK $2 \ldots \ldots \ldots \ldots . \ldots . \ldots . \ldots . \ldots 243$

153 KODAK 2 - digit correlation . . . . . . . . . . . . . . . 244

154 KODAK 2 - upper case correlation . . . . . . . . . . . 245

155 KODAK 2 - lower case correlation . . . . . . . . . . . . . 246

156 Error rate versus rejection rate for MIME . . . . . . . . . . . . . 249

157 Error rate per writer of MIME . . . . . . . . . . . . . . . . . 249

158 MIME - digit correlation . . . . . . . . . . . . . . . 250 
159 MIME - upper case correlation . . . . . . . . . . . . . . . 251

160 MIME - lower case correlation . . . . . . . . . . . . . . . . . 252

161 Error rate versus rejection rate for NESTOR . . . . . . . . . . 255

162 Error rate per writer of NESTOR . . . . . . . . . . . . 255

163 NESTOR - digit correlation . . . . . . . . . . . . . 256

164 NESTOR - upper case correlation . . . . . . . . . . . . . . 257

165 NESTOR - lower case correlation . . . . . . . . . . . . . 258

166 Error rate versus rejection rate for NIST_1 . . . . . . . . . . . 261

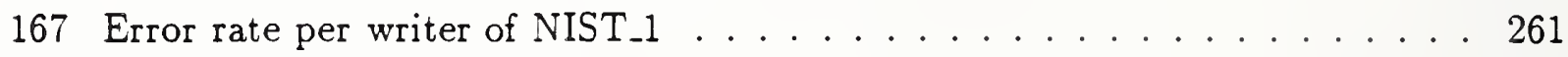

168 NIST_1 - digit correlation . . . . . . . . . . . . . . 262

169 NIST_1 - upper case correlation . . . . . . . . . . . . . . 263

170 NIST_1 - lower case correlation . . . . . . . . . . . . . . . 264

171 Error rate versus rejection rate for NIST $2 \ldots \ldots \ldots . \ldots . \ldots 267$

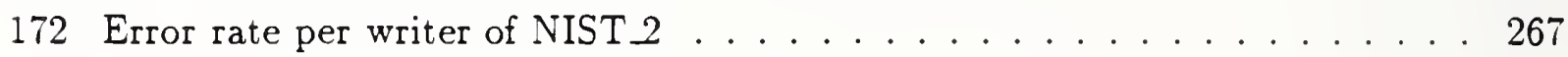

173 NIST 2 - digit correlation . . . . . . . . . . . . 268

174 NIST 2 - upper case correlation . . . . . . . . . . . . . . 269

175 NIST 2 - lower case correlation . . . . . . . . . . . . . 270

176 Error rate versus rejection rate for NIST_3 . . . . . . . . . . . . . 273

177 Error rate per writer of NIST_3 . . . . . . . . . . . . . 273

178 NIST_3 - digit correlation . . . . . . . . . . . . . . . . 274

179 NIST_3 - upper case correlation . . . . . . . . . . . . . . . 275

180 NIST_3 - lower case correlation . . . . . . . . . . . . . . . 276

181 Error rate versus rejection rate for NYNEX . . . . . . . . . . . . 279

182 Error rate per writer of NYNEX . . . . . . . . . . . . . . . . . . 279

183 NYNEX - digit correlation . . . . . . . . . . . . . . . 280

184 NYNEX - upper case correlation . . . . . . . . . . . . . . . . 281

185 NYNEX - lower case correlation . . . . . . . . . . . . . . . 282

186 Error rate versus rejection rate for OCRSYS . . . . . . . . . . . . 285

187 Error rate per writer of OCRSYS . . . . . . . . . . . . . . 285

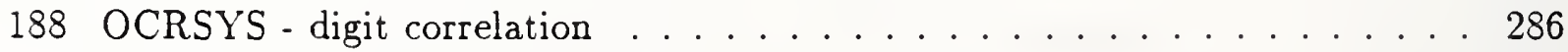

189 OCRSYS - upper case correlation . . . . . . . . . . . . . 287

190 OCRSYS - lower case correlation . . . . . . . . . . . . . . . 288

191 Error rate versus rejection rate for REI . . . . . . . . . . . . . . . 291 
192 Error rate per writer of REI . . . . . . . . . . . . . . . . . 291

193 REI - digit correlation . . . . . . . . . . . . . . . . . . 292

194 REI - upper case correlation . . . . . . . . . . . . . . . . . 293

195 REI - lower case correlation . . . . . . . . . . . . . . . . . . 294

196 Error rate versus rejection rate for RISO . . . . . . . . . . . . . . 297

197 Error rate per writer of RISO . . . . . . . . . . . . . . . . . 297

198 RISO - digit correlation . . . . . . . . . . . . . . . . 298

199 RISO - upper case correlation . . . . . . . . . . . . . . . . . . . 299

200 RISO - lower case correlation . . . . . . . . . . . . . . . 300

201 Error rate versus rejection rate for SYMBUS . . . . . . . . . . . . 303

202 Error rate per writer of SYMBUS . . . . . . . . . . . . . . 303

203 SYMBUS - digit correlation . . . . . . . . . . . . . . . 304

204 SYMBUS - upper case correlation . . . . . . . . . . . . 305

205 SYMBUS - lower case correlation . . . . . . . . . . . . 306

206 Error rate versus rejection rate for THINK_1 . . . . . . . . . . . . 309

207 Error rate per writer of THINK_1 . . . . . . . . . . . . . . 309

208 THINK_1 - digit correlation . . . . . . . . . . . . . . 310

209 THINK_1 - upper case correlation . . . . . . . . . . . . . . 311

210 THINK_1 - lower case correlation . . . . . . . . . . . . . . 312

211 Error rate versus rejection rate for UBOL . . . . . . . . . . . . . . 315

212 Error rate per writer of UBOL . . . . . . . . . . . . . . . . 315

213 UBOL - digit correlation . . . . . . . . . . . . . . . . . . 316

214 UBOL - upper case correlation . . . . . . . . . . . . . . . . 317

215 UBOL - lower case correlation . . . . . . . . . . . . . . . . 318

216 Error rate versus rejection rate for UPENN . . . . . . . . . . . . . . 321

217 Error rate per writer of UPENN . . . . . . . . . . . . . . . . 321

218 UPENN - digit correlation . . . . . . . . . . . . . . . . . 322

219 UPENN - upper case correlation . . . . . . . . . . . . . . . 323

220 UPENN - lower case correlation . . . . . . . . . . . . . . . . . 324

221 Error rate versus rejection rate for VALEN_1 . . . . . . . . . . . . 327

222 Error rate per writer of VALEN $1 \ldots \ldots \ldots . \ldots \ldots 327$

223 VALEN_1 - digit correlation . . . . . . . . . . . . . . 328

224 VALEN_1 - upper case correlation . . . . . . . . . . . . . . . 329 
225 VALEN_1 - lower case correlation . . . . . . . . . . . . . . 330

226 Error rate versus rejection rate for $\mathrm{NIST}_{4} 4 \ldots \ldots \ldots . \ldots . \ldots . . \ldots 334$

227 Error rate per writer of NIST_4 . . . . . . . . . . . . . 334

228 NIST_4 - digit correlation . . . . . . . . . . . . . 335

229 NIST_4 - upper case correlation . . . . . . . . . . . . . 336

230 NIST_4 - lower case correlation . . . . . . . . . . . . . 337

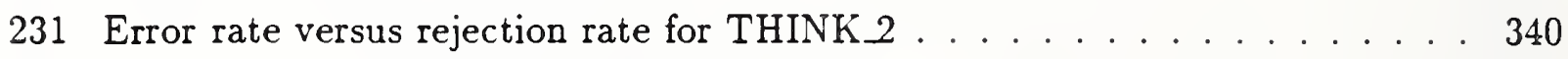

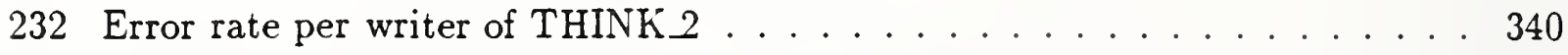

233 THINK 2 - digit correlation . . . . . . . . . . . . . 341

234 THINK 2 - upper case correlation . . . . . . . . . . . . . . 342

235 THINK 2 - lower case correlation . . . . . . . . . . . . . . 343

236 Error rate versus rejection rate for $\mathrm{UMICH}_{-1} \ldots \ldots \ldots \ldots$

237 Error rate per writer of UMICH_1 . . . . . . . . . . . . . 346

238 UMICH_1 - digit correlation . . . . . . . . . . . . 347

239 UMICH_1 - upper case correlation . . . . . . . . . . . . . . 348

240 UMICH_1 - lower case correlation . . . . . . . . . . . . . . . . . 349

241 Error rate versus rejection rate for VALEN $2 \ldots \ldots \ldots 352$

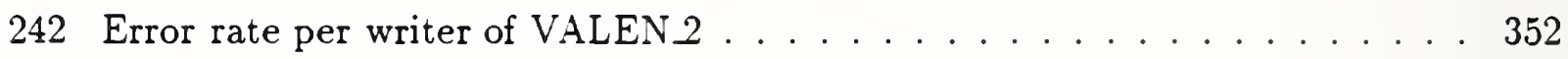

243 VALEN 2 - digit correlation . . . . . . . . . . . . . 353

244 VALEN 2 - upper case correlation . . . . . . . . . . . . . 354

245 VALEN 2 - lower case correlation . . . . . . . . . . . . . . 355 


\section{List of Tables}

1 List of participants, system names, tests, and references . . . . . . . . 7

2 List of participants, system names, tests, and references . . . . . . . . . 8

3 Mean zero-rejection-rate error rates and standard deviations in percent calculated over 10 partitions of TD1. . . . . . . . . . . . 9

4 Inter and Intra database Cross Validation Recognition Errors for Digits . . . 25

5 Inter and Intra database Cross Validation Recognition Errors for Uppers . . 26

$6 \quad$ Inter and Intra database Cross Validation Recognition Errors for Lowers . . 27

7 Parameters of fit over range from 0 to $14 \%$ of model to error versus rejection rate curves for systems submitting classifications and confidences values for the digit test.

8 Parameters of fit over range from 0 to $14 \%$ of model to error versus rejection rate curves for systems submitting classifications and confidences values for the upper case letter test.

9 Parameters of fit over range from 0 to $14 \%$ of model to error versus rejection rate curves for systems submitting classifications and confidences values for the lower case letter test. . . . . . . . . . . . . . . . . . . 35

10 System times in seconds for 2100 forms on a parallel computer. . . . . . . . . 43

11 AEG correlation graph key for digits. . . . . . . . . . . . . . 76

12 AEG correlation graph key for uppers. . . . . . . . . . . . . . 77

13 AEG correlation graph key for lowers. . . . . . . . . . . . . . 78

14 ASOL correlation graph key for digits. . . . . . . . . . . . . 82

15 ASOL correlation graph key for uppers. . . . . . . . . . . . . . . 83

16 ASOL correlation graph key for lowers. . . . . . . . . . . . . . 84

17 ATT_1 correlation graph key for digits. . . . . . . . . . . . 88

18 ATT_1 correlation graph key for uppers. . . . . . . . . . . . . . . . 89

19 ATT_1 correlation graph key for lowers. . . . . . . . . . . . . . . 90

20 ATT 2 correlation graph key for digits. . . . . . . . . . . . . . 94

21 ATT 2 correlation graph key for uppers. . . . . . . . . . . . . 95

22 ATT 2 correlation graph key for lowers. . . . . . . . . . . . . . . . 96

23 ATT_3 correlation graph key for digits. . . . . . . . . . . . . . . 100

24 ATT_3 correlation graph key for uppers. . . . . . . . . . . . . . . . 101

25 ATT_3 correlation graph key for lowers. . . . . . . . . . . . . . . . . 102

26 ATT_4 correlation graph key for digits. . . . . . . . . . . . . . . 106 
27 ATT_4 correlation graph key for uppers. . . . . . . . . . . . . . . 107

28 ATT_4 correlation graph key for lowers. . . . . . . . . . . . . . . 108

29 COMCOM correlation graph key for digits. . . . . . . . . . . . . . . 112

30 COMCOM correlation graph key for uppers. . . . . . . . . . . . . . 113

31 COMCOM correlation graph key for lowers. . . . . . . . . . . . . . . 114

32 ELSAGB_1 correlation graph key for digits. . . . . . . . . . . . . 118

33 ELSAGB_1 correlation graph key for uppers. . . . . . . . . . . . . . . . 119

34 ELSAGB_1 correlation graph key for lowers. . . . . . . . . . . . . . . 120

35 ELSAGB_2 correlation graph key for digits. . . . . . . . . . . . . . . . 124

36 ELSAGB_2 correlation graph key for uppers. . . . . . . . . . . . . 125

37 ELSAGB_2 correlation graph key for lowers. . . . . . . . . . . . . 126

38 ELSAGB_3 correlation graph key for digits. . . . . . . . . . . . . 130

39 ELSAGB_3 correlation graph key for uppers. . . . . . . . . . . . . . . 131

40 ELSAGB_3 correlation graph key for lowers. . . . . . . . . . . . . . . 132

41 ERIM_1 correlation graph key for digits. . . . . . . . . . . . . . 136

42 ERIM_1 correlation graph key for uppers. . . . . . . . . . . . . . 137

43 ERIM_1 correlation graph key for lowers. . . . . . . . . . . . . . . 138

44 ERIM_2 correlation graph key for digits. . . . . . . . . . . . . . . . . . . 142

45 ERIM_2 correlation graph key for uppers. . . . . . . . . . . . . . . . 143

46 ERIM_2 correlation graph key for lowers. . . . . . . . . . . . . . . . . . 144

47 GMD_1 correlation graph key for digits. . . . . . . . . . . . . . . . 148

48 GMD_1 correlation graph key for uppers. . . . . . . . . . . . . . . . . 149

49 GMD_1 correlation graph key for lowers. . . . . . . . . . . . . . . 150

50 GMD_2 correlation graph key for digits. . . . . . . . . . . . . . . . 154

51 GMD_2 correlation graph key for uppers. . . . . . . . . . . . . . . . 155

52 GMD_2 correlation graph key for lowers. . . . . . . . . . . . . . 156

53 GMD_3 correlation graph key for digits. . . . . . . . . . . . . . 160

54 GMD_3 correlation graph key for uppers. . . . . . . . . . . . . . . . 161

55 GMD_3 correlation graph key for lowers. . . . . . . . . . . . . . . 162

56 GMD_4 correlation graph key for digits. . . . . . . . . . . . . 166

57 GMD_4 correlation graph key for uppers. . . . . . . . . . . . 167

58 GMD_4 correlation graph key for lowers. . . . . . . . . . . . . . . 168

59 GTESS_1 correlation graph key for digits. . . . . . . . . . . . . 172 
60 GTESS_1 correlation graph key for uppers. . . . . . . . . . . . . . . 173

61 GTESS _1 correlation graph key for lowers. . . . . . . . . . . . . . . . . . 174

62 GTESS_2 correlation graph key for digits. . . . . . . . . . . . . . . 178

63 GTESS_2 correlation graph key for uppers. . . . . . . . . . . . . . . . 179

64 GTESS_2 correlation graph key for lowers. . . . . . . . . . . . . . . . 180

65 HUGHES_1 correlation graph key for digits. . . . . . . . . . . . . . . . . 184

66 HUGHES _1 correlation graph key for uppers. . . . . . . . . . . . . . 185

67 HUGHES_1 correlation graph key for lowers. . . . . . . . . . . . . . 186

68 HUGHES_2 correlation graph key for digits. . . . . . . . . . . . . . 190

69 HUGHES_2 correlation graph key for uppers. . . . . . . . . . . . . . . 191

70 HUGHES 2 correlation graph key for lowers. . . . . . . . . . . . . . . . 192

71 IBM correlation graph key for digits. . . . . . . . . . . . . . . . 196

72 IBM correlation graph key for uppers. . . . . . . . . . . . . . . . 197

73 IBM correlation graph key for lowers. . . . . . . . . . . . . . . . 198

74 IFAX correlation graph key for digits. . . . . . . . . . . . . . . 202

75 IFAX correlation graph key for uppers. . . . . . . . . . . . . . . . 203

76 IFAX correlation graph key for lowers. . . . . . . . . . . . . . . . . 204

77 KAMAN_1 correlation graph key for digits. . . . . . . . . . . . . . . 208

78 KAMAN_l correlation graph key for uppers. . . . . . . . . . . . . . . 209

79 KAMAN_1 correlation graph key for lowers. . . . . . . . . . . . . . . 210

80 KAMAN2 correlation graph key for digits. . . . . . . . . . . . . . . 214

81 KAMAN_2 correlation graph key for uppers. . . . . . . . . . . . . . 215

82 KAMAN 2 correlation graph key for lowers. . . . . . . . . . . . . . 216

83 KAMAN_3 correlation graph key for digits. . . . . . . . . . . . . . 220

84 KAMAN_3 correlation graph key for uppers. . . . . . . . . . . . . . . 221

85 KAMAN_3 correlation graph key for lowers. . . . . . . . . . . . . . . . 222

86 KAMAN_4 correlation graph key for digits. . . . . . . . . . . . . . 226

87 KAMAN_4 correlation graph key for uppers. . . . . . . . . . . . . . 227

88 KAMAN_4 correlation graph key for lowers. . . . . . . . . . . . . . . 228

89 KAMAN_5 correlation graph key for digits. . . . . . . . . . . . . . . 232

90 KAMAN_5 correlation graph key for uppers. . . . . . . . . . . . . . . 233

91 KAMAN_5 correlation graph key for lowers. . . . . . . . . . . . . . . 234

92 KODAK $\_$correlation graph key for digits. . . . . . . . . . . . 238 
93 KODAK $\_$correlation graph key for uppers. . . . . . . . . . . . . . 239

$94 \mathrm{KODAK} \_$correlation graph key for lowers. . . . . . . . . . . . . 240

95 KODAK 2 correlation graph key for digits. . . . . . . . . . . . . . . 244

96 KODAK 2 correlation graph key for uppers. . . . . . . . . . . . 245

97 KODAK 2 correlation graph key for lowers. . . . . . . . . . . 246

98 MIME correlation graph key for digits. . . . . . . . . . . . . . 250

99 MIME correlation graph key for uppers. . . . . . . . . . . . . . . . . 251

100 MIME correlation graph key for lowers. . . . . . . . . . . . . . . . 252

101 NESTOR correlation graph key for digits. . . . . . . . . . . . . 256

102 NESTOR correlation graph key for uppers. . . . . . . . . . . . . 257

103 NESTOR correlation graph key for lowers. . . . . . . . . . . . . 258

104 NIST_1 correlation graph key for digits. . . . . . . . . . . . . . . 262

105 NIST_1 correlation graph key for uppers. . . . . . . . . . . . . . . . 263

106 NIST_1 correlation graph key for lowers. . . . . . . . . . . . . . . 264

107 NIST 2 correlation graph key for digits. . . . . . . . . . . . . 268

108 NIST 2 correlation graph key for uppers. . . . . . . . . . . . . . 269

109 NIST 2 correlation graph key for lowers. . . . . . . . . . . . . . 270

110 NIST_3 correlation graph key for digits. . . . . . . . . . . . . . . . . 274

111 NIST_3 correlation graph key for uppers. . . . . . . . . . . . . . . . 275

112 NIST_3 correlation graph key for lowers. . . . . . . . . . . . . 276

113 NYNEX correlation graph key for digits. . . . . . . . . . . . . . 280

114 NYNEX correlation graph key for uppers. . . . . . . . . . . . . . 281

115 NYNEX correlation graph key for lowers. . . . . . . . . . . . . . 282

116 OCRSYS correlation graph key for digits. . . . . . . . . . . . 286

117 OCRSYS correlation graph key for uppers. . . . . . . . . . . . 287

118 OCRSYS correlation graph key for lowers. . . . . . . . . . . . . . 288

119 REI correlation graph key for digits. . . . . . . . . . . . . . . . . . . 292

120 REI correlation graph key for uppers. . . . . . . . . . . . . . 293

121 REI correlation graph key for lowers. . . . . . . . . . . . . . . . . 294

122 RISO correlation graph key for digits. . . . . . . . . . . . . . . . . 298

123 RISO correlation graph key for uppers. . . . . . . . . . . . . . . . . . 299

124 RISO correlation graph key for lowers. . . . . . . . . . . . . . . . 300

125 SYMBUS correlation graph key for digits. . . . . . . . . . . . . 304 
126 SYMBUS correlation graph key for uppers. . . . . . . . . . . . . . 305

127 SYMBUS correlation graph key for lowers. . . . . . . . . . . . . . 306

128 THINK_1 correlation graph key for digits. . . . . . . . . . . . . . 310

129 THINK_1 correlation graph key for uppers. . . . . . . . . . . . . . 311

130 THINK_1 correlation graph key for lowers. . . . . . . . . . . . . . 312

131 UBOL correlation graph key for digits. . . . . . . . . . . . . 316

132 UBOL correlation graph key for uppers. . . . . . . . . . . . . . 317

133 UBOL correlation graph key for lowers. . . . . . . . . . . . . . . . 318

134 UPENN correlation graph key for digits. . . . . . . . . . . . . . 322

135 UPENN correlation graph key for uppers. . . . . . . . . . . . . . 323

136 UPENN correlation graph key for lowers. . . . . . . . . . . . . . . . 324

137 VALEN_1 correlation graph key for digits. . . . . . . . . . . . . . 328

138 VALEN_l correlation graph key for uppers. . . . . . . . . . . . . . . . . 329

139 VALEN_1 correlation graph key for lowers. . . . . . . . . . . . . 330

140 NIST_4 correlation graph key for digits. . . . . . . . . . . . . 335

141 NIST_4 correlation graph key for uppers. . . . . . . . . . . . . . . . 336

142 NIST_4 correlation graph key for lowers. . . . . . . . . . . . . . . 337

143 THINK_2 correlation graph key for digits. . . . . . . . . . . . . . . 341

144 THINK_2 correlation graph key for uppers. . . . . . . . . . . . . . . 342

145 THINK 2 correlation graph key for lowers. . . . . . . . . . . . . . . 343

146 UMICH_1 correlation graph key for digits. . . . . . . . . . . . . 347

147 UMICH_l correlation graph key for uppers. . . . . . . . . . . . . . . 348

148 UMICH_1 correlation graph key for lowers. . . . . . . . . . . . . . . . 349

149 VALEN 2 correlation graph key for digits. . . . . . . . . . . . 353

150 VALEN 2 correlation graph key for uppers. . . . . . . . . . . . . 354

151 VALEN 2 correlation graph key for lowers. . . . . . . . . . . . 355 



\section{Executive Summary}

Bob Hammond

\subsection{Background}

Since 1790, the United States has conducted a decennial census, or head count, of the American population. Over the last century, growth in the population and demand for quicker tabulations have presented very strenuous tasks for data capture and information technology. In the late 1800 's, tabulating machines with punched cards were invented for Census use. In the 1950's, staff at Census and NBS helped develop the UNIVAC for general purpose computing. About the same time, they jointly developed the first optical scanning device for high speed mark recognition of microfilm.

For almost three decades, staff at the Census Bureau have heard claims that machine recognition of handwriting was just around the technological corner. However, a careful review of most claims showed that the corner was still a long way off. Recent advances in recognition of machine print and improvements in microprocessor performance have renewed optimism for machine recognition of hand print. In the late 1980's, the Census Bureau enlisted the Image Recognition Group (IRG) at the National Institute for Standards and Technology (NIST) to help evaluate these claims more closely.

\subsection{The Conference}

After several years of research, the NIST/IRG had developed a working prototype along with various methods to measure the performance of other systems. After the 1990 Census, NIST and Census decided to sponsor a scientific experiment and conference (hereafter referred to as the Conference) to determine the state of the art in this industry. NIST and Census formed a Committee having representatives from government, industry, and academia to organize the Conference, and NIST personnel ran the Conference.

Twenty nine different groups from North America and Europe responded to the call for participation. Each party received an image data base of segmented, hand-printed, alpha and numeric characters for training their systems. Later, each party received a similar database for test purposes. Each attempted to recognize the characters, and all but three submitted their results to NIST for scoring. In late May 1992, all parties that submitted results convened in Gaithersburg, Maryland to discuss the results. Scientific and academic participation was encouraged, and marketing interests were discouraged. Attendance was strictly limited to sponsors, participants, and up to two associates designated by each participant, along with a few observers from federal agencies (FBI, IRS, USPS) that are currently sponsoring work in the field.

The Conference and related exercises focused on a single step in the process: machine recognition of individual (or segmented) characters with no context. With the single variable nature of this study, no valid comparisons can be made regarding cost or performance of 
systems designed to process entire forms or documents. Further, the efforts of participants were not proctored or monitored in any way by Census or NIST staff.

\subsection{Conclusions}

NIST and Census are in no way responsible for how these results may be used. NIST made every effort to assure the accuracy of the measures computed from the submissions by the participants. Nevertheless, NIST and Census are aware that different tests, which may be more pertinent to real applications, might give different results than those reported here, and that other analyses of the submissions might give more complete results than those reported here.

While some results from this Conference may appear in marketing literature, under no circumstances should potential buyers use data from this study as a primary basis for purchasing decisions. Census and NIST can make only one recommendation to potential buyers: use your own application-specific data to thoroughly test the performance of any system (or component) in a realistic setting.

The Conference resulted in the following general conclusions:

A bout half of the systems correctly recognized over $95 \%$ of the digits, over $90 \%$ of the upper case letters, and over $80 \%$ of the lower case letters in the test. For comparison, a human correctly recognized about $98.5 \%$ of the test digits. (Chapters 3 and 4 discuss the test data, scoring, and error rates in detail.)

While machine recognition of segmented digits appears to be approaching the level of human performance, one should not extrapolate this conclusion to the performance on unconstrained input, which is a much more difficult problem.

Further research, development, and testing on realistic sources of hand-printing is needed to determine the cost and practicality of this technology. Many participants said they learned of new techniques at the Conference that will help them improve their system's performances, but potential buyers should use their unique, application-specific data to thoroughly test the performance of any system (or component) in a realistic setting. Discussions about differences in the training data and the test data suggests that various systems may perform differently with only slight changes in the source data.

For scientific reasons, future efforts to measure performance of competing systems would isolate the source and extent of error resulting from each step of the recognition process. However, given the wide variety of approaches to various steps in the process, this incremental approach to the research may be impractical.

\subsection{Organization of this Report}

The Introduction in Chapter 2 provides an overview of the Conference, materials, theories, and methods used in the effort. Chapters 3 and 4 discuss in more technical detail the theory and results of metrics used for scoring and evaluation of results. Chapter 5 attempts to 
lay out a taxonomy of approaches to optical character recognition systems, while Chapter 6 qualifies and discusses various considerations on the speed measures of the Conference. The various appendices offer original copy from the initial call for participation and instructional material, discussions of lessons learned (and problems encountered), and detailed descriptions and scoring results of all 118 submissions. 


\section{Introduction}

Jon Geist

The goals of the First Census Optical Character Recognition (OCR) Systems Conference were scientific in nature. The first goal was to gauge the state of the art of OCR of handprinted characters with respect to the particular problems associated with entering census data into a computer database. The second was to learn what is currently limiting the state of the art. The third goal was to determine whether new databases of handprinted characters for use either in training or in testing could be expected to help to improve the state of the art of OCR for applications such as the census, and if so, what types of new databases are needed.

It was decided that a test open to organizations having strong OCR programs would be a cost-efficient tool for meeting these goals. This would allow comparison of the results from a wide variety of systems, algorithms, features, and preprocessing. Unfortunately, it would not be possible to control the variables as well as might otherwise be desirable with this type of experiment, but comparison of the results from a broad range of systems was thought to be more important than comparison of the results obtained from different variations of a single type of system.

The full Census OCR task consists of document handling, form identification, field isolation, character segmentation, character recognition, and context-based field correction. However, the recognition of segmented characters has been considered the bellwether of OCR progress for some time. Therefore, it seemed desirable to limit the test to this subtask, both to establish a baseline for this capability before considering more complex combinations of subtasks, and to test recent thinking that the recognition of segmented characters is no longer the accuracy-limiting subtask for hand-print OCR.

This decision required postponing tests that are more typical of the full Census OCR task for future conferences. The test that was implemented consisted of classifying about 85000 binary images of segmented characters that were distributed on a CD-ROM. All participants received identical tests, and none had seen any of the images on the CD before receiving it. The Conference had no marketing goals. In particular, the test was not proctored, and neither the capabilities tested nor the test images were representative of any commercial application. Also, participants were implicitly encouraged to carry out experiments that promoted the scientific goals of the Conference, but which might not contribute to optimum system performance. For instance, a training $C D$ that contained over 300000 binary images of segmented characters was provided to the participants in advance of the test $C D$, but the participants were not required to train their OCR systems on the characters on this CD. Instead they could use their own training data, and use the training $C D$ only to experiment with the data formats that would be used on the test CD. Nevertheless, many of the participants chose to train on subsets of the characters on the training CD rather than on internal databases that might have given better results.

The results of this test should not be used as the basis for purchasing an OCR system. Anyone who does base a purchase on these results will probably encounter a number of 
serious problems. Decisions regarding applying an OCR system to some specific task should be based on the results of proctored tests with test materials that are typical of that task. On the other hand, the methodologies developed for this Conference and the results obtained from this Conference should prove quite useful in designing tests, both large and small, to support purchasing decisions.

\subsection{Organization of the Conference}

The Conference was organized by a Committee consisting of the following individuals:

Bob Hammond, Robert Creecy, and Norman W. Larsen, US Bureau of the Census

Charles L. Wilson and Jon Geist, National Institute of Standards and Technology

Dr. Jonathan J. Hull, Center of Excellence for Document Analysis And Recognition

Dr. Thomas P. Vogl, Environmental Research Institute of Michigan

Dr. Christopher J. C. Burges, AT\&T Bell Laboratories

Jon Geist, the Committee Chariman, handled the planning of the Conference and the majority of the interaction with the participants. The Conference was run for the Committee by the Image Recognition Group (IRG) at the National Institute of Standards and Technology (NIST) under contract to the US Bureau of the Census. Bob Hammond administered the contract supporting this Conference.

The following individuals from the NIST IRG were instrumental in carrying out the work of the Conference. Charles Wilson, the Leader of the NIST IRG, assured that resources were available when needed. Allen Wilkinson coordinated the technical activities of the Conference including the preparation of training and test materials, the receipt of participant submissions, and software trouble shooting. Stan Janet scored all of the submissions. Michael Garris designed and helped implement a new procedure for classifying the character images on the test CD to take maximum advantage of the NIST IRG OCR system while still assuring that every classification was checked by a human. Patrick Grother provided valuable advice on various aspects of the Conference based on his role as a participant representing the NIST IRG OCR system.

A Call for Participation was prepared and issued on behalf of the Committee as the first activity of the Conference. A version of the Call is reproduced in Appendix C. NIST Special Database 3 (SD3)[1] was sent to the participants to familiarize them with the test data formats and for possible use as training data. A preprint of the documentation for that database was sent as instructional material at the same time. NIST Test Data 1 (TD1)[2] was sent to each of the participants as the test data. Instructions for the test phase of the Conference were sent with TD1. These are reproduced in Appendix D.

Twenty nine organizations agreed to participate in the Conference. Three organizations: HNC, San Diego, CA; Scan Optics, East Hartford, CT; and the University of Massachusetts at Lowell, chose not to return test results after receiving the training and test materials. The remaining twenty six participants, representing over 45 different systems, returned over 115 submissions for scoring. These participants, the names assigned by NIST to the systems for 
which they submitted results, the type of results submitted, and pertinent references where available are summarized in Tables 1 and 2 . The detailed activities that occurred before the meeting phase of the Conference are described in the Appendices mentioned above.

Since this was the first conference of its kind being run by the NIST IRG, a number of problems were encountered. These are discussed in Appendices A and B of this report. The former is a list of issues raised by the participants during the Conference meeting. The latter presents, from the NIST perspective, a short list of problems along with possible solutions proposed by various individuals. A short discussion is also included for each problem, both to provide background information, and to indicate how practical each proposed solutions appears.

\subsection{Summary of Results}

Classification, rejection, confidence, and error are general ideas of importance in OCR. The following definitions of these and related terms will be used throughout this report.

A classification process assigns an ASCII character to an image of a character. The classification may be correct or incorrect.

A rejection process divides a set of classifications into rejected classifications and accepted classifications. Only the accepted classifications are considered useful.

Usually, the rejection mechanism is applied after the classification process. However, some rejection processes work in parallel with the classification process, and no classification is assigned when a character is rejected. Most systems that carry out the rejection process after the classification process produce what is called a confidence for each classification. This is a number (usually between zero and one) that orders the classifications according to expected reliability.

The rejection rate for a set of character classifications is defined as the ratio of the number of characters rejected by the rejection process to the total number of characters presented for classification. For convenience, this report will refer to classifications that are not rejected by the classification process at any given rejection rate as unrejected or accepted classifications.

If a confidence is associated with each classification, any desired rejection rate can be obtained by choosing the correct value for the confidence threshold and rejecting any classifications having confidences less than or equal to the threshold and accepting any classifications having confidences greater than the threshold.

The error rate for a set of classifications is defined as the fraction of the unrejected (accepted) characters that are classified incorrectly. Therefore, the error rate varies as a function of the rejection rate.

Table 3 lists the zero-rejection-rate error rates for all of the OCR results submitted to the Conference. As a very rough summary, about half of the systems produced error rates of less than $5 \%$ at zero rejection rate for digits, about half produced error rates of less than $10 \%$ at zero rejection rate for upper case letters, and about half produced error rates of less than $20 \%$ at zero rejection rate for lower case letters. 


\begin{tabular}{|c|c|c|c|c|c|}
\hline $\begin{array}{l}\text { PARTICIPATING } \\
\text { ORGANIZATION }\end{array}$ & SYSTEM & $\overline{\overline{\text { DIGIT }}}$ & $\overline{\text { UPPER }}$ & $\overline{\text { LOWER }}$ & REFERENCES \\
\hline $\begin{array}{l}\overline{A E} \bar{G} \text { Electrocom GmbH } \\
\text { Konstanz, Germany }\end{array}$ & $\overline{\mathrm{AEG}}$ & $\overline{\mathrm{X}}$ & $\overline{\bar{X}}$ & $\overline{\bar{X}}$ & \\
\hline $\begin{array}{l}\text { Adaptive Solutions, Inc. } \\
\text { Beaverton, OR }\end{array}$ & ASOL & $\mathrm{X}$ & $\mathrm{X}$ & $\mathrm{X}$ & {$[3][4]$} \\
\hline AT\&T Bell Laboratories & ATT_1 & $\mathrm{X}$ & $\mathrm{X}$ & $\mathrm{X}$ & {$[5][6][7][8]$} \\
\hline \multirow[t]{3}{*}{ Holmdel, NJ } & $\mathrm{ATT} 2$ & $\mathrm{X}$ & $\mathrm{X}$ & $\mathrm{X}$ & {$[5][6][7][8]$} \\
\hline & ATT_3 & $\mathrm{x}$ & $\mathrm{X}$ & $\mathrm{X}$ & {$[9][10]$} \\
\hline & ATT_4 & $\mathrm{X}$ & $\mathrm{X}$ & $\mathrm{X}$ & {$[5][6][7][8]$} \\
\hline $\begin{array}{l}\text { Com Com Systems, Inc. } \\
\text { Clearwater, FL }\end{array}$ & COMCOM & $\mathrm{X}$ & $\mathrm{X}$ & $\mathrm{X}$ & \\
\hline ELSAG BAILEY, INC. & ELSAGB_1 & $\mathrm{X}$ & & & \\
\hline Conshohocken, PA & ELSAGB 2 & $\mathrm{X}$ & & & \\
\hline & ELSAGB_3 & $\mathrm{X}$ & & & \\
\hline Environmental Research & ERIM_1 & $\mathrm{X}$ & $\mathrm{X}$ & $\mathrm{X}$ & {$[11][12][13][14]$} \\
\hline $\begin{array}{l}\text { Institute of Michigan } \\
\text { Ann Arbor, MI }\end{array}$ & ERIM_2 & $\mathrm{X}$ & & & {$[11][12][13][14]$} \\
\hline Gesellschaft für & GMD_1 & $\mathrm{X}$ & $\mathrm{X}$ & $\mathrm{X}$ & {$[15][16]$} \\
\hline Mathematik und & GMD 2 & $\mathrm{X}$ & $\mathrm{x}$ & $\mathrm{X}$ & {$[17][18]$} \\
\hline Datenverarbeitung & GMD_3 & $\mathrm{X}$ & $\mathrm{X}$ & $\mathrm{x}$ & {$[15][16]$} \\
\hline Sankt Augustin, Germany & GMD_4 & $\mathrm{X}$ & $\mathrm{X}$ & $\mathrm{X}$ & {$[15][16]$} \\
\hline GTESS Corporation & GTESS_1 & $\overline{\mathrm{X}}$ & $\mathrm{X}$ & $\mathrm{X}$ & \\
\hline Richardson, TX & GTESS_2 & $\mathrm{x}$ & $\mathrm{X}$ & $\mathrm{X}$ & \\
\hline Hughes Aircraft Company & HUGHES_I & $\overline{\mathrm{X}}$ & $\bar{X}$ & $\bar{X}$ & [19] \\
\hline Canoga Park, CA & HUGHES_2 & $\mathrm{X}$ & $\mathrm{X}$ & $\mathrm{X}$ & [19] \\
\hline $\begin{array}{l}\text { IBM Almaden } \\
\text { Research Center, } \\
\text { San Jose, CA }\end{array}$ & IBM & $\mathrm{X}$ & $\mathrm{X}$ & $\mathrm{X}$ & $\begin{array}{l}{[20][21][22]} \\
{[23][24][25][26]}\end{array}$ \\
\hline $\begin{array}{l}\text { InterFax, Inc. } \\
\text { Sunnyvale, } \mathrm{CA}\end{array}$ & IFAX & $\overline{\mathrm{X}}$ & $\mathrm{X}$ & & \\
\hline Kaman Sciences & KAMAN_1 & $\mathrm{X}$ & $\mathrm{X}$ & $\bar{X}$ & \\
\hline Corporation & KAMAN_2 & $\mathrm{X}$ & $\mathrm{X}$ & $\mathrm{X}$ & \\
\hline Utica, NY & KAMAN_3 & $\mathrm{X}$ & $\mathrm{x}$ & $\mathrm{X}$ & \\
\hline & KAMAN_4 & $\mathrm{X}$ & $\mathrm{X}$ & $\mathrm{X}$ & \\
\hline & KAMAN_5 & $\mathrm{X}$ & $\mathrm{X}$ & $\mathrm{X}$ & \\
\hline
\end{tabular}

Table 1: List of participants, system names, tests, and references 


\begin{tabular}{|c|c|c|c|c|c|}
\hline $\begin{array}{l}\text { PARTICIPATING } \\
\text { ORGANIZATION }\end{array}$ & SYSTEM & $\overline{\text { DIGIT }}$ & UPPER & LOWER & REFERENCES \\
\hline $\begin{array}{l}\text { Eastman Kodak Co. } \\
\text { Rochester, NY }\end{array}$ & $\begin{array}{l}\text { KODAK_1 } \\
\text { KODAK_2 }\end{array}$ & $\begin{array}{l}\bar{X} \\
\mathrm{X}\end{array}$ & $\overline{\bar{X}}$ & $\overline{\bar{X}}$ & $\begin{array}{l}{[5][27][28]} \\
{[5][27][28]}\end{array}$ \\
\hline $\begin{array}{l}\text { Mimetics } \\
\text { Chatenay Malabry, } \\
\text { France }\end{array}$ & MIME & $\mathrm{X}$ & $\mathrm{X}$ & & {$[29][30]$} \\
\hline $\begin{array}{l}\text { Nestor, Inc. } \\
\text { Providence, RI }\end{array}$ & NESTOR & $\bar{X}$ & $\bar{X}$ & $\bar{X}$ & $\begin{array}{l}{[19][31][32]} \\
{[10][33][34][35]} \\
{[36][37][38]}\end{array}$ \\
\hline $\begin{array}{l}\text { National Institute of } \\
\text { Standards and Tech. } \\
\text { Gaithersburg, MD }\end{array}$ & $\begin{array}{l}\text { NIST_1 } \\
\text { NIST_2 } \\
\text { NIST_3 } \\
\text { NIST_4 }\end{array}$ & $\begin{array}{l}X \\
X \\
X \\
X\end{array}$ & $\begin{array}{l}\mathrm{X} \\
\mathrm{X} \\
\mathrm{X} \\
\mathrm{X}\end{array}$ & $\begin{array}{l}\mathrm{X} \\
\mathrm{X} \\
\mathrm{X} \\
\mathrm{X}\end{array}$ & $\begin{array}{l}{[39]} \\
{[40]} \\
{[41]} \\
{[42]} \\
\end{array}$ \\
\hline $\begin{array}{l}\text { NYNEX Sciences } \\
\text { \& Technology, Inc. } \\
\text { White Plains, NY }\end{array}$ & NYNEX & $\bar{X}$ & $\bar{X}$ & $\bar{X}$ & \\
\hline $\begin{array}{l}\text { OCR SYSTEMS, Inc. } \\
\text { Huntingdon Valley, PA }\end{array}$ & OCRSYS & $\bar{X}$ & $\bar{X}$ & $\bar{X}$ & \\
\hline $\begin{array}{l}\text { Recognition Equip. Inc. } \\
\text { Dallas, TX }\end{array}$ & REI & $\bar{X}$ & $\bar{X}$ & & \\
\hline $\begin{array}{l}\text { Riso National Lab. } \\
\text { Roskilde, Denmark }\end{array}$ & RISO & $\bar{X}$ & $\mathrm{X}$ & $\bar{X}$ & \\
\hline $\begin{array}{l}\text { Symbus Technology } \\
\text { Waltham, MA }\end{array}$ & SYMBUS & $\bar{X}$ & $\bar{X}$ & & \\
\hline $\begin{array}{l}\text { Thinking Machines } \\
\text { Corporation } \\
\text { Cambridge, MA }\end{array}$ & $\begin{array}{l}\text { THINK_1 } \\
\text { THINK_2 }\end{array}$ & $\begin{array}{l}\mathrm{X} \\
\mathrm{X}\end{array}$ & & & \\
\hline $\begin{array}{l}\text { University of Bologna } \\
\text { Bologna, Italy }\end{array}$ & $\overline{\mathrm{UBOL}}$ & $\mathrm{X}$ & $\mathrm{X}$ & $\bar{X}$ & $\begin{array}{l}{[43][44][45]} \\
{[46][47][48]} \\
{[49][12][6]}\end{array}$ \\
\hline $\begin{array}{l}\text { University of } \\
\text { Michigan-Dearborn } \\
\text { Dearborn, MI }\end{array}$ & UMICH_1 & & $\mathrm{X}$ & $\bar{X}$ & \\
\hline $\begin{array}{l}\text { University of Penn. } \\
\text { Philadelphia, PA }\end{array}$ & UPENN & $\mathrm{X}$ & $\mathrm{X}$ & $\mathrm{X}$ & $\begin{array}{l}{[50][51][37]} \\
{[52][5][53]} \\
{[54][55][56][57]}\end{array}$ \\
\hline $\begin{array}{l}\text { Universidad Politecnica } \\
\text { de Valencia } \\
\text { Valencia, Spain }\end{array}$ & $\begin{array}{l}\text { VALEN_1 } \\
\text { VALEN_2 }\end{array}$ & $\begin{array}{l}\mathrm{X} \\
\mathrm{X}\end{array}$ & $\bar{X}$ & $\mathrm{X}$ & $\begin{array}{l}{[58]} \\
{[59]}\end{array}$ \\
\hline
\end{tabular}

Table 2: List of participants, system names, tests, and references 


\begin{tabular}{|c|c|c|c|}
\hline Entered & \multicolumn{3}{|c|}{ Percentage Classification Error } \\
\hline System & Digits & Uppers & Lowers \\
\hline$\overline{\mathrm{AEG}}$ & $3.43 \pm 0.23$ & $3.74 \pm 0.82$ & $12.74 \pm 0.75$ \\
\hline ASOL & $8.91 \pm 0.39$ & $11.16 \pm 1.05$ & $21.25 \pm 1.36$ \\
\hline ATT_1 & $3.16 \pm 0.29$ & $6.55 \pm 0.66$ & $13.78 \pm 0.90$ \\
\hline ATT 2 & $3.67 \pm 0.23$ & $5.63 \pm 0.63$ & $14.06 \pm 0.95$ \\
\hline ATT 3 & $4.84 \pm 0.24$ & $6.83 \pm 0.86$ & $16.34 \pm 1.11$ \\
\hline ATT_4 & $4.10 \pm 0.16$ & $5.00 \pm 0.79$ & $14.28 \pm 0.98$ \\
\hline COMCOM & $4.56 \pm 0.91$ & $16.94 \pm 0.99$ & $48.00 \pm 1.87$ \\
\hline ELSAGB_1 & $5.07 \pm 0.32$ & & \\
\hline ELSAGB_2 & $3.38 \pm 0.20$ & & \\
\hline ELSAGB_3 & $3.35 \pm 0.21$ & & \\
\hline ERIM_1 & $3.88 \pm 0.20$ & $5.18 \pm 0.67$ & $13.79 \pm 0.80$ \\
\hline ERIM 2 & $3.92 \pm 0.24$ & & \\
\hline GMD_1 & $8.73 \pm 0.35$ & $14.04 \pm 1.00$ & $22.54 \pm 1.22$ \\
\hline GMD_2 & $15.45 \pm 0.64$ & $24.57 \pm 0.91$ & $28.61 \pm 1.25$ \\
\hline GMD_3 & $8.13 \pm 0.39$ & $14.22 \pm 1.09$ & $20.85 \pm 1.25$ \\
\hline GMD_4 & $10.16 \pm 0.35$ & $15.85 \pm 0.95$ & $22.54 \pm 1.22$ \\
\hline GTESS_1 & $6.59 \pm 0.18$ & $8.01 \pm 0.59$ & $17.53 \pm 0.75$ \\
\hline GTESS_2 & $6.75 \pm 0.30$ & $8.14 \pm 0.59$ & $18.42 \pm 1.09$ \\
\hline HUGHES_1 & $4.84 \pm 0.38$ & $6.46 \pm 0.52$ & $15.39 \pm 1.10$ \\
\hline HUGHES 2 & $4.86 \pm 0.35$ & $6.73 \pm 0.64$ & $15.59 \pm 1.08$ \\
\hline IBM & $3.49 \pm 0.12$ & $6.41 \pm 0.80$ & $15.42 \pm 0.95$ \\
\hline IFAX & $17.07 \pm 0.34$ & $19.60 \pm 1.26$ & \\
\hline KAMAN_1 & $11.46 \pm 0.41$ & $15.03 \pm 0.79$ & $31.11 \pm 1.15$ \\
\hline KAMAN_2 & $13.38 \pm 0.49$ & $20.74 \pm 0.88$ & $35.11 \pm 1.09$ \\
\hline KAMAN_3 & $13.13 \pm 0.45$ & $19.78 \pm 0.60$ & $33.55 \pm 1.37$ \\
\hline KAMAN_4 & $20.72 \pm 0.44$ & $27.28 \pm 1.30$ & $46.25 \pm 1.23$ \\
\hline KAMAN_5 & $15.13 \pm 0.41$ & $33.95 \pm 1.22$ & $42.20 \pm 0.96$ \\
\hline KODAK_1 & $4.74 \pm 0.37$ & $6.92 \pm 0.78$ & $14.49 \pm 0.77$ \\
\hline KODAK 2 & $4.08 \pm 0.26$ & & \\
\hline MIME & $8.57 \pm 0.34$ & $10.07 \pm 0.81$ & \\
\hline NESTOR & $4.53 \pm 0.20$ & $5.90 \pm 0.68$ & $15.39 \pm 0.90$ \\
\hline NIST_l & $7.74 \pm 0.31$ & $13.85 \pm 0.83$ & $18.58 \pm 1.12$ \\
\hline NIST_2 & $9.19 \pm 0.32$ & $23.10 \pm 0.88$ & $31.20 \pm 1.16$ \\
\hline NIST_3 & $9.73 \pm 0.29$ & $16.93 \pm 0.90$ & $20.29 \pm 0.99$ \\
\hline NIST_4 & $4.97 \pm 0.30$ & $10.37 \pm 1.28$ & $20.01 \pm 1.06$ \\
\hline NYNEX & $4.32 \pm 0.22$ & $4.91 \pm 0.79$ & $14.03 \pm 0.96$ \\
\hline OCRSYS & $1.56 \pm 0.19$ & $5.73 \pm 0.63$ & $13.70 \pm 0.93$ \\
\hline REI & $4.01 \pm 0.26$ & $11.74 \pm 0.90$ & \\
\hline RISO & $10.55 \pm 0.43$ & $14.14 \pm 0.88$ & $21.72 \pm 0.98$ \\
\hline SYMBUS & $4.71 \pm 0.38$ & $7.29 \pm 1.07$ & \\
\hline THINK_1 & $4.89 \pm 0.24$ & & \\
\hline THINK_2 & $3.85 \pm 0.33$ & & \\
\hline UBOL & $4.35 \pm 0.20$ & $6.24 \pm 0.66$ & $15.48 \pm 0.81$ \\
\hline UMICH_1 & & $5.11 \pm 0.94$ & $15.08 \pm 0.92$ \\
\hline UPENN & $9.08 \pm 0.37$ & & \\
\hline VALEN_l & $17.95 \pm 0.59$ & $24.18 \pm 1.00$ & $31.60 \pm 1.33$ \\
\hline VALEN_2 & $15.75 \pm 0.32$ & & \\
\hline
\end{tabular}

Table 3: Mean zero-rejection-rate error rates and standard deviations in percent calculated over 10 partitions of TD1. 
Figures 1 through 6 plot the error rate versus the rejection rate for digits, for upper case letters, and for lower case letters, respectively, for all of the systems. The odd numbered figures plot the error versus rejection curves that were obtained by applying thresholds to the confidence data provided for most of the systems. The even number figures plot the points obtained directly from rejection data provided for the remaining systems. Summaries describing the individual systems and more detailed results are included in Appendices $\mathrm{E}$ and $F$.

Table 3 contains uncertainties for the error rates presented there. These uncertainties were calculated by dividing the characters in Test Data 1 into ten sets of characters, each set being a contiguous block of the test materials. Each set contained a fixed number of classification hypotheses. Each set was scored separately for each submission and the mean and sample standard deviation of those means are recorded in the table. The recognition error percentages are the definitive scores for each conference participant. They are exact performance measures over the whole database. The standard deviation is a measure of the change expected for the given classifiers on alternative subsets of the test data. All results refer to zero-rejection-rate classification.

\subsection{Differences between Test and Training Databases}

Most participants pointed out that the characters in NIST TD1 seemed different from and harder to recognize than those in NIST SD3. One participant suggested a cross validation data study. The study is described in detail in Section 3. The results suggest that TD1 is significantly harder than SD3 for digits, but not significantly harder, only different, for upper and lower case letters. A more definitive study and supplemental studies using other systems seem warranted.

A possible explanation for the different levels of difficulty for the two sets of digit images is the different way that the two sets were obtained. NIST SD3 and NIST TD1 were obtained by segmenting the characters filled out in boxes on forms that were variations of that shown in Figure 7. The forms for SD3 were filled out and returned by 2100 of 3400 permanent Census field workers as part of the 1990 Census program. The forms for TD1 were filled out by math and science students in a high school as a short exercise during class. The 2100 Census workers who actually returned filled out forms to their employer were clearly more motivated than the 1300 who did not, and may be more motivated than the 500 high school students who were forced to fill out and return the forms in class. The attitudes of the high school students are probably more representative of the attitudes of the general population when filling out a Census form.

The individual characters on the forms obtained from the Census workers were isolated by a different segmenter than that used with the forms obtained from the high school students. The segmentor used for SD3 failed to successfully segment a much larger fraction of the characters presented to it than did the segmenter used to create TD1. If segmenters fail on the most difficult characters, then this could be another reason why TD1 appeared more difficult. 
All participants who used NIST SD3 for training thought that their error rate for TD1 would have been lower had they used a better training set. The Kodak and AEG systems both demonstrated this point in different ways. The only difference between the KODAK_1 and KODAK 2 system results in Appendix $E$ is the addition of sevens with crosses to SD3 for training. This one change reduced the zero-rejection-rate digit error rate from $4.7 \%$ to $4.1 \%$. AEG used SD3 for the Conference submission, but reran the digit test after the Conference using an internal database. This reduced the zero-rejection-rate error rate from $3.4 \%$ to $2.9 \%$. Both of these results are consistent with the cross validation results for digits described in Section 3 .

\subsection{Error Rates as a Function of Rejection Rates}

A casual glance at the curves in Figures 1 through 6 suggests that they all have very similar shapes. Section 4 derives the ideal error rate versus rejection rate curve, fits an empirical equation to the data in Figures 1,3, and 5, and derives the probability distributions that generate the ideal and empirical error rate versus rejection rate curves. As discussed in Section 4 there is a lot of room for improvement in the shapes of the error rate versus rejection rate curves produced by the OCR systems before they start to be limited by the ideal shape. First their slope at zero rejection rate is less negative than the ideal slope at zero rejection rate, and secondly the curvature on a log plot is in the wrong direction.

The last column in Tables 7,8 , and 9 in Section 4 lists the ratios of the slopes of the error rates at zero-rejection rate to the optimum slopes for all of the curves in Figures 1, 3, and 5. Notice that it apparently easy to get the ratio of the actual slope to the ideal slope greater than $30 \%$, but quite difficult to get it greater than $80 \%$.

The first 10000 digits of TD1 were presented without any indication as to the correct class and classified by a human. This process was carried out at about 2 characters per second for periods from one to two hours with many hours between the classification periods. The result was a zero-rejection-rate error rate of $1.57 \%$, which is very close to the digit rate for all of the digits in TD1 for the OCRSYS system. It is very interesting that this system obtained approximately the same zero-rejection-rate error rate as a human, while producing an error rate versus rejection rate that is much less satisfactory than those for the other OCR systems in the Conference.

\subsection{Other Topics}

Many different types of classifiers, feature extractors, and preprocessors were used (including no feature extractors or preprocessors). The only general conclusion that is evident so far is that good people can make almost anything work. Section 5 presents a taxonomy for OCR systems and some more detailed observations about the different types of systems tested.

Determining the state of the art with respect to system speed was not a goal of the Conference. Nevertheless, the participants were asked to provide the time from first CD-ROM access to last CD-ROM access for their system runs. Some participants provided these times, 
some provided times that were associated only with the actual OCR recognition task, some provided both, and some provided some intermediate times. The results are not meaningful enough to warrant any figures, but some general conclusions are listed in Section 6 .

\subsection{Conclusion}

Some preliminary conclusions of the Conference are listed below.

The state of the art of machine OCR of segmented, hand-printed digits is approaching human performance with respect to the zero-rejection-rate error rate. The results for upper case letters and lower case letters are probably not as good relative to human performance as the performance for digits, but no human classifications under the conditions of the Conference test have been conducted to address question.

The digits in NIST SD3 do not represent a heterogeneous enough sample of hand-printed digits for optimum training of OCR systems. The same is probably true for the letters in SD3, but studies comparable to that reported in Section 3 have yet to be carried out.

The fact that almost all of the systems give similar shape error rate versus rejection rate curves for the digit, upper case letter, and lower case letters suggests that these curves come about as close as can be expected to optimum with the data in NIST TD1. On the other hand, theoretical studies suggest that there is room for considerable improvement. Further research will be needed to resolve this paradox.

Many of the participants indicated that the Conference was a useful learning experience. This includes the NIST IRG. Following the Conference, several simple changes were made to the NIST_1 system. These changes converted a K-Nearest Neighbor (KNN) system to a Probabilistic Neural Network (PNN) system using Karhunen-Loève (KL) features on binary images obtained from simple preprocessing, and produced the NIST_4 system summarized in Appendix F. The improvement in performance, a $35 \%$ decrease in the zero-rejection-rate error rate for digits from $7.7 \%$ down to $5.0 \%$, is striking. It is noteworthy that the changes giving this level of improvement were not only easily implemented, but they were more of the nature of improvements in what was already being done rather than the introduction of new or different approaches.

Most of the participants and Committee members believe that the results of this Conference were more than sufficient to justify a Conference on isolated fields, but there was less of a consensus on exactly what sort of isolated fields were appropriate for the next test. When forced to choose between two extremes, the participants overwhelmingly preferred digital images of the microfilmed occupation and industry fields on real Census forms along with a dictionary of allowed answers rather than artificial fields of random letters digitized from forms such as were used for NIST SD3 and TD1. 


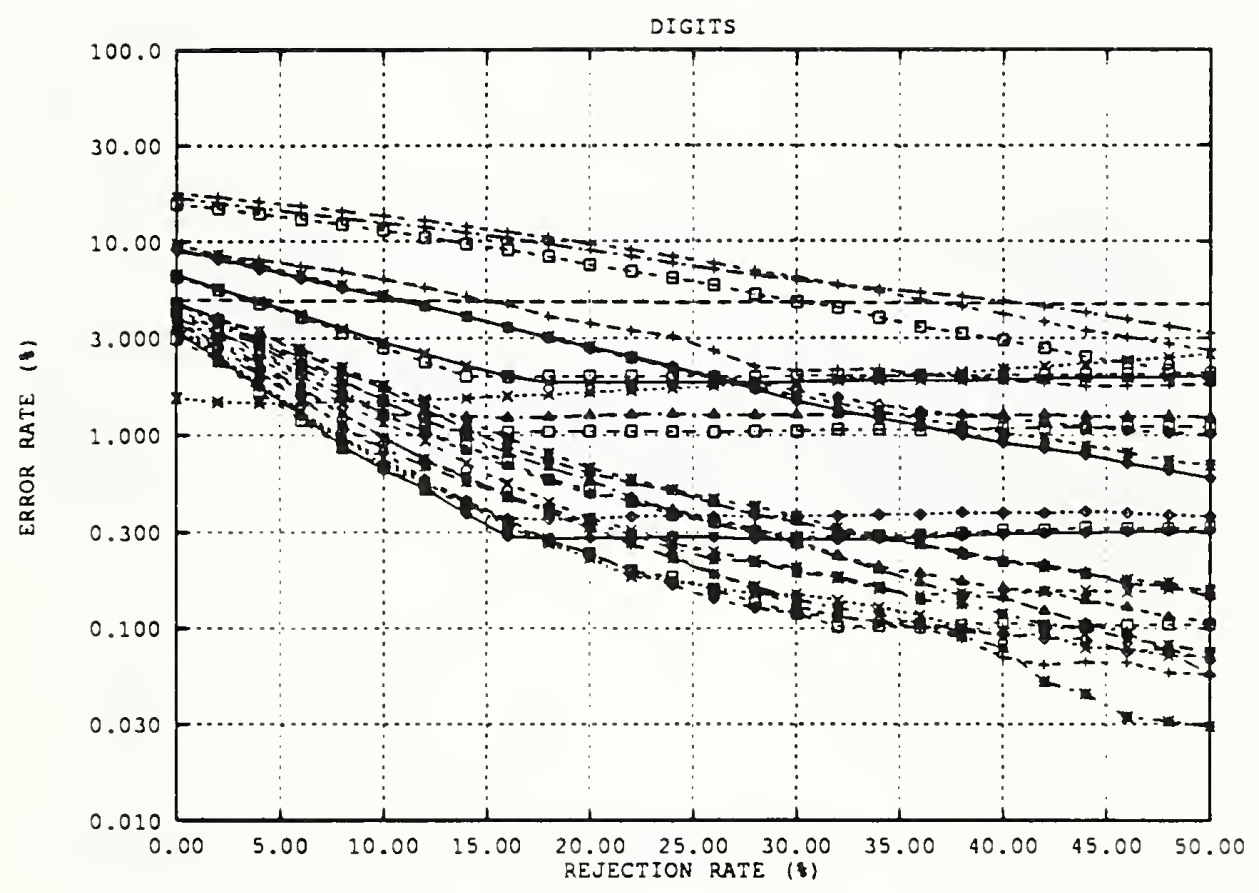

Figure 1: Error rate versus rejection rate for all systems providing confidence data with their classifications for the digit test. 


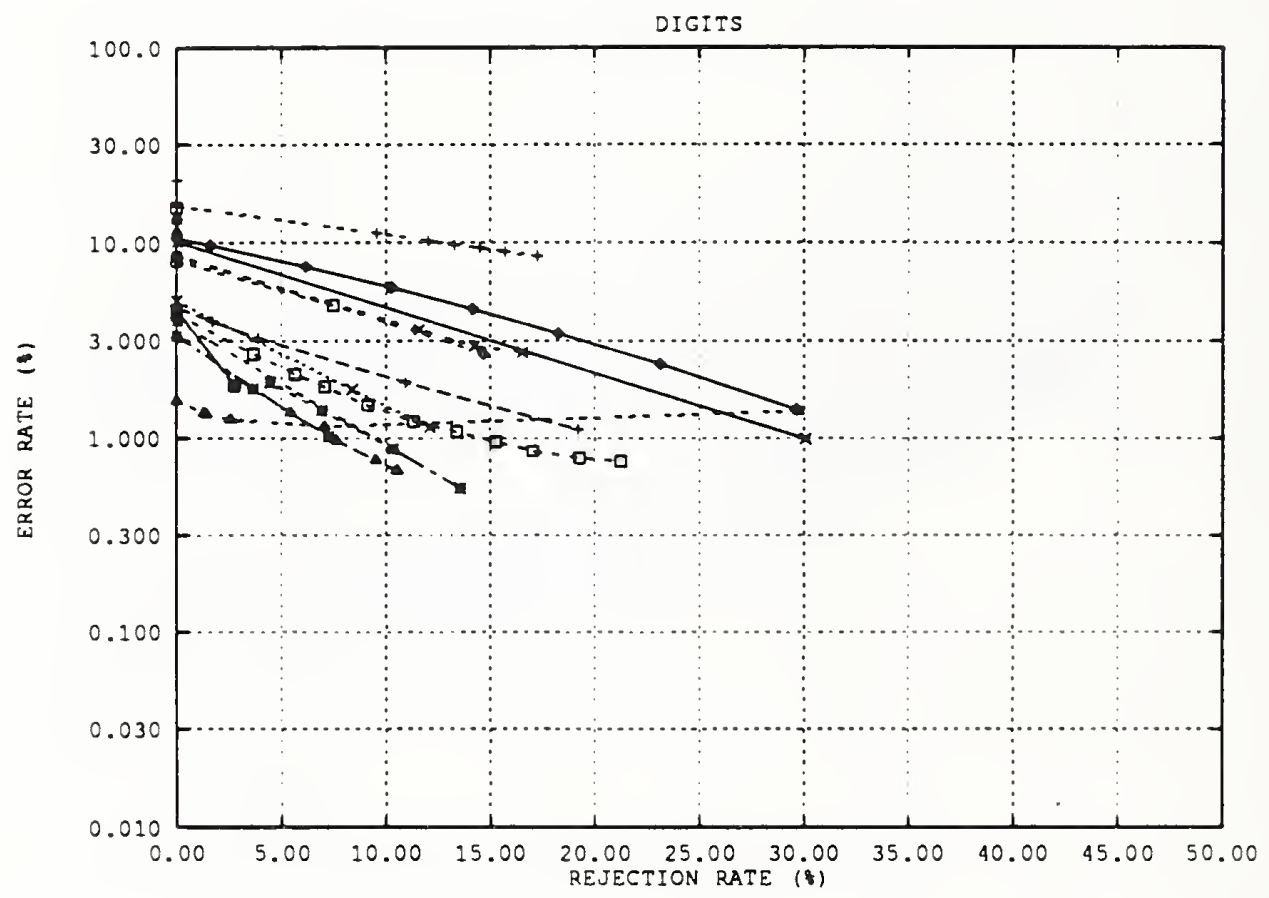

Figure 2: Error rate versus rejection rate for all systems providing rejection data with their classifications for the digit test. 


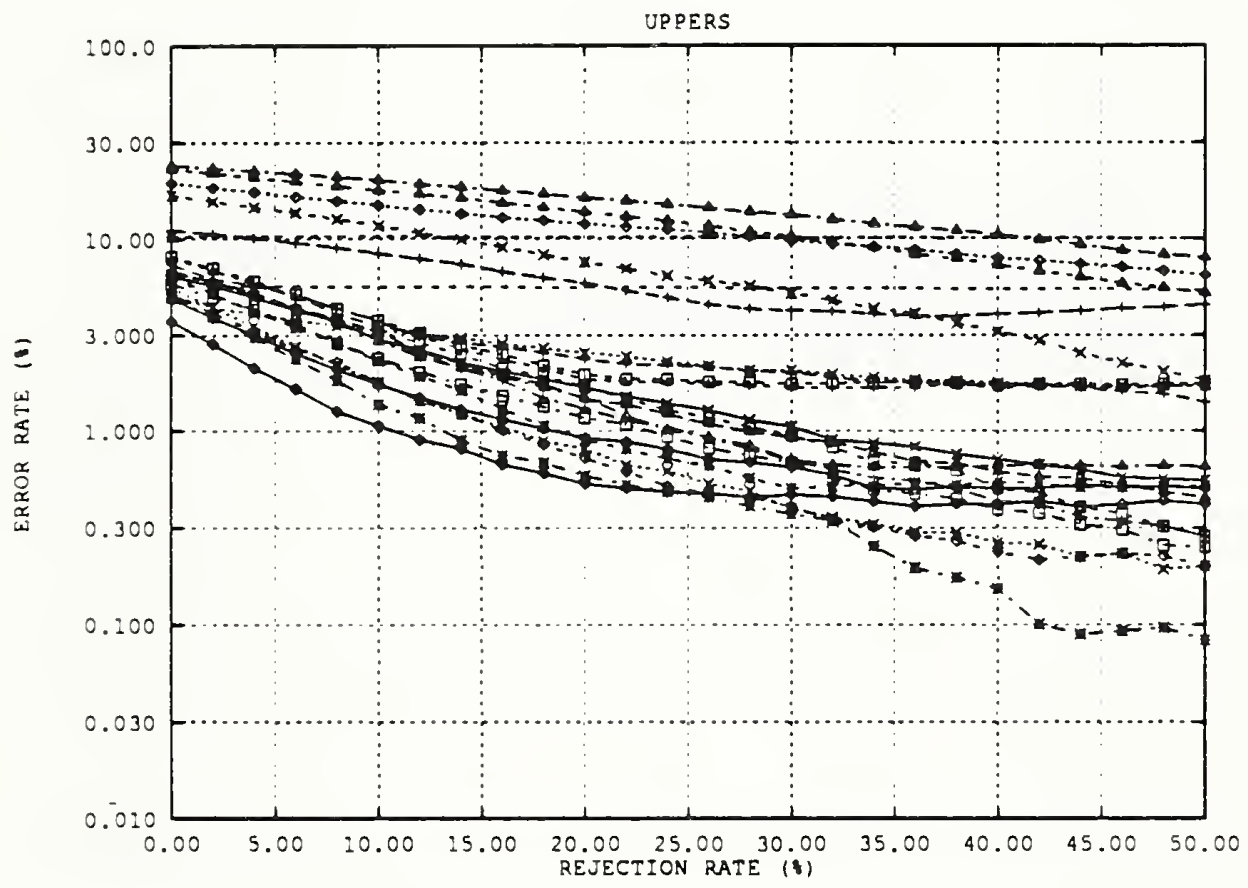

Figure 3: Error rate versus rejection rate for all systems providing confidence data with their classifications for the upper case test. 


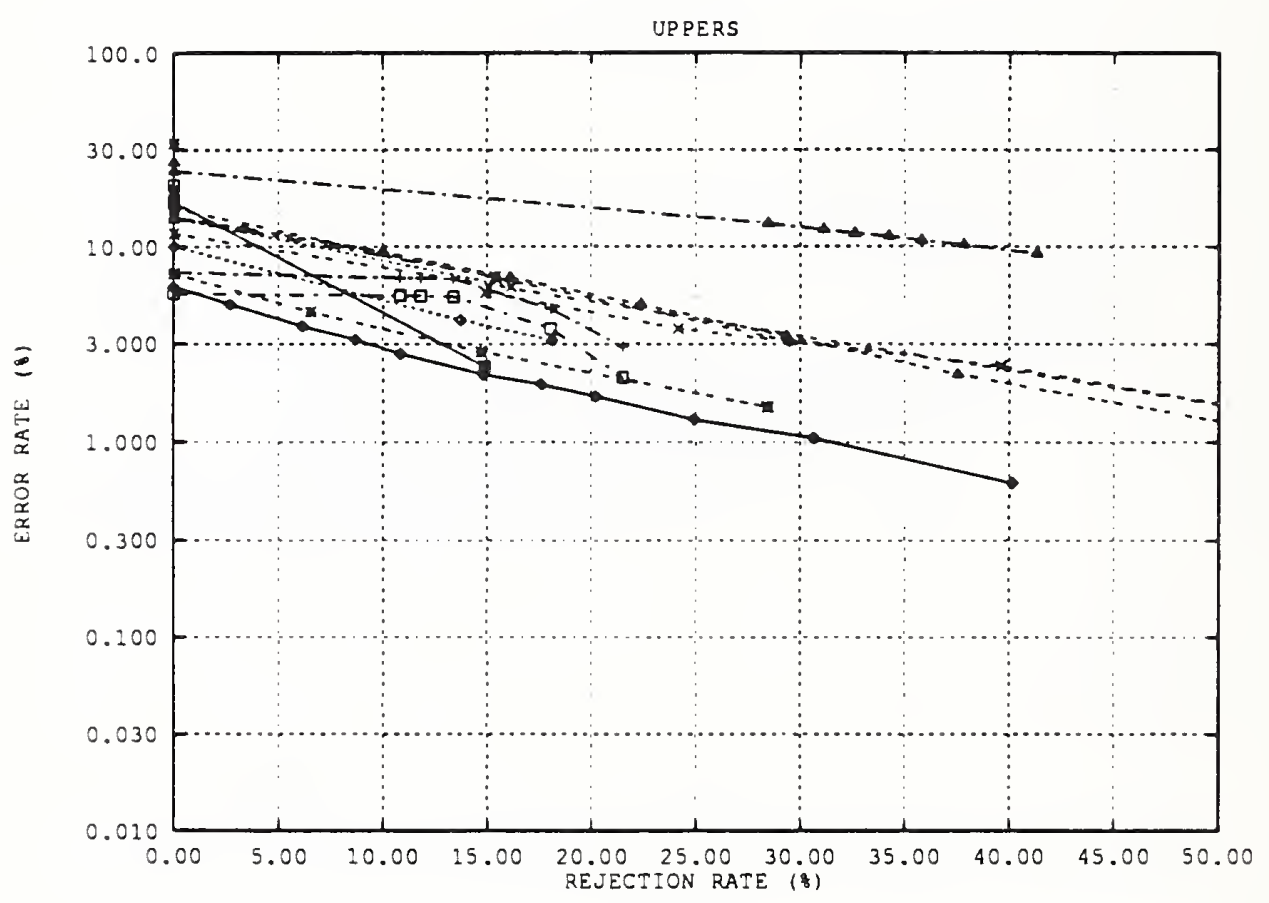

Figure 4: Error rate versus rejection rate for all systems providing rejection data with their classifications for the upper case test. 


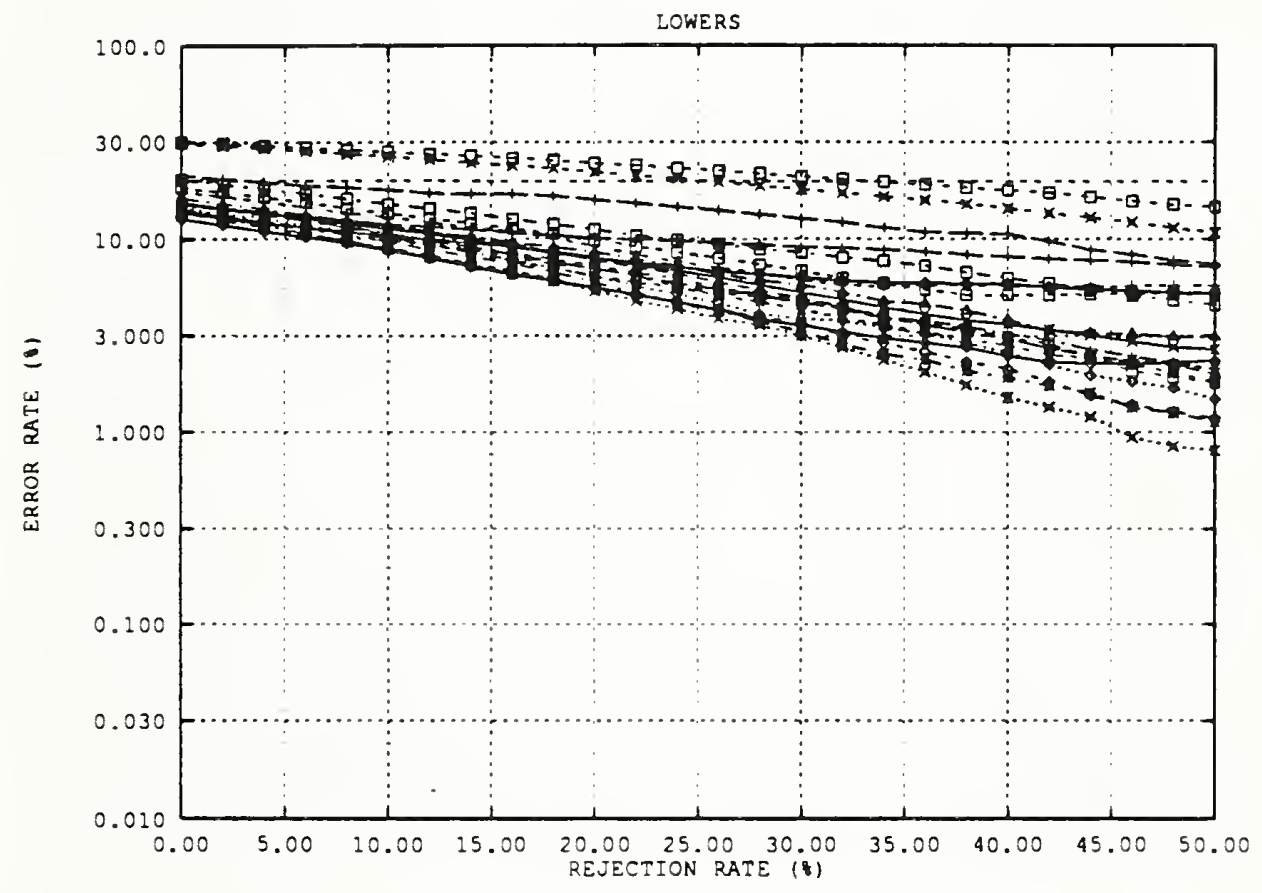

Figure 5: Error rate versus rejection rate for all systems providing confidence data with their classifications for the lower case test. 


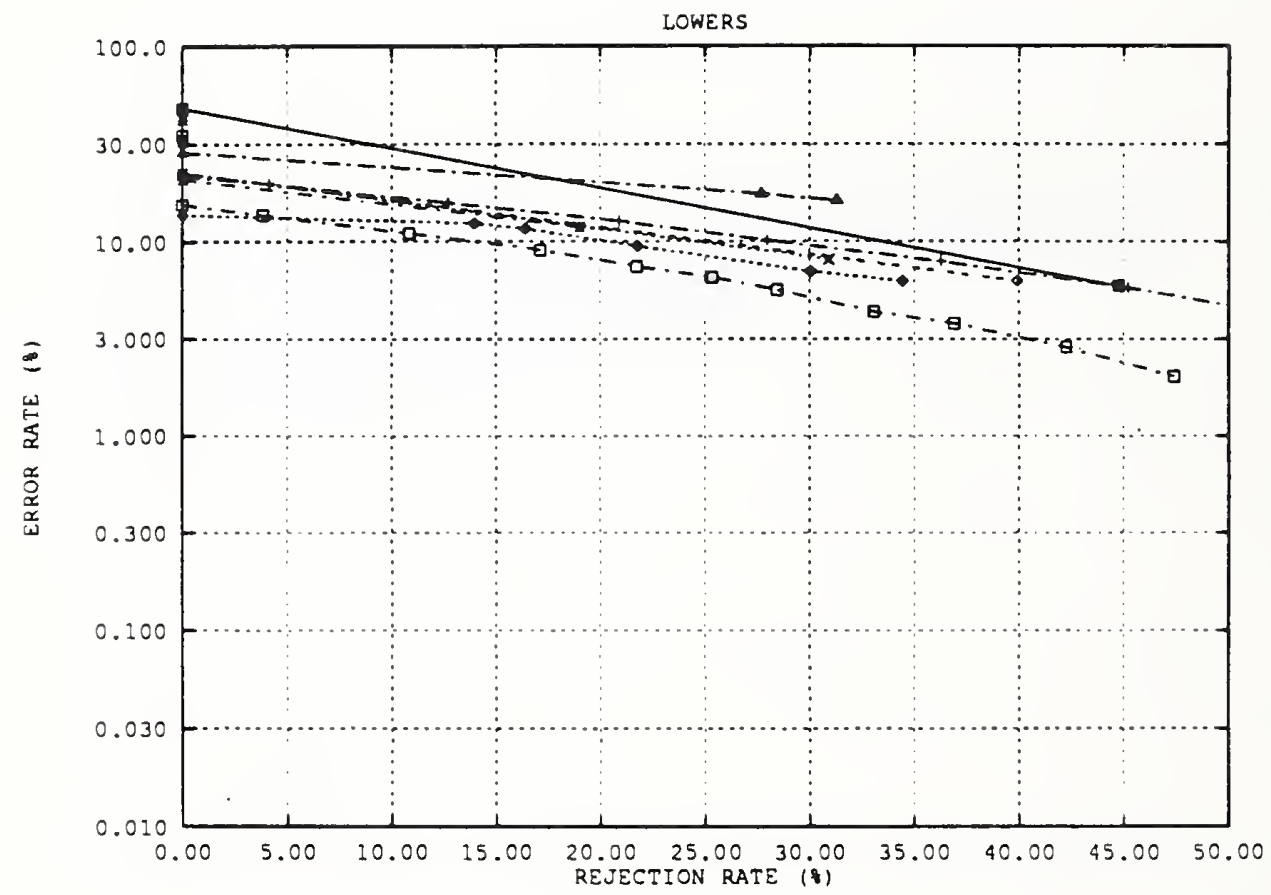

Figure 6: Error rate versus rejection rate for all systems providing rejection data with their classifications for the lower case test. 


\section{HANDWRITING SAMPLE FORM}

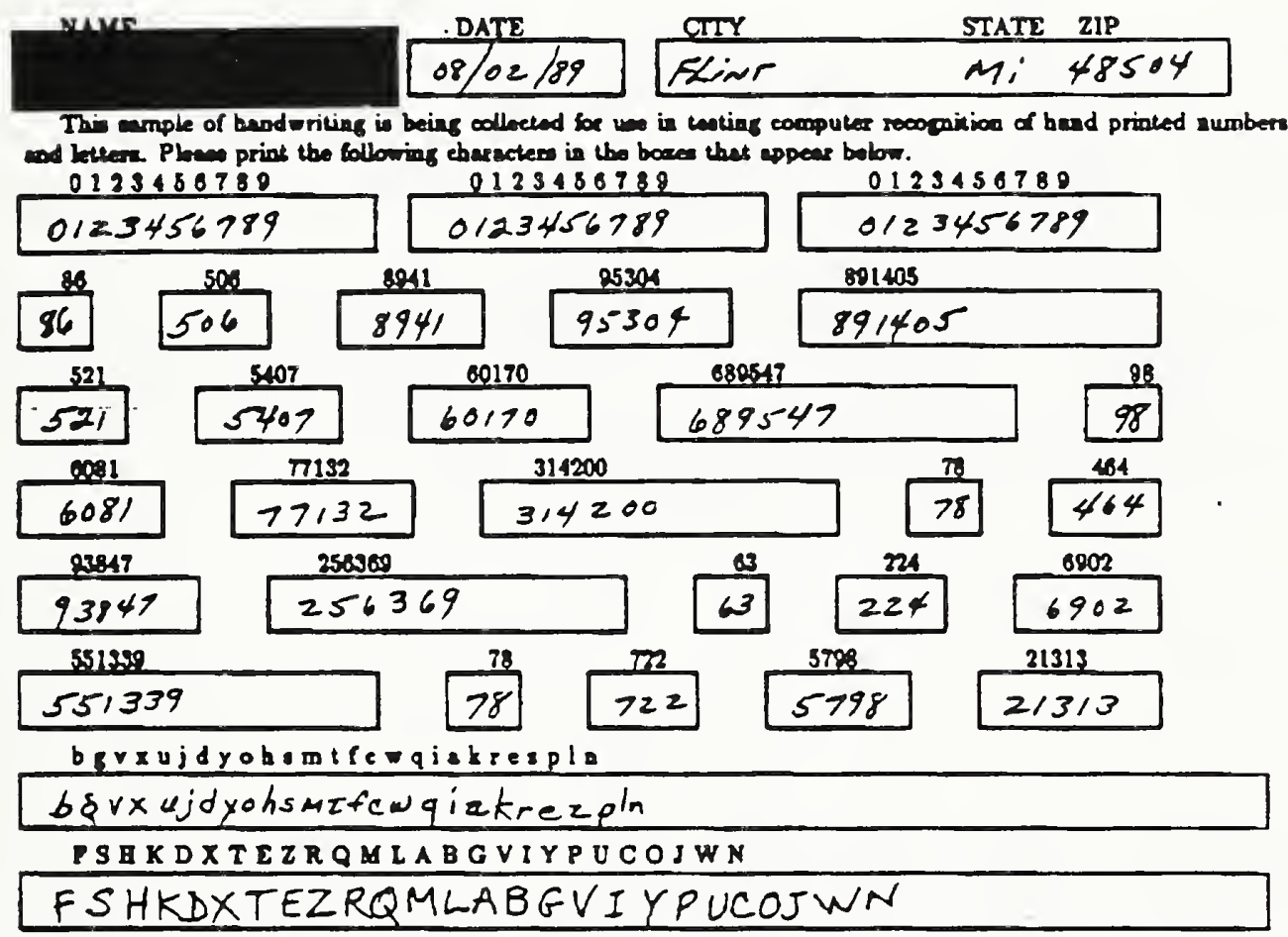

Place print the following teat is the bex below:

He, the People of the Uaited States, in order to form a more perfect Jaion, entablinb Jatice, insure doneatic Tranquillty, provide for the common Defense, promote the gened Welfare, and ecure tho Blening of Liberty wo arratres and our paterity, do oxdain ead extablisb thi CONSTITUTION for the United Stake of America.

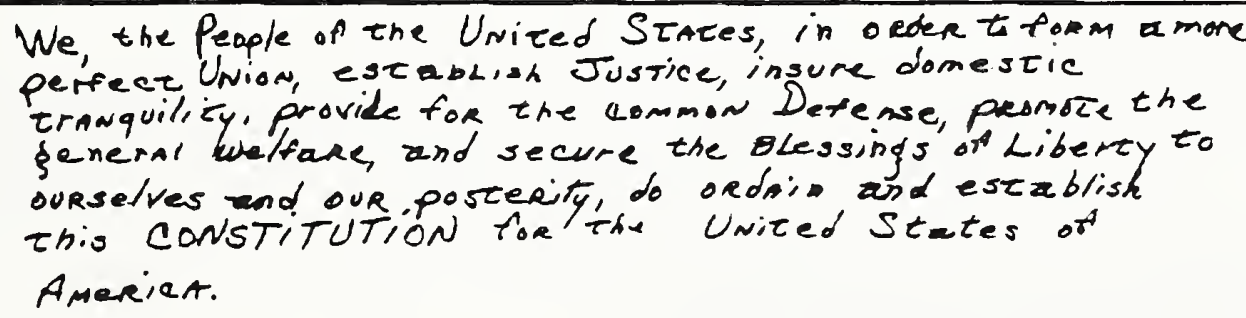

Figure 7: A typical filled-out sample form 


\title{
3 Cross Validation Studies
}

\author{
Patrick J. Grother
}

\subsection{Introduction}

Participants in the Conference agreed to classify unlabelled images using their own recognition systems and submit their classifications to NIST for scoring. NIST provided two databases to all entrants. The first, SD3, contained the segmented characters of 2100 writers and the "known" class files. This constituted an optional training set. The second database, TD1, contained unlabelled characters from 500 writers, and it constituted the test materials.

One result of the Conference was that those recognition systems trained solely on the SD3 database generally displayed inferior TD1 recognition to those trained on a superset of this data, i.e. one including SD3 as a subset, or other, possibly proprietary, datasets. The notion that SD3 was "clean" or "constrained" relative to the TD1 dataset was suggested by the writer profiles; SD3 was obtained from motivated permanent Census field personnel whereas TD1 was obtained from variously motivated, more diverse and cosmopolitan high school students. An example is that the European crossed seven is far more abundant in TD1 than SD3.

A study was initiated to formally investigate the relative differences between the two databases. The intent was to obtain some classifier-independent measures of the relative database difficulty - to obtain results that pertain to the properties of the data, and not the particular recognition algorithm. Cross validation [60] [61] has long been used as a method of obtaining more "mileage" from a data set. By partitioning the data into disjoint subsets, one for parameter estimation (i.e. training) and the other for performance measurement (i.e. testing). more robust estimates of performance statistics are available.

\subsection{Theory}

Cross validation is a method for accumulating a statistic, which in this section is the classification error obtained using a nearest neighbor classifier. Moody [62] expressed cross validation in terms of the mapping error associated between inputs and targets to a multilayer perceptron (MLP), but the concept of cross validation is in no way restricted to neural network classifiers or function approximators.

A problem associated with MLP networks is that patterns (for example, crossed sevens) present in a training set in small numbers are only weakly represented by the estimated weights, such that generalization is poor. Algorithms that do not aggregate information from the training data are not usually prone to this problem. Such a method is the ubiquitous K-Nearest Neighbor (KNN) algorithm [63]. The distances of an unknown pattern to elements of a prototype set are calculated using a suitable metric (often euclidean). Voting between the classes of the $\mathrm{K}$-closest patterns implies the class of the unknown pattern. Numerous extensions to the scheme have been used effectively including an elaboration, 
termed "Probabilistic Neural Network" (PNN), developed by Donald Specht [42] in which all prototypes are included in a gaussian distance weighted metric. The advantage of the method is that an a posteriori probability is attached to each possible class; the unknown is classed as that with the highest probability. NIST has used nearest neighbor classifiers that significantly outperform MLP networks given identical features.

\subsection{Classification}

The first stage of classification for purposes of cross validation between the two sets used the Karhunen Loève ( $\mathrm{KL}$ ) expansion of the images as a reduced dimensionality, optimally compact, representation. The use of such features in OCR has been described[64] [39].

The hand printed binary characters are isolated and represented as the \pm 1 elements of a column vector by some consistent ordering of the rectangular image. The mean vector of $P$ such images is subtracted from each and an ensemble matrix, $U$ is formed with the $P$ vectors as its columns. The symmetric covariance matrix, $R$, which can be expressed as

$$
\mathbf{R}=\mathbf{U U}^{T},
$$

gives the mean of all the interpixel correlations over all images in the ensemble. This can be used to statistically describe how handprinted character images vary. The covariance matrix $\mathrm{R}$ has eigenvectors as the columns of $\Psi$ :

$$
\mathbf{R} \Psi=\Psi \Lambda
$$

where the only non-zero elements of $\Lambda$ are the eigenvalues on its diagonal. The eigenvectors are the directions of maximum variance in the image space and form a complete orthonormal set termed the principal axes of a hyperellipse in that space. The eigenvalues define the statistical "length" of these axes; thus the first column of $\Psi$ corresponding to the largest eigenvalue is the major axis. The eigensolution of the covariance matrix provides a variance expansion of the image ensemble ordered from largest to smallest eigenvectors. The eigenvectors having the smaller values and therefore describing very little variance in the images, are discarded, thus affording useful dimensionality reduction. Any image vector as a column of a new matrix $U$ is a linear superposition of the basis vectors:

$$
\mathrm{U}=\Psi \mathrm{V}
$$

where the inversion of this formula, $\mathbf{V}$, defines the Karhunen Loève Transform (KLT) [65], the elements of which are the projection of the image vector onto the principal axes:

$$
\mathrm{V}=\Psi^{T} \mathrm{U}
$$

These feature vectors are classified using the PNN nearest neighbor technique[42]. Although many variations are described, the NIST_4 implementation reported in Appendix $F$ is as follows. The square euclidean distance of an unknown pattern, $\tau$, to the $i^{\text {th }}$ prototype in the training set, $t_{i}$, is

$$
d_{i}^{2}=\sum_{j=1}^{N}\left(\tau_{j}-t_{i j}\right)^{2}
$$


where the subscript $j$ spans the $N$ highest $\mathrm{KL}$ eigenvectors retained for the expansion. The distances $d_{i}$ are expressed as a function of the standard deviations of normal distributions centered on each of the prototypes. A gaussian is applied as a kernel weighting function.

$$
g_{i}=e^{-d_{i}^{2} / 2 \sigma^{2}}
$$

The weighted distances are then accumulated by class over the $K$ classes, to which the prototypes belong.

$$
v_{k}=\sum_{i}^{P} g_{i} \delta_{i k}
$$

where $\delta_{i k}$ is unity if the $i^{\text {th }}$ prototype is of class $k$ and zero otherwise. Interestingly this vector may be normalized to give a posteriori probabilities by dividing by $\sum_{k} v_{k}$. The unknown is assigned the class with the largest attached probability. For optimal classification it is necessary to survey over the gaussian width $\sigma$; for digits the best value was taken as 3.0, whereas for uppers and lowers a value of 4.0 was adopted.

Rather than use classifiability as a measure of database homogeneity it is possible to obtain a priori measures. Consider the databases as image ensembles for which the KLT is defined. The variances of the transform coefficients are the eigenvalues. Since the eigenvectors, the basis of the KLT, form a complete orthonormal set, any image (including those of the ensemble from which the covariance matrix is calculated) is exactly a linear superposition of those bases. If the eigenvalue spectrum is relatively flat, then the variance in an image ensemble is distributed over many eigenvectors and more eigenvectors are needed for an adequate representation, as for instance in achieving a low reconstruction mean square error level.

Interestingly, this total image variance is related to the scatter of the data, $S$, defined as

$$
S=E\left\{\left\|\mathbf{u}_{i}-\mathbf{u}_{j}\right\|\right\}
$$

where the expectation, $E\{\cdot\}$ of the underlying distribution is replaced by the sample mean whence

$$
\begin{gathered}
S=\frac{1}{P^{2}} \sum_{i=1}^{P} \sum_{j=1}^{P}\left(\mathbf{u}_{i}-\mathbf{u}_{j}\right)^{T}\left(\mathbf{u}_{i}-\mathbf{u}_{j}\right) \\
S=\frac{1}{P^{2}} \sum_{i=1}^{P} \sum_{j=1}^{P}\left(\mathbf{u}_{i}^{T} \mathbf{u}_{i}+\mathbf{u}_{j}^{T} \mathbf{u}_{j}\right)-\frac{1}{P^{2}} \sum_{i=1}^{P} \sum_{j=1}^{P}\left(\mathbf{u}_{i}^{T} \mathbf{u}_{j}+\mathbf{u}_{j}^{T} \mathbf{u}_{i}\right)-
\end{gathered}
$$

Given that the $\mathbf{u}$ are mutually independent and from a single distribution the double sums are replaceable thus

$$
S=\frac{2}{P} \sum_{i=1}^{P} \mathbf{u}_{i}^{T} \mathbf{u}_{i}-\frac{2}{P} \sum_{i=1}^{P} \mathbf{u}_{\mathbf{i}}^{\mathrm{T}} \frac{1}{P} \sum_{j=1}^{P} \mathbf{u}_{\mathbf{j}}
$$


By considering these inner products as the traces of the outer products of the ensemble matrices then the scatter becomes twice the difference in the traces of the autocorrelation and mean outer product matrices. This matrix is identical to the covariance matrix of equation 1.

$$
S=2 \operatorname{Trace}\left\{\mathbf{U U}^{T}\right\}
$$

The diagonal elements of the covariance matrix are the variances of the image pixels. The total variance is conserved under any unitary transformation:

$$
\sum_{i} \mathbf{R}_{i i} \equiv \text { Trace } \mathbf{R}=\text { Trace } \Lambda
$$

It is found that the scatter statistic is proportional to the sum of the eigenvalues. Further expressing eigenvalues as a percentage of their total yields the percentage of the ensemble variance represented by a subset of $N$ eigenvectors. For comparison of the two databases the difference in the percentages as a function of $N$ is discussed. Some eigenfunctions describe image variance that is relevant to classification, and others describe variation that is representative of noise. If an eigenspectrum is wide, then the percentage variance described by the $N$ leading eigenvectors will be small. If the cross validation percentage recognition is high, then the information discarded by using an incomplete KLT is irrelevant even though there is much of it. Alternatively, if the eigenspectrum is narrow, with much of the variance captured, then a low recognition rate implies that the discarded transform coefficients are valuable. This latter sensitivity to the high-order $\mathrm{KL}$-transforms is undesirable since the motivation for feature extraction is reduced dimensionality.

\subsection{Cross Validation Results}

This study generated a Validation Comparison Matrix. The matrix has rank two and dimension equal to the number of databases in the comparison which in this case is also two. The row and column indices of the matrix denote, respectively, the databases used for training and testing. The absolute classification error rates in the matrix are taken as irrelevant since the entries were all produced using the same classifier which was not particularly well optimized. The interesting features are the relative percentages discussed below.

The on-diagonal terms, $c_{i i}$, indicate the mean error rates for standard $v$-fold cross validation of the $i^{t h}$ database. The off-diagonal elements, $c_{i j} i \neq j$, result from cross-cross validation. The first $u$ partitions of database $i$ are used as training sets for the $v$-fold cross validation of the $j^{\text {th }}$ database. In the case of $v$-fold partitioning of the training set there will be $u v$ results the mean of which is $c_{i j}$.

All mean elements have an attached sample standard deviation.

$$
\sigma=\sqrt{\frac{1}{N-1} \sum_{i=1}^{N}\left(x_{i}-\mu\right)}
$$


If an homogeneous dataset is large enough then this quantity will approach zero. The standard deviation is also a function of the data set redundancy. For instance, consider a database to which a copy of itself is appended, and which is classified with, for example, a single nearest neighbor algorithm. Perfect recognition could then be achieved if, as in the cross validation scheme used here, the partitions are contiguous blocks from the dataset.

The standard error is accessible by dividing the standard deviations by a further $\sqrt{N}$ where $N=10$. The discussion of the comparisons of the means and the variances is aided by invoking the results of Student's " $t$ test" and the " $f$ test" (see for example [66]).

They are used to assess whether two distributions have the same mean and the same variances. The entire corpus of human hand-printed characters may be considered as one distribution of which SD3 and TD1 are subsets, but for this study the two sets are extracted from different distributions, namely the two social writer groups outlined in the introduction. The $t$-test quantifies the difference in two means as a function of their mutual root mean square standard error.

$$
t=\frac{\mu_{1}-\mu 2}{\sqrt{\frac{\sigma_{1}^{2}}{N_{1}}+\frac{\sigma_{2}^{2}}{N_{2}}}}
$$

Attached to $t$ is a significance, $0 \leq p \leq 1$, giving the probability that $|t|$ could be at least this large by chance. That is if $p$ takes on a "small" value then the distributions have significantly different means. Similarly the $f$-test quantifies two variances as a ratio taken to be greater than 1 (i.e. either $\sigma_{1}^{2} / \sigma_{2}^{2}$ or its reciprocal). The value of $f$ directly indicates differing variances. The attached significance, $p$ is again a probability. Small values indicate significantly different variances.

The statistics are derived from the two samples obtained by testing the 10 partitions of SD3 and TD1 data using one or other training set. In all cases, digits, upper-case and lower-case letters, the calculated value of the $t$ is found with very low significance indicating the mean differences are not at all spurious. However in no case does the attached probability for the $f$-test indicate that the variances are significantly different.

\subsubsection{Digits}

The handprinted digits of the first 500 writers of SD3 were partitioned into blocks each containing digits from 50 writers. The numbers of characters in these ten sets were not identical but varied by only $0.2 \%$. The number of SD3 digits totalled 53449 . The 500 writers of TD1 were similarly partitioned. The number of TD1 digits totalled 58646. The pure 10 fold cross validations for SD3 and TD1 were obtained using the characters of $90 \%$ of the writers as training prototypes with the characters of the remaining $10 \%$ used for testing. The mean of the zero-rejection-rate error rates for the cross validations are quoted on the diagonal of the Table below.

The first partition of SD3 (450 writers) was used as prototypes for the classification of all 10 sets of characters of TD1, and vice versa. The off-diagonal elements of the validation 


\begin{tabular}{|c|c|c|l|}
\hline $\begin{array}{c}\text { Correct \% } \\
\pm \sigma\end{array}$ & $\begin{array}{c}\text { Test SD3 } \\
50 \text { writers }\end{array}$ & $\begin{array}{c}\text { Test TD1 } \\
50 \text { writers }\end{array}$ & \\
\hline Train SD3 & $1.7 \% \pm 0.3$ & $6.8 \% \pm 0.4$ & $\mathrm{t}=28.5 \mathrm{p}=0.0$ \\
450 writers & & $3.5 \% \pm 0.5$ & $\mathrm{t}=1.4 \mathrm{p}=0.3$ \\
\hline $\begin{array}{l}\text { Train TD1 } \\
450 \text { writers }\end{array}$ & $3.5 \% \pm 0.3$ & $\mathrm{f}=2.1 \mathrm{p}=0.3$ \\
\hline
\end{tabular}

Table 4: Inter and Intra database Cross Validation Recognition Errors for Digits

comparison matrix, so obtained, are also given in the Table below.

The most relevant result from this table is that, using the classifier as described above, training solely on SD3 implies a 5\% loss when classifying TD1. This is effectively NIST's experience with its NIST_1 and NIST_3 systems reported in Appendix E.

The on-diagonal elements of the cross validation matrix show that SD3 is a less diverse digit set than TD1. That is the test partitions of SD3 are more like their training sets, in the nearest neighbor sense, than is the case with TD1. Greater on-diagonal terms indicate a higher intrinsic diversity for that database. If we relate the low TD1 classification to the width of the eigenvalue spectrum or the volume of the eigenspace, it is apparent that TD1 would benefit from the use of a more complete KLT as input to the classifier.

Figure 8 shows the eigenspectra of the SD3 and TD1 characters. Note in particular that the total variances for the 1024 pixel images are 575.5 (SD3) and 636.8 (TD1) indicating that $\mathrm{TD} 1$ is absolutely more diverse (larger scatter). Approximately $6.6 \%$ more of the variance of SD3 is described with $48 \mathrm{KL}$ eigenvectors (as used by the classifier) than is the case for TD1.

The off-diagonal terms show that use of SD3 as a training set for testing with TD1 is markedly inferior to use of TD1 as a training set for testing with SD3. The implication is that TD1 is a superset of the SD3 set, i.e. TD1 contains sufficiently distributed prototypes to classify SD3 - whereas TD1 contains exemplars that are not "closely" present in SD3. That TD1 classifies itself and SD3 equally (to within one standard deviation) implies that TD1 is a more general dataset.

\subsubsection{Uppers}




\begin{tabular}{|c|c|c|l|}
\hline $\begin{array}{c}\text { Correct } \% \\
\pm \sigma\end{array}$ & $\begin{array}{c}\text { Test SD3 } \\
48 \text { writers }\end{array}$ & $\begin{array}{c}\text { Test TD1 } \\
50 \text { writers }\end{array}$ & \\
\hline Train SD3 & $14.2 \% \pm 1.4$ & $19.4 \% \pm 1.4$ & $\mathrm{t}=7.9 \mathrm{p}=0.0$ \\
432 writers & & $16.0 \mathrm{p}=0.8$ \\
\hline $\begin{array}{c}\text { Train TD1 } \\
450 \text { writers }\end{array}$ & $19.3 \% \pm 1.7$ & $16.5 \% \pm 1.4$ & $\mathrm{t}=3.8 \mathrm{p}=0.0$ \\
& & & $\mathrm{f}=1.5 \mathrm{p}=0.4$ \\
\hline
\end{tabular}

Table 5: Inter and Intra database Cross Validation Recognition Errors for Uppers

The handprinted upper case letters of the first $480^{1}$ writers of SD3 were partitioned into blocks from 48 writers. The upper case letters totalled 10790 examples. The 500 writers of TD1, similarly partitioned, yielded 11941 characters. As in the case of digits, there is a $5 \%$ difference between the classification of SD3 on itself and on TD1. Again, TD1 is more diverse in classification of itself than is the case with SD3. On the other hand, the total variances are 734.0 (SD3) and 650.2 (TD1) indicating that SD3 is absolutely more diverse. With classification using $96 \mathrm{KL}$ coefficients the percentage variance captured for SD3 was $4.8 \%$ less than that for TD1.

The off-diagonal elements, however, are the same indicating that neither set is more general than the other. That the off-diagonal elements are lower than the on-diagonals indicates the databases contain unique subsets that require specialist knowledge contained only in that database.

\subsubsection{Lowers}

The handprinted lower case letters of the first $490^{2}$ writers of SD3 were partitioned into blocks from 49 writers. The lower case letters totalled 10968. The 500 writers of TD1, similarly partitioned, yielded 12000 characters. As with the upper case letters, but not with the digits, the total variances are 740.4 (SD3) and 637.9 (TD1) indicating that SD3 is absolutely more diverse. With classification using $96 \mathrm{KL}$ coefficients the percentage variance captured for SD3 was $2.3 \%$ less than the that for TD1. The cross validation matrix shows

\footnotetext{
${ }^{1}$ None of the upper case letters of twenty writers were segmented in the preparation of SD3. See subsection 3.5 .

${ }^{2}$ None of the lowercase letters of 10 writers were segmented in the preparation of SD3. See subsection 3.5 .
} 


\begin{tabular}{|c|c|c|c|}
\hline $\begin{array}{c}\text { Correct } \% \\
\pm \sigma\end{array}$ & $\begin{array}{l}\text { Test SD3 } \\
49 \text { writers }\end{array}$ & $\begin{array}{l}\text { Test TD1 } \\
50 \text { writers }\end{array}$ & \\
\hline $\begin{array}{l}\text { Train SD3 } \\
441 \text { writers }\end{array}$ & $19.6 \% \pm 1.4$ & $23.5 \% \pm 1.4$ & $\begin{array}{l}\mathrm{t}=5.9 \mathrm{p}=0.0 \\
\mathrm{f}=1.1 \mathrm{p}=0.8\end{array}$ \\
\hline $\begin{array}{l}\text { Train TD1 } \\
450 \text { writers }\end{array}$ & $25.9 \% \pm 1.8$ & $19.2 \% \pm 1.1$ & $\begin{array}{l}\mathrm{t}=9.6 \mathrm{p}=0.0 \\
\mathrm{f}=2.5 \mathrm{p}=0.1\end{array}$ \\
\hline
\end{tabular}

Table 6: Inter and Intra database Cross Validation Recognition Errors for Lowers

that the lower case datasets are equally difficult and yet different - they are insufficiently general to classify each other as well as they classify themselves.

\subsection{Caveats}

\subsubsection{Segmentation}

This initial study reports work NIST conducted immediately after the Conference. As such it is a provisional investigation of database quality; it is not experimentally flawless and therefore the conclusion that SD3 is cleaner than TD1, at least for digits, does not necessarily apply to the forms from which the two databases of characters were segmented.

One reason for this is that SD3 and TD1, both obtained from fields of full page forms, were arrived at with different character segmenters. From a possible 65000 characters on each 500 form set, final numbers of human-checked characters were 53449 (SD3) and 58646 (TD1). The SD3 segmentor, an old version, produced $9 \%$ fewer isolated characters than the updated model used for TD1. The principal reason for failure of the SD3 segmenter was the inability to segment connected or overlapping characters. If the characters from SD3 that were not segmented resemble the difficult digit images that apparently characterize TD1, then the difference between the two databases may not be writer-letter dependent at all. Instead it could represent writer-connectivity/overlap differences between the two different writer groups, or the differences in ability of the different segmenters to segment connected or overlapping characters that tend to be difficult in other ways as well.

This problem can be negated by resegmenting and rechecking the characters in SD3 using the identical algorithms applied to TD1. A new database, a superset of SD3, would then 
obtained, which could then be used in a more controlled comparison with TD1.

\subsubsection{Classifier Dependence}

The eigenvalue spectrum describes the information loss suffered when only the KL eigenvectors having the largest eigenvalues are used in classification. The classification of incomplete KLT's is peculiar in that variance ordered information is discarded. Using a much higher number of coefficients in the digit classification might equalize the on-diagonal cross validation entries. Even with a higher dimensional (but lower variance) KL space, aggregated functionally approximated MLP classifiers are not able to recognize minority patterns. Under these conditions, the nearest neighbor schemes do better.

Instead of using a "lossy" incomplete feature classifier it is possible to use a full description of the image; the complete $\mathrm{KL}$ transform. Variance equalization may be more reasonable - choose the number of KL features corresponding to either an absolute level of described variance or percentage thereof. Thus in the case of the digits in SD3, 43 eigenevectors describe $75 \%$ of the variance, whereas to reach this level with TD1, $70 \mathrm{KL}$ coefficients are required. Alternatively, features that do not bias information loss may be used. For example, image row and column pixel histograms or orthogonal moments are known to be classifiable.

\subsection{Conclusions}

Given the experimental scheme described, it appears that TD1 does contain a more diverse and general digit set than SD3. The latter classified disjoint digit subsets of itself with a $5 \%$ lower error rate than that with which it classified TD1. Furthermore, TD1 yields a $3 \%$ improvement over SD3 in the classification of disjoint digit subsets of the former. The hypothesis that differing writer populations are responsible for this difference has not been proven. Indeed, the fact that the cross validations for the upper and lower case letters yield very different results seems to weaken this argument for digits.

Further, more controlled experimentation is necessary and on-going. The a priori measures of image variance are similarly inconclusive. 

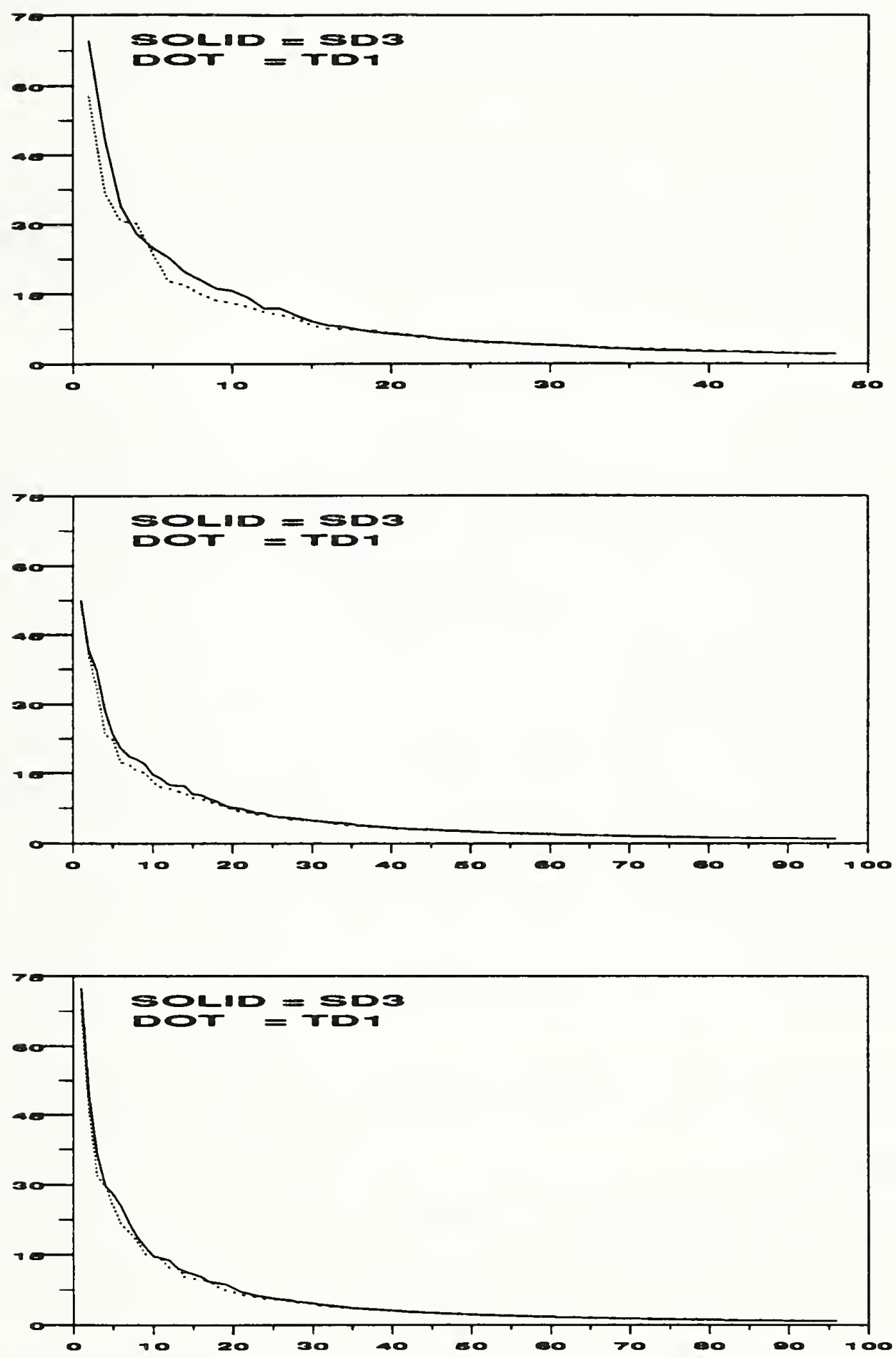

Figure 8: Eigenvalue vs Index for SD3 and TD1. From top: digits, uppers and lowers. All writers were used. 


\section{System Error Rates Versus Rejection Rates}

Jon Geist and R. Allen Wilkinson

\subsection{Theory}

Let $q(r)$ be the probability as a function of rejection rate $r$ that a rejected classification is an incorrect classification. In this case, the error rate $e(r)$, which is defined as the ratio of accepted (unrejected) classifications that are incorrect to the total number of accepted classifications, is given by

$$
e(r)=\frac{e(0)-f(r)}{1-r}
$$

where

$$
f(r)=\int_{0}^{r} q(s) d s,
$$

is the fraction of the rejected classifications as a function of $r$ that are actually incorrect. Equations 16 and 17 may be combined to give the slope of the error rate,

$$
e^{\prime}(r)=\frac{e(r)-q(r)}{1-r}
$$

If $e^{\prime}(r)$ is zero in eq. 18 , then

$$
q(r)=e(r)=e_{0},
$$

where $e_{0}$ is a constant. This means that the probability of rejecting an incorrect classification is equal to the fraction of incorrect classifications remaining in the unrejected sample. In this case, the rejection mechanism just rejects classifications at random.

If $q(r)$ is equal to the constant $q_{0}$ over some subrange of $r$, then

$$
e(r)=\frac{e(0)-q_{0} r}{1-r}
$$

and

$$
e^{\prime}(r)=\frac{-q_{0}+e(0)}{(1-r)^{2}}
$$

over the same subrange.

Therefore, a perfect rejection mechanism is characterized by $q(r)=1$ for $0 \leq r \leq e(0)$, and $q(r)=0$, for $r>e(0)$, in which case,

$$
e(r)=\frac{e(0)-r}{1-r}
$$

for $0 \leq r \leq e(0)$, and

$$
e(r)=0,
$$

for $r>e(0)$. 
It is clear from a cursory investigation of Figures 1 through 6 in Section 2 that none of the submissions to the Conference come close to a perfect rejection mechanism, yet all of their $e(r)$ curves seem to have similar shapes. The next section describes an experiment that was carried out to test this observation.

\subsection{Fit of Model to Experimental Data}

A visual examination of the curves in Figures 1 through 6 in Section 2 suggests that they might be well described by

$$
e(r)=\frac{\left(e_{0}-e_{\min }\right) \exp \left(-r / r_{0}\right)+e_{\min }}{1-r} .
$$

To test this conjecture, we fit the logarithms of the measured $e(r)$ curves to the logarithm of eq. 24 over the range $0 \leq r \leq 0.14$, where $e_{0}, e_{\min }$, and $r_{0}$ were adjusted in the fit. Logarithms were used to optimize the shape of the fits, rather than the values near the maximum of $e(r)$.

The results of the fits are summarized in Tables 7,8 , and 9 , which list for the digit, the upper case, and the lower case test, respectively, the values of $e_{0}, e_{\min }$, and $r_{0}$ for each system that participated in each test. These tables also list the residual standard deviation of each fit, and the ratio of the actual value of $e^{\prime}(0)$ for each system to the ideal value of $e^{\prime}(0)$ for a perfect rejection process for that system. It is also noteworthy that it appears easy for a system to obtain a value of $e^{\prime}(0)$ that is greater than $30 \%$ of the ideal value, but it appears very difficult for a system to obtain a value of $e^{\prime}(0)$ that is greater than $80 \%$ of the ideal value.

Eight data points were used in each fit. Three parameters were estimated. This leaves five degrees of freedom in each fit. Because the fits were carried out on the logarithms of the data, the residual standard deviations of the fits are actually the standard deviations of the relative differences between the measured error rates and those predicted by eq. 24 . Thus a residual standard deviation of 0.01 corresponds to a standard deviation of the relative errors of the fit of $1 \%$ over the range of the fit.

It is remakable how well eq. 24 can be fit to the $e(r)$ curves for the various different systems over the 0 to $14 \%$ subrange of $r$. In fact, most of the $e(r)$ curves are well described by eq. 24 over a subrange $0 \leq r \leq r_{s 1}$, and by

$$
e(r)=e_{s}
$$

over a subrange $r_{s 1} \leq r \leq r_{s 2}$, where $e_{s}, r_{s 1}$, and $r_{s 2}$ are system dependent constants, $r_{s 1} \leq r_{s 2}$, and $r_{s 2} \gg 14 \%$.

\subsection{Conclusion}

Equation 25 corresponds to the case where the rejection process has degenerated to a random sampling of the unrejected classifications, as described in connection with eq. 19. Equation 
24 , on the other hand, corresponds to the case where the probability of rejecting a classifcation that is actually incorrect is given by

$$
q(r)=\frac{e_{0}-e_{\min }}{r_{0}} \exp \left(-r / r_{0}\right),
$$

which can be rewritten in terms of $e(r)$ as

$$
q(r)=\frac{e(r)(1-r)-e_{\min }}{r_{0}}
$$

and which is bounded above by

$$
q(r)=e(r) / r_{0} .
$$

For most of the systems in the Conference, $e_{\min } \ll e(0)$. Therefore, for small r, eq. 28 is a good approximation to to the probability distribution of eq. 27 for most of the systems. This distribution is an improvement by a factor of $1 / r_{0}$ over the probability distribution for a completely random rejection process, but it is still greatly inferior to the ideal distribution. In fact, no probability distribution that is proportional to $e(r)$ can be efficient, because the very act of reducing $e(r)$ through the rejection process reduces the efficiency with which incorrect classifications are rejected.

The fact that eq. 24 describes all of the $e(r)$ curves reasonably well would seem to suggest that some limiting behavior is being approached. On the other hand, the fact that there is so much difference between the shapes of the $e(r)$ curves for the systems in the Conference and the ideal curve for a perfect rejection process suggests that there is considerable room for improvement. This is a paradox that will not be resolved without further research. 


\begin{tabular}{|l|l|l|l|l|l|}
\hline \hline SYSTEM & sigma & $e_{0}$ & $e_{\min }$ & $r_{0}$ & ratio \\
\hline AEG & 0.028356 & 0.034726 & 0.001083 & 0.052480 & 0.585633 \\
ASOL & 0.031873 & 0.092238 & 0.000000 & 0.203180 & 0.241507 \\
ATT_1 & 0.029338 & 0.032628 & 0.001808 & 0.050910 & 0.447927 \\
ATT_2 & 0.015908 & 0.036287 & 0.001320 & 0.053330 & 0.673430 \\
ATT_3 & 0.072066 & 0.050545 & 0.007738 & 0.048070 & 0.666328 \\
ATT_4 & 0.019877 & 0.041735 & 0.001156 & 0.060660 & 0.583642 \\
ERIM_1 & 0.020684 & 0.039069 & 0.000177 & 0.059683 & 0.634640 \\
ERIM_2 & 0.015108 & 0.039497 & 0.000880 & 0.063479 & 0.561363 \\
GTESS_1 & 0.012625 & 0.066724 & 0.000000 & 0.104407 & 0.560962 \\
GTESS_2 & 0.006790 & 0.067664 & 0.002970 & 0.102696 & 0.592692 \\
HUGHES_1 & 0.028769 & 0.050092 & 0.000000 & 0.084586 & 0.462246 \\
HUGHES_2 & 0.029808 & 0.049664 & 0.000000 & 0.090055 & 0.519539 \\
IBM & 0.014380 & 0.034863 & 0.001592 & 0.052278 & 0.629884 \\
IFAX & 0.003250 & 0.170347 & 0.019570 & 0.206207 & 0.710072 \\
KODAK_1 & 0.041515 & 0.049025 & 0.000850 & 0.076357 & 0.468308 \\
KODAK_2 & 0.019072 & 0.041296 & 0.000600 & 0.070753 & 0.467999 \\
NESTOR & 0.016548 & 0.045243 & 0.002240 & 0.064995 & 0.609639 \\
NIST_2 & 0.004107 & 0.091833 & 0.000004 & 0.146915 & 0.600810 \\
NIST_3 & 0.005298 & 0.097302 & 0.000017 & 0.138602 & 0.691096 \\
NYNEX & 0.024356 & 0.044058 & 0.002230 & 0.067403 & 0.540163 \\
OCRSYS & 0.004160 & 0.015539 & 0.013377 & 0.034779 & 0.038724 \\
THINK_1 & 0.009330 & 0.049349 & 0.001670 & 0.072030 & 0.586927 \\
THINK_2 & 0.019543 & 0.038205 & 0.002250 & 0.053868 & 0.717975 \\
UPENN & 0.003870 & 0.090498 & 0.000393 & 0.148420 & 0.587952 \\
VALEN_1 & 0.007752 & 0.181158 & 0.000001 & 0.252546 & 0.547717 \\
VALEN_2 & 0.012972 & 0.159487 & 0.000000 & 0.222795 & 0.542634 \\
\hline
\end{tabular}

Table 7: Parameters of fit over range from 0 to $14 \%$ of model to error versus rejection rate curves for systems submitting classifications and confidences values for the digit test. 


\begin{tabular}{|l|l|l|l|l|l|}
\hline \hline SYSTEM & sigma & $e_{0}$ & $e_{\min }$ & $r_{0}$ & ratio \\
\hline AEG & 0.017454 & 0.038010 & 0.004459 & 0.053350 & 0.527693 \\
ASOL & 0.012070 & 0.113362 & 0.000000 & 0.248373 & 0.282966 \\
ATT_1 & 0.011175 & 0.065826 & 0.000000 & 0.126673 & 0.487130 \\
ATT_2 & 0.011336 & 0.056636 & 0.002750 & 0.071867 & 0.699079 \\
ATT_3 & 0.032949 & 0.070109 & 0.000000 & 0.104839 & 0.530980 \\
ATT_4 & 0.027155 & 0.050839 & 0.000950 & 0.070190 & 0.604783 \\
ERIM_1 & 0.020207 & 0.051538 & 0.000940 & 0.083857 & 0.600994 \\
GTESS_1 & 0.012625 & 0.066724 & 0.000000 & 0.104407 & 0.560962 \\
GTESS_2 & 0.006790 & 0.067664 & 0.002970 & 0.102696 & 0.592692 \\
HUGHES_1 & 0.027327 & 0.066304 & 0.000000 & 0.116388 & 0.420525 \\
HUGHES_2 & 0.042265 & 0.070535 & 0.000000 & 0.111462 & 0.376435 \\
IBM & 0.005457 & 0.064087 & 0.001980 & 0.085055 & 0.700729 \\
IFAX & 0.001717 & 0.195923 & 0.017783 & 0.236433 & 0.697279 \\
KODAK_1 & 0.023934 & 0.070835 & 0.000000 & 0.104786 & 0.561767 \\
NESTOR & 0.028858 & 0.060172 & 0.000000 & 0.100379 & 0.511940 \\
NIST_2 & 0.001366 & 0.231152 & 0.014857 & 0.257046 & 0.759154 \\
NIST_3 & 0.010560 & 0.170368 & 0.000000 & 0.205900 & 0.790337 \\
NYNEX & 0.005835 & 0.049212 & 0.004903 & 0.071414 & 0.584223 \\
OCRSYS & 0.000000 & 0.057283 & 0.010000 & 0.085924 & 0.006016 \\
UMICH_1 & 0.013518 & 0.050340 & 0.020245 & 0.089622 & 0.386150 \\
VALEN_1 & 0.007327 & 0.243245 & 0.000028 & 0.339330 & 0.560223 \\
\hline
\end{tabular}

Table 8: Parameters of fit over range from 0 to $14 \%$ of model to error versus rejection rate curves for systems submitting classifications and confidences values for the upper case letter test. 


\begin{tabular}{|l|l|l|l|l|l|}
\hline \hline SYSTEM & sigma & $e_{0}$ & $e_{\min }$ & $r_{0}$ & ratio \\
\hline AEG & 0.018673 & 0.129064 & 0.000002 & 0.197466 & 0.549637 \\
ASOL & 0.006330 & 0.212562 & 0.070999 & 0.219958 & 0.519595 \\
ATT_1 & 0.011495 & 0.139576 & 0.000000 & 0.232988 & 0.431578 \\
ATT_2 & 0.006518 & 0.141519 & 0.000000 & 0.175623 & 0.710256 \\
ATT_3 & 0.006362 & 0.163227 & 0.000000 & 0.229147 & 0.733748 \\
ATT_4 & 0.011273 & 0.144687 & 0.000000 & 0.195346 & 0.590847 \\
ERIM_1 & 0.006800 & 0.137500 & 0.000000 & 0.185982 & 0.793400 \\
GTESS_1 & 0.005090 & 0.176155 & 0.007665 & 0.229252 & 0.562496 \\
GTESS_2 & 0.006994 & 0.185672 & 0.000000 & 0.240376 & 0.600176 \\
HUGHES_1 & 0.009387 & 0.153990 & 0.000020 & 0.240259 & 0.690638 \\
HUGHES_2 & 0.013273 & 0.156073 & 0.000000 & 0.247943 & 0.689370 \\
IBM & 0.004598 & 0.154840 & 0.000008 & 0.206804 & 0.674157 \\
KODAK_1 & 0.013933 & 0.147603 & 0.000000 & 0.206858 & 0.493963 \\
NESTOR & 0.010327 & 0.155805 & 0.000003 & 0.207386 & 0.582884 \\
NIST_2 & 0.004220 & 0.313207 & 0.000015 & 0.366355 & 0.720330 \\
NIST_3 & 0.004278 & 0.203856 & 0.000000 & 0.251538 & 0.711280 \\
NYNEX & 0.008670 & 0.140270 & 0.000002 & 0.219168 & 0.581997 \\
OCRSYS & 0.002186 & 0.136838 & 0.038749 & 0.171823 & 0.500069 \\
UMICH_1 & 0.002900 & 0.150510 & 0.043706 & 0.197158 & 0.489376 \\
VALEN_1 & 0.003552 & 0.317315 & 0.000000 & 0.438767 & 0.514008 \\
\hline
\end{tabular}

Table 9: Parameters of fit over range from 0 to $14 \%$ of model to error versus rejection rate curves for systems submitting classifications and confidences values for the lower case letter test. 


\title{
5 Types of Algorithms Used
}

\author{
Charles L. Wilson
}

\subsection{Rule-based versus Machine learning}

In the past few years neural networks have become important as a possible method for constructing computer programs that can solve problems, such as speech and character recognition, where "human-like" response or artificial intelligence is needed. The most useful characteristics of neural networks are their ability to learn from examples, their ability to operate in parallel, and their ability to perform well using data that are noisy or incomplete. Many of these characteristics are shared by various statistical pattern recognition methods. These characteristics of pattern recognition systems are important for solving real problems from the field of character recognition exemplified by this report, as opposed to "toy" problems. The goal of this section is to summarize the different methods used at the Census OCR Conference in a way that will illustrate why neural networks and rule based methods achieved the level of performance that they did. The various methods used are summarized in Figure 9 for classification and feature extraction. Most of the systems presented at the Conference, but not all, used separate methods of feature extraction and classification. In the discussion presented here any image processing which preceded the feature extraction is combined with feature extraction.

The discriminant function and classification sections of the systems are of two types: adaptive learning based and rule-based. The most common approach to machine learning based systems used at the Conference was neural networks. The neural approach to machine learning was originally devised by Rosenblat [6i] by connecting together a layer of artificial neurons [68] on a perceptron network. The weaknesses which were present in this approach were analyzed by Minski and Papert [69]. The results of this Conference suggest that many of these weaknesses are still important. The advent of new methods for network construction and training during the last ten years led to rapid expansions in neural network research in the late 1980s. Many of the methods referred to in Figure 9 were developed in this period.

Adaptive learning is further subdivided in to two types, supervised learning and self-organization. The material presented in this report does not cover the mathematical detail of these methods, but the bibliographic references provided with many of the systems discuss these methods in detail. A good source of general information on neural networks is Lippmann's review [70]. The primary research sources for neural networks are available in Anderson and Rosenfeld [71]. More detailed information on the supervised learning methods discussed here is given in [72]; self-organizing methods are discussed by Kohonen [73] and Grossberg [74].

The principal difference between neural network methods and rule-based methods is that the former attempts to simulate intelligent behavior by using adaptive learning and the latter uses logical symbol manipulation. The two most common rule-based approaches at the Conference were those derived from mathematical image processing and those derived from statistics. With adaptive learning, once the learning phase has been completed the network 
response is automatic and similar in nature to reflex responses in living organisms. The processes where these methods have been most successful are in areas where human responses are automatic, such as touching ones nose or recognizing characters. With mathematical approaches, fixed operations are performed on individual images or on statistical samples of images.

The alternate approach to artificial intelligence is rule-based. Rather than teaching the program to differentiate between characters, a rule-based program is constructed to distinguish among the various characters by writing rules to be followed by the system. These are explicitly programmed in the system in the form of mathematical formulas.

Most of the OCR implementations discussed in this report combine several methods to carry out preprocessing (filtering) and feature extraction. Many of the filtering methods used are based on methods described in texts on image processing such as [65] and on a method based on $\mathrm{KL}$ transforms [39]. In these methods, the recognition is done using features extracted from the primary image by rule based techniques. The filtering and feature extraction processes start with an image of a character. The features produced are then used as the input for classification.

In a self-organizing method, such as [19], data is applied directly to the neural network and any filtering is learned as features are extracted. In a supervised method, the features are extracted using either rule-based or adaptive methods and classification is carried out using either type of method. Systems with all four possible combinations of rules and adaptive learning were used at the Conference.

\subsection{Statistical Rules versus Mathematical Rules}

In Figure 9, rules based on mathematical image processing are distinguished from rules based on statistics. These two types of rules are similar in that they both derive features based on a model of the images. Statistical rules derive these model parameters based on the data presented. For example, typical model parameters might be sample means and variances. Mathematical rules operate on the data based on external model parameters or on the specific data being analyzed. The model parameters might be designed to detect strokes, curvature, holes, or concave or convex surfaces.

\subsection{Linear versus Non-linear Methods}

All of the methods shown in Figure 9 can also be classed broadly into linear methods, such as perceptrons [67], and nonlinear methods, such as Multi-Layer Perceptrons (MLPs) [72]. This separation into linear and non-linear algorithms also extends to mathematical and statistical methods. Many of the convolution and transform methods, such as Walsh transforms [75] or combinations of Gabor transforms [28] are linear. Other method start with linear operations such as correlation matrices and become non-linear by removing information with low statistical significance; $\mathrm{KL}$ transforms [65] and principal component analysis (PCA) [64] are examples of this. 


\subsection{Statistical and Neural Methods}

When training data is used to adjust statistical model parameters to train MLPs, certain methods may be classed as either neural network or statistical methods. The probabilistic neural network (PNN) is an example of this type of method. In another context PNN methods can be regarded as one class of a radial basis function (RBF) method. The information in Figure 9 classifies methods of this kind in an arbitrary way when statistical accumulation or neural network models of a given method are equivalent.

\subsection{Role of Learning and Rules in Feature Extraction and Clas- sification}

The systems submitted for testing at the Conference used all of the four combinations of rule-based and learning-based feature extraction and classification. All possible combinations yielded at least one low error rate system. The most common combination was the use of a mathematically based feature extractor with a MLP classifier. At least one system combined feature extraction with classification [6]. One major surprise was that linear methods, such as Learned Vector Quantitatization (LVQ) [73] and PNN performed as well as highly non-linear methods such as MLPs.

A possible explanation for this can be found in Bayesian models of the learning and recognition process [76], [77], and [78]. The relationship between testing error, $E_{t s t}$ and training error $E_{t r n}$ is given by:

$$
E_{t s t}=E_{t r n}+2 \sigma_{e f f}^{2} \frac{p_{e f f}}{n}
$$

where $\sigma_{\text {eff }}^{2}$ is the effective noise in the network variables, $p_{\text {eff }}$ is the effective number of network parameters, and $n$ is the size of the training sample.

The noise in the network is learned from the training sample and should be similar for all participants. Most participants achieved training errors of less than $0.5 \%$. The strong similarity of accuracy results suggest that all of the methods used maintain a fixed ratio of complexity to sample size. This would suggest that, in noisy samples of the kind used in the Conference tests, learning can not remove sample noise injected into the classification system from the training data because the excess complexity of the network is used to track the noise in the data. This is not unexpected since the systems have no mechanism for evaluating "bad" writing except by statistical frequency. 

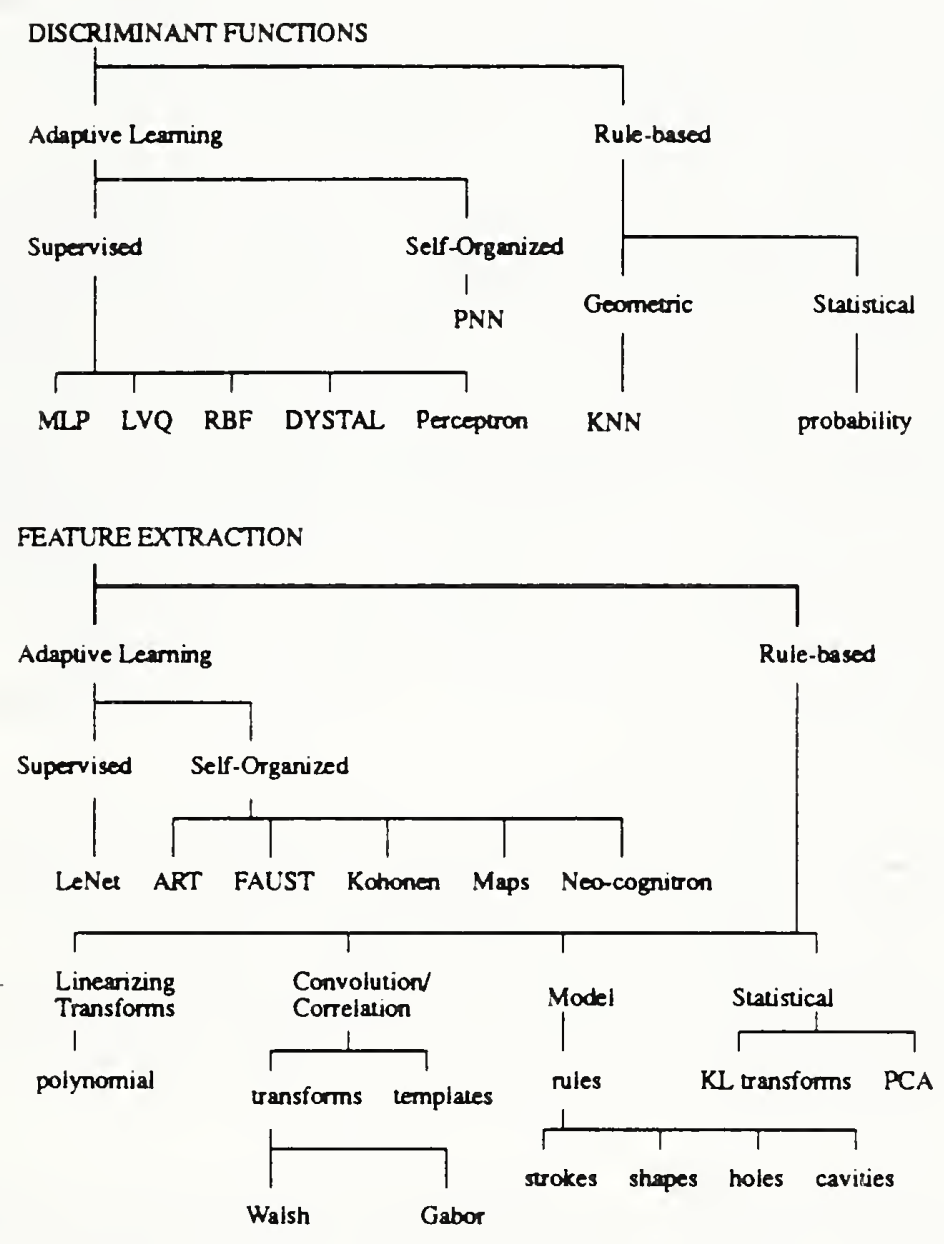

Figure 9: Types of methods used for feature extraction and classification. 


\section{$6 \quad$ System Speed}

\section{Charles L. Wilson}

Figure 10 shows the flow of data through a typical page level OCR system. The details of the particular system are discussed in [79]. The tests run for this Conference were conducted on a simplified problem in which the characters were isolated and segmented prior to being used by the Conference participants so that the only modules used for Conference testing are normalization, filtering/feature extraction, recognition, and rejection. The load and store modules are present in either the full system or the simplified test system. This Conference did not address field isolation and character segmentation.

Typical timings for a system of the type shown in Figure 10 are given in Table 10. The dominant times in this table are image loading, field isolation, and character segmentation times. In the Conference systems, field isolation and character segmentation times were not required so that the dominant time for the Conference systems is the image loading time. In the system summaries in Appendices $E$ and F, two rates are listed: the total system time and the recognition time. In most cases, total system rate is much longer than recognition rate. This speed difference increases as recognition time decreases. Most systems have similar load times but recognition times vary by several orders of magnitude. The minimum recognition time is less than $1 \mathrm{~ms} /$ character. The typical load time is near $100 \mathrm{~ms} /$ character. These two times place distinct bounds on system performance. The recognition rate of the faster systems is near the present state-of-the-art for recognition performance. The system rate is near the typical speed that can be achieved loading and decompressing image data on common present-day desk-top systems.

In order to evaluate the performance bounds of possible systems, some knowledge of both algorithmic complexity and the importance of the algorithm in the overall system performance are needed. This can not be accomplished without breaking the system into separate components each of which contains only one dominant algorithmic process. The importance of the scaling of algorithms has been known since the early work on neural networks [69]. The second factor which contributes to Table 10 is the data volume which each module encounters during operation.

Most theories of numerical algorithm complexity such as those given in [80], [69], and [81] express complexity results in notation of the form $O\left(n^{p}\right)$ where $n$ is a measure of the size of a specific type of objects such as $n$ weights, $n$ pixels, or $n$ classes and $p$ is a measure of a specific polynomial complexity. As data flows through the recognition process, $n$ decreases very rapidly. The characters used for testing in this Conference were scanned at six line pairs per $\mathrm{mm}$. For a $5 \mathrm{~mm}$ square character, this results in an input image having 3600 pixels. The system outputs were a single class. This reduces $n$ from 3600 to one. In order to separate the $O\left(n^{p}\right)$ effects from changes in the size of $n$, the exact proportionality constant for each type of algorithm is important. A $n^{3}$ algorithm working on 10 data items may still be fast if compared to an linear algorithm working on 3600 data items.

The systems that were submitted to the Conference for testing used a wide variety of hardware. These ranged from PC's to a Connection Machine. Several types of special purpose 
systems were used. These included VLSI based hardware [10] and three kinds of massively parallel computer: Connection Machine, Adaptive Solutions, and AMT DAP. Several of these systems achieved recognition rates over 500 characters/second. At these rates, all of these systems were limited by image loading requirements. While high rates were achieved using special hardware, at least one system implemented on a PC platform achieved comparable speeds. This was possible by programing critical dot product routines as 8-bit calculations in assembly language. The algorithm used was a MLP with the usual complexity for this method but the speed achieved was dominated by reducing the basic calculation time.

The speed measurements presented in this report show that high recognition rates can be achieved either by using powerful hardware or by clever implementation. Algorithmic complexity cannot be separated from data flow requirements unless each algorithm is separated from the other system components during testing. High speed systems are limited by the ability to provide them with image data. None of these variables has been separated in the data presented here. NIST has measured system performance at the level of detail required to separate the effects of the various modules [79] but evaluation of such a system is much more complex than the bounding times given here. The Conference did show that systems on slow platforms or with slow implementations run at less than a character per second and that systems implemented on high speed hardware can run at 1000 character per second if images can be supplied at this rate. 


\section{System Data Flow}

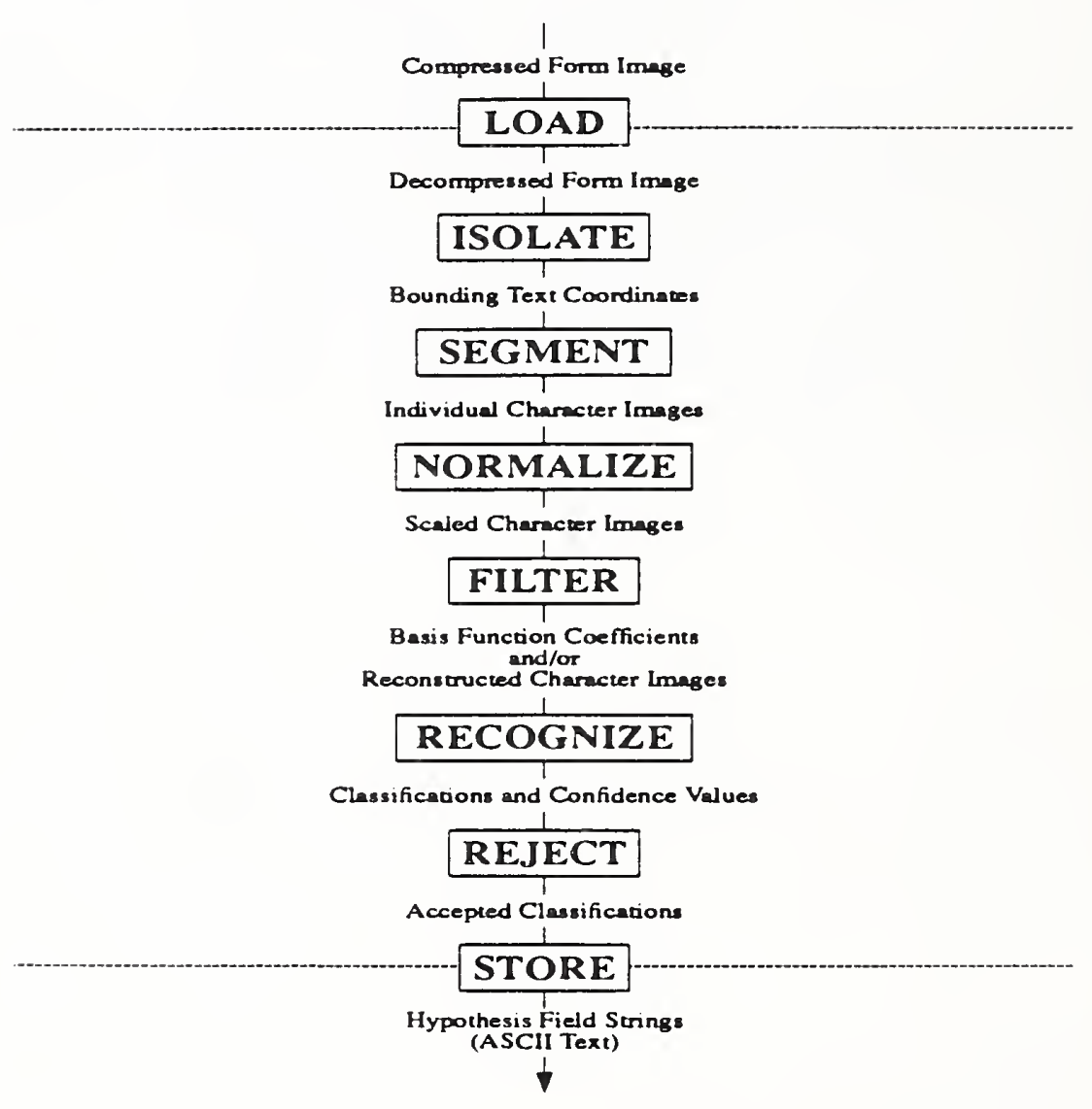

Figure 10: Data flow in a complete recognition system. 


\begin{tabular}{||c|r|cc||}
\hline COMPONENT & OVERALL & PER FORM & \\
\hline Load: & 18668.328 & 8.889680 & $(58.54 \%)$ \\
Isolate: & 3669.375 & 1.747321 & $(11.51 \%)$ \\
Segment: & 4773.691 & 2.273186 & $(14.97 \%)$ \\
Normalize: & 854.941 & 0.407115 & $(2.68 \%)$ \\
Filter: & 3013.547 & 1.435023 & $(9.45 \%)$ \\
Recognize: & 250.982 & 0.119515 & $(0.79 \%)$ \\
Reject: & 50.900 & 0.024238 & $(0.16 \%)$ \\
Store: & 609.079 & 0.290038 & $(1.91 \%)$ \\
\hline Total: & 31890.845 & 15.186117 & $(100.00 \%)$ \\
\hline
\end{tabular}

Table 10: System times in seconds for 2100 forms on a parallel computer. 


\section{References}

[1] M. D. Garris and R. A. Wilkinson. Handwritten segmented characters database. Technical Report Special Database 3, HWSC, National Institute of Standards and Technology, February 1992.

[2] R. A. Wilkinson. Handprinted segmented characters database. Technical Report Test Database 1, TST1, National Institute of Standards and Technology, April 1992.

[3] Thomas E. Baker. Artificial neural network and image processing on the adaptive solutions architecture. In SPIE/SPSE Symposium on Electronic Imaging Science and Technology, Conference 1452 Image Processing Algorithms and Techniques II, 1991.

[4] Thomas E. Baker and Hal McCartor. Comparison of neural network classifiers for optical character recognition. In SPIE/SPSE Symposium on Electronic Imaging Science and Technology, Conference 1661 Machine Vision Applications in Character Recognition and Industrial Inspection, 1992.

[5] Y. le Cun. Generalization and network design strategies. Technical Report CGR-TR89-4, University of Toronto, Dept. of Computer Science, 1989.

[6] Y. Le Cun, B. Boser, J. S. Denker, D. Henderson, R. E. Howard, W. Hubbard, and L. D. Jackel. Handwritten digit recognition with a back-propagation network. In D. Touretzky, editor, Advances in Neural Information Processing Systems, volume 2, pages 396-404. Morgan Kaufman, 1990.

[7] I. Guyon, V. N. Vapnick, B. E. Boser, L. Y. Botton, and S. A. Solla. Structural risk minimization for character recognition. In R. Lippmann, editor, Advances in Neural Information Processing System, volume 4, pages 471-479. Morgan Kauffman, 1992.

[8] L. D. Jackel, H. P. Graf, W. Hubbard, J. S. Denker, D. Henderson. and Isabelle Guyon. An application of neural net chips: Handwritten digit recognition. In IEEE International Conference on Neural Networks, volume II, pages 107-115, San Diego, 1988.

[9] H. P. Graf, C. Nohl, and J. Ben. Image segmentation with networks of variable scale. In J. Moody, S. Hanson, and R. Lippmann, editors, Advances in Neural Information Processing Systems, volume IV, pages 480-487. Morgan Kaufmann, Denver, December 1991.

[10] L. D. Jackel, B. Boser, J. S. Denker, H. P. Graf, Y. Le Cun, I. Guyon, D. Henderson, R. E. Howard, W. Hubbard, and S. A. Solla. Hardware requirements for neural-net optical character recognition. In Proceedings of IJCNN, volume 2, pages 855-861, San Diego, 1990.

[11] D. O. Hepp. Advanced research in contextual analysis of addresses: Phase IVa Report. Technical Report 210600-52-T, ERIM, 1992. 
[12] P. Gader, B. Forester, M. Ganzberger, A. Gillies, B. Mitchell, M. Whalen, and T. Yocum. Recognition of handwritten digits using template and model matching. Pattern Recognition, 24(5):421-431, 1991.

[13] P. D. Gader. Pipelined Systems for Recognition of Handwritten digits in USPS ZIP Codes. In Proceedings of the United States Postal Service Advanced Technology Conference, Washington, DC, 1990.

[14] A. G. Gillies and B. T. Mitchell. A model-based approach to handwritten digit recognition. Machine Vision and Applications, 2:231-243, 1989.

[15] H. Muehlenbein. Evolution in time and space - the parallel genetic algorithm. In G. Rawlins, editor, Foundations of Genetic Algorithms, pages 316-337, San Mateo, 1991. Morgan-Kaufman.

[16] H. Muhlenbein, M. Schomisch, and J. Born. The parallel genetic algorithm as function optimizer. Parallel Computing, 17:619-632, 1991.

[17] F. J. Smieja. Multiple network systems (MIN0S) modules: Task division and module discrimination. In Proceedings of the 8th AISB conference on Artificial Intelligence, April 1991.

[18] F. Smieja and H. Muhlenbein. Reflective modular neural networks. GMD Report to be published, 1992.

[19] K. Fukushima. Neocognitron: A self-organizing neural network model for mechanism of pattern recognition unaffected by shift in position. Biological Cybernetics, 36:193-202, 1980.

[20] H. Takahashi. A Neural Net OCR using Geometrical and Zonal Pattern Features. In Proceedings of International Conference on Document Analysis and Recognition, pages 821-828, France, 1991.

[21] R. G. Casey, D. R. Ferguson, K. M. Mohiuddin, and E. Walach. An intelligent forms processing subsystem. Machine Vision and Applications, 1992. to appear.

[22] R. G. Casey and K. Y. Wong. Document analysis systems and techniques. In Image Analysis Applications. Marcel Dekker, Inc., New York, 1990.

[23] R. G. Casey and D. R. Ferguson. Intelligent forms processing. IBM Systems Journal, 29(3):435-450, 1990.

[24] R. G. Casey and H. Takahashi. Experience in segmenting and recognizing the nist database. In Proceedings of the International Workshop on Frontiers of Handwriring Recognition, France, 1991.

[25] H. Takahashi, N. Itoh, T. Amano, and A. Yamashita. A Spelling Correction Method and Its Applications to an OCR System. Pattern Recognition, 23(3/4), 1990. 
[26] S. Katob and $\mathrm{H}$. Takahashi. A multi-font kanji recognition method using a layered template dictionary. IEICE Transactions, D-II, 1991.

[2i] Uma Shrinivasan. Polynomial discriminant method for handwritten digit recognition. Technical report, SUNY Buffalo, 1989.

[28] J. G. Daugman. Complete discrete 2-d Gabor transform by neural networks for image analysis and compression. IEEE Trans. on Acoustics, Speech, and Signal Processing, 36:1169-1179, 1988.

[29] J. Loncelle, N. Derycke, and F. Fogelman Soulie. Optical character recognition and cooperating neural networks techniques. ICSSN '92, 1992. to appear.

[30] J. Loncelle. Detection de contours par retro-propagation de gradient. Neuro-Nimes '89, pages 373-393, 1989.

[31] D. L. Reilly, C. L. Scofield, C. Elbaum, and L. N. Cooper. Learning system architectures composed of multiple learning modules. In Proceedings of IEEE First International Conference on Neural Networks, volume II, pages 495-503, San Diego CA, June 1987.

[32] C. L. Scofield, L. Kenton, and J. C. Chang. Multiple neural net architectures for character recognition. Comp Conf, pages 487-491, 1991. invited paper.

[33] D. L. Reilly, L. N. Cooper, and C. Elbaum. A neural model for category learning. Biological Cybernetics, 45:35-41, 1982.

[34] D. E. Rumelhart, G. E. Hinton, and R. J. Williams. Learning representations by backpropagating errors. Nature, 332:533-536, 1986.

[35] C. L. Scofield, D. L. Reilly, C. Elbaum, and L. N. Cooper. Pattern class degeneracy in an unrestricted storage density memory. In D. Z. Anderson, editor, Neural Information Processing Systems, pages 674-682. American Institute of Physics, New York NY, 1988.

[36] C. L. Wilson and M. D. Garris. Handprinted character database. National Institute of Standards and Technology, Special Database 1, HWDB, April 18, 1990.

[37] J. S. Denker, W. R. Gardner, H. P. Graf, D. Henderson, R. E. Howard, W. Hubbard, L. D. Jackel, H. S. Baird, and I. Guyon. Neural network recognizer for hand-written zip code digits. In D. Touretzky, editor, Advances in Neural Information Processing Systems, volume 1, pages 323-331. Morgan Kaufmann, 1989.

[38] C. L. Scofield. Learning internal representations in the coulomb energy network. IEEE First International Conference on Neural Networks, 1:271-276, 1987.

[39] P. J. Grother. Karhunen Loève feature extraction for neural handwritten character recognition. In Proceedings: Applications of Artificial Neural Networks III. Orlando, SPIE, April 1992. 
[40] J. L. Blue and P. J. Grother. Training Feed Forward Networks Using Conjugate Gradients. In Conference on Character Recognition and Digitizer Technologies, volume 1661, pages 179-190, San Jose California, February 1992. SPIE.

[41] M. F. Møller. A scaled conjugate gradient algorithm for fast supervised learning. Neural Networks, 1991.

[42] Donald F. Specht. Probalistic neural networks. Neural Networks, 3(1):109-118, 1990.

[43] Zs M. Kovacs, R. Guerrieri, and G. Baccarani. A novel metric for nearest-neighbor classification of hand-written digits. In 11th ICPR, The Netherlands, Aug. - Sept. 1992. to be presented.

[44] Zs. M. Kovacs and R. Guerrieri. A generalization technique for nearest-neighbor classifiers. In Proceedings of IJCNN '91, volume 2, pages 1782-1788, Singapore, 1991.

[45] R. G. Casey. Moment normalization of handprinted characters. IBM J. Res. Dev, page $548,1970$.

[46] Z. Guo and R. W. Hall. Parallel thinning with two-subiteration algorithms. Image Processing and Computer Vision, 32:359-373, 1989.

[47] K. Fukunaga. Introduction to Statistical Pattern Recognition. New York: Academic Press, second edition, 1990.

[48] L. Lam and C. Y. Suen. Structural classification and relaxation matching of totally unconstrained handwritten zip-code numbers. Pattern Recognition, 21:19-31, 1988.

[49] A. Rosenfeld and J. L. Pfaltz. Distance functions on digital pictures. Pattern Recognition, 1(1):33-61, 1968.

[50] T. Fontaine and L. Shastri. Character recognition using a modular spatiotemporal connectionist model. Technical Report MS-CIS-92-24/Linc Lab 219, University of Pennsylvania, 1992. to appear.

[51] R. Bakis, N. Herbst, and G. Nagy. An experimental study of machine recognition of hand-printed numerals. IEEE Transactions on Systems Science and Cybernetics, 4-2:119-132, 1968.

[52] H. S. Hou. Digital Document Processing. John Wiley and Sons, 1983.

[53] G. Martin and J. Pittman. Recognizing hand-printed letters and digits. In D. Touretzky, editor, Advances in Neural Information Processing Systems, volume 2, pages 405-414. Morgan Kaufmann, 1990.

[54] N. J. Naccache and R. Shinghal. Spta: a proposed algorithm for thinning binary patterns. IEEE Transactions on Systems, Man and Cybernetics, SMC-14:409-418, 1984.

[55] R. Watrous. Speech Recognition Using Connectionist Networks. PhD thesis, University of Pennsylvania, 1988. 
[56] R. Fletcher. Practical Methods of Optimization. John Wiley and Sons, 1980.

[57] T. Fontaine. A Hybrid Procedural-Connectionist Word Recognition System. PhD thesis, University of Pennsylvania, 1991.

[58] J. C. Prez, E. Vidal, and L. Sanchez. Un sistema geometrico de reconocimiento optico de caracteres. In Simposium Nacional de Reconocimiento de Formas y Analisis de Imagenes, 1992. A technical report in English is currently under preparation.

[59] E. Vidal, H. Rulot, J. M. Valiente, and G. Andreau. Recognition of planar shapes through the error-correcting grammatical inference algorithm (ecgi). ICPR-92, The Hague, 1992. extended version available as Technical Report.

[60] M. Stone. Cross validatory choice and assessment of statistical procedures. Royal Statistical Society, B36, 1974.

[61] J. W. Tukey and F Mosteller. Data analysis, including statists. In G. Lindzey and E. Aronson, editors, Handbook of Social Psychology, volume 2. Addison-Wesley, 1968.

[62] J. Moody and J. Utans. Selection of neural net architectures via the prediction risk: Application to corporate bond rating prediction. In Proceedings of the First Intl. Conference on Artificial Intelligence Applications on Wall St. IEEE Computer Society Press, 1991.

[63] T. M. Cover and P. E. Hart. Nearest neighbour pattern classification. IEEE Transactions on Information Processing, IT-13:21-27, 1967.

[64] T. P. Vogl, K. L. Blackwell, S. D. Hyman, G. S. Barbour, and D. L. Alkon. Classification of Japanese Kanji using principal component analysis as a preprocessor to an artificial neural etwork. In International Joint Conference on Neural Networks, volume 1, pages 233-238. IEEE and International Neural Network Society, 71991.

[65] Anil K. Jain. Fundamentals of Digital Image Processing, chapter 5.11, pages 163-174. Prentice Hall Inc., prentice hall international edition, 1989.

[66] S. A. Teukolsky W. H. Press, B. P. Flannery and W. T. Vetterling. Numerical Recipes, chapter 13, pages 464-469. Cambridge University Press, 1989.

[67] F. Rosenblatt. The perceptron: a probabilistic model for information storage and organization in the brain. Psychological Review, 65:386-408, 1958.

[68] W. S. McCulloch and W. Pitts. A logical calculus of the ideas immanent in nervous activity. Bull. Math. Biophysics, 9:115-133, 1943.

[69] M. Minsky and S. Papert. Perceptrons. MIT Press, Cambridge, MA, 1969.

[70] R. P. Lippman. An introduction to computing with neural nets. IEEE ASSP Magazine, pages 4-22, April 1987. 
[71] J. A. Anderson and E. Rosenfeld. Neurocomputing. MIT Press, Cambridge, MA, 1989.

[72] D. E. Rumelhart, G. E. Hinton, and R. J. Williams. Learning internal representations by error propagation. In D. E. Rumelhart and J. L. McClelland, et al., editors, Parallel Distributed Processing: Explorations in the Microstructure of Cognition. Volume 1: Foundations, chapter 8, pages 318-362. MIT Press, Cambridge, MA, 1988.

[73] T. Kohonen. Self-Organization and Associative Memory. Springer-Verlag, Berlin, second edition, 1988.

[74] S. Grossberg. On learning and energy-entropy dependence in recurrent and nonrecurrent signed networks. J. Statistical Physics, 1:319-350, 1969.

[75] K. G. Beauchamp. Walsh Functions and Their Applications. Academic Press, London, 1975.

[76] David J. C. MacKay. Bayesian model comparison and backprop nets. In R. Lippmann. editor, Advances in Neural Information Processing System, volume 4, pages 839-846. Morgan Kauffman, 1992.

[77] V. Vapnik. Principals of risk minimization for learning theory. In R. Lippmann, editor, Advances in Neural Information Processing System, volume 4, pages 831-838. Morgan Kauffman, 1992.

[78] J. E. Moody. The effective numbers of parameters: an analysis of generalization and regularization in nonlinear systems. In R. Lippmann, editor, Advances in Neural Information Processing System, volume 4, pages 847-854. Morgan Kauffman, 1992.

[79] M. D. Garris. C. L. Wilson, J. L. Blue, G. T. Candela, P. Grother, S. Janet, and R. A. Wilkinson. Massivelly parallel implementation of character recognition systems. In Proceedings Febuary 1992, pages 269-280. SPIE, San Jose California, February 1992.

[80] R. P. Brent. Algorithms for Minimization Without Derivitives. Prentice-Hall, New York, 1973.

[81] A. S. Householder. The Theory of Matrices in Numerical Analysis. Dover, New York, 1964. 


\section{A Issues Raised by Participants}

\section{Christopher J. C. Burges and Thomas P. Vogl}

This section contains a list of issues that Conference participants raised during the course of their presentations. Feedback from participants is a very important part of our effort to make future Systems Conferences as effective and useful to the community as possible. The issues listed here will be seriously considered in the planning of the next System Conference. Some of these issues and possible problems anticipated in addressing them are described in the next appendix. It should be noted that the following does not represent a majority view of participants; it is merely a list of items that individual participants felt to be important.

(1) The long range goal of the enterprise should be "Goal Directed Document Understanding". Only when the overall goal is kept in mind will we have meaningful end-to-end performance measures.

(2) The next Systems Conference should involve recognition of isolated fields: strings of digits and printed, unsegmented words, perhaps including cursive words.

(3) Tests should be run at NIST, or somehow proctored by NIST, since whether we like it or not, results will be used extensively for marketing purposes.

(4) NIST should always provide a "submission received" message when materials are received from participants. This will prevent confusion in the event that, for example, electronic mail is lost.

(5) Participants should be given a mini-training set long before anything else happens, so that they can get their systems and software in place and ready to process large amounts of training/test materials in limited time. (In the test just passed, this process ate into the time available for training). Sufficient time should be given to participants so that all problems regarding data formatting and data exchange can be resolved, so that no time need be wasted in pursuing these issues at the following Conference.

(6) The NIST Test Data 1 should be split (by NIST) into training and test subsets, so that participants can compare the performance of systems trained on a portion of the test database.

(7) Two separate tests should be performed: one in which the test data is taken from the same distribution as the training data, and one in which it is not. This should be done because in some applications, the former may hold, while in others, the latter may hold.

(8) Participants should be told what part of the country and world the test samples are from, so that they might take advantage of (learned) "Handwriting Dialects".

(9) A writer index to databases should be provided, since in a real-world application like form reading, it is a good bet that the same writer filled out the whole form, and some systems might take advantage of this fact. Similarly, writer implement identification should be given.

(10) For word recognition, lexicons should be made available, even if they are as large as the English language. Nonsense words constructed from individual characters would not be 
a useful test.

(11) Use of contextual information (in addition to lexical information) should eventually be tested. For example, in form recognition, there is often valuable contextual information available, such as how a particular writer prints the 1 and the 9 in a date.

(12) NIST, and other users, should settle on a standardized resolution for images so that results of tests performed elsewhere in the community (outside of OCR Systems Conferences) can be more easily compared.

(13) Single character OCR systems should also be tested for their rejection of NON-characters (junk), since that is extremely important when segmenting fields.

(14) Systems should be allowed to classify an image as ambiguous. Systems should give several top choice candidate answers for a given image. Such information could be used by a contextual-analysis "supersystem". In addition, a system that does not get the right top answer, but gets the right answer in the top few, should be given credit over a system that does not get the correct answer anywhere.

(15) A proposal was made for an error rate metric: error rate $=$ Sum ( $F$ (character) $\times$ error (character)), where $F$ (character) is the frequency of the character in the English language. Another proposal was to use the integral of the error rate as a function of rejection rate instead of the zero-rejection-rate error rate.

(16) Ranking test results by a single measurement was not a good idea; several measurements should be used to get fairer analyses (e.g. raw recognition rate, throughput, unit cost, punting, latency if any, flexibility to different applications). Tests should be done with and without both lexical and non-lexical context, and the scores for each reported.

(17) People who do NOT take part in a Conference should still be able to be subjected to the same kind of test by NIST, for example by some publicly acknowledged arrangement for submitting a request to NIST, getting test materials, and having to return the scored. OCR classified test materials within a fixed, short time. These "post conference" tests should support the just-completed segmented character tests for some reasonable minimum time (say 3 years). This would help many who would otherwise not be able to be involved, and would give a more accurate representation of the state of the art at any time.

(18) While it is likely that in future Conferences all applicants will be allowed to participate in the testing and scoring aspects of the Conference, only those who are willing to divulge information about their methods should be allowed to speak, or even to attend the meeting where other participants are going to describe their methods. 


\section{B NIST Perspective on Perceived Problems}

Jon Geist

Some NIST staff members, some Conference Committee members, and some participants pointed out and suggested solutions to problems that occured at the first Conference. Problems of the most concern to the NIST personnel running the Conference are addressed in this Appendix. These are not necessarily the problems of the most concern to the participants. The information in this and Appendix $A$ is included to help in the planning of future Conferences.

\section{B.1 Perceived Problem 1}

The plan to make all results public was inadequately formulated and inadequately stated in the Call for Participation.

\section{B.1.1 Proposed Solutions}

State this aspect of the plan very clearly in future Calls for Participation.

\section{B.1.2 Discussion}

From the earliest stages of planning for the Conference, it appeared that the goals of the Conference could not be met without disclosing the scores obtained by each system. Otherwise, it would not be possible to ask specific questions about aspects of the performance of a particular system. Therefore, there was at least a weak consensus among the Committee to distribute all of the scores for all of the systems to each participant, and to publish the results in a report that would enter the public domain. During the final preparations for the Conference meeting, the consensus grew in strength as the problems with keeping the scores confidential were brought clearly into focus.

\section{B.2 Perceived Problem 2}

The attempt to restrict the number of participants was a mistake.

\section{B.2.1 Proposed Solutions}

Open the Conferences to all applicants. If logistic considerations make it necessary, then restrict the actual number of attendees at the Conference meeting rather than the number of participants in the Conference test. 


\section{B.2.2 Discussion}

Much more was learned by having 26 participants than would have been learned with only 15. It is unlikely that the Committee would have chosen an optimum combination of 15 participants from the 29 applications it received. By following a more relaxed schedule, it should be possible to close the application period before a room is reserved for the meeting. If not, actual attendance at the meeting can be limited based on factors listed in item 18 in Appendix A, on the basis of the scores obtained, or by further restricting the number of nonparticipant attendees, if necessary.

\section{B.3 Perceived Problem 3}

Too much time was required of each participant to prepare a proposal to participate and to respond to requests from the Committee for more information. In addition, the Committee and NIST staff found it very time consuming to abstract useful information from the proposals.

\section{B.3.1 Proposed Solutions}

A simple application form requesting all of the information desired by the Committee could be submitted by the participants. This form could be included in the Call for Participation.

\section{B.3.2 Discussion}

The proposals did not prove useful in choosing among the applicants for participation, and the effort the participants expended in preparing their proposals varied greatly. Futhermore, the Committee found it necessary to solicit more information from the participants in order to prepare the system summaries in Appendix C.

\section{B.4 Perceived Problem 4}

The test was not proctored.

\section{B.4.1 Proposed Solutions}

Possible solutions include:

1) Publicly disclose the participant's scores in such a way that no one can identify a specific score with a specific participant.

2) Privately disclose each participant's scores only to that participant.

3) Set up a means by which participants can have their tests proctored if they so desire. 


\section{B.4.2 Discussion}

The idea behind the first and second proposed solutions is that there would be less motivation for the participants to cheat under these conditions. However, this proposal effectively prevents open discussion at the meeting, so there would be no point to the meeting. The Committee learned much at the meeting that would not have been learned otherwise, and many participants claimed that they learned a lot at the meeting. It seems that undisclosed or secretly disclosed scores without meetings, and meeting about openly disclosed scores are the only practical alternatives. Since one of the purposes of a systems conference is to stimulate improvements in the state of the art, the first two proposed solutions do not seem workable.

Also, secretly disclosed scores do not really remove the motivation to cheat, but only modify it. With unproctored tests and openly disclosed scores, the participants might be tempted to supplement their results with human classification in order to get a better score, so that they could advertise that score either to their sponsor to continue funding or to potential customers to encourage sales. With proctored tests and secretly disclosed scores, the participants might be tempted to lie about their scores to their sponsors or their potential customers for exactly the same reasons. This would be possible because there would be no independent way for anyone to verify that any particular participant actually received the score claimed unless the Committee were brought into all such discussions in a police role. This does not seem practical.

The idea behind the third proposed solution is that those participants who chose to enter the proctored section of the test would be protected from comparison with those who did not by the fact that the latter were apparently afraid to be proctored. Various ways that proctored tests could be conducted without requiring an undue amount of proctor time were proposed. For instance, the tests could be run on one or a limited number of different platforms, and they could be submitted as executable code with a (yet to be specified) standard interface to a (yet to be specified) central location where the test would be conducted. It remains an open question how practical this would actually be, but the developement of a standard interface for proctored tests might be a useful activity.

\section{B.5 Perceived Problem 5}

Some participants were not as open as they should have been for a Conference of this nature.

\section{B.5.1 Proposed Solutions}

Possible solutions include:

1) Request all information required of the participants in the application form, and reject any participants who do not provide it.

2) Make open discussion a prerequisite for attendence at the Conference meeting, but not for participation in the test, as discussed in item 18 of Appendix A. 


\section{B.5.2 Discussion}

These solutions might reduce participation, particularly the first, which would reduce the usefulness of the Conference as discussed above under Perceived Problem 2. The Committee appreciates the openness that many participants showed, and hopes that their example will help other participants to be more open in the future.

\section{B.6 Perceived Problem 6}

E-mail did not prove suitable for returning test results to NIST.

\section{B.6.1 Proposed Solutions}

Possible solutions include:

1) Require all submissions on disk or tape.

2) Set up an anonymous FTP on a computer at NIST to receive the test results.

3) Set up participant accounts on a computer at NIST to receive the test results.

\section{B.6.2 Discussion}

It is most convenient for NIST IRG personnel to receive test results directly on a computer at NIST rather than to have to read a disk or tape. The people responsible for choosing E-mail did not know that DARPANET and BITNET E-mail network nodes truncate Email messages to $100 \mathrm{k}$ or $300 \mathrm{k}$ bytes and cannot handle the volume of messages that can be encountered from a number of different participants all submitting their returns at the same time through various network nodes. To use E-mail, some participants had to split tarred and uuencoded files into 190 separate files for submission. The E-mail spooler on the IRG network node cannot handle this many messages at one time. To solve this problem. a software switch was written to intercept Conference returns, and to redirect them to a large buffer. Unfortunately, the NIST IRG computers went down over the weekend before the last day to return the results. When the computers were restored many of the E-mailed returns were waiting at various E-mail nodes, and they all tried to enter the NIST node at the same time. This caused the buffer to crash. An anonymous FTP is clearly the best solution.

\section{B.7 Perceived Problem 7}

Some submissions and some corrections of errors in the format or content of CON and RJX (described in Appendices C and D files, that could not be easily carried out at NIST, were accepted and scored after April 27, which was the cutoff date for submission of test results. 


\section{B.7.1 Proposed Solutions}

Possible solutions include:

1) Don't enforce any time limits.

2) Strictly enforce a time limit.

3) Provide all participants with $\mathrm{C}$ source code for a package that checks the structure of the results before they are submitted to NIST, and only score those submissions that are received on time at NIST and that pass the same check at NIST.

4) List all of the results that were obtained on time and in the correct format in one section, and all of the other results in a separate section to distinguish them in this respect.

\section{B.7.2 Discussion}

The third proposed solution seems the best compromise for addressing this problem for future Conferences. The fourth proposed solution was adopted for summarizing the results of this Conference. That section also contains a few results submitted after the Conference meeting to address specific questions brought up during the meeting.

The time limit was imposed mainly to assure that most of the results would be received in time for scoring at NIST before the Conference meeting. If the time limit is not enforced, then the participants will not make the effort to adhere to it, and it will not serve its main purpose. On the other hand, a number of useful submissions would have been rejected had the time limit been strictly enforced due to the problems that the participants had in conforming to the data formats specified for the classification results. Since this was the first Conference, and since the E-mail procedure for returning the results was fraught with its own problems, it was decided to request resubmissions of lost or incomplete results submitted by E-mail. This led to requests for resubmission in correct format of CON and RJX files that did not conform to the specified formats. This, in turn, led to a toleration for results that were submitted late, but before all of the CON and RJX were received in correct format. 


\title{
C The Call for Participation
}

\author{
CALL FOR PARTICIPATION
}

FIRST CENSUS OPTICAL CHARACTER RECOGNITION SYSTEMS CONFERENCE

\author{
February 1992 - May 1992 \\ Sponsored by: \\ US Bureau of the Census
}

Conducted by:

National Institute of Standards and Technology

(NIST)

1. Background of the CALL FOR PARTICIPATION:

The Bureau of the Census has requested the National Institute of Standards and Technology (NIST) to run a Systems Conference on Optical Character Recognition (OCR) of handprinted characters. One goal of the Systems Conference is to give the Bureau a sense of the current state-of-the-art in OCR of hand printed characters and the directions of near term R\&D. Another goal is to provide a forum through which participants can influence 1) the creation of large data bases of hand printed characters for uniform testing of OCR systems, 2) the development of uniform methods for scoring tests based on such databases, and 3 ) the development of standards for evaluating the performance of OCR systems.

A registration fee of about $\$ 50$ per person will be charged to cover lunches and coffees. All aspects of participation in this Systems Conference will be carried out at no cost to the Government. No contracts or grants will be awarded in connection with, or as a result of this Systems Conference. The Conference will be run through the First Census OCR Systems Conference Committee consisting of the following members:

Jon Geist, NIST, chairman 
Charles Wilson, NIST

Bob Hammond, Census Bureau

Robert Creecy, Census Bureau

Tom Vogl, ERIM

Christopher Burges, ATI

Jonathan Hull, U. of Buffalo

Norman Larsen, Census Bureau

2. Activities of the Systems Conference:

2.1 The Committee will review with respect to the criteria stated in Section 6 below all applications for participation that are received at NIST before Close of Business (COB) on March 4 , 1992. The Committee reserves the right to review applications received after this date.

2.2 The Committee will divide the applications into two categories with respect to the criteria stated in Section 6 , qualified and unqualified.

2.3 If there are more than about fifteen qualified applications, the Committee will rank them according to the criteria stated in Section 6 , and will select about fifteen applications for participation in the Conference. Otherwise, all qualified applications will be selected for participation.

2.4 NIST will inform the participants of their selection and will send them training materials on behalf of the Committee before COB March 23, 1992.

2.5 NIST will send test materials to the participants on behalf of the Committee before COB April 13, 1992.

2.6 The participants will return the completed test materials to NIST before COB April 27, 1992.

2.7 NIST will score the returned tests materials.

2.8 The participants and the Committee will attend a meeting on May 27 and 28, 1992. At this meeting

1) NIST will describe all participants' test results.

2) each participant will present a 15 minute talk outlining 
the OCR approach used in completing the test, and any other information that they deem pertinent, and

3) the Committee and the participants will attempt to reach a consensus about what sort of test materials should be provided for the Second Census OCR Systems Conference, and what other issues should be addressed to make the second Conference more useful to the participants.

3. Specification of Formats:

3.1 Training and Test materials supplied by NIST:

Both the training and test materials will consist of digital images of segmented numbers, upper case letters, and lower case letters on an ISO-9660 formatted CD ROM disc. For the training and test materials, the numbers, the upper case letters, and the lower case letters will be in separate files. However, as many as $30 \%$ of the letters in the lower case training files will actually be little upper case letters that were printed when lower case letters were requested. The participants are requested to return their test results by E-Mail, but they may also return them on $8 \mathrm{~mm}$ magnetic tape, or on IBM PC compatible 5.25 inch floppy disks.

The format for the image data for both the training and test materials will be in the Multiple Image Set (MIS) format, and the format for the classification data for the training materials will be the Multiple Feature Set (MFS) format. These formats are used by the NIST Image Recognition Group for Standard Reference Databases. More details of the MIS and MFS file formats, and the test result formats are given in the Appendix to this document.

3.2 Test Results supplied by Participants:

Participants will be required to return their test results in a Classification file (HYP) and either a Confidence file (CON) or a set of Rejection (RJX) file, if confidence levels are not readily available. All of theses files, HYP and CON or HYP and multiple RJX, must be in the MFS format. More details about the specifications for these files are given in the Appendix along with the specifications of the MIS and 
MFS file formats. Participants vill also be required to report the elapsed time for the OCR process and minimal specifications of the system used to obtain the results. Up to five different sets of test results will be accepted from each participant, but the participants must prioritize the results according to the format described in the Appendix.

4. Application Format:

Applications to participate in the First Census OCR Systems Conference should be no longer than 3 pages of text, and should conform to the following format:

4.1 The first section should briefly describe the proposing organization. This section should identify the person who will be the point of contact for the Systems Conference, including the mailing address, phone number, FAX number and E-Mail address, as appropriate, and up to two other attendees.

4.2 The second section of the application should state that the proposing organization agrees to participate by following instructions for the training, testing, and meeting phases of the Conference, provided that NIST supplies the materials before the dates stated in Section 2 of this CALL, as summarized below:

$$
\begin{aligned}
& 03 / 04 \text { - deadline for receipt of application at NIST } \\
& 03 / 23 \text {-- deadline for receipt of training material from NIST } \\
& 04 / 13 \text {-- deadline for receipt of testing material from NIST } \\
& 04 / 27 \text { - - deadline for return of completed test to NIST }
\end{aligned}
$$

4.3 The third section should concisely describe the state of the art in OCR of handprinted characters in the proposing organization by reporting at least one data point for the error rate and the rejection rate for some subset of NIST Special Database 1 or some other database of handprinted characters. The nature of the database used and exactly which OCR functions were performed automatically for the results presented should also be indicated.

4.4 The fourth section should concisely outline the approach to OCR used for the results reported in the third section. 
5. Submission of Applications:

Applications should be submitted to:

Jon Geist

B316/225

ASD/NCSL/NIST

Gaithersburg, MD 20899

Applications may be submitted by regular mail, express mail, courier service, FAX to (301) 948-4081, or

E-Mail to geist@sed.eeel.nist.gov or geist@magi.ncsl.nist.gov.

6. Rating Criteria:

Applications not meeting the requirements stated in Section 4 of this CALL may be eliminated from further consideration at the discretion of the Committee. The remaining Applications will be be divided into qualified and unqualified categories on the basis of sections 4.3 and 4.4 . These sections should demonstrate in a concise manner both a thorough understanding of the basic ideas of OCR of handwritten characters, and a state-of-the-art competence in this area.

If more than 15 qualified proposals are received, they will be divided into categories based on the similarity of the OCR techniques described, and the applications in each category will be ranked according to the performance claimed in the third section of each application. The fifteen applications will be selected by choosing the top ranked application from each category, then the second ranked, and so forth, until about 15 applications are chosen. The Committee reserves the right to reject all but one application from a single organization. 


\section{Instructions to Participants}

INDEX

1. INTRODUCTION

2. DIRECTORY FORMAT

3. RETURN FILE FORMATS

3.1 ASCII STRING (ASR) AND LINE (ALR) REPRESENTATIONS

3.2 MFS FILE FORMAT

3.3 HYP FILE FORMAT

3.4 RJX FILE FORMAT

3.5 CON FILE FORMAT

4. TEST RESULTS FORMAT

4.1 E-MAIL

4.2 EXABYTE UNIX 8MM MAGNETIC MEDIA

4.3 IBM PC 1.2 MEGABYTE 5.25" FLOPPY DISK

1. INTRODUCTION

This report contains information about how to return the OCR test results obtained for the MIS files in subdirectory TEST1 to NIST for scoring.

For the purposes of this test you have been assigned the site name: 


\section{DIRECTORY FORMAT}

The main directory on the CD-ROM called TEST DISK 1 has three subdirectories, DOS, DOC, and TEST1. DOS contains files that are needed only if the test results will be returned on an IBM PC compatible, 1.2 Mbyte, 5.25" floppy disk.

The actual test files are stored in subdirectory TEST1, which is organized as follows:

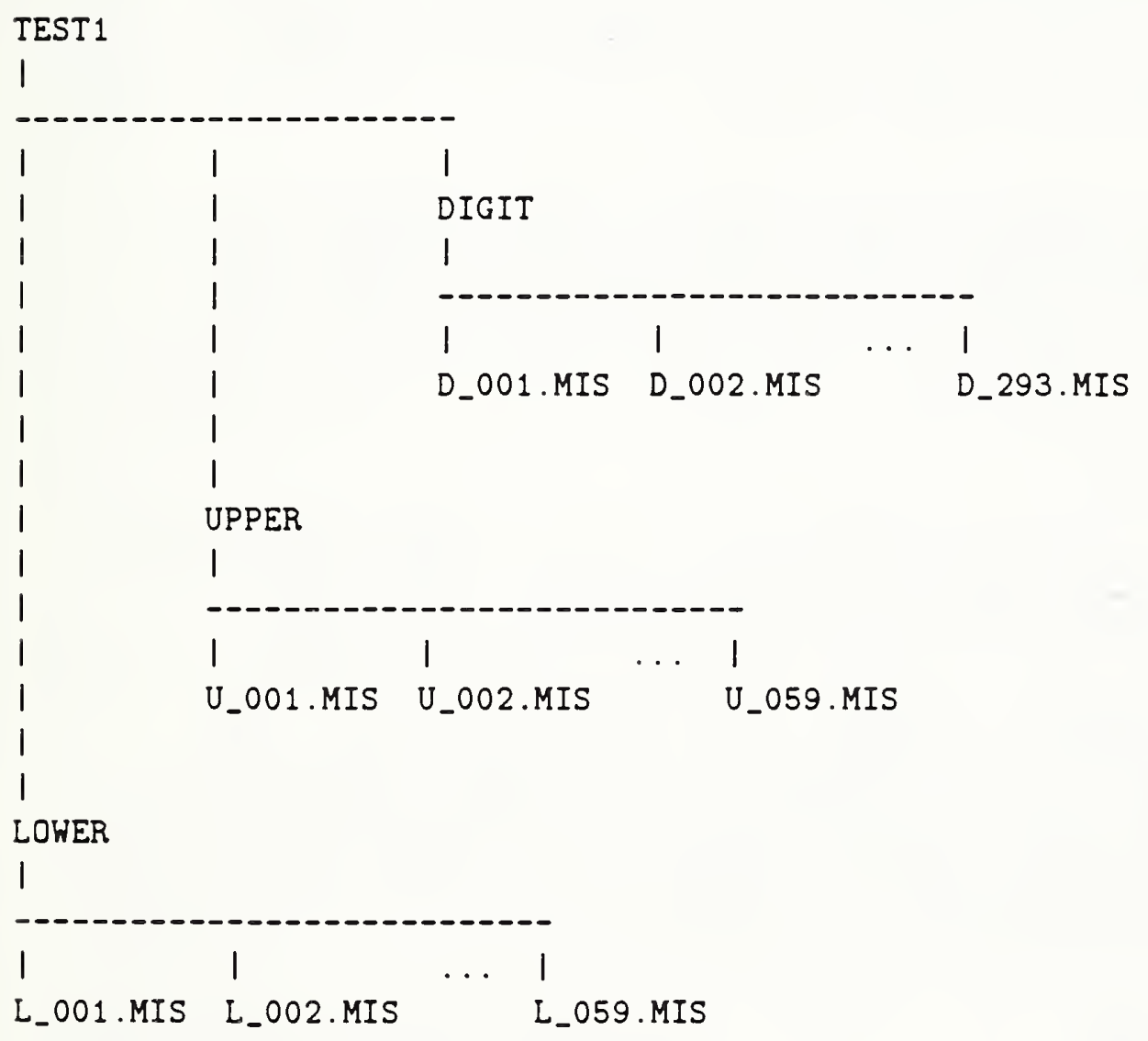

Each file except the last in each of the subdirectories DIGIT, UPPER, and LOWER has 200 images in it, while the last file has less than 200 images.

All of the information on the test file formats and programs to read these file formats was included on and/or with NIST Special Database 3, which was sent to you as the training 
materials for this test.

\section{RETURN FILE FORMATS}

This section explains the various file formats for use in returning your OCR classifications of the MIS file images in subdirectory TEST1 to NIST for scoring.

\subsection{ASCII STRING (ASR) AND LINE (ALR) REPRESENTATIONS}

An ASCII String Representation (ASR) is a buffer of variable length containing any number of printable ASCII characters, where the printable ASCII characters include all characters in the hexadecimal range 20 to $7 \mathrm{E}$.

An ASCII line Representation (ALR) is an ASR terminated by the ASCII LF character, hexadecimal OA. This means that the ASCII CR character OD cannot occur anywhere in an ALR, or in place of, or in combination with the ASCII LF character OA at the end of the ALR.

\section{2 MFS FILE FORMAT}

A Multiple Feature Set (MFS) file is a file of ALRs. Each MFS file is associated with a unique MIS file. The first line of the MFS file contains the ASR of a decimal number, which is the number of lines in the file minus one, and which is also the number of images in the associated MIS file. No ASCII SPACE characters are allowed in the ASR for the first line. Each line following the first line of an MFS file is an ALR containing information about the corresponding image in the associated MIS file.

\subsection{HYP FILE FORMAT}

A Hypothesis (HYP) file is a file in the MFS file format. Each line following the first line contains an ASR of the correct class assigned to the corresponding image in the associated MIS file. The ASR in each line consists of two ASCII characters. These are the ASCII characters that represent the hexadecimal number that represents the ASCII character of the class. No space characters are allowed on 
any line of this type of file.

The name of a HYP file must be the same as the name of the associated MIS file, except that the extension must be. HYP. For example, consider an MIS file called ALPHAS.MIS that contains images of the five characters $G, I, L, S$, and $w$. An ASCII dump (that recognized the convention that $O A$ is the end of line marker) of the associated file ALPHAS.HYP would look like:

5

47

72

$4 \mathrm{C}$

53

77

Similarly, a HEX dump of the same CLS file would look like:

$\begin{array}{lllllllllllllllll}35 & O A & 34 & 37 & O A & 37 & 32 & O A & 34 & 63 & O A & 35 & 33 & O A & 37 & 37 & O A\end{array}$

(A lower case "C" (hex 43 instead of hex 63) would be OK.)

You will return one HYP file for each MIS file in the subdirectory TEST1.

\subsection{RJX FILE FORMAT}

A Rejection (RJX) file is a file in the MFS file format in which the ASR on each line following the first line is an ASCII 0 or an ASCII 1. A 1 indicates that the classification should be scored as a Reject rather than as a Correct or an Incorrect. A 0 indicates that the classification should be scored Correct if identical with the correct classification and scored Incorrect otherwise.

The name of an R.JX file must be the same as the name of the associated MIS file except that it must end in one of the ten extensions .RJo, .RJ1, ..., .RJ8, .RJ9. Again as an example, consider the same MIS file used for the last example. An ASCII dump of one of the associated RJX files, ALPHAS.RJ3, for instance, might look like: 
Similarly, a HEX dump of the same CON file would look like:

You may use the RJX file format to return information on the reliability of the hypothetical classifications obtained from your OCR system. This format is useful if your system does not provide confidence levels or activations. Also, if your system has an accept/reject criterion that is more complex than setting a threshold on the highest confidence level or activation, this is the preferred format. If you choose to use this format, you should provide up to ten RJX files for each MIS file, and should try to include some rejection rates in the range from $5 \%$ to $50 \%$.

\subsection{CON FILE FORMAT}

A Confidence (CON) file is a file in the MFS file format in which the ASR on each line after the first line gives the decimal representation of the confidence level (or activation) assigned to the classification on the corresponding line of the HYP file that is associated with the same MIS file. The confidence level must be a number ranging from 0.0 through 1.0 . The number of digits to the right of the decimal point must be less than 17.

The name of a CON file must be the same as the name of the associated MIS file except that the extension must be .CON. For example, consider the same MIS file used for the last example. An ASCII dump of the associated file ALPHAS.CON might look like:

$$
\begin{aligned}
& 5 \\
& 0.375 \\
& .9 \\
& .7
\end{aligned}
$$


Similarly, a HEX dump of the same CON file would look like:

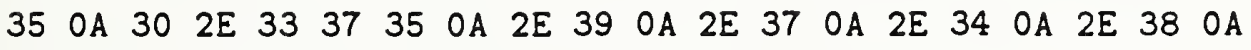

(Leading zeros are optional.)

You may use the CON file format to return the confidence levels assigned by your OCR system to the hypothetical classifications obtained from your OCR systems, provided that such information is available, and provided that your system makes it accept/reject decisions by comparing the contents of these files with a user specified threshold.

\section{TEST RESULTS FORMAT}

This section describes how the test results are to be returned to NIST. Three media are supported. The preferred media is E-Mail, the next choice is an Exabyte UNIX 8mm Magnetic Tape, and last choice is an IBM PC compatible 5.25" floppy disk.

No matter which format is used, the same directory tree structure will be used for organizing the test results. The tree will look as follows:

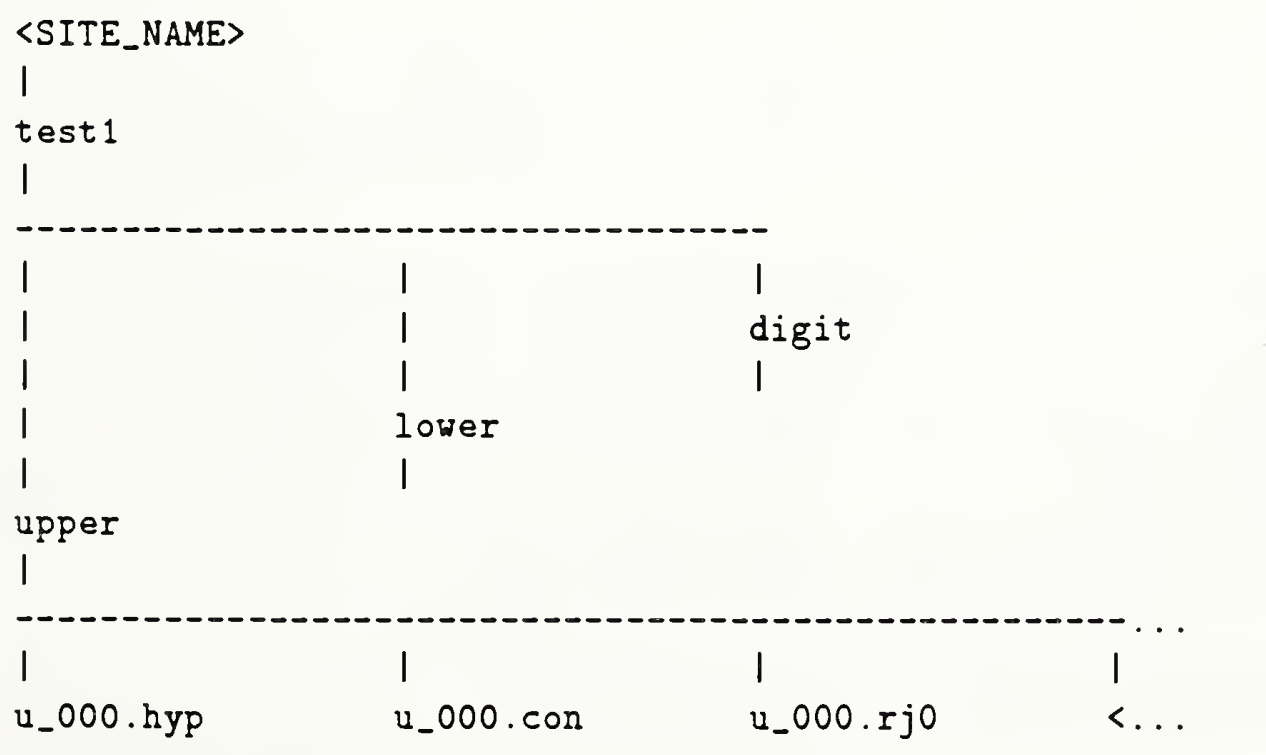


where 〈SITE_NAME〉 is the name assigned to your site in Section 1 above, if you are only reporting one set of results. If you are reporting more than one set of results (for instance, results for different systems, or results for the same system that are based on different sets of training materials such as your proprietary training materials and NIST Special Database 3 , then 〈SITE_NAME〉 is obtained from the name assigned your site by adding an underscore followed by a single digit 1 through 5 to your assigned site name. For instance if your assigned site name is $X Y Z$, and you are reporting only one set of results, then 〈SITE_NAME〉 $=X Y Z$, but if you are reporting two sets of results, then $\left\langle\right.$ SITE_NAME $=X Y Z_{-} 1$ for the first set, and $\left\langle S I T E_{-} N A M E\right\rangle=X Y Z_{-} 2$ for the second set. Note that NIST will assign a higher priority to XYZ_1 than to XYZ_2 for scoring and reporting purposes. Each separate set of results having a different 〈SITE_NAME〉 must be sent on a separate floppy disk, on a separate $8 \mathrm{~mm}$ tape, or in a separate E-mail message. Your $8 \mathrm{~mm}$ tapes (and floppy disks, if you want them back) will be returned at the Conference.

The next three sections describe how to send the test results to NIST. Of the following three options, E-Mail is preferred, $8 \mathrm{~mm}$ tape is next, and 5.25" floppy disks can be used as a last resort. The results of each test are to be sent only once unless NIST requests that they be resent.

\subsection{E-MAIL}

This is the preferred way of getting the test results to NIST.

With a UNIX system, the directory tree described above will be turned into one file using a tar utility and a uuencode utility. The command line to turn the directory tree into one file is as follows and should be run from the directory above 〈SITE_NAME〉:

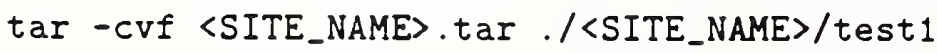

This command will generate a file, 〈SITE_NAME〉.tar, containing everything in the directory test 1. 
The file must be uuencoded to send it by E-Mail. To uuencode the tar file use the following command:

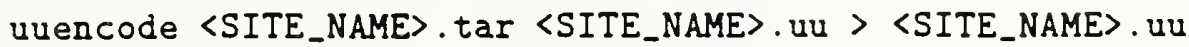

This will create the file 〈SITE_NAME〉. uu which can be E-mailed to NIST. The mail command may vary from machine to machine. Please be sure to include the subject line

"test1 results from <SITE_NAME>"

and to send the results to urtomagi.ncsl.nist.gov. On a Unix machine this can be done as follows:

$$
\begin{aligned}
& \text { mail -s "test1 results from 〈SITE_NAME>" । } \\
& \text { urt@magi.ncsl.nist.gov 〈〈SITE_NAME〉.uu }
\end{aligned}
$$

Refer to Section 4.3 to see how to use a MS-DOS based tar utility, which will be provided with the test materials, to prepare the directory tree for E-Mailing. Again, the mail command will vary from machine to machine.

\subsection{EXABYTE UNIX 8MM MAGNETIC MEDIA}

This option requires an Exabyte 8mm tape drive having no compress hardware, and a machine running UNIX.

The directory tree above will be turned into one file using a tar utility. The command line to turn the directory tree into one file is as follows and should be run from the directory above 〈SITE_NAME〉:

tar -cvf $\langle$ TAPE_DEVICE〉 ./ $\langle$ SITE_NAME / test 1

where, $\langle$ TAPE_DEVICE> is the $8 \mathrm{~mm}$ tape drive.

This command will generate a tar tape, containing everything in the directory test 1.

Send the $8 \mathrm{~mm}$ tape by Express Mail or Federal Express to:

R. Allen Wilkinson

Room A216 TECH 
NIST/NCSL/ASD/IRG

Gaithersburg, MD 20899

\subsection{IBM PC 1.2 MEGABYTE 5.25" FLOPPY DISK}

This disk should be readable on an machine running MS-DOS.

The directory tree above will be turned into one file using a public domain, MS-DOS tar utility, which was tested and shown to produce directory structures that can be handled at NIST. This public domain utility can be found in the DOS subdirectory under the TEST1 directory on the TEST DATA 1 CD-ROM.

The command line to turn the directory tree into one file is as follows and should be run from the directory above 〈SITE_NAME>:

tar - cvf $\left\langle\right.$ SITE_NAME〉.tar. $\backslash\left\langle S I T E_{-}\right.$NAME $\backslash$ test 1

This command will generate a file, 〈SITE_NAME〉.tar, containing everything in the directory TEST1 only.

This file will be too large to put on the floppy. The PKware software that can be found in the DOS subdirectory under the TEST1 directory on the TEST DATA 1 CD-ROM will be used to compress the file into a size that should fit onto the floppy. The command to compress the file is

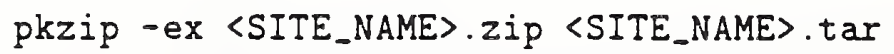

This will create a file 〈SITE_NAME〉.zip which will contain the compressed version of 〈SITE_NAME〉,tar

Copy the file 〈SITE_NAME .zip to the floppy and send it by Express Mail or by Federal Express to:

R. Allen Hilkinson

Room A216 TECH

NIST/NCSL/ASD/IRG

Gaithersburg, MD 20899 


\section{E System Summaries For Results Submitted On Time}

Jon Geist, Jonathan J. Hull, Stanley Janet, R. Allen Wilkinson, and Charles L. Wilson

This appendix contains summaries for all system results that were received on time. The first page of each summary lists pertinent information about the system such as the type of preprocessing, the type of feature extraction, the type of classification, and the training data used, whenever such information was provided by the participants. This page also summarizes the error rate as a function of rejection rate and the OCR rate in characters per second (CPS) for the digit, upper case, and lower case tests.

The second page of each system summary gives references to pertinent publications for the system and optional comments by the participants where such were provided. The DARPA Systems Conferences upon which this Conference was modeled provide a page for comments, so such a page was provided here. Very few participants in the Conference took advantage of this page, and some of those that did used it more for advertising than for information exchange. Bear in mind that the information given under the heading COMMENTS was provided by the participants, and does not necessarily represent the opinions of the Bureau of the Census, NIST, or the Committee.

The first graph on the third page of each system summary plots the logarithm of the system error rate versus the rejection rate for each test (digits $=$ diamonds, upper case letters $=$ plus signs, and lower case letters = squares) for which results were submitted.

The second graph on the third page of each summary is a little more difficult to explain. The abscissa of this graph is the zero-rejection-rate error rate for all of the test characters produced by a single writer for a given test (digits, upper case letters, lower case letters). The ordinate of this graph is the number of writers for which the single-writer zero-rejectionrate error rate is less than the percentage given on the abscissa. Again there is one curve for each test for which results were submitted. The three curves for digits, upper case letters, and lower case letters are not labeled, but they are readily distinguished. The curves for the upper and lower case letters are characterized by large steps near 4 and $8 \%$ rejection rate, corresponding to one and two incorrect characters out of a maximum of 26 letters per writer. The rounding of these steps is caused by the fact not all writers are represented by all 26 upper or lower case letters. Some letters were lost as a result of segmentation errors. The lower (upper) of the two curves with the large steps is always the curve for the upper (lower) case letters. The curve for digits has much smaller steps because there are many more digits (a maximum of 130) per writer than letters per writer.

The fourth through sixth pages of each summary contain three pseudo-correlation graphs. These show the correlations between the classifications produced by the system in question and the classifications produced by all of the other systems. The plus and minus signs in the graphs report two different correlation measures, whereas the continuous lines are for reference purposes. These graphs are also somewhat difficult to explain.

System number 1 in each graph is the system that is the subject of the particular system summary being read. Each plus sign reports the ratio of the zero-rejection-rate classifications that were identical for system number 1 and for the system corresponding to the number on 
the abscissa to the total number of characters to be classified. Each minus sign reports the ratio of the zero-rejection-rate classifications that were correct for system number 1 and for the system corresponding to the number on the abscissa to the total number of characters to be classified. The systems are ordered and numbered along the abscissa according to their plus-sign pseudo-correlation with system number 1 . This means that the ordering could be different for the digit, upper case letter, and lower case letter tests within every system summary and between system summaries. Therefore, a key to the numbers on the abscissa and the correlation data is provided for each graph on the same page of the summary. The key also contains the numerical values for the pseudo correlations.

The upper continuous line in the pseudo-correlation graphs is the zero-rejection-rate accuracy rate (one minus the error rate) for each of the systems listed along the abscissa. The lower continuous line is the upper continuous line displaced downward by the zero-rejection-rate error rate for system number 1 , the system in question. The lower and upper lines are lower and upper bounds, respectively, for the minus signs. The minus signs are lower bounds for the plus signs.

The pseudo-correlation graphs are useful for determining which systems might be used together to produce a lower error rate than either system alone. For example, there is little use to combining the two HUGHES_1 and HUGHES_2 systems, which produced virtually identical zero-rejection-rate error rates, because they are so strongly correlated. On the other hand, the U_PENN and NIST_2 systems also produce virtually identical results, but are much less strongly correlated. Therefore, combining their results might give a better system, at least as a function of rejection rate, if not for the zero-rejection-rate error rate.

The List of Figures and the List of Tables at the beginning of the Report following the Table of Contents can be used as an index of the system summaries given in this Appendix. 
SYSTEM: AEG

PARTICIPANT: Juergen Franke

ORGANIZATION: AEG Electrocom GmbH, Konstanz, Germany

PREPROCESSING: normalization for size, stroke width, and slant

FEATURES: KL transform into 256 features

CLASSIFICATION: adaptive statistical polynomial classification (POLYFONT)

HARDWARE: VAX 6510 without 6510 vector processor

TRAINING: DIGITS UPPERS LOWERS DATABASE
all
all
al1 NSDB3

STATUS :

on time

RESULTS: -- DIGITS -- -- UPPERS -- -- LOWERS -- DATABASE

$\begin{array}{lllllll}\text { REJ. } & \text { ERR. } & \text { REJ. } & \text { ERR. } & \text { REJ. } & \text { ERR. } & \text { TESTDATA1 } \\ \text { RATE } & \text { RATE-- } & \text { RATE } & \text { RATE-- } & \text { RATE } & \text { RATE-- } & \\ 0.00 & 0.0343 & 0.00 & 0.0374 & 0.00 & 0.1274 & \\ 0.10 & 0.0067 & 0.10 & 0.0107 & 0.10 & 0.0876 & \\ 0.20 & 0.0029 & 0.20 & 0.0053 & 0.20 & 0.0562 & \\ 0.30 & 0.0029 & 0.30 & 0.0047 & 0.30 & 0.0358 & \\ 0.40 & 0.0031 & 0.40 & 0.0042 & 0.40 & 0.0249 & \\ 0.50 & 0.0032 & 0.50 & 0.0042 & 0.50 & 0.0237 & \end{array}$

OCR RATE (CPS): DIGITS UPPERS LOWERS

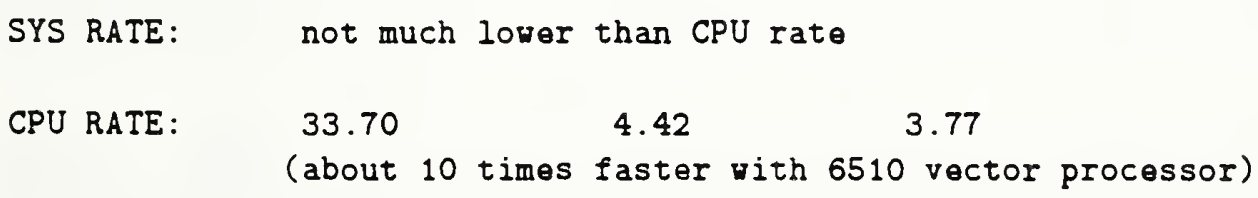


SYSTEM: AEG

\section{BIBLIOGRAPHY:}

The following references have been provided for this system:

none

COMMENTS: AEG

COMPANY CAPABILITIES:

AEG Electrocom $\mathrm{GmbH}$ is a Constance based subsidiary of the AEG Group. AEG represents one of the four main branches of the Daimler Benz Group. At AEG Electrocom currently approximately 1400 employees are responsible for an annual turnover of approximately 250 million DM.

AEG Electrcom's mission is to qualify as an efficient partner for high tech systems in automation, information technology and communications with precision mechanics, advanced electronics and customer specific software. AEG Electrocom is sharing R\&D efforts for character recognition with the Daimler Benz Research Institute at Ulm, Germany.

The product range includes - Letter sorting systems - Parcel and flat sorters - Recognition systems: various form readers, reading electronics, scanners for OCR/ICR applications

Today, AEG is successfully addressing the US market with solutions for address and form reading (including hand print). AEG is represented in the US market by our subsidiary: AEG Washinton 1350 Connecticut Avenue NW Washington, DC 20036 Phone: (202) 835-2003 FAX : (202) 835-2022

\section{STATE OF THE ART IN OCR OF HANDPRINTED CHARACTERS}

AEG Electrocom has sold world-wide many thousands of systems for postal address reading and forms reading applications.

\section{AEG'S CHARACTER RECOGNITION TECHNOLOGY}

AEG's ICR technology, called POLYFONT, is based on a mathematical statistical approach and applies a polynomila classifier for the recognition task. The basis for the recognition process is a bit-map of the characters to be recognized. On this bit map, a primary segment calculation (black connected components) is applied. Primary segments are clustered together into compound objects which reflect single characters. These compound objects are normalized in to a matrix. This matrix is represented afterwards by a vector with 256 dimensions. The vector is fed into the classifier. The classifier will produce a confidence level indicating the probability to which an input pattern does belong to a shape class which is stored in the classifier. The shape classes, which resemble the "typical" representation of a character to be recognized, are determined during the training of the classifier.

The core recognition algorithms are similar for the complete AEG product range. Performance of the different products varies in throughput and in the methods for image handling and preprocessing, as well as for the determination of the actual meaning of a shape class assigned by the recognizer. These processes are combined from a large toolbox to match customer requirements. 


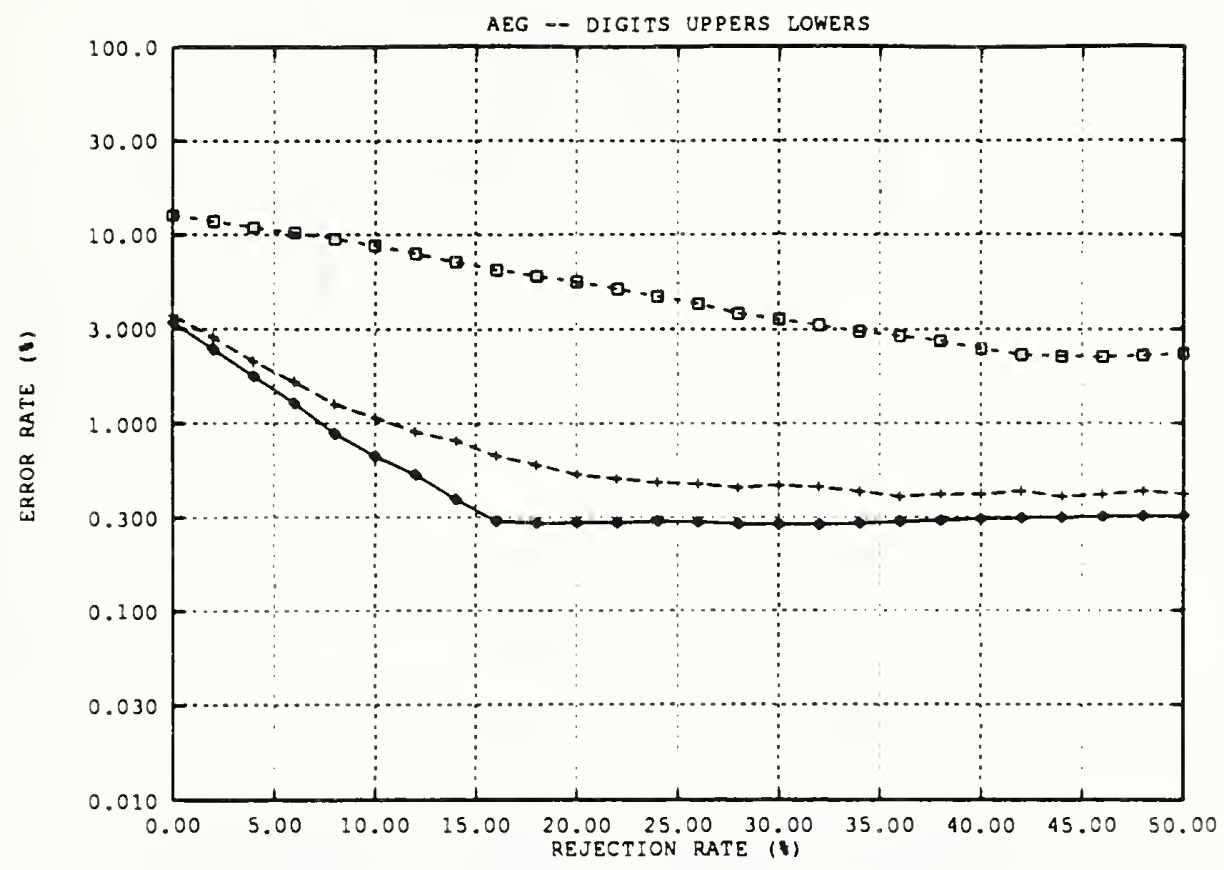

Figure 11: Error rate versus rejection rate for $A E G$

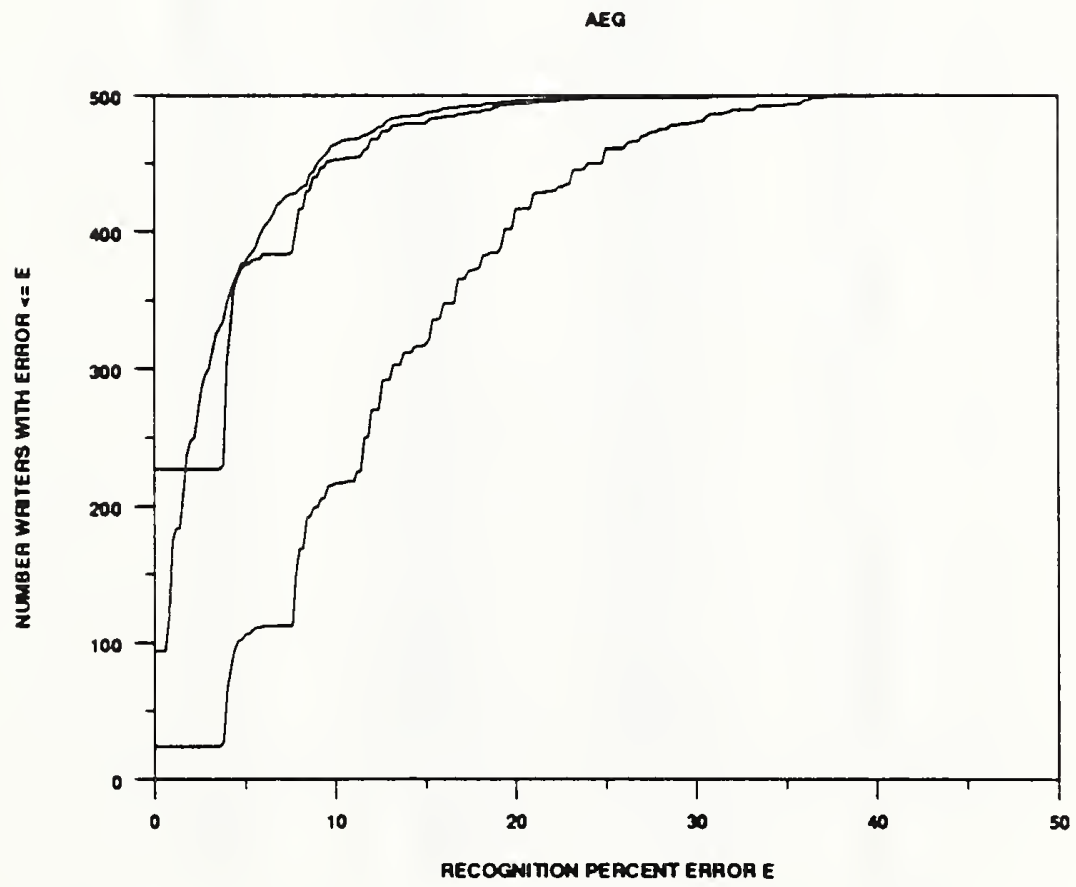

Figure 12: Error rate per writer of AEG 


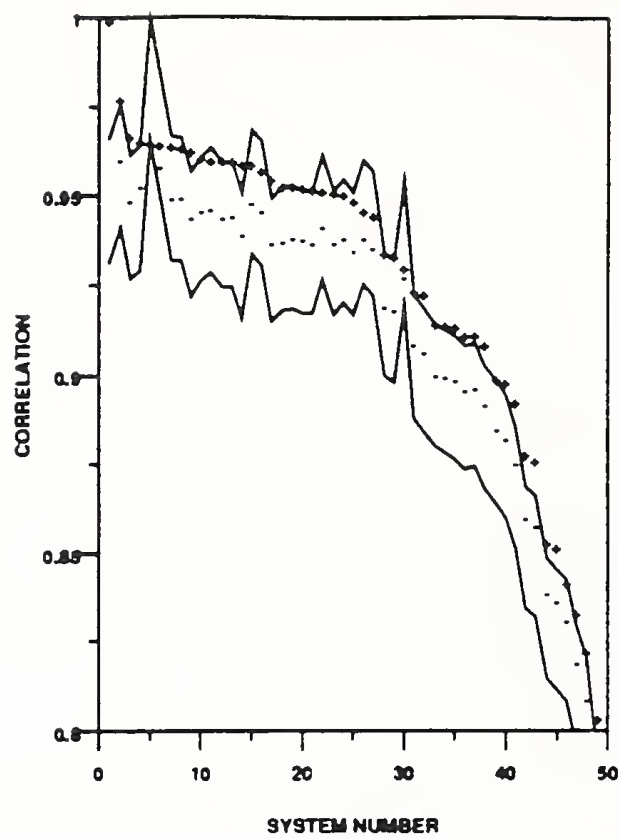

Figure 13: AEG - digit correlation

\begin{tabular}{|c|c|c|c|}
\hline System Number & System Name & Correlation (ail) & Correlation (correct) \\
\hline 1 & AEG & 10000 & 1.0000 \\
\hline 2 & VOTE_M & 0.9781 & 0.9606 \\
\hline 3 & ERIM-1 & 0.9674 & 09492 \\
\hline 4 & VOTEP & 09659 & 09534 \\
\hline 5 & REFERENCE & 0.2657 & 02657 \\
\hline 6 & OCRSYS & 0.9652 & 0.9586 \\
\hline 7 & ELSAGB 3 & 0.9645 & 09502 \\
\hline 8 & ELSAGB 2 & 09642 & 0.3499 \\
\hline 9 & UBOL & 09632 & 09446 \\
\hline 10 & ERIMI2 & 09616 & 09465 \\
\hline 11 & A T T 2 & 09606 & 09469 \\
\hline 12 & KODAKב & 09605 & 0.3448 \\
\hline 13 & $\mathrm{ATT}_{-4}$ & 09605 & 09446 \\
\hline 14 & ATT」 & 09598 & 09487 \\
\hline 15 & NIST-4 & 0.9598 & 09397 \\
\hline 16 & IBM & 0.9578 & 0.9464 \\
\hline 17 & ELSAGB_l & 09535 & 0.9378 \\
\hline 18 & SYMBUS & 09536 & 09388 \\
\hline 19 & KODAK_ & 0.9536 & 09383 \\
\hline 20 & ATT J & 0.2533 & 0.9384 \\
\hline 21 & HUGHES-1 & 09526 & 0.9378 \\
\hline 22 & THINK 2 & 0.3523 & 0.9420 \\
\hline 23 & HUGHES_2 & 03520 & 03375 \\
\hline 24 & NESTOR & 0.9516 & 0.9389 \\
\hline 25 & THINK_1 & 0.9496 & 03356 \\
\hline 26 & REI & 0.9468 & 0.9389 \\
\hline 27 & NYNEX & 09454 & 0.9363 \\
\hline 28 & GTESS_l & 0.9352 & 0.9203 \\
\hline 29 & GTESS_2 & 09346 & 0.9193 \\
\hline 30 & COMCOM & 02314 & 0.9286 \\
\hline 31 & NIST_l & 09247 & 0.9095 \\
\hline 32 & GMD_3 & 09239 & 09075 \\
\hline 33 & MIME & 0.9155 & 0.9011 \\
\hline 34 & GMD 1 & 09152 & 0.9005 \\
\hline 35 & ASOL & 0.9148 & 08996 \\
\hline 36 & UPENN & 09124 & 0.8974 \\
\hline 37 & $\mathrm{NIST}_{2}$ & 09124 & 08968 \\
\hline 38 & NIST & 0.9036 & 08928 \\
\hline 39 & GMD_4 & 0.8998 & 08858 \\
\hline 40 & RISO & 0.8990 & 08828 \\
\hline 41 & KAMAN_l & 0.8937 & 0.8762 \\
\hline 42 & KAMAN_3 & 0.8789 & 0.8608 \\
\hline 43 & KAMAN-2 & 0.8768 & 08587 \\
\hline 44 & KAMAN_5 & 0.8540 & 0.8391 \\
\hline 45 & GMD_2 & 0.8525 & 0.8369 \\
\hline 46 & VALEN 2 & 0.8427 & 0.8315 \\
\hline 47 & IFAX & 0.8336 & 0.8194 \\
\hline 48 & VALEN_I & 08229 & 0.8093 \\
\hline 49 & KAMAN_4 & 0.8043 & 07867 \\
\hline
\end{tabular}

Table 11: AEG correlation graph key for digits. 


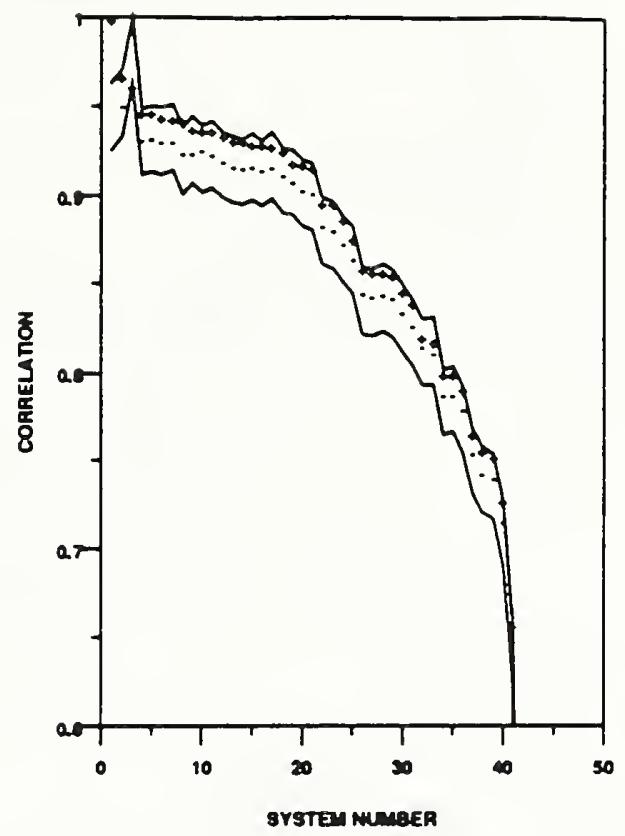

Figure 14: AEG - upper case correlation

\begin{tabular}{|c|c|c|c|}
\hline Syatem Number & System Name & Correlation (all) & Correlasion (correct) \\
\hline$\overline{1}$ & AEG & 1.0000 & 1.0000 \\
\hline 2 & VOTE_M & 0.9680 & 0.9518 \\
\hline 3 & REFERENCE & 0.9626 & 0.9626 \\
\hline 4 & ERIM_I & 0.9477 & 0.9323 \\
\hline 5 & ATT_A & 0.9473 & 0.9330 \\
\hline 6 & UMICH_I & 0.9430 & 0.9313 \\
\hline 7 & NYNEX & 0.8140 & 0.9312 \\
\hline 8 & UBOL & 0.9433 & 0.9246 \\
\hline 8 & $\operatorname{ATT} 2$ & 0.9384 & 0.9252 \\
\hline 10 & VOTEP & 0.8374 & 0.9270 \\
\hline 11 & NESTOR & 0.0374 & 0.9238 \\
\hline 12 & HUGHES-1 & 0.9348 & 0.9198 \\
\hline 13 & HUGHES 2 & 0.9324 & 0.8173 \\
\hline 14 & ATT 3 & 0.0322 & 0.9170 \\
\hline 15 & ATT 1 & 0.9303 & 0.9172 \\
\hline 16 & KODAK_ & 0.9302 & 0.9158 \\
\hline 17 & IBM & 0.2293 & 0.9173 \\
\hline 18 & SYMBUS & 0.9268 & 0.9126 \\
\hline 19 & OCRSYS & 0.2203 & 0.9091 \\
\hline 20 & GTESS 1 & 0.9189 & 0.9042 \\
\hline 21 & GTESS 2 & 0.8172 & 0.9024 \\
\hline 22 & MIME & 0.8977 & 0.8841 \\
\hline 23 & NIST -4 & 0.8972 & 0.8820 \\
\hline 24 & ASOL & 0.8885 & 0.8740 \\
\hline 25 & REI & 0.8771 & 0.8638 \\
\hline 26 & GMD_1 & 08601 & 0.8467 \\
\hline 27 & RISO & 08587 & 0.8446 \\
\hline 28 & NIST 1 & 0.8585 & 0.8457 \\
\hline 29 & GMD_3 & 0.8573 & 0.8444 \\
\hline 30 & KAMAN_1 & 0.8478 & 0.8354 \\
\hline 31 & GMD_4 & 08409 & 0.8282 \\
\hline 32 & COMCOM & 0.8222 & 0.8168 \\
\hline 33 & NIST 3 & 0.8196 & 0.8125 \\
\hline 34 & KAMAN_J & 0.8007 & 0.7892 \\
\hline 35 & IFAX & 0.8007 & 0.7889 \\
\hline 36 & KAMAN_2 & 0.7822 & 0.7802 \\
\hline 37 & $N_{1 S T} 2$ & 0.7687 & 0.7581 \\
\hline 38 & VALEN-1 & 0.7372 & 0.7450 \\
\hline 39 & GMD_2 & 0.7542 & 0.7424 \\
\hline 10 & $K_{A M A N}-4$ & 0.7290 & 0.7169 \\
\hline 41 & KAMAN_3 & 0.6585 & 0.8491 \\
\hline 42 & $\mathrm{UMICH}_{2}$ & 00337 & 0.0228 \\
\hline
\end{tabular}

Table 12: AEG correlation graph key for uppers. 


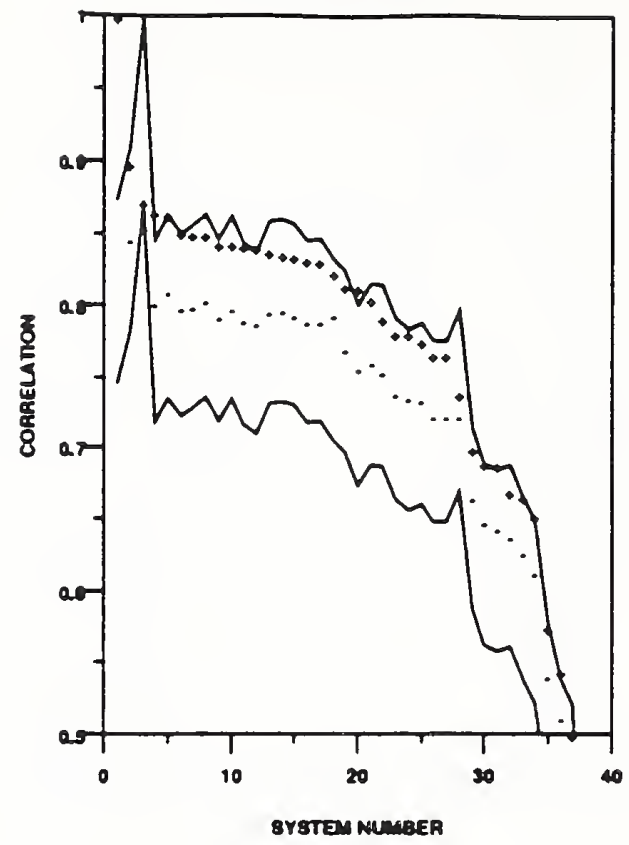

Figure 15: AEG - lower case correlation

\begin{tabular}{|c|c|c|c|}
\hline System Number & System Name & Correlation (ali) & Corpelation (coprect) \\
\hline 1 & AEG & 1.0000 & 1.0000 \\
\hline 2 & VOTE_M & 0.8987 & 0.8468 \\
\hline 3 & REFERENCE & 08728 & 0.8726 \\
\hline 4 & UBOL & 0.8683 & 0.8026 \\
\hline 3 & ERIM-1 & 0.8644 & 0.8112 \\
\hline 6 & UMICH-1 & 0.8521 & 0.7987 \\
\hline 7 & OCRSYS & 0.8508 & 0.8048 \\
\hline 8 & KODAK」 & 0.8508 & 0.8008 \\
\hline 9 & HUGHES-1 & 0.8440 & 0.7933 \\
\hline 10 & ATT $\perp$ & 0.8438 & 0.7989 \\
\hline 11 & HUGHES_2 & 0.8427 & 0.7916 \\
\hline 12 & ATT 3 & 0.8422 & 0.7886 \\
\hline 13 & ATT2 2 & 0.8398 & 0.7975 \\
\hline 14 & NYNEX & 0.8373 & 0.7979 \\
\hline 18 & ATT_A & 0.8362 & 0.7946 \\
\hline 16 & IBM & 0.8339 & 0.7897 \\
\hline 17 & NESTOR & 0.8331 & 0.7900 \\
\hline 18 & VOTEP & 0.8247 & 0.7980 \\
\hline 19 & GTESS -1 & 0.8148 & 0.7702 \\
\hline 20 & NIST _-4 & 0.8144 & 0.7365 \\
\hline 21 & GTESS_2 & 0.8067 & 0.7617 \\
\hline 22 & NIST_I & 0.7924 & 0.7542 \\
\hline 23 & GMD_3 & 0.7826 & 0.7391 \\
\hline 24 & RISO & 0.7817 & 0.7360 \\
\hline 25 & ASOL & 0.7763 & 0.7347 \\
\hline 26 & GMD_4 & 0.7688 & 07233 \\
\hline 27 & GMD_1 & 0.7668 & 0.7235 \\
\hline 28 & NIST_3 & 0.7394 & 0.7235 \\
\hline 29 & GMD_2 & 0.7000 & 0.6839 \\
\hline 30 & KAMAN_1 & 0.6899 & 0.6487 \\
\hline 31 & VALEN_1 & 0.6883 & 0.6446 \\
\hline 32 & NIST 2 & 0.6702 & 0.6384 \\
\hline 33 & KAMAN_J & 0.6677 & 0.6266 \\
\hline 34 & KAMAN_2 & 0.6531 & 0.6137 \\
\hline 35 & KAMAN -5 & 0.3735 & 0.5407 \\
\hline 36 & KAMAN_4 & 0.5438 & 0.3117 \\
\hline 37 & COMCOM & 0.3011 & 0.4882 \\
\hline 38 & $\mathrm{UMICH}_{2}$ & 0.0898 & 0.0505 \\
\hline
\end{tabular}

Table 13: AEG correlation graph key for lowers. 
SYSTEM: ASOL

PARTICIPANT: Thomas Baker

ORGANIZATION: Adaptive Solutions, Inc., Beaverton, OR

PREPROCESSING: size normalization to $8 \times 8$

FEATURES: Digits: raw

Uppers: raw and histograms from four directions

Lowers: raw and histograms from four directions

CLASSIFICATION: One layer Learning Vector Quantization NN

HARDWARE: CNAPS computer, digital SIMD processor array, 64 processors per chip, multiple chips per board.

Each processor is similar to DSP.

TRAINING :

$\begin{array}{llcl}\text { DIGITS } & \text { UPPERS } & \text { LOWERS } & \text { DATABASE } \\ 65000 & 44951 & 45313 & \text { NSDB3 }\end{array}$

STATUS: $\quad$ on time

RESULTS: -- DIGITS -- -- UPPERS -- -- LOWERS -- DATABASE

REJ. ERR. REJ. ERR. REJ. ERR. TESTDATA1

RATE RATE-- RATE RATE-- RATE RATE--

$\begin{array}{lllllll}0.00 & 0.0891 & 0.00 & 0.1116 & 0.00 & 0.2125\end{array}$

$\begin{array}{lllllll}0.10 & 0.0636 & 0.10 & 0.0842 & 0.10 & 0.1795\end{array}$

$\begin{array}{lllllll}0.20 & 0.0377 & 0.20 & 0.0592 & 0.20 & 0.1597\end{array}$

$\begin{array}{lllllll}0.30 & 0.0215 & 0.30 & 0.0423 & 0.30 & 0.1280\end{array}$

$\begin{array}{lllllll}0.40 & 0.0192 & 0.40 & 0.0407 & 0.40 & 0.1062\end{array}$

$\begin{array}{lllllll}0.50 & 0.0184 & 0.50 & 0.0457 & 0.50 & 0.0745\end{array}$

OCR RATE (CPS): DIGITS UPPERS LOHERS

$\begin{array}{llll}\text { SYS RATE: } & 77.06 & 51.92 & 51.72\end{array}$

$\begin{array}{llll}\text { CPU RATE: } & 1303.24 & 459.27 & 461.54\end{array}$

NOTE: Output is the Euclidean distance between nodes in the network and the input vector. Net reported top three classes. 
SYSTEM: ASOL

BIBLIOGRAPHY: [3][4]

COMMENTS:

System Description:

The OCR system submitted by Adaptive Solutions used a Learning Vector Quantization (LVQ) neural network classifier. LVQ is a single layer, winner-take-all network. Each weight vector in the network is assigned to a class. There can be more than one weight vector assigned to each class. The network uses the lowest euclidean distance between the weight vectors and the input vector to determine the winning class. The digit network had 224 output nodes, and the upper and lower case networks each had 416 output nodes.

The digits were normalized to an $8 \times 8$ array and input to the network. The inputs to the upper and lower case networks were a combination of the $8 \times 8$ normalized data and a histogram of the characters taken from the top, bottom, left and right of a 16x16 scale normalized array.

To report the confidence of the classification the three closest weight vectors were used. Statistics were accumulated based on the ordering of the outputs. The statistics were put into a table for reporting the confidence of the test data.

The neural network classifier was trained and tested on an Adaptive Solutions neurocomputer using a CNAPS parallel array of processors. The system that was used for the conference results had 32 processors. A system that used 64 processors for the preprocessing and classification of the test digits achieved a speed of over 1400 characters per second.

For questions or comments please contact:

Thomas Baker INTERNET: tom@asi.com Adaptive Solutions, Inc. UUCP: uunet!adaptive!tom 1400 N.W. Compton Drive, Suite 340 PHONE: (503) 690-1236 Beaverton, Oregon 97006 FAX: (503) $690-1249$ 


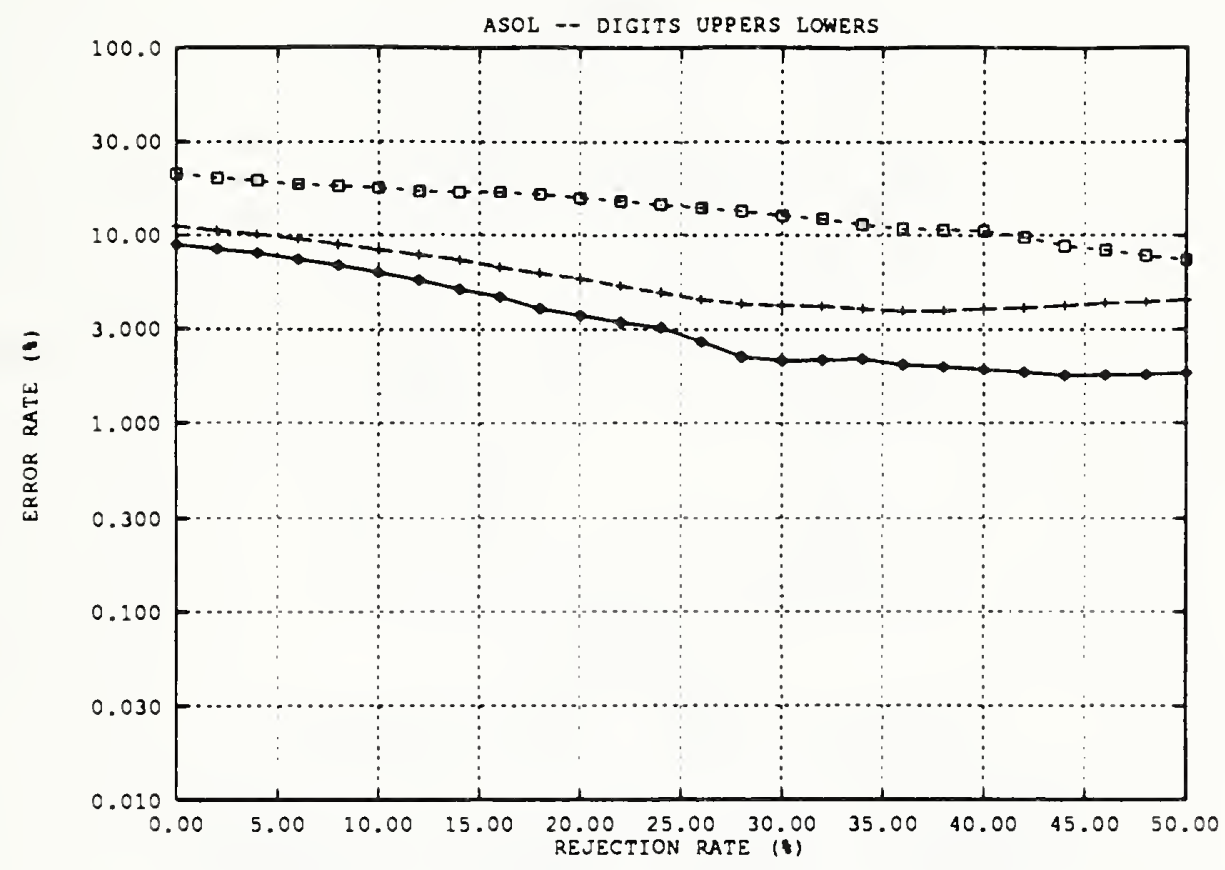

Figure 16: Error rate versus rejection rate for ASOL

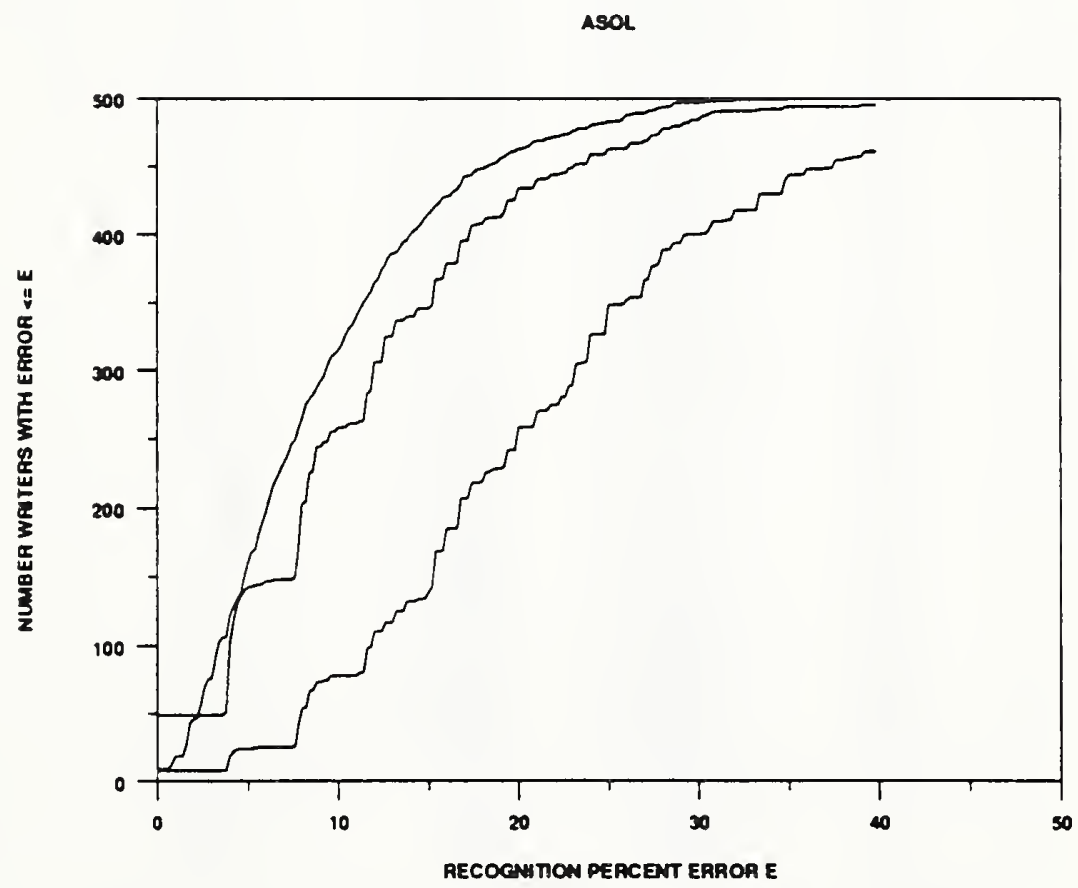

Figure 17: Error rate per writer of ASOL 


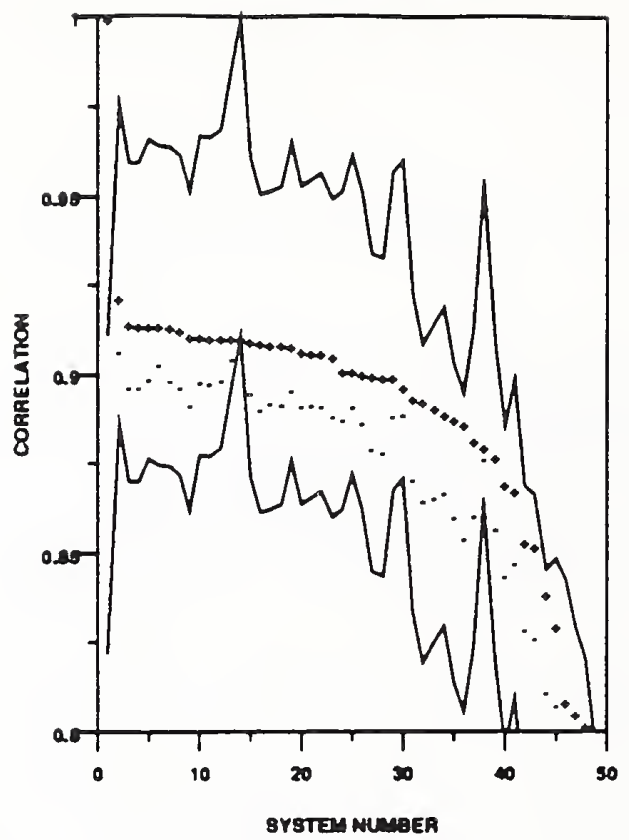

Figure 18: ASOL - digit correlation

\begin{tabular}{|c|c|c|c|}
\hline System Number & Sygtem Name & Corpelation (all) & Correlasion (correct) \\
\hline 1 & ASOL & 1.0000 & 1.0000 \\
\hline 2 & VOTE_M & 0.2225 & 0.2071 \\
\hline 3 & ATT 4 & 0.2153 & 08974 \\
\hline 4 & AEG & 0.2148 & 0.8998 \\
\hline 3 & KODAK2 2 & 02148 & 0.8973 \\
\hline 6 & VOTEP & 0.2147 & 0.8037 \\
\hline 7 & $\mathrm{ATT}_{2}$ & 0.2143 & 08989 \\
\hline 8 & ERIM_I & 0.9134 & 08972 \\
\hline 9 & THINK_I & 0.2115 & 08922 \\
\hline 10 & ELSAGBA & 0.9114 & 08985 \\
\hline 11 & ELSAGB2 & 0.2112 & 08983 \\
\hline 12 & ATT 1 & 0.8111 & 08990 \\
\hline 13 & REFERENCE & 0.2109 & 0.9109 \\
\hline 14 & OCRSYS & 0.9109 & 0.9030 \\
\hline 15 & ERIM 2 & 0.8100 & 0.8954 \\
\hline 18 & NIST_4 & 0.8097 & 0.8807 \\
\hline 17 & ATT 3 & 09093 & 08924 \\
\hline 18 & KODAK」 & 0.2093 & 08921 \\
\hline 19 & IBM & 0.9085 & 08865 \\
\hline 20 & SYMBUS & 0.9075 & 08915 \\
\hline 21 & NESTOR & 0.8087 & 0.8921 \\
\hline 22 & UBOL & 02067 & 08918 \\
\hline 23 & ELSAGB-1 & 0.8057 & 08890 \\
\hline 24 & THINK 2 & 0.9018 & 08917 \\
\hline 25 & HUGHES 1 & 0.9018 & 0.8878 \\
\hline 26 & HUGHES_2 & 0.2011 & 08873 \\
\hline 27 & GTESS_L & 09006 & 08799 \\
\hline 28 & GTESS 2 & 0.9001 & 0.8790 \\
\hline 29 & NYNEX & 0.8000 & 08889 \\
\hline 30 & REI & 0.8972 & 0.8893 \\
\hline 31 & NIST_1 & 08942 & 0.8717 \\
\hline 32 & $N_{2}$ & 0.8228 & 0.8855 \\
\hline 33 & MIME & 08913 & 08884 \\
\hline 34 & GMD_3 & 0.8893 & 0.8877 \\
\hline 35 & בـ NIST & 08881 & 08810 \\
\hline 36 & RISO & 08887 & 0.8351 \\
\hline 37 & GMD_1 & $0.882 \mathrm{~L}$ & 0.8615 \\
\hline 38 & COMCOM & 0.8802 & 0.8772 \\
\hline 39 & UPENN & 0.8773 & 0.8577 \\
\hline 40 & KAMAN_1 & $0.870 \mathrm{~L}$ & 08446 \\
\hline 41 & GMD.4 & 0.8883 & 0.8479 \\
\hline 42 & KAMAN_J & 0.8541 & 0.8291 \\
\hline 43 & KAMAN_2 & 0.8527 & 0.8271 \\
\hline 44 & GMD_2 & 0.8392 & 0.8118 \\
\hline 45 & KAMAN_S & 0.8302 & 0.8080 \\
\hline 46 & VALEN 2 & 0.8092 & 0.7944 \\
\hline 47 & IFAX & 0.8057 & 0.7860 \\
\hline 48 & VALEN-1 & 0.8028 & 0.7802 \\
\hline 49 & KAMAN-4 & 0.7865 & 0.7392 \\
\hline
\end{tabular}

Table 14: ASOL correlation graph key for digits. 


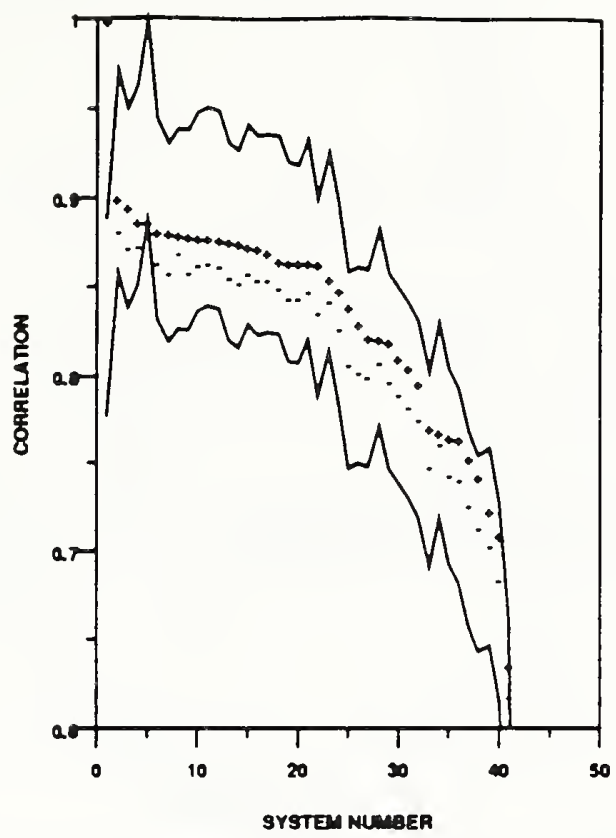

Figure 19: ASOL - upper case correlation

\begin{tabular}{|c|c|c|c|}
\hline Syssem Number & Syatem Name & Correlation (sll) & Correlstion (correct) \\
\hline$i$ & ASOL & 1.0000 & 1.0000 \\
\hline 2 & VOTE_M & 0.9008 & 08827 \\
\hline 3 & ATT 4 & 08967 & 0.8734 \\
\hline 4 & $A E G$ & 0.8885 & 0.8740 \\
\hline 3 & REFERENCE & 08884 & 0.8884 \\
\hline 6 & ATT2 2 & 08824 & 08647 \\
\hline 7 & KODAK_ & 08818 & 08582 \\
\hline 8 & VOTEP & 0.8808 & 0.8703 \\
\hline 9 & UBOL & 08797 & 0.8598 \\
\hline 10 & ERIM-1 & 0.8789 & 0.8639 \\
\hline 11 & NYNEX & 0.8787 & 0.8647 \\
\hline 12 & UMICH-1 & 08781 & 0.8633 \\
\hline 13 & ATT 3 & 08769 & 0.8570 \\
\hline 14 & SYMBUS & 08761 & 08545 \\
\hline 15 & NESTOR & 08747 & 08599 \\
\hline 16 & $\mathrm{ATT}\lrcorner$ & 08735 & 08558 \\
\hline 17 & IBM & 08712 & 08557 \\
\hline 18 & HUGHES.I & 08673 & 08518 \\
\hline 19 & GTESS -1 & 08662 & 0.8462 \\
\hline 20 & GTESS 2 & 08658 & 08453 \\
\hline 21 & HUGHES_2 & 08656 & 0.8498 \\
\hline 22 & MIME & 08648 & 08372 \\
\hline 23 & OCRSYS & 0.8565 & 08441 \\
\hline 24 & NIST_4 & 0.8508 & 0.8283 \\
\hline 23 & RISO & 08409 & 0.8085 \\
\hline 26 & NIST $T_{-1}$ & 0.8308 & 08036 \\
\hline 27 & GMD_1 & 08235 & 0.8004 \\
\hline 28 & REI & 0.8223 & 0.8092 \\
\hline 29 & GMD_3 & 0.8208 & 07981 \\
\hline 30 & $K_{A M A N}-1$ & 0.8116 & 07903 \\
\hline 31 & GMD_A & 08061 & 07836 \\
\hline 32 & NIST_3 $_{3}$ & 07972 & 07761 \\
\hline 33 & KAMAN_-3 & 0.7711 & 0.7495 \\
\hline 34 & COMCOM & 07684 & 07623 \\
\hline 35 & IFAX & 0.7658 & 0.7449 \\
\hline 36 & KAMAN_2 & 0.7650 & 07421 \\
\hline 37 & NIST 2 & 07540 & 0.7270 \\
\hline 38 & GMD_2 & 07438 & 07148 \\
\hline 39 & VALEN-1 & 07249 & 0.7043 \\
\hline 40 & KAMAN_4 & 0.7107 & 0.6855 \\
\hline 41 & KAMAN_S & 06360 & 0.6189 \\
\hline 42 & $\mathrm{UMICH}_{2}$ & 00310 & 00188 \\
\hline
\end{tabular}

Table 15: ASOL correlation graph key for uppers. 


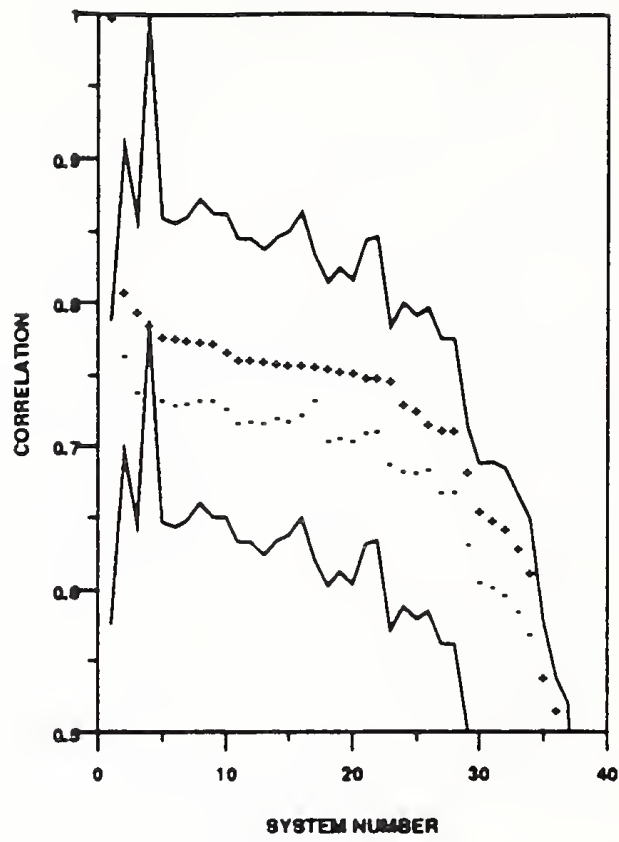

Figure 20: ASOL - lower case correlation

\begin{tabular}{|c|c|c|c|}
\hline System Number & System Name & Correlation (ali) & Correlation (correct) \\
\hline $\mathrm{T}$ & ASOL & 1.0000 & 1.0000 \\
\hline 2 & VOTE_M & 08108 & 0.7660 \\
\hline 3 & ATT -4 & 07969 & 07408 \\
\hline 4 & REFERENCE & 0.7875 & 0.7875 \\
\hline 5 & $\operatorname{ATT} 2$ & 0.7801 & 0.7355 \\
\hline 6 & KODAK_ & 0.7788 & 0.7320 \\
\hline 7 & NYNEX & 07773 & 07328 \\
\hline 8 & $A E G$ & 0.7763 & 0.7347 \\
\hline 2 & ERIM-1 & 0.7737 & 0.7345 \\
\hline 10 & ATT $\perp$ & 0.7690 & 0.7290 \\
\hline 11 & UBOL & 07634 & 0.7189 \\
\hline 12 & IBM & 0.7633 & 0.7203 \\
\hline 13 & ATTA & 0.7628 & 0.7193 \\
\hline 14 & NESTOR & 0.7617 & 0.7222 \\
\hline 15 & UMICHAL & 0.7603 & 07198 \\
\hline 16 & OCRSYS & 0.7800 & 07242 \\
\hline 17 & VOTEP & 07593 & 07349 \\
\hline 18 & NIST_L & 0.7581 & 0.7065 \\
\hline 19 & GTESS -1 & 07561 & 07084 \\
\hline 20 & GTESS 2 & 07547 & 0.7058 \\
\hline 21 & HUGHES -1 & 0.7512 & 0.7128 \\
\hline 22 & HUGHES -2 & 0.7512 & 0.7119 \\
\hline 23 & RISO & 0.7493 & 0.6898 \\
\hline 24 & NIST_4 & 07323 & 06857 \\
\hline 25 & GMD_3 & 07283 & 06841 \\
\hline 26 & הב & 0.7187 & 0.6867 \\
\hline 27 & GMD_4 & 0,7146 & 0.6706 \\
\hline 28 & GMD_l & 0.7146 & 0.6706 \\
\hline 29 & GMD_2 & 0.6852 & 0.6344 \\
\hline 30 & NIST 2 & 0.6581 & 0.6076 \\
\hline 31 & KAMAN_1 & 0.6506 & 06039 \\
\hline 32 & VALEN_I & 06450 & 0.5987 \\
\hline 33 & KAMAN_J & 06314 & 0.5863 \\
\hline 34 & KAMAN_2 & 0.8149 & 0.5710 \\
\hline 35 & KAMAN 5 & 0.5412 & 0.5042 \\
\hline 36 & $K A M A N-4$ & 0.5184 & 0.4772 \\
\hline 37 & СОMСOM & 0.4631 & 0.4545 \\
\hline 38 & $\mathrm{UMICH}_{2}$ & 0.1101 & 0.0513 \\
\hline
\end{tabular}

Table 16: ASOL correlation graph key for lowers. 
SYSTEM: ATT_1

PARTICIPANT: Dr. Craig R. Nohl

ORGANIZATION: AT\&T Bell Laboratories, Holmdel, NJ

FEATURES: gray levels in rescaled image

CLASSIFICATION: $k$-NN with specially-designed distance measure that can compensate for some common distortions such as translation.

HARDWARE: $\quad$ SPARC2

TRAINING: DIGITS UPPERS LOWERS DATABASE

$220000 \quad 44000 \quad 44000 \quad$ NSDB3

STATUS: $\quad$ on time

RESULTS: -- DIGITS -- -- UPPERS -- -- LOWERS -- DATABASE

REJ. ERR. REJ. ERR. REJ. ERR. TESTDATA1

RATE RATE-- RATE RATE-- RATE RATE--

$\begin{array}{lllllll}0.00 & 0.0316 & 0.00 & 0.0655 & 0.00 & 0.1378\end{array}$

$\begin{array}{lllllll}0.10 & 0.0069 & 0.10 & 0.0331 & 0.10 & 0.1013\end{array}$

$\begin{array}{lllllll}0.20 & 0.0025 & 0.20 & 0.0171 & 0.20 & 0.0706\end{array}$

$\begin{array}{lllllll}0.30 & 0.0012 & 0.30 & 0.0094 & 0.30 & 0.0473\end{array}$

$\begin{array}{lllllll}0.40 & 0.0011 & 0.40 & 0.0050 & 0.40 & 0.0310\end{array}$

$\begin{array}{lllllll}0.50 & 0.0011 & 0.50 & 0.0028 & 0.50 & 0.0182\end{array}$

OCR RATE (CPS): DIGITS UPPERS LOWERS

SYS RATE: $\quad 0.30 \quad 0.32 \quad 0.20$

CPU RATE: 
SYSTEM: ATT 1

BIBLIOGRAPHY:

The following references have been provided for this system:

$[5][6][7][8]$ 


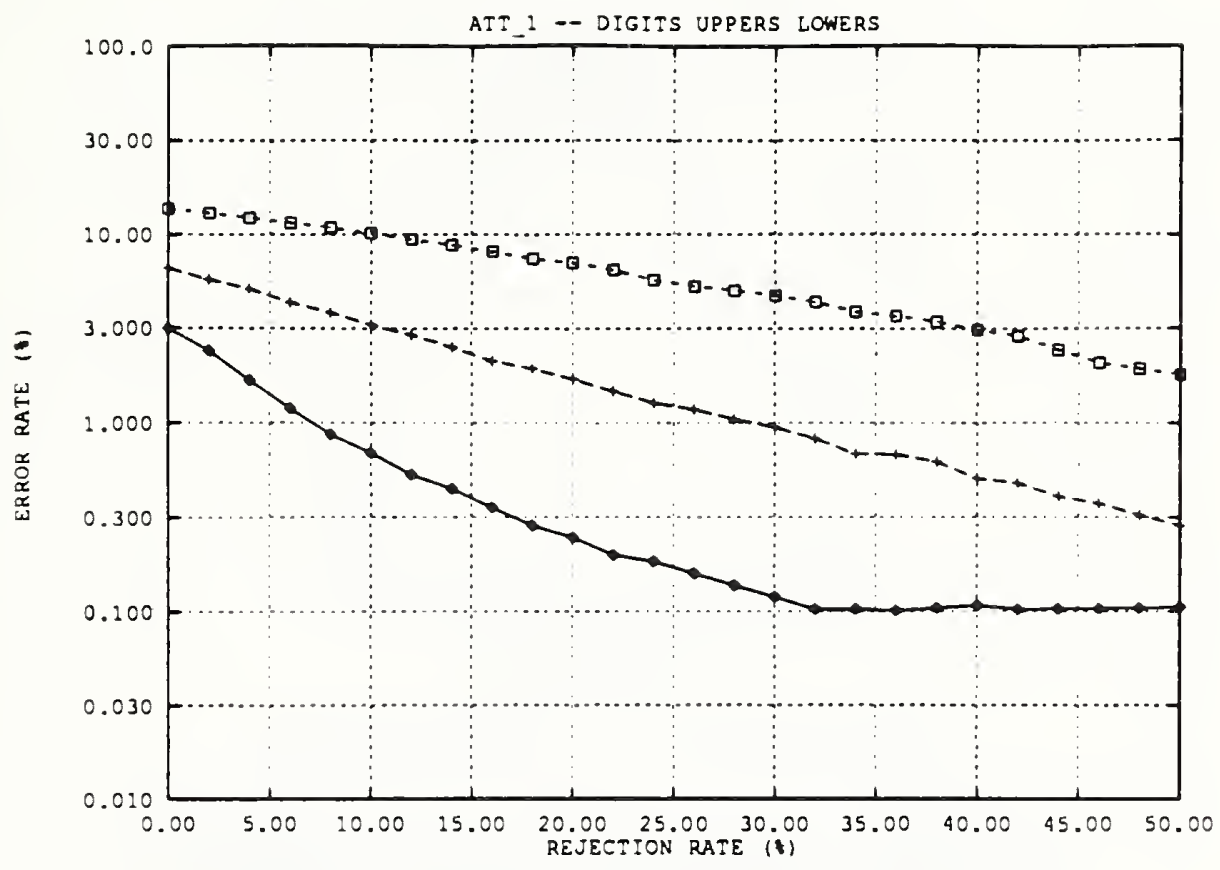

Figure 21: Error rate versus rejection rate for ATT_1

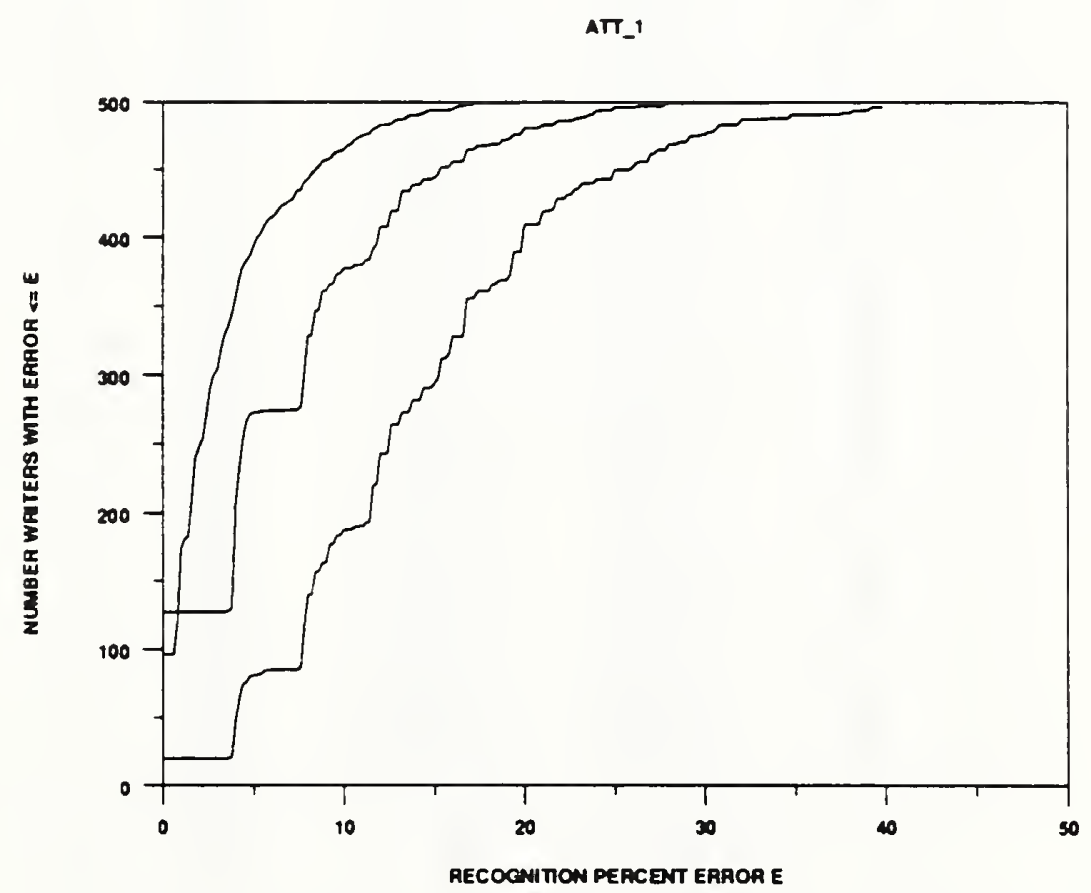

Figure 22: Error rate per writer of ATT_1 


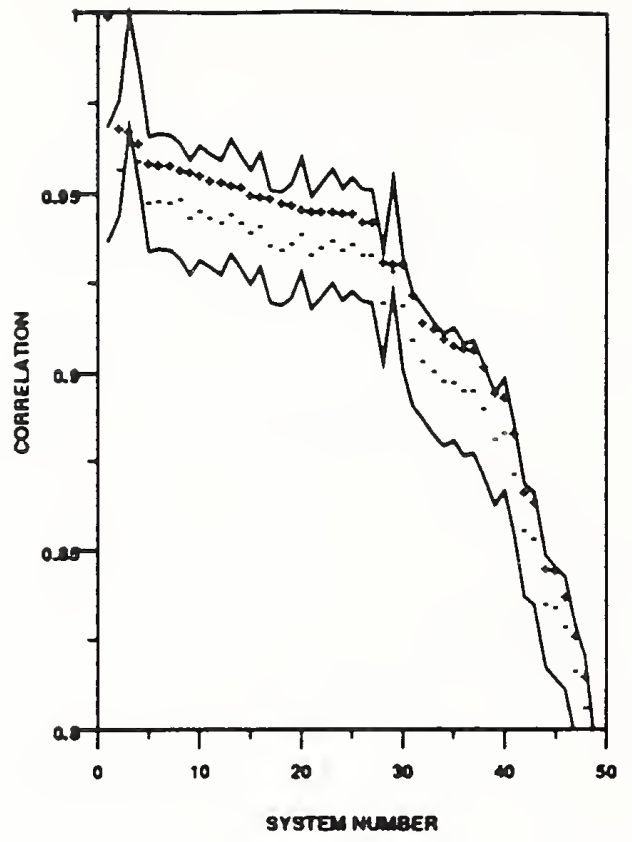

Figure 23: ATT_1 - digit correlation

\begin{tabular}{|c|c|c|c|}
\hline System Number & Sysiem Name & Correistion (all) & Correlation (correct) \\
\hline 1 & ATT_ & 1.0000 & 1.0000 \\
\hline 2 & VOTE_M & 0.2693 & 0.9580 \\
\hline 3 & REFERENCE & 0.9684 & 0.9684 \\
\hline 4 & OCRSYS & 0.9653 & 09601 \\
\hline 3 & AEG & 09598 & 0.8487 \\
\hline 6 & ELSAGBA & 0.9594 & 0.9491 \\
\hline 7 & ELSAGB2 2 & 0.9581 & 0.8488 \\
\hline 8 & VOTEP & 0.8580 & 0.9496 \\
\hline 9 & ATT-4 & 09373 & 0.9445 \\
\hline 10 & ATT 2 & 0.8566 & 0.9462 \\
\hline 11 & ERIM-1 & 0.2553 & 0.9416 \\
\hline 12 & KODAKב & 0.8544 & 0.9431 \\
\hline 13 & IBM & 02535 & 0.9456 \\
\hline 14 & ERIM_2 & 0.9534 & 0.8433 \\
\hline 13 & UBOL & 0.8511 & 0.8403 \\
\hline 16 & THINK 2 & 0.8506 & 0.9422 \\
\hline 17 & THINK-1 & 0.8300 & 0.9369 \\
\hline 18 & NIST_-4 & 09485 & 0.9357 \\
\hline 19 & KODAK」 & 02484 & 0.9371 \\
\hline 20 & REI & 0.9467 & 0.9400 \\
\hline 21 & NYNEX & 0.8462 & 0.9380 \\
\hline 22 & SYMBUS & 0.8462 & 0.8361 \\
\hline 23 & ELSAGB-1 & 0.2462 & 0.9345 \\
\hline 24 & NESTOR & 02459 & 0.9372 \\
\hline 25 & ATTS & 0.8459 & 0.9339 \\
\hline 26 & HUGHES $\_1$ & 0.9435 & 0.9342 \\
\hline 27 & HUGHES 2 & 0.8434 & 0.9342 \\
\hline 28 & GTESS_l & 0.9327 & 0.9209 \\
\hline 29 & СОМСOM & 0.9323 & 0.2298 \\
\hline 30 & GTESS 2 & 0.8322 & 09200 \\
\hline 31 & NIST -1 & 0.2235 & 0.9105 \\
\hline 32 & GMD_3 & 0.8155 & 0.9044 \\
\hline 33 & MIME & 0.9139 & 0.9017 \\
\hline 34 & ASOL & 0.2111 & 0.8990 \\
\hline 35 & GMD_1 & 0.9091 & 0.8985 \\
\hline 36 & $\mathrm{NIST}_{2}$ & 0.9080 & 0.8961 \\
\hline 37 & UPENN & 0.9079 & 0.8961 \\
\hline 38 & נה & 0.8032 & 0.8912 \\
\hline 39 & RISO & 0.8959 & 0.8827 \\
\hline 40 & GMD -4 & 0.8846 & 0.8843 \\
\hline 41 & KAMAN_1 & 0.8843 & 0.8730 \\
\hline 42 & KAMAN_3 & 0.8679 & 0.8569 \\
\hline 43 & KAMAN_2 & 0.8631 & 0.8543 \\
\hline 44 & KAMAN_5 & 0.8463 & 0.8363 \\
\hline 43 & GMD -2 & 0.8437 & 0.8350 \\
\hline 46 & VALEN_2 & 0.8382 & 0.8288 \\
\hline 47 & IF AX & 0.8273 & 0.8172 \\
\hline 48 & VALEN_1 & 0.8161 & 0.8071 \\
\hline 49 & KAMAN-4 & 0.7922 & 0.7821 \\
\hline
\end{tabular}

Table 17: ATT_1 correlation graph key for digits. 


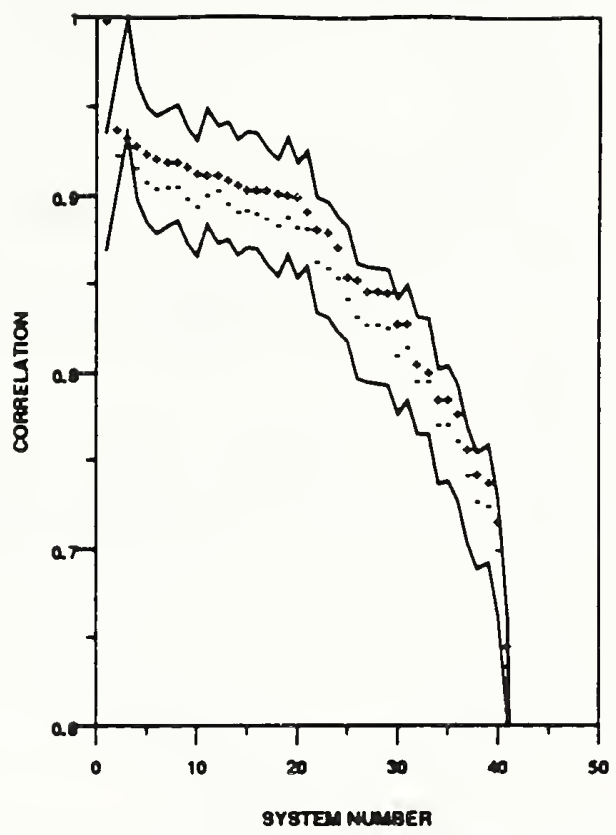

Figure 24: ATT_1 - upper case correlation

\begin{tabular}{|c|c|c|c|}
\hline System Number & System Name & Corretacion (all) & Correlation (correct) \\
\hline 1 & ATT_ & 1.0000 & 1.0000 \\
\hline 2 & VOTE_M & 0.9397 & 0.9245 \\
\hline $\mathbf{3}$ & REFERENCE & 0.8345 & 0.9345 \\
\hline 4 & $A E G$ & 0.9305 & 0.9172 \\
\hline 3 & ATT -4 & 0.9258 & 0.9094 \\
\hline 6 & ATT 2 & 0.9228 & 0.9051 \\
\hline 7 & ERIM_I & 0.9213 & 0.9066 \\
\hline 8 & NYNEX & 0.9209 & 0.9069 \\
\hline 9 & UBOL & 0.9186 & 0.8998 \\
\hline 10 & KODAKA & 0.8143 & 0.8957 \\
\hline 11 & UMICH.I & 0.9140 & 0.9022 \\
\hline 12 & VOTEP & 0.9139 & 0.9048 \\
\hline 13 & NESTOR & 0.9111 & 0.8977 \\
\hline 14 & $\mathrm{ATT}_{3}$ & 0.8085 & 0.8929 \\
\hline is & IBM & 0.9059 & 0.8934 \\
\hline 16 & HUGHES - 1 & 0.9053 & 0.8921 \\
\hline 17 & SYMBUS & 0.2051 & 08887 \\
\hline 18 & GTESS-1 & 09034 & 08855 \\
\hline 19 & HUGHES_2 & 0.9032 & 08897 \\
\hline 20 & GTESSב & 0.8017 & 0.8841 \\
\hline 21 & OCRSYS & 0.8938 & 08832 \\
\hline 22 & MIME & 08839 & 0.8653 \\
\hline 23 & NIST_A & 0.8818 & 0.8617 \\
\hline 24 & A SOL & 0.8735 & 0.8558 \\
\hline 23 & REI & 0.8571 & 0.8442 \\
\hline 26 & NIST_l & 0.8530 & 0.8338 \\
\hline 27 & GMD_1 & 0.8485 & 0.8295 \\
\hline 28 & RISO & 0.8484 & 0.8296 \\
\hline 29 & GMD_3 & 0.8474 & 0.8277 \\
\hline 30 & KAMAN_1 & 0.8304 & 0.8160 \\
\hline 31 & GMD_4 & 0.8304 & 0.8113 \\
\hline 32 & NIST 3 & 0.8076 & 0.7972 \\
\hline 33 & СОМСОМ & 0.8031 & 0.7968 \\
\hline 34 & KAMAN_3 & 0.7872 & 0.7723 \\
\hline 33 & IFAX & 0.7870 & 07723 \\
\hline 36 & KAMAN_2 & 0.7785 & 0.7628 \\
\hline 37 & NIST_2 & 0.7588 & 0.7441 \\
\hline 38 & GMD_2 & 0.7450 & 0.7291 \\
\hline 39 & VALEN-1 & 0.7404 & 0.7262 \\
\hline 40 & KAMAN_4 & 0.7178 & 0.7012 \\
\hline 41 & KAMAN_S & 0.6478 & 0.6336 \\
\hline 12 & UMICH_ & 0.0453 & 0.0234 \\
\hline
\end{tabular}

Table 18: ATT_1 correlation graph key for uppers. 


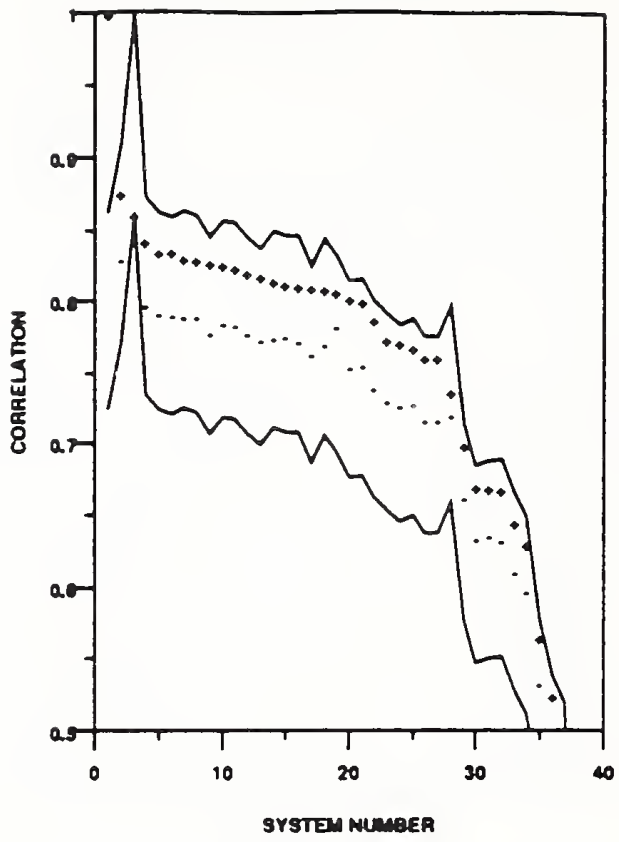

Figure 25: ATT_1 - lower case correlation

\begin{tabular}{|c|c|c|c|}
\hline System Number & System Name & Correlation (sil) & Correlation (correct) \\
\hline $\mathrm{l}$ & ATTd & 1.0000 & 1.0000 \\
\hline 2 & VOTE_M & 0.8773 & 0.8315 \\
\hline 3 & REFERENCE & 0.8622 & 0.8622 \\
\hline 4 & $A E G$ & 0.8438 & 0.7989 \\
\hline 5 & ERIM_L & 0.8375 & 07938 \\
\hline 6 & ATT 2 & 0.8370 & 07927 \\
\hline 7 & OCRSYS & 08325 & 0.7908 \\
\hline 8 & NYNEX & 0.8318 & 0.7912 \\
\hline 9 & UBOL & 0.8288 & 0.7796 \\
\hline 10 & A T T _4 & 0.8275 & 0.7864 \\
\hline 11 & KODAK」 & 0.8261 & 0.7853 \\
\hline 12 & IBM & 0.8224 & 07793 \\
\hline 13 & ATT 3 & 0.8202 & 0.7749 \\
\hline 14 & UMICH-L & 0.8168 & 07767 \\
\hline 15 & NESTOR & 0.8142 & 0.7780 \\
\hline 16 & HUGHES_L & 0.8129 & 07738 \\
\hline 17 & GTESS-1 & 0.8114 & 0.7647 \\
\hline 18 & HUGHES_2 & 08105 & 0.7717 \\
\hline 10 & VOTE $P$ & 08088 & 0.7841 \\
\hline 20 & NIST_1 & 0.8035 & 07554 \\
\hline 21 & $\operatorname{GTESS}_{2}$ & 08018 & 0.7563 \\
\hline 22 & NIST_4 & 07885 & 0.7403 \\
\hline 23 & GMD_3 & 0.7753 & 07320 \\
\hline 24 & RISO & 07724 & 0.7281 \\
\hline 25 & ASOL & 07690 & 0.7290 \\
\hline 26 & GMD_4 & 0.7623 & 0.7176 \\
\hline 27 & GMD_l & 07623 & 0.7176 \\
\hline 28 & NIST & 07383 & 0.7210 \\
\hline 29 & GMD_2 & 0.7010 & 0.6637 \\
\hline 30 & VALEN_L & 0.6718 & 06346 \\
\hline 31 & $\mathrm{NIST}_{2}$ & 06705 & 06374 \\
\hline 32 & KAMAN & 06697 & 06336 \\
\hline 33 & KAMAN-3 & 06461 & 06118 \\
\hline 34 & KAMAN_2 & 06311 & 0.5983 \\
\hline 35 & KAMAN_S & 05658 & 0.5338 \\
\hline 36 & $\mathrm{KAMAN}_{-4}$ & 05254 & 0.4979 \\
\hline 37 & COMCOM & 0.4951 & 0.4848 \\
\hline 38 & $\mathrm{UMICH}_{2}$ & 0.1023 & 0.0582 \\
\hline
\end{tabular}

Table 19: ATT_1 correlation graph key for lowers. 
SYSTEM : ATT_2

PARTICIPANT: Dr. Craig R. Nohl

ORGANIZATION: ATeT Bell Laboratories, Holmdel, NJ

FEATURES: raw?

CLASSIFICATION: five layer NN with local receptive fields and replicated weights

HARDWARE: $\quad$ SPARC2

TRAINING: DIGITS UPPERS LOWERS DATABASE

$156000 \quad 31000 \quad 31000 \quad$ NSDB3

STATUS: $\quad$ on time

RESULTS: -- DIGITS -- -- UPPERS -- -- LOWERS -- DATABASE

REJ. ERR. REJ. ERR. REJ. ERR. TESTDATA1

RATE RATE-- RATE RATE-- RATE RATE--

$\begin{array}{lllllll}0.00 & 0.0367 & 0.00 & 0.0563 & 0.00 & 0.1406\end{array}$

$\begin{array}{lllllll}0.10 & 0.0076 & 0.10 & 0.0180 & 0.10 & 0.0893\end{array}$

$\begin{array}{lllllll}0.20 & 0.0023 & 0.20 & 0.0081 & 0.20 & 0.0538\end{array}$

$\begin{array}{lllllll}0.30 & 0.0015 & 0.30 & 0.0039 & 0.30 & 0.0317\end{array}$

$\begin{array}{lllllll}0.40 & 0.0009 & 0.40 & 0.0027 & 0.40 & 0.0151\end{array}$

$\begin{array}{llllll}0.50 & 0.0007 & 0.50 & 0.0020 & 0.50 & 0.0080\end{array}$

OCR RATE (CPS): DIGITS UPPERS LOWERS
SYS RATE:
5.10
1.95
1.99

CPU RATE: 
SYSTEM: ATT 2

BIBLIOGRAPHY:

The following references have been provided for this system:

$[5][6][7][8]$ 


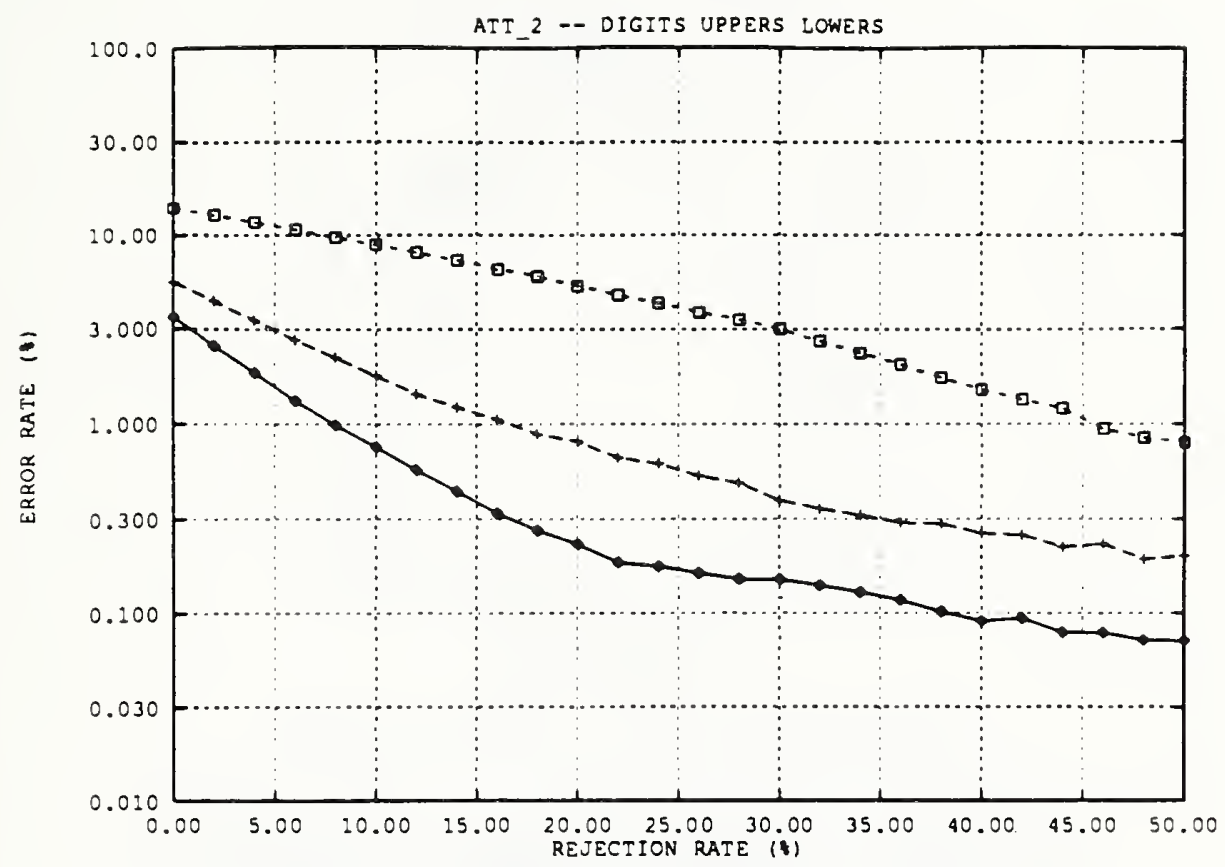

Figure 26: Error rate versus rejection rate for ATT_2

ATT_2

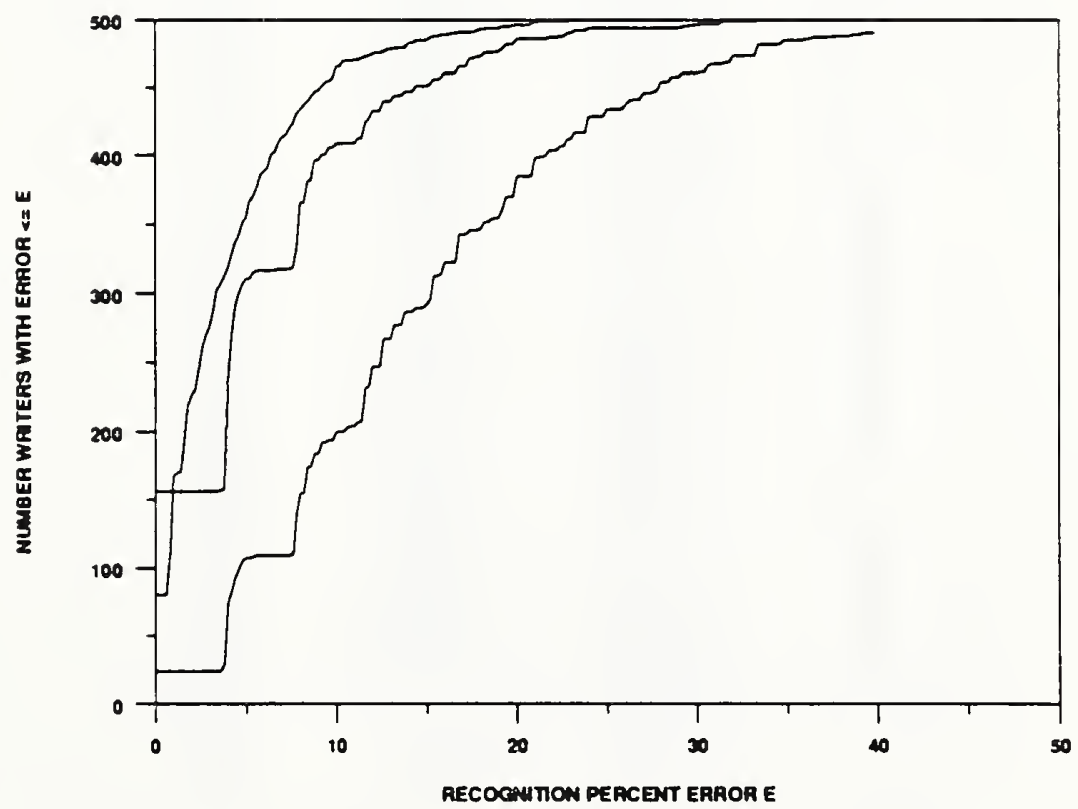

Figure 27: Error rate per writer of ATT_2 


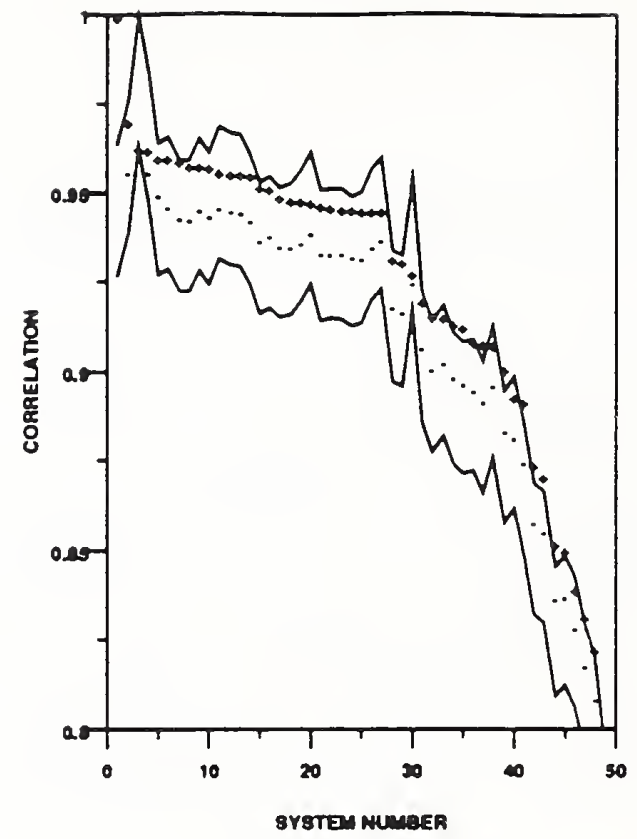

Figure 28: ATT_2 - digit correlation

\begin{tabular}{|c|c|c|c|}
\hline Syatem Number & System Name & Correlation (all) & Correlation (correct) \\
\hline $\bar{l}$ & ATT2 & 1.0000 & 1.0000 \\
\hline 2 & VOTE_M & 0.9709 & 0.9566 \\
\hline 3 & REFERENCE & 0.9633 & 0.9633 \\
\hline 4 & OCRSYS & 0.2627 & 0.9563 \\
\hline 3 & VOTEP $P$ & 0.2606 & 0.2498 \\
\hline 6 & $A E G$ & 0.2606 & 0.9468 \\
\hline 7 & ATT 4 & 0.9595 & 0.9436 \\
\hline 8 & KODAK 2 & 0.9585 & 0.9431 \\
\hline 9 & IBM & 0.8583 & 0.8480 \\
\hline 10 & ERIM-1 & 0.8580 & 0.8440 \\
\hline 11 & ATT」 & 0.9566 & 0.9462 \\
\hline 12 & ELSAGBA & 0.9562 & 0.9454 \\
\hline 13 & ELSAGB 2 & 0.8558 & 0.8450 \\
\hline 14 & ERIM_ & 0.2557 & 0.9428 \\
\hline is & KODAK_ & 0.8523 & 0.9371 \\
\hline 16 & NESTOR & 0.2518 & 0.9384 \\
\hline 17 & ATTH & 0.9497 & 0.9360 \\
\hline 18 & SYMBUS & 0.9488 & 09336 \\
\hline 18 & UBOL & 0.9486 & 0.9368 \\
\hline 20 & THINK 2 & 0.9484 & 0.9324 \\
\hline 21 & THINK_L & 0.8474 & 09341 \\
\hline 22 & HUGHES_1 & 0.2468 & 0.9341 \\
\hline 23 & HUGHES -2 & 0.8464 & 0.9340 \\
\hline 24 & ELSAGB_l & 0.9463 & 0.9328 \\
\hline 25 & $\mathrm{NIST}_{4}$ & 0.8460 & 0.9327 \\
\hline 26 & REI & 0.9459 & 0.9377 \\
\hline 27 & NYNEX & 0.8439 & 0.9360 \\
\hline 28 & GTESS_L & 0.9323 & 0.9186 \\
\hline 28 & GTESS_2 & 0.9318 & 0.9176 \\
\hline 30 & COMCOM & 0.9285 & 0.9238 \\
\hline 31 & NIST .1 & 0.8204 & 0.9072 \\
\hline 32 & MIME & 0.9163 & 0.9013 \\
\hline 33 & GMD_3 & 0.2161 & 0.9033 \\
\hline 34 & ASOL & 0.8143 & 0.8989 \\
\hline 35 & NIST 2 & 0.2134 & 0.8974 \\
\hline 36 & UPENN & 0.9091 & 0.8983 \\
\hline 37 & NIST 3 & 0.2089 & 0.8923 \\
\hline 38 & GMD +1 & 0.2082 & 0.8967 \\
\hline 39 & RISO & 0.2015 & 0.8839 \\
\hline 40 & GMD _4 & 08933 & 0.8822 \\
\hline 41 & KAMAN-L & 0.8922 & 0.8752 \\
\hline 42 & KAMAN_3 & 0.8747 & 0.8587 \\
\hline 43 & KAMAN_2 & 0.8715 & 0.8539 \\
\hline 44 & GMD -2 & 0.8525 & 0.8370 \\
\hline 45 & KAMAN_S & 0.8508 & 0.8375 \\
\hline 46 & VALEN 2 & 0.8396 & 0.8288 \\
\hline 47 & IFAX & 0.8319 & 0.8181 \\
\hline 48 & $V_{A L E N}-1$ & 0.8228 & 0.8091 \\
\hline 49 & KAMAN_4 & 0.7983 & 0.7834 \\
\hline
\end{tabular}

Table 20: ATT 2 correlation graph key for digits. 


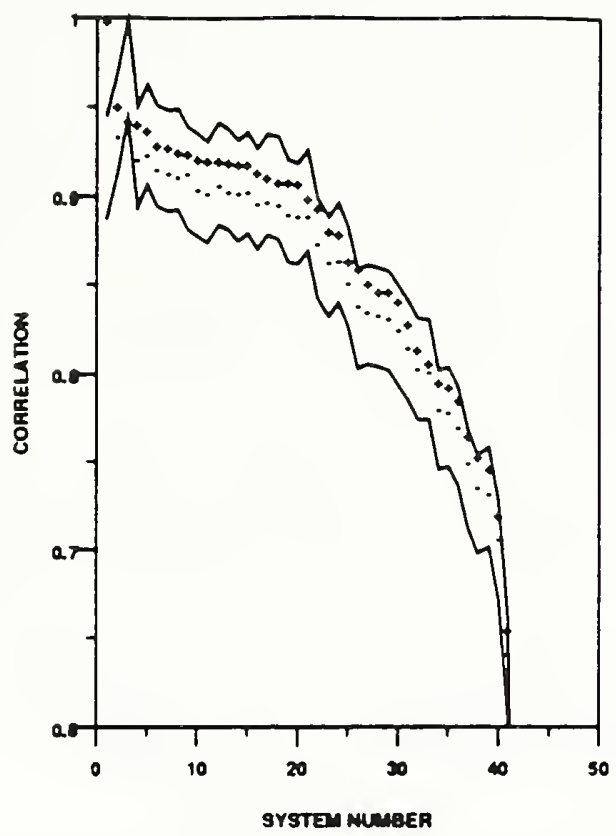

Figure 29: ATT 2 - upper case correlation

\begin{tabular}{|c|c|c|c|}
\hline System Number & Sybiem Name & Corpelation (ali) & Corretation (coprect) \\
\hline $\bar{I}$ & ATT2 & 1.0000 & 1.0000 \\
\hline 2 & VOTE_M & 09528 & 09354 \\
\hline 3 & REFERENCE & 08437 & 09437 \\
\hline 4 & A T T -4 & 0.9422 & 0.9223 \\
\hline 3 & $A E G$ & 0.8384 & 09252 \\
\hline 6 & NYNEX & 08307 & 0.9165 \\
\hline 7 & ERIM-1 & 09290 & 0.9143 \\
\hline 8 & UMICH-I & 0.9265 & 0.9131 \\
\hline 9 & VOTE_P & 0.9254 & 09148 \\
\hline 10 & ATT $\perp$ & 0.9228 & 0.9051 \\
\hline 11 & KODAK」 & 0.9225 & 0.9039 \\
\hline 12 & NESTOR & 09224 & 0.9080 \\
\hline 13 & UBOL & 09210 & 0.9049 \\
\hline 14 & ATT 3 & 08206 & 09033 \\
\hline 15 & IBM & 09198 & 09048 \\
\hline 16 & SYMBUS & 0.9158 & 08985 \\
\hline 17 & HUGHES_I & 0.9131 & 08995 \\
\hline 18 & HUGHES 2 & 0.9106 & 08969 \\
\hline 19 & GTESS_L & 0.9101 & 08921 \\
\hline 20 & GTESS 2 & 0.9091 & 08912 \\
\hline 21 & OCRSYS & 0.9010 & 08905 \\
\hline 22 & MIME & 08952 & 08756 \\
\hline 23 & ASOL & 0.8824 & 08647 \\
\hline 24 & NIST_t & 0.8812 & 08638 \\
\hline 25 & REI & 0.8658 & 08531 \\
\hline 26 & RISO & 08619 & 08406 \\
\hline 27 & NIST_L & 0.8529 & 0.8364 \\
\hline 28 & GMD_1 & 08488 & 08349 \\
\hline 29 & GMD_3 & 08482 & 08333 \\
\hline 30 & KAMAN_1 & 08429 & 08261 \\
\hline 31 & GMD_4 & 08303 & 08164 \\
\hline 32 & נה NIST & 0.8159 & 08049 \\
\hline 33 & COMCOM & 0.8084 & 08024 \\
\hline 34 & KAMAN_3 & 0.7973 & 07813 \\
\hline 35 & IFAX & 0.7946 & 0.7799 \\
\hline 36 & KAMAN_2 & 07870 & 07712 \\
\hline 37 & NIST_2 & 0.7668 & 07511 \\
\hline 38 & GMD_2 & 0.7546 & 0.7376 \\
\hline 39 & VALEN_1 & 0.7487 & 0.7342 \\
\hline 10 & KAMAN-4 & 0.7222 & 0.7077 \\
\hline 41 & KAMAN_5 & 0.6562 & 0.6429 \\
\hline 42 & $\mathrm{UMICH}_{2}$ & 0.0408 & 00231 \\
\hline
\end{tabular}

Table 21: ATT_2 correlation graph key for uppers. 


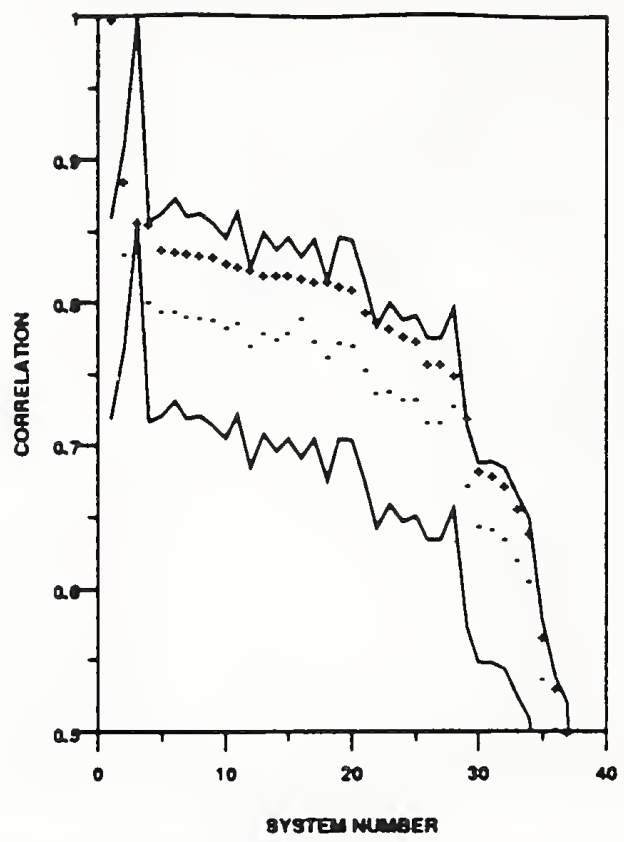

Figure 30: ATT 2 - lower case correlation

\begin{tabular}{|c|c|c|c|}
\hline System Number & Syslem Name & Corretation (all) & Correlation (correct) \\
\hline 1 & ATT2 & 1.0000 & 1.0000 \\
\hline 2 & VOTE_M & 0.8879 & 0.8372 \\
\hline 3 & REFERENCE & 0.8594 & 0.8594 \\
\hline 4 & ATT 4 & 0.8580 & 0.8033 \\
\hline 3 & ERIM_ & 0.8408 & 0.7985 \\
\hline 6 & AEG & 0.8396 & 0.7973 \\
\hline 7 & NYNEX & 0.8378 & 0.7939 \\
\hline 8 & ATT $\mathrm{S}$ & 0.8370 & 0.7927 \\
\hline 9 & KODAKג & 0.8357 & 0.7911 \\
\hline 10 & IBM & 0.8313 & 0.7858 \\
\hline 11 & OCRSYS & 0.8291 & 0.7893 \\
\hline 12 & GTESS -1 & 0.8267 & 0.7734 \\
\hline 13 & UMICH.I & 0.8239 & 0.7818 \\
\hline 14 & NESTOR & 0.8235 & 0.7818 \\
\hline 13 & ATT & 08235 & 0.7780 \\
\hline 16 & VOTEP & 0.8216 & 0.7928 \\
\hline 17 & UBOL & 08188 & 0.7763 \\
\hline 18 & GTESS 2 & 0.8184 & 0.7652 \\
\hline 19 & HUGHES_1 & 0.8154 & 0.7747 \\
\hline 20 & HUGHES_2 & 0.8128 & 0.7728 \\
\hline 21 & NIST -1 & 0.7974 & 0.7352 \\
\hline 22 & RISO & 0.7902 & 0.7393 \\
\hline 23 & NIST-4 & 0.7851 & 0.7402 \\
\hline 24 & ASOL & 0.7801 & 0.7355 \\
\hline 23 & GMD_3 & 0.7762 & 0.7352 \\
\hline 26 & GMD_-1 & 0.7397 & 0.7193 \\
\hline 27 & GMD_1 & 0.7597 & 0.7193 \\
\hline 28 & נה NIST & 0.7527 & 0.7306 \\
\hline 29 & GMD_2 & 0.7219 & 0.8758 \\
\hline jo & NIST $_{2}$ & 0.6860 & 0.8465 \\
\hline 31 & $\operatorname{KAMAN}_{-1}$ & 0.6825 & 0.6142 \\
\hline 32 & VALEN_I & 0.6747 & 0.8376 \\
\hline 33 & KAMAN_-3 & 0.6596 & 0.6227 \\
\hline 34 & KAMAN_2 & 0.6416 & 0.6073 \\
\hline 33 & KAMAN_S & 0.5680 & 0.3385 \\
\hline 36 & KAMAN_4 & 05334 & 0.3043 \\
\hline 37 & COMCOM & 0.5013 & 0.4896 \\
\hline 38 & $\mathrm{UMICH}_{2}$ & 00944 & 0.0588 \\
\hline
\end{tabular}

Table 22: ATT 2 correlation graph key for lowers. 
SYSTEM: ATT_3

PARTICIPANT: Dr. Craig R. Nohl

ORGANIZATION: AT\&T Bell Laboratories, Holmdel, NJ

FEATURES : Iaw?

CLASSIFICATION: hybrid of feature-based and NN classifiers. The commercial NCR product.

HARDWARE: proprietary board based on Analog Devices 2901

TRAINING: DIGITS UPPERS LOWERS DATABASE

$140000 \quad 26000 \quad 23000 \quad$ NSDB3

STATUS: $\quad$ on time

RESULTS: -- DIGITS -- -- UPPERS -- -- LOWERS -- DATABASE

REJ. ERR. REJ. ERR. REJ. ERR. TESTDATA1

RATE RATE-- RATE RATE-- RATE RATE--

$\begin{array}{llllll}0.00 & 0.0484 & 0.00 & 0.0683 & 0.00 & 0.1634\end{array}$

$\begin{array}{lllllll}0.10 & 0.0129 & 0.10 & 0.0297 & 0.10 & 0.1176\end{array}$

$\begin{array}{lllllll}0.20 & 0.0126 & 0.20 & 0.0150 & 0.20 & 0.0856\end{array}$

$\begin{array}{lllllll}0.30 & 0.0127 & 0.30 & 0.0071 & 0.30 & 0.0582\end{array}$

$\begin{array}{lllllll}0.40 & 0.0128 & 0.40 & 0.0066 & 0.40 & 0.0382\end{array}$

$\begin{array}{llllll}0.50 & 0.0124 & 0.50 & 0.0065 & 0.50 & 0.0313\end{array}$

OCR RATE (CPS): DIGITS UPPERS LOWERS

$\begin{array}{llll}\text { SYS RATE : } & 146.38 & 142.82 & 146.82\end{array}$

CPU RATE: 
SYSTEM: ATT_3

BIBLIOGRAPHY:

The following references have been provided for this system:

[9][10] 


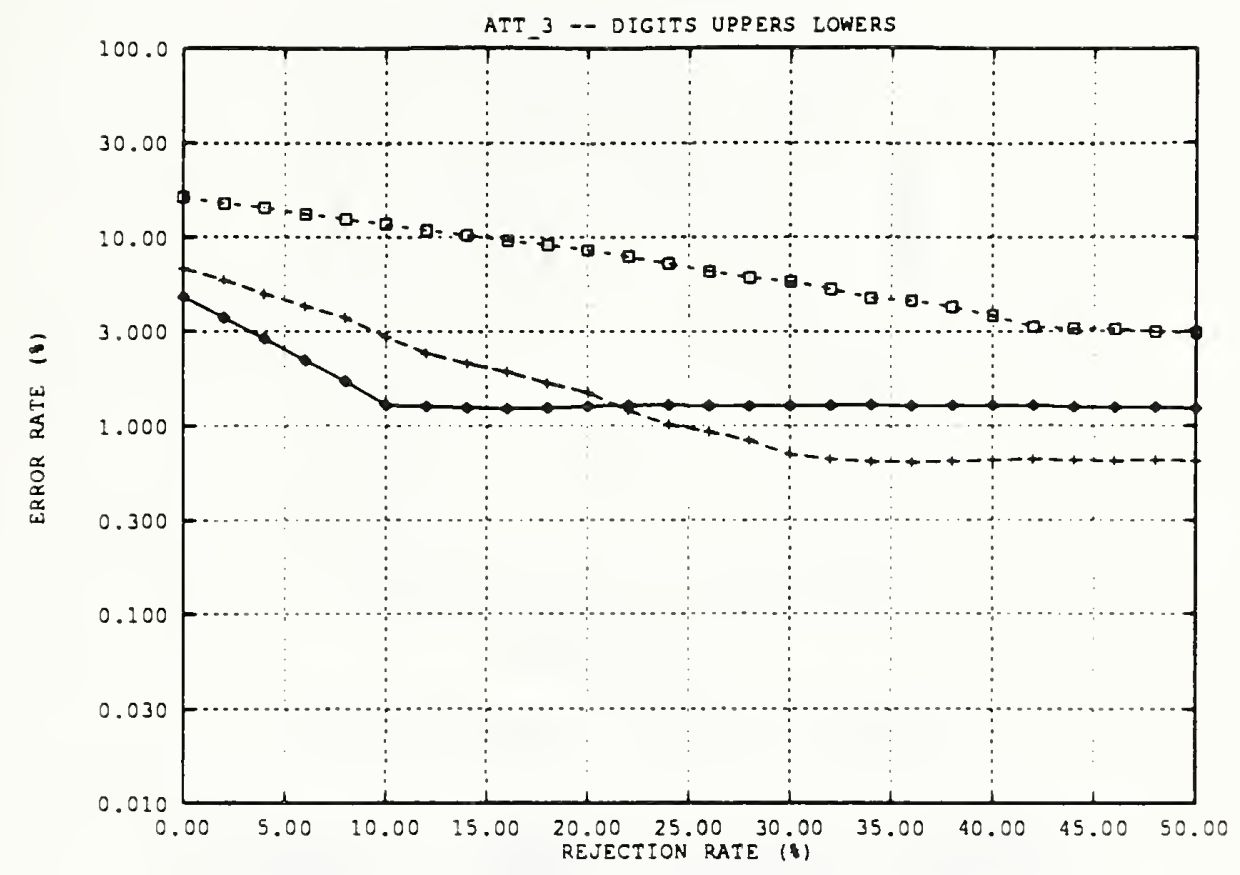

Figure 31: Error rate versus rejection rate for ATT_3

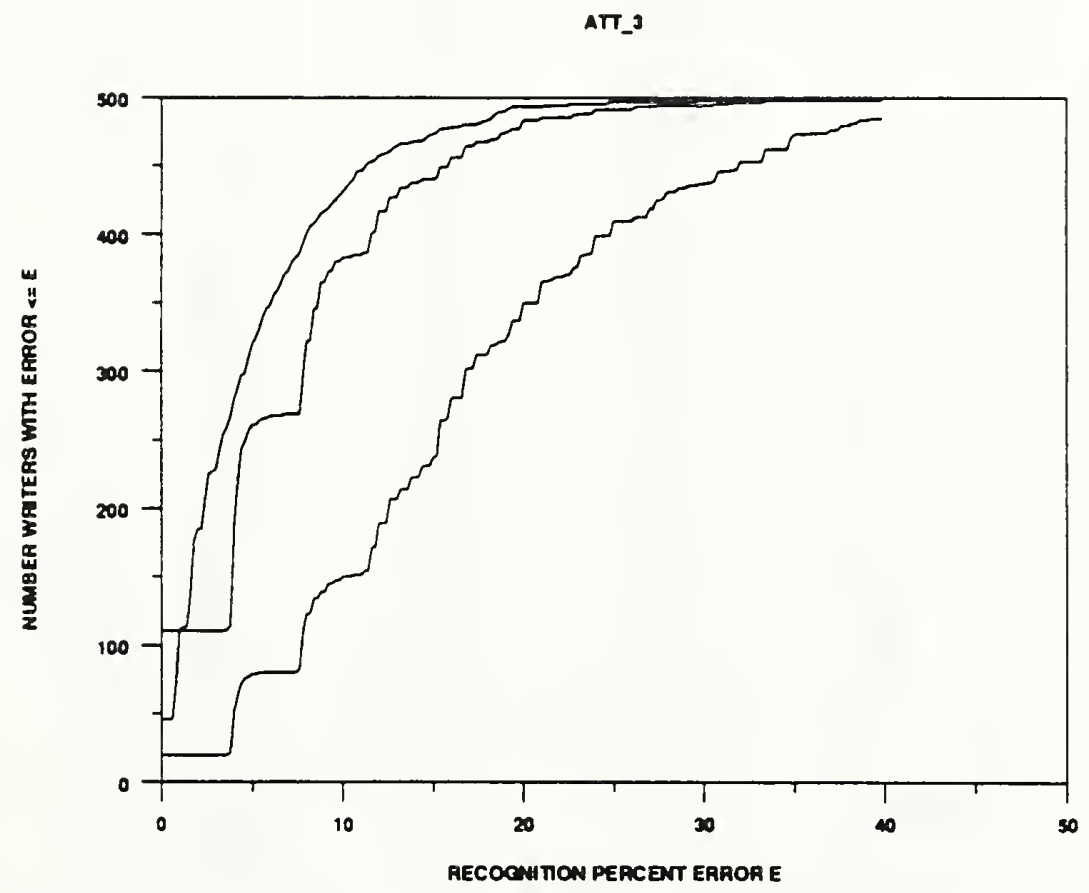

Figure 32: Error rate per writer of ATT_3 


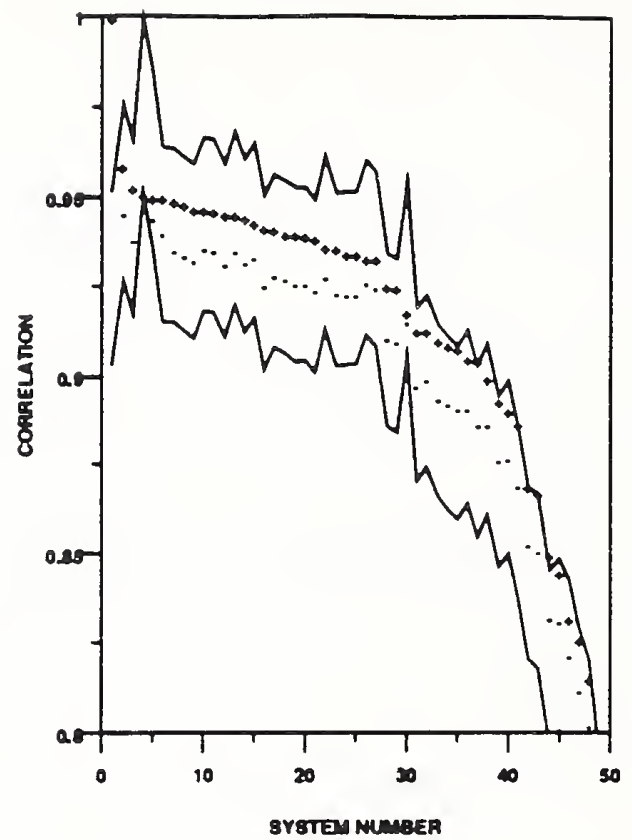

Figure 33: ATT_3 - digit correlation

\begin{tabular}{|c|c|c|c|}
\hline System Number & System Name & Correlation (all) & Correlation (correet) \\
\hline$T$ & ATTS & 1.0000 & 1.0000 \\
\hline 2 & VOTE_M & 0.9593 & 0.9457 \\
\hline 3 & AEG & 0.8533 & 0.9384 \\
\hline 4 & REFERENCE & 02516 & 0.9516 \\
\hline 5 & OCRSYS & 0.9506 & 09447 \\
\hline B & VOTEP & 0.8503 & 0.9404 \\
\hline 7 & ATT 2 & 0.9497 & 0.9360 \\
\hline 8 & ERIM-1 & 0.8486 & 0.9345 \\
\hline 9 & ATT_4 & 0.9473 & 0.9329 \\
\hline 10 & ELSAGB 3 & 0.8472 & 0.9362 \\
\hline 11 & ELSAGBZ & 0.9468 & 0.9358 \\
\hline 12 & KODAK2 & 0.8460 & 0.9323 \\
\hline 13 & ATT $\perp$ & 08459 & 0.8359 \\
\hline 14 & ERIM 2 & 0.8450 & 0.8326 \\
\hline 15 & IBM & 0.2436 & 0.9338 \\
\hline 16 & NIST 4 & 0.9420 & 0.9262 \\
\hline 17 & UBOL & 0.8417 & 0.8289 \\
\hline 18 & NESTOR & 0.9404 & 0.9282 \\
\hline 19 & KODAK」 & 0.8403 & 0.8267 \\
\hline 20 & SYMBUS & 0.2328 & 0.9268 \\
\hline 21 & ELSAGB-1 & 09391 & 0.8247 \\
\hline 22 & THINK 2 & 0.9369 & 0.9284 \\
\hline 23 & THINK-I & 0.8364 & 0.9240 \\
\hline 24 & HUGHES -2 & 0.9348 & 02235 \\
\hline 25 & HUGHES_1 & 0.8347 & 0.8233 \\
\hline 26 & REI & 0.8337 & 0.9268 \\
\hline 27 & NYNEX & 0.9336 & 0.9251 \\
\hline 28 & GTESS -1 & 0.9258 & 0.8111 \\
\hline 29 & GTESS_2 & 0.8252 & 0.9101 \\
\hline 30 & COMCOM & 0.8181 & 0.8155 \\
\hline 31 & GMD_3 & 0.8133 & 0.8977 \\
\hline 32 & NIST -1 & 0.8132 & 0.8995 \\
\hline 33 & MIME & 0.8103 & 0.8942 \\
\hline 34 & ASOL & 09093 & 0.8924 \\
\hline 35 & NIST 2 & 02084 & 0.8911 \\
\hline 36 & GMD-1 & 0.9056 & 08911 \\
\hline 37 & נה AIST & 08048 & 0.8866 \\
\hline 38 & UPENN & 0.8998 & 0.8867 \\
\hline 39 & RISO & 0.8936 & 0.8763 \\
\hline 40 & GMD _4 & 0.8907 & 0.8768 \\
\hline 41 & $K_{A} M_{-1}$ & 0.8869 & 0.8695 \\
\hline 42 & KAMAN_J & 0.8696 & 0.8530 \\
\hline 43 & $\mathrm{KAMAN}_{-2}$ & 0.8680 & 0.8612 \\
\hline 44 & GMD_2 & 0.8504 & 0.8325 \\
\hline 45 & KAMAN 3 & 0.8455 & 0.8314 \\
\hline 46 & VALEN 2 & 0.8325 & 0.8218 \\
\hline 47 & IFAX & 0.8264 & 0.8123 \\
\hline 48 & VALEN_l & 0.8156 & 0.8026 \\
\hline 49 & KAMAN_4 & 0.7975 & 0.7802 \\
\hline
\end{tabular}

Table 23: ATT_3 correlation graph key for digits. 


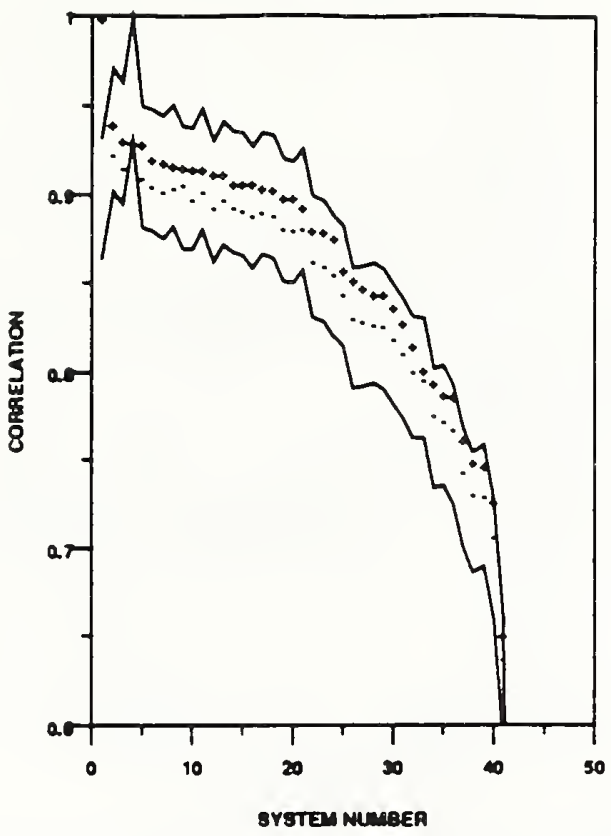

Figure 34: ATT_3 - upper case correlation

\begin{tabular}{|c|c|c|c|}
\hline System Number & System Name & Correlation (all) & Correlation (correct) \\
\hline 1 & ATTA & 1.0000 & 1.0000 \\
\hline 2 & VOTE_M & 0.9413 & 0.9240 \\
\hline 3 & AEG & 0.8322 & 0.9170 \\
\hline 4 & REFERENCE & 09317 & 0.9317 \\
\hline 3 & ATT_4 & 0.8300 & 0.9111 \\
\hline 6 & ERIM-1 & 0.8223 & 0.9064 \\
\hline 7 & ATT 2 & 0.9206 & 0.9033 \\
\hline 8 & NYNEX & 0.8183 & 0.9032 \\
\hline 9 & VOTEP & 09173 & 0.9070 \\
\hline 10 & UBOL & 0.8169 & 0.8989 \\
\hline 11 & UMICH_l & 09166 & 0.8034 \\
\hline 12 & KODAK_ & 09141 & 0.8946 \\
\hline 13 & NESTOR & 09139 & 0.8991 \\
\hline 14 & IBM & 09087 & 0.8949 \\
\hline 13 & ATT $\perp$ & 0.8083 & 08929 \\
\hline 16 & SYMBUS & 0.8082 & 08897 \\
\hline 17 & HUGHES - 1 & 09036 & 08916 \\
\hline 18 & HUGHES -2 & 09044 & 0.8896 \\
\hline 19 & GTESS 1 & 09003 & 08824 \\
\hline 20 & GTESS 2 & 0.8999 & 0.8813 \\
\hline 21 & OCRSYS & 08943 & 0.8827 \\
\hline 22 & MIME & 08813 & 0.8643 \\
\hline 23 & NIST_4 & 08807 & 0.8619 \\
\hline 24 & ASOL & 08769 & 08370 \\
\hline 23 & REI & 0.8596 & 0.8436 \\
\hline 26 & RISO & 08637 & 0.8321 \\
\hline 27 & GMD - 1 & 0.8493 & 0.8303 \\
\hline 28 & NIST_A & 08463 & 0.8284 \\
\hline 29 & GMD_` & 08457 & 0.8278 \\
\hline 30 & KAMAN_L & 0.8383 & 0.8204 \\
\hline 31 & GMD_4 & 0.8294 & 0.8119 \\
\hline 32 & בـ NIST & 0.8168 & 0.8013 \\
\hline 33 & COMCOM & 0.8029 & 0.7973 \\
\hline 34 & KAMAN_3 & 0.7951 & 0.7787 \\
\hline 33 & IFAX & $0.7 \mathrm{Ags}$ & 07731 \\
\hline 36 & KAMAN.2 & 0.7880 & 0.7684 \\
\hline 37 & NIST 2 & 0.7831 & 07432 \\
\hline 38 & GMD .2 & 0.7504 & 0.7322 \\
\hline 32 & VALEN-I & 0.7489 & 0.7308 \\
\hline 40 & KAM AN-4 & 0.7286 & 0.7078 \\
\hline 41 & KAMAN_S & 0.6516 & 0.6384 \\
\hline 42 & UMICH 2 & 0.0423 & 0.0210 \\
\hline
\end{tabular}

Table 24: ATT_3 correlation graph key for uppers. 


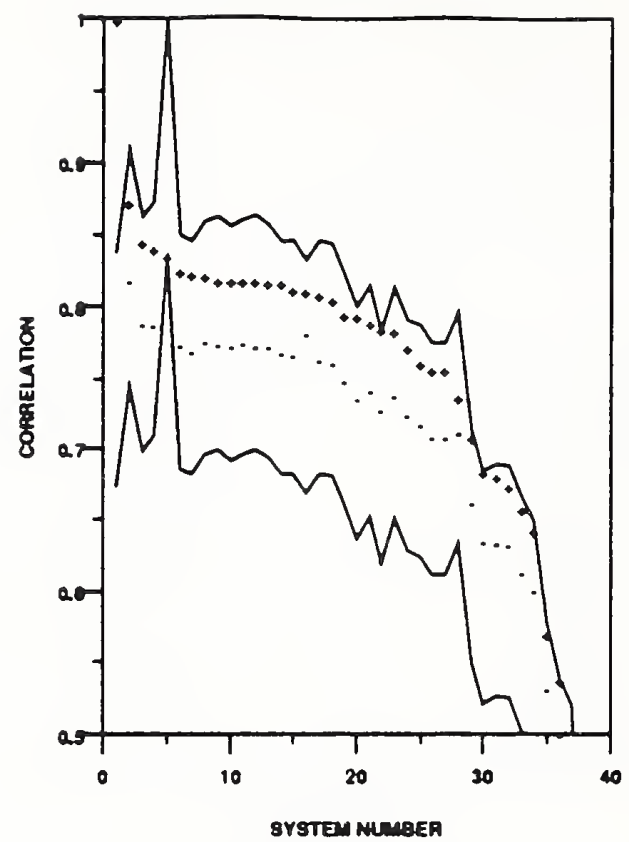

Figure 35: ATT_3 - lower case correlation

\begin{tabular}{|c|c|c|c|}
\hline System Number & System Name & Correlation (all) & Correlation (correct) \\
\hline $\mathrm{T}$ & ATT3 & 1.0000 & 1.0000 \\
\hline 2 & VOTE_M & 0.8740 & 0.8197 \\
\hline 3 & ERIM-I & 08464 & 0.7898 \\
\hline 4 & AEG & 0.8422 & 0.7886 \\
\hline $\mathbf{3}$ & REFERENCE & 0.8366 & 0.8366 \\
\hline B & UMICH_I & 0.8273 & 0.7747 \\
\hline 7 & UBOL & 0.8248 & 0.7704 \\
\hline 8 & ATT 2 & 0.8235 & 07780 \\
\hline 9 & ATT_ $\perp$ & 0.8202 & 0.7749 \\
\hline 10 & KODAKג & 0.8189 & 0.7733 \\
\hline 11 & NYNEX & 0.8187 & 07768 \\
\hline 12 & OCRSYS & 0.8194 & 0.7744 \\
\hline 13 & ATT 4 & 0.8182 & 0.7743 \\
\hline 14 & IBM & 0.8183 & 07699 \\
\hline 13 & NESTOR & 0.8141 & 0.7681 \\
\hline 16 & VOTEP $P$ & 08133 & 0.7827 \\
\hline 17 & HUGHES_1 & 0.8108 & 0.7648 \\
\hline 18 & HUGHES_2 & 0.8070 & 0.7622 \\
\hline 19 & GTESS_l & 07972 & 07496 \\
\hline 20 & NIST_t & 0.7960 & 0.7376 \\
\hline 21 & GTESS 2 & 0.7918 & 0.7431 \\
\hline 22 & RISO & 07869 & 0.7289 \\
\hline 23 & NIST 1 & 0.7837 & 0.7390 \\
\hline 24 & GMD_3 & 07738 & 0.7260 \\
\hline 23 & ASOL & 0.7628 & 0.7193 \\
\hline 26 & GMD_4 & 0.7374 & 0.7100 \\
\hline 27 & GMD_L & 0.7374 & 0.7100 \\
\hline 28 & SIST & 0.7382 & 0.7129 \\
\hline 29 & GMD_2 & 0.7100 & 0.6837 \\
\hline 30 & VALEN_1 & 0.6881 & 0.6339 \\
\hline 31 & KAMAN_L & 0.6822 & 0.6333 \\
\hline 32 & NIST 2 & 0.6736 & 0.6342 \\
\hline 33 & KAMAN_J & 0.6397 & 06146 \\
\hline 34 & KAMAN_2 & 0.6442 & 0.8013 \\
\hline 33 & KAMAN_3 & 0.3709 & 0.3332 \\
\hline 36 & KAMAN_-1 & 0.3381 & 0.3007 \\
\hline 37 & СомСом & 0.4940 & 04818 \\
\hline 38 & UMICH_2 & 0.0832 & 00436 \\
\hline
\end{tabular}

Table 25: ATT_3 correlation graph key for lowers. 
SYSTEM: ATT_4

PARTICIPANT: Dr. Craig R. Nohl

ORGANIZATION: AT\&T Bell Laboratories, Holmdel,NJ

FEATURES : IaW?

CLASSIFICATION: vote of three ? layer NNs with local receptive fields and replicated weights

HARDHARE: $\quad$ SPARC2

TRAINING: DIGITS UPPERS LOHERS DATABASE

$210000 \quad 40000 \quad 40000 \quad$ NSDB3

$\begin{array}{llll}10000 & 0 & 0 & \text { USPS }\end{array}$

STATUS: $\quad$ on time

RESULTS: -- DIGITS -- -- UPPERS -- -- LOWERS -- DATABASE

REJ . ERR . REJ . ERR . REJ . ERR . TESTDATA 1

RATE RATE-- RATE RATE-- RATE RATE--

$\begin{array}{lllllll}0.00 & 0.0410 & 0.00 & 0.0500 & 0.00 & 0.1428\end{array}$

$\begin{array}{lllllll}0.10 & 0.0098 & 0.10 & 0.0138 & 0.10 & 0.0968\end{array}$

$\begin{array}{lllllll}0.20 & 0.0034 & 0.20 & 0.0059 & 0.20 & 0.0596\end{array}$

$\begin{array}{lllllll}0.30 & 0.0014 & 0.30 & 0.0037 & 0.30 & 0.0334\end{array}$

$\begin{array}{lllllll}0.40 & 0.0008 & 0.40 & 0.0015 & 0.40 & 0.0193\end{array}$

$\begin{array}{lllllll}0.50 & 0.0003 & 0.50 & 0.0008 & 0.50 & 0.0113\end{array}$

OCR RATE (CPS): DIGITS

UPPERS

LOWERS

SYS RATE:

1.15

1.03

1.50

CPU RATE: 
SYSTEM: ATT_4

BIBLIOGRAPHY:

The following references have been provided for this system:

$[5][6][7][8]$ 


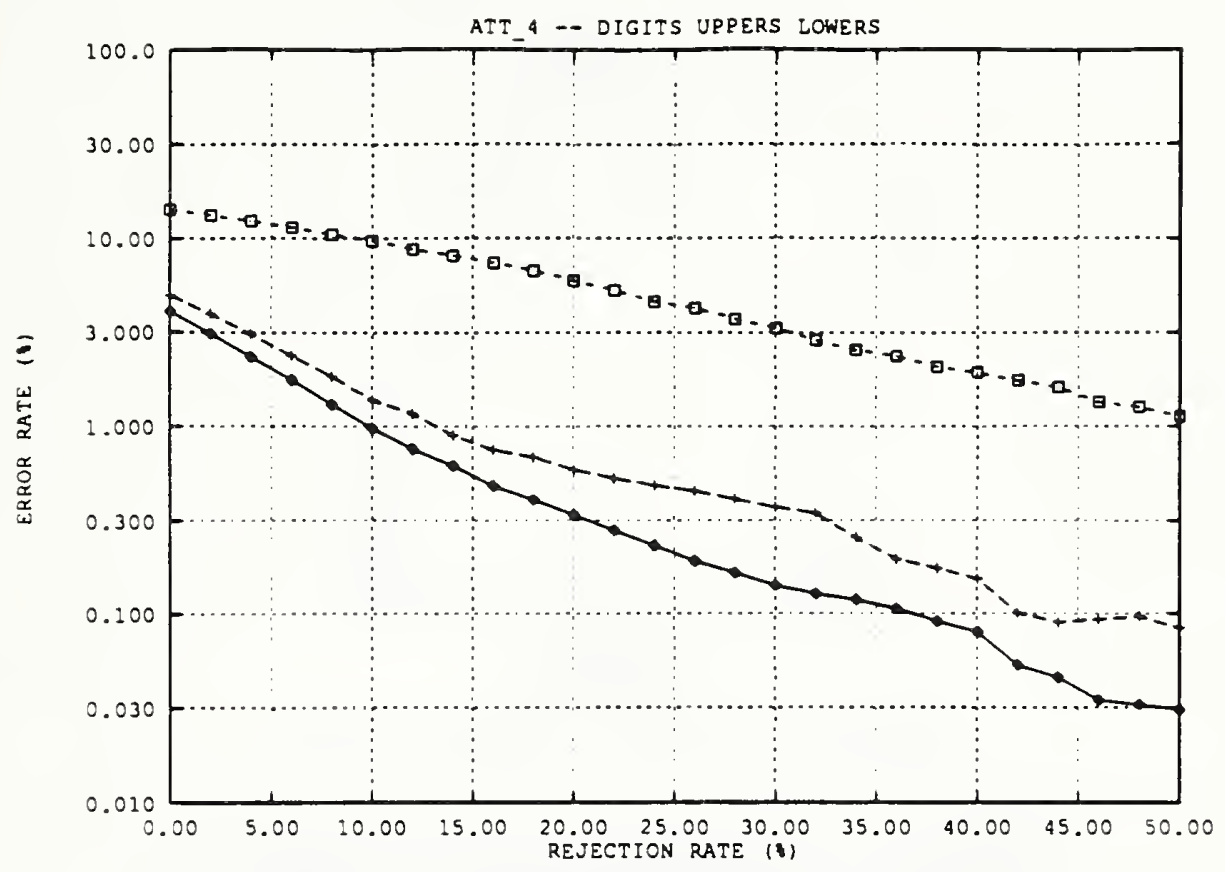

Figure 36: Error rate versus rejection rate for ATT_4

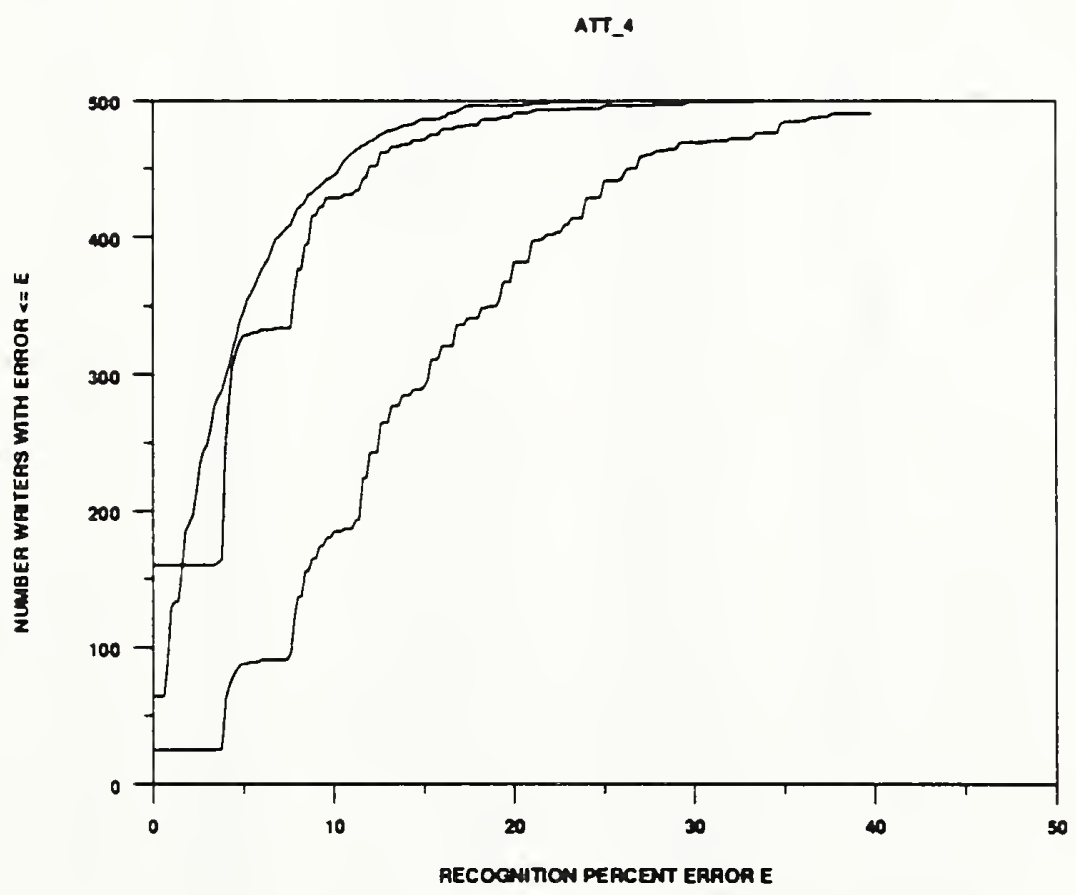

Figure 37: Error rate per writer of ATT_4 


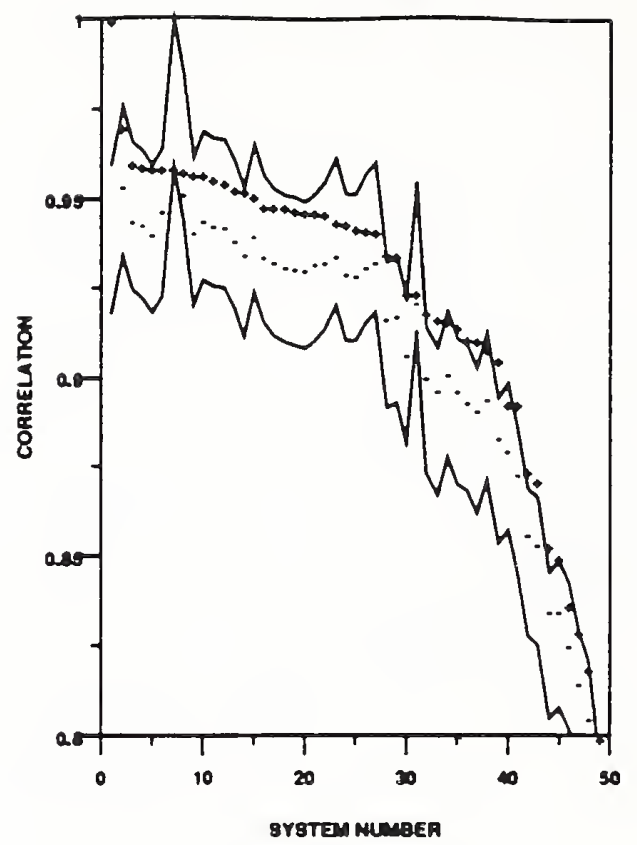

Figure 38: ATT_4 - digit correlation

\begin{tabular}{|c|c|c|c|}
\hline System Number & System Name & Correlation (ail) & Correlation (correct) \\
\hline 1 & ATT-4 & 1.0000 & 1.0000 \\
\hline 2 & VOTE M & 0.9708 & 0.9341 \\
\hline $\mathbf{3}$ & $A E G$ & 0.9605 & 0.9446 \\
\hline 4 & ATT 2 & 09593 & 0.9436 \\
\hline 3 & VOTEP & 0.9591 & 0.9473 \\
\hline B & KODAKב & 0.9591 & 0.9410 \\
\hline 7 & REFERENCE & 0.8590 & 0.9590 \\
\hline 8 & OCRSYS & 09383 & 0.9520 \\
\hline 9 & ERIM-I & 09574 & 0.9413 \\
\hline 10 & ATT $\perp$ & 0.8373 & 0.8445 \\
\hline 11 & ELSAGB-3 & 09358 & 0.9432 \\
\hline 12 & ELSAGB 2 & 0.9352 & 0.8428 \\
\hline 13 & ERIM_2 & 0.9531 & 0.8391 \\
\hline 14 & KODAK」 & 09526 & 0.9351 \\
\hline 15 & IBM & 09514 & 0.8404 \\
\hline 16 & UBOL & 0.9488 & 0.8348 \\
\hline 17 & SYMBUS & 0.8486 & 0.9333 \\
\hline 18 & THINK-L & 09485 & 0.9322 \\
\hline 19 & NIST_4 & 0.8478 & 09315 \\
\hline 20 & ELSAGB -1 & 0.9474 & $0.831 \mathrm{l}$ \\
\hline 21 & ATT 3 & 0.9473 & 0.8329 \\
\hline 22 & NESTOR & 09468 & 0.9334 \\
\hline 23 & THINK_2 & 08443 & 0.9331 \\
\hline 24 & HUGHES 1 & 0.9439 & 0.8303 \\
\hline 25 & HUGHES_2 & 09429 & 0.9299 \\
\hline 26 & NYNEX & 09422 & 0.9321 \\
\hline 27 & REI & 09417 & 0.9333 \\
\hline 28 & GTESS_ & 0.9356 & 0.9175 \\
\hline 29 & GTESS.1 & $0.933 \mathrm{~s}$ & 0.8181 \\
\hline 30 & COMCOM & 0.9247 & 0.9218 \\
\hline JI & NIST_I & 0.9247 & 09072 \\
\hline 32 & MIME & 0.9193 & 0.9007 \\
\hline 33 & NIST 2 & 09172 & 08973 \\
\hline 34 & GMD 3 & 0.9170 & 09016 \\
\hline 35 & ASOL & 0.9133 & 0.8974 \\
\hline 36 & UPENN & 0.9120 & 08942 \\
\hline 37 & NIST & 09114 & 0.8915 \\
\hline 38 & GMD_l & 0.9090 & 0.8949 \\
\hline 39 & RISO & 09060 & 0.8838 \\
\hline 40 & GMD _4 & 0.8935 & 0.8801 \\
\hline 41 & KAMAN_l & 0.8934 & 0.8739 \\
\hline 42 & KAMAN_3 & 0.8746 & 0.8566 \\
\hline 43 & KAMAN_2 & 0.8721 & 0.8342 \\
\hline 44 & GMD -2 & 0.8335 & 0.8353 \\
\hline 45 & KAMAN_S & 0.8303 & 0.8352 \\
\hline 46 & VALEN_2 & 08370 & 0.8257 \\
\hline 47 & IFAX & 0.8297 & 0.8152 \\
\hline 48 & VALEN-1 & 0.8191 & 0.8052 \\
\hline 49 & KAMAN_4 & 08000 & 0.7826 \\
\hline
\end{tabular}

Table 26: ATT -4 correlation graph key for digits. 


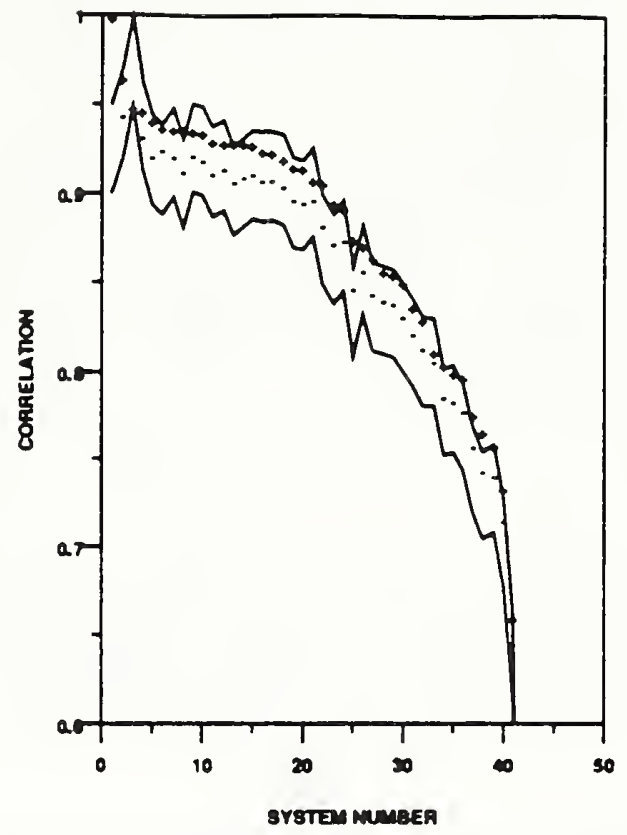

Figure 39: ATT_4 - upper case correlation

\begin{tabular}{|c|c|c|c|}
\hline System Number & System Name & Correlation (all) & Corpelation (correct) \\
\hline 1 & ATT_4 & 1.0000 & 1.0000 \\
\hline 2 & VOTE_M & 0.9863 & 0.9451 \\
\hline 3 & REFERENCE & 0.9500 & 0.9300 \\
\hline 4 & AEG & 09473 & 0.9330 \\
\hline 5 & ATT 2 & 08422 & 0.9223 \\
\hline 6 & VOTEP & 0.8385 & 0.9258 \\
\hline 7 & ERIM-1 & 0.9379 & 0.9219 \\
\hline 8 & KODAK」 & 0.8374 & 0.9140 \\
\hline 9 & NYNEX & 09371 & 0.8227 \\
\hline 10 & UMICH.1 & 0.9361 & 0.8206 \\
\hline 11 & UBOL & 0.9313 & 0.8132 \\
\hline 12 & NESTOR & 0.9308 & 0.9133 \\
\hline 13 & SYMBUS & 0.2306 & 0.9081 \\
\hline 14 & ATT 3 & 0.9300 & 0.8111 \\
\hline 15 & IBM & 0.9298 & 09127 \\
\hline 16 & ATT 2 & 0.2238 & 0.8094 \\
\hline 17 & HUGHES -I & 0.9245 & 0.9088 \\
\hline 18 & HUGHES_2 & 0.9216 & 0.9060 \\
\hline 18 & GTESS 1 & 0.9168 & 0.8981 \\
\hline 20 & GTESS 2 & 02157 & 08967 \\
\hline 21 & OCRSYS & 0.8091 & 08983 \\
\hline 22 & MIME & 0.9077 & 08838 \\
\hline 23 & ASOL & 0.8967 & 0.8734 \\
\hline 24 & NIST_A & 0.8237 & 0.8733 \\
\hline 23 & RISO & 0.8760 & 0.8488 \\
\hline 26 & REI & 08726 & 08389 \\
\hline 27 & NIST_l & 0.8664 & 08460 \\
\hline 28 & GMD_l & 08587 & 08421 \\
\hline 29 & GMD.3 & 08570 & 08401 \\
\hline so & KAMAN_1 & 08323 & 08333 \\
\hline 31 & GMD.4 & 0.8386 & 08230 \\
\hline 32 & NIST & 0.8307 & 08148 \\
\hline 33 & COMCOM & 0.8132 & 0.8073 \\
\hline 34 & KAMAN_3 & 08033 & 0.7873 \\
\hline 33 & IFAX & 08004 & 07846 \\
\hline 36 & KAMAN_2 & 0.7977 & 0.7789 \\
\hline 37 & NIST 2 & 0.7770 & 0.7386 \\
\hline 38 & GMD_2 & 0.7671 & 0.7452 \\
\hline 39 & VALEN_L & 0.7396 & 0.7416 \\
\hline 10 & KAMAN_4 & 0.7331 & 0.7162 \\
\hline 11 & KAMAN_5 & 0.6616 & 0.6469 \\
\hline 12 & $\mathrm{UM}_{1 \mathrm{CH}} 2$ & 00384 & 0.0218 \\
\hline
\end{tabular}

Table 27: ATT_4 correlation graph key for uppers. 


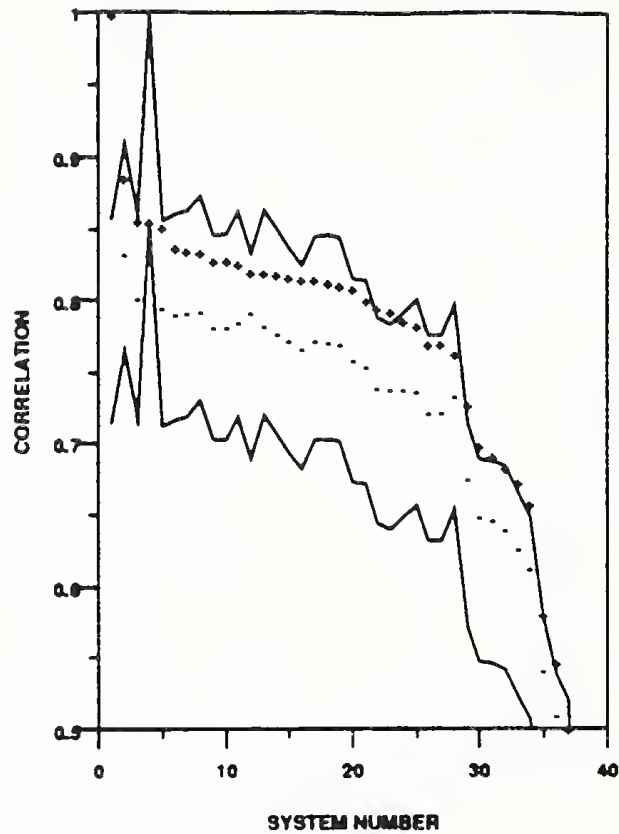

Figure 40: ATT_4 - lower case correlation

\begin{tabular}{|c|c|c|c|}
\hline Syssem Number & System Name & Correlation (all) & Correlation (correct) \\
\hline 1 & ATT_4 & 10000 & 1.0000 \\
\hline 2 & VOTE $\triangle$ & 08873 & 0.8351 \\
\hline 3 & ATT 2 & 08880 & 0.8033 \\
\hline 4 & REFERENCE & 08572 & 0.8572 \\
\hline 5 & KODAK」 & 08528 & 0.7872 \\
\hline 6 & NYNEX & 08395 & 07828 \\
\hline 7 & ERIM_L & 0.8374 & 07234 \\
\hline 8 & $A E G$ & 08362 & 0.7946 \\
\hline 9 & IBM & 08303 & 0.7828 \\
\hline 10 & NESTOR & 0.8301 & 0.7837 \\
\hline 11 & ATT_ & 0.8275 & 07864 \\
\hline 12 & VOTE_P & 08223 & 0.7938 \\
\hline 13 & OCRSYS & 0.8218 & 0.7849 \\
\hline 14 & UMICH-L & 08210 & 07791 \\
\hline 15 & ATT 3 & 08192 & 0.7745 \\
\hline 16 & GTESS_1 & 0.8176 & 0.7678 \\
\hline 17 & UBOL & 0.8173 & 07740 \\
\hline 18 & HUGHES - 1 & 08153 & 0.7734 \\
\hline 19 & HUGHES_2 & 08130 & 07718 \\
\hline 20 & GTESS 2 & 08112 & 0.7605 \\
\hline 21 & NIST -1 & 0.8030 & 0.7559 \\
\hline 22 & ASOL & 07969 & 0.7408 \\
\hline 23 & RISO & 0.7847 & 0.7391 \\
\hline 24 & G MD_3 & 0.7878 & 0.7390 \\
\hline 25 & NIST 4 & 0.7847 & 0.7387 \\
\hline 26 & GMD_4 & 0.7718 & 0.7232 \\
\hline 27 & GMD_l & 0.7718 & 07232 \\
\hline 28 & NIST 3 & 0.7650 & 0.7349 \\
\hline 29 & GMD _2 & 0.7287 & 0.6779 \\
\hline 30 & KAMAN_L & 0.7004 & 0.6510 \\
\hline 31 & $\mathrm{NIST}_{2}$ & 0.6933 & 08484 \\
\hline 32 & VALEN_l & 0.6851 & 06419 \\
\hline 33 & KAMAN_3 & 0.6750 & 06283 \\
\hline 34 & KAMAN_2 & 0.6608 & 0.6148 \\
\hline 35 & KAMAN_5 & 0.5823 & 0.5420 \\
\hline 36 & KAMAN_4 & 0.5477 & 0.5107 \\
\hline 37 & COMCOM & 0.5023 & 0.4896 \\
\hline 38 & $\mathrm{UMICH}_{2}$ & 0.1085 & 0.0583 \\
\hline
\end{tabular}

Table 28: ATT_4 correlation graph key for lowers. 
SYSTEM : COMCOM

PARTICIPANTS: Mr. Eberhard Kuehl, Perry Riggs

ORGANIZATION: Com Com Systems, Inc., Clearwater, FL

PREPROCESSING: thinning.

FEATURES : ?

CLASSIFICATION: proprietary: not NN, not pixel comparison, nor vector analysis. Positional information and features matched against database of "tables".

HARDWARE: 386

TRA INING :

DIGITS

UPPERS

LOWERS DATABASE

-.-- Number used is proprietary -.. NSDB3

-.-- Number used is proprietary -.-- INTERNAL

STATUS: $\quad$ on time, $O$ 's and 1 's interchanged in RJX files

RESULTS: -- DIGITS -- -- UPPERS -- -- LOWERS -- DATABASE

REJ . ERR. REJ. ERR. REJ. ERR. TESTDATA 1

RATE RATE-- RATE RATE-- RATE RATE--

$\begin{array}{llllll}0.00 & 0.0456 & 0.00 & 0.1694 & 0.00 & 0.4800\end{array}$

$\begin{array}{llllll}0.03 & 0.0186 & 0.15 & 0.0242 & 0.45 & 0.0590\end{array}$

OCR RATE (CPS): DIGITS

UPPERS

LOWERS
SYS RATE:
12.68
11.71
9.09

CPU RATE:

NOTE: Internal database contains 110000 hand printed digits, 220000 upper case letters, and at least 60000 mixed uppers and lowers. 
SYSTEM: COMCOM

The following references have been provided for this system: 


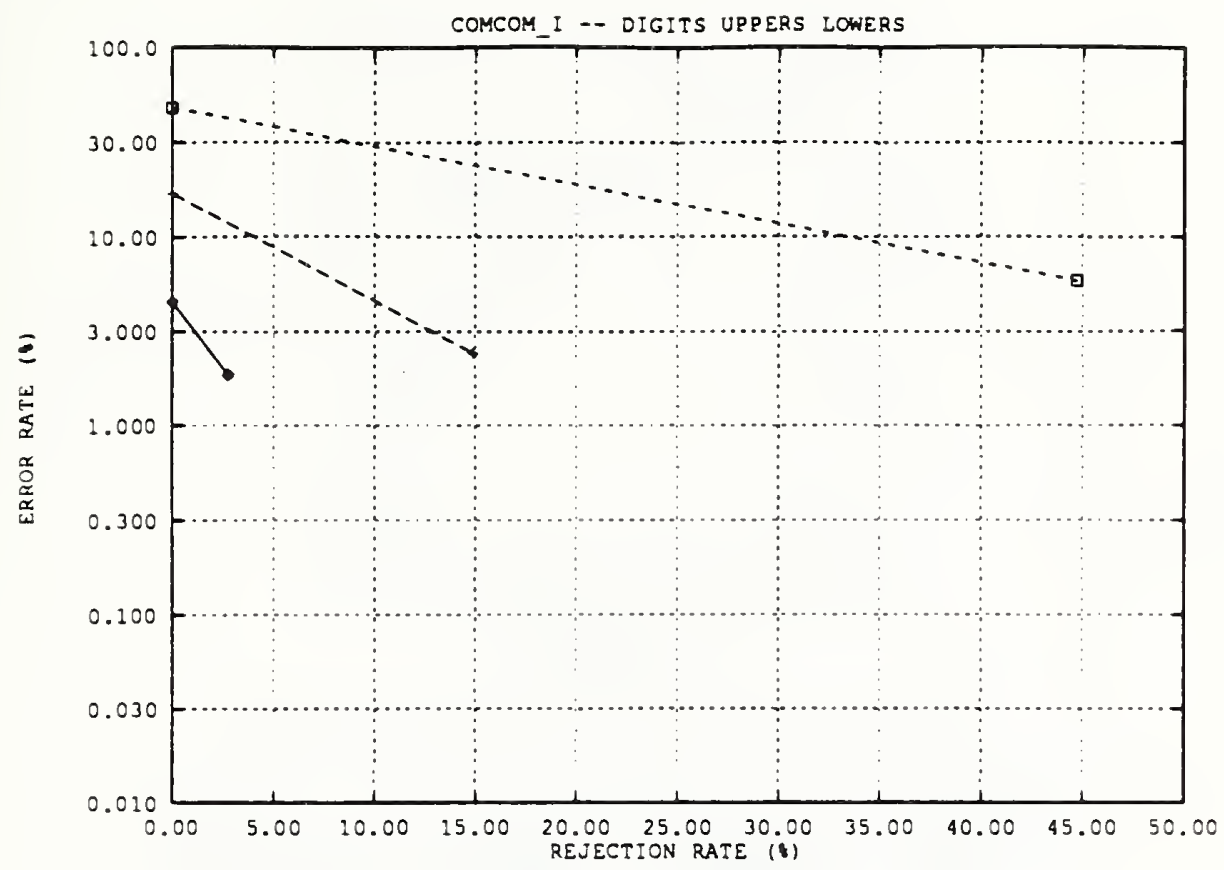

Figure 41: Error rate versus rejection rate for COMCOM

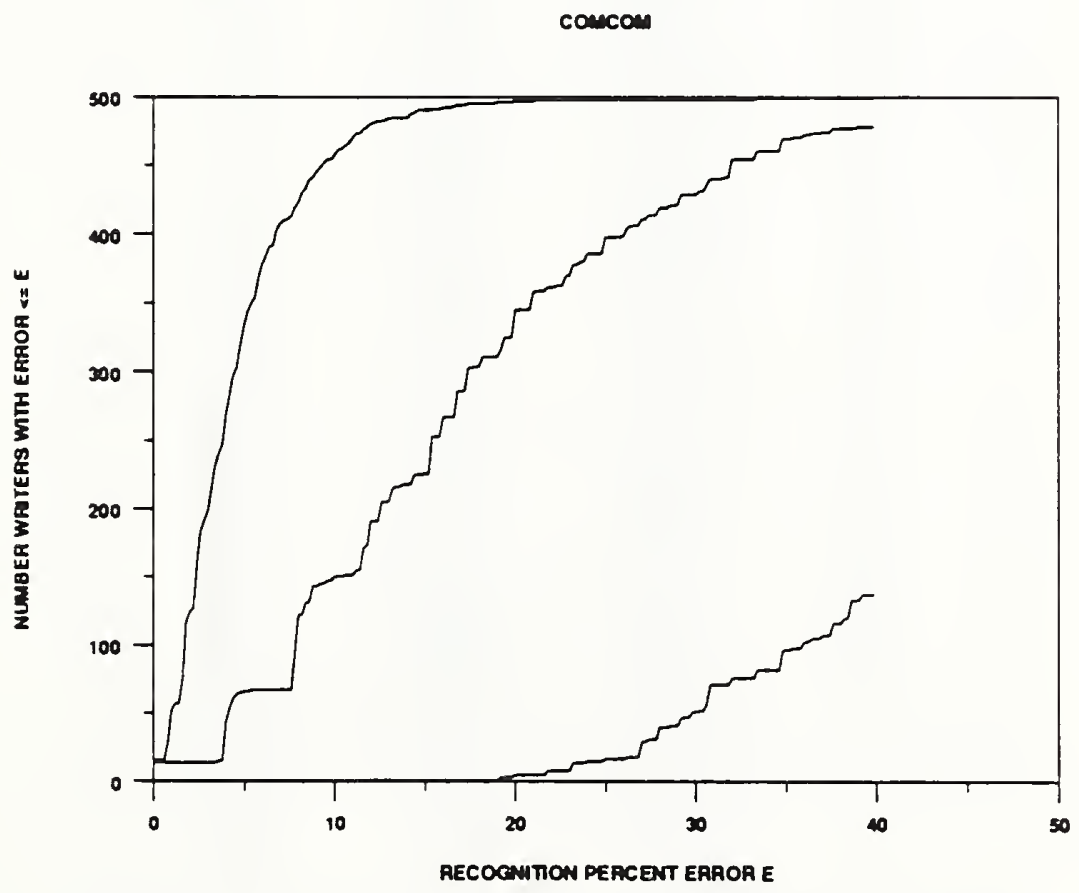

Figure 42: Error rate per writer of COMCOM 


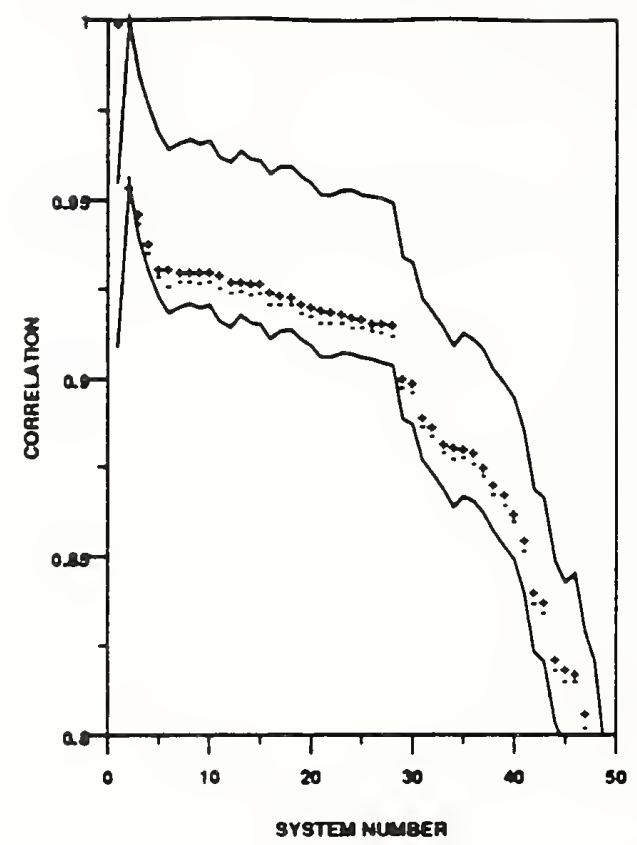

Figure 43: COMCOM - digit correlation

\begin{tabular}{|c|c|c|c|}
\hline System Number & System Name & Correiation (all) & Correlation (correct) \\
\hline ! & COMCOM & 1.0000 & 1.0000 \\
\hline 2 & REFERENCE & 0.8544 & 0.9544 \\
\hline 3 & OCRSYS & 0.9471 & 0.9445 \\
\hline 4 & VOTE_M & 0.9390 & 0.9364 \\
\hline$s$ & ATT_ & 0.9323 & 0.9298 \\
\hline 6 & VOTEP & 0.9321 & 0.9271 \\
\hline 7 & $A E G$ & 0.8314 & 0.9286 \\
\hline 8 & ELSAGBA & 0.9313 & 0.9286 \\
\hline 9 & ELSAGB 2 & 0.9311 & 0.9284 \\
\hline 10 & IBM & 0.9311 & 0.9280 \\
\hline 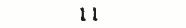 & THINK 2 & 0.9302 & 0.9264 \\
\hline 12 & ATT 2 & 0.9285 & 0.9258 \\
\hline 13 & REI & 0.9283 & 0.9251 \\
\hline 14 & ERIM_1 & 0.9279 & 0.9249 \\
\hline 15 & ERIM_2 & 0.9278 & 0.9250 \\
\hline 16 & NYNEX & 0.9255 & 0.9219 \\
\hline 17 & A T T -4 & 0.9247 & 0.3219 \\
\hline 18 & KODAK2 & 0.9244 & 0.9218 \\
\hline 19 & UBOL & 0.9224 & 0.9198 \\
\hline 20 & NESTOR & 0.9215 & 0.9186 \\
\hline 21 & HUGHES - 1 & 0.9206 & 0.9170 \\
\hline 22 & HUGHES_2 & 09203 & 09168 \\
\hline 23 & SYMBUS & 0.9197 & 0.9169 \\
\hline 24 & KODAK」 & 0.9186 & 0.9158 \\
\hline 25 & $\operatorname{ATT}_{3}$ & 09181 & 0.9155 \\
\hline 26 & THINK_1 & 0.9171 & 0.9145 \\
\hline 27 & NIST_4 & 0.9170 & 0.9141 \\
\hline 28 & ELSAGB-I & 0.9163 & 0.3133 \\
\hline 29 & GTESS_1 & 0.9013 & 08986 \\
\hline 30 & GTESS_2 & 0.9000 & 0.8972 \\
\hline 31 & NIST.1 & 0.8903 & 0.8875 \\
\hline 32 & GMD_3 & 0.8875 & 08847 \\
\hline 33 & MIME & 0.8831 & 08800 \\
\hline 34 & UPENN & 0.8819 & 08782 \\
\hline 35 & GMD_1 & 0.8818 & 08789 \\
\hline 36 & ASOL & 0.8802 & 0.8772 \\
\hline 37 & NIST 2 & 0.8762 & 0.8739 \\
\hline 38 & NIST & 0.8713 & 0.8689 \\
\hline 39 & GMD_-4 & 0.8686 & 0.8637 \\
\hline 40 & RISO & 0.8633 & 0.8607 \\
\hline 41 & KAMAN-L & 0.8557 & 0.8527 \\
\hline 42 & KAMAN_3 & 0.8410 & 0.8378 \\
\hline 43 & KAMAN_2 & 0.8386 & 0.8353 \\
\hline 44 & KAMAN_5 & 0.8223 & 0.8189 \\
\hline 45 & VALEN 2 & 0.8196 & 0.8159 \\
\hline 46 & GMD -2 & 08183 & 0.8159 \\
\hline 47 & IFAX & 0.8072 & 0.8032 \\
\hline 48 & VALEN_I & 0.7946 & 0.7914 \\
\hline 49 & KAMAN_4 & 0.7676 & 0.7646 \\
\hline
\end{tabular}

Table 29: COMCOM correlation graph key for digits. 


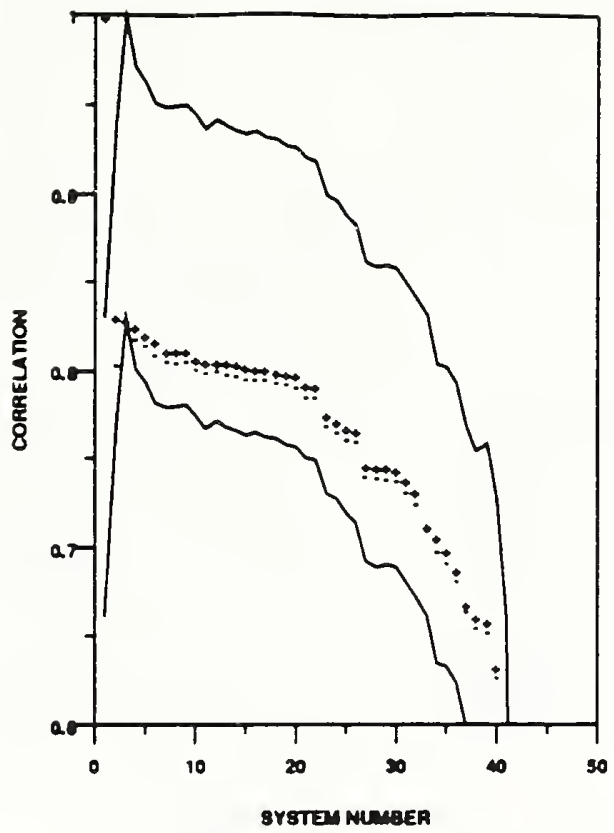

Figure 44: COMCOM - upper case correlation

\begin{tabular}{|c|c|c|c|}
\hline System Number & Sydtem Name & Correlation (all) & Correlation (correct) \\
\hline $\mathrm{I}$ & COMCOM & 1.0000 & 1.0000 \\
\hline 2 & VOTE $P$ & 0.8324 & 08052 \\
\hline 3 & REFERENCE & 0.8306 & 08306 \\
\hline 4 & VOTE_M & 08261 & 0.8205 \\
\hline 5 & AEG & 0.8222 & 0.8168 \\
\hline 6 & NYNEX & 0.8180 & 08113 \\
\hline 7 & ATT_-4 & 08132 & 08075 \\
\hline 8 & ERIM-I & 08132 & 08071 \\
\hline 9 & UMICH-I & 0.8132 & 08066 \\
\hline 10 & ATT 2 & 0.8084 & 0.8024 \\
\hline 11 & NESTOR & 0.8085 & 08014 \\
\hline 12 & IBM & 0.8065 & 0.8004 \\
\hline 13 & UBOL & 0.8060 & 0.7999 \\
\hline 14 & HUGHES-1 & 08053 & 0.7986 \\
\hline 15 & HUGHES-2 & 0.8040 & 07971 \\
\hline 16 & ATT $\perp$ & 0.8031 & 0.7968 \\
\hline 17 & ATT 3 & 0.8029 & 0.7973 \\
\hline 18 & KODAK_ & 0.8007 & 0.7949 \\
\hline 19 & SYMBUS & 0.7995 & 07941 \\
\hline 20 & OCRSYS & 07987 & 0.7927 \\
\hline 21 & GTESS -1 & 0.7931 & 0.7875 \\
\hline 22 & GTESS 2 & 07930 & 0.7870 \\
\hline 23 & MIME & 07764 & 0.7709 \\
\hline 24 & NIST_4 & 0.7728 & 07669 \\
\hline 25 & ASOL & 0.7684 & 0.7623 \\
\hline 26 & REI & 0.7868 & 0.7612 \\
\hline 27 & NIST & 0.7474 & 0.7421 \\
\hline 28 & RISO & 07469 & 07409 \\
\hline 29 & GMD_l & 07462 & 07402 \\
\hline 30 & GMD_3 & 0.7432 & 0.7391 \\
\hline 31 & KAMAN_I & 07391 & 0.7327 \\
\hline 32 & GMD _4 & 0.7324 & 0.7261 \\
\hline 33 & בה NIST & 0.7133 & 0.7119 \\
\hline 34 & IFAX & 0.7068 & 0.6995 \\
\hline 35 & KAMAN_J & 0.6997 & 0.6937 \\
\hline 36 & KAMAN_2 & 0.6890 & 0.6833 \\
\hline 37 & NIST 2 & 0.6697 & 0.6660 \\
\hline 38 & GMD_2 & 0.8618 & 0.6569 \\
\hline 39 & VALEN-I & 0.6598 & 0.6340 \\
\hline 40 & KAMAN_4 & 0.6335 & 0.6282 \\
\hline 41 & KAMAN_S & 0.5768 & 05718 \\
\hline 42 & $\mathrm{UMICH}_{2}$ & 00274 & 0.0175 \\
\hline
\end{tabular}

Table 30: COMCOM correlation graph key for uppers. 


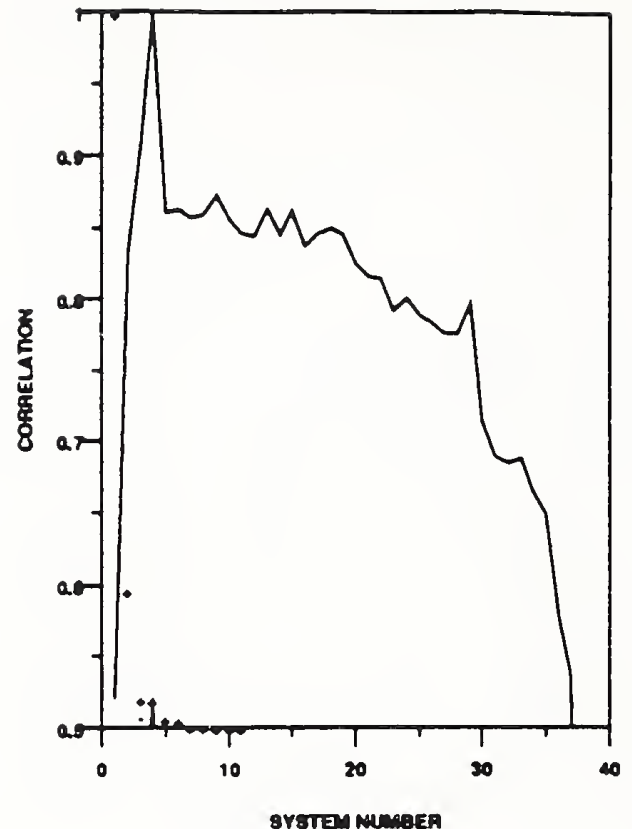

Figure 45: COMCOM - lower case correlation

\begin{tabular}{|c|c|c|c|}
\hline System Number & System Name & Cotrelation (all) & Cortelation (correct) \\
\hline 1 & COMCOM & 1.0000 & 1.0000 \\
\hline 2 & VOTE_P & 0.5066 & 0.4902 \\
\hline 3 & VOTE_M & 0.5209 & 0.5082 \\
\hline 4 & REFERENCE & 05200 & 0.3200 \\
\hline 3 & NYNEX & 0.5074 & 0.4937 \\
\hline$B$ & ERIM-1 & 0.8061 & 0.1923 \\
\hline 7 & ATT-A & 0.5023 & 0.4896 \\
\hline 8 & ATT2 2 & 05013 & 0.4896 \\
\hline 9 & AEG & 0.5011 & 0.4882 \\
\hline 10 & KODAK_ & 0.5007 & 0.4876 \\
\hline 11 & HUGHES_- & 0.3004 & 0.4886 \\
\hline 12 & HUGHES_2 & 04983 & 0.4872 \\
\hline 13 & OCRSYS & 04980 & 0.4882 \\
\hline 14 & IBM & 04968 & 0.4856 \\
\hline 15 & ATT $\perp$ & 04981 & 0.4848 \\
\hline 16 & ATTS & 0.4940 & 0.4818 \\
\hline 17 & NESTOR & 0.4833 & 0.4822 \\
\hline 18 & UMICH-I & 0.4923 & 0.4823 \\
\hline 18 & UBOL & 0.4018 & 0.4798 \\
\hline 20 & GTESS_l & 0.4873 & 0.4757 \\
\hline 21 & GTESS 2 & 04830 & 0.4712 \\
\hline 22 & NIST_I & 0,1782 & 0.4678 \\
\hline 23 & GMD.3 & 04712 & 0.4594 \\
\hline 24 & NIST_A & 0.4688 & 0.4364 \\
\hline 25 & ASOL & 0.4681 & 0.4545 \\
\hline 28 & RISO & 0.4627 & 0.4540 \\
\hline 27 & GMD_4 & 04622 & 0.4513 \\
\hline 28 & GMD_1 & 04622 & 0.4513 \\
\hline 29 & NIST & 0.4555 & 0.4323 \\
\hline 30 & GMD_2 & 04342 & 0.4247 \\
\hline 31 & KAMAN_l & 04218 & 0.4098 \\
\hline 32 & VALEN-I & 0.4204 & 0.1100 \\
\hline 33 & NIST 2 & 04168 & 0.4075 \\
\hline 34 & KAMAN_3 & 04097 & 0.3981 \\
\hline 35 & KAMAN_2 & 03975 & 0.3869 \\
\hline 36 & KAMAN_S & 03382 & 0.3483 \\
\hline 37 & $K A M A N-4$ & 0.3302 & 0.3223 \\
\hline 38 & $\mathrm{UMICH}_{2}$ & 0.0438 & 0.0306 \\
\hline
\end{tabular}

Table 31: COMCOM correlation graph key for lowers. 
SYSTEM: ELSAGB_1

PARTICIPANT: Mr. Francesco Fignoni

ORGANIZATION: ELSAG BAILEY, INC., Conshohocken, PA

PREPROCESSING: noise removal and size normalization to $24 \times 36$.

FEATURES: shape function of the character bit maps having the same size as the character.

CLASSIFICATION: KNN with respect to shape function distance from references representing clusters of shape functions in training sample

HARDWARE: 33 MHz 386 running tight assembly code

IRAINING: DIGITS UPPERS LOWERS DATABASE

85491 NA NA $\quad$ NSDB3

STATUS: $\quad$ on time

RESULTS: -- DIGITS -- -- UPPERS -- -- LOWERS -- DATABASE

REJ. ERR. REJ. ERR. REJ. ERR. TESTDATA1

RATE RATE-- RATE RATE-- RATE RATE--

$0.00 \quad 0.0507$

$0.08 \quad 0.0179$

$0.12 \quad 0.0114$

OCR RATE (CPS): DIGITS

UPPERS

LOWERS

SYS RATE:

$\begin{array}{llll}\text { CPU RATE: } & 65.00 & \text { NA }\end{array}$

NOTE: This is the system used in their postal OCR. Few details of the recognition algorithm were provided. 
SYSTEM: ELSAGB 1

BIBLIOGRAPHY:

The following references have been provided for this system:

none

COMMENTS: ELSAG BAILEY

SPECIFIC ABOUT ELSAG BAILEY

- AFTER receiving the TESTDATA1 CD ROM, that is the test set, Elsag Bailey neither modified in any part or tuned in any way the recognition units and associated data-bases produced from training for the tests ELSAGB_1, ELSAGB_2, and ELSAGB_3.

Elsag Bailey is aware of the fact that given the poor relationship between training and test sets, these countermeasures could prove useful.

- ELSAG_1 had some troubles dealing with the thickness range of characters: about $4 \%$ the training digits have an average thickness of less than 2 or more than 9 pixels.

\section{GENERAL ABOUT THE CONFERENCE}

- Elsag Bailey appreciated the way the test and Conference were set up by NIST. It was something between an acceptance test and a scientific conference and proved itself both useful and interesting.

- The test set for digits was both very difficult and very "far" from the training set; this fact produced rather conservative recognition results.

One reason is the fact that the training set did not contain examples of the difficult test characters. If it had, performance would have been higher. The other reason is that the test characters are poor in quality, probably representing the low end in a real environment.

While these points do not weaken the relative comparisons among the participants, nevertheless. they compromise the absolute meaning of the recognition performance.

- A good estimate of segmentation performance, that is, the next important part of the whole OCR process, is an open question.

In fact, the scoring procedure should be independent from the recognition unit and automatic. Otherwise, the two procedures are mixed together. 


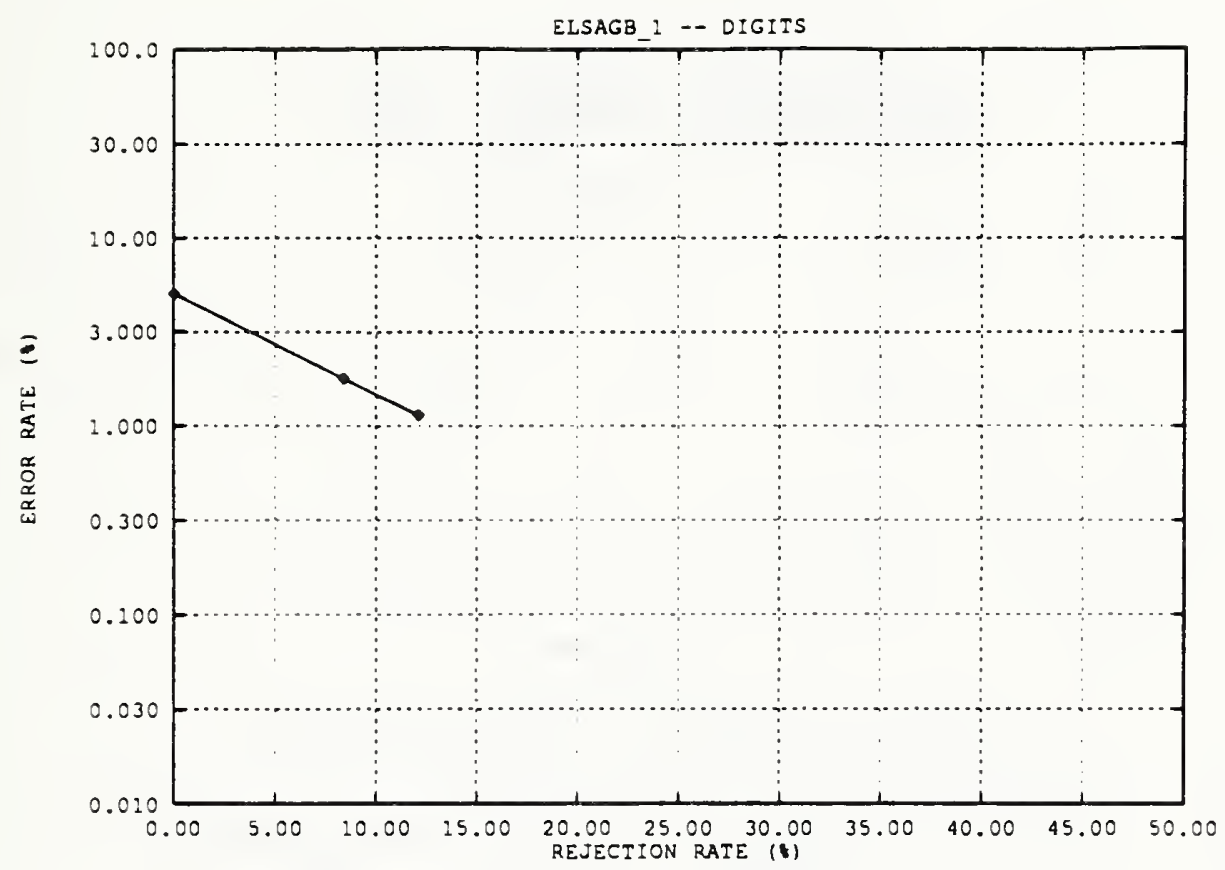

Figure 46: Error rate versus rejection rate for ELSAGB_1

ELSAOB_1

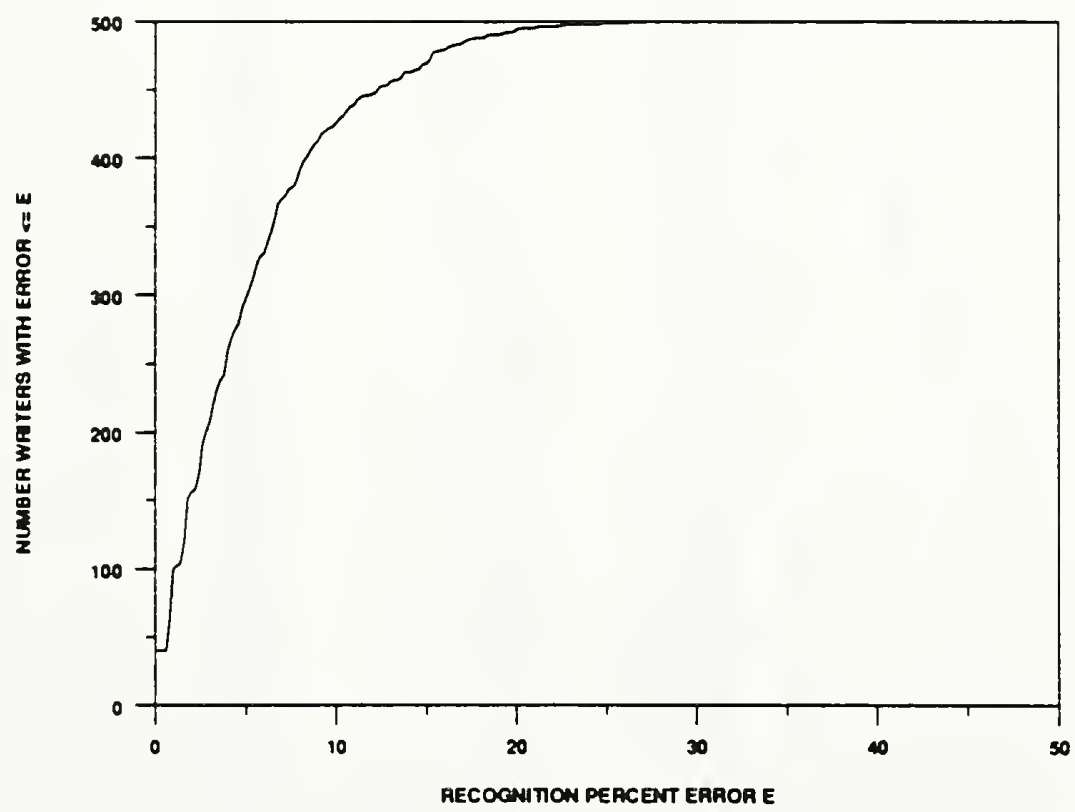

Figure 47: Error rate per writer of ELSAGB_1 


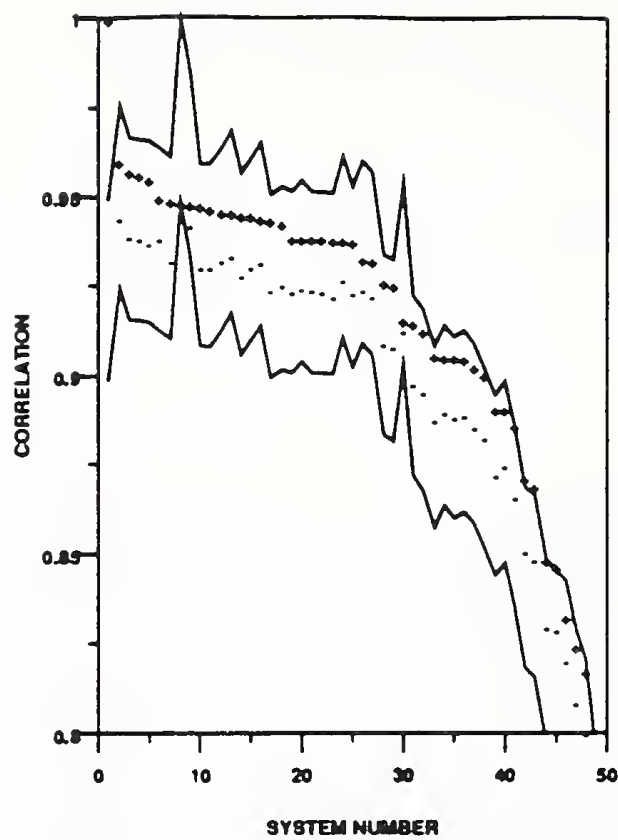

Figure 48: ELSAGB_1 - digit correlation

\begin{tabular}{|c|c|c|c|}
\hline System Number & System Name & Correlation (all) & Corfelstion (correct) \\
\hline 1 & $\overline{\text { ELSAGB.1 }}$ & 1.0000 & 1.0000 \\
\hline 2 & VOTE_M & 09606 & 09444 \\
\hline 3 & ELSAGB & 0.9378 & 0.9393 \\
\hline 4 & ELSAGB2 & 0.9370 & 09388 \\
\hline 5 & $A E G$ & 09353 & 0.9378 \\
\hline 8 & VOTEP & 0.9306 & 09390 \\
\hline 7 & ERIM_1 & 0.9493 & 0.9331 \\
\hline 8 & REFERENCE & 09493 & 09493 \\
\hline 9 & OCRSYS & 09485 & 09423 \\
\hline 10 & KODAK2 2 & 09480 & 0.3313 \\
\hline 11 & AT T -4 & 09474 & 0.9311 \\
\hline 12 & ATT 2 & 0.8463 & 0.8328 \\
\hline 13 & $\mathrm{ATT} \perp$ & 08462 & 09345 \\
\hline 14 & UBOL & 09453 & 09287 \\
\hline 13 & ERIM 2 & 09454 & 0.9312 \\
\hline 16 & IBM & 09447 & 0.9323 \\
\hline 17 & NIST_4 & 09439 & 0.9249 \\
\hline 18 & KODAK_ & 0.9431 & 0.8260 \\
\hline 19 & NESTOR & 09392 & 0.9254 \\
\hline 20 & HUGHES -1 & 0.9392 & 0.9243 \\
\hline 21 & ATT 3 & 0.9391 & 09247 \\
\hline 22 & HUGHES -2 & 09389 & 0.9241 \\
\hline 23 & THINK-I & 0.9386 & 0.9228 \\
\hline 24 & THINK 2 & 0.9384 & 0.9277 \\
\hline 25 & SYMBUS & 09379 & 02240 \\
\hline 26 & REI & 09334 & 0.9249 \\
\hline 27 & NYNEX & 09329 & 0.9228 \\
\hline 28 & GTESS_1 & 09270 & 09097 \\
\hline 29 & GTESS 2 & 0.9263 & 0.9087 \\
\hline 30 & COMCOM & 09163 & 0.9133 \\
\hline 31 & NIST 1 & 0.9133 & 08981 \\
\hline 32 & GMD_3 & 0.9133 & 08958 \\
\hline 33 & $\mathrm{NIST}_{2}$ & 09065 & 08881 \\
\hline 34 & MIME & 0.9039 & 08902 \\
\hline 35 & ASOL & 0.9057 & 0.8890 \\
\hline 36 & GMD - 1 & 09036 & 08894 \\
\hline 37 & UPENN & 09033 & 0.8861 \\
\hline 38 & נב & 0.9010 & 08830 \\
\hline 39 & RISO & 08912 & 0.8730 \\
\hline 40 & GMD_4 & 0.8910 & 0.8751 \\
\hline 41 & KAMAN-1 & 0.8865 & 08666 \\
\hline 42 & KAMAN_3 & 08720 & 08313 \\
\hline 43 & KAMAN_2 & 0.8693 & 08480 \\
\hline 44 & KAMAN_3 & 0.8491 & 08303 \\
\hline 45 & GMD -2 & 08470 & 0.8291 \\
\hline 46 & VALEN_2 & 0.8327 & 08203 \\
\hline 47 & IFAX & 0.8247 & 0.8092 \\
\hline 48 & VALEN_1 & 08178 & 0.8003 \\
\hline 49 & KAMAN_4 & 0.7964 & 07775 \\
\hline
\end{tabular}

Table 32: ELSAGB_1 correlation graph key for digits. 


\section{No Data Available}

Figure 49: ELSAGB_1 - upper case correlation

There was no data for this evaluation

Table 33: ELSAGB_1 correlation graph key for uppers. 


\section{No Data Available}

Figure 50: ELSAGB.1 - lower case correlation

There was no data for this evaluation.

Table 34: ELSAGB_1 correlation graph key for lowers. 
SYSTEM: EISAGB_2

PARTICIPANT: Mr. Francesco Fignoni

ORGANIZATION: ELSAG BAILEY, INC., Conshohocken, PA

PREPROCESSING: noise removal and size normalization to $24 \times 36$.

FEATURES: shape function of the character bit maps having the same size as the character.

CLASSIFICATION: KNN with respect to shape function distance from references representing clusters of shape functions in training sample (the classifier used vith ELSAGB_1) for preclassification followed by the same classifier using a more sophisticated distance measure and many more references

HARDWARE: $33 \mathrm{MHz} 386$ running tight assembly for preclassification VAX $6000 / 410$ under VMS running FORTRAN for classification

TRAINING: DIGITS UPPERS LOWERS DATABASE

85491 NA NA $\quad$ NSDB3

STATUS: $\quad$ on time

RESULTS: -- DIGITS -- -- UPPERS -- -- LOWERS -- DATABASE

REJ. ERR. REJ. ERR. REJ. ERR. TESTDATA1

RATE RATE-- RATE RATE-- RATE RATE--

$0.00 \quad 0.0338$

$0.05 \quad 0.0135$

$0.08 \quad 0.0097$

$0.10 \quad 0.0077$

0.110 .0068

OCR RATE (CPS): DIGITS

UPPERS LOWERS

SYS RATE:

CPU RATE: $\quad 0.30 \quad$ NA $\quad$ NA

NOTE: This is a laboratory research system. Few details of the recognition algorithm were provided. 
SYSTEM: ELSAGB 2

BIBLIOGRAPHY:

The following references have been provided for this system:

none 


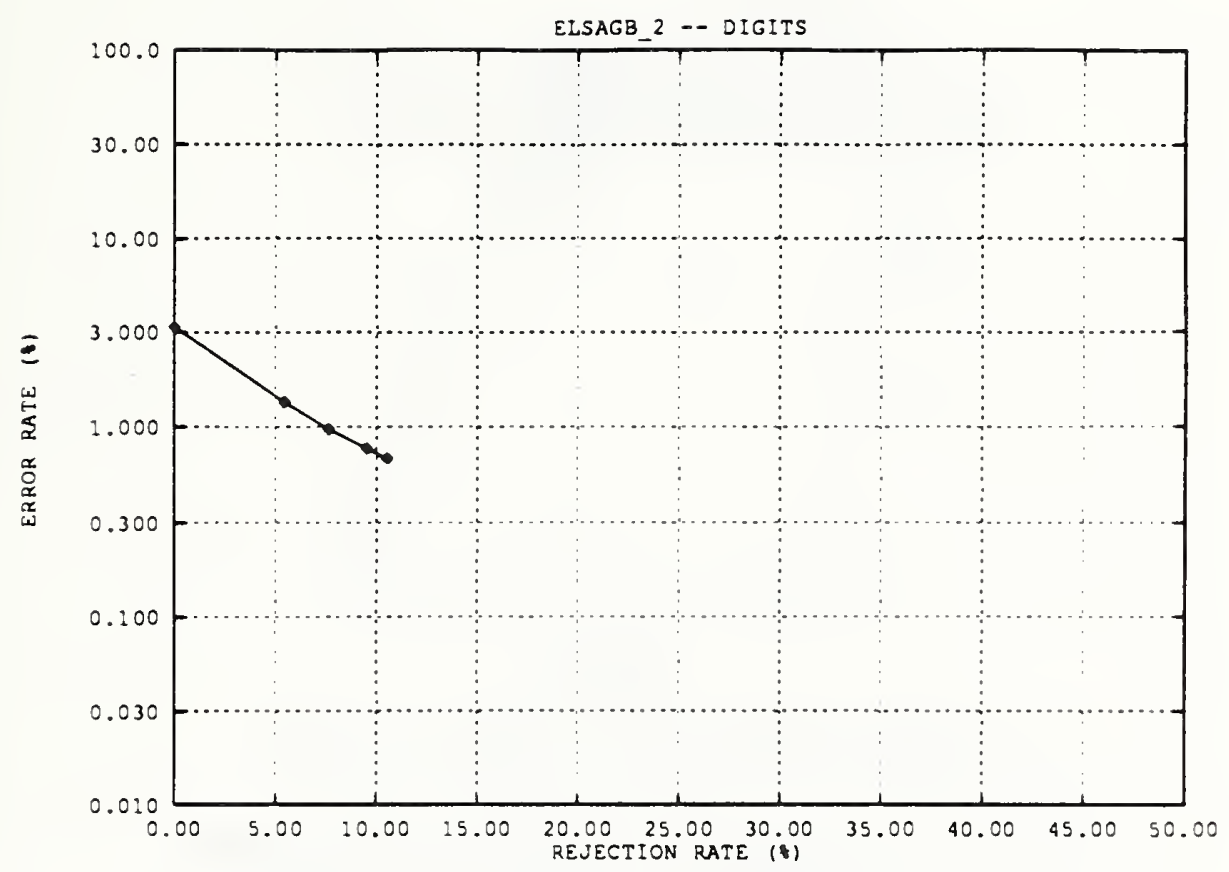

Figure 51: Error rate versus rejection rate for ELSAGB 2

ELSAOB_2

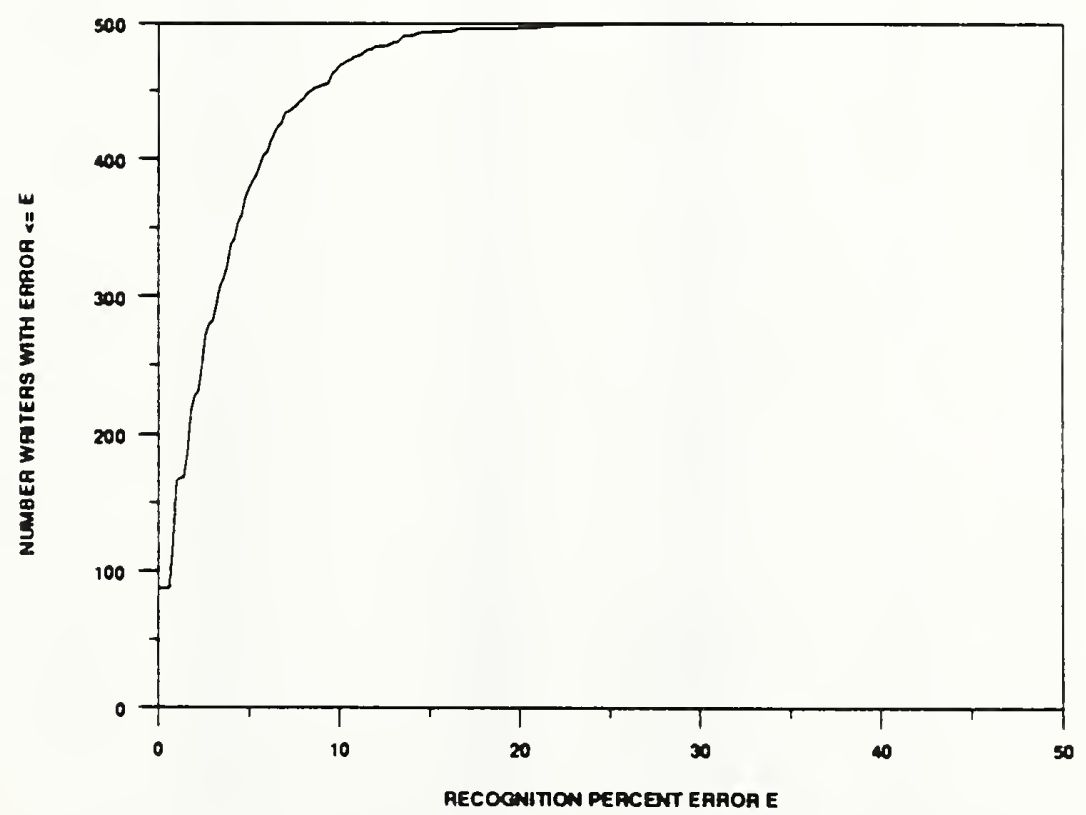

Figure 52: Error rate per writer of ELSAGB_2 


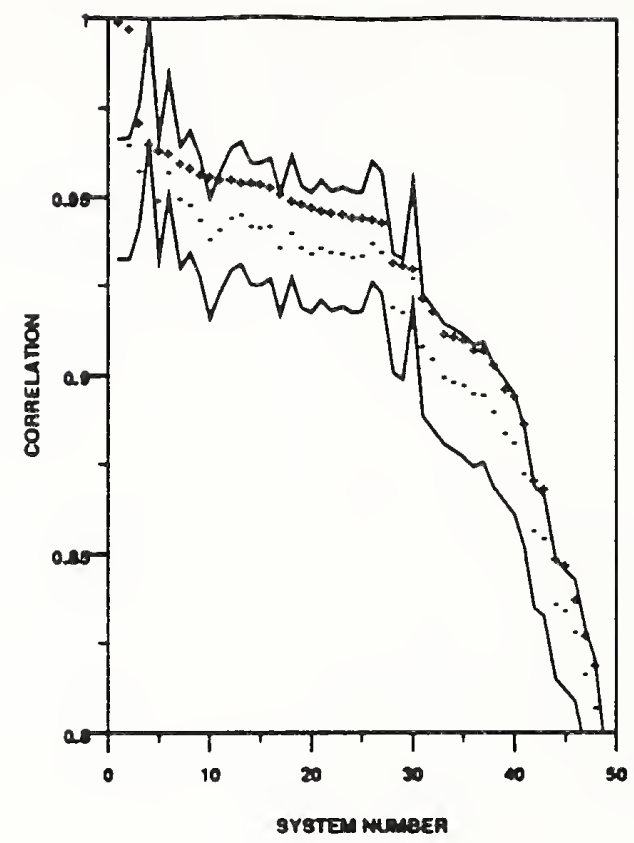

Figure 53: ELSAGB 2 - digit correlation

\begin{tabular}{|c|c|c|c|}
\hline System Numbet & System Name & Correiation (all) & Cortelation (correct) \\
\hline 1 & ELSAGB2 & 1.0000 & 1.0000 \\
\hline 2 & ELSAGB 3 & 0.8983 & 0.8635 \\
\hline 3 & VOTE_M & 0.8721 & 0.8383 \\
\hline 4 & REFERENCE & 0.9682 & 0.8662 \\
\hline 3 & AEG & 0.9642 & 0.8489 \\
\hline 6 & OCRSYS & 0.8632 & 0.8578 \\
\hline 7 & VOTEP & 0.9606 & 0.8506 \\
\hline 8 & ATT $\perp$ & 0.8591 & 0.2488 \\
\hline$\theta$ & ERIM-1 & 0.9372 & 0.8447 \\
\hline 10 & ELSAGBAL & 0.8570 & 0.8388 \\
\hline 11 & UBOL & 0.8561 & 0.8416 \\
\hline 12 & $\operatorname{ATT} 2$ & 0.9538 & 0.9430 \\
\hline 13 & IBM & 0.8553 & 0.9457 \\
\hline 14 & ATT_ 4 & 0.8352 & 0.9428 \\
\hline 15 & KODAK2 & 0.8345 & 0.9423 \\
\hline 16 & ERIM 2 & 0.8338 & 0.8428 \\
\hline 17 & NIST_4 & 0.0817 & 0.8365 \\
\hline 18 & THINK 2 & 0.8489 & 0.9410 \\
\hline 19 & KODAK」 & 0.9482 & 0.9368 \\
\hline 20 & THINK-I & 0.8482 & 0.9352 \\
\hline 21 & NESTOR & 0.9471 & 0.8368 \\
\hline 22 & ATT 3 & 0.9488 & 0.9358 \\
\hline 23 & SYMBUS & 0.8462 & 0.9352 \\
\hline 24 & HUGHES_2 & 0.9434 & 0.9345 \\
\hline 25 & HUGHES_l & 0.8453 & 0.8347 \\
\hline 26 & REI & 0.2451 & 0.8383 \\
\hline 27 & NYNEX & 0.8440 & 0.9360 \\
\hline 28 & GTESS_l & 0.8328 & 0.9200 \\
\hline 29 & GTESS 2 & 0.8318 & 0.8186 \\
\hline 30 & COMCOM & 0.8311 & 0.2284 \\
\hline 31 & NIST_I & 09230 & 0.9090 \\
\hline 32 & GMD_3 & 0.8184 & 0.9055 \\
\hline 33 & MIME & 0.8128 & 0.9006 \\
\hline 34 & GMD_1 & 0.9118 & 0.8890 \\
\hline 35 & ASOL & 09112 & 0.8883 \\
\hline 36 & NIST 2 & 0.9083 & 0.8958 \\
\hline 37 & UPENN & 0.8080 & 0.8853 \\
\hline 38 & NIST 3 & 0.9040 & 0.8909 \\
\hline 38 & GMD_4 & 0.8870 & 0.8846 \\
\hline 40 & RISO & 0.8955 & 0.8820 \\
\hline 41 & KAMAN-1 & 0.8876 & 0.8739 \\
\hline 42 & KAMAN_3 & 0.8720 & 0.8878 \\
\hline 43 & KAMAN_2 & 0.8686 & 0.8534 \\
\hline 44 & KAMAN_5 & 0.8498 & 0.8368 \\
\hline 45 & GMD_2 & 0.8479 & 0.8334 \\
\hline 46 & VALEN 2 & 0.8384 & 0.8293 \\
\hline 47 & IFAX & 0.8281 & 0.8172 \\
\hline 48 & VALEN_I & 0.8201 & 0.8079 \\
\hline 49 & KAMAN_4 & 0.7985 & 0.7831 \\
\hline
\end{tabular}

Table 35: ELSAGB_2 correlation graph key for digits. 


\section{No Data Available}

Figure 54: ELSAGB 2 - upper case correlation

There was no dats for this evaluation.

Table 36: ELSAGB_2 correlation graph key for uppers. 


\section{No Data Available}

Figure 55: ELSAGB 2 - lower case correlation

There was no dats for this evaluation

Table 37: ELSAGB_2 correlation graph key for lowers. 
SYSTEM: ELSAGB_3

PARTICIPANT: Mr. Francesco Fignoni

ORGANIZATION: ELSAG BAILEY, INC., Conshohocken, PA

PREPROCESSING: noise removal and size normalization to $24 \times 36$.

FEATURES: shape function of the character bit maps having the same size as the character.

CLASSIFICATION: KNN with respect to shape function distance from references representing clusters of shape functions in training sample (the classifier used with ELSAGB_1) for preclassification followed by the same classifier using a more sophisticated distance measure and many more references

HARDWARE: $33 \mathrm{MHz} 386$ running tight assembly for preclassification VAX 6000/410 under VMS running FORTRAN for classification

TRAINING: DIGITS UPPERS LOHERS DATABASE

85491 NA NA $\quad$ NSDB3

STATUS: on time, lost at NIST until after Conference

RESULTS: -- DIGITS -- -- UPPERS -- -- LOWERS -- DATABASE

REJ. ERR. REJ. ERR. REJ. ERR. TESTDATA1

RATE RATE-- RATE RATE-- RATE RATE--

$0.00 \quad 0.0335$

$0.04 \quad 0.0180$

$0.07 \quad 0.0102$

OCR RATE (CPS): DIGITS

UPPERS

LOWERS

SYS RATE:

CPU RATE: $\quad 0.30 \quad$ NA NA

NOTE: This is a laboratory research system. Few details of the recognition algorithm were provided. 
SYSTEM: ELSAGB_3

\section{BIBLIOGRAPHY:}

The following references have been provided for this system:

none 


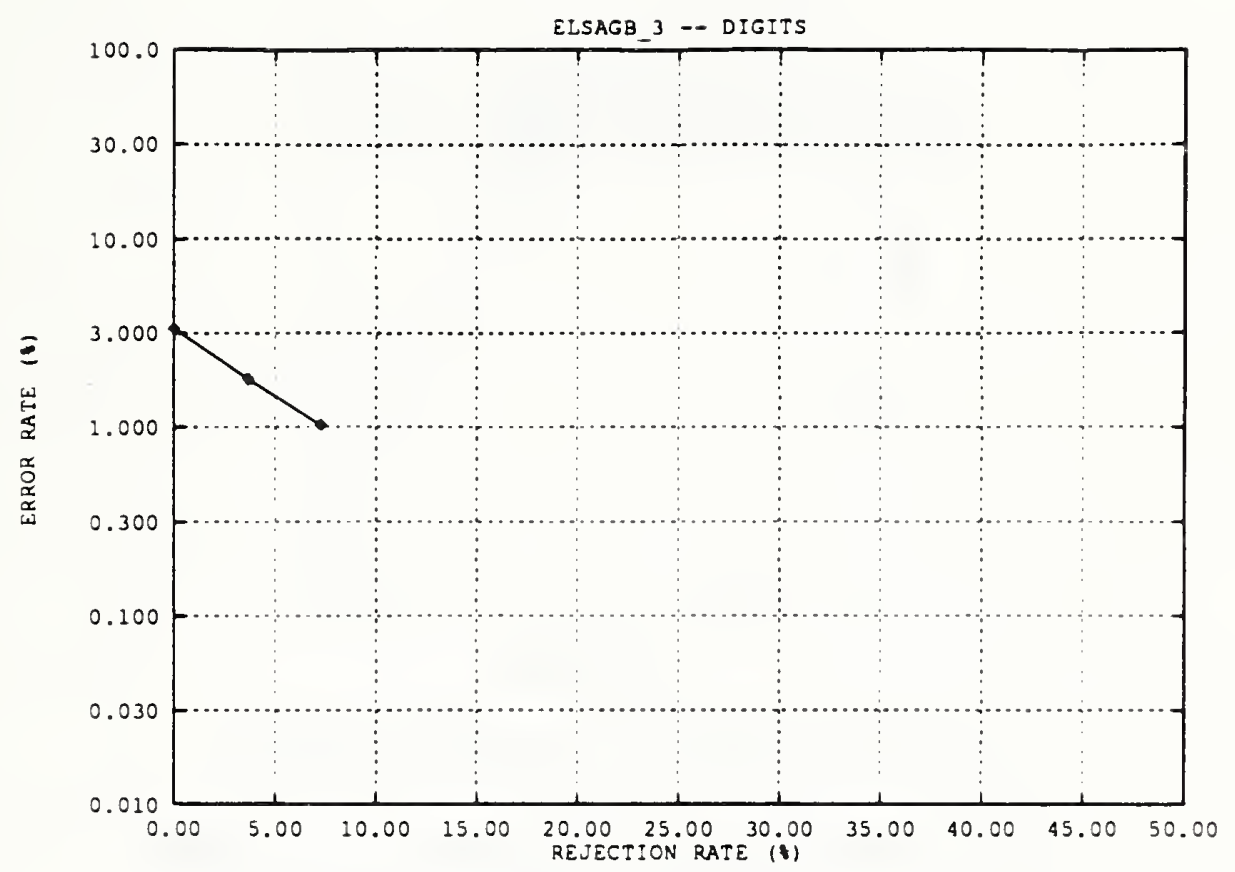

Figure 56: Error rate versus rejection rate for ELSAGB_3

ELSAOB_J

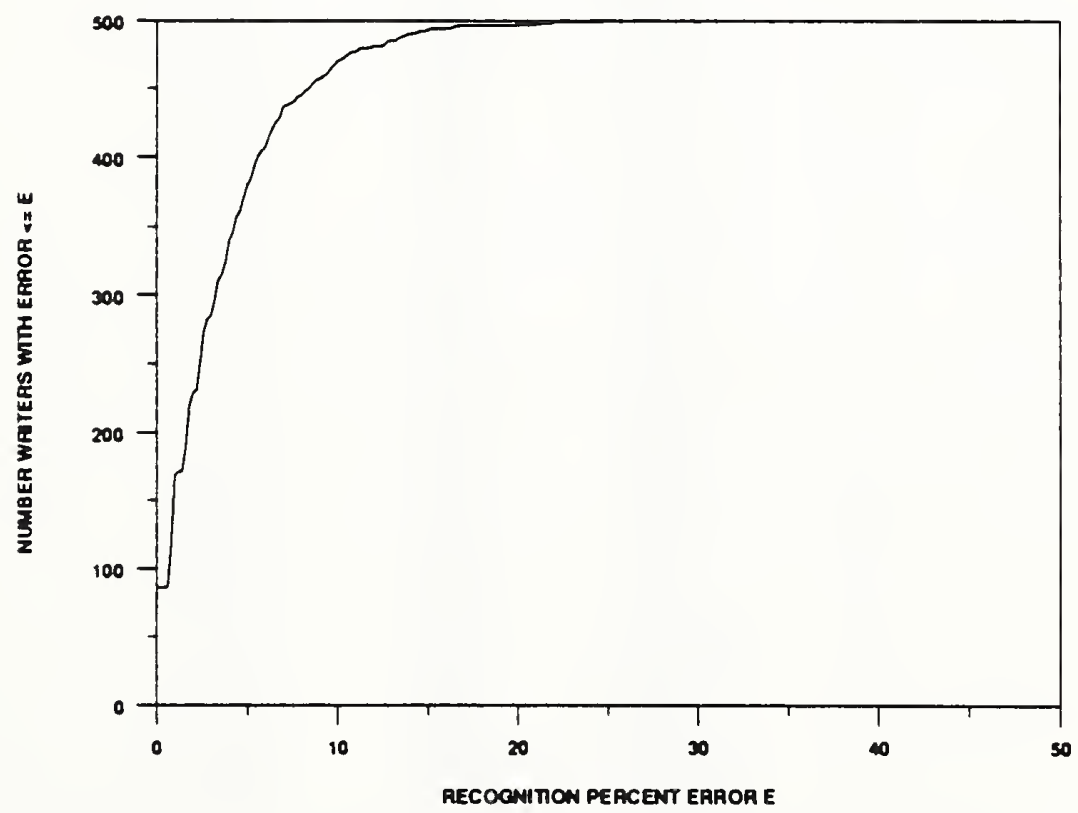

Figure 57: Error rate per writer of ELSAGB_3 


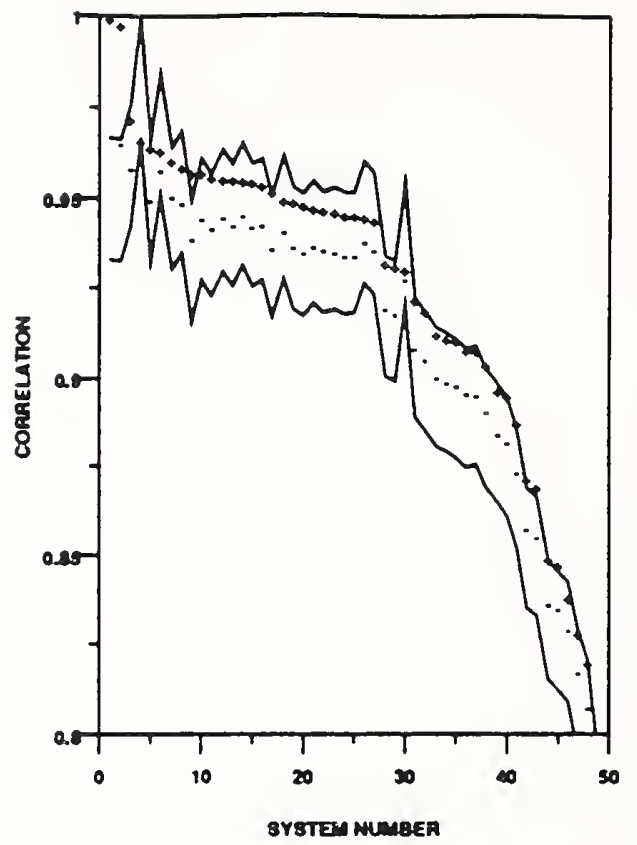

Figure 58: ELSAGB_3 - digit correlation

\begin{tabular}{|c|c|c|c|}
\hline System Number & System Name & Correlasion (all) & Correlation (correcs) \\
\hline 1 & ELSAGBA & 1.0000 & 10000 \\
\hline 2 & ELSAGB 2 & 0.9083 & 09855 \\
\hline $\mathbf{3}$ & VOTE_M & 0.9726 & 0.9587 \\
\hline 4 & REFERENCE & 09685 & 09865 \\
\hline 3 & $A E G$ & 0.9645 & 0.9502 \\
\hline 6 & OCRSYS & 09638 & 0.9583 \\
\hline 7 & VOTEP & 09811 & 09510 \\
\hline 8 & ATT」 & 0.9594 & 0.9491 \\
\hline 9 & ERIM-1 & 0.8578 & 09431 \\
\hline 10 & ELSAGB-1 & 09578 & 0.9393 \\
\hline 11 & UBOL & 09586 & 0.9420 \\
\hline 12 & ATT 2 & 09562 & 09434 \\
\hline 13 & AT T -4 & 09538 & 0.9432 \\
\hline 14 & IBM & 0.9586 & 0.9460 \\
\hline 13 & KODAK 2 & 0.9550 & 0.9427 \\
\hline 16 & ERIM 2 & 09543 & 09433 \\
\hline 17 & NIST -4 & 09622 & 0.9369 \\
\hline 18 & THINK_2 & 09502 & 0.9413 \\
\hline 19 & KODAK」 & 09497 & 0.9371 \\
\hline 20 & THINK_l & 09486 & 0.9356 \\
\hline 21 & NESTOR & 09477 & 09373 \\
\hline 22 & ATT 3 & 09472 & 0.9362 \\
\hline 23 & SYMBUS & 0.9467 & 0.9336 \\
\hline 24 & HUGHES -2 & 0.9439 & 0.9349 \\
\hline 25 & HUGHES_l & 0.9457 & 0.9380 \\
\hline 26 & REI & 0.9453 & 0.9387 \\
\hline 27 & NYNEX & 0.9443 & 0.9363 \\
\hline 28 & GTESS_L & 09330 & 09203 \\
\hline 29 & GTESS 2 & 0.9322 & 0.9189 \\
\hline 30 & COMCOM & 0.9313 & 0.9286 \\
\hline 31 & NIST_I & 0.9231 & 0.9092 \\
\hline 32 & GMD_3 & 0.9198 & 0.9059 \\
\hline 33 & MIME & 09132 & 0.9010 \\
\hline 34 & GMD_I & 0.9120 & 08993 \\
\hline 33 & ASOL & 09114 & 0.8985 \\
\hline 36 & NIST 2 & 09087 & 0.8962 \\
\hline 37 & UPENN & 09086 & 0.8958 \\
\hline 38 & נה & 0.9044 & 0.8913 \\
\hline 39 & GMD_4 & 0.8972 & 0.8849 \\
\hline 40 & RISO & 08958 & 0.8823 \\
\hline 41 & KAMAN_l & 0.8879 & 0.8742 \\
\hline 12 & KAMAN_3 & 0.8723 & 0.8582 \\
\hline 43 & KAMAN.2 & 0.8699 & 0.8557 \\
\hline 14 & KAMAN_S & 0.8501 & 0.8371 \\
\hline 15 & GMD_2 & 0.8482 & 0.8336 \\
\hline 46 & VALEN_2 & 0.8387 & 0.8296 \\
\hline 47 & IFAX & 0.8287 & 08177 \\
\hline 48 & VALEN_L & 0.8204 & 0.8082 \\
\hline 19 & KAMAN-1 & 07968 & 0.7834 \\
\hline
\end{tabular}

Table 38: ELSAGB_3 correlation graph key for digits. 


\section{No Data Available}

Figure 59: ELSAGB_3 - upper case correlation

There was no data for this evaluation.

Table 39: ELSAGB_3 correlation graph key for uppers. 


\section{No Data Available}

Figure 60: ELSAGB_3 - lower case correlation

There was no data for this evaluation

Table 40: ELSAGB_3 correlation graph key for lowers. 
SYSTEM: ERIM_1

PARTICIPANT: Steven Schlosser

ORGANIZATION: Environmental Research Institute of Michigan (ERIM) Ann Arbor, Michigan

PREPROCESSING: filtering and size normalization

FEATURES: stroke detection, morphological

feature extraction.

CLASSIFICATION: four layer NN with BP. For digits, 245 input units, and two hidden layers with 25 and 15 hidden units, 10 output units. For characters, 120 input units, and two hidden layers with 65 and 39 hidden units, 26 output units.

HARDHARE: SUN -4

TRAINING: DIGITS UPPERS LOHERS DATABASE

$61000 \quad 40300 \quad 36400 \quad$ NSDB3

STATUS: $\quad$ on time, submitted as ERIM_O

RESULTS: -- DIGITS -- -- UPPERS -- -- LOHERS -- DATABASE

REJ. ERR. REJ. ERR. REJ. ERR. TESTDATA1

RATE RATE-- RATE RATE-- RATE RATE--

$\begin{array}{lllllll}0.00 & 0.0388 & 0.00 & 0.0518 & 0.00 & 0.1379\end{array}$

$\begin{array}{lllllll}0.10 & 0.0082 & 0.10 & 0.0180 & 0.10 & 0.0897\end{array}$

$\begin{array}{lllllll}0.20 & 0.0025 & 0.20 & 0.0072 & 0.20 & 0.0554\end{array}$

$\begin{array}{lllllll}0.30 & 0.0012 & 0.30 & 0.0041 & 0.30 & 0.0368\end{array}$

$\begin{array}{lllllll}0.40 & 0.0009 & 0.40 & 0.0024 & 0.40 & 0.0214\end{array}$

$\begin{array}{lllllll}0.50 & 0.0007 & 0.50 & 0.0020 & 0.50 & 0.0118\end{array}$

OCR RATE (CPS): DIGITS

UPPERS

LOHERS

SYS RATE:

0.24

0.24

0.24

CPU RATE:

0.91

0.91

0.91 
SYSTEM: ERIM_1

BIBLIOGRAPHY:

The following references have been provided for this system:

$[11][12][13][14]$ 


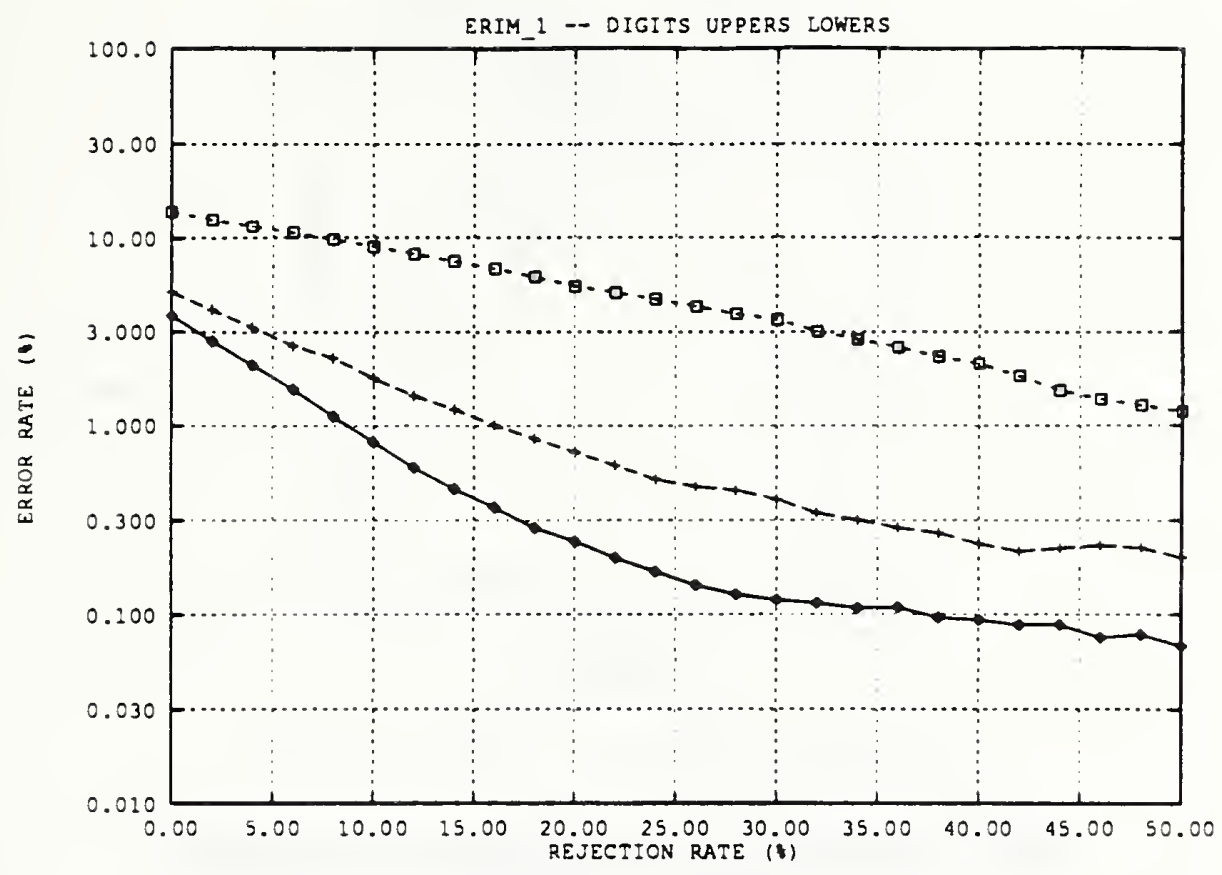

Figure 61: Error rate versus rejection rate for ERIM_1

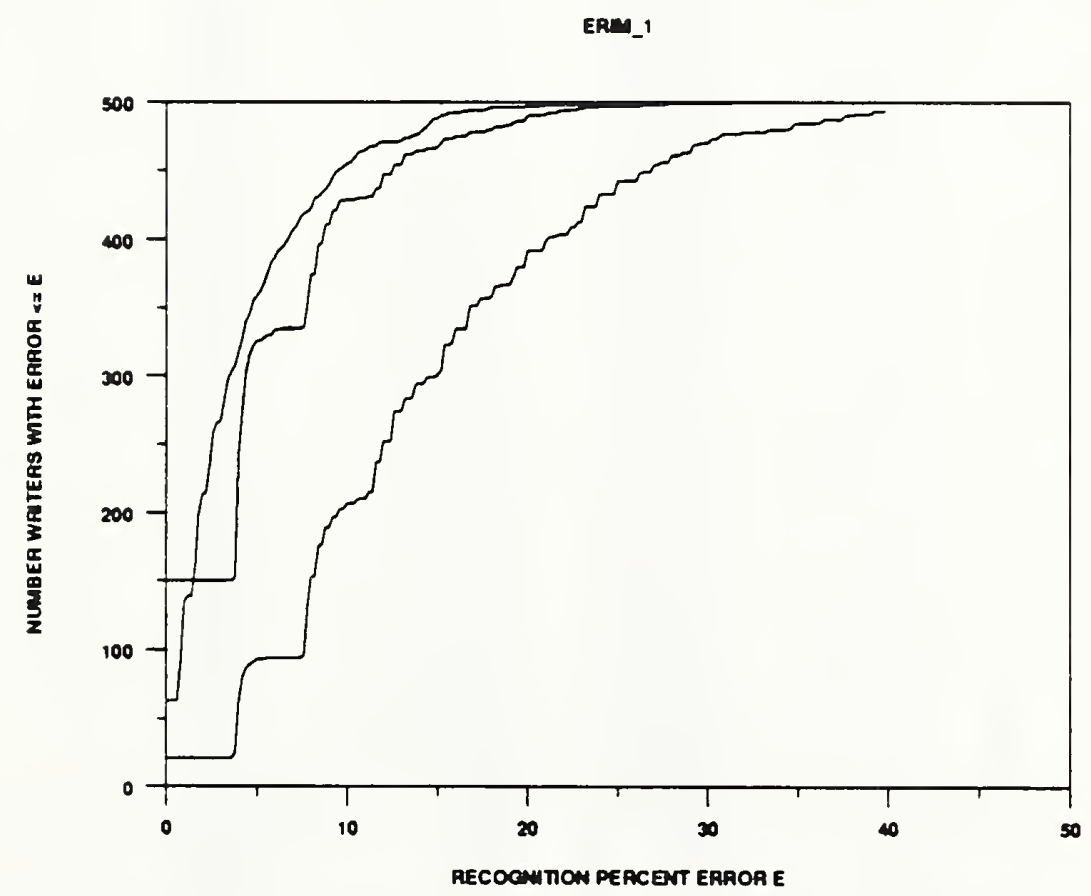

Figure 62: Error rate per writer of ERIM_1 


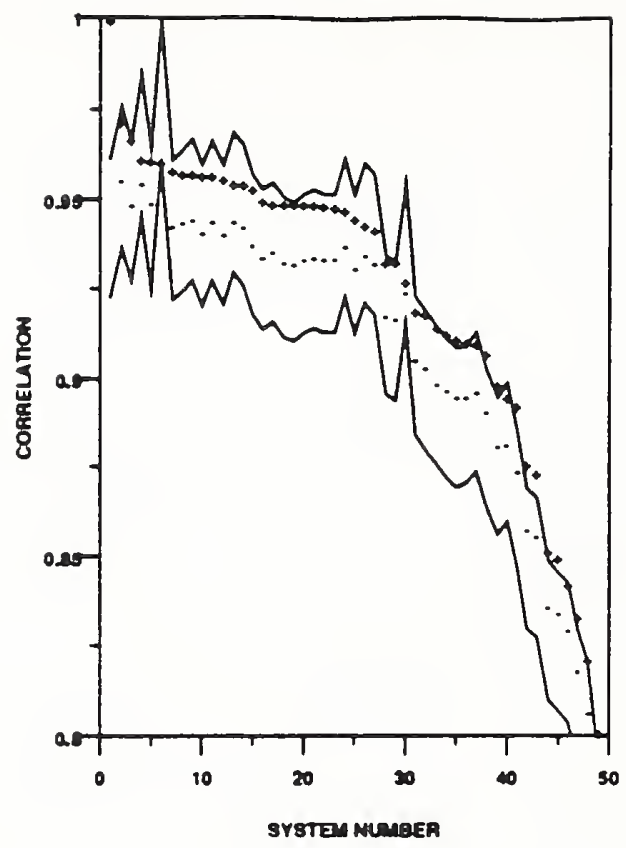

Figure 63: ERIM_1 - digit correlation

\begin{tabular}{|c|c|c|c|}
\hline System Number & Sysiem Name & Cofrelabion (all) & Correistion (correct) \\
\hline$T$ & ERIM-I & 1.0000 & 1.0000 \\
\hline 2 & VOTE_M & 0.8725 & 0.9561 \\
\hline 3 & $A E G$ & 09674 & 0.9492 \\
\hline 4 & OCRSYS & 09619 & 09550 \\
\hline 5 & VOTE_P & 0.8613 & 0.9494 \\
\hline 6 & REFERENCE & 0.8612 & 09612 \\
\hline 7 & ERIM 2 & 0.8587 & 0.2433 \\
\hline 8 & A TT 2 & 0.8580 & 0.9440 \\
\hline 8 & ELSAGBA & 08578 & 0.9451 \\
\hline 10 & A TT -4 & 0.9574 & 0.9415 \\
\hline 11 & ELSAGB 2 & 0.9572 & 09447 \\
\hline 12 & KODAK 2 & 0.8566 & 09410 \\
\hline 13 & AT T $\perp$ & 0.9553 & 0.9446 \\
\hline 14 & IBM & 08549 & 0.9432 \\
\hline 13 & UBOL & 0.8536 & 0.9382 \\
\hline 16 & KODAK」 & 0.8504 & 0.9348 \\
\hline 17 & NESTOR & 0.9497 & 0.9361 \\
\hline 18 & NIST_4 & 0.8486 & 0.9334 \\
\hline 18 & HUGHES - 1 & 0.8485 & 09345 \\
\hline 20 & ELSAGB_1 & 0.8485 & 09331 \\
\hline 21 & SYMBUS & 0.9494 & 0.9350 \\
\hline 22 & HUGHES.2 & 0.9481 & 0.3344 \\
\hline 23 & ATT 3 & 0.8485 & 0.9345 \\
\hline 24 & THINK 2 & 0.8477 & 0.9376 \\
\hline 25 & THINK_-1 & 0.8453 & 0.9316 \\
\hline 26 & REI & 0.8436 & 09355 \\
\hline 27 & NYNEX & 0.8421 & 0.9331 \\
\hline 28 & GTESS 1 & 09334 & 0.9182 \\
\hline 29 & GTESS 2 & 0.9333 & 0.9175 \\
\hline 30 & СОМСОМ & 0.9279 & 0.3249 \\
\hline 31 & NIST -1 & 0.8189 & 0.3057 \\
\hline 32 & GMD_3 & 0.8186 & 0.9036 \\
\hline 33 & MIME & 09150 & 08992 \\
\hline 34 & ASOL & 0.8134 & 0.8972 \\
\hline 35 & NIST 2 & 0.9117 & 0.8954 \\
\hline 36 & UPENN & 0.8110 & 0.8952 \\
\hline 37 & GMD -1 & 0.2108 & 08968 \\
\hline 38 & NIST & 0.9078 & 0.8910 \\
\hline 39 & RISO & 0.8890 & 0.8818 \\
\hline 40 & GMD_4 & 0.8952 & 0.8821 \\
\hline 41 & KAMAN-1 & 0.8830 & 0.8746 \\
\hline 42 & $K A M A N=3$ & 0.8764 & 0.8583 \\
\hline 43 & KAMAN_2 & 08742 & 0.8561 \\
\hline 44 & KAMAN_S & 0.8521 & 0.8367 \\
\hline 43 & GMD -2 & 0.8504 & 0.8347 \\
\hline 46 & VALEN 2 & 0.8428 & 0.8301 \\
\hline 47 & IFAX & 0.8340 & 0.8185 \\
\hline 48 & VALEN_I & 0.8217 & 0.8073 \\
\hline 49 & KAMAN_4 & 0.8016 & 0.7844 \\
\hline
\end{tabular}

Table 41: ERIM_1 correlation graph key for digits. 


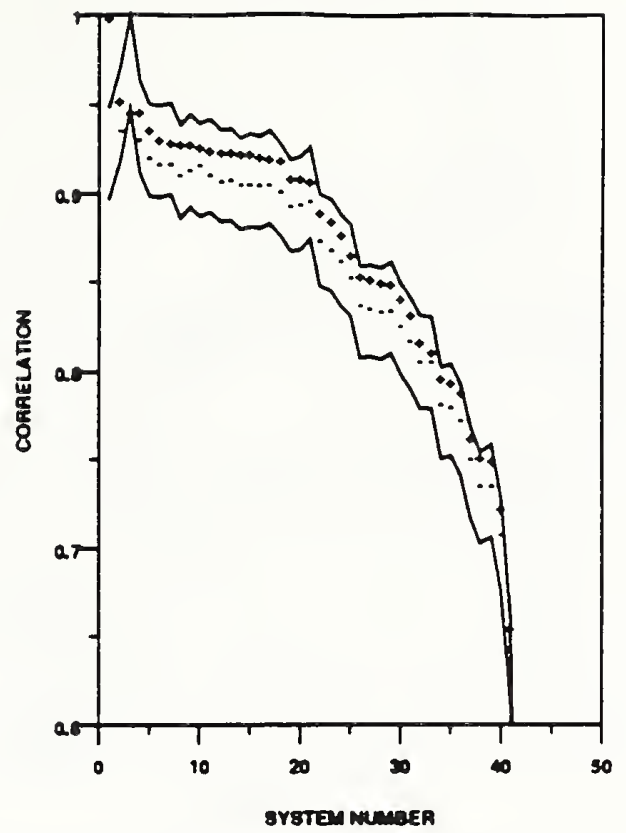

Figure 64: ERIM_1 - upper case correlation

\begin{tabular}{|c|c|c|c|}
\hline System Number & Syblem Name & Correlation (ali) & Correlasion (correct) \\
\hline 1 & ERIM-1 & 1.0000 & 1.0000 \\
\hline 2 & VOTE_M & 0.9839 & 09379 \\
\hline 3 & REFERENCE & 0.9482 & 0.9482 \\
\hline 4 & $A E G$ & 0.9477 & 0.8323 \\
\hline 5 & ATT_4 & 0.9378 & 02219 \\
\hline 6 & UMICH.I & 0.9323 & 0.9181 \\
\hline 7 & NYNEX & 09305 & 0.9182 \\
\hline 8 & ATT 2 & 0.9290 & 0.2143 \\
\hline 9 & UBOL & 0.9290 & 0.9116 \\
\hline 10 & VOTEP & 0.9278 & 0.9173 \\
\hline 11 & NESTOR & 0.9255 & 0.9117 \\
\hline 12 & HUGHES -1 & 0.9252 & $0.908 \mathrm{~J}$ \\
\hline 13 & IBM & 0.9245 & 0.9090 \\
\hline 14 & KODAK」 & 0.9237 & 0.2066 \\
\hline 18 & HUGHES -2 & 0.9237 & 0.9063 \\
\hline 16 & ATT 3 & 0.8223 & 0.9064 \\
\hline 17 & ATT $\perp$ & 0.9215 & 0.9066 \\
\hline 18 & SYMBUS & 0.9200 & 0.9029 \\
\hline 19 & GTESS_l & 0.9101 & 0.8950 \\
\hline 20 & GTESS_2 & 0.2101 & 0.8942 \\
\hline 21 & OCRSYS & 0.9080 & 0.8972 \\
\hline 22 & MIME & 0.8911 & 0.8750 \\
\hline 23 & NIST_A & 0.8865 & 0.8705 \\
\hline 24 & ASOL & 0.8789 & 0.8639 \\
\hline 25 & REI & 0.8673 & 08552 \\
\hline 26 & RISO & 0.8562 & 08390 \\
\hline 27 & GMD_l & 0.8338 & 0.8375 \\
\hline 28 & GMD_3 & 0.8519 & 08358 \\
\hline 29 & NIST_1 & 0.8516 & 0.8371 \\
\hline so & KAMAN_L & 0.8434 & 0.8279 \\
\hline 31 & GMD_A & 08341 & 08192 \\
\hline 32 & נב_TIST & 0.8179 & 0.8072 \\
\hline 33 & СОМСОM & 0.8132 & 0.8071 \\
\hline 34 & KAMAN_J & 0.7983 & 0.7833 \\
\hline 35 & IFAX & 07949 & 0.7814 \\
\hline 36 & KAMAN_2 & 0.7901 & 0.7740 \\
\hline 37 & NIST 2 & 0.7640 & 07519 \\
\hline 38 & GMD_2 & 0.7829 & 0.7377 \\
\hline 39 & VALEN 1 & 0.7513 & 0.7372 \\
\hline 40 & KAMAN_4 & 0.7248 & 0.7097 \\
\hline 41 & KAMAN_S & 0.6866 & 0.6441 \\
\hline 42 & $\mathrm{UMICH}_{2} 2$ & 0.0390 & 00219 \\
\hline
\end{tabular}

Table 42: ERIM_1 correlation graph key for uppers. 


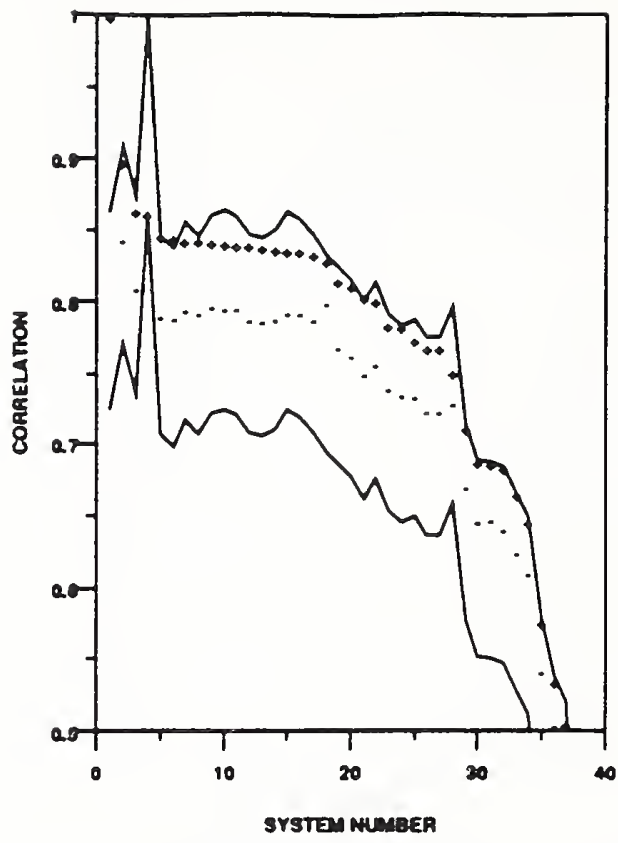

Figure 65: ERIM_1 - lower case correlation

\begin{tabular}{|c|c|c|c|}
\hline System Number & System Name & Correlation (all) & Correlasion (correct) \\
\hline 1 & ERIM_1 & 1.0000 & 1.0000 \\
\hline 2 & VOTE_M & 0.8993 & 0.8441 \\
\hline 3 & AEG & 0.8644 & 0.8112 \\
\hline 4 & REFERENCE & 0.8621 & 0.8621 \\
\hline$s$ & UBOL & 0.8471 & 0.7914 \\
\hline 6 & ATT 3 & 0.8464 & 0.7899 \\
\hline 7 & KODAK」 & 0.8445 & 0.7961 \\
\hline 8 & IBM & 08443 & 0.7932 \\
\hline 9 & NYNEX & 0.8429 & 0.7985 \\
\hline 10 & OCRSYS & 0.8417 & 0.7969 \\
\hline 11 & ATT 2 & 0.8405 & 0.7965 \\
\hline 12 & HUGHES - 1 & 0.8404 & 0.7893 \\
\hline 13 & HUGHES 2 & 0.8398 & 0.7882 \\
\hline 14 & UMICH_L & 0.8378 & 0.7883 \\
\hline 15 & ATT」 & 0.8375 & 0.7938 \\
\hline 16 & ATT $A$ & 0.8374 & 0.7934 \\
\hline 17 & NESTOR & 0.8381 & 0.7889 \\
\hline 18 & VOTEP & 0.8308 & 0.7989 \\
\hline 18 & GTESS -1 & 0.8167 & 0.7691 \\
\hline 20 & GTESS 2 & 0.8125 & 0.7641 \\
\hline 21 & NIST -4 & 0.8052 & 0.7508 \\
\hline 22 & NIST_I & 0.8030 & 0.7584 \\
\hline 23 & GMD_3 & 0.7855 & 0.7403 \\
\hline 24 & RISO & 0.7838 & 0.7358 \\
\hline 25 & ASOL & 0.7757 & 0.7345 \\
\hline 26 & GMD_4 & 0.7693 & 07247 \\
\hline 27 & GMD_1 & 07693 & 0.7247 \\
\hline 28 & $\mathrm{NIST}_{3}$ & 0.7521 & 0.7307 \\
\hline 29 & GMD 2 & 0.7135 & 06723 \\
\hline 30 & KAMAN.1 & 0.6892 & 0.6481 \\
\hline 31 & NIST 2 & 0.6877 & 0.6483 \\
\hline 32 & VALEN_1 & 0.6859 & 0.6423 \\
\hline 33 & KAMAN_3 & 0.6668 & 06262 \\
\hline 34 & $K_{A M A N}-2$ & 0.8482 & 06113 \\
\hline 35 & KAMAN_5 & 0.5763 & 0.5423 \\
\hline 36 & KAMAN_4 & 0.5356 & 0.5058 \\
\hline 37 & COMCOM & 0.5061 & 0.4923 \\
\hline 38 & $\mathrm{UMICH}_{2}$ & 0.0910 & 0.0539 \\
\hline
\end{tabular}

Table 43: ERIM_1 correlation graph key for lowers. 


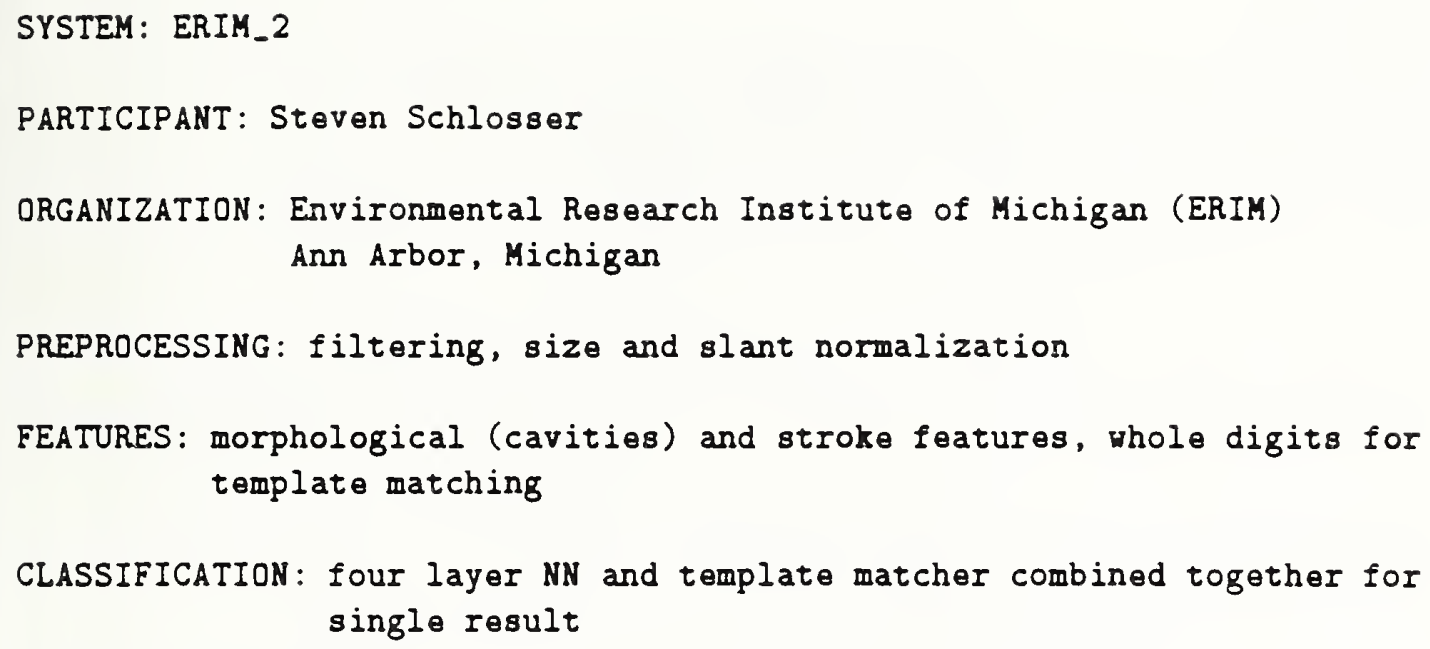

OCR RATE (CPS): DIGITS

UPPERS LOHERS

SYS RATE:

10.0

NA

NA

CPU RATE: 
SYSTEM: ERIM_2

BIBLIOGRAPHY:

The following references have been provided for this system:

[11][12][13][14] 


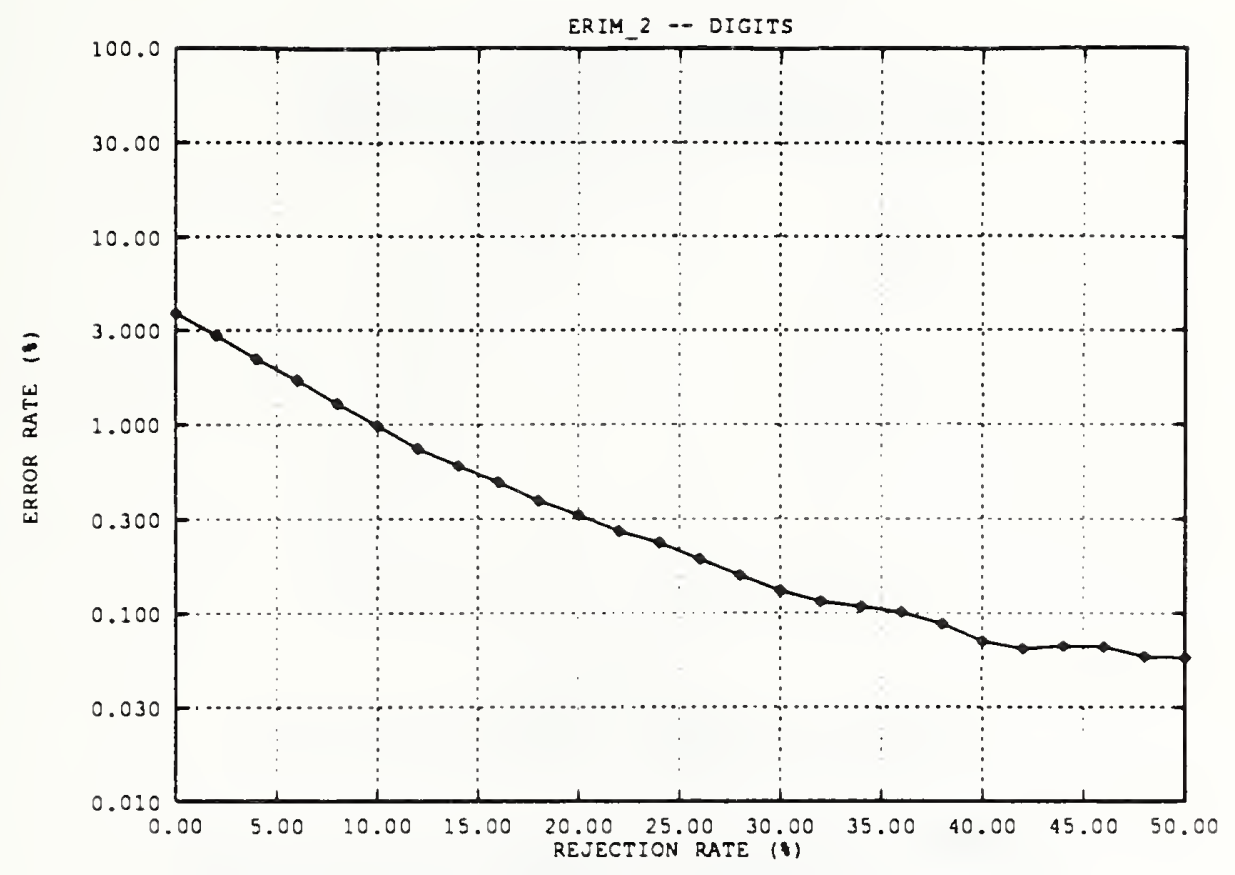

Figure 66: Error rate versus rejection rate for ERIM_2

ERI-2

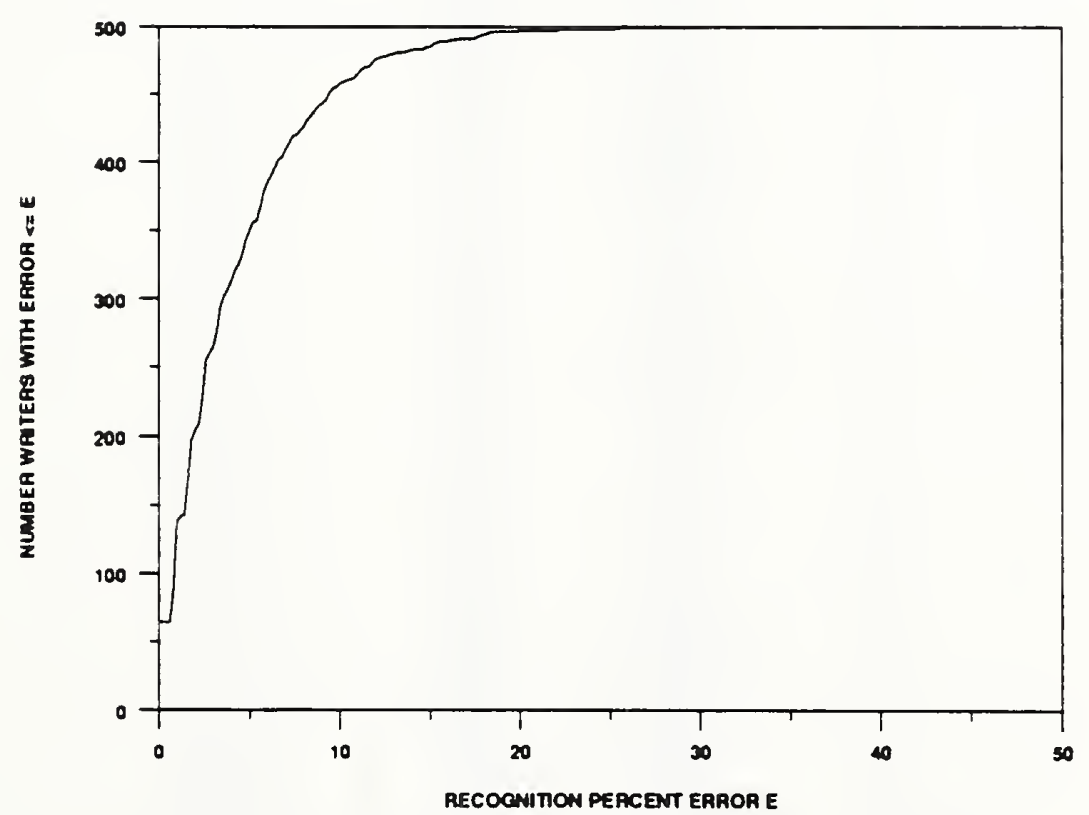

Figure 67: Error rate per writer of ERIM_2 


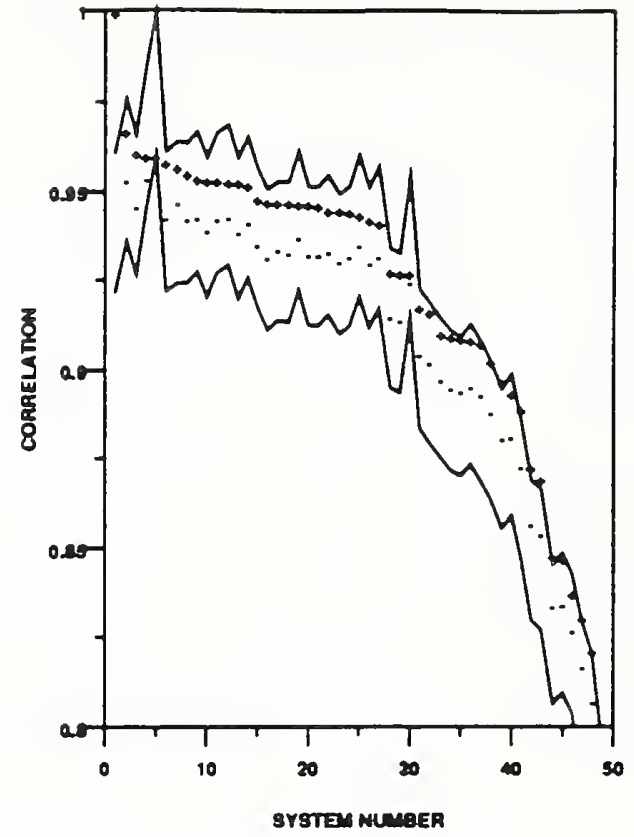

Figure 68: ERIM_2 - digit correlation

\begin{tabular}{|c|c|c|c|}
\hline System Number & System Nome & Correlstion (sll) & Correlstion (correct) \\
\hline 1 & हRIM 2 & 1.0000 & 1.0000 \\
\hline 2 & VOTE_M & 02677 & 0.9537 \\
\hline 3 & $A E G$ & 0.2616 & 0.9463 \\
\hline 4 & REFERENCE & 09608 & 0.2608 \\
\hline 3 & OCRSYS & 0.9608 & 09342 \\
\hline 6 & ERIM-1 & 0.2587 & 0.9433 \\
\hline 7 & VOTE $P$ & 09576 & 09472 \\
\hline 8 & ATT 2 & 0.9537 & 0.9428 \\
\hline 2 & ELSAGB 3 & 0.2543 & 0.2433 \\
\hline 10 & KODAK 2 & 02539 & 0.9396 \\
\hline 11 & ELSAGB 2 & 09335 & 0.9428 \\
\hline 12 & ATT $\perp$ & 0.9534 & 0.9433 \\
\hline 13 & A T T -4 & 09531 & 0.8321 \\
\hline 14 & IBM & 0.2523 & 0.2417 \\
\hline 15 & UBOL & 0.9487 & 0.8359 \\
\hline 16 & SYMBUS & 09479 & 0.9345 \\
\hline 17 & NIS T _4 & 09479 & 0.9323 \\
\hline 18 & KODAK」 & 0.9476 & 0.9335 \\
\hline 19 & THINK 2 & 02474 & 0.2373 \\
\hline 20 & HUGHES - 1 & 02473 & 0.9329 \\
\hline 21 & HUGHES_2 & 0.9469 & 0.2328 \\
\hline 22 & NESTOR & 02453 & 0.9339 \\
\hline 23 & ELSAGB-L & 09434 & 0.9312 \\
\hline 24 & ATT 3 & 0.2430 & 0.9326 \\
\hline 25 & REI & 0.2439 & 0.9357 \\
\hline 26 & THINK_I & 09429 & 0.9306 \\
\hline 27 & NYNEX & 09417 & 0.9325 \\
\hline 28 & GTESS - 1 & 0.9283 & 0.9135 \\
\hline 29 & GTESS 2 & 0.9280 & 0.2147 \\
\hline 30 & COMCOM & 0.9278 & 0.2250 \\
\hline 31 & $\mathrm{NIST}_{-1}$ & 0.9182 & 0.9048 \\
\hline 32 & GMD_3 & 0.2170 & 0.2026 \\
\hline 33 & MIME & 0.9111 & 0.8276 \\
\hline 34 & ASOL & 0.2100 & 0.8954 \\
\hline 35 & UPENN & 0.2094 & 0.8945 \\
\hline 36 & GMD - 1 & 0.9093 & 0.8960 \\
\hline 37 & NIST 2 & 0.2080 & 0.8936 \\
\hline 38 & NIST 3 & 0.8031 & 0.8883 \\
\hline 39 & RISO & 0.8974 & 0.8812 \\
\hline 40 & GMD_- 1 & 0.8942 & 0.8813 \\
\hline 41 & $K A M A N_{-1}$ & 0.8896 & 0.8735 \\
\hline 42 & KAMAN_J & 0.8733 & 0.8572 \\
\hline 43 & KAMAN.2 & 0.8701 & 0.8545 \\
\hline 44 & GMD -2 & 0.8483 & 0.8341 \\
\hline 45 & KAMAN_S & 0.8482 & 0.8349 \\
\hline 46 & VALEN 2 & 0.8379 & 0.8273 \\
\hline 47 & IFAX & 0.8312 & 0.8173 \\
\hline 48 & VALEN-1 & 0.8218 & 0.8077 \\
\hline 49 & KAMAN_A & 07976 & 0.7823 \\
\hline
\end{tabular}

Table 44: ERIM_2 correlation graph key for digits. 


\section{No Data Available}

Figure 69: ERIM_2 - upper case correlation

There was no data for this evaluation

Table 45: ERIM 2 correlation graph key for uppers. 


\section{No Data Available}

Figure 70: ERIM_2 - lower case correlation

There was no data for this evaluation.

Table 46: ERIM_2 correlation graph key for lowers. 
SYSTEM: GMD_1

PARTICIPANT: Frank Smieja

ORGANIZATION: Gesellshcaft fuer Mathematik und Datenverarbeitung (GMD), Sankt Augustin, Germany

PREPROCESSING : size normalization to $16 \times 24$

FEATURES: genetically optimized polynomial filter, 384 features extracted.

Feature optimization by PGA (parallel genetic algorithm).

CLASSIFICATION: statistical, nearest neighbor

HARDWARE: $\quad$ SPARC2

TRAINING: DIGITS UPPERS LOWERS DATABASE

$15000 \quad 22000 \quad 22000 \quad$ NSDB3

STATUS: $\quad$ on time

RESULTS: -- DIGITS -- -- UPPERS -- -- LOWERS -- DATABASE

REJ. ERR. REJ. ERR. REJ. ERR. TESTDATA 1

RATE RATE-- RATE RATE-- RATE RATE--

$\begin{array}{lllllll}0.00 & 0.0873 & 0.00 & 0.1404 & 0.00 & 0.2254\end{array}$

$\begin{array}{lllllll}0.15 & 0.0272 & 0.16 & 0.0625 & 0.31 & 0.0809\end{array}$

OCR RATE (CPS): DIGITS UPPERS LOHERS
SYS RATE:
1.18
0.48
0.40

CPU RATE: 
SYSTEM: GMD 1

BIBLIOGRAPHY:

The following references have been provided for this system:

[15][16]

COMMENTS: GMD_1,3,4

PARTICIPANT: Frank Śmieja

ORGANIZATION: Gesellschaft für Mathematik und Datenverarbeitung (GMD), Sankt Augustin, Germany.

The algorithm works in several steps.

1. Normalization of the image to $16 \times 24$ pixels.

2. From a training set, 64 features are computed by Karhunen-Loeve transformation.

3. Distance and variance of the clusters are optimized by the genetic algorithm.

Future developments:

- Reduction of the training set required to be stored.

- Employment of geometric learning. 


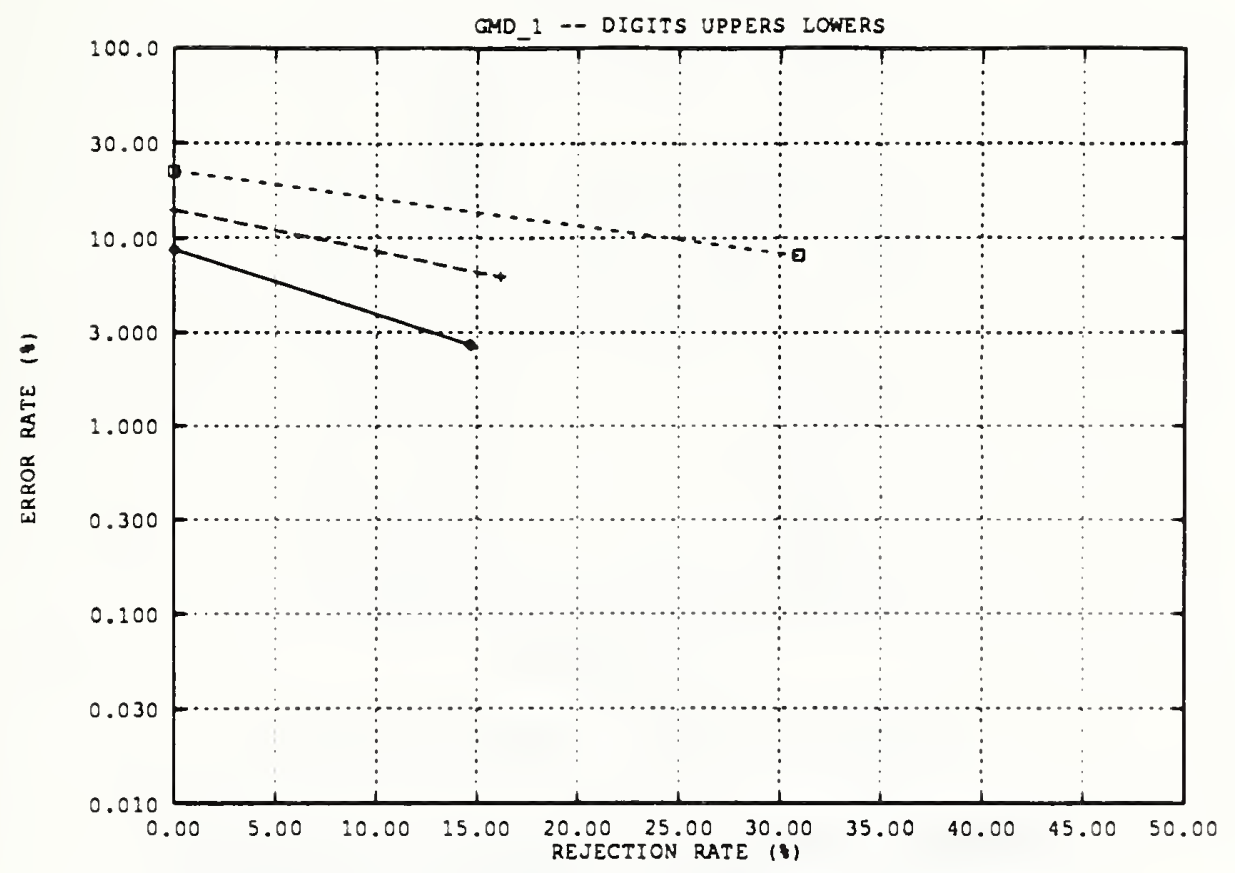

Figure 71: Error rate versus rejection rate for GMD_1

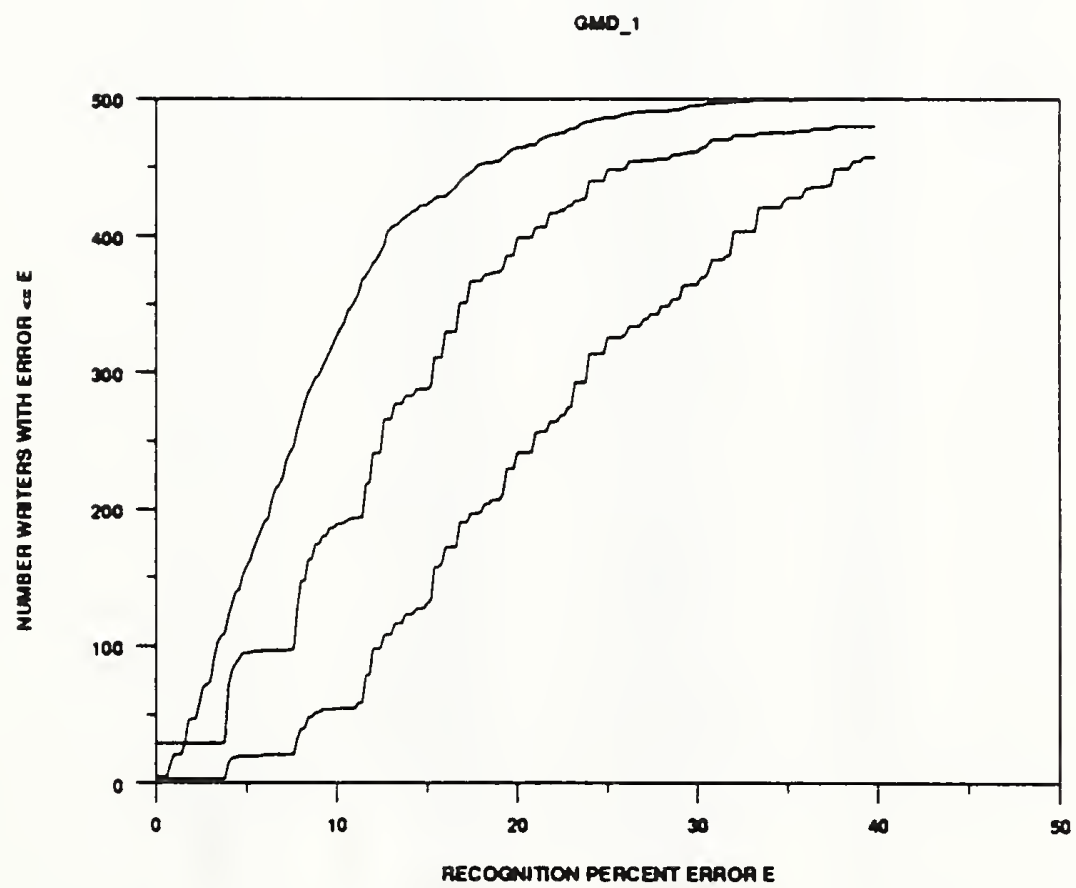

Figure 72: Error rate per writer of GMD_1 


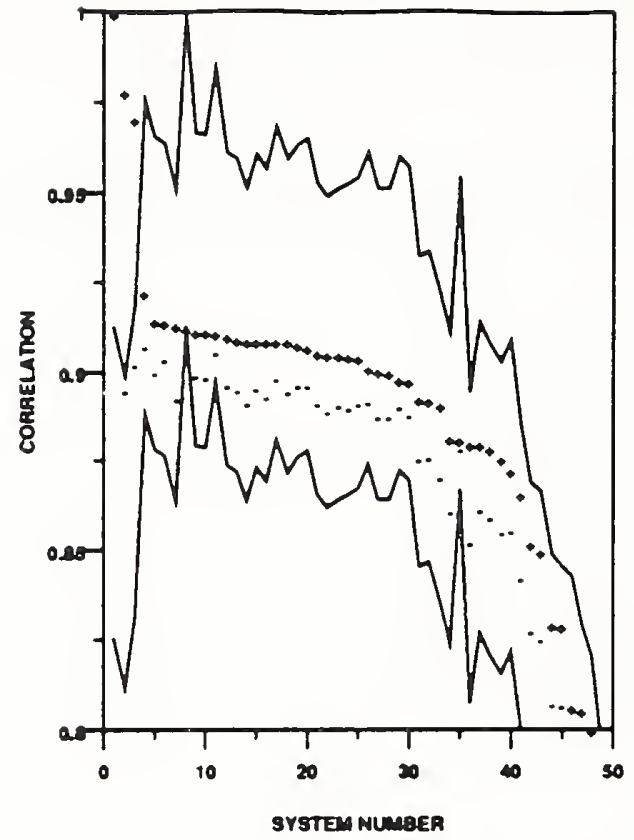

Figure 73: GMD_1 - digit correlation

\begin{tabular}{|c|c|c|c|}
\hline System Number & System Name & Correlation (all) & Correlstion (correct) \\
\hline $\mathrm{I}$ & GMD_1 & 1.0000 & 10000 \\
\hline 2 & GMD_A & 0.9786 & 0.8954 \\
\hline 3 & GMD_J & 09712 & 0.2027 \\
\hline 4 & VOTE_M & 0.9227 & 08079 \\
\hline 3 & $A E G$ & 0.9152 & 0.2003 \\
\hline 6 & VOTEP & 09146 & 09040 \\
\hline 7 & NIST_A & 0.8138 & 08932 \\
\hline $\mathbf{8}$ & REFERENCE & 09127 & 09127 \\
\hline 9 & ELSAGB 3 & 0.9120 & 0.8993 \\
\hline 10 & ELSA GB 2 & 0.9118 & 08890 \\
\hline 11 & OCRSYS & 09114 & 0.9060 \\
\hline 12 & ERIM-1 & 0.9106 & 0.8968 \\
\hline 13 & KODAK 2 & 09096 & 08933 \\
\hline 14 & ERIM_2 & 0.9093 & 08960 \\
\hline 13 & THINK_I & 09093 & 0.8916 \\
\hline 16 & ATT」 $\perp$ & 0.8091 & 0.8885 \\
\hline 17 & UBOL & 0.8081 & 08933 \\
\hline 18 & A T T -4 & 0.9090 & 0.8949 \\
\hline 18 & ATT2 2 & 09082 & 0.8967 \\
\hline 20 & IBM & 08075 & 0.8967 \\
\hline 21 & SYMBUS & 0.9038 & 0.8913 \\
\hline 22 & ATT 3 & 09056 & 0.8911 \\
\hline 23 & ELSAGB-1 & 09036 & 08894 \\
\hline 24 & KODAK」 & 0.8052 & 0.8804 \\
\hline 25 & NESTOR & 0.9044 & 0.8916 \\
\hline 26 & THINK 2 & 0.9018 & 0.8920 \\
\hline 27 & HUGHES_1 & 0.9010 & 0.8881 \\
\hline 28 & HUGHES 2 & 09004 & 0.8879 \\
\hline 29 & REI & 08984 & 0.8906 \\
\hline 30 & NYNEX & 0.8982 & 0.8883 \\
\hline 31 & $\operatorname{GTESS} 2$ & 08931 & 0.8761 \\
\hline 32 & GTESS_l & 08924 & 0.8764 \\
\hline 33 & NIST 1 & 08913 & 0.8709 \\
\hline 34 & ASOL & 08821 & 0.8613 \\
\hline 35 & COMCOM & 08818 & 0.8789 \\
\hline 36 & MIME & 08800 & 08620 \\
\hline 37 & RISO & 08800 & 0.8526 \\
\hline 38 & NIST 2 & 08786 & 08596 \\
\hline 39 & NIST & 08739 & 0.8556 \\
\hline 40 & UPENN & 0.8727 & 0.8558 \\
\hline 41 & $K_{A M A N}-1$ & 0.8639 & 0.8425 \\
\hline 42 & KAMAN_3 & 0.8520 & 0.8280 \\
\hline 43 & KAMAN_2 & 08498 & 0.8257 \\
\hline 44 & KAMAN_5 & 0.8297 & 0.8074 \\
\hline 45 & GMD.2 & 0.8291 & 08072 \\
\hline 16 & VALEN_? & 08069 & 0.7934 \\
\hline 17 & IFAX & 0.8039 & 0.7863 \\
\hline 48 & VALEN_-1 & 0.8002 & 0.7782 \\
\hline 49 & KAMAN-1 & 07832 & 07585 \\
\hline
\end{tabular}

Table 47: GMD_1 correlation graph key for digits. 


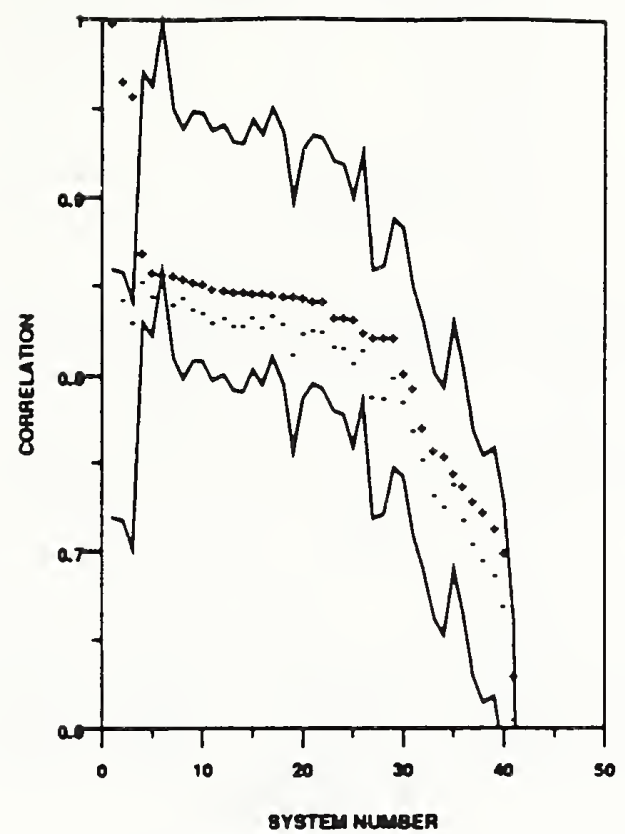

Figure 74: GMD_1 - upper case correlation

\begin{tabular}{|c|c|c|c|}
\hline System Numbet & System Name & Correlation (ali) & Correlasion (correct) \\
\hline 1 & GMD.1 & 1.0000 & 1.0000 \\
\hline 2 & GMD_3 & 0.9679 & 0.8446 \\
\hline 3 & GMD_4 & 0.9595 & 0.8318 \\
\hline 4 & VOTE_M & 0.8715 & 0.8549 \\
\hline 3 & $A E G$ & 0.8601 & 0.8467 \\
\hline 6 & REFERENCE & 0.8396 & 0.8596 \\
\hline 7 & ATT_A & 0.8587 & 0.8421 \\
\hline 8 & VOTE_P & 0.8570 & 0.8461 \\
\hline 9 & UMICH-1 & 0.8551 & 0.8390 \\
\hline 10 & ERIM-1 & 0.8538 & 0.8375 \\
\hline 11 & UBOL & 0.8510 & 0.8324 \\
\hline 12 & NESTOR & 0.8508 & 0.8350 \\
\hline 13 & ATT 3 & 0.8493 & 0.8305 \\
\hline 14 & KODAK_ & 08491 & 0.8304 \\
\hline is & $\mathrm{ATT}_{2}$ & 08488 & 0.8349 \\
\hline 16 & ATTد & 0.8485 & 0.8295 \\
\hline 17 & NYNEX & 08478 & 0.8359 \\
\hline 18 & IBM & 0.8470 & 0.8308 \\
\hline 19 & NIST _4 & 08464 & 0.8135 \\
\hline 20 & SYMBUS & 0.8460 & 0.8253 \\
\hline 21 & HUGHES.I & 0.8442 & 08272 \\
\hline 22 & HUGHES - 2 & 0.8440 & 0.8261 \\
\hline 23 & GTESS-1 & 0.8350 & 0.8186 \\
\hline 24 & GTESS2 & 08345 & 0.8178 \\
\hline 25 & MIME & 0.8341 & 0.8095 \\
\hline 26 & OCRSYS & 08267 & 0.8163 \\
\hline 27 & RISO & 0.8241 & 0.7899 \\
\hline 28 & NIST_l & 0.8239 & 0.7892 \\
\hline 29 & ASOL & 0.8235 & 0.8004 \\
\hline 30 & REI & 0.8037 & 0.7870 \\
\hline 31 & $K A M A N_{-1}$ & 0.7949 & 0.7708 \\
\hline 32 & נב TIST & 0.7719 & 0.7537 \\
\hline 33 & KAMAN_3 & 0.7597 & 0.7339 \\
\hline 34 & KAMAN_2 & 07555 & 0.7271 \\
\hline 35 & СОМСOM & 0.7482 & 0.7402 \\
\hline 36 & IFAX & 0.7392 & 0.7198 \\
\hline 37 & NIST 2 & 0.7313 & 0.7063 \\
\hline 38 & GMD_2 & 07245 & 0.6968 \\
\hline 39 & VALEN_1 & 0.7152 & 0.6886 \\
\hline 40 & KAMAN_4 & 0.7012 & 0.6710 \\
\hline 41 & KAMAN_S & 0.6319 & 0.6073 \\
\hline 42 & $\mathrm{UMICH}_{2}$ & 0.0528 & 0.0154 \\
\hline
\end{tabular}

Table 48: GMD_1 correlation graph key for uppers. 


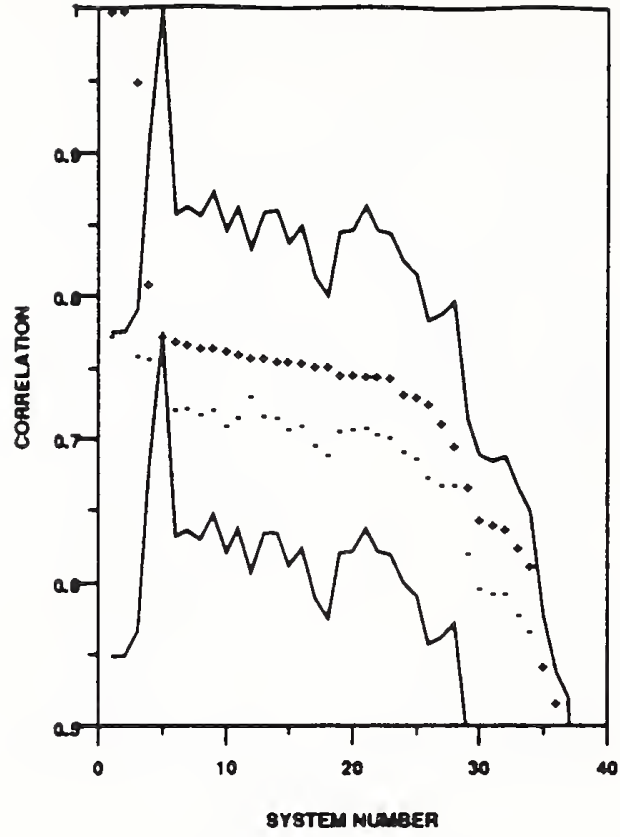

Figure 75: GMD_1 - lower case correlation

\begin{tabular}{|c|c|c|c|}
\hline System Number & System Name & Correlation (all) & Correlation (correct) \\
\hline 1 & GMD-1 & 1.0000 & 10000 \\
\hline 2 & GMD -4 & 1.0000 & 07746 \\
\hline 3 & GMD_3 & 0.9522 & 0.7613 \\
\hline 4 & VOTE_M & 0.8117 & 0.7591 \\
\hline 3 & REFERENCE & 0.7746 & 0.7746 \\
\hline 6 & ATT $=4$ & 0.7718 & 0.7232 \\
\hline 7 & ERIM -1 & 07693 & 0.7247 \\
\hline 8 & KODAK」 & 0.7873 & 07199 \\
\hline 8 & AEG & 07668 & 07235 \\
\hline 10 & UBOL & 07644 & 0.7122 \\
\hline 11 & ATT_ & 0.7623 & 0.7176 \\
\hline 12 & VOTE.P & 0.7802 & 0.7325 \\
\hline 13 & A TT 2 & 0.7597 & 0.7193 \\
\hline 14 & NYNEX & 0.7382 & 07182 \\
\hline 15 & ATT 3 & 0.7574 & 07100 \\
\hline 16 & $\mathrm{UMICH}_{-1}$ & 0.7371 & 0.7117 \\
\hline 17 & NIST -1 & 07542 & 0.6981 \\
\hline 18 & NIST_A & 07341 & 0.6915 \\
\hline 19 & IBM & 07489 & 0.7088 \\
\hline 20 & NESTOR & 0.7485 & $0.709 \mathrm{~s}$ \\
\hline 21 & OCRSYS & 07473 & 0.7110 \\
\hline 22 & HUGHES.I & 07472 & 0.7057 \\
\hline 23 & HUGHES -2 & 0.7459 & 0.7040 \\
\hline 24 & GTESS_l & 0.7352 & 0.6931 \\
\hline 25 & GTESS 2 & 0.7327 & 0.6888 \\
\hline 26 & RISO & 0.7285 & 06765 \\
\hline 27 & ASOL & 0.7146 & 0.6706 \\
\hline 28 & ב_ NIST & 0.6983 & 0.6709 \\
\hline 29 & GMD_2 & 06692 & 0.6226 \\
\hline 30 & KAMAN_1 & 0.6470 & 0.5987 \\
\hline 31 & VALEN_l & 06428 & 0.5945 \\
\hline 32 & $\mathrm{NIST}_{2}$ & 06395 & 0.5954 \\
\hline 33 & KAMAN_S & 0.6270 & 0.5795 \\
\hline 34 & KAMAN-2 & 06148 & 0.5684 \\
\hline 35 & KAMAN_5 & 05438 & 05020 \\
\hline 36 & KAMAN_A & 05192 & 0.4757 \\
\hline 37 & COMCOM & 04622 & 0.4513 \\
\hline 38 & $\mathrm{UMICH}_{2}$ & 0.1098 & 0.0478 \\
\hline
\end{tabular}

Table 49: GMD_1 correlation graph key for lowers. 
SYSTEM: GMD_2

PARTICIPANT: Frank Smieja

ORGANIZATION: Gesellschaft fuer Mathematik und Datenverarbeitung (GMD), Sankt Augustin, Germany

PREPROCESSING: scaled, centered, contrast filtered images?

FEATURES: pixel representation only

CLASSIFICATION: network of worker, monitor, and decision NN in pandemonium system of MINOS modules, "pandemonium reflective system". Output is chosen from network with maximum confidence value by decision NN.

HARDWARE: $\quad$ SPARC2

TRAINING :

UPPERS LOHERS DATABASE

$\begin{array}{llll}4180 & 8979 & 9355 & \text { NSDB3 } \\ -420 & -420 & -420 & \text { writers }\end{array}$

STATUS: $\quad$ on time

RESULTS: -- DIGITS -- -- UPPERS -- -- LOWERS -- DATABASE

$\begin{array}{llllll}\text { REJ. } & \text { ERR. } & \text { REJ. } & \text { ERR. } & \text { REJ. } & \text { ERR. } \\ \text { RATE } & \text { RATE-- } & \text { RATE } & \text { RATE-- } & \text { RATE } & \text { RATE-- } \\ 0.00 & 0.1545 & 0.00 & 0.2457 & 0.00 & 0.2861 \\ 0.10 & 0.1120 & 0.28 & 0.1321 & 0.28 & 0.1752 \\ 0.12 & 0.1023 & 0.31 & 0.1219 & 0.31 & 0.1625 \\ 0.13 & 0.0979 & 0.33 & 0.1172 & & \\ 0.14 & 0.0941 & 0.34 & 0.1132 & & \\ 0.16 & 0.0904 & 0.36 & 0.1080 & & \\ 0.17 & 0.0855 & 0.38 & 0.1022 & & \end{array}$

OCR RATE (CPS): DIGITS UPPERS LOWERS
SYS RATE:
0.68
0.43
0.32

CPU RATE: 
SYSTEM: GMD 2

BIBLIOGRAPHY:

The following references have been provided for this system:

$[17][18]$

COMMENTS: GMD 2

\section{PARTICIPANT: Frank Smieja}

ORGANIZATION: Gesellschaft für Mathematik und Datenverarbeitung (GMD), Sankt Augustin, Germany.

The data was learnt by a system of modular neural networks, described in the reports cited below. The individual patterns to be learnt are automatically decomposed over the modular system, such that the Worker neural network that learns to map a particular pattern to a target is the one that is most specialized at that time to learn it. In order that the appropriate network can be believed, when a test session is in process, a partner Monitor network is employed. The Monitor network partnered to the Worker network allocated the pattern to learn is trained to produce a positive output when it sees the pattern. The other Monitor networks, associated with Workers that do not learn the current pattern, are trained to produce a negative output on seeing this pattern.

Various confidence values are derived from the outputs from the Monitor networks during the test sessions. An ambiguity measure is also derived from degree of closeness of the two most positive Monitor outputs. Both the confidences and the ambiguity are then used to filter off the answers that are not provided with sufficient commitment (the "rejected" patterns).

Insofar as the NIST test results are concerned, it was observed that the training on so few examples (see above) was quite a disadvantage. The system was able to model the NIST training set well enough to produce good generalization for this set, but in general 10 worse for the NIST test sets. BIBLIOGRAPHY:

F. J. Śmieja, Multiple network systems (MINOS) modules: task division and module discrimination, Proc. 8th AISB conference on Artificial Intelligence, Leeds, April 1991.

F. J Śmieja and H. Mühlenbein, Reflective modular neural networks, submitted to Machine Learning, available as GMD report number 633 (1992). 


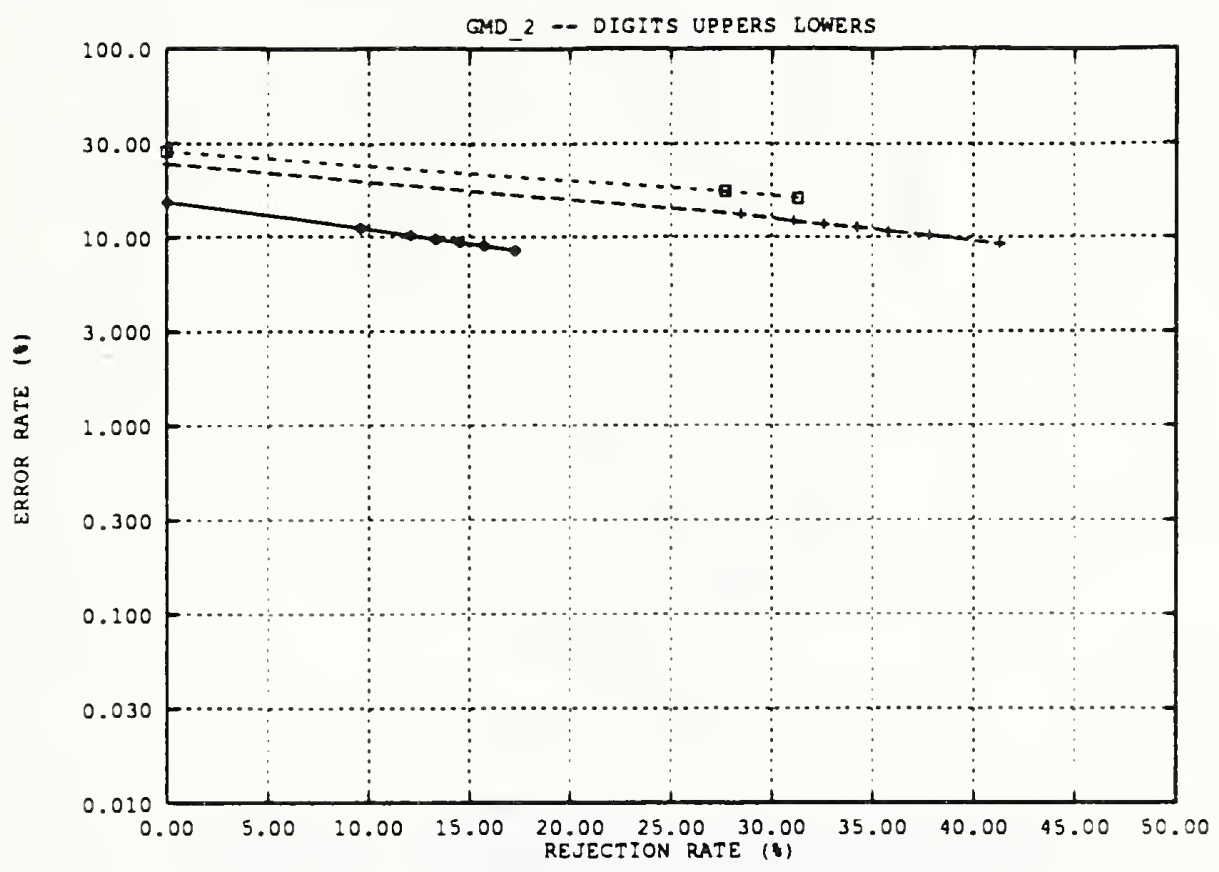

Figure 76: Error rate versus rejection rate for GMD_2

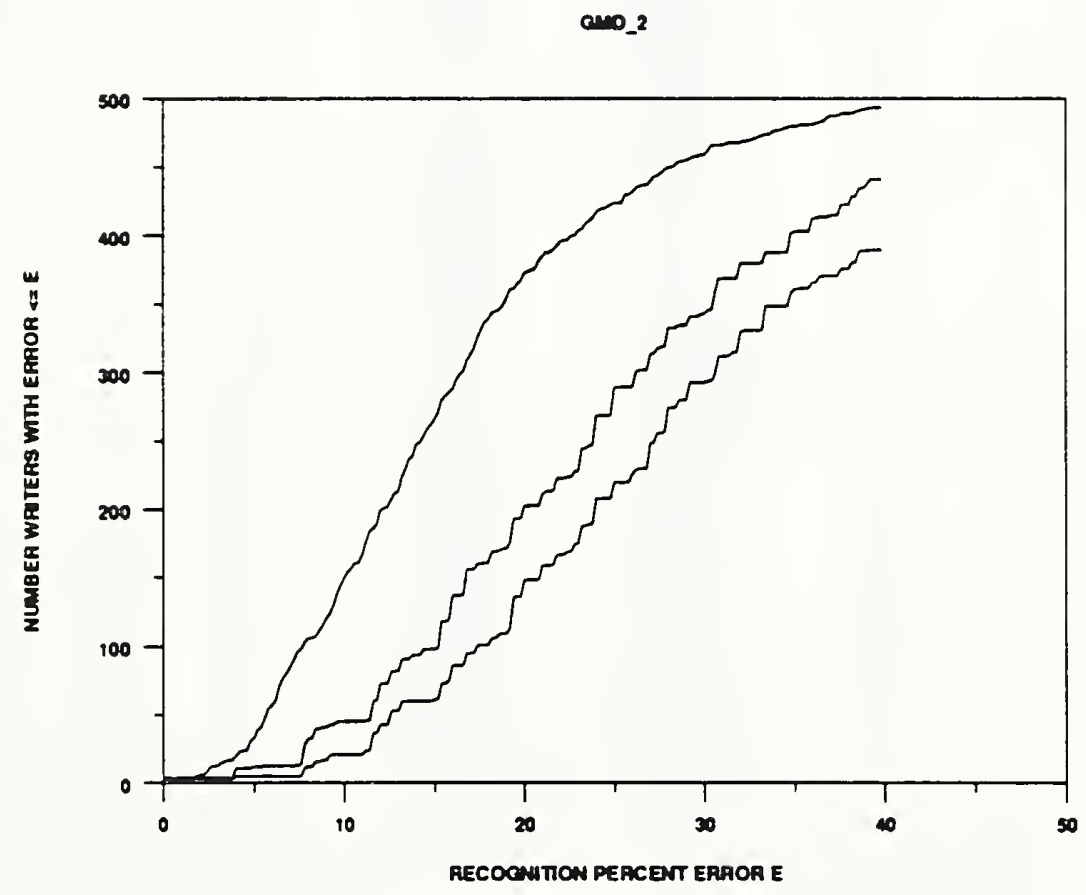

Figure 77: Error rate per writer of GMD_2 


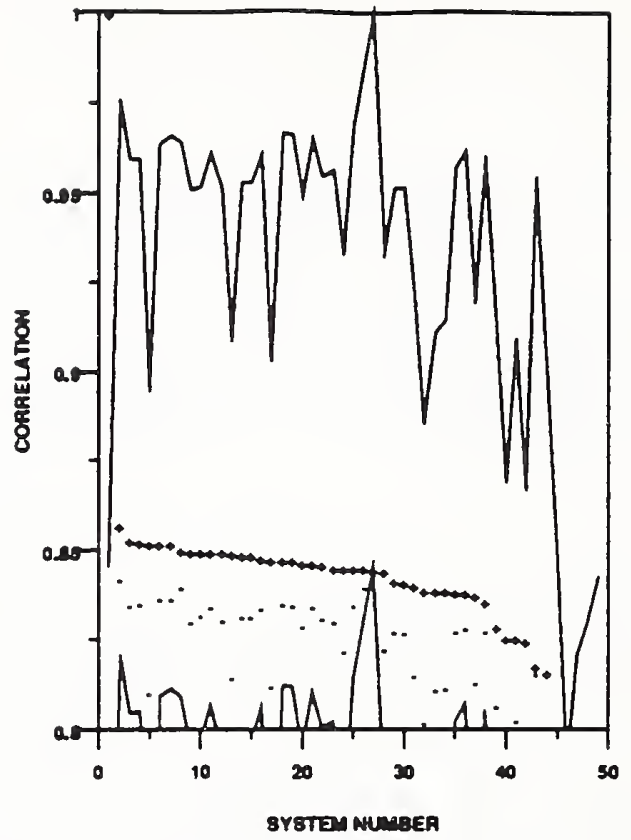

Figure 78: GMD 2 - digit correlation

\begin{tabular}{|c|c|c|c|}
\hline System Number & System Name & Coppelation (all) & Correlation (correct) \\
\hline 1 & GMD2 & 1.0000 & 1.0000 \\
\hline 2 & VOTE_M & 0.8575 & 0.8425 \\
\hline 3 & ATT -4 & 0.8535 & 0.8353 \\
\hline 4 & KODAK 2 & 0.8530 & 0.8356 \\
\hline 3 & ATT 2 & 0.8525 & 0.8370 \\
\hline 6 & AEG & 0.8525 & 0.8368 \\
\hline 7 & RISO & 0.8525 & 0.8110 \\
\hline 8 & VOTEP & 0.8509 & 0.8402 \\
\hline 9 & ERIM_L & 0.8504 & 0.8347 \\
\hline 10 & ATT 3 & 0.8504 & 0.8325 \\
\hline 11 & NIST _ 4 & 0.8504 & 0.8305 \\
\hline 12 & THINK -1 & 0.8502 & 0.8312 \\
\hline 13 & NIST 2 & 0.8497 & 0.8152 \\
\hline 14 & SYMBUS & 0.8495 & 0.8320 \\
\hline 15 & KODAK」 & 0.8494 & 0.8319 \\
\hline 16 & ERIM_2 & 0.8483 & 0.8341 \\
\hline 17 & בـ ELSAGB & 0.8482 & 0.8358 \\
\hline 18 & NIST 3 & 0.8482 & 0.8126 \\
\hline 19 & ELSAGB 2 & 0.8479 & 0.8354 \\
\hline 20 & ELSAGB_1 & 0.8470 & 0.8291 \\
\hline 21 & IBM & 0.8469 & 0.8349 \\
\hline 22 & NESTOR & 0.8466 & 0.8316 \\
\hline 23 & UBOL & 0.8458 & 0.8307 \\
\hline 24 & OCRSYS & 0.8457 & 0.8402 \\
\hline 25 & ATT $\perp$ & 0.8457 & 0.8350 \\
\hline 26 & GTESS_2 & 0.8457 & 0.8225 \\
\hline 27 & REFERENCE & 0.8455 & 0.8455 \\
\hline 28 & GTESS_L & 0.8450 & 0.8226 \\
\hline 28 & HUGHES_2 & 0.8421 & 0.8278 \\
\hline 30 & HUGHES _1 & 0.8416 & 0.8275 \\
\hline 31 & NIST _1 & 0.8406 & 0.8155 \\
\hline 32 & KAMAN_L & 0.8395 & 0.8024 \\
\hline 33 & ASOL & 0.8392 & 0.8116 \\
\hline 34 & MIME & 0.8391 & 0.8124 \\
\hline 35 & NYNEX & 0.8389 & 0.8277 \\
\hline 36 & THINK 2 & 0.8387 & 0.8289 \\
\hline 37 & GMD_J & 0.8381 & 0.8138 \\
\hline 38 & REI & 08362 & 0.8280 \\
\hline 39 & GMD_1 & 0.8291 & 0.8072 \\
\hline 40 & UPENN & 0.8258 & 0.8031 \\
\hline 41 & KAMAN_3 & 0.8258 & 0.7887 \\
\hline 42 & KAMAN_2 & 0.8248 & 0.7868 \\
\hline 43 & СОМСом & 0.8183 & 0.8159 \\
\hline 14 & GMD_-1 & 0.8165 & 0.7948 \\
\hline 45 & KAMAN_5 & 0.7975 & 0.7664 \\
\hline 16 & KAMAN_4 & 0.7727 & 0.7280 \\
\hline 47 & VALEN_1 & 0.7659 & 0.7377 \\
\hline 48 & IFAX & 07631 & 0.7403 \\
\hline 49 & VALEN 2 & 0.7594 & 0.7438 \\
\hline
\end{tabular}

Table 50: GMD_2 correlation graph key for digits. 


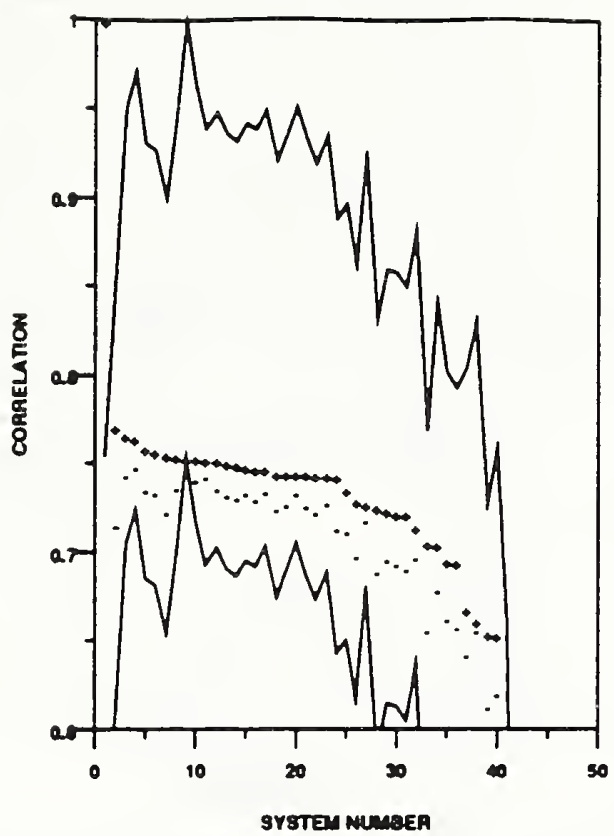

Figure 79: GMD_2 - upper case correlation

\begin{tabular}{|c|c|c|c|}
\hline Syslem Number & System Name & Correlation (all) & Correlation (correct) \\
\hline 1 & GMD.2 & 1.0000 & 1.0000 \\
\hline 2 & RISO & 0.7710 & 0.7188 \\
\hline 3 & A T T -4 & 0.7671 & 0.7432 \\
\hline 4 & VOTE_M & 07649 & 0.7493 \\
\hline $\mathbf{s}$ & KODAK, 1 & 0.7591 & 0.7364 \\
\hline 6 & SYMBUS & 07579 & 0.7344 \\
\hline 7 & MIME & 0.7561 & 0.7236 \\
\hline 8 & ATT 2 & 0.7546 & 0.7376 \\
\hline 9 & REFERENCE & 0.7543 & 0.7543 \\
\hline 10 & AEG & 0.7542 & 0.7424 \\
\hline 11 & VOTEP & 0.7532 & 0.7442 \\
\hline 12 & ERIM-1 & 07529 & 0.7377 \\
\hline 13 & IBM & 0.7511 & 0.7334 \\
\hline 14 & ATT3 & 0.7504 & 0.7322 \\
\hline 15 & NESTOR & 0.7494 & 0.7343 \\
\hline 16 & UMICH_I & 0.7488 & 0.7356 \\
\hline 17 & UBOL & 07488 & 0.7311 \\
\hline 18 & GTESS_1 & 0.7458 & 0.7253 \\
\hline 19 & NYNEX & 0.7457 & 0.7344 \\
\hline 20 & HUGHES -1 & 07437 & 0.7283 \\
\hline 21 & HUGHES -2 & 0.7454 & 0.7277 \\
\hline 22 & ATT $\perp$ & 0.7430 & 0.7291 \\
\hline 23 & GTESS_2 & 0.7430 & 0.7239 \\
\hline 24 & ASOL & 0.7438 & 0.7148 \\
\hline 23 & NIST_A & 0.7363 & 0.7123 \\
\hline 26 & NIST_l & 0.7304 & 06986 \\
\hline 27 & OCRSYS & 0.7287 & 07187 \\
\hline 28 & NIST_3 & 0.7286 & 06893 \\
\hline 29 & GMD_l & 07243 & 0.6968 \\
\hline 30 & GMD.3 & 0.7226 & 0.6947 \\
\hline 31 & KAMAN_1 & 07223 & 0.6917 \\
\hline 32 & REI & 0.7134 & 0.6975 \\
\hline 33 & NIST 2 & 0.7063 & 06369 \\
\hline 34 & GMD_4 & 0.7051 & 0.6796 \\
\hline 35 & KAMAN_3 & 0.6963 & 0.6633 \\
\hline 36 & KAMAN_2 & 0.6954 & 0.6586 \\
\hline 37 & IFAX & 0.6686 & 0.6429 \\
\hline 38 & СOMCOM & 0.6618 & 0.6569 \\
\hline 39 & KAMAN_4 & 0.6543 & 0.6134 \\
\hline 40 & VALEN_L & 0.6540 & 06203 \\
\hline 41 & KAMAN_S & 0.5801 & 0.5490 \\
\hline 42 & UMICH 2 & 00803 & 0.0143 \\
\hline
\end{tabular}

Table 51: GMD_2 correlation graph key for uppers. 


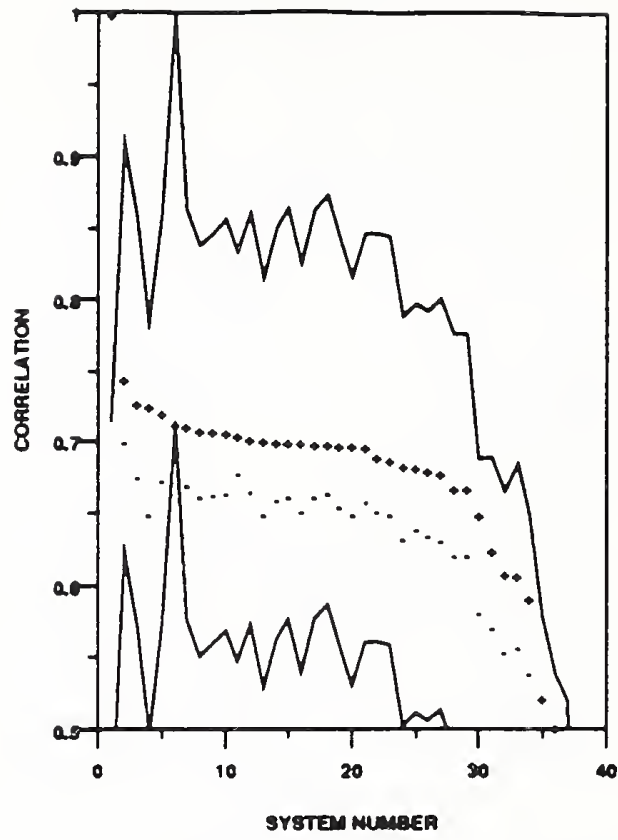

Figure 80: GMD_2 - lower case correlation

\begin{tabular}{|c|c|c|c|}
\hline System Number & System Name & Correiation (all) & Correlation (correct) \\
\hline $\mathrm{I}$ & GMD_2 & 1.0000 & 1.0000 \\
\hline 2 & VOTE_M & 0.7470 & 0.7013 \\
\hline 3 & ATT_A & 07287 & 0.6779 \\
\hline 4 & RISO & 0.7265 & 0.6507 \\
\hline 5 & ATT 2 & 0.7219 & 0.6758 \\
\hline 6 & REFERENCE & 07139 & 0.7139 \\
\hline 7 & ERIM.1 & 07135 & 0.6723 \\
\hline $\mathrm{g}$ & ATT 3 & 07100 & 0.6637 \\
\hline 9 & $18 \mathrm{M}$ & 0.7097 & 06646 \\
\hline 10 & KODAK」 & 0.7081 & 0.8662 \\
\hline 11 & VOTEP & 0.7068 & 0.6801 \\
\hline 12 & NYNEX & 0.7035 & 0.6671 \\
\hline 13 & NIST_l & 0.7031 & 0.6815 \\
\hline 14 & $\mathrm{UMICH}_{-1}$ & 0.7022 & 0.8610 \\
\hline 13 & OCRSYS & 0.7021 & 0.6642 \\
\hline 16 & GTESS-1 & 07016 & 0.6337 \\
\hline 17 & ATT $\perp$ & 0.7010 & 0.8637 \\
\hline 18 & $A E G$ & 0.7000 & 0.6659 \\
\hline 19 & UBOL & 06998 & 0.6568 \\
\hline 20 & $\mathrm{GTESS}_{2}$ & 06992 & 0.6507 \\
\hline 21 & NESTOR & 0.6977 & 0.6598 \\
\hline 22 & HUGHES_- & 0.6917 & 06536 \\
\hline 23 & HUGHES - 2 & 0.6889 & 0.6511 \\
\hline 24 & ASOL & 06852 & 0.6344 \\
\hline 23 & NIST 3 & 06850 & 06412 \\
\hline 28 & GMD_3 & 0.6822 & 0.6359 \\
\hline 27 & NIST_4 & 0.6798 & 0.6330 \\
\hline 28 & GMD_4 & 06692 & 0.6226 \\
\hline 29 & GMD_1 & 06892 & 0.6226 \\
\hline 30 & NIST 2 & 0 BS13 & 0.5825 \\
\hline 31 & $\mathrm{KAM} \mathrm{N}_{-1}$ & 06262 & 0.5715 \\
\hline 32 & KAMAN_3 & 0.6093 & 0.5548 \\
\hline 33 & VALEN -1 & 0.8081 & 05585 \\
\hline 34 & $K A M A N_{-2}$ & 03921 & 0.3398 \\
\hline 35 & KAMAN S & 03239 & 04779 \\
\hline 36 & KAMAN-4 & 05033 & 04527 \\
\hline 37 & COMCOM & 04342 & 0.4247 \\
\hline 38 & $\mathrm{UMICH}_{2}$ & 0.1047 & 0.0412 \\
\hline
\end{tabular}

Table 52: GMD_2 correlation graph key for lowers. 


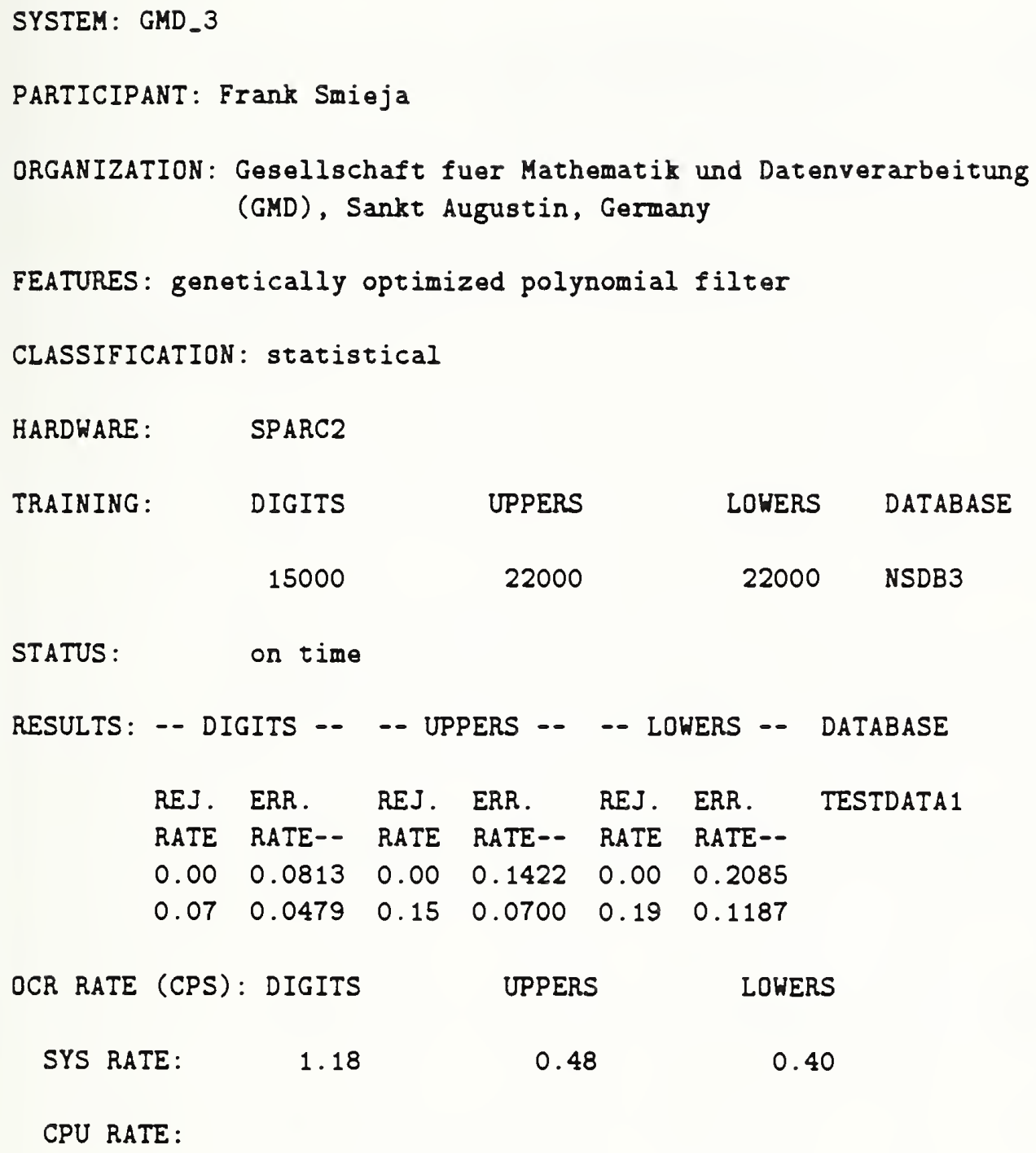

1.18

0.48

0.40

CPU RATE: 
SYSTEM: GMD_3

BIBLIOGRAPHY:

The following references have been provided for this system:

[15][16] 


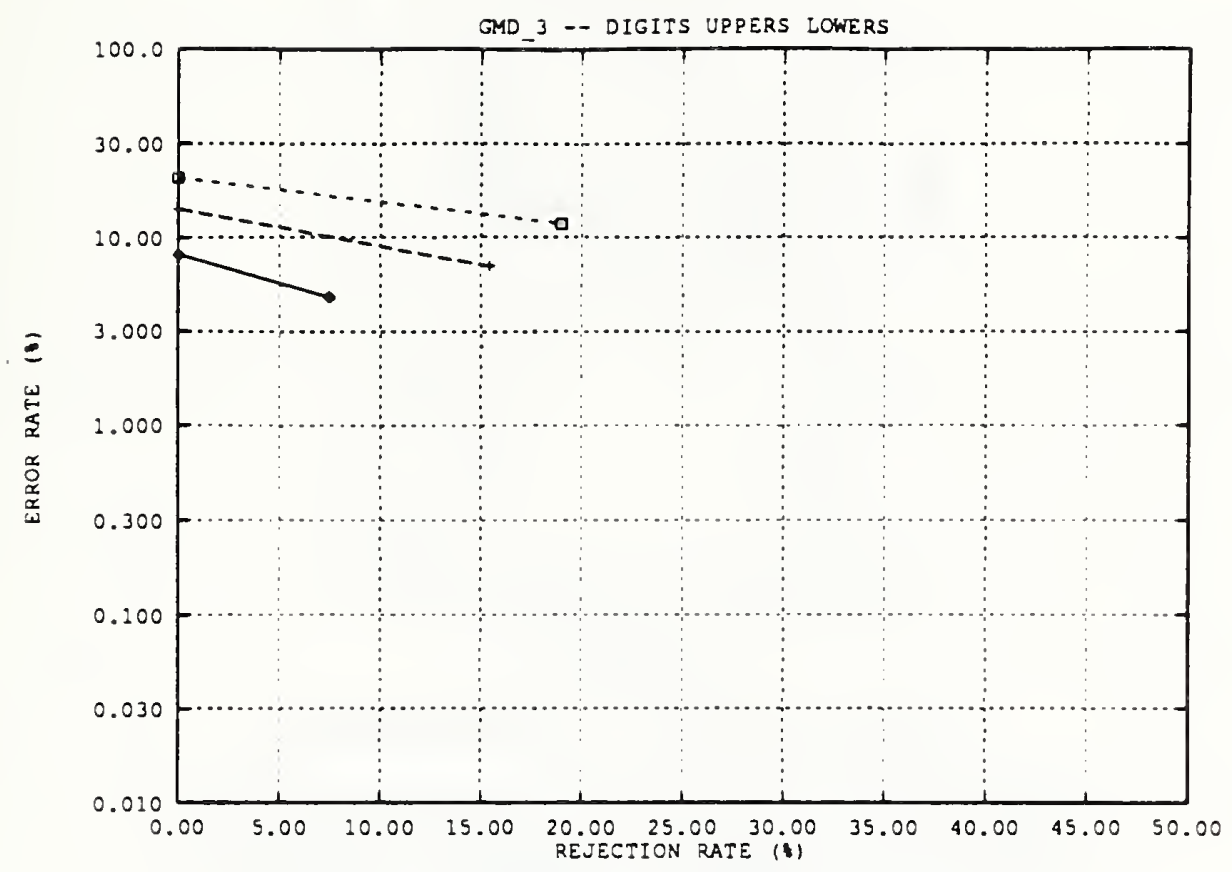

Figure 81: Error rate versus rejection rate for GMD_3

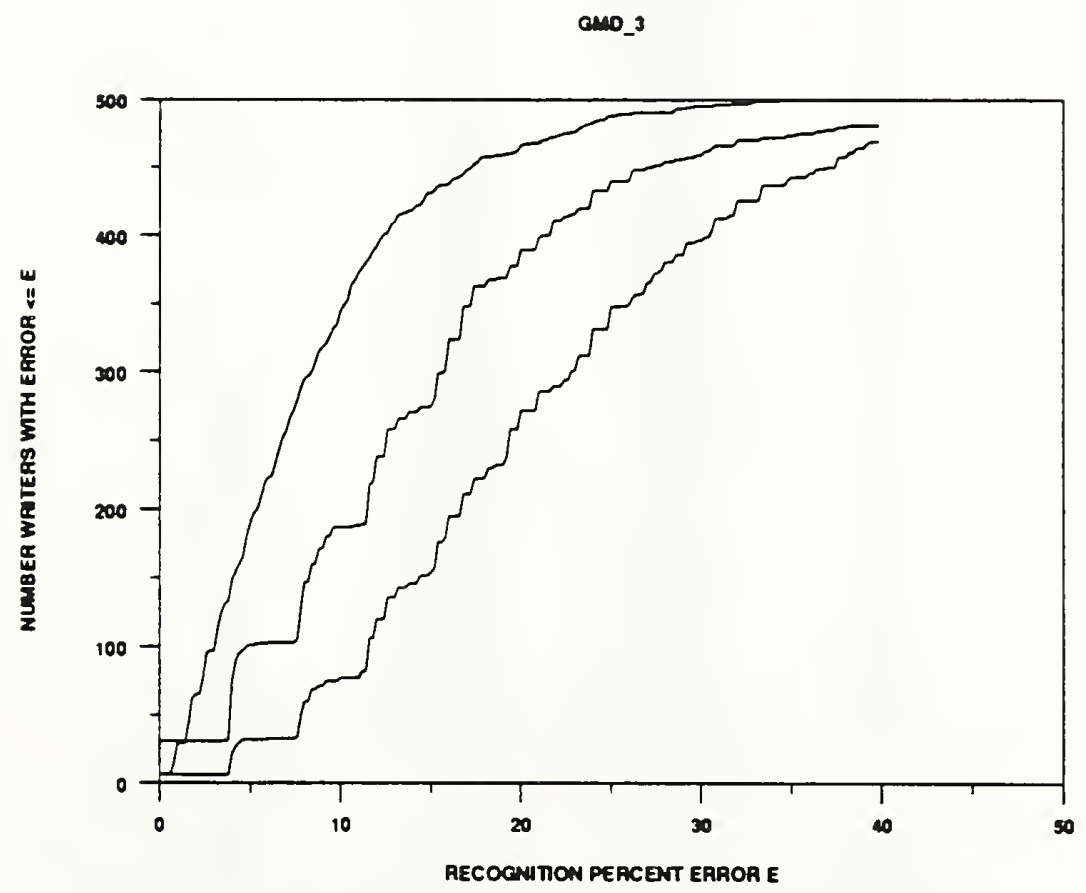

Figure 82: Error rate per writer of GMD_3 


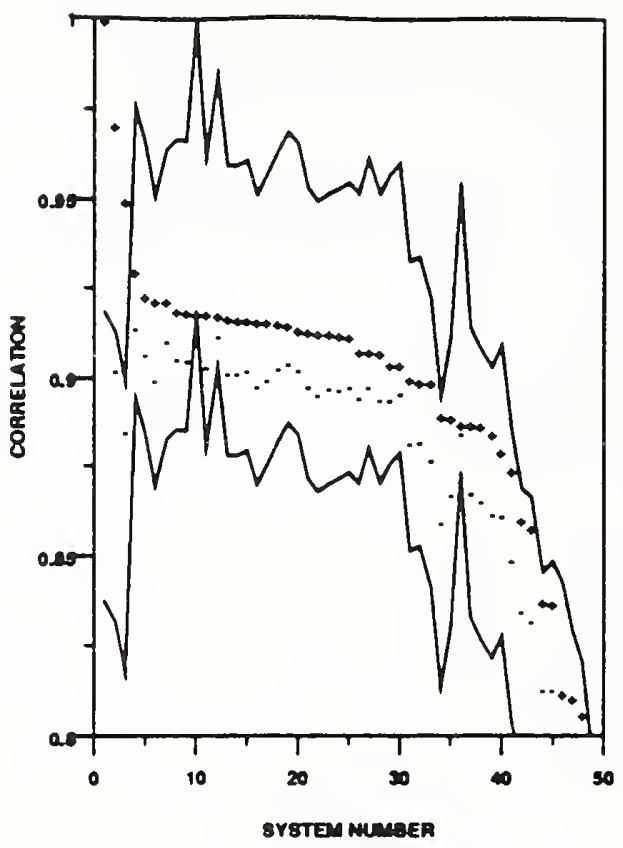

Figure 83: GMD_3 - digit correlation

\begin{tabular}{|c|c|c|c|}
\hline Sygtem Number & System Name & Correlation (aII) & Correlation (correct) \\
\hline $\mathrm{T}$ & GMD_3 & 1.0000 & 1.0000 \\
\hline 2 & GMD.1 & 0.9712 & 0.9027 \\
\hline 3 & GMD.4 & 0.9498 & 08834 \\
\hline 4 & VOTE_M & 0.8308 & 0.9146 \\
\hline 3 & $A E G$ & 0.8230 & 09075 \\
\hline 6 & NIST.4 & 0.9223 & 0.9001 \\
\hline 7 & VOTEP & 0.9224 & 0.9109 \\
\hline 8 & SLSAGBA & 0.9198 & 0.8059 \\
\hline 9 & ELSAGB 2 & 0.9194 & 0.9055 \\
\hline 10 & REFERENCE & 0.8187 & 0.9187 \\
\hline 11 & ERIM-1 & 0.8186 & 0.9036 \\
\hline 12 & OCRSYS & 0.9181 & 0.9122 \\
\hline 13 & KODAK 2 & 0.9172 & 0.9019 \\
\hline 14 & ERIM 2 & 0.8170 & 0.8026 \\
\hline 15 & ATT_A & 09170 & 0.9016 \\
\hline 16 & THINK_ & 0.8167 & 0.8879 \\
\hline 17 & $\mathrm{UBOL}$ & 0.9165 & 0.8999 \\
\hline 18 & ATT2 2 & 0.8161 & 0.9033 \\
\hline 18 & ATT $\downarrow$ & 0.2155 & 0.8044 \\
\hline 20 & IBM & 09141 & 0.9029 \\
\hline 21 & SYMBUS & 0.9136 & 0.8981 \\
\hline 22 & ATT 3 & 0.9133 & 0.8977 \\
\hline 23 & ELSAGB-1 & 09133 & 0.8938 \\
\hline 24 & KODAKA & 0.8130 & 0.8973 \\
\hline 25 & NESTOR & 0.9122 & 0.8982 \\
\hline 26 & HUGHES_1 & 0.8082 & 0.8947 \\
\hline 27 & THINK 2 & 0.9081 & 0.8980 \\
\hline 28 & HUGHES_2 & 0.8078 & 0.8944 \\
\hline 29 & NYNEX & 0.8047 & 0.8944 \\
\hline 30 & REI & 0.9046 & 0.8963 \\
\hline 31 & GTESS 2 & 0.9002 & 0.8821 \\
\hline 32 & GTESS_1 & 0.8996 & 0.8823 \\
\hline 33 & NIST $_{-1}$ & 0.8995 & 0.8775 \\
\hline 34 & RISO & 0.8898 & 0.8598 \\
\hline 35 & ASOL & 0.8893 & 0.8677 \\
\hline 36 & СОМСОМ & 0.8875 & 0.8847 \\
\hline 37 & MIME & 0.8874 & 0.8883 \\
\hline 38 & NIST 2 & 0.8870 & 0.8661 \\
\hline 39 & NIST & 0.8848 & 0.8624 \\
\hline 40 & UPENN & 0.8798 & 0.8620 \\
\hline 41 & KAMAN.1 & 0.8745 & 0.8493 \\
\hline 42 & KAMAN_J & 0.8610 & 0.8351 \\
\hline 43 & KAMAN 2 & 0.8586 & 0.8324 \\
\hline 44 & GMD.2 & 0.8381 & 0.8138 \\
\hline 43 & KAMAN_S & 0.8374 & 0.8138 \\
\hline 46 & VALEN 2 & 0.8128 & 0.7990 \\
\hline 47 & IFAX & 08113 & 0.7915 \\
\hline 48 & VALEN_1 & 0.8089 & 0.7839 \\
\hline 49 & KAMAN_4 & 0.7915 & 07646 \\
\hline
\end{tabular}

Table 53: GMD_3 correlation graph key for digits. 


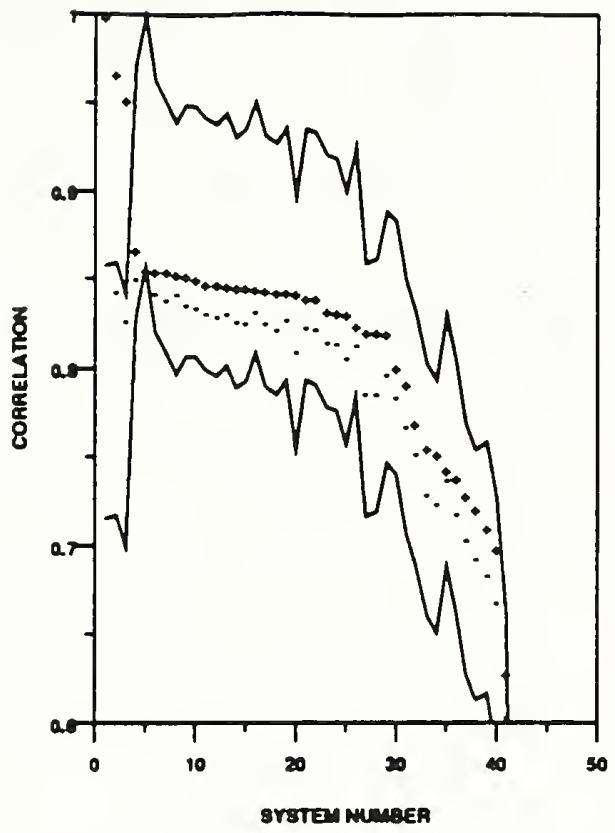

Figure 84: GMD_3 - upper case correlation

\begin{tabular}{|c|c|c|c|}
\hline System Number & Syotem Name & Correlastion (all) & Correlation (correct) \\
\hline 1 & GMD_3 & 1.0000 & 1.0000 \\
\hline 2 & GMD_1 & 0.9679 & 0.8446 \\
\hline 3 & GMD-4 & 0.9832 & 0.8280 \\
\hline 4 & VOTEAM & 0.8689 & 0.8525 \\
\hline 3 & REFERENCE & 0.8578 & 0.8878 \\
\hline 6 & AEG & 0.8373 & 0.8444 \\
\hline 7 & ATT_4 & 0.8570 & 0.8401 \\
\hline 8 & VOTEP & 0.8547 & 0.8437 \\
\hline 9 & UMICH_l & 0.8539 & 0.8372 \\
\hline 10 & ERIM_1 & 0.8318 & 0.8358 \\
\hline 11 & NESTOR & 0.8497 & 0.8333 \\
\hline 12 & UBOL & 0.8496 & 0.8308 \\
\hline 13 & ATT 2 & 0.8482 & 0.8333 \\
\hline 14 & KODAK」 & 0.8478 & 0.8287 \\
\hline 15 & ATT_ 1 & 0.8474 & 0.8277 \\
\hline 16 & NYNEX & 0.8467 & 0.8343 \\
\hline 17 & בה ATT & 0.8487 & 0.8278 \\
\hline 18 & IBM & 0.8453 & 0.8290 \\
\hline 19 & SYMBUS & 0.8453 & 0.8240 \\
\hline 20 & NIST 44 & 0.8442 & 0.8113 \\
\hline 21 & HUGHES - 1 & 0.8416 & 0.8250 \\
\hline 22 & HUGHES_2 & 0.8414 & 0.8238 \\
\hline 23 & GTESS 1 & 0.8341 & 0.8169 \\
\hline 24 & GTESS 2 & 0.8331 & 0.8188 \\
\hline 25 & MIME & 0.8323 & 0.8076 \\
\hline 26 & OCRSYS & 0.8259 & 0.8150 \\
\hline 27 & RISO & 0.8220 & 0.7875 \\
\hline 28 & NIST_l & 08213 & 0.7870 \\
\hline 29 & ASOL & 0.8208 & 0.7981 \\
\hline 30 & REI & 0.8015 & 0.7851 \\
\hline 31 & KAMAN_1 & 0.7927 & 0.7685 \\
\hline 32 & ב ב & 0.7705 & 0.7528 \\
\hline 33 & KAMAN 3 & 0.7564 & 0.7313 \\
\hline 34 & $K A M A N-2$ & 0.7335 & 0.7252 \\
\hline 33 & СOMCOM & 0.7452 & 0.7391 \\
\hline 36 & IFAX & 07401 & 0.7196 \\
\hline 37 & NIST 2 & 0.7302 & 0.7050 \\
\hline 38 & GMD_2 & 0.7226 & 0.6947 \\
\hline 39 & VALEN-1 & 0.7117 & 0.6853 \\
\hline 40 & KAMAN_4 & 0.6998 & 0.6696 \\
\hline 41 & KAMAN_S & 0.6286 & 0.6049 \\
\hline 42 & $\mathrm{UMICH}_{2}$ & 0.0544 & 0.0149 \\
\hline
\end{tabular}

Table 54: GMD_3 correlation graph key for uppers. 


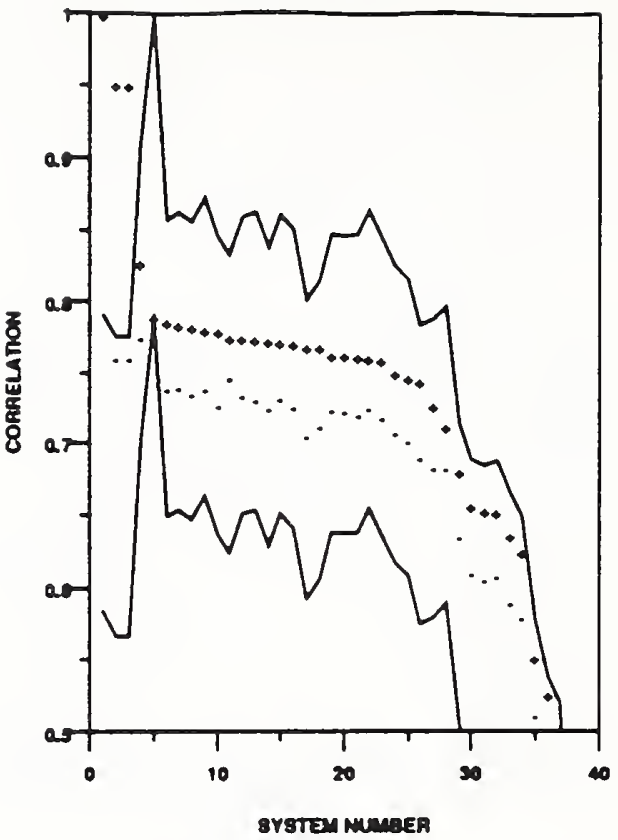

Figure 85: GMD_3 - lower case correlation

\begin{tabular}{|c|c|c|c|}
\hline Syorem Number & Syatem Name & Cortetalion (all) & Copreiation (coprect) \\
\hline $\mathrm{I}$ & GMD_3 & 1.0000 & 1.0000 \\
\hline 2 & GMD_4 & 0.9522 & 0.7613 \\
\hline $\mathbf{3}$ & GMD_1 & 0.9522 & 0.7613 \\
\hline 4 & VOTEM & 0.8292 & 0.7758 \\
\hline 5 & REFERENCE & 0.7915 & 0.7915 \\
\hline 6 & ATT_4 & 07878 & 0.7390 \\
\hline 7 & ERIM-1 & 0.7855 & 0.7403 \\
\hline 8 & KODAK_ & 0.7843 & 0.7362 \\
\hline 9 & $A E G$ & 0.7826 & 0.7391 \\
\hline 10 & UBOL & 0.7809 & 0.7283 \\
\hline 11 & VOTE_P & 0.7783 & 0.7477 \\
\hline 12 & ATT 2 & 0.7762 & 0.7352 \\
\hline 13 & ATT $\perp$ & 0.7753 & 0.7320 \\
\hline 14 & ATT J & 0.7738 & 0.7260 \\
\hline 15 & NYNEX & 0.7728 & 0.7327 \\
\hline 16 & UMICH-d & 0.7713 & 0.7265 \\
\hline 17 & NIST_4 & 0.7698 & 07067 \\
\hline 18 & NIST_l & 0.7692 & 0.7132 \\
\hline 19 & NESTOR & 0.7642 & 0.7247 \\
\hline 20 & IBM & 0.7636 & 0.7236 \\
\hline 21 & HUGHES - 1 & 0.7629 & 0.7212 \\
\hline 22 & OCRSYS & $0.761 \mathrm{~J}$ & 0.7256 \\
\hline 23 & HUGHES_2 & 0.7607 & 0.7190 \\
\hline 24 & GTESS_1 & 0.7515 & 0.7084 \\
\hline 25 & GTESS 2 & 0.7474 & 0.7032 \\
\hline 26 & Riso & 0.7448 & 0.6917 \\
\hline 27 & ASOL & 0.7283 & 0.6841 \\
\hline 28 & $N_{1 S T} 3$ & 0.7129 & 0.6849 \\
\hline 29 & GMD_2 & 0.6822 & 0.6359 \\
\hline 30 & $\mathrm{KAMAN}_{-1}$ & 0.6584 & 0.6108 \\
\hline 31 & VALEN-1 & 0.6542 & 0.6062 \\
\hline 32 & $\mathrm{NIST}_{2}$ & 0.6538 & 06083 \\
\hline 33 & KAMAN_3 & 0.6373 & 0.3907 \\
\hline 34 & KAMAN_2 & 0.6261 & 0.5800 \\
\hline 35 & KAMAN_S & 0.5522 & 0.3116 \\
\hline 36 & KAMAN_4 & 0.5268 & 0.4839 \\
\hline 37 & COMCOM & 04712 & 0.4594 \\
\hline 38 & $\mathrm{UMICH}_{2}$ & 0.1073 & 0.0498 \\
\hline
\end{tabular}

Table 55: GMD_3 correlation graph key for lowers. 


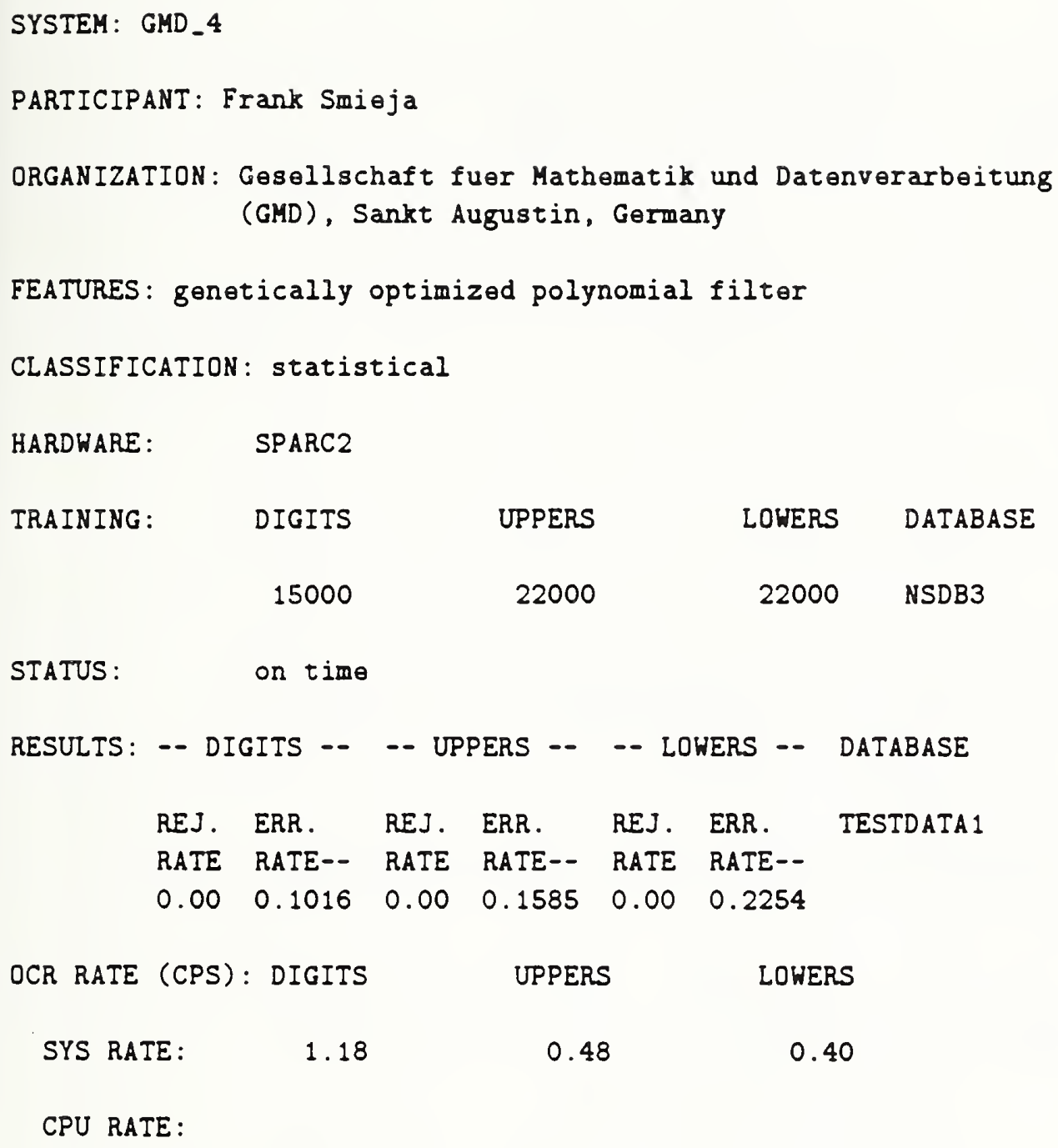


SYSTEM: GMD_4

BIBLIOGRAPHY:

The following references have been provided for this system:

[15][16] 


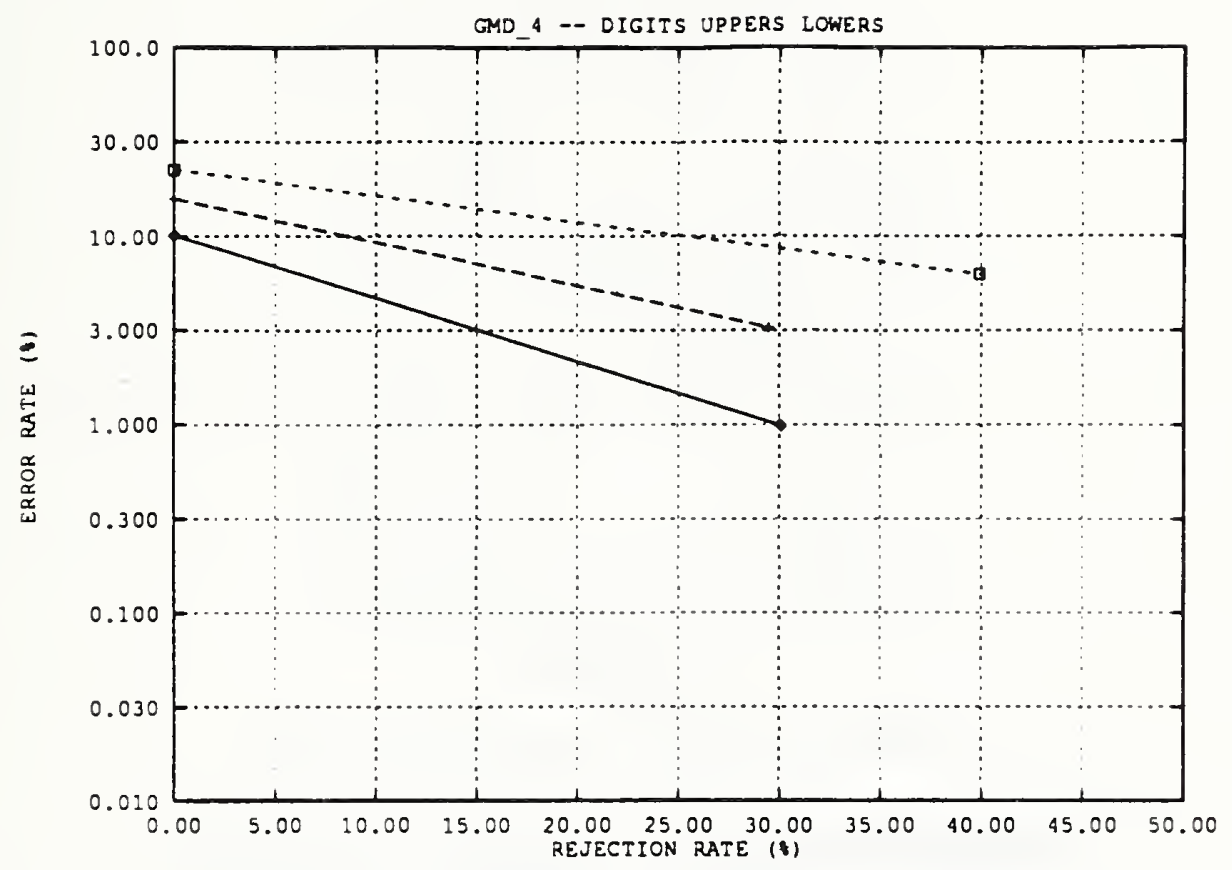

Figure 86: Error rate versus rejection rate for GMD_4

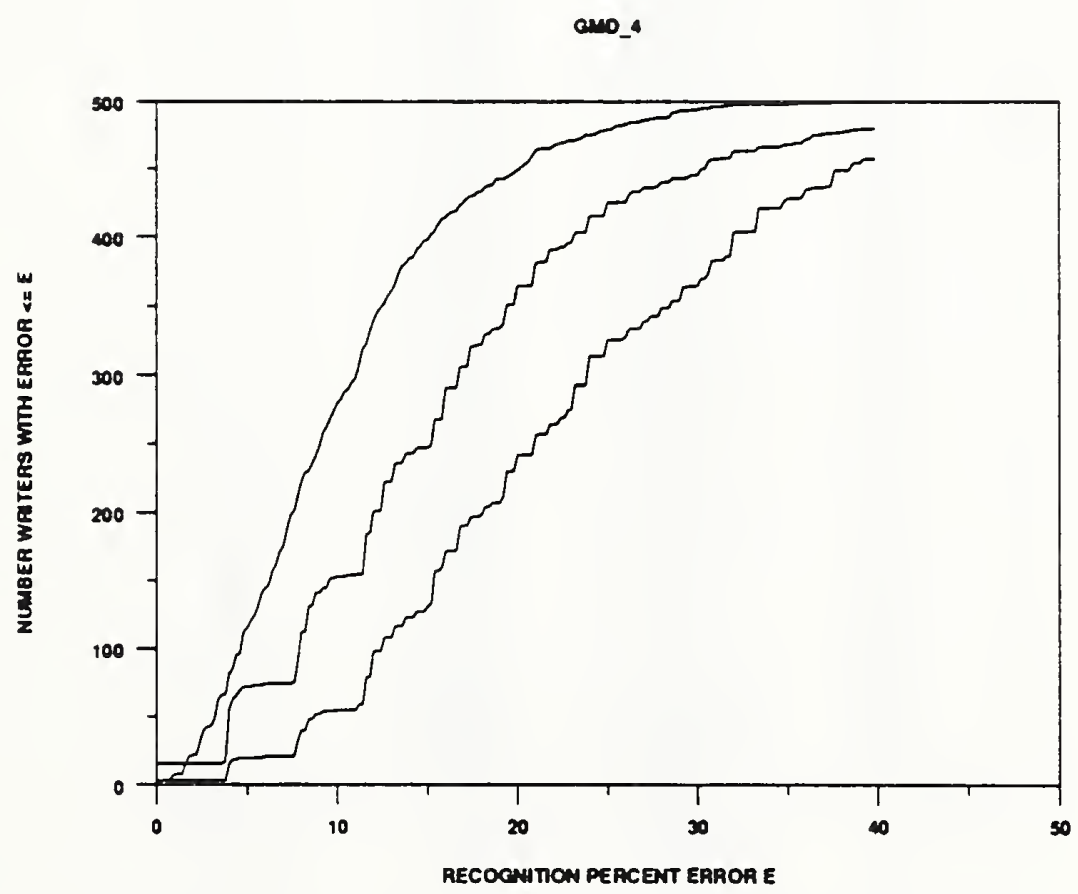

Figure 87: Error rate per writer of GMD_4 


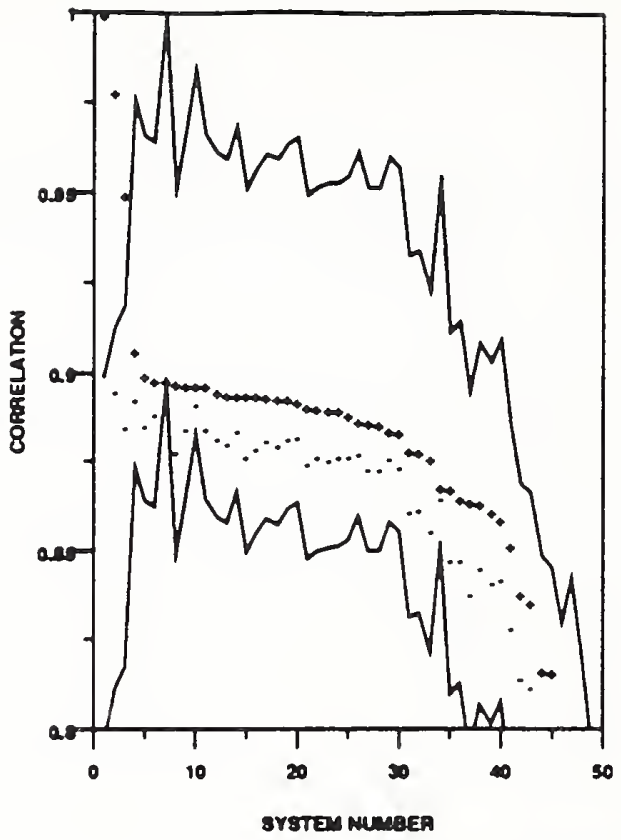

Figure 88: GMD_4 - digit correlation

\begin{tabular}{|c|c|c|c|}
\hline Syalem Number & System Name & Correlation (all) & Coprelation (correct) \\
\hline $\mathrm{T}$ & GMD_t & 1.0000 & 1.0000 \\
\hline 2 & GMD_-1 & 0.8786 & 0.8954 \\
\hline $\mathbf{3}$ & GMD & 0.9498 & 0.8854 \\
\hline 4 & VOTEM & 0.2068 & 0.8930 \\
\hline 3 & $A E G$ & 0.8988 & 03858 \\
\hline 6 & VOTEP & 0.8988 & 0.8890 \\
\hline 7 & REFERENCE & 0.8984 & 08984 \\
\hline 8 & NIST_4 & 0.8977 & 0.8783 \\
\hline 9 & ELSAGBA & 0.8972 & 0.8848 \\
\hline 10 & OCRSYS & 0.8871 & 0.8218 \\
\hline 11 & ELSAGB_2 & 0.8970 & 0.8846 \\
\hline 12 & ERIM_1 & 0.8252 & 0.8821 \\
\hline 13 & $\mathbf{A T T} \perp$ & 0.8246 & 0.8843 \\
\hline 14 & KODAK 2 & 0.8946 & 0.8807 \\
\hline 15 & UBOL & 08944 & 0.8792 \\
\hline 16 & THINK-1 & 0.8944 & 0.8771 \\
\hline 17 & ERIM_2 & 0.8942 & 0.8813 \\
\hline 18 & ATT_4 & 0.8935 & 0.8801 \\
\hline 19 & ATT 2 & 08833 & 0.8822 \\
\hline 20 & IBM & 0.8928 & 0.8823 \\
\hline 21 & ELSAGB.1 & 0.8910 & 0.8751 \\
\hline 22 & ATT 3 & 0.8207 & 0.8768 \\
\hline 23 & SYMBUS & 0.8904 & 0.8768 \\
\hline 24 & KODAK」 & 0.8904 & 0.8760 \\
\hline 25 & NESTOR & 0.8891 & 0.8769 \\
\hline 26 & THINK 2 & 0.8873 & 0.8778 \\
\hline 27 & HUGHES-1 & 0.8866 & 0.8740 \\
\hline 28 & HUGHES_2 & 0.8860 & 0.8738 \\
\hline 28 & REI & 0.8844 & 0.8768 \\
\hline 30 & NYNEX & 0.8840 & 0.8743 \\
\hline 31 & GTESS_2 & 0.8788 & 0.8620 \\
\hline 32 & GTESS -1 & 08783 & 0.8625 \\
\hline 33 & NIST _L & 0.8763 & 0.8564 \\
\hline 34 & COMCOM & 0.8686 & 0.8657 \\
\hline 35 & ASOL & 0.8683 & 0.8478 \\
\hline 36 & MIME & 0.8655 & 0.8480 \\
\hline 37 & RISO & 0.8648 & 0.8382 \\
\hline 38 & NIST 2 & 0.8643 & 0.8456 \\
\hline 39 & NIST & 0.8617 & 0.8417 \\
\hline 40 & UPENN & 0.8594 & 0.8423 \\
\hline 41 & KAMAN-1 & 0.8520 & 0.8289 \\
\hline 42 & KAMAN_3 & 0.8384 & 0.8147 \\
\hline 43 & KAMAN_2 & 0.8363 & 0.8123 \\
\hline 44 & KAMAN_S & 0.8170 & 0.7946 \\
\hline 45 & GMD_2 & 0.8163 & 0.7948 \\
\hline 46 & IFAX & 0.7945 & 0.7744 \\
\hline 47 & VALEN 2 & 0.7844 & 0.7807 \\
\hline 48 & VALEN_l & 0.7888 & 0.7662 \\
\hline 48 & KAMAN_4 & 0.7713 & 0.7463 \\
\hline
\end{tabular}

Table 56: GMD_4 correlation graph key for digits. 


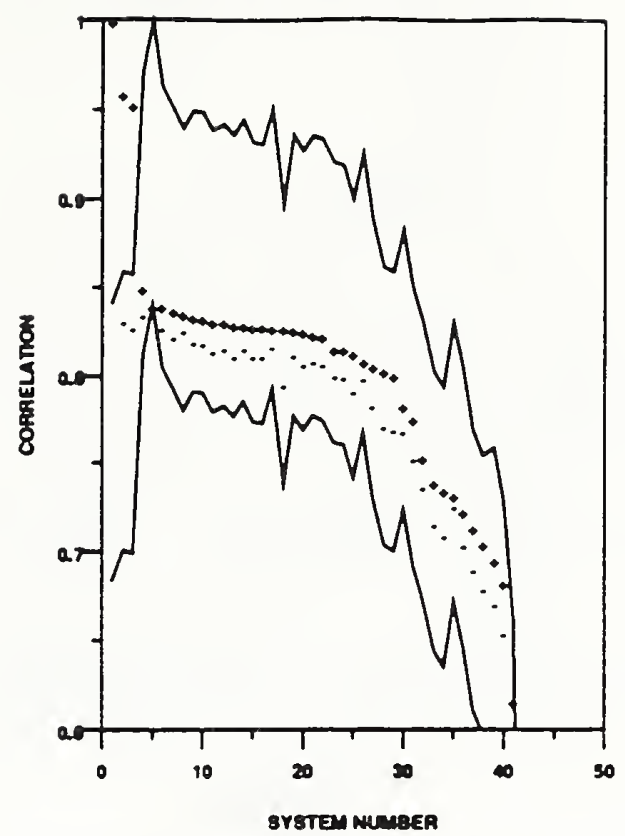

Figure 89: GMD_4 - upper case correlation

\begin{tabular}{|c|c|c|c|}
\hline Syolem Number & System Name & Corfelation (ail) & Correlation (correct) \\
\hline$T$ & GMD_4 & 1.0000 & 1.0000 \\
\hline 2 & GMD_I & 0.8595 & 0.8318 \\
\hline 3 & GMDS & 0.9532 & 0.8280 \\
\hline 4 & VOTE_M & 0.8513 & 0.8338 \\
\hline s & REFERENCE & 08415 & $0.841 \mathrm{~s}$ \\
\hline 6 & $A E G$ & 08409 & 0.8282 \\
\hline 7 & AT T 44 & 0.8386 & 0.8230 \\
\hline 8 & VOTEP $P$ & $0.836 \theta$ & 0.8266 \\
\hline$\theta$ & UMICH_L & 0.8344 & 0.8127 \\
\hline 10 & ERIM-1 & 0.8341 & 0.8182 \\
\hline 11 & UBOL & 0.8323 & 0.8144 \\
\hline 12 & NESTOR & 0.8318 & 0.8168 \\
\hline 13 & ATT $\perp$ & 0.8304 & 0.8115 \\
\hline 14 & ATT 2 & 08303 & 0.8164 \\
\hline 15 & ATT & 0.8284 & 0.8118 \\
\hline$: 6$ & KODAKد & 0.8292 & 0.8118 \\
\hline 17 & NYNEX & 0.8288 & 08174 \\
\hline 18 & NIST -4 & 0.8282 & 0.7957 \\
\hline 19 & IBM & 0.8277 & 08124 \\
\hline 20 & SYMBUS & 08270 & 08070 \\
\hline 21 & HUGHES 1 & 0.8246 & 0.8089 \\
\hline 22 & HUG HES_2 & 0.8241 & 0.8076 \\
\hline 23 & GTESS-1 & 08160 & 0.8004 \\
\hline 24 & GTESS2 & 0.8161 & 0.7996 \\
\hline 25 & MIME & 0.8140 & 0.7213 \\
\hline 26 & OCRSYS & 0.8090 & 07988 \\
\hline 27 & ASOL & 0.8061 & 0.7836 \\
\hline 28 & NIST_I & 0.8035 & 0.7711 \\
\hline 29 & RISO & 0.8009 & 0.7627 \\
\hline 30 & REI & 0.7834 & 0.7688 \\
\hline 31 & KAMAN_1 & 0.7763 & 0.7532 \\
\hline 32 & נב NIST & 0.7542 & 0.7370 \\
\hline 33 & KAMAN_J & 0.7406 & 0.7168 \\
\hline 34 & KAMAN -2 & 0.7360 & 07097 \\
\hline 35 & COMCOM & 0.7324 & 07261 \\
\hline 36 & IFAX & 0.7240 & 0.7042 \\
\hline 37 & NIST_2 & 07142 & 0.6904 \\
\hline 38 & GMD_2 & 0.7051 & 0.6796 \\
\hline 39 & VALEN_I & 0.6965 & 0.6716 \\
\hline 40 & KAMAN-4 & 0.6836 & 0.6550 \\
\hline 41 & KAMAN_S & 06171 & 0.5928 \\
\hline 42 & $\mathrm{UMICH} 2$ & 0.0605 & 0.0158 \\
\hline
\end{tabular}

Table 57: GMD_4 correlation graph key for uppers. 


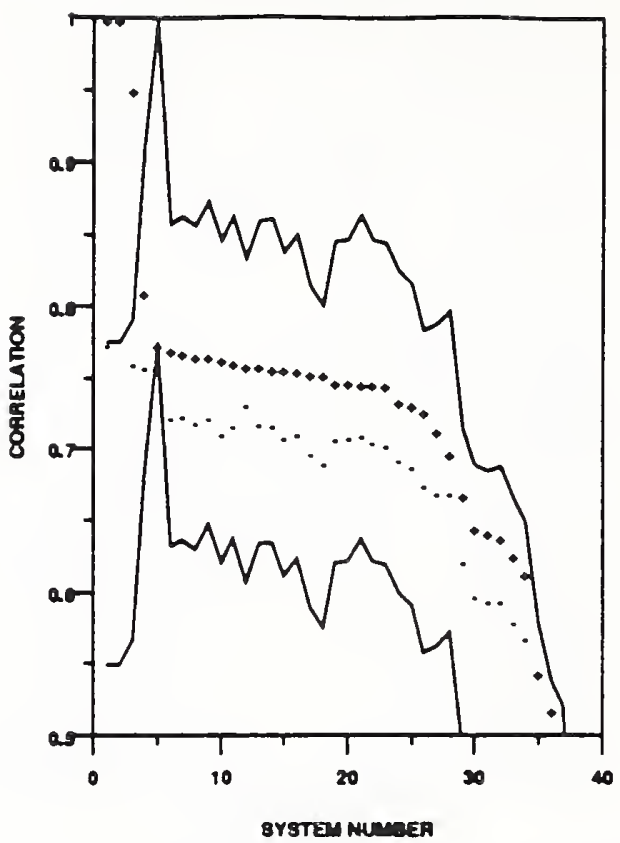

Figure 90: GMD_4 - lower case correlation

\begin{tabular}{|c|c|c|c|}
\hline System Number & System Name & Correlation (all) & Correlation (correct) \\
\hline$i$ & GMD_A & 1.0000 & 1.0000 \\
\hline 2 & GMD_1 & 1.0000 & 0.7746 \\
\hline 3 & GMD_3 & 0.9522 & 0.7813 \\
\hline 4 & VOTE_M & 0.8117 & 0.7391 \\
\hline 3 & REFERENCE & 0.7746 & 0.7746 \\
\hline 6 & ATT -4 & 0.7718 & 0.7232 \\
\hline 7 & ERIM-1 & 0.7893 & 0.7247 \\
\hline 8 & KODAK」 & 0.7673 & 0.7199 \\
\hline 9 & AEG & 0.7668 & 0.7235 \\
\hline 10 & UBOL & 0.7644 & 0.7122 \\
\hline 11 & ATT $\lrcorner$ & 0.7623 & 0.7176 \\
\hline 12 & VOTE_P & 0.7802 & 0.7325 \\
\hline 13 & ATT 2 & 0.7597 & 0.7183 \\
\hline 14 & NYNEX & 0.7382 & $0.71 \mathrm{B2}$ \\
\hline 15 & ATTS & 0.7574 & 0.7100 \\
\hline 16 & UMICH-1 & 0.7571 & 07117 \\
\hline 17 & NIST_1 & 0.7542 & 0.6981 \\
\hline 18 & NIST - 4 & 0.7541 & 06915 \\
\hline 19 & IBM & 07489 & 0.7088 \\
\hline 20 & NESTOR & 07486 & 0.7095 \\
\hline 21 & OCRSYS & 0.7473 & 0.7110 \\
\hline 22 & HUGHES_- & 0.7472 & 0.7057 \\
\hline 23 & HUGHES-2 & 0.7439 & 0.7040 \\
\hline 24 & GTESS -1 & 0.7352 & 0.6931 \\
\hline 23 & GTESS 2 & 0.7327 & 0.6888 \\
\hline 26 & RISO & 0.7288 & 0.6768 \\
\hline 27 & ASOL & 0.7146 & 0.6706 \\
\hline 28 & בנ NIST & 0.6983 & 0.6709 \\
\hline 29 & GMD 22 & 0.6692 & 0.6226 \\
\hline 30 & $K A M A N-1$ & 0.6470 & 0.5987 \\
\hline 31 & VALEN-1 & 0.8128 & 0.5945 \\
\hline 32 & NIST 2 & 0.6398 & 0.5954 \\
\hline 33 & KAMAN_J & 0.6270 & 0.5795 \\
\hline 34 & KAMAN-2 & 06148 & 0.5884 \\
\hline 35 & KAMAN_S & 0.5438 & 0.5020 \\
\hline 36 & KAMAN_4 & 05192 & 04757 \\
\hline 37 & COMCOM & 0.4622 & 0.4513 \\
\hline 38 & $\mathrm{UMICH}_{2}$ & 0.1098 & 0.0478 \\
\hline
\end{tabular}

Table 58: GMD_4 correlation graph key for lowers. 
SYSTEM: GTESS 1

PARTICIPANT: Dr. Vadim Anshelevich

ORGANIZATION: GTESS CORPORATION, Richardson, IX

PREPROCESSING: size normalization, deskewing, and dimension reduction.

FEATURES: vectors from non-linear transformations, result is 200-400 dimensional vector.

CLASSIFICATION: MLP, training performed with variant of perceptron training algorithm modified for feed-forward network.

HARDHARE: $\quad 50 \mathrm{MHz} 486$

TRAINING: DIGITS UPPERS LOWERS DATABASE

$\begin{array}{rrrr}1 / 4 & 3 / 4 & 3 / 4 & \text { NSDB3 } \\ 4983 & 8217 & 7103 & \text { INTERNAL } \\ -70 & -70 & -70 & \text { writers }\end{array}$

STATUS: $\quad$ on time, corrected CON files 9 days late

RESULTS: -- DIGITS -- -- UPPERS -- -- LOWERS -- DATABASE

REJ. ERR. REJ. ERR. REJ. ERR. TESTDATA 1

RATE RATE-- RATE RATE-- RATE RATE--

$\begin{array}{llllll}0.00 & 0.0659 & 0.00 & 0.0801 & 0.00 & 0.1753\end{array}$

$\begin{array}{lllllll}0.10 & 0.0284 & 0.10 & 0.0374 & 0.10 & 0.1296\end{array}$

$\begin{array}{lllllll}0.20 & 0.0202 & 0.20 & 0.0186 & 0.20 & 0.0918\end{array}$

$\begin{array}{lllllll}0.30 & 0.0202 & 0.30 & 0.0169 & 0.30 & 0.0613\end{array}$

$\begin{array}{llllll}0.40 & 0.0205 & 0.40 & 0.0167 & 0.40 & 0.0575\end{array}$

$\begin{array}{lllllll}0.50 & 0.0210 & 0.50 & 0.0172 & 0.50 & 0.0580\end{array}$

OCR RATE (CPS): DIGITS UPPERS LOWERS
SYS RATE:
15.51
3.43
3.51

CPU RATE: 
SYSTEM: GTESS_1

\section{BIBLIOGRAPHY:}

The following references have been provided for this system:

none

COMMENTS: GTESS

COMPANY INFORMATION

GTESS Corporation was founded in January, 1991. It currently employs 6 people and is in the business of providing inexpensive $\mathrm{PC}$-based hand print and machine print form recognition systems. Areas of interest are: character recognition, character segmentation, form pre-processing, form post-processing (context), case independence (lower/upper), style independence (machine/hand).

\section{RECOGNITION TECHNOLOGY DESCRIPTION SUMMARY}

We use a two stage isolated character recognition engine composed of 1) reduction and normalization 2) neural classification

Instead of back propagation, we use a modified perceptron training algorithm which allows us to retrain our network in a matter of hours rather than weeks. Training and production algorithms do not require floating point, are portable, run on PC platforms without special hardware and recognize at the rate of 10-100 characters/sec on a $50 \mathrm{MHz} 486$, depending upon the alphabet. Inexpensive DSP implementations are also being developed for high performance systems.

\section{INTERPRETATION OF NIST CONFERENCE RESULTS}

We feel that our current algorithms offer an attractive compromise between reliability of recognition and economy of implementation. The Conference results indicate to us that we are able to achieve one of the best overall reliability recognition rates among the participants which relied only on NIST supplied training data.

\section{PRODUCTS}

Within the next few months, GTESS will start distributing two products:

1) A PC-based, all software form recognition subsystem;

2) A field recognition engine under Windows $3 . x$ to be used in form processing applications. 


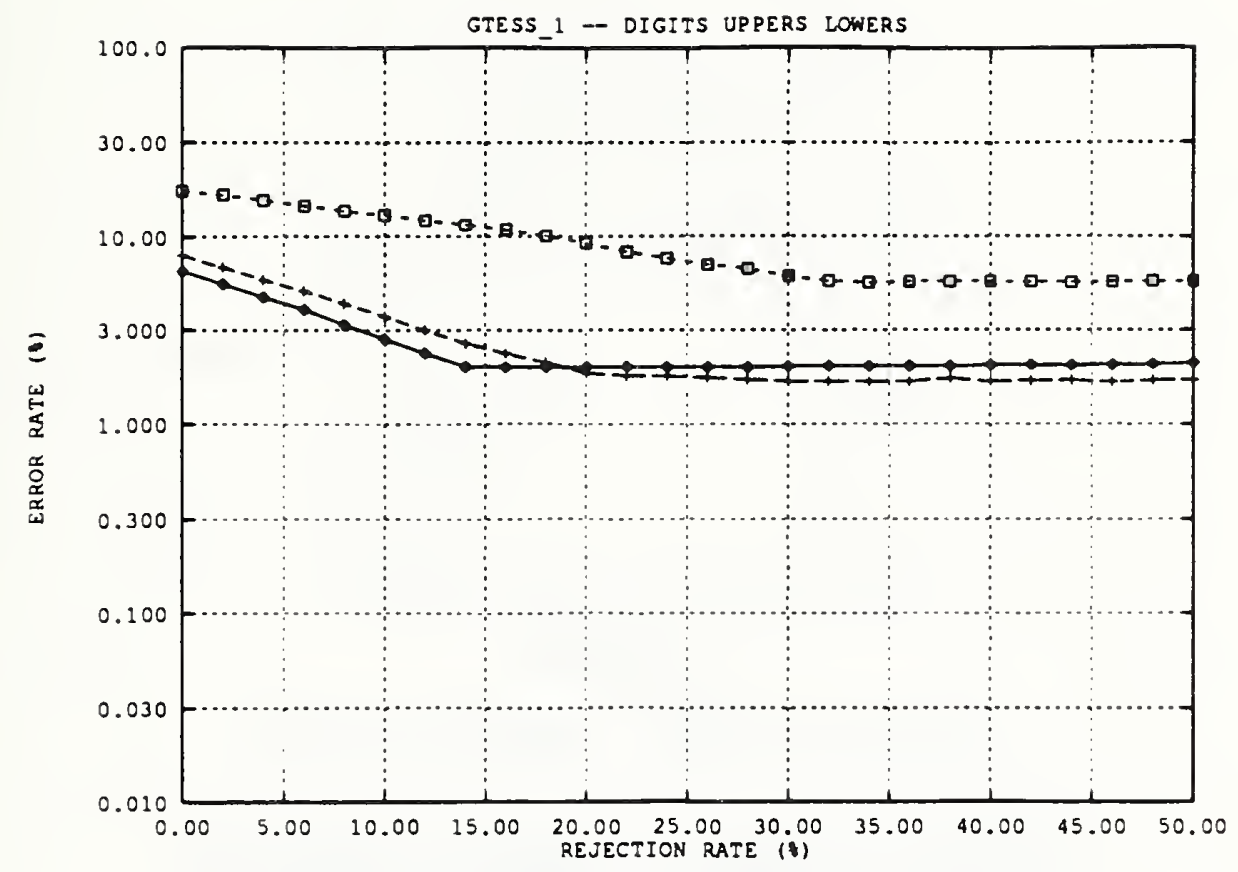

Figure 91: Error rate versus rejection rate for GTESS_1

OTESO_1

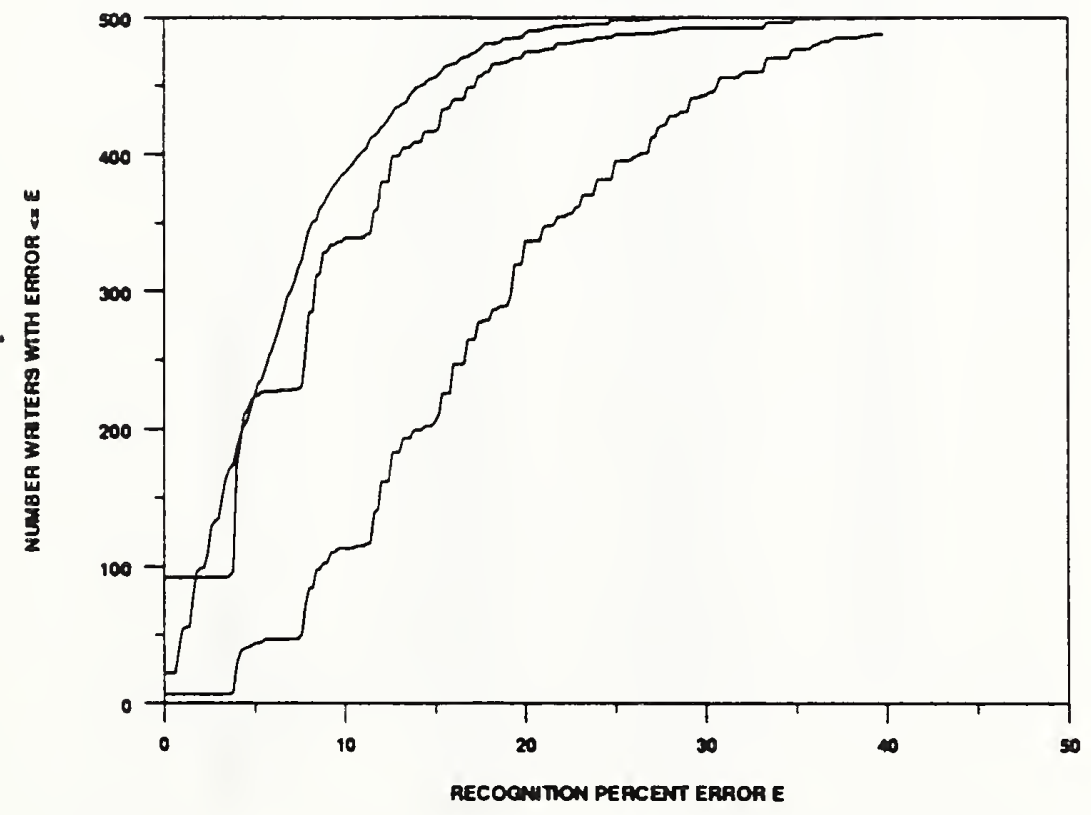

Figure 92: Error rate per writer of GTESS_1 


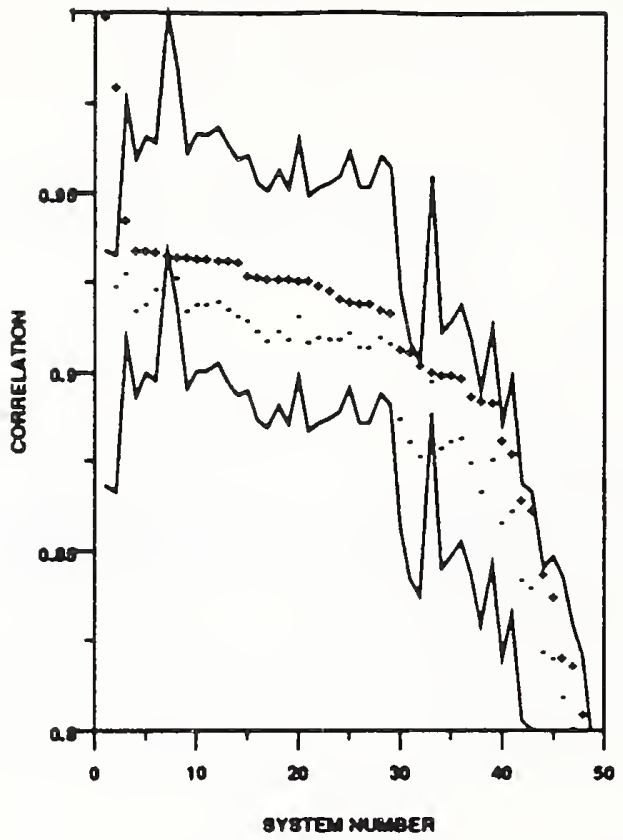

Figure 93: GTESS_1 - digit correlation

\begin{tabular}{|c|c|c|c|}
\hline System Number & System Name & Correlation (ali) & Correlation (correct) \\
\hline 1 & GTESS-1 & 1.0000 & 1.0000 \\
\hline 2 & GTESS 2 & 0.9806 & 0.9230 \\
\hline 3 & VOTE_M & 0.9435 & 0.9287 \\
\hline 4 & ATT -4 & 09358 & 0.9181 \\
\hline 3 & $A E G$ & 0.8352 & 09203 \\
\hline 6 & VOTEP & 0.9347 & 0.9244 \\
\hline 7 & REFERENCE & 0.8341 & $0.934 t$ \\
\hline 8 & OCRSYS & 0.9336 & 0.9277 \\
\hline 8 & ERIM-1 & 0.9334 & 0.9182 \\
\hline 10 & ELSAGBS & 0.9330 & 0.9203 \\
\hline 11 & ELSAGB_2 & 0.9328 & 0.8200 \\
\hline 12 & ATT 1 & 09327 & 0.9209 \\
\hline 13 & ATT 2 & 09323 & 02186 \\
\hline 14 & KODAK 2 & 0.9322 & 0.8170 \\
\hline 15 & ERIM 2 & 0.9283 & 0.9135 \\
\hline 16 & KODAK $\perp$ & $0.927 \theta$ & 0.8126 \\
\hline 17 & NIST -4 & 0.9277 & 0.8101 \\
\hline 18 & UBOL & 0.9276 & 0.8129 \\
\hline 19 & THINK_-1 & 0.9273 & 08107 \\
\hline 20 & IBM & 09272 & 0.9170 \\
\hline 21 & ELSAGB-1 & 09270 & 0.8097 \\
\hline 22 & ATT 3 & 0.9259 & 0.8111 \\
\hline 23 & SYMBUS & 0.9243 & 0.8103 \\
\hline 24 & NESTOR & 0.9222 & 0.8107 \\
\hline 23 & THINK 2 & 09213 & 0.9124 \\
\hline 26 & HUGHES 1 & 0.9207 & 0.9081 \\
\hline 27 & HUGHES_2 & 0.9207 & 0.9080 \\
\hline 28 & REI & 0.9188 & 0.9109 \\
\hline 29 & NYNEX & 0.9179 & 0.9093 \\
\hline 30 & NIST I & 0.9077 & 0.8882 \\
\hline 31 & NIST 2 & 0.9068 & 0.8816 \\
\hline 32 & NIST & 0.9032 & 0.8773 \\
\hline 33 & COMCOM & 0.9013 & 0.8986 \\
\hline 34 & ASOL & 0.9006 & 0.8799 \\
\hline 33 & MIME & 0.9004 & 0.8814 \\
\hline 36 & GMD_3 & 0.8996 & 0.8823 \\
\hline 37 & UPENN & 0.8945 & 0.8754 \\
\hline 38 & RISO & 0.8928 & 0.8677 \\
\hline 39 & GMD_1 & 0.8924 & 0.8764 \\
\hline 40 & KAMAN_1 & $0.882 t$ & 0.8593 \\
\hline 41 & GMD_-1 & 0.8783 & 0.8623 \\
\hline 42 & KAMAN_3 & 0.8653 & 0.8432 \\
\hline 43 & $K A M A N \_2$ & 0.8626 & 0.8406 \\
\hline 44 & GMD 22 & 0.8430 & 0.8226 \\
\hline 43 & KAMAN_S & 0.8382 & 0.8211 \\
\hline 46 & VALEN_2 & 0.8212 & 0.8102 \\
\hline 47 & IFAX & 0.8192 & 0.8018 \\
\hline 48 & VALEN_I & 0.8038 & 0.7899 \\
\hline 49 & $K A M A N_{-4}$ & 07946 & 0.7713 \\
\hline
\end{tabular}

Table 59: GTESS_1 correlation graph key for digits. 


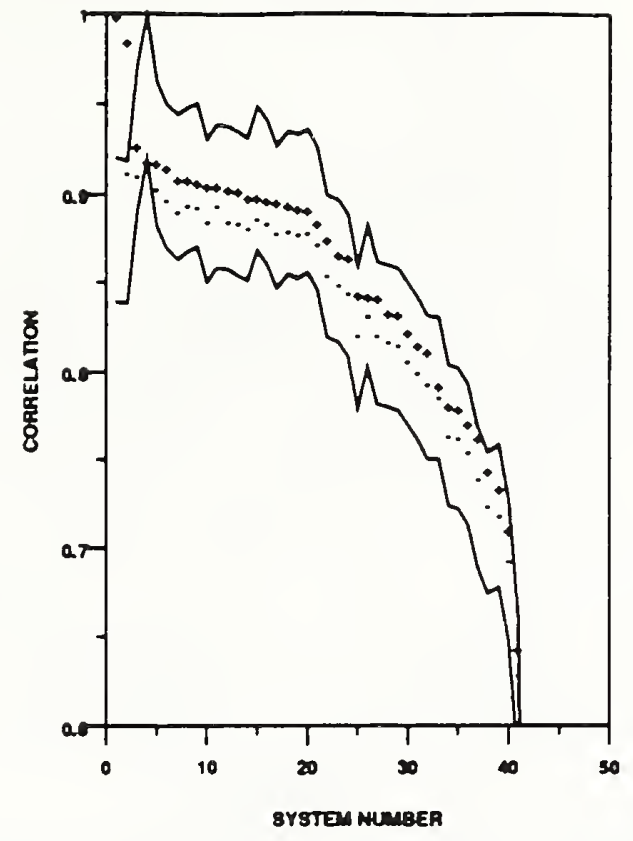

Figure 94: GTESS_1 - upper case correlation

\begin{tabular}{|c|c|c|c|}
\hline System Number & System Name & Corpelation (ali) & Cortelation (correct) \\
\hline 1 & GTESS -1 & 1.0000 & 1.0000 \\
\hline 2 & GTESS_ & 0.9864 & 0.9138 \\
\hline $\mathbf{3}$ & VOTE_M & 0.9286 & 0.8118 \\
\hline 4 & REFERENCE & 0.9190 & 0.9199 \\
\hline 3 & $A E G$ & 0.8188 & 0.8042 \\
\hline 6 & A T T -4 & 0.9168 & 0.8981 \\
\hline 7 & ERIM-I & 0.8101 & 0.8850 \\
\hline 8 & ATT 2 & 0.8101 & 0.8921 \\
\hline 9 & NYNEX & 0.8078 & 0.8942 \\
\hline 10 & KODAK」 & 0.9064 & 0.8864 \\
\hline 11 & VOTE_P & 0.9063 & 0.8958 \\
\hline 12 & UBOL & 0.9044 & 0.8865 \\
\hline 13 & ATT $\perp$ & 0.9034 & 0.8855 \\
\hline 14 & ATT & 0.8005 & 0.8824 \\
\hline 15 & UMICH.I & 0.8997 & 0.8883 \\
\hline 16 & NESTOR & 0.8885 & 0.8853 \\
\hline 17 & SYMBUS & 08977 & 0.8794 \\
\hline 18 & HUGHES_I & 0.8986 & 0.8812 \\
\hline 19 & HUGHES 2 & 08940 & 0.8791 \\
\hline 20 & IBM & 0.8926 & 0.8802 \\
\hline 21 & OCRSYS & 0.8858 & 0.8737 \\
\hline 22 & MIME & 0.8764 & 0.8568 \\
\hline 23 & NIST 4 & 0.8683 & 0.8501 \\
\hline 24 & ASOL & 0.8662 & 0.8462 \\
\hline 25 & RISO & 0.8448 & 0.8218 \\
\hline 26 & REI & 0.8142 & 0.8327 \\
\hline 27 & $N_{1 S T}$ & 0.8428 & 0.8217 \\
\hline 28 & GMD_1 & 0.8350 & 0.8186 \\
\hline 29 & GMD_3 & 0.8341 & 0.8169 \\
\hline 30 & KAMAN_1 & 0.8240 & 0.8076 \\
\hline 31 & GMD_4 & 0.8166 & 0.8004 \\
\hline 32 & NIST 3 & 0.8123 & 0.7844 \\
\hline 33 & СOMCOM & 0.7831 & 0.7873 \\
\hline 34 & IFAX & 0.7822 & 0.7648 \\
\hline 33 & KAMAN_3 & 0.7807 & 0.7643 \\
\hline 36 & KAMAN_2 & 0.7723 & 0.7554 \\
\hline 37 & NIST 2 & 0.7643 & 0.7414 \\
\hline 38 & GMD_2 & 07458 & 0.7253 \\
\hline 39 & VALEN_I & 07337 & 0.7196 \\
\hline 10 & KAMAN_A & 0.7116 & 0.6939 \\
\hline 41 & KAMAN_S & 0.6443 & 0.6303 \\
\hline 12 & $\mathrm{UMICH}_{2}$ & 0.0497 & 0.0231 \\
\hline
\end{tabular}

Table 60: GTESS_1 correlation graph key for uppers. 


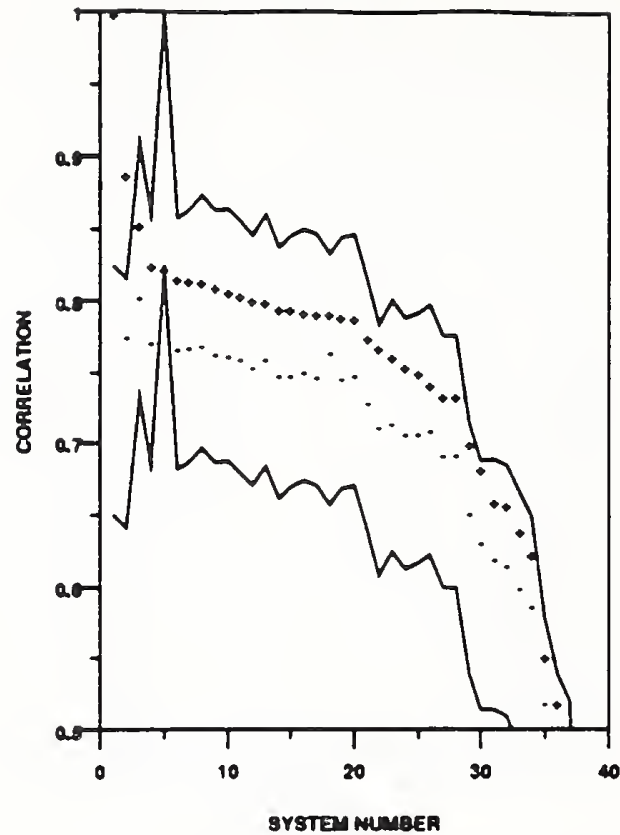

Figure 95: GTESS_1 - lower case correlation

\begin{tabular}{|c|c|c|c|}
\hline System Number & System Name & Correlation (ail) & Correlalion (correct) \\
\hline I & GTESS_l & 1.0000 & 1.0000 \\
\hline 2 & GTESS 2 & 0.8892 & 07779 \\
\hline 3 & VOTE_M & 0.8547 & 0.8048 \\
\hline 4 & ATT2 & 0.8267 & 07734 \\
\hline 3 & REFERENCE & 0.8248 & 0.8248 \\
\hline 6 & ATT_4 & 0.8176 & 0.7678 \\
\hline 7 & ERIM_l & 0.8167 & 0.7691 \\
\hline 8 & $A E G$ & 0.8148 & 0.7702 \\
\hline 9 & ATT 1 & 0.8114 & 07647 \\
\hline 10 & OCRSYS & 0.8083 & 0.7641 \\
\hline 11 & KODAKA & 0.8038 & 0.7612 \\
\hline 12 & IBM & 0.8023 & 0.7556 \\
\hline 13 & NYNEX & 0.8015 & 0.7619 \\
\hline 14 & ATT 3 & 0.7972 & 0.7496 \\
\hline is & UBOL & 0.7965 & 0.7504 \\
\hline 16 & UMICH_l & 0.7943 & 0.7517 \\
\hline 17 & HUGHES-1 & 0.7937 & 07493 \\
\hline 18 & VOTE_P & 0.7931 & 0.7882 \\
\hline 19 & HUGHES_2 & 0.7917 & 07473 \\
\hline 20 & NESTOR & 0.7898 & 07498 \\
\hline 21 & NIST -1 & 0.7783 & 0.7307 \\
\hline 22 & RISO & 07690 & 07131 \\
\hline 23 & NIST_A & 0.7628 & 0.7133 \\
\hline 24 & ASOL & 0.7361 & 0.7084 \\
\hline 25 & GMD_3 & 07515 & 0.7084 \\
\hline 26 & SIST & 0.7429 & 0.7107 \\
\hline 27 & GMD_4 & 07352 & 0.6931 \\
\hline 28 & GMD_1 & 0.7382 & 0.6931 \\
\hline 29 & GMD_2 & 0.7016 & 08537 \\
\hline 30 & NIST 2 & 0.6843 & 06331 \\
\hline 31 & $K_{A M A N}-1$ & 0.6619 & 0.6208 \\
\hline 32 & VALEN_L & 0.6587 & 06168 \\
\hline 33 & $K A M A N, 3$ & 0.6413 & 0.6007 \\
\hline 34 & KAMAN-2 & 0.8245 & 0.5880 \\
\hline 35 & KAMAN_5 & 0.5527 & 0.5203 \\
\hline 36 & KAMAN-4 & 0.5203 & 0.4888 \\
\hline 37 & COMCOM & 0.4873 & 0.4757 \\
\hline 38 & $\mathrm{UMICH}_{2}$ & 0.1026 & 0.0318 \\
\hline
\end{tabular}

Table 61: GTESS_1 correlation graph key for lowers. 
SYSTEM: GTESS_2

PARTICIPANT: Dr. Vadim Anshelevich

ORGANIZATION: GTESS CORPORATION, RichardsOn, TX

FEATURES: vectors from non-linear transformations

CLASSIFICATION : MLP

HARDWARE: $\quad 50 \mathrm{MHz} 486$

TRAINING: DIGITS UPPERS LONERS DATABASE

$\begin{array}{llll}1 / 4 & 3 / 4 & 3 / 4 & \text { NSDB3 }\end{array}$

$\begin{array}{rrrr}4983 & 8217 & 7103 & \text { INTERNAL } \\ -70 & -70 & -70 & \text { writers }\end{array}$

STATUS: on time, corrected con files 9 days late

RESULTS: -- DIGITS -- -- UPPERS -- -- LOWERS -- DATABASE

REJ. ERR. REJ. ERR. REJ. ERR. TESTDATA1

RATE RATE-- RATE RATE-- RATE RATE--

$\begin{array}{lllllll}0.00 & 0.0675 & 0.00 & 0.0814 & 0.00 & 0.1842\end{array}$

$\begin{array}{lllllll}0.10 & 0.0301 & 0.10 & 0.0381 & 0.10 & 0.1358\end{array}$

$\begin{array}{lllllll}0.20 & 0.0188 & 0.20 & 0.0198 & 0.20 & 0.0992\end{array}$

$\begin{array}{lllllll}0.30 & 0.0189 & 0.30 & 0.0176 & 0.30 & 0.0684\end{array}$

$\begin{array}{lllllll}0.40 & 0.0194 & 0.40 & 0.0173 & 0.40 & 0.0515\end{array}$

$\begin{array}{lllllll}0.50 & 0.0203 & 0.50 & 0.0176 & 0.50 & 0.0522\end{array}$

OCR RATE (CPS): DIGITS

UPPERS LOWERS
SYS RATE:
18.80
3.37
3.39

CPU RATE: 
SYSTEM: GTESS 2

BIBLIOGRAPHY:

The following references have been provided for this system:

none 


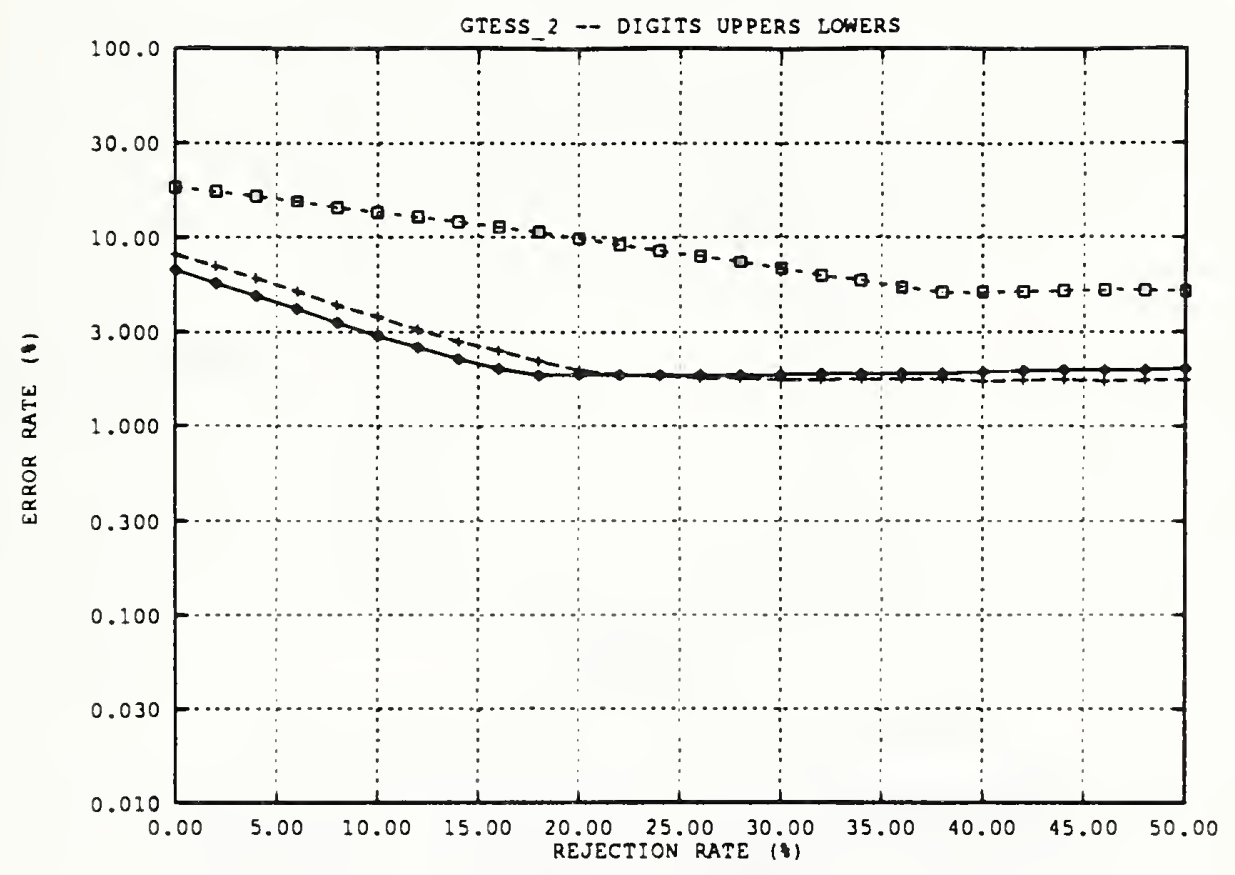

Figure 96: Error rate versus rejection rate for GTESS_2

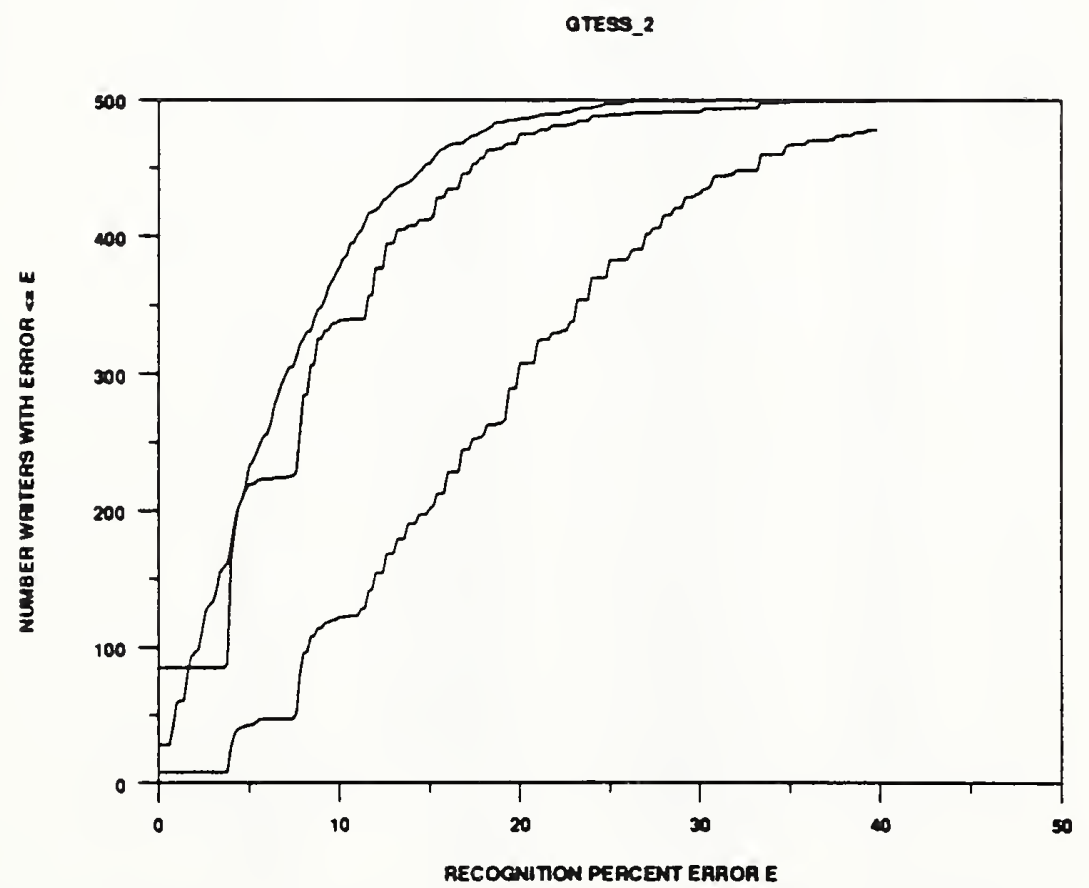

Figure 97: Error rate per writer of GTESS_2 


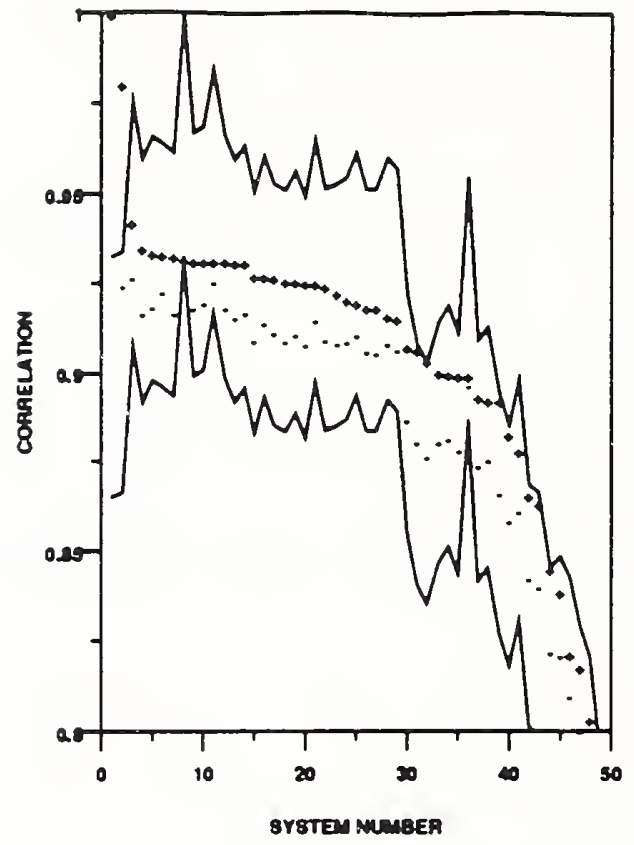

Figure 98: GTESS_2 - digit correlation

\begin{tabular}{|c|c|c|c|}
\hline System Number & System Name & Corretation (ali) & Correlation (correct) \\
\hline 1 & GTESS 2 & 1.0000 & 1.0000 \\
\hline 2 & GTESS_1 & 0.9806 & 0.9250 \\
\hline 3 & VOTE_M & 0.9429 & 0.9277 \\
\hline 4 & ATT_A & 0.9356 & 0.8175 \\
\hline 5 & $A E G$ & 0.9346 & 0.2193 \\
\hline 6 & VOTE_P & 0.9341 & 0.9234 \\
\hline 7 & ERIM-1 & 0.9333 & 0.9175 \\
\hline 8 & REFERENCE & 0.9323 & 0.9325 \\
\hline 9 & ATT_ & 0.9322 & 0.9200 \\
\hline 10 & ELSAGBA & 0.9322 & 0.8189 \\
\hline 11 & OCRSYS & 0.9320 & 0.9261 \\
\hline 12 & ELSAGB2 & 0.8319 & 0.9186 \\
\hline 13 & ATT2 2 & 0.9318 & 0.9176 \\
\hline 14 & KODAK 2 & 0.2318 & 0.9162 \\
\hline 15 & ERIM 2 & 0.9280 & 0.9147 \\
\hline 16 & NIST -4 & 0.9280 & 0.9096 \\
\hline 17 & KODAK_ & 0.9275 & 0.9119 \\
\hline 18 & THINK_I & 09268 & 09098 \\
\hline 19 & UBOL & 0.9265 & 0.9116 \\
\hline 20 & ELSAGB-L & 09263 & 0.9087 \\
\hline 21 & IBM & 09262 & 0.9157 \\
\hline 22 & ATT 3 & 0.9252 & 0.9101 \\
\hline 23 & SYMBUS & 0.9234 & 0.9093 \\
\hline 24 & NESTOR & 0.9215 & 09096 \\
\hline 25 & THINK 2 & 0.9206 & 0.2113 \\
\hline 26 & HUGHES-1 & 0.9193 & 0.9067 \\
\hline 27 & HUGHES.2 & 0.9192 & 0.9066 \\
\hline 28 & REI & 0.9169 & 0.9092 \\
\hline 29 & NYNEX & 0.9159 & 0.9074 \\
\hline 30 & NIST_I & 0.9080 & 0.8878 \\
\hline 31 & NIST 2 & 0.9071 & 0.8810 \\
\hline 32 & NIST_3 & 0.9043 & 0.8772 \\
\hline 33 & MIME & 0.9007 & 08809 \\
\hline 34 & GMD_3 & 0.9002 & 0.8821 \\
\hline 35 & ASOL & 0.9001 & 08790 \\
\hline 36 & COMCOM & 0.9000 & 08972 \\
\hline 37 & UPENN & 0.8941 & 08748 \\
\hline 38 & GMD.L & 08931 & 08761 \\
\hline 39 & RISO & 0.8930 & 0.8669 \\
\hline 40 & KAMAN - L & 0.8833 & 0.8593 \\
\hline 41 & GMD_4 & 0.8788 & 0.8620 \\
\hline 42 & KAMAN_3 & 0.8662 & 0.8432 \\
\hline 43 & KAMAN_2 & 08642 & 0.8408 \\
\hline 44 & GMD_2 & 0.8457 & 0.8225 \\
\hline 45 & KAMAN_S & 0.8392 & 0.8212 \\
\hline 16 & VALEN_? & 0.8220 & 0.8102 \\
\hline 47 & IFAX & 0.8183 & 0.8007 \\
\hline 48 & VALEN_1 & 0.8040 & 0.7884 \\
\hline 49 & KAMAN_- & 0.7963 & 0.7715 \\
\hline
\end{tabular}

Table 62: GTESS_2 correlation graph key for digits. 


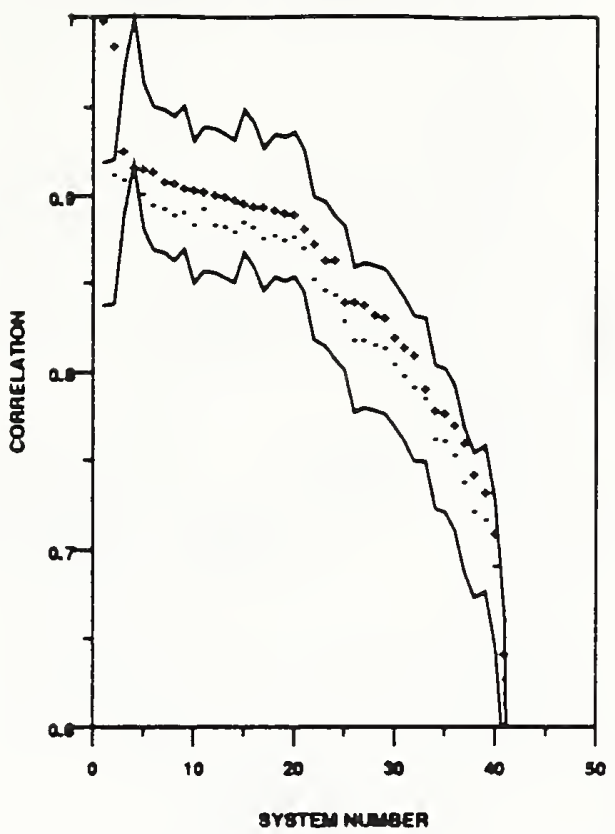

Figure 99: GTESS_2 - upper case correlation

\begin{tabular}{|c|c|c|c|}
\hline System Number & System Name & Correlation (ali) & Correlation (correct) \\
\hline I & GTESS 2 & 1.0000 & 1.0000 \\
\hline 2 & GTESS_l & 0.9864 & 0.9139 \\
\hline 3 & VOTEM & 09274 & 0.8107 \\
\hline 4 & REFERENCE & 0.9186 & 0.9186 \\
\hline 3 & $A E G$ & 0.9172 & 0.8024 \\
\hline 6 & ATT_4 & 0.9137 & 08967 \\
\hline 7 & ERIM-1 & 0.9101 & 0.8942 \\
\hline 8 & ATT 2 & 0.9091 & 0.8912 \\
\hline 9 & NYNEX & 0.9085 & 0.8928 \\
\hline 10 & KODAK_ & 0.8080 & 0.8854 \\
\hline 11 & VOTE_P & 0.9044 & 0.8943 \\
\hline 12 & UBOL & 0.9031 & 0.8849 \\
\hline 13 & ATT_ & 0.2017 & 0.8841 \\
\hline 14 & ATT 3 & 08999 & 0.8815 \\
\hline 13 & UMICH_L & 0.8986 & 0.8869 \\
\hline 16 & NESTOR & 0.8968 & 0.8840 \\
\hline 17 & SYMBUS & 08985 & 0.8781 \\
\hline 18 & HUGHES_1 & 0.8946 & 0.8798 \\
\hline 18 & HUGHES_2 & 0.8924 & 0.8775 \\
\hline 20 & 18M & 0.8914 & 0.8791 \\
\hline 21 & OCRSYS & 0.8839 & 0.8723 \\
\hline 22 & MIME & 0.8731 & 0.8546 \\
\hline 23 & NIST -4 & 08663 & 0.8488 \\
\hline 24 & ASOL & 08658 & 0.8435 \\
\hline 25 & REI & 08426 & 0.8312 \\
\hline 26 & RISO & 0.8425 & 0.8204 \\
\hline 27 & NIST_l & 0.8404 & 0.8203 \\
\hline 28 & GMD_l & 08345 & 08178 \\
\hline 29 & GMD_3 & 08331 & 0.8158 \\
\hline 30 & KAMAN_1 & 0.8223 & 0.8063 \\
\hline 31 & GMD_4 & 0.8161 & 0.7996 \\
\hline 32 & NIST_3 & 0.8117 & 0.7934 \\
\hline 33 & COMCOM & 07930 & 0.7870 \\
\hline 34 & IFAX & 07808 & 0.7640 \\
\hline 35 & KAMAN_J & 07792 & 0.7633 \\
\hline 36 & KAMAN_2 & 0.7722 & 0.7546 \\
\hline 37 & NIST 2 & 0.7625 & 07406 \\
\hline 38 & GMD_2 & 0.7450 & 0.7239 \\
\hline 39 & VALEN_L & 0.7350 & 0.7187 \\
\hline 40 & KAMAN-4 & 0.7117 & 0.6933 \\
\hline 41 & KAMAN_S & 0.6434 & 0.6294 \\
\hline 42 & $\mathrm{UMICH}_{2}$ & 00491 & 0.0229 \\
\hline
\end{tabular}

Table 63: GTESS_2 correlation graph key for uppers. 


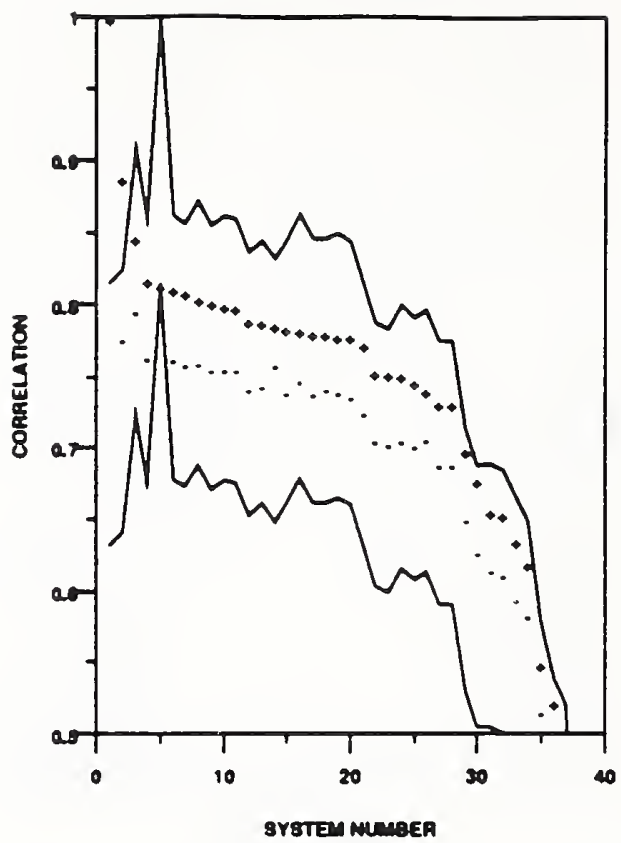

Figure 100: GTESS_2 - lower case correlation

\begin{tabular}{|c|c|c|c|}
\hline System Number & System Name & Correlation (all) & Correistion (correct) \\
\hline $\mathrm{I}$ & GTESS 2 & 1.0000 & 1.0000 \\
\hline 2 & GTESS_I & 0.8892 & 0.7779 \\
\hline 3 & VOTE_M & 0.8470 & 0.7968 \\
\hline 4 & ATT 2 & 0.8184 & 0.7682 \\
\hline 5 & REFERENCE & 0.8138 & 0.8138 \\
\hline 6 & ERIM_l & 0.8125 & 0.7641 \\
\hline 7 & ATT_4 & 0.8112 & 0.7603 \\
\hline 8 & $A E G$ & 0.8067 & 0.7617 \\
\hline 9 & KODAK- & 0.8040 & 0.7373 \\
\hline 10 & ATT」 & 0.8018 & 0.7363 \\
\hline 11 & NYNEX & 0.8000 & 0.7370 \\
\hline 12 & ATT 3 & 0.7918 & 0.7431 \\
\hline 13 & IBM & 0.7900 & 0.7486 \\
\hline 14 & VOTEP & 0.7878 & 0.7601 \\
\hline is & UBOL & 0.7831 & 0.7410 \\
\hline 16 & OCRSYS & 0.7843 & 0.7483 \\
\hline 17 & NESTOR & 0.7822 & 0.7429 \\
\hline 18 & HUGHES_l & 0.7822 & 07393 \\
\hline 19 & UMICH.I & 0.7799 & 07407 \\
\hline 20 & HUGHES_2 & 0.7796 & 0.7370 \\
\hline 21 & NIST 1 & 0.7740 & 07262 \\
\hline 22 & ASOL & 0.7547 & 0.7038 \\
\hline 23 & RISO & 0.7338 & 0.7039 \\
\hline 24 & NIST_4 & 0.7323 & 0.7067 \\
\hline 23 & GMD_3 & 0.7474 & 0.7032 \\
\hline 26 & NIST & 0.7413 & 07079 \\
\hline 27 & GMD_4 & 0.7327 & 0.6888 \\
\hline 28 & GMD -1 & 0.7327 & 06888 \\
\hline 29 & GMD _2 & 0.6992 & 0.6307 \\
\hline 30 & NIST 2 & 0.6783 & 0.6279 \\
\hline 31 & KAMAN -1 & 0.6371 & 0.6137 \\
\hline 32 & VALEN_I & 0.6830 & 0.6117 \\
\hline 33 & KAMAN_3 & 0.6363 & 0.3949 \\
\hline 34 & KAMAN_2 & 0.6198 & 0.5828 \\
\hline 33 & KAMAN_S & 0.5490 & 0.3136 \\
\hline 36 & KAMAN_4 & 0.3224 & 0.4880 \\
\hline 37 & COMCOM & 0.4830 & 0.4712 \\
\hline 38 & $\mathrm{UMICH}_{2}$ & 0.1070 & 0.0342 \\
\hline
\end{tabular}

Table 64: GTESS_2 correlation graph key for lowers. 
SYSTEM: HUGHES_ 1

PARTICIPANT: Tony Baraghimian

ORGANIZATION: Hughes Aircraft Company, Canoga Park, CA

FEATURES : ?

CLASSIFICATION: fusion of results of multiple nonparametric algorithms (neocognitron)

HARDHARE: single Intel 1860 in a Datacube computer

TRAINING: DIGITS UPPERS LOHERS DATABASE

$\begin{array}{llll}10000 & 7800 & 7800 & \text { NSDB3 }\end{array}$

STATUS: $\quad$ on time

RESULTS: -- DIGITS -- -- UPPERS -- -- LOWERS -- DATABASE

REJ. ERR. REJ. ERR. REJ. ERR. TESTDATA1

RATE RATE-- RATE RATE-- RATE RATE--

$\begin{array}{lllllll}0.00 & 0.0484 & 0.00 & 0.0646 & 0.00 & 0.1539\end{array}$

$\begin{array}{lllllll}0.10 & 0.0173 & 0.10 & 0.0301 & 0.10 & 0.1129\end{array}$

$\begin{array}{lllllll}0.20 & 0.0064 & 0.20 & 0.0169 & 0.20 & 0.0806\end{array}$

$\begin{array}{lllllll}0.30 & 0.0036 & 0.30 & 0.0105 & 0.30 & 0.0529\end{array}$

$\begin{array}{llllll}0.40 & 0.0022 & 0.40 & 0.0071 & 0.40 & 0.0362\end{array}$

$\begin{array}{lllllll}0.50 & 0.0015 & 0.50 & 0.0055 & 0.50 & 0.0270\end{array}$

OCR RATE (CPS): DIGITS UPPERS LOWERS

SYS RATE:

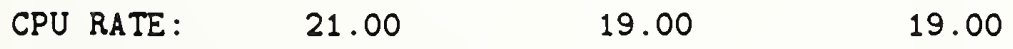

NOTE: proprietary architecture using a neural net classifier. Few details of recognition algorithm provided. 


\section{SYSTEM: HUGHES_1}

\section{BIBLIOGRAPHY:}

The following references have been provided for this system:

[19]

\section{COMMENTS: HUGHES}

HUGHES Recognition Systems brings complete document image processing solutions based on its proven success in advanced imaging and recognition technology. We provide a wide range of technology, solutions, and services from system analysis through system integration, training, and support.

HUGHES develops sophisticated subsystem solutions easily tailorable to your application for preprocessing, intelligent recognition, contextual analysis, and more. We accommodate image lift from a variety of sources directly into our pre-processing subcomponent. We apply unique pre-processing techniques such as image quality control, registration, and enhancement, as well as form identification, suppression, and field isolation. The result feeds immediately in to HIGHES' intelligent recognition subcomponent, or any other you provide. With technologies such as artificial networks and fuzzy logic, our pre-processing in concert with our intelligent recognizer provides maximum performance. The flexible pre-processing also enables higher performance of your own recognition system. Further enhancements to recognition performance is accomplished by contextual analysis in our post-processing subcomponent.

We also offer traditional subsystems for image acquisition, format conversion, work flow, forms editing, image storage, and much more.

HUGHES Recognition Systems participating in the First Census OCR Systems Conference in May 1992. Our test results were highly competitive, among the top performing group of participants.

For more information, please contact Tony Baraghimian at (818) 702-1580. 


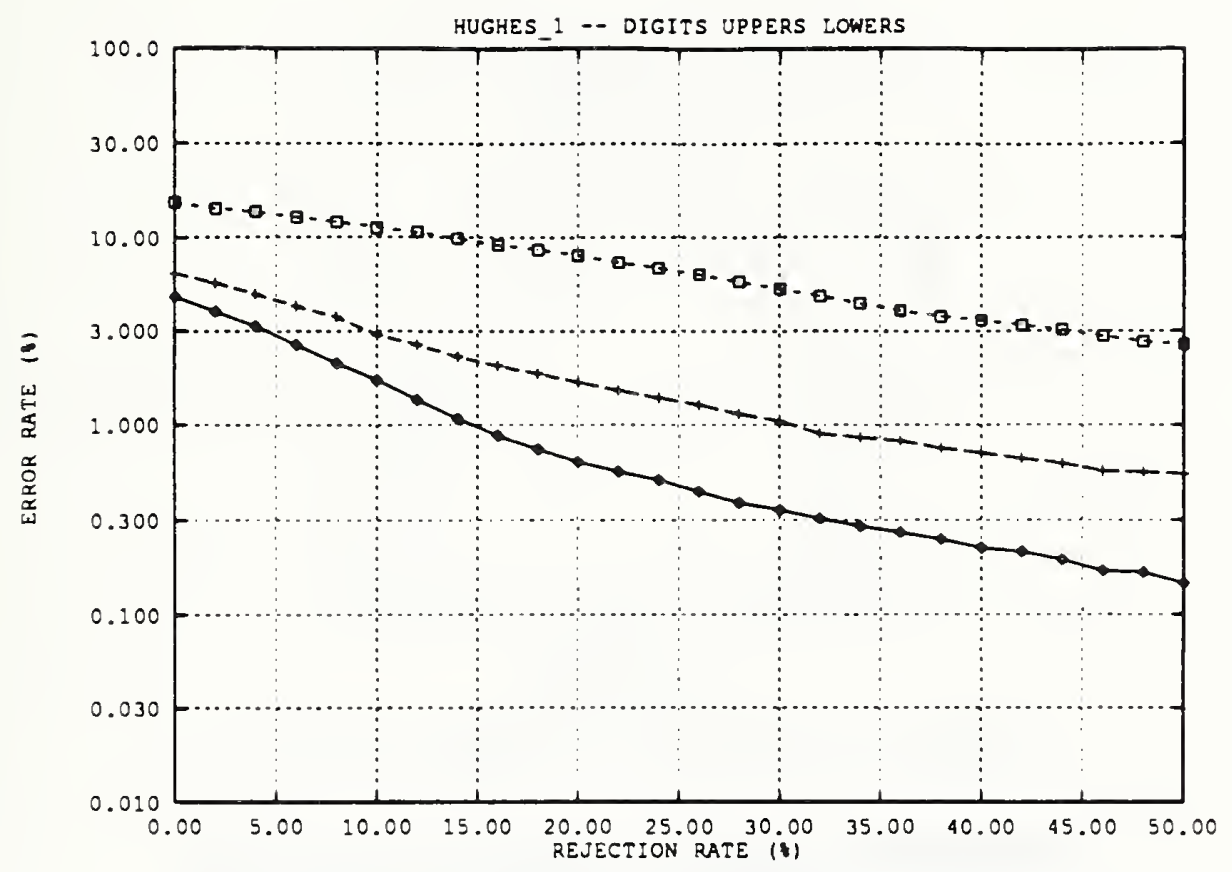

Figure 101: Error rate versus rejection rate for HUGHES_1

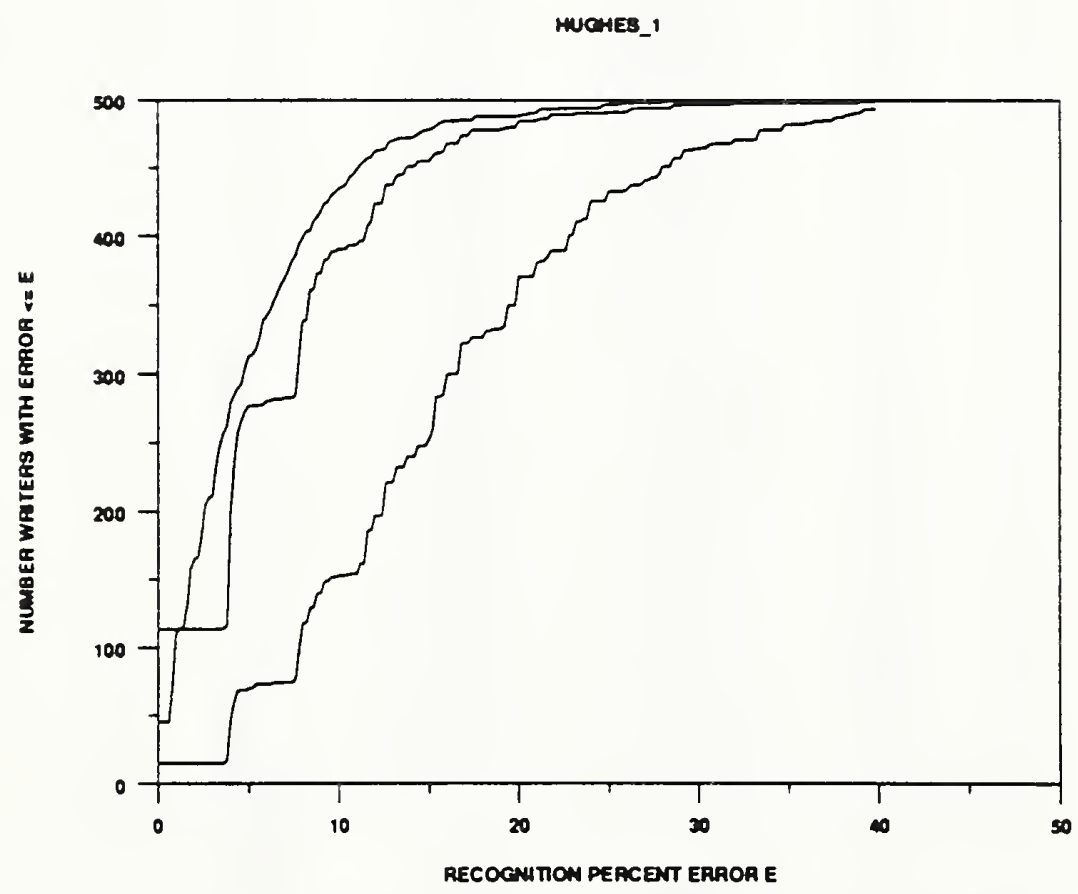

Figure 102: Error rate per writer of HUGHES_1 


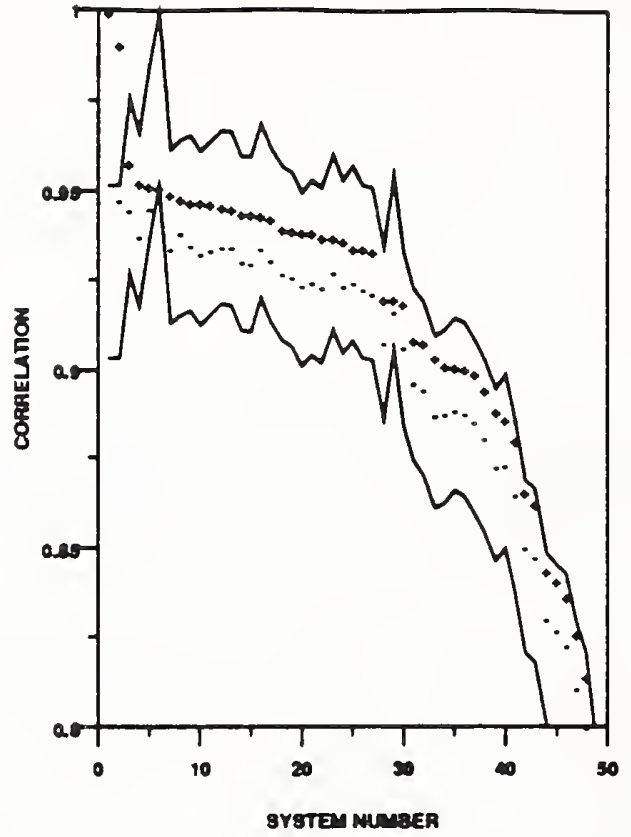

Figure 103: HUGHES_1 - digit correlation

\begin{tabular}{|c|c|c|c|}
\hline System Number & System Name & Correlation (all) & Correlation (correct) \\
\hline 1 & HUGHES_L & 1.0000 & 1.0000 \\
\hline 2 & HUGHES_2 & 0.9913 & 0.9478 \\
\hline 3 & VOTE_M & 0.8583 & 0.9448 \\
\hline 4 & $A E G$ & 0.9326 & 0.8378 \\
\hline s & OCRSYS & 0.9320 & 0.9453 \\
\hline 6 & REFERENCE & 0.9516 & 0.9516 \\
\hline 7 & ERIM_l & 0.9498 & 0.9345 \\
\hline 8 & VOTEP & 0.9483 & 0.2385 \\
\hline 8 & IBM & 0.9474 & 0.9351 \\
\hline 10 & ERIM $_{2}$ & 0.9473 & 0.9329 \\
\hline 11 & ATT 2 & 0.8468 & 0.2341 \\
\hline 12 & ELSAGBA & 0.9457 & 0.8350 \\
\hline 13 & ELSAGB_2 & 0.9453 & 0.9347 \\
\hline 14 & KODAK_ 2 & 0.9442 & 0.9306 \\
\hline 15 & ATT -4 & 0.8438 & 0.9303 \\
\hline 16 & ATT $\perp$ & 0.9435 & 0.9342 \\
\hline 17 & THINK 2 & 0.9426 & 0.9311 \\
\hline 18 & UBOL & 0.8401 & 0.9273 \\
\hline 18 & NESTOR & 0.9393 & 0.9268 \\
\hline 20 & ELSAGB_I & 0.9392 & 0.9243 \\
\hline 21 & KODAK」 & 0.9388 & 0.9231 \\
\hline 22 & REI & 0.9377 & 0.2282 \\
\hline 23 & NIST_4 & 0.9377 & 0.9237 \\
\hline 24 & SYMBU'S & 0.9366 & 0.9242 \\
\hline 25 & NYNEX & 0.9349 & 0.9230 \\
\hline 26 & ATT 3 & 0.9347 & 0.9233 \\
\hline 27 & THINK_L & 0.9341 & 0.9220 \\
\hline 28 & GTESS -1 & 0.8207 & 0.9081 \\
\hline 29 & COMCOM & 0.9206 & 0.9170 \\
\hline 30 & GTESS 2 & 0.9193 & 0.9067 \\
\hline 31 & NIST_l & 0.9091 & 0.8986 \\
\hline 32 & GMD_3 & 0.9082 & 0.8947 \\
\hline 33 & UPENN & 0.9043 & 0.8877 \\
\hline 34 & ASOL & 0.9018 & 0.8878 \\
\hline 35 & MIME & 0.9014 & 0.8890 \\
\hline 36 & GMD_l & 0.8010 & 0.8881 \\
\hline 37 & NIST 2 & 0.8993 & 0.8857 \\
\hline 38 & ה & 0.8949 & 0.8811 \\
\hline 39 & RISO & 0.8890 & 0.8732 \\
\hline 40 & GMD_- & 0.8866 & 0.8740 \\
\hline 41 & KAMAN_L & 0.8805 & 0.8653 \\
\hline 42 & KAMAN_3 & 0.8664 & 0.8508 \\
\hline 43 & KAMAN_2 & 0.8631 & 0.8480 \\
\hline 44 & KAMAN_5 & 0.8443 & 0.8304 \\
\hline 45 & GMD_2 & 0.8416 & 0.8273 \\
\hline 46 & VALEN 2 & 0.8371 & 0.8234 \\
\hline 47 & IFAX & 0.8266 & 0.8118 \\
\hline 48 & VALEN_I & 0.8144 & 0.8003 \\
\hline 49 & KAMAN-4 & 0.7894 & 0.7760 \\
\hline
\end{tabular}

Table 65: HUGHES_1 correlation graph key for digits. 


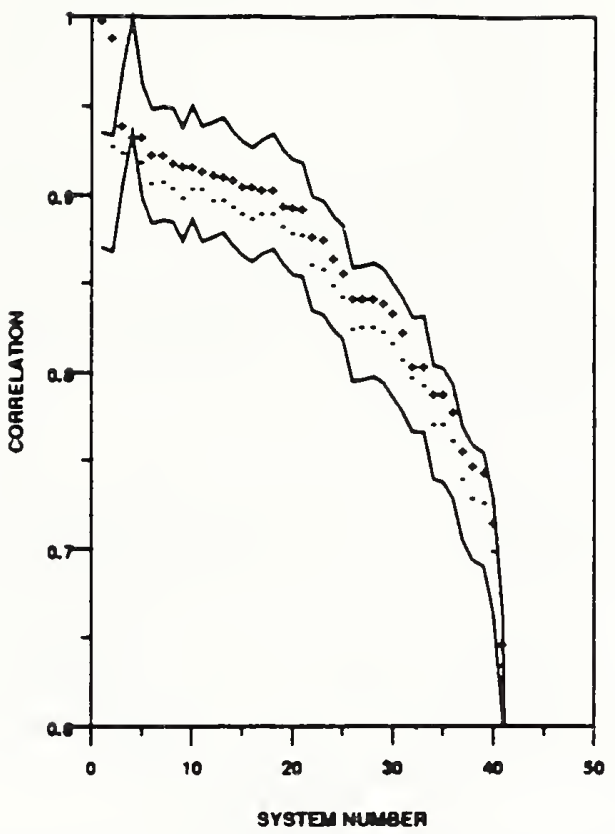

Figure 104: HUGHES_1 - upper case correlation

\begin{tabular}{|c|c|c|c|}
\hline System Number & Syolem Name & Cortelation (ail) & Cotrelation (correct) \\
\hline 1 & HUGHES_I & 1.0000 & 1.0000 \\
\hline 2 & HUGHES_2 & 0.9907 & 0.9296 \\
\hline 3 & VOTE_M & 0.8411 & 0.2234 \\
\hline 4 & REFERENCE & 0.9354 & 0.9334 \\
\hline 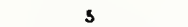 & AEG & 09348 & 0.9188 \\
\hline 6 & ERIM-1 & 09252 & 0.9083 \\
\hline 7 & ATT_A & 0.8245 & 09088 \\
\hline 8 & UMICH_L & 0.9198 & 0.9056 \\
\hline $\boldsymbol{9}$ & UBOL & 0.2188 & 08987 \\
\hline 10 & NYNEX & 0.8182 & 0.9060 \\
\hline 11 & VOTEP & 0.8158 & 0.9053 \\
\hline 12 & NESTOR & 0.8140 & 08991 \\
\hline 13 & ATT 2 & 0.9131 & 0.8993 \\
\hline 14 & IBM & 0.2114 & 08958 \\
\hline is & KODAK」 & 0.9073 & 0.8817 \\
\hline 16 & SYMBUS & 0.8070 & 0.8893 \\
\hline 17 & ATT 3 & 0.9036 & 08816 \\
\hline 18 & ATT」 & 0.8053 & 0.8821 \\
\hline 19 & OCRSYS & 0.8967 & 08848 \\
\hline 20 & GTESS_L & 0.8936 & 0.8812 \\
\hline 21 & GTESS_2 & 0.8946 & 0.8798 \\
\hline 22 & MIME & 0.8793 & 0.8631 \\
\hline 23 & NIST_t & 0.8774 & 0.8602 \\
\hline 24 & ASOL & 0.8673 & 0.8518 \\
\hline 23 & REI & 0.8591 & 0.8447 \\
\hline 26 & RISO & 0.8443 & 0.8263 \\
\hline 27 & GMD_1 & 0.8442 & 0.8272 \\
\hline 28 & NIS T_ 1 & 0.8439 & 0.8273 \\
\hline 29 & GMD_3 & 0.8416 & 08230 \\
\hline 30 & KAMAN_I & 0.8361 & 0.8184 \\
\hline 31 & GMD_4 & 0.8246 & 0.8088 \\
\hline 32 & COMCOM & 0.8053 & 0.7986 \\
\hline 33 & NIST 3 & 0.3051 & 0.7847 \\
\hline 34 & IFAX & 0.7898 & 07727 \\
\hline 35 & KAMAN_3 & 0.7894 & 07728 \\
\hline 36 & KAMAN_2 & 0.7798 & 0.7633 \\
\hline 37 & NIST? & 0.7573 & 0.7421 \\
\hline 38 & VALEN_1 & 0.7496 & 0.7308 \\
\hline 39 & GMD_2 & 0.7457 & 0.7283 \\
\hline 40 & $\mathrm{KAMAN}_{-4}$ & 0.7174 & 0.7010 \\
\hline 41 & KAMAN_S & 0.6481 & 0.6360 \\
\hline 42 & $\mathrm{UMICH}_{2}$ & 0.0438 & 0.0204 \\
\hline
\end{tabular}

Table 66: HUGHES_1 correlation graph key for uppers. 


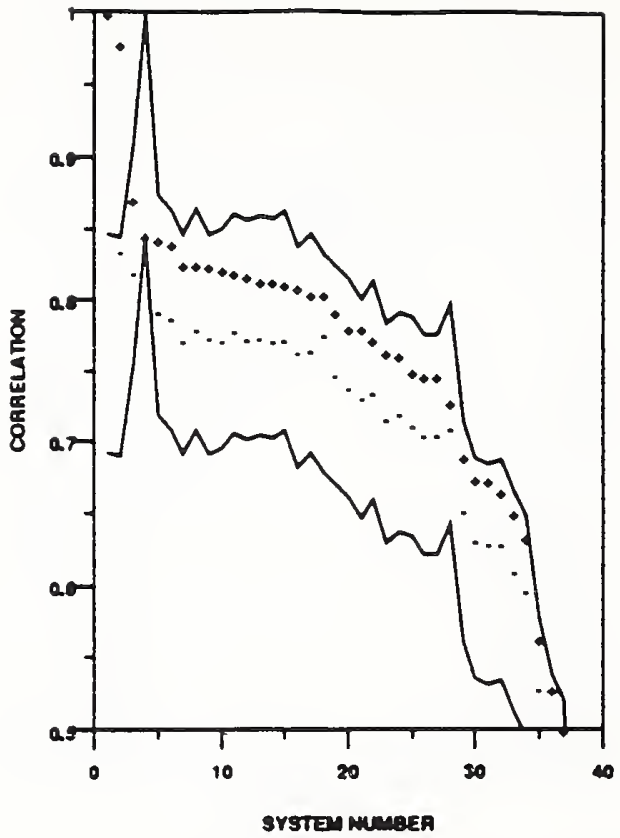

Figure 105: HUGHES_1 - lower case correlation

\begin{tabular}{|c|c|c|c|}
\hline System Number & Syatem Name & Correlation (all) & Correlation (correct) \\
\hline I & HUGHES - 1 & 1.0000 & 1.0000 \\
\hline 2 & HUGHES 2 & 0.2800 & 08383 \\
\hline 3 & VOTE_M & 02715 & 0.8215 \\
\hline 4 & REFERENCE & 0.8461 & 0.8461 \\
\hline 5 & $A E G$ & 0.8440 & 0.7933 \\
\hline 6 & ERIM-1 & 0.8404 & 0.7893 \\
\hline 7 & UBOL & 0.8273 & 0.7732 \\
\hline 8 & OCRSYS & 0.8267 & 0.7810 \\
\hline 9 & IBM & 0.8252 & 0.7747 \\
\hline 10 & UMICH-I & 0.8232 & 07732 \\
\hline 11 & NYNEX & 0.8208 & 0.7795 \\
\hline 12 & KODAK_ & 0.8183 & 0.7738 \\
\hline 13 & $\mathrm{ATT}_{2}$ & 0.8154 & 0.7747 \\
\hline 14 & ATT_A & 0.8153 & 0.7734 \\
\hline 15 & ATT_ & 0.8129 & 0.7738 \\
\hline 16 & ATT & 0.8108 & 0.7648 \\
\hline 17 & NESTOR & 0.8062 & 0.7664 \\
\hline 18 & VOTEP & 0.8056 & 0.7771 \\
\hline 19 & GTESS_l & 0.7937 & 0.7493 \\
\hline 20 & GTESS 2 & 0.7822 & 0.7393 \\
\hline 21 & NIST_A & 0.7817 & 0.7323 \\
\hline 22 & NIST.1 & 0.7744 & 0.7364 \\
\hline 23 & RISO & 0.7647 & 0.7173 \\
\hline 24 & GMD.3 & 0.7629 & 0.7212 \\
\hline 25 & ASOL & 07512 & 0.7128 \\
\hline 26 & GMD.4 & 0.7472 & 0.7057 \\
\hline 27 & GMD_- & 0.7472 & 0.7057 \\
\hline 28 & נה & 0.7293 & 0.7105 \\
\hline 29 & GMD_2 & 0.6917 & 0.6536 \\
\hline 30 & $\mathrm{KAMAN}_{-1}$ & 0.6762 & 0.6332 \\
\hline 31 & VALEN_I & 0.6757 & 0.6309 \\
\hline 32 & NIST 2 & 0.6675 & 0.6302 \\
\hline 33 & $K A M A N_{-3}$ & 0.6525 & 0.6112 \\
\hline 34 & KAMAN_2 & 0.6351 & 0.5970 \\
\hline 35 & $K A M A N=3$ & 0.5642 & 0.5293 \\
\hline 36 & KAMAN_4 & 03293 & 0.4966 \\
\hline 37 & COMCOM & 0.5004 & 0.4886 \\
\hline 38 & UMICH_2 & 0.1015 & 0.0514 \\
\hline
\end{tabular}

Table 67: HUGHES_1 correlation graph key for lowers. 
SYSTEM : HUGHES_2

PARTICIPANT: Tony Baraghimian

ORGANIZATION: Hughes Aircraft Company, Missiles Systems Group, Canoga Park, CA

FEATURES :

CLASSIFICATION: fusion of results of multiple nonparametric algorithms (neocognitron)

HARDWARE: single Intel $i 860$ in a Datacube computer

TRAINING: DIGITS UPPERS LOHERS DATABASE

NIST SPECIAL DATABASE 3

$10000 \quad 7800 \quad 7800$ random

STATUS: $\quad$ on time

RESULTS: -- DIGITS -- -- UPPERS -- -- LOWERS -- DATABASE

REJ . ERR. REJ. ERR. REJ. ERR. TESTDATA 1

RATE RATE-- RATE RATE-- RATE RATE--

$\begin{array}{lllllll}0.00 & 0.0486 & 0.00 & 0.0673 & 0.00 & 0.1559\end{array}$

$\begin{array}{lllllll}0.10 & 0.0181 & 0.10 & 0.0332 & 0.10 & 0.1176\end{array}$

$\begin{array}{lllllll}0.20 & 0.0068 & 0.20 & 0.0147 & 0.20 & 0.0781\end{array}$

$\begin{array}{lllllll}0.30 & 0.0038 & 0.30 & 0.0092 & 0.30 & 0.0493\end{array}$

$\begin{array}{lllllll}0.40 & 0.0022 & 0.40 & 0.0061 & 0.40 & 0.0307\end{array}$

$\begin{array}{lllllll}0.50 & 0.0015 & 0.50 & 0.0045 & 0.50 & 0.0202\end{array}$

OCR RATE (CPS): DIGITS UPPERS LOWERS

SYS RATE:

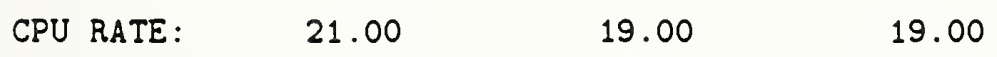


SYSTEM: HUGHES_2

BIBLIOGRAPHY:

The following references have been provided for this system:

[19] 


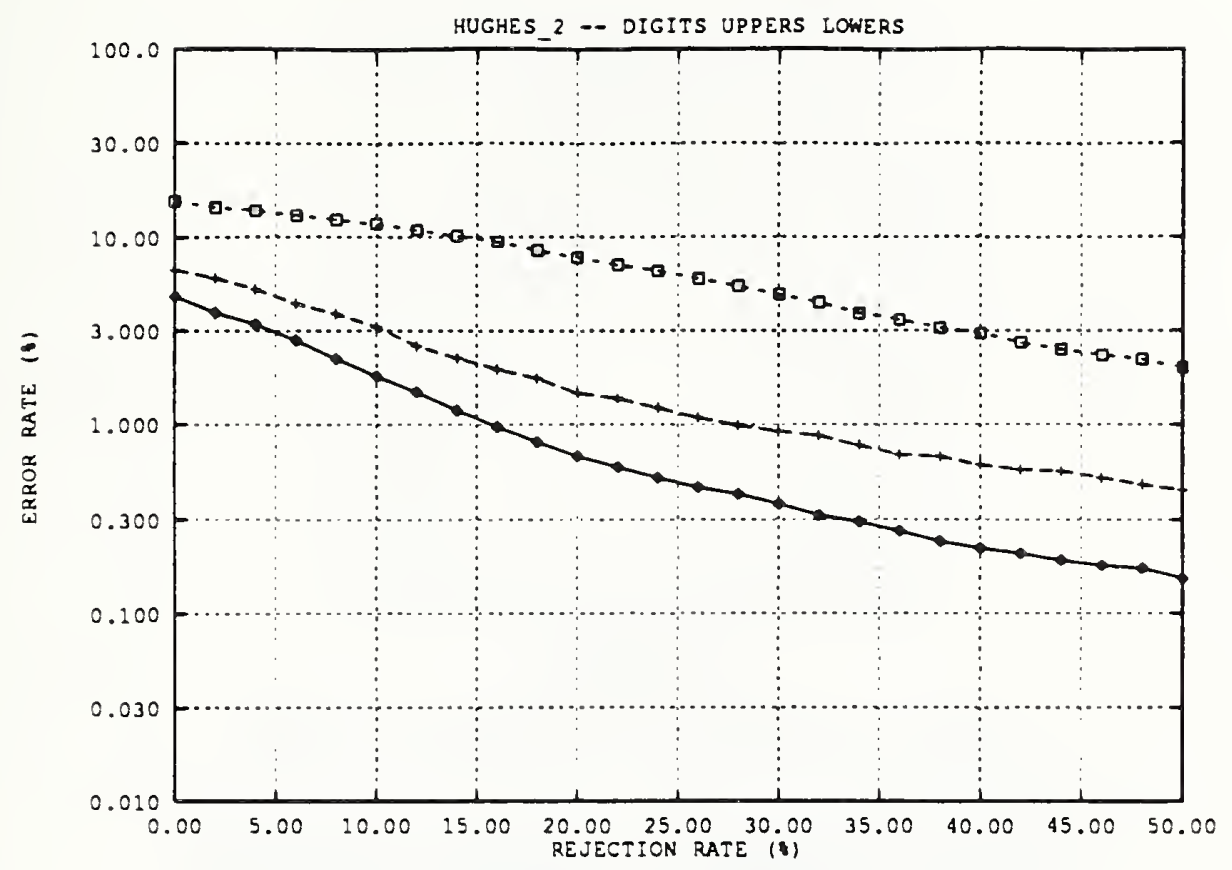

Figure 106: Error rate versus rejection rate for HUGHES_2

HUCHES_2

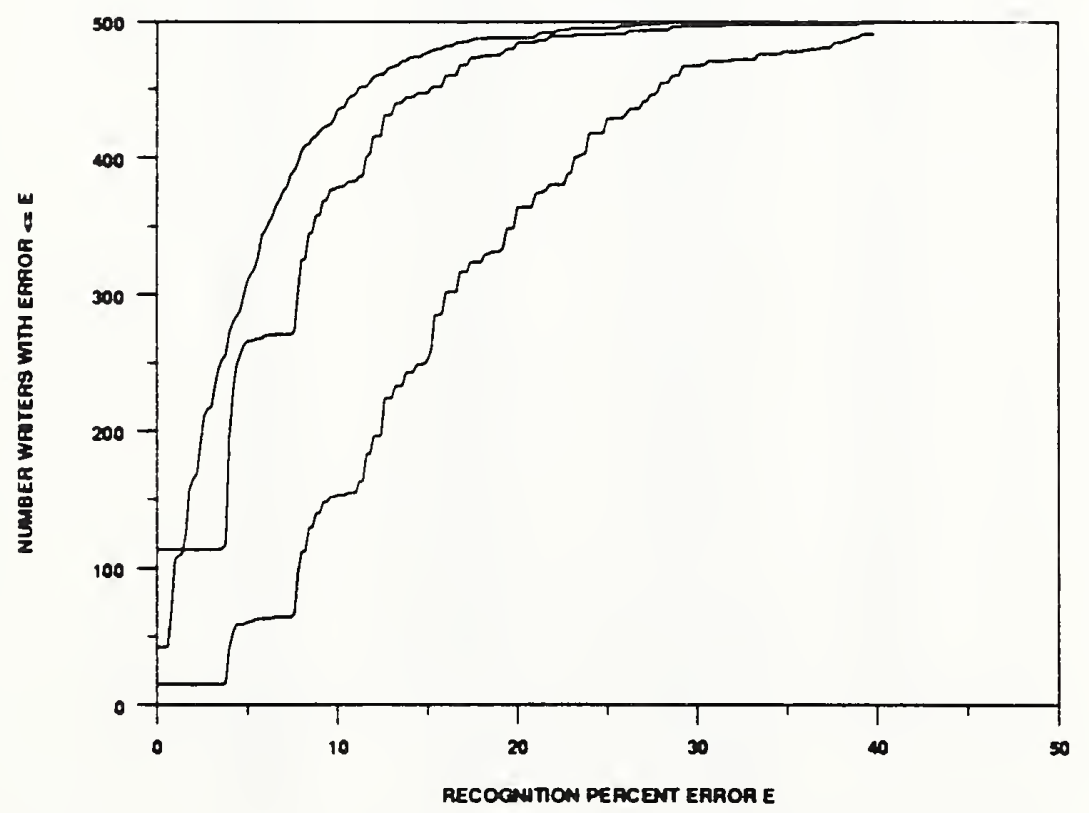

Figure 107: Error rate per writer of HUGHES_2 


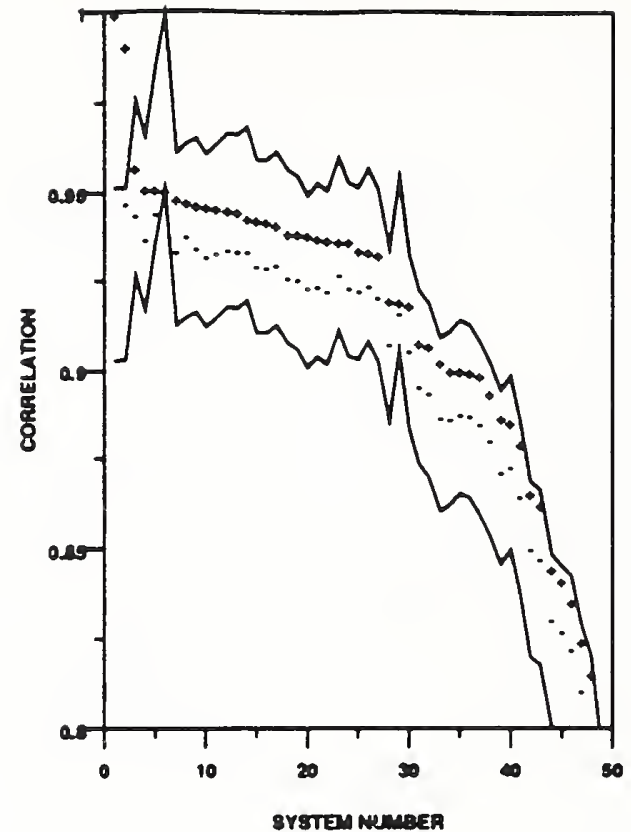

Figure 108: HUGHES_2 - digit correlation

\begin{tabular}{|c|c|c|c|}
\hline System Number & System Name & Correlation (all) & Correlation (correct) \\
\hline 1 & HUGHES.2 & 1.0000 & 1.0000 \\
\hline 2 & HUGHES -1 & 0.9918 & 0.9479 \\
\hline 3 & VOTE_M & 0.9378 & 0.9446 \\
\hline 4 & AEG & 0.9520 & 0.9375 \\
\hline s & OCRSYS & 0.9519 & 0.9451 \\
\hline 6 & REFERENCE & 0.9814 & 0.9814 \\
\hline 7 & ERIM_L & 0.9491 & 0.9344 \\
\hline 8 & VOTE $P$ & 0.9480 & 0.9384 \\
\hline 9 & IBM & 0.9474 & 0.9381 \\
\hline 10 & ERIM_2 & 0.9469 & 0.9328 \\
\hline 11 & ATT 2 & 0.9464 & 0.9340 \\
\hline 12 & ELSAGBA & 0.9459 & 0.9349 \\
\hline 13 & ELSAGB_ & 0.9434 & 0.9345 \\
\hline 14 & ATTد & 0.9434 & 0.9342 \\
\hline is & KODAK_2 & 0.9432 & 0.9301 \\
\hline 16 & ATT -4 & 0.0429 & 0.9289 \\
\hline 17 & THINK_ 2 & 0.9416 & 0.9306 \\
\hline 18 & UBOL & 0.9396 & 0.9272 \\
\hline 19 & NESTOR & 0.9395 & 0.9288 \\
\hline 20 & ELS A GB-1 & 0.9389 & 0.9241 \\
\hline 21 & KODAK_- & 0.9379 & 0.9248 \\
\hline 22 & NIST_-4 & 0.9374 & 0.9235 \\
\hline 23 & REI & 0.9372 & 0.9278 \\
\hline 24 & SYMBUS & 0.9370 & 0.9244 \\
\hline 25 & ATT & 0.9349 & 0.9233 \\
\hline 26 & NYNEX & 0.9344 & 0.9247 \\
\hline 27 & THINK_L & 0.9336 & 0.9217 \\
\hline 28 & GTESS_l & 0.9207 & 0.9080 \\
\hline 29 & COMCOM & 0.9203 & 0.9168 \\
\hline 30 & GTESS 2 & 0.9192 & 0.9066 \\
\hline 31 & NIST_-1 & 0.9089 & 08963 \\
\hline 32 & GMD_3 & 0.9076 & 0.8944 \\
\hline 33 & UPENN & 0.9031 & 0.8874 \\
\hline 34 & ASOL & 0.9011 & 0.8873 \\
\hline 35 & MIME & 0.9008 & 0.8887 \\
\hline 36 & GMD_1 & 0.9004 & 0.8879 \\
\hline 37 & NIST 2 & 0.8996 & 0.8837 \\
\hline 38 & NIST 3 & 0.8945 & 0.8809 \\
\hline 39 & RISO & 0.8875 & 0.8728 \\
\hline 40 & GMD_4 & 0.8860 & 0.8738 \\
\hline 41 & KAMAN_L & 08802 & 0.8633 \\
\hline 42 & KAMAN 3 & 0.8663 & 0.8807 \\
\hline 43 & KAMAN_2 & 0.8630 & 0.8480 \\
\hline 44 & KAMAN -5 & 0.8452 & 0.8309 \\
\hline 45 & GMD.2 & 0.8421 & 0.8278 \\
\hline 46 & VALEN_2 & 0.8360 & 0.8228 \\
\hline 47 & IFAX & 0.8233 & 0.8111 \\
\hline 48 & VALEN-1 & 0.8157 & 0.8010 \\
\hline 49 & KAMAN_4 & 0.7891 & 0.7739 \\
\hline
\end{tabular}

Table 68: HUGHES_2 correlation graph key for digits. 


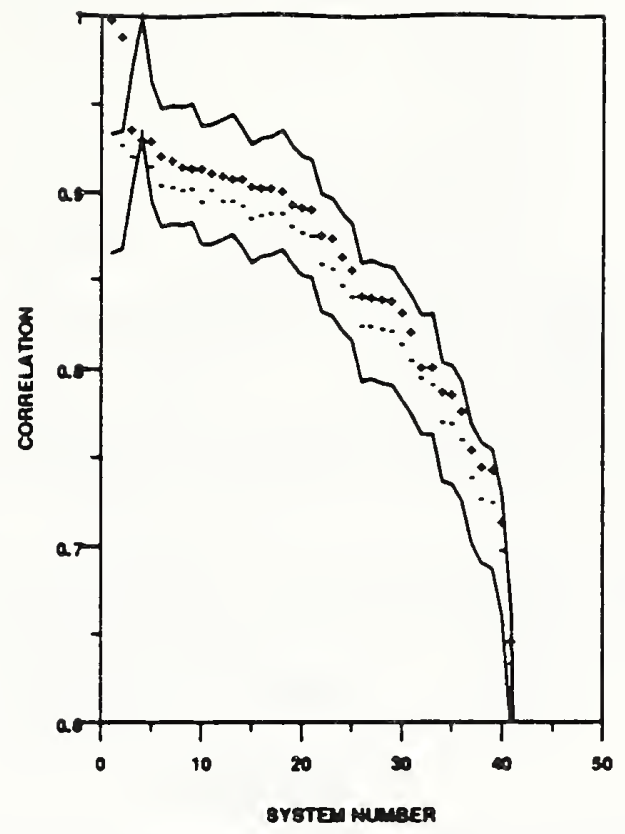

Figure 109: HUGHES 2 - upper case correlation

\begin{tabular}{|c|c|c|c|}
\hline Sydtem Number & Sydtem Name & Correlation (all) & Coprelasion (correct) \\
\hline$\overline{1}$ & HUGHES_2 & 1.0000 & 1.0000 \\
\hline 2 & HUGHES -1 & 0.2907 & 0.8296 \\
\hline 3 & VOTE_M & 0.9389 & 0.9230 \\
\hline 4 & REFERENCE & 0.8327 & 09327 \\
\hline 3 & $A E G$ & 09324 & 0.9173 \\
\hline 6 & ERIM-1 & 0.9237 & 0.9063 \\
\hline 7 & A T T -4 & 08216 & 0.8060 \\
\hline 8 & $\mathrm{UMICH}_{-}$ & 0.9179 & 0.9034 \\
\hline 8 & NYNEX & 0.9169 & 0.9042 \\
\hline 10 & UBOL & 0.9168 & 0.8873 \\
\hline 11 & VOTE_P & 0.9138 & 0.9034 \\
\hline 12 & NESTOR & 0.2121 & 0.8970 \\
\hline 13 & $\mathrm{ATT}_{2} 2$ & 0.2106 & 0.8969 \\
\hline 14 & IBM & 0.8105 & 0.8943 \\
\hline 15 & SYMBUS & 0.9054 & 0.8872 \\
\hline 16 & KODAK」 & 0.9047 & 0.8891 \\
\hline 17 & ATT 3 & 0.8044 & 0.8898 \\
\hline 18 & ATT_ & 0.8032 & 0.8897 \\
\hline 18 & OCRSYS & 0.8952 & 0.8828 \\
\hline 20 & GTESS_1 & 0.8940 & 0.8791 \\
\hline 21 & GTESS 2 & 0.8924 & 0.8775 \\
\hline 22 & MIME & 0.8778 & 0.8612 \\
\hline 23 & NIST_4 & 0.8759 & 0.8584 \\
\hline 24 & ASOL & 0.8656 & 0.8498 \\
\hline 23 & REI & 0.8587 & 0.8433 \\
\hline 26 & GMD_-1 & 0.8440 & 0.8281 \\
\hline 27 & NIST_I & 0.8427 & 0.8261 \\
\hline 28 & RISO & 0.8425 & 0.8245 \\
\hline 29 & GMD_3 & 0.8414 & 0.8238 \\
\hline 30 & KAMAN_I & 0.8349 & 0.8168 \\
\hline 31 & GMD_4 & 0.8241 & 0.8076 \\
\hline 32 & COMCOM & 0.8040 & 0.7971 \\
\hline 33 & הIST & 0.8034 & 0.7931 \\
\hline 34 & IFAX & 0.7801 & 0.7723 \\
\hline 35 & KAMAN 3 & 0.7881 & 0.7714 \\
\hline 36 & KAMAN 2 & 0.7792 & 0.7622 \\
\hline 37 & NIST_2 & 0.7571 & 0.7409 \\
\hline 38 & VALEN-I & 0.7478 & 0.7283 \\
\hline 39 & GMD_2 & 0.7454 & 0.7277 \\
\hline 40 & KAMAN_4 & 0.7162 & 0.6997 \\
\hline 41 & KAMAN_S & 0.6479 & 0.6331 \\
\hline 42 & UMICH 2 & 0.0458 & 0.0203 \\
\hline
\end{tabular}

Table 69: HUGHES 2 correlation graph key for uppers. 


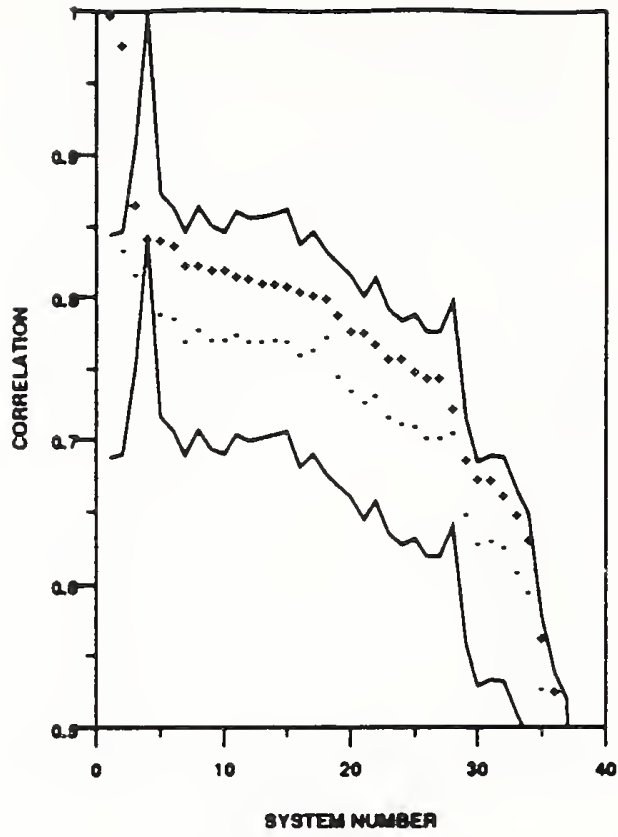

Figure 110: HUGHES 2 - lower case correlation

\begin{tabular}{|c|c|c|c|}
\hline System Number & System Name & Correlation (all) & Cortelation (correct) \\
\hline I & HUGHES 2 & 1.0000 & 1.0000 \\
\hline 2 & HUGHES -1 & 0.9800 & 0.8363 \\
\hline 3 & VOTE_M & 0.8679 & 0.8190 \\
\hline 4 & REFERENCE & 0.8441 & 0.8441 \\
\hline 3 & $A E G$ & 0.8427 & 0.7916 \\
\hline 6 & ERIM-1 & 0.8398 & 0.7882 \\
\hline 7 & UBOL & 0.8257 & 0.7715 \\
\hline 8 & OCRSYS & 0.8252 & 0.7794 \\
\hline 9 & UMICH_1 & 0.8227 & 0.7723 \\
\hline 10 & IBM & 0.8220 & 0.7724 \\
\hline 11 & NYNEX & 0.8172 & 0.7768 \\
\hline 12 & KODAK_ & 0.8163 & 0.7718 \\
\hline 13 & ATT_4 & 0.8130 & 0.7718 \\
\hline 14 & ATT 2 & 0.8128 & 0.7728 \\
\hline 13 & ATT_1 & 0.8105 & 0.7717 \\
\hline 16 & ATT 3 & 0.8070 & 0.7622 \\
\hline 17 & NESTOR & 0.8032 & 0.7654 \\
\hline 18 & VOTE_ & 0.8028 & 0.7748 \\
\hline 19 & GTESS -1 & 0.7817 & 0.7473 \\
\hline 20 & GTESS 2 & 0.7796 & 0.7370 \\
\hline 21 & NIST_4 & 07782 & 0.7298 \\
\hline 22 & NIST 4 & 0.7707 & 0.7337 \\
\hline 23 & GMD_3 & 0.7607 & 0.7190 \\
\hline 24 & RISO & 0.7602 & 0.7145 \\
\hline 25 & ASOL & 0.7512 & 0.7119 \\
\hline 26 & GMD_1 & 0.7439 & 0.7040 \\
\hline 27 & GMD_I & 0.7459 & 0.7040 \\
\hline 28 & NIST 3 & 0.7251 & 0.7074 \\
\hline 29 & GMD 2 & 06889 & 0.8511 \\
\hline so & VALEN_1 & 0.6768 & 06508 \\
\hline 31 & $K A M A N_{-1}$ & 0.6747 & 0.6324 \\
\hline 32 & $\mathrm{NIST}_{2}$ & 0.6653 & 0.6284 \\
\hline 3 & KAMAN $\bar{N}_{-3}$ & 0.6315 & 06109 \\
\hline 34 & KAMAN_2 & 0.6342 & 0.3968 \\
\hline 3s & KAMAN_3 & 03648 & 0.5293 \\
\hline 36 & KAMAN_A & 0.3288 & 0.4958 \\
\hline 37 & COMCOM & 0.4993 & 0.4872 \\
\hline 38 & $\mathrm{UMICH} 2$ & 0.1008 & 0.0303 \\
\hline
\end{tabular}

Table 70: HUGHES 2 correlation graph key for lowers. 
SYSTEM: IBM

PARTICIPANT: Dr. K. M. Mohiuddin

ORGANIZATION: IBM Almaden Research Center, San Jose, CA

FEATURES: geometrical and zonal patterns, including bending points

and areas of significant direction change around the contour.

CLASSIFICATION: 3-layer NN: 184 input units ( 96 for bending points and 88 for direction changes), 40 hidden units (static for all experiments), 10 output units for digits, 26 for upper case and 26 for lower case.

HARDHARE: RS/6000 Model 530 running AIX

TRAINING :

$$
\text { DIGITS }
$$

UPPERS

LOWERS DATABASE

$$
80 \backslash \%
$$

$100 \backslash \%$

$100 \backslash \%$

NSDB3

STATUS: on time

RESULTS: -- DIGITS -- -- UPPERS -- -- LOHERS -- DATABASE

$\begin{array}{lllllll}\text { REJ. } & \text { ERR. } & \text { REJ. } & \text { ERR. } & \text { REJ. } & \text { ERR. } & \text { TESTDATA1 } \\ \text { RATE } & \text { RATE-- } & \text { RATE } & \text { RATE-- } & \text { RATE } & \text { RATE-- } & \\ 0.00 & 0.0349 & 0.00 & 0.0641 & 0.00 & 0.1542 & \\ 0.10 & 0.0071 & 0.10 & 0.0234 & 0.10 & 0.1061 & \\ 0.20 & 0.0037 & 0.20 & 0.0090 & 0.20 & 0.0730 & \\ 0.30 & 0.0038 & 0.30 & 0.0050 & 0.30 & 0.0482 & \\ 0.40 & 0.0040 & 0.40 & 0.0054 & 0.40 & 0.0307 & \\ 0.50 & 0.0038 & 0.50 & 0.0052 & 0.50 & 0.0183 & \end{array}$

OCR RATE (CPS): DIGITS

UPPERS

LOWERS

SYS RATE:

86.95

80.97

89.04

CPU RATE: 200 .

194.17

194.17 
SYSTEM: IBM

BIBLIOGRAPHY:

The following references have been provided for this system:

[20][24][21][22][23] [24] [25][26] 


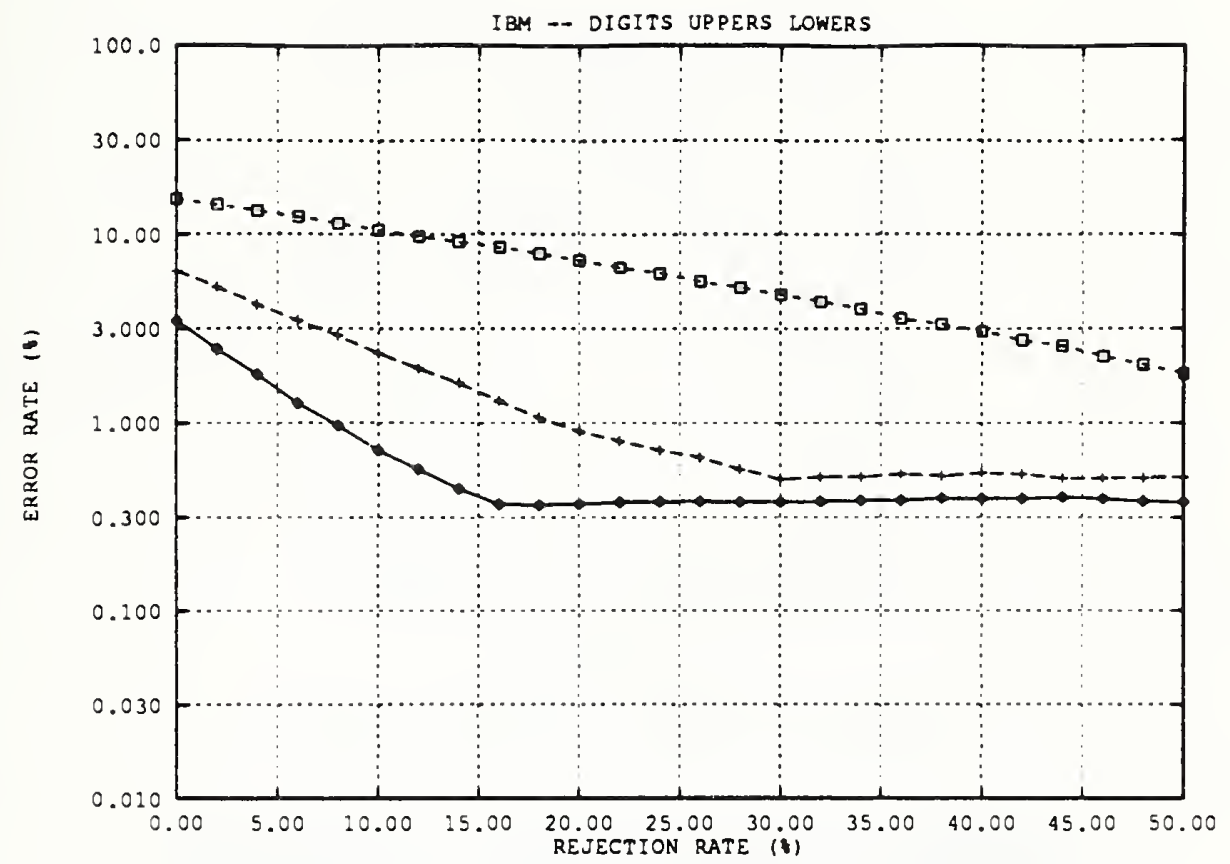

Figure 111: Error rate versus rejection rate for IBM

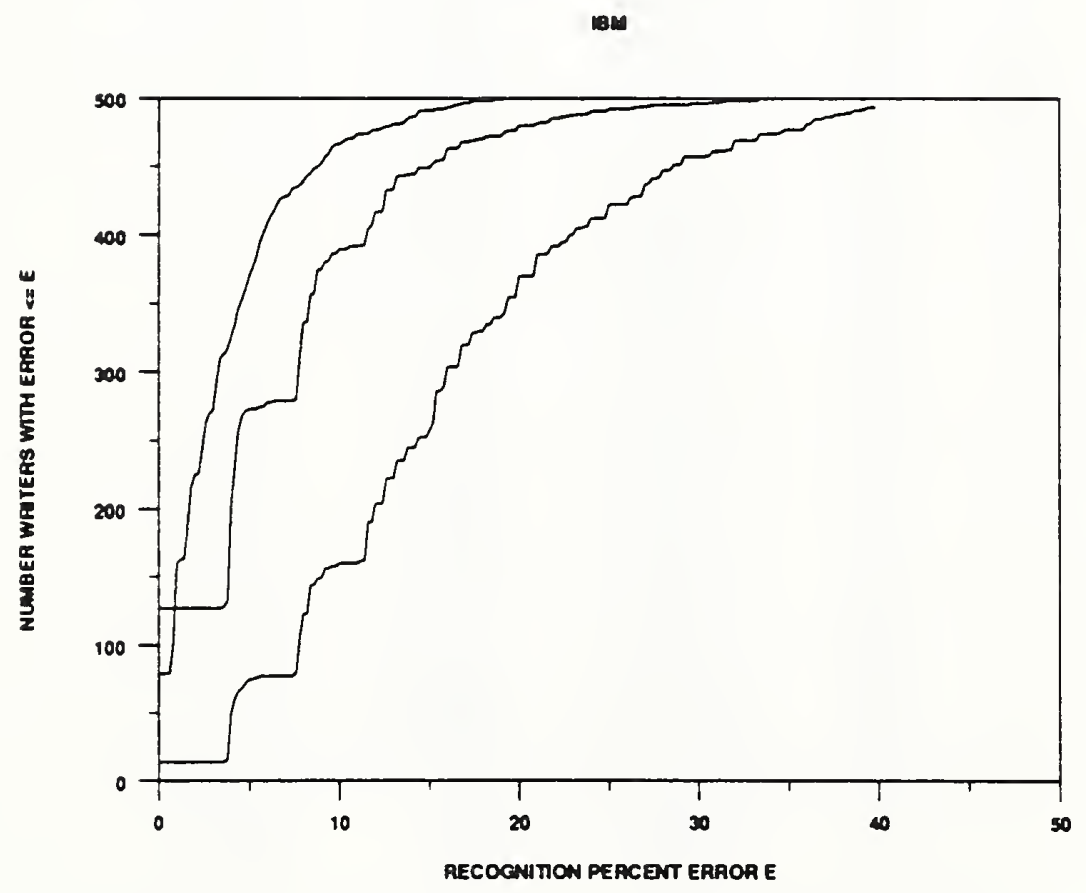

Figure 112: Error rate per writer of IBM 


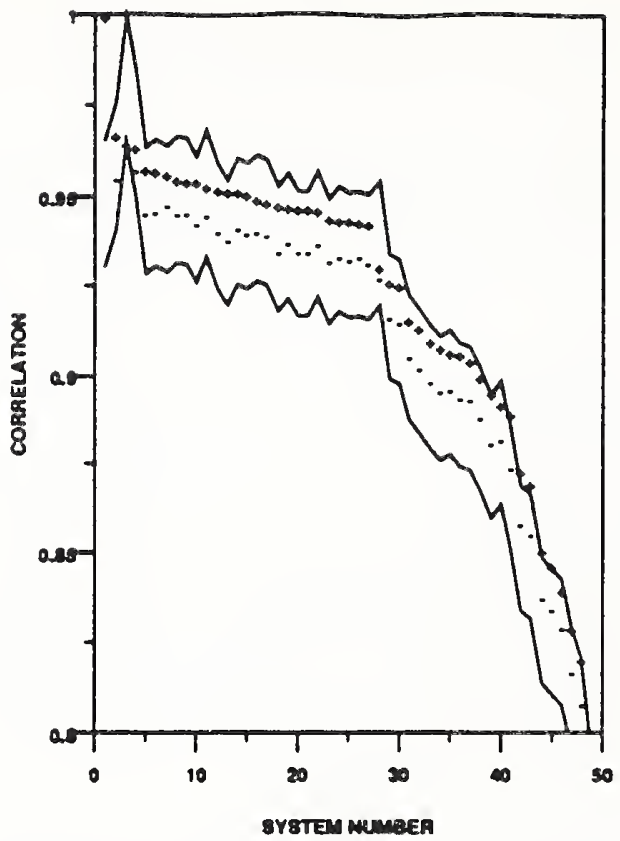

Figure 113: IBM - digit correlation

\begin{tabular}{|c|c|c|c|}
\hline System Number & System Name & Copreletion (sil) & Correlation (correct) \\
\hline$T$ & IBM & 1.0000 & 1.0000 \\
\hline 2 & VOTEM & 0.9677 & 0.8857 \\
\hline 3 & REFERENCE & 0.8681 & 0.8681 \\
\hline 4 & OCRSYS & 0.2641 & 0.2577 \\
\hline 3 & ATT 2 & 0.0883 & 0.8460 \\
\hline 6 & AEG & 0.9578 & 0.9464 \\
\hline 7 & VOTEP $P$ & 0.9370 & 0.8483 \\
\hline 8 & ELSAGB_3 & 0.9858 & 0.8160 \\
\hline 9 & ELSAGBב & 0.9883 & 0.9457 \\
\hline 10 & ERIM-I & 0.9849 & 0.8432 \\
\hline il & ATT_ & 0.9835 & 0.8456 \\
\hline 12 & KODAK_2 & 0.9529 & 0.8408 \\
\hline 13 & ERIM 2 & 0.0823 & 0.9417 \\
\hline 14 & NESTOR & 0.9523 & 0.8386 \\
\hline 15 & ATT_A & 0.0314 & 0.9404 \\
\hline 16 & THINK_ 2 & 0.9501 & 0.8407 \\
\hline 17 & REI & 0.9489 & 0.9400 \\
\hline 18 & KODAK」 & 0.9480 & 0.9354 \\
\hline 19 & UBOL & 0.8477 & 0.2374 \\
\hline 20 & HUGHES 2 & 0.9474 & 0.9331 \\
\hline 21 & HUGHES_I & 0.9474 & 0.9381 \\
\hline 22 & NYNEX & 0.8470 & 0.9371 \\
\hline 23 & ELSAGB_l & 0.0447 & 0.9323 \\
\hline 24 & SYMBUS & 0.9442 & 0.9341 \\
\hline 25 & THINK_L & 0.9440 & 0.9328 \\
\hline 26 & $\mathrm{ATT}_{3}$ & 0.8456 & 0.9358 \\
\hline 27 & NIST_4 & 0.8433 & 0.9323 \\
\hline 28 & COMCOM & 0.9311 & 0.8280 \\
\hline 29 & GTESS_L & 0.0272 & 0.8170 \\
\hline 30 & GTESS 2 & 0.9262 & 0.8137 \\
\hline 31 & NIST_1 & 0.2163 & 0.0059 \\
\hline 32 & GMD_3 & 0.9141 & 0.9029 \\
\hline 33 & MIME & 0.9103 & 0.8989 \\
\hline 34 & ASOL & 0.9085 & 0.8968 \\
\hline 35 & GMD_1 & 0.8075 & 0.8967 \\
\hline 36 & UPENN & 0.9089 & 0.8945 \\
\hline 37 & NIST 2 & 0.8051 & 0.8938 \\
\hline 38 & NIST & 0.8003 & 0.8889 \\
\hline 39 & RISO & 0.8987 & 0.8816 \\
\hline 40 & GMD_4 & 0.8928 & 0.8823 \\
\hline 41 & KAMAN_L & 0.8898 & 0.8745 \\
\hline 42 & KAMAN & 0.8737 & 0.8584 \\
\hline 43 & KAMAN_2 & 0.8701 & 0.8558 \\
\hline 44 & KAMAN_S & 0.8513 & 0.8380 \\
\hline 45 & GMD -2 & 0.8469 & 0.8349 \\
\hline 46 & VALEN_ & 0.8403 & 0.8296 \\
\hline 47 & IFAX & 0.8293 & 0.8174 \\
\hline 48 & VALEN_l & 0.8209 & 0.8086 \\
\hline 49 & KAMAN-A & 0.7845 & 0.7824 \\
\hline
\end{tabular}

Table 71: IBM correlation graph key for digits. 


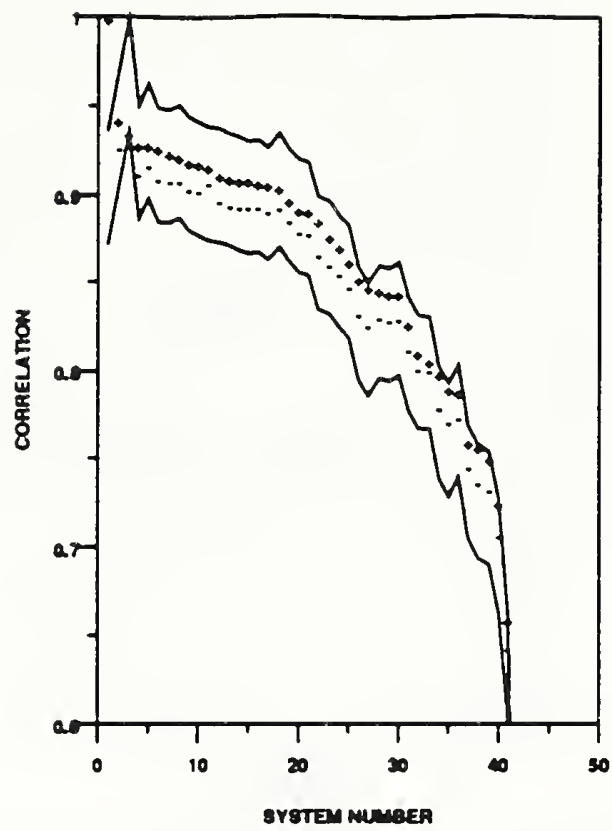

Figure 114: IBM - upper case correlation

\begin{tabular}{|c|c|c|c|}
\hline System Number & System Name & Correlation (all) & Corretasion (correci) \\
\hline 1 & IBM & 1.0000 & 1.0000 \\
\hline 2 & VOTE_M & 0.9430 & 0.8272 \\
\hline 3 & REFERENCE & 0.9359 & 0.9369 \\
\hline 4 & ATT -4 & 0.9298 & 0.9127 \\
\hline 3 & AEG & 0.9295 & 0.9173 \\
\hline 6 & UMICH.I & 0.9274 & 0.9106 \\
\hline 7 & ERIM-1 & 0.2245 & 0.9090 \\
\hline 8 & NYNEX & 0.9231 & 0.9094 \\
\hline 9 & ATT2 2 & 0.9198 & 0.9048 \\
\hline 10 & NESTOR & 0.9191 & 0.9038 \\
\hline 11 & VOTEP & 0.9178 & 0.9081 \\
\hline 12 & UBOL & 0.9128 & 0.8979 \\
\hline 13 & HUGHES-I & 0.9114 & 0.8959 \\
\hline 14 & HUGHES-2 & 0.9105 & 0.8945 \\
\hline 15 & $K O D A K \perp$ & 0.9101 & 0.8947 \\
\hline 16 & ATTA & 0.9087 & 0.8949 \\
\hline 17 & SYMEUS & 0.9071 & 0.8915 \\
\hline 18 & ATT 1 & 0.9059 & 0.8934 \\
\hline 19 & OCRSYS & 0.8982 & 08866 \\
\hline 20 & GTESS-1 & 0.8926 & 0.8802 \\
\hline 21 & GTESS_ 2 & 0.8914 & 0.8791 \\
\hline 22 & MIME & 0.8860 & 0.8673 \\
\hline 23 & NIST_4 & 0.8773 & 0.8617 \\
\hline 24 & ASOL & 0.8712 & 0.8557 \\
\hline 25 & REI & 0.8634 & 0.8480 \\
\hline 26 & RISO & 0.8528 & 0.8331 \\
\hline 27 & KAMAN_I & 08482 & 0.8269 \\
\hline 28 & GMD_1 & 0.8470 & 0.8308 \\
\hline 29 & GMD_J & 0.8453 & 0.8290 \\
\hline 30 & NIST_1 & 0.8449 & 0.8302 \\
\hline 31 & GMD_4 & 0.8277 & 0.8124 \\
\hline 32 & נה & 0.8112 & 0.8013 \\
\hline 33 & COMCOM & 0.8065 & 0.8004 \\
\hline 34 & KAMAN_3 & 0.7988 & 0.7801 \\
\hline 35 & KAMAN_2 & 0.7905 & 07710 \\
\hline 36 & IFAX & 0.7893 & 0.7746 \\
\hline 37 & NIST 2 & 0.7607 & 0.7463 \\
\hline 38 & VALEN_1 & 0.7574 & 0.7378 \\
\hline 39 & GMD -2 & 0.7511 & 0.7334 \\
\hline 40 & $K_{A M} M_{-4}$ & 0.7264 & 0.7076 \\
\hline 41 & KAMAN_S & 0.6603 & 0.6435 \\
\hline 42 & $\mathrm{UMICH}_{2}$ & 0.0408 & 0.0193 \\
\hline
\end{tabular}

Table 72: IBM correlation graph key for uppers. 


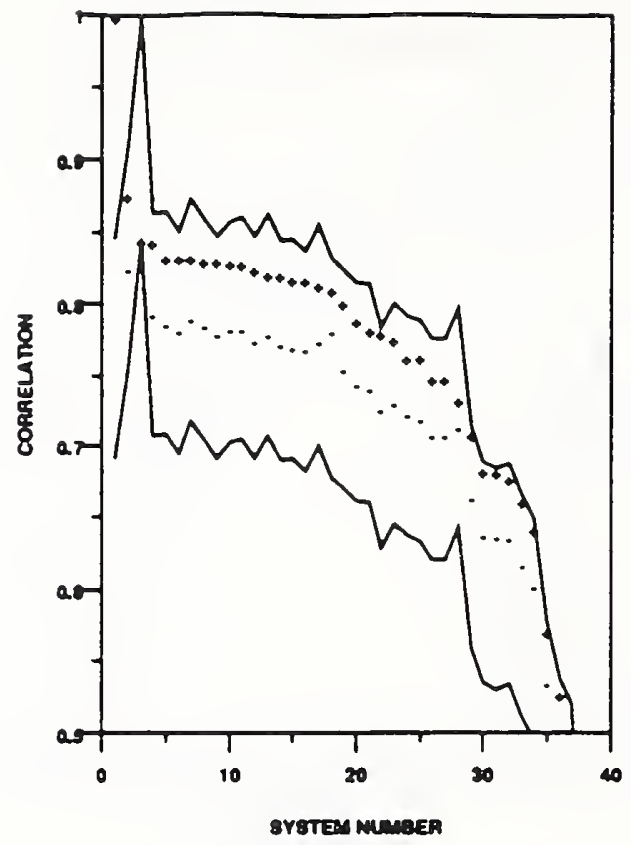

Figure 115: IBM - lower case correlation

\begin{tabular}{|c|c|c|c|}
\hline Sydtem Number & Syatem Name & Corretation (alf) & Correlation (correcs) \\
\hline 1 & TBM & 1.0000 & 1.0000 \\
\hline 2 & VOTEM & 0.8760 & 0.8254 \\
\hline $\mathbf{3}$ & REFERENCE & 0.8457 & 0.8457 \\
\hline 4 & ERIM_- & 0.8443 & 0.7932 \\
\hline 5 & OCRSYS & 0.8342 & 0.7863 \\
\hline 6 & AEG & 0.8339 & 0.7897 \\
\hline 7 & UMICH.1 & 0.8339 & 0.7817 \\
\hline 8 & ATT2 2 & 0.8315 & 0.7858 \\
\hline 9 & NESTOR & 0.8311 & 0.7797 \\
\hline 10 & ATT 4 & 0.8303 & 0.7828 \\
\hline 11 & NYNEX & 0.8296 & 0.7837 \\
\hline 12 & HUGHES - 1 & 0.8252 & 0.7747 \\
\hline 13 & ATT $\perp$ & 0.8224 & 0.7793 \\
\hline 14 & HUGHES_2 & 0.8220 & 0.7724 \\
\hline 15 & UBOL & 0.8184 & 0.7706 \\
\hline 16 & ATT $\mathrm{T}$ & 0.8183 & 0.7699 \\
\hline 17 & KODAK」 & 0.8150 & 0.7748 \\
\hline 18 & VOTE_P & 0.8114 & 0.7823 \\
\hline 19 & GTESS_1 & 0.8023 & 0.7356 \\
\hline 20 & GTESS 2 & 0.7800 & 0.7436 \\
\hline 21 & NIST_A & 0.7828 & 0.7413 \\
\hline 22 & RISO & 0.7811 & 0.7272 \\
\hline 23 & NIST_4 & 0.7762 & 0.7312 \\
\hline 24 & GMD_3 & 0.7636 & 0.7236 \\
\hline 25 & ASOL & 0.7633 & 0.7203 \\
\hline 26 & GMD_4 & 0.7489 & 0.7088 \\
\hline 27 & GMD.1 & 0.7488 & 0.7088 \\
\hline 28 & NIST 3 & 0.7337 & 0.7142 \\
\hline 28 & GMD_2 & 0.7097 & 0.6646 \\
\hline 30 & KAMAN_1 & 0.6848 & 0.6390 \\
\hline 31 & VALEN_l & 0.6837 & 0.6368 \\
\hline 32 & NIST_2 & 0.6783 & 0.6337 \\
\hline 33 & KAMAN_3 & 0.6624 & 0.6177 \\
\hline 34 & KAMAN_2 & 0.6436 & 0.6032 \\
\hline 35 & KAMAN S & 0.5724 & 0.5352 \\
\hline 36 & KAMAN_4 & 0.5284 & 0.4976 \\
\hline 37 & COMCOM & 0.4965 & 0.4856 \\
\hline 38 & $\mathrm{UMICH}_{2}$ & 0.0922 & 0.0482 \\
\hline
\end{tabular}

Table 73: IBM correlation graph key for lowers. 
SYSTEM: IFAX

PARTICIPANT: Leonid Nilva

ORGANIZATION: InterFax, Inc., Sunnyvale, CA

FEATURES: Shape and Histogram based,

adaptively selected relevant subset of over 500 features

CLASSIFICAIION: series of adaptive affine transformations

HARDWARE :

TRAINING: DIGITS UPPERS LOWERS DATABASE

$\begin{array}{llll}? & ? & \text { NA } & \text { NSDB3 }\end{array}$

STATUS: on time

RESULTS: -- DIGITS -- -- UPPERS -- -- LOHERS -- DATABASE

REJ. ERR. REJ. ERR. REJ. ERR. TESTDATA1

RATE RATE-- RATE RATE-- RATE RATE--

$0.00 \quad 0.1707 \quad 0.00 \quad 0.1960$

$\begin{array}{lllll}0.10 & 0.1249 & 0.10 & 0.1498\end{array}$

$\begin{array}{lllll}0.20 & 0.0897 & 0.20 & 0.1198\end{array}$

$\begin{array}{llll}0.30 & 0.0626 & 0.30 & 0.0974\end{array}$

$\begin{array}{lllll}0.40 & 0.0491 & 0.40 & 0.0794\end{array}$

$\begin{array}{lllll}0.50 & 0.0335 & 0.50 & 0.0648\end{array}$

OCR RATE (CPS): DIGITS UPPERS LOHERS

SYS RATE: $\quad 20.00 \quad 12.00 \quad$ NA

CPU RATE:

NOTE: Few details of features or classification provided. 


\section{SYSTEM: IFAX}

\section{BIBLIOGRAPHY:}

The following references have been provided for this system:

none

COMMENTS:

InterFax, headquartered in Sunnyvale, California, develops and markets an integrated family of robust application development tools for fax information processing.

One of InterFax's new products, code-named Harvest, is an object-oriented development environment that automates the reading and entering of data from faxed forms into host transaction systems. These forms can have hand-printed numbers or letters, machine-printed characters, mark sense boxes, graphics, or other images. Harvest reads and interprets forms from fax machines or scanners. Once a form is read and verified, the information is automatically sent to the host computer application and a fax response or confirmation is generated.

Harvest will be available for commercial use in the fourth quarter of 1992 . Initial release of the product will support IBM mainframe and AS/400 host computers. The implementation platform is 486 IBM compatible computers with OS/2 2.0 operating system and C++ programming language. The hand-printed character recognition used in the First Census OCR Systems Conference is a prototype algorithm, one of a couple that InterFax may pursue. The engine utilizes geometric feature extraction and modified a $k$-nearest neighbor classifier. 


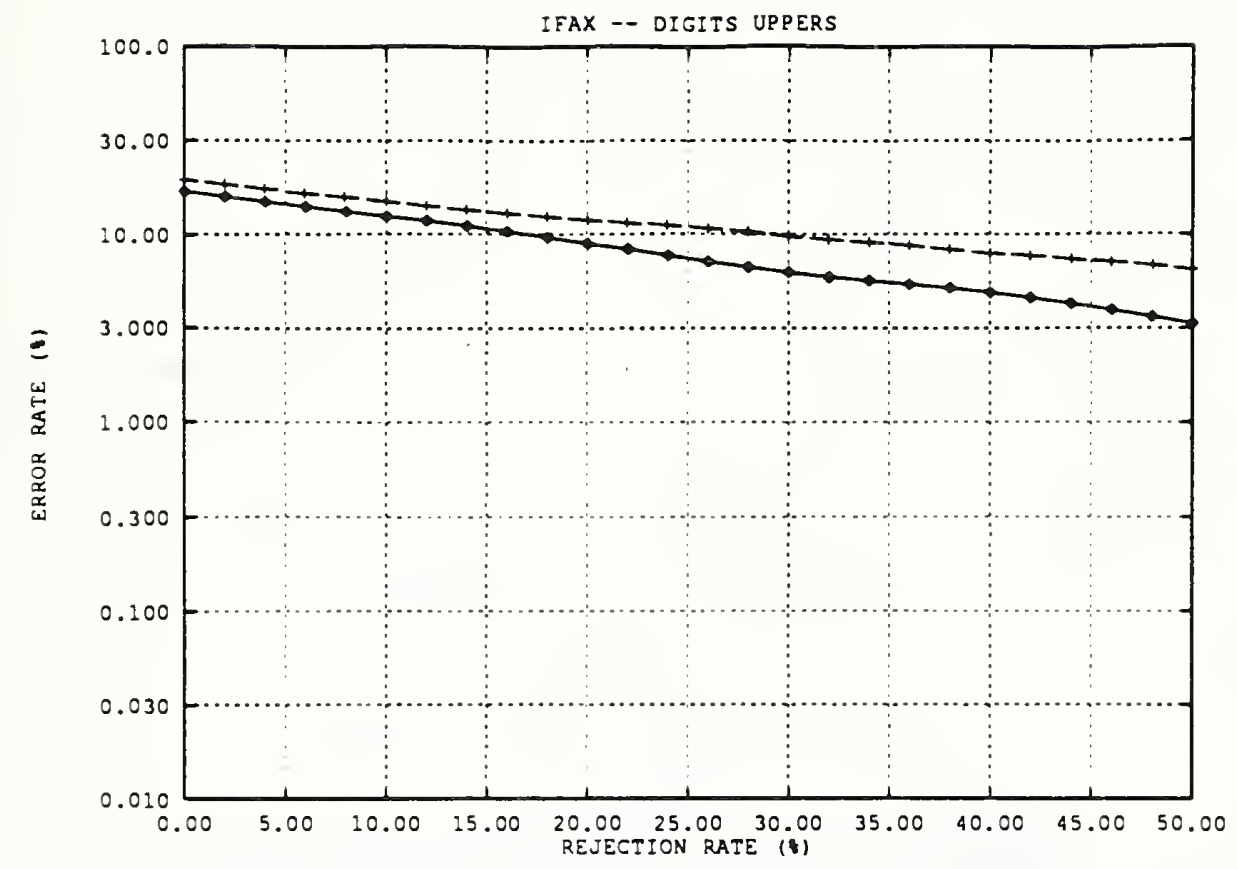

Figure 116: Error rate versus rejection rate for IFAX

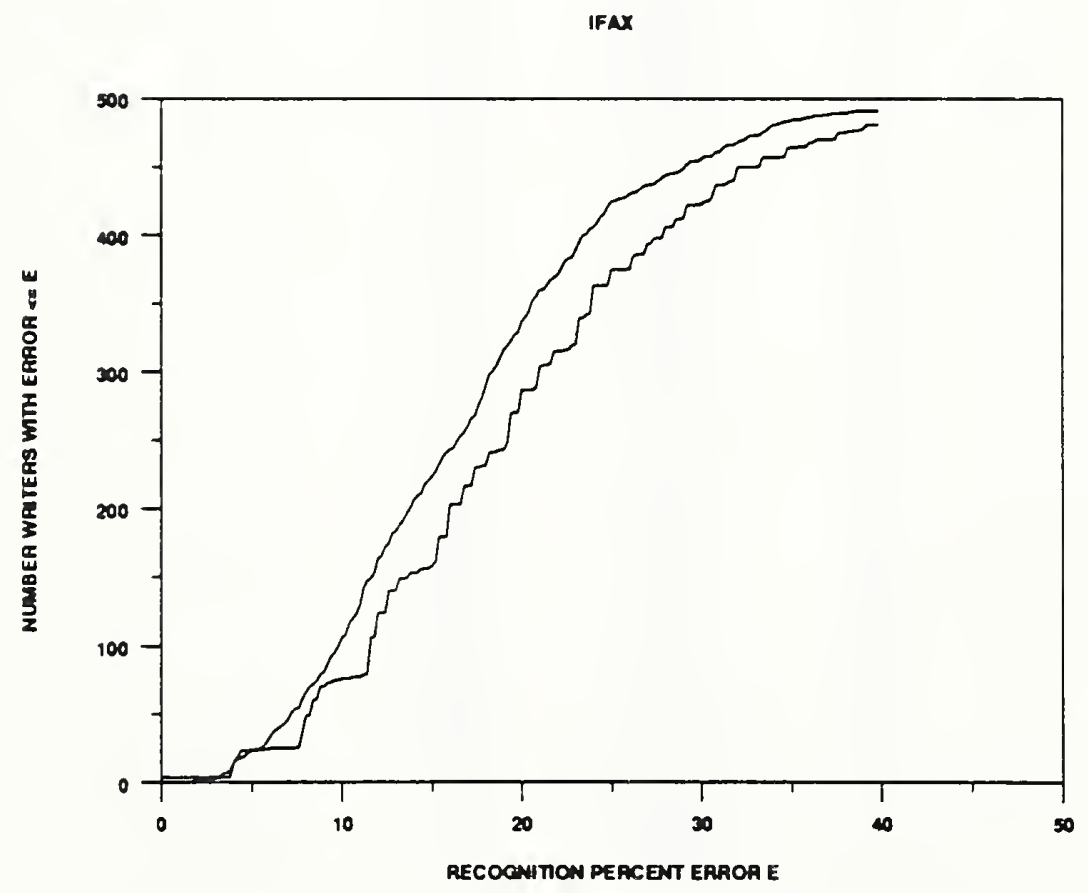

Figure 11T: Error rate per writer of IFAX 


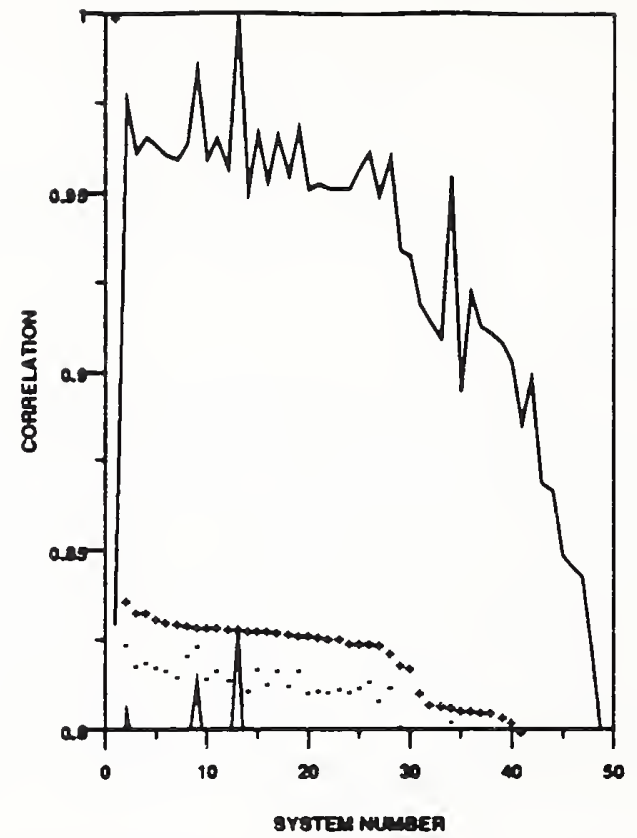

Figure 118: IFAX - digit correlation

\begin{tabular}{|c|c|c|c|}
\hline System Number & Syscem Name & Correlalion (all) & Correlation (correct) \\
\hline 1 & TFAX & 1.0000 & 1.0000 \\
\hline 2 & VOTE_M & 0.8370 & 0.8248 \\
\hline $\mathbf{3}$ & ERIM_l & 0.8340 & 0.8183 \\
\hline 4 & $A E G$ & 0.8396 & 0.8194 \\
\hline 3 & ATT 2 & 0.8319 & 0.8181 \\
\hline 6 & ERIM 2 & 0.8312 & 0.8173 \\
\hline 7 & KODAK2 & 0.8304 & 0.8153 \\
\hline 8 & VOTEP & 0.8301 & 0.8212 \\
\hline 9 & OCRSYS & 0.8298 & 0.8241 \\
\hline 10 & ATT_4 & 0.8297 & 0.8182 \\
\hline 11 & IBM & $0.829 \mathrm{~s}$ & 08174 \\
\hline 12 & REFERENCE & 0.8293 & 0.8293 \\
\hline 13 & UBOL & 0.8293 & 0.8143 \\
\hline 14 & NIST_-4 & 0.8289 & 0.8118 \\
\hline 13 & SـSAGB & 0.8287 & 0.8177 \\
\hline 16 & SYMBUS & 0.8286 & 0.8134 \\
\hline 17 & ELSAGB2 & 08281 & 08172 \\
\hline 18 & NESTOR & 0.8279 & 0.8131 \\
\hline 19 & ATT $\lrcorner$ & 0.8273 & 08172 \\
\hline 20 & THINK-1 & 0.8273 & 0.8114 \\
\hline 21 & 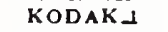 & 0.8271 & 0.8117 \\
\hline 22 & HUGHES-1 & 0.8266 & 0.8115 \\
\hline 23 & ATT 3 & 08264 & 08123 \\
\hline 24 & HUGHES .2 & 0.8253 & 08111 \\
\hline 23 & NYNEX & 0.8232 & 0.8127 \\
\hline 26 & THINK 2 & 0.8249 & 08139 \\
\hline 27 & ELSAGB_l & 0.8247 & 0.8092 \\
\hline 28 & REI & 0.8221 & 0.8128 \\
\hline 29 & GTESS_l & 0.8192 & 0.8013 \\
\hline 30 & GTESS2 & 0.8183 & 0.8007 \\
\hline 31 & GMD_3 & 0.8113 & 07813 \\
\hline 32 & MIME & 0.8082 & 0.7881 \\
\hline 33 & UPENN & 0.8078 & 0.7862 \\
\hline 34 & COMCOM & 0.8072 & 0.8032 \\
\hline 33 & RISO & 0.8064 & 0.7802 \\
\hline 36 & NIST -1 & 0.8062 & 0.7898 \\
\hline 37 & GMD - 1 & 0.8039 & 07863 \\
\hline 38 & ASOL & 0.8057 & 07860 \\
\hline 39 & NIST_2 & 0.8044 & 0.7843 \\
\hline 40 & NIST & 0.8028 & 0.7823 \\
\hline 41 & KAMAN.1 & 0.8003 & 0.7734 \\
\hline 42 & GMD - 4 & $0.794 \mathrm{~s}$ & 0.7744 \\
\hline 43 & KAMAN_3 & 0.7878 & 0.7603 \\
\hline 44 & KAMAN-2 & 0.7848 & 0.7381 \\
\hline 45 & $K A M A N \_5$ & 0.7633 & 0.7412 \\
\hline 46 & GMD_2 & 0.7631 & 0.7403 \\
\hline 47 & VALEN_2 & 0.7623 & 0.7391 \\
\hline 48 & VALEN_1 & 0.7479 & 0.7196 \\
\hline 49 & KAMAN_4 & 0.7267 & 0.6971 \\
\hline
\end{tabular}

Table 74: IFAX correlation graph key for digits. 


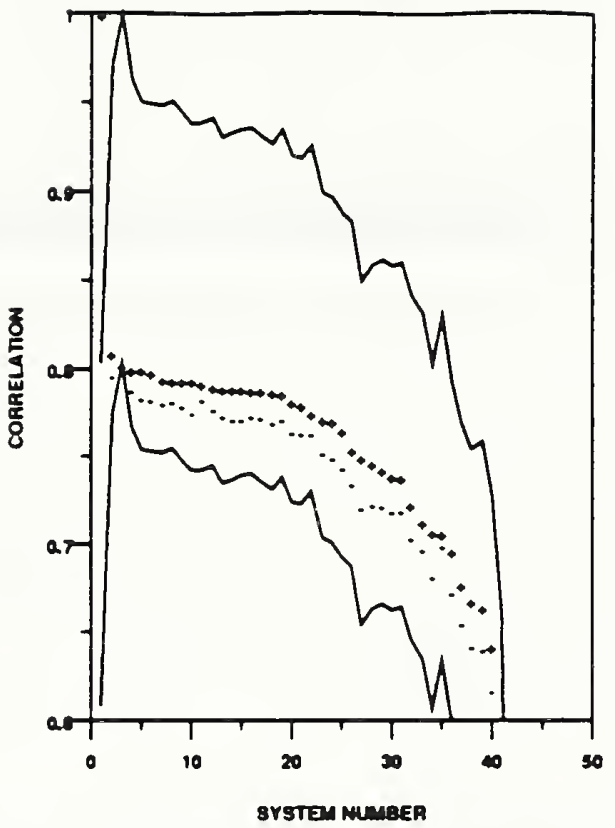

Figure 119: IFAX - upper case correlation

\begin{tabular}{|c|c|c|c|}
\hline System Number & System Name & Correlation (all) & Correlation (correct) \\
\hline 1 & IFAX & 1.0000 & 1.0000 \\
\hline 2 & VOTE_M & 0.8100 & 0.7967 \\
\hline 3 & REFERENCE & 0.8040 & 0.8040 \\
\hline 4 & $A E G$ & 0.8007 & 0.7889 \\
\hline $\mathbf{s}$ & A T T -4 & 0.8004 & 0.7846 \\
\hline B & UMICH-I & 07992 & 0.7834 \\
\hline 7 & ERIM-1 & 0.7948 & 0.7814 \\
\hline $\mathbf{B}$ & NYNEX & 0.7947 & 0.7828 \\
\hline$\theta$ & ATT 2 & 0.7946 & 0.7799 \\
\hline 10 & UBOL & 0.7948 & 0.7762 \\
\hline 11 & VOTEP & 0.7927 & 0.7838 \\
\hline 12 & NESTOR & 0.7908 & 0.7775 \\
\hline 13 & KODAK & 0.7901 & 0.7738 \\
\hline 14 & HUGHES 2 & 0.7901 & 0.7723 \\
\hline 13 & HUGHES . 1 & 0.7898 & 0.7727 \\
\hline 16 & IBM & 0.7893 & 0.7746 \\
\hline 17 & ATT 3 & 0.7885 & 0.7731 \\
\hline 18 & SYMBUS & 0.7877 & $0.770 B$ \\
\hline 19 & ATT $\perp$ & 0.7870 & 0.7723 \\
\hline 20 & GTESS-L & 0.7822 & 0.7649 \\
\hline 21 & GTESS_Z & 0.7808 & 0.7640 \\
\hline 22 & OCRSYS & 0.7758 & 0.7642 \\
\hline 23 & MIME & 0.7726 & 0.7329 \\
\hline 24 & NIST_4 & $0.771 \mathrm{~s}$ & 0.7507 \\
\hline 25 & ASOL & 0.7658 & 0.7449 \\
\hline 26 & REI & 0.7549 & 0.7357 \\
\hline 27 & KAMAN-1 & 0.7301 & 0.7217 \\
\hline 28 & RISO & 0.7479 & 0.7241 \\
\hline 29 & NIST_l & 0.7436 & 0.7231 \\
\hline 30 & GMD_3 & 0.7401 & 0.7198 \\
\hline 31 & GMD - 1 & 0.7392 & 0.7198 \\
\hline 32 & GMD_4 & 0.7240 & 0.7042 \\
\hline 33 & נה TIST & 0.7140 & 0.6981 \\
\hline 34 & KAMAN_J & 0.7081 & 0.6824 \\
\hline 33 & COMCOM & 0.7088 & 0.6995 \\
\hline 36 & KAMAN_2 & 0.8973 & 0.8731 \\
\hline 37 & $\mathrm{NIST}_{2}$ & 0.6773 & 0.6536 \\
\hline 38 & GMD_2 & 0.8686 & 0.6428 \\
\hline 39 & VALEN_L & 0.6650 & 0.6406 \\
\hline 40 & $K A M A N-4$ & 0.6431 & 0.6176 \\
\hline 41 & KAMAN_S & 0.5874 & 0.3646 \\
\hline 42 & $\mathrm{UMICH}_{2}$ & 0.0837 & 0.0157 \\
\hline
\end{tabular}

Table 75: IFAX correlation graph key for uppers. 


\section{No Data Available}

Figure 120: IFAX - lower case correlation

There was no date for this evaluation.

Table 76: IFAX correlation graph key for lowers. 
SYSTEM: KAMAN_1

PARTICIPANT: Mark G. Costello

ORGANIZATION: Kaman Sciences Corporation, Utica, NY

FEATURES :

CLASSIFICATION :

HARDWARE: SPARC2, mUItiuser

TRAINING: DIGITS UPPERS LOWERS DATABASE

$8002080 \quad 2080 \quad$ NSDB1?

STATUS: $\quad$ on time

RESULTS: -- DIGITS -- -- UPPERS -- -- LOWERS -- DATABASE

REJ. ERR. REJ. ERR. REJ. ERR. TESTDATA1

RATE RATE-- RATE RATE-- RATE RATE--

$\begin{array}{llllll}0.00 & 0.1146 & 0.00 & 0.1503 & 0.00 & 0.3111\end{array}$

OCR RATE (CPS): DIGITS UPPERS LOWERS

$\begin{array}{llll}\text { SYS RATE : } & 0.50 & 0.38 & 0.43\end{array}$

CPU RATE :

NOTE: The CON files for the KAMAN systems had numbers greater than 1, which is not allowed by the NIST scoring package, so no rejection-rate data was calculated.

NOTE: No details of recognition algorithms provided. 
SYSTEM: KAMAN_1

BIBLIOGRAPHY:

The following references have been provided for this system: 


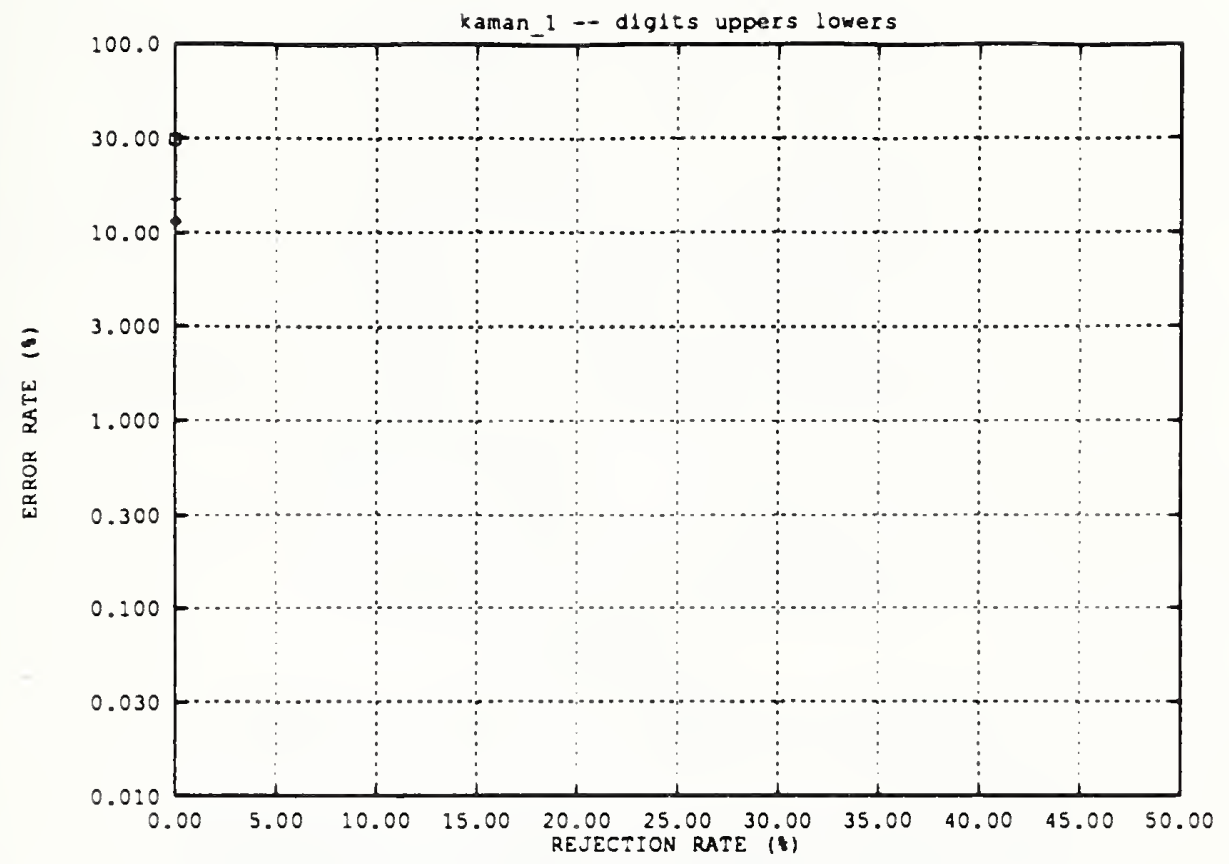

Figure 121: Error rate versus rejection rate for KAMAN_1

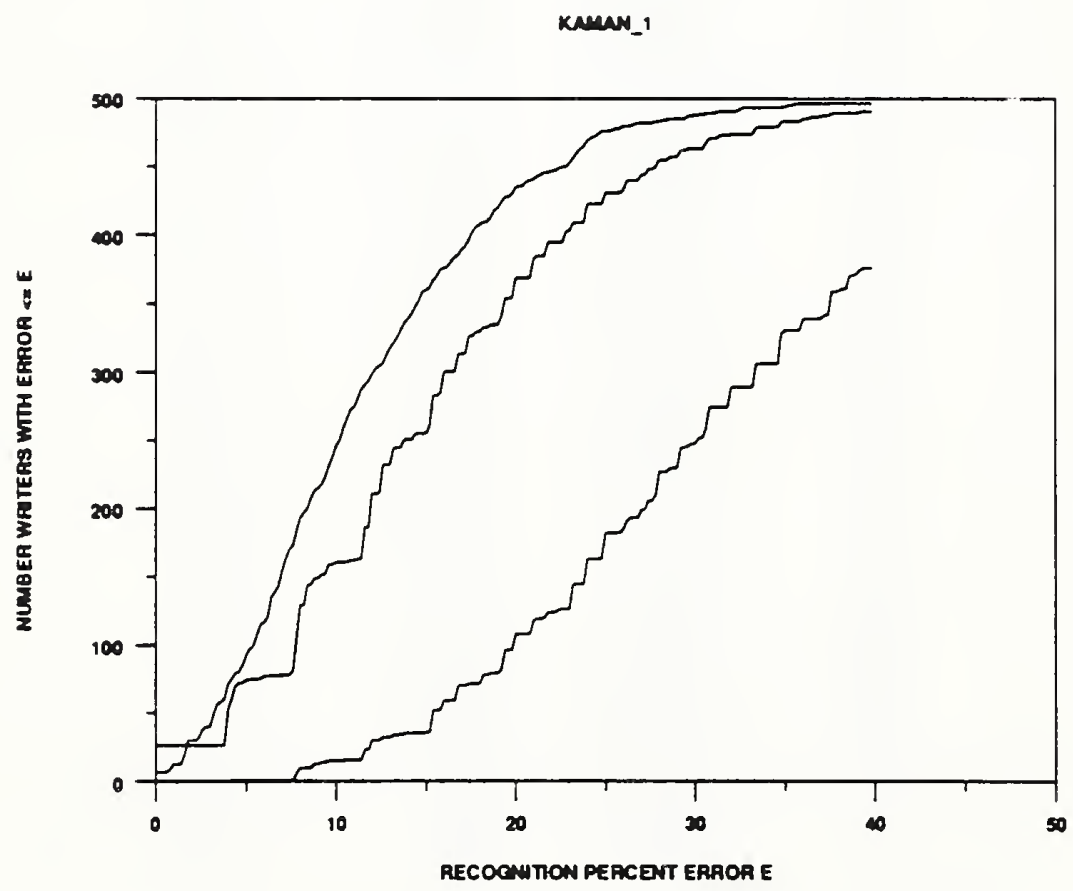

Figure 122: Error rate per writer of KAMAN_1 


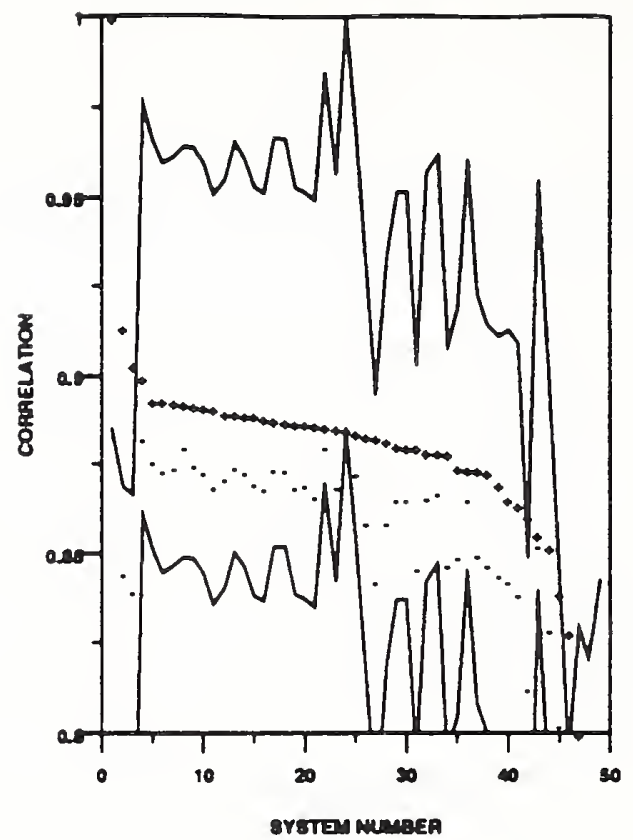

Figure 123: KAMAN_1 - digit correlation

\begin{tabular}{|c|c|c|c|}
\hline System Number & System Name & Coprelabion (All) & Correlation (correcs) \\
\hline 1 & KAMAN-I & 1.0000 & 1.0000 \\
\hline 2 & KAMAN 3 & 0.9142 & 0.8448 \\
\hline $\mathbf{3}$ & KAMAN_2 & 0.9036 & 0.8398 \\
\hline 4 & VOTE_M & 0.8908 & 0.8827 \\
\hline s & AEG & 0.8937 & 0.8762 \\
\hline 6 & ATT 4 & 0.8934 & 0.8738 \\
\hline 7 & ERIM d & 0.8930 & 0.8746 \\
\hline$B$ & VOTE P & 0.8924 & 0.8804 \\
\hline 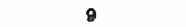 & ATT 2 & 0.8922 & 0.8782 \\
\hline 10 & KODAK2 & 0.8918 & 0.8733 \\
\hline 11 & NIST-4 & 0.8914 & 0.8694 \\
\hline 12 & IBM & 0.8898 & 0.8745 \\
\hline 13 & NESTOR & 0.8898 & 0.8713 \\
\hline 14 & ERIM 2 & 0.8896 & 0.8733 \\
\hline 15 & KODAK_ & 0.8893 & 08699 \\
\hline 16 & THINK-1 & 0.8887 & 0.8687 \\
\hline 17 & ELSAGBA & 0.8879 & 0.8742 \\
\hline 18 & ELSAGB2 & 0.8876 & 0.8739 \\
\hline 19 & SYMBUS & 0.8873 & 0.8693 \\
\hline 20 & ATT 3 & 0.8869 & 0.8698 \\
\hline 21 & ELSAGB_I & 0.8868 & 0.8666 \\
\hline 22 & OCRSYS & 0.8864 & 0.8800 \\
\hline 23 & UBOL & 0.8857 & 0.8691 \\
\hline 24 & REFERENCE & 0.8854 & 0.8854 \\
\hline 25 & $\boldsymbol{A T T} \perp$ & 0.8845 & 0.8730 \\
\hline 26 & GTESS 2 & 0.8833 & 0.8593 \\
\hline 27 & RISO & 0.8828 & 0.8426 \\
\hline 28 & GTESS 1 & 0.8821 & 0.8593 \\
\hline 29 & HUGHES_L & 0.8808 & 0.8683 \\
\hline 30 & HUGHES_2 & 0.8802 & 0.8683 \\
\hline 31 & NIST & 0.8801 & 0.8463 \\
\hline 32 & THINK 2 & 0.8786 & 0.8674 \\
\hline 33 & NYNEX & 0.8786 & 0.8660 \\
\hline 34 & NIST 2 & 0.8788 & 0.8470 \\
\hline 35 & GMD 3 & 0.8748 & 0.8493 \\
\hline 36 & REI & 08743 & 0.8655 \\
\hline 37 & NIST_l & 0.8741 & 0.8498 \\
\hline 38 & MIME & 0.8733 & 0.8469 \\
\hline 38 & ASOL & 0.8701 & 0.8446 \\
\hline 40 & GMD - 1 & 0.8659 & 0.8425 \\
\hline 41 & UPENN & 0.8640 & 0.8387 \\
\hline 42 & KAMAN 3 & 0.8610 & 0.8127 \\
\hline 43 & СOMCOM & 0.8567 & 0.8627 \\
\hline 44 & GMD_4 & 0.8520 & 0.8289 \\
\hline 45 & GMD 2 & 0.8393 & 0.8024 \\
\hline 46 & KAMAN_A & 0.8284 & 0.7696 \\
\hline 47 & IFAX & 0.8003 & 0.7734 \\
\hline 48 & VALEN_1 & 0.7973 & 0.7678 \\
\hline 48 & VALEN 2 & 0.7961 & 0.7782 \\
\hline
\end{tabular}

Table 77: KAMAN_1 correlation graph key for digits. 


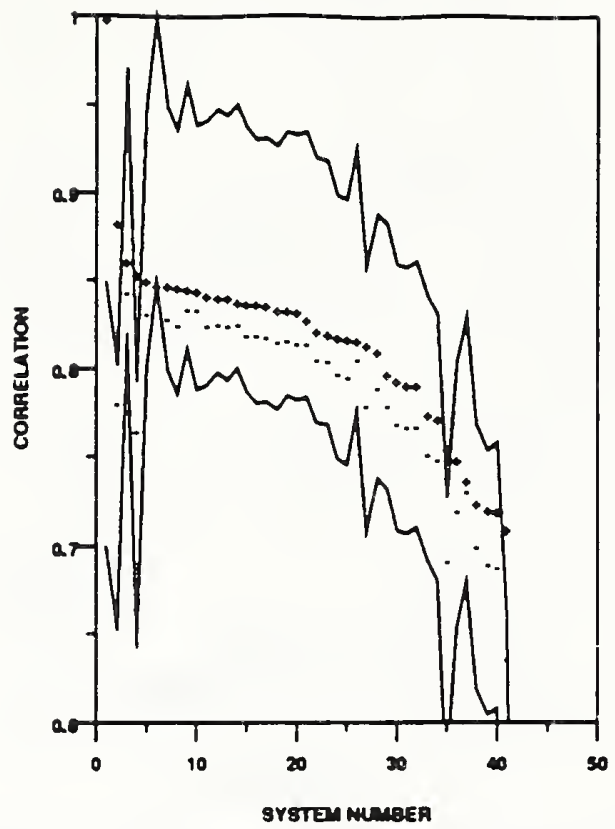

Figure 124: KAMAN_1 - upper case correlation

\begin{tabular}{|c|c|c|c|}
\hline System Number & System Name & Correlation (all) & Correlation (correct) \\
\hline 1 & KAMAN-1 & 1.0000 & 1.0000 \\
\hline 2 & KAMAN3 & 0.8844 & 0.7818 \\
\hline 3 & VOTE_M & 0.8836 & 0.8432 \\
\hline 4 & KAMAN.2 & 0.8588 & 0.7681 \\
\hline $\mathrm{s}$ & A T T -4 & 08523 & 0.8333 \\
\hline 6 & REFERENCE & 0.8497 & 0.8497 \\
\hline 7 & UMICH_l & 0.8491 & 0.8306 \\
\hline 8 & IBM & 0.8482 & 0.8268 \\
\hline 9 & AEG & 0.8478 & 0.8354 \\
\hline 10 & VOTEP & 0.8470 & 0.8360 \\
\hline 11 & NESTOR & 0.8436 & 0.8268 \\
\hline 12 & ERIM-1 & 0.8434 & 0.8279 \\
\hline 13 & ATT2 2 & 0.8429 & 0.8261 \\
\hline 14 & NYNEX & 0.8400 & 0.8272 \\
\hline 15 & UBOL & 0.8393 & 0.8213 \\
\hline 16 & KODAK_ & 0.8393 & 0.8209 \\
\hline 17 & ATT 3 & 0.8385 & 0.8204 \\
\hline 18 & HUGHES - 1 & 08361 & 0.8184 \\
\hline 19 & SYMBUS & 08361 & 0.8178 \\
\hline 20 & HUGHES .2 & 08349 & 0.8168 \\
\hline 21 & ATT $\perp$ & 0.8304 & 0.8160 \\
\hline 22 & GTESS-1 & 0.8240 & 0.8076 \\
\hline 23 & $\operatorname{GTESS} 2$ & 0.8223 & 0.8065 \\
\hline 24 & MIME & 0.8204 & 0.7987 \\
\hline 25 & NIST 4 & 08189 & 0.7967 \\
\hline 26 & OCRSYS & 0.8180 & 0.8070 \\
\hline 27 & RISO & 0.8133 & 0.7803 \\
\hline 28 & ASOL & 0.8116 & 0.7903 \\
\hline 29 & REI & 0.7991 & 07803 \\
\hline 30 & GMD_1 & 0.7949 & 0.7708 \\
\hline 31 & GMD_3 & 0.7927 & $0.768 \mathrm{~s}$ \\
\hline 32 & NIST 1 & 0.7821 & 0.7689 \\
\hline 33 & GMD_4 & 0.7763 & 0.73332 \\
\hline 34 & ב & 0.7729 & 0.7499 \\
\hline 35 & KAMAN-4 & 07366 & 0.6932 \\
\hline 36 & IFAX & 0.7501 & 0.7217 \\
\hline 37 & СOMCOM & 0.7391 & 0.7327 \\
\hline 38 & NIS T 2 & 0.7268 & 0.7012 \\
\hline 39 & GMD _2 & 0.7223 & 0.6917 \\
\hline 40 & VALEN,1 & 0.7218 & 0.6901 \\
\hline 41 & KAMAN.3 & 0.7117 & 0.6372 \\
\hline 42 & $\mathrm{UMICH}_{2}$ & 0.0648 & 0.0149 \\
\hline
\end{tabular}

Table 78: KAMAN_1 correlation graph key for uppers. 


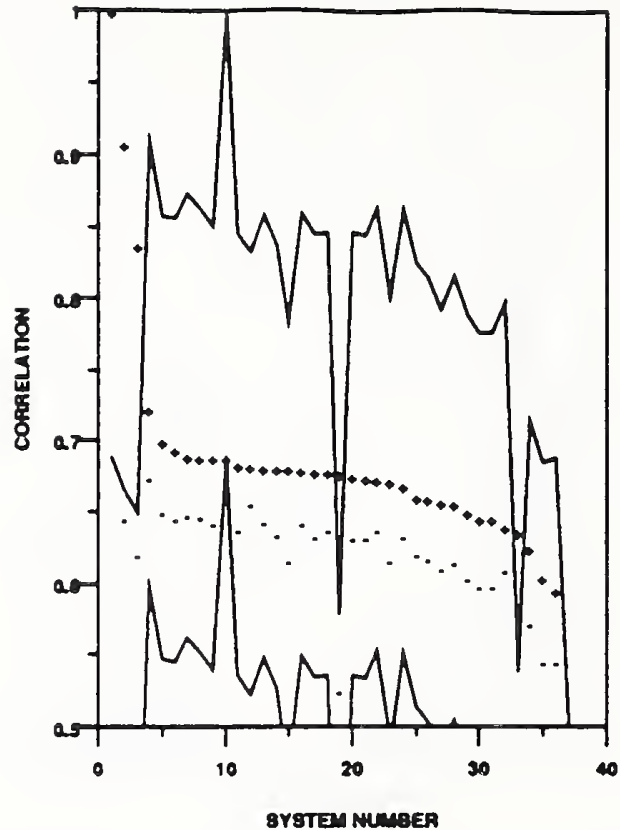

Figure 125: KAMAN_1 - lower case correlation

\begin{tabular}{|c|c|c|c|}
\hline System Number & Syotem Name & Corpelation (ail) & Corretation (correct) \\
\hline 1 & KAMAN-I & 1.0000 & 1.0000 \\
\hline 2 & KAMAN_a & 0.9088 & 0.0464 \\
\hline 3 & $\mathrm{KAMAN}_{2}$ & 0.8382 & 0.6208 \\
\hline 4 & VOTE_M & 0.7220 & 0.6749 \\
\hline 3 & ATT_4 & 0.7004 & 0.6510 \\
\hline 6 & KODAK」 & 0.6947 & 0.6460 \\
\hline 7 & AEG & 06899 & 0.6487 \\
\hline 8 & ERIM_I & 0.6892 & 0.6481 \\
\hline 9 & UMICH-I & 0.6890 & 0.6428 \\
\hline 10 & REFERENCE & 0.6889 & 0.6889 \\
\hline 11 & IBM & 0.6848 & 0.6390 \\
\hline 12 & VOTEP & 0.6834 & 0.6568 \\
\hline 13 & ATT2 2 & 0.6825 & 0.6442 \\
\hline 14 & ATT 3 & 0.6822 & 0.6355 \\
\hline is & RISO & 0.6821 & 06172 \\
\hline 16 & NYNEX & 0.8809 & 0.6431 \\
\hline 17 & UBOL & 0.6802 & 0.6338 \\
\hline 18 & NESTOR & 0.6800 & 0.6382 \\
\hline 19 & KAMAN_-3 & 0.6787 & 0.5261 \\
\hline 20 & HUGHES - 1 & 0.8762 & 0.6332 \\
\hline 21 & HUGHES-2 & 0.6747 & 0.6324 \\
\hline 22 & OCRSYS & 0.6744 & 0.6381 \\
\hline 23 & NIST_4 & 0.6733 & 0.6166 \\
\hline 24 & ATT」 & 0.6697 & 0.6336 \\
\hline 25 & GTESS -1 & 0.8618 & 0.6208 \\
\hline 26 & NIST_l & 0.6604 & 0.6181 \\
\hline 27 & GMD_3 & 0.6384 & 0.6108 \\
\hline 28 & GTESS 2 & 0.6371 & 0.6157 \\
\hline 29 & ASOL & 0.8506 & 0.8039 \\
\hline 30 & GMD_4 & 0.6470 & 0.5987 \\
\hline 31 & GMD_1 & 0.6470 & 0.5987 \\
\hline 32 & NIST & 0.6411 & 0.6094 \\
\hline 33 & KAMAN-4 & 0.6373 & 0.4952 \\
\hline 34 & $\mathrm{GMD} \_$ & 0.6262 & 0.5715 \\
\hline 35 & VALEN_I & 0.6037 & 0.5438 \\
\hline 36 & NIST $_{2}$ & 0.3961 & 0.3460 \\
\hline 37 & COMCOM & 0.4218 & 0.4098 \\
\hline 38 & UMICH 2 & 0.1263 & 0.0362 \\
\hline
\end{tabular}

Table 79: KAMAN_ 1 correlation graph key for lowers. 
SYSTEM: KAMAN_2

PARTICIPANT: Mark G. Costello

ORGANIZATION: Kaman Sciences Corporation, Utica, NY

FEATURES :

CLASSIFICATION :

HARDWARE: SPARC2, multiuser

TRAINING: DIGITS UPPERS LOWERS DATABASE

$800 \quad 2080 \quad 2080 \quad$ NSDB1?

STATUS: $\quad$ on time

RESULTS: -- DIGITS -- -- UPPERS -- -- LOWERS -- DATABASE

REJ. ERR. REJ. ERR. REJ. ERR. TESTDATA1

RATE RATE-- RATE RATE-- RATE RATE--

$\begin{array}{lllllll}0.00 & 0.1338 & 0.00 & 0.2074 & 0.00 & 0.3511\end{array}$

OCR RATE (CPS): DIGITS UPPERS LOWERS

$\begin{array}{llll}\text { SYS RATE: } & 0.76 & 0.47 & 0.47\end{array}$

CPU RATE: 
SYSTEM: KAMAN 2

BIBLIOGRAPHY:

The following references have been provided for this system: 


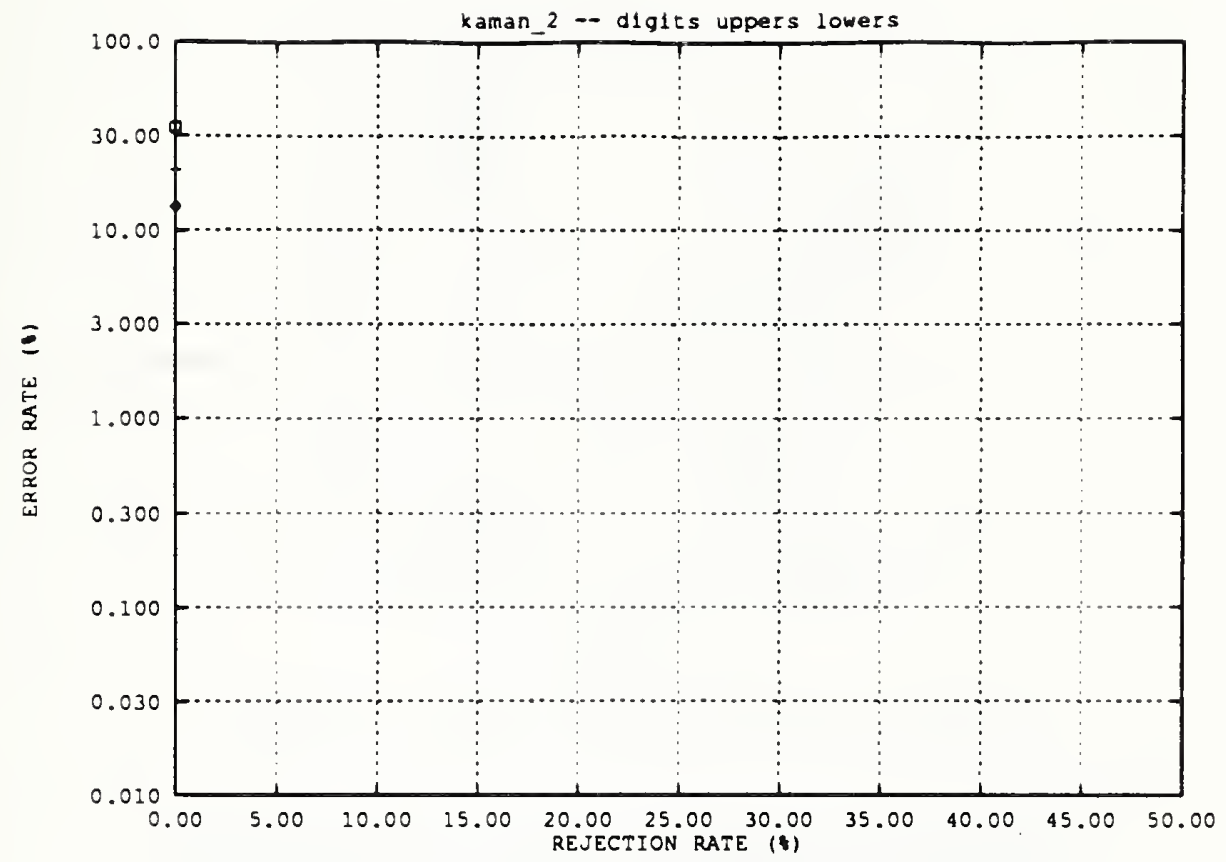

Figure 126: Error rate versus rejection rate for KAMAN 2

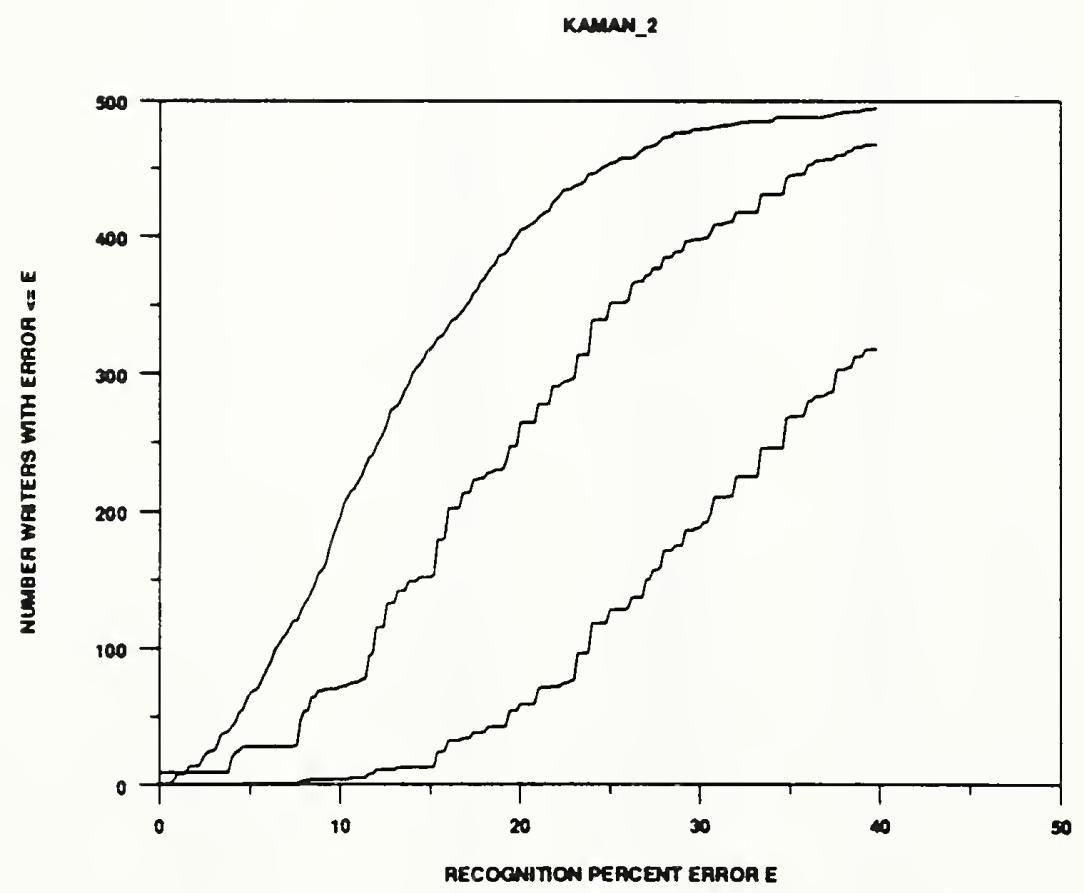

Figure 127: Error rate per writer of KAMAN_2 


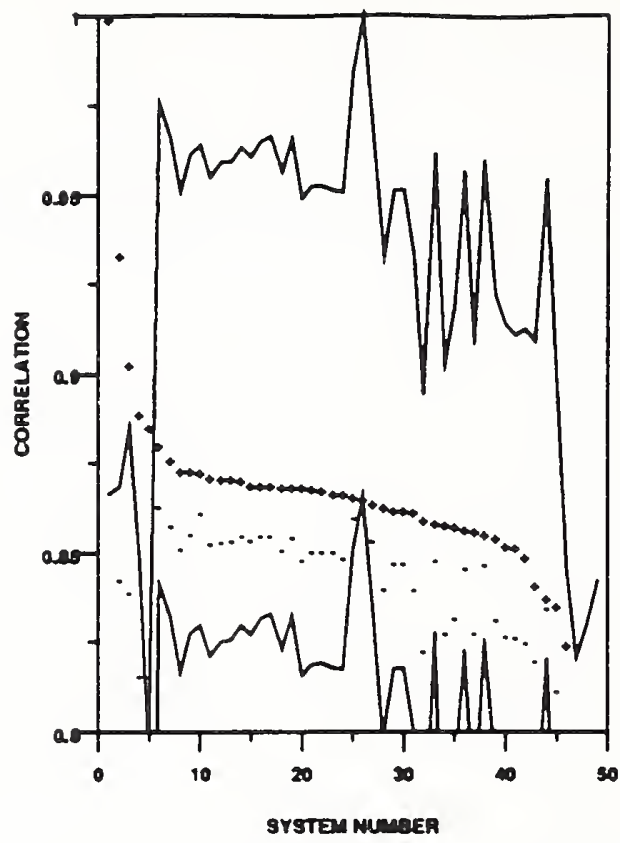

Figure 128: KAMAN 2 - digit correlation

\begin{tabular}{|c|c|c|c|}
\hline System Number & Syatem Name & Correlation (all) & Correlation (correct) \\
\hline 1 & KAMAN_2 & 1.0000 & 1.0000 \\
\hline 2 & KAMAN_S & 0.9343 & 08436 \\
\hline 3 & KAMAN-1 & 0.8036 & 0.8398 \\
\hline 4 & KAMAN_S & 0.8899 & 0.8162 \\
\hline 5 & KAMAN-4 & 0.8864 & 0.7807 \\
\hline B & VOTE $M$ & 08809 & 0.8639 \\
\hline 7 & AEG & 0.8768 & 0.8387 \\
\hline 8 & ERIM-1 & 0.8742 & 0.8561 \\
\hline 9 & NIST -4 & 0.8742 & 08320 \\
\hline 10 & VOTEP & 08739 & 0.8621 \\
\hline 11 & NESTOR & 08726 & 0.8337 \\
\hline 12 & ATT-4 & 0.8721 & 0.8542 \\
\hline 13 & KODAK2 2 & 0.8718 & 0.8546 \\
\hline 14 & ATT 2 & 08713 & 0.8339 \\
\hline 15 & IBM & 08701 & 0.8338 \\
\hline 16 & ERIM 2 & 0.8701 & 08345 \\
\hline 17 & ELSAGBA & 08698 & 08357 \\
\hline 18 & UBOL & 0.8698 & 0.8319 \\
\hline 18 & ELSAGB 2 & 08696 & 08534 \\
\hline 20 & ELSAGB-1 & 0.8693 & 08480 \\
\hline 21 & KODAK_ & 0.8692 & 0.8512 \\
\hline 22 & SYMBUS & 0.8683 & 08312 \\
\hline 23 & ATT 3 & 08680 & 0.8312 \\
\hline 24 & THINK_l & 08678 & 0.8494 \\
\hline 23 & OCRSYS & 08671 & 0.8608 \\
\hline 26 & REFERENCE & 0.8662 & 0.8862 \\
\hline 27 & ATT $\perp$ & 0.8631 & 0.8343 \\
\hline 28 & GTESS 2 & 0.8642 & 0.8408 \\
\hline 29 & HUGHES 1 & 0.8631 & 08480 \\
\hline 30 & HUGHES 2 & 08630 & 0.8480 \\
\hline 31 & GTESS.1 & 08626 & 08406 \\
\hline 32 & RISO & 0.8603 & 0.8233 \\
\hline 33 & THINK 2 & 0.8595 & 0.8489 \\
\hline 34 & NIST & 0.8591 & 0.8284 \\
\hline 35 & GMD_3 & 08386 & 0.8324 \\
\hline 36 & NYNEX & 08376 & 0.8468 \\
\hline 37 & $\mathrm{NIST}_{2}$ & 08571 & 0.8283 \\
\hline 38 & REI & 08364 & 08478 \\
\hline 39 & NIST.1 & 08556 & 0.8319 \\
\hline 40 & MIME & 0.8330 & 0.8276 \\
\hline 41 & ASOL & 0.8527 & 0.8271 \\
\hline 42 & GMD_1 & 0.8498 & 0.8257 \\
\hline 43 & UPENN & 0.8423 & 0.8205 \\
\hline 44 & COMCOM & 0.8386 & 0.8333 \\
\hline 43 & GMD - 4 & 0.8363 & 0.8123 \\
\hline 46 & GMD .2 & 0.8249 & 0.7868 \\
\hline 47 & VALEN_1 & 0.7921 & 0.7367 \\
\hline 48 & IFAX & 0.7848 & 0.7581 \\
\hline 49 & VALEN 2 & 0.7834 & 0.7836 \\
\hline
\end{tabular}

Table 80: KAMAN_2 correlation graph key for digits. 


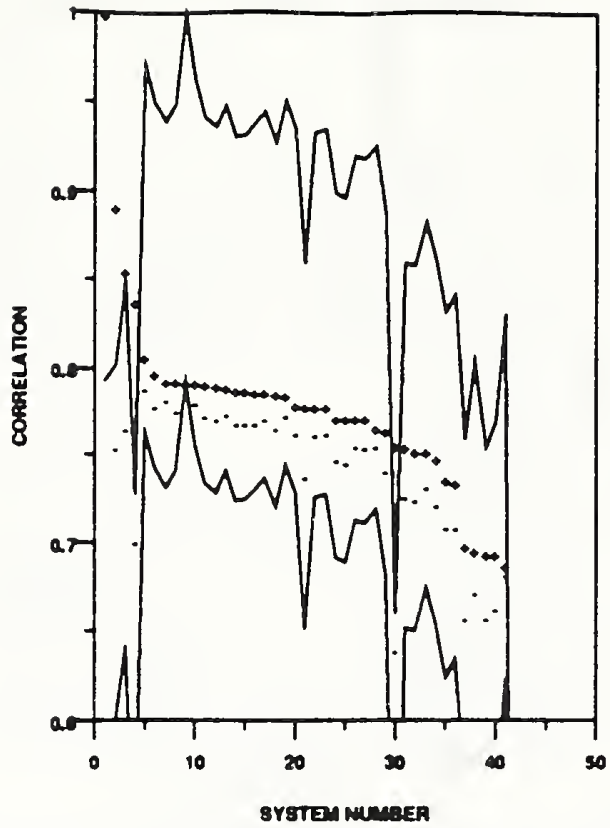

Figure 129: KAMAN 2 - upper case correlation

\begin{tabular}{|c|c|c|c|}
\hline System Number & Sysiem Name & Corselation (all) & Correlstion (correct) \\
\hline 1 & KAMAN2 & 1.0000 & 1.0000 \\
\hline 2 & KAMAN] & 0.8918 & 0.7846 \\
\hline $\mathbf{3}$ & KAMAN -1 & 0.8588 & 0.7661 \\
\hline 4 & KAMAN-4 & 0.8382 & 0.7018 \\
\hline s & VOTE_M & 08068 & 0.7889 \\
\hline 6 & ATT 4 & 0.7977 & 0.7789 \\
\hline 7 & VOTEP & 0.7935 & 0.7826 \\
\hline 8 & UMICH-1 & 0.7931 & 0.7736 \\
\hline 9 & REFERENCE & 0.7926 & 0.7926 \\
\hline 10 & $A E G$ & 0.7922 & 0.7802 \\
\hline 11 & NESTOR & 0.7916 & 0.7736 \\
\hline 12 & IBM & 0.7908 & 0.7710 \\
\hline 13 & ERIM-1 & 0.7901 & 0.7740 \\
\hline 14 & KODAK」 & 0.7884 & 0.7683 \\
\hline is & ATT & 0.7880 & 0.7684 \\
\hline 16 & ATT 2 & 0.7870 & 0.7712 \\
\hline 17 & UBOL & 07870 & 0.7684 \\
\hline 18 & SYMBUS & 0.7882 & 0.7639 \\
\hline 19 & NYNEX & 07856 & 0.7731 \\
\hline 20 & HUGHES -1 & 0.7798 & 0.7633 \\
\hline 21 & HUGHES.2 & 0.7792 & 0.7622 \\
\hline 22 & RISO & 0.7792 & 0.7381 \\
\hline 23 & ATT $\perp$ & 0.7788 & 0.7628 \\
\hline 24 & MIME & 0.7728 & 0.7488 \\
\hline 23 & NIST_A & 0.7726 & 0.7470 \\
\hline 26 & GTESS -1 & 0.7728 & 0.7354 \\
\hline 27 & GTESS2 & 0.7722 & 0.7346 \\
\hline 28 & OCRSYS & 0.7672 & 0.7335 \\
\hline 29 & ASOL & 0.7630 & 0.7421 \\
\hline 30 & $\mathrm{KAMAN}_{-3}$ & 0.7366 & 0.6400 \\
\hline 31 & GMD_1 & 0.7338 & 0.7271 \\
\hline 32 & GMD_s & 07333 & 0.7232 \\
\hline 33 & REI & 0.7333 & 0.7328 \\
\hline 34 & $\mathrm{NIST}_{-1}$ & 0.7494 & 0.7229 \\
\hline 33 & נב & 0.7374 & 07100 \\
\hline 36 & GMD_4 & 0.7360 & 0.7097 \\
\hline 37 & VALEN-1 & 0.6998 & 0.6388 \\
\hline 38 & IFAX & 0.6973 & 0.6731 \\
\hline 39 & GMD_2 & 0.6984 & 0.6886 \\
\hline 40 & NIST 2 & 0.6983 & 0.6640 \\
\hline 11 & COMCOM & 0.6890 & 0.6833 \\
\hline 12 & UMICH 2 & 0.0728 & 0.0131 \\
\hline
\end{tabular}

Table 81: KAMAN 2 correlation graph key for uppers. 


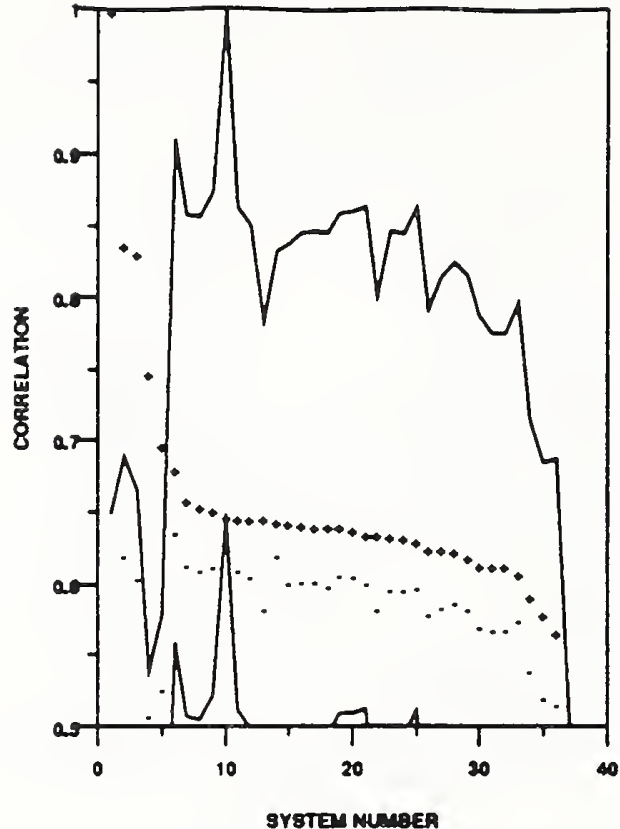

Figure 130: KAMAN2 - lower case correlation

\begin{tabular}{|c|c|c|c|}
\hline System Number & Syslem Name & Coprelation (all) & Correlasion (correct) \\
\hline 1 & KAMAN_2 & 1.0000 & 1.0000 \\
\hline 2 & KAMAN_1 & 0.8382 & 0.6208 \\
\hline $\mathbf{3}$ & KAMAN_J & 0.8320 & 0.6050 \\
\hline 4 & KAMAN-4 & 0.7484 & 0.5091 \\
\hline 5 & KAMAN_S & 06985 & 0.5268 \\
\hline 6 & VOTE_M & 0.6818 & 0.6378 \\
\hline 7 & ATT_4 & 06608 & 0.6148 \\
\hline 8 & KODAK_ & 0.6356 & 0.6112 \\
\hline 9 & AEG & 0.6831 & 0.6137 \\
\hline 10 & REFERENCE & 0.6489 & 0.6489 \\
\hline 11 & ERIM-1 & 0.6482 & 0.6113 \\
\hline 12 & UMICH_I & 0.6476 & 0.6062 \\
\hline 13 & RISO & 06472 & 0.5833 \\
\hline 14 & VOTEP & 0.6437 & 0.6209 \\
\hline 15 & ATT3 & 0.6442 & 0.6013 \\
\hline 16 & IBM & 0.6436 & 0.6032 \\
\hline 17 & NESTOR & 0.6418 & 0.6028 \\
\hline 18 & UBOL & 0.6417 & 0.5992 \\
\hline 19 & ATT2 2 & 0.8416 & 0.6073 \\
\hline 20 & NYNEX & 0.6398 & 0.6059 \\
\hline 21 & OCRSYS & 0.6362 & 0.6022 \\
\hline 22 & NIST-4 & 0.6360 & 0.5839 \\
\hline 23 & HUGHES_1 & 0.6351 & 0.5970 \\
\hline 24 & HUGHES 2 & 0.6342 & 0.5968 \\
\hline 25 & ATT_ & 0.8311 & 0.3983 \\
\hline 26 & GMD_3 & 0.6261 & 0.5800 \\
\hline 27 & NIST_l & 0.6255 & 0.5847 \\
\hline 28 & GTESS_- & 0.6245 & 0.5880 \\
\hline 29 & GTESS 2 & 0.6198 & 0.5828 \\
\hline J0 & ASOL & 0.6149 & 0.5710 \\
\hline 31 & GMD_4 & 0.6148 & 0.5684 \\
\hline 32 & GMD_1 & 0.6148 & 0.5884 \\
\hline 33 & ה הIST & 0.6083 & 0.5733 \\
\hline 34 & GMD _2 & 0.5921 & 0.5398 \\
\hline 33 & $V_{A L E N}-1$ & 0.3794 & 0.5218 \\
\hline 36 & NIST 2 & 0.3673 & 0.5173 \\
\hline 37 & СOMCOM & 0.3973 & 0.3868 \\
\hline 38 & UMICH 2 & 0.1227 & 0.0334 \\
\hline
\end{tabular}

Table 82: KAMAN 2 correlation graph key for lowers. 
SYSTEM: KAMAN_3

PARTICIPANT: Mark G. Costello

ORGANIZATION: Kaman Sciences Corporation, Utica, NY

FEATURES :

CLASSIFICATION :

HARDWARE: SPARC2, multiuser

TRAINING: DIGITS UPPERS LOWERS DATABASE

$8002080 \quad 2080 \quad$ NSDB1?

STATUS: $\quad$ on $\operatorname{time}$

RESULTS: -- DIGITS -- -- UPPERS -- -- LOWERS -- DATABASE

REJ. ERR. REJ. ERR. REJ. ERR. TESTDATA1

RATE RATE-- RATE RATE-- RATE RATE--

$\begin{array}{lllllll}0.00 & 0.1313 & 0.00 & 0.1978 & 0.00 & 0.3355\end{array}$

OCR RATE (CPS): DIGITS

UPPERS

LOWERS

SYS RATE:

0.76

0.47

0.47

CPU RATE: 
SYSTEM: KAMAN_3

\section{BIBLIOGRAPHY:}

The following references have been provided for this system: 


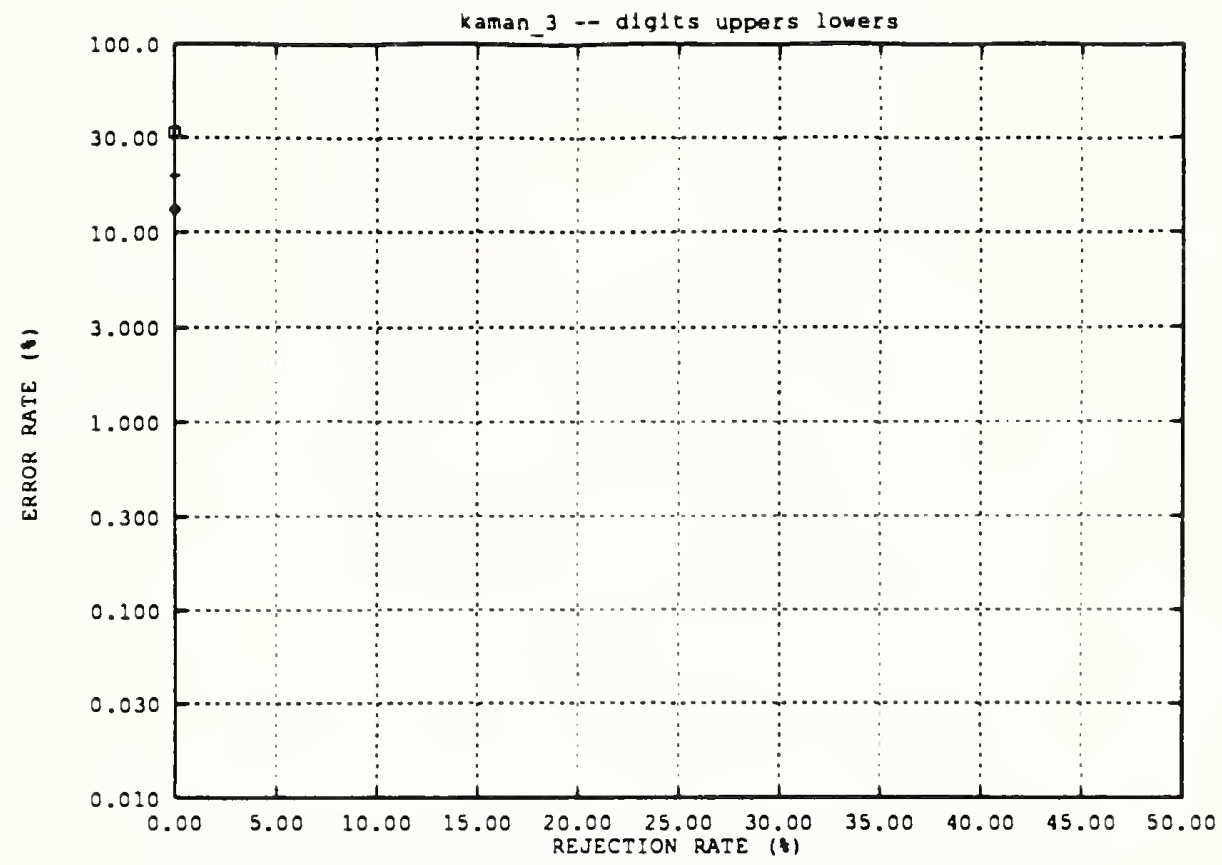

Figure 131: Error rate versus rejection rate for KAMAN_3

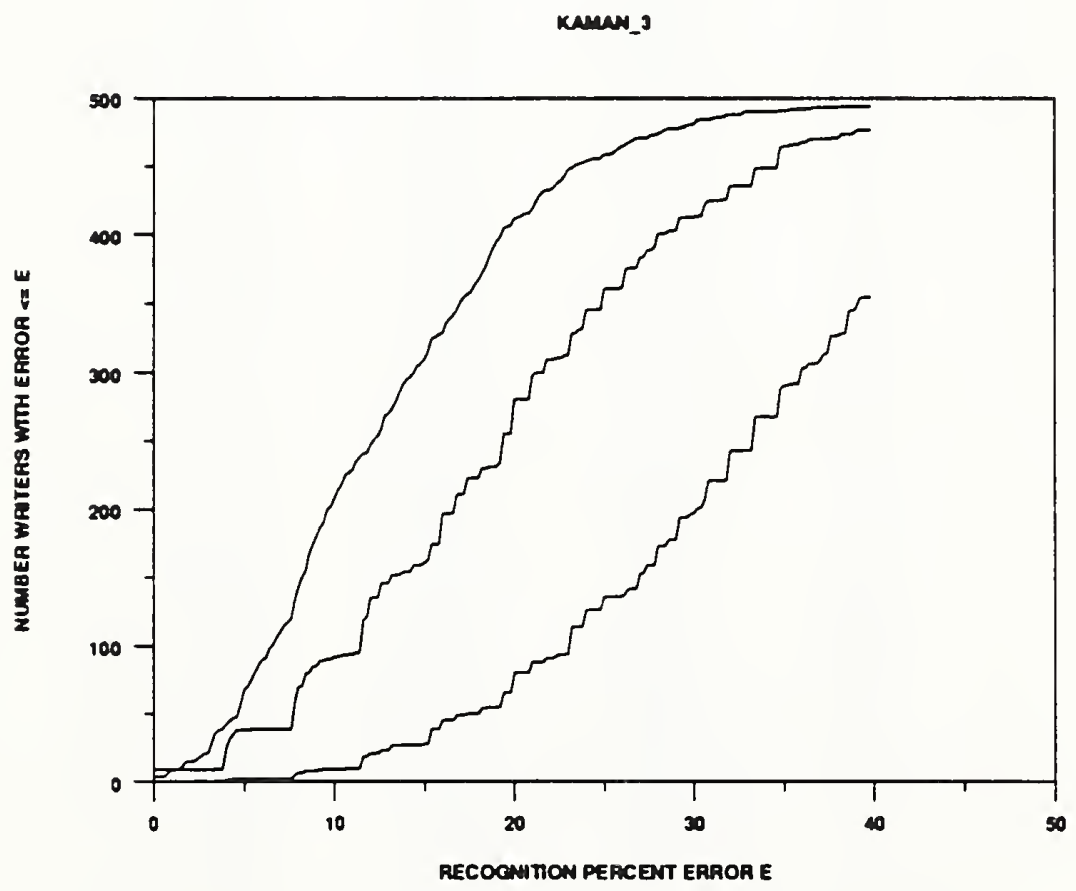

Figure 132: Error rate per writer of KAMAN_3 


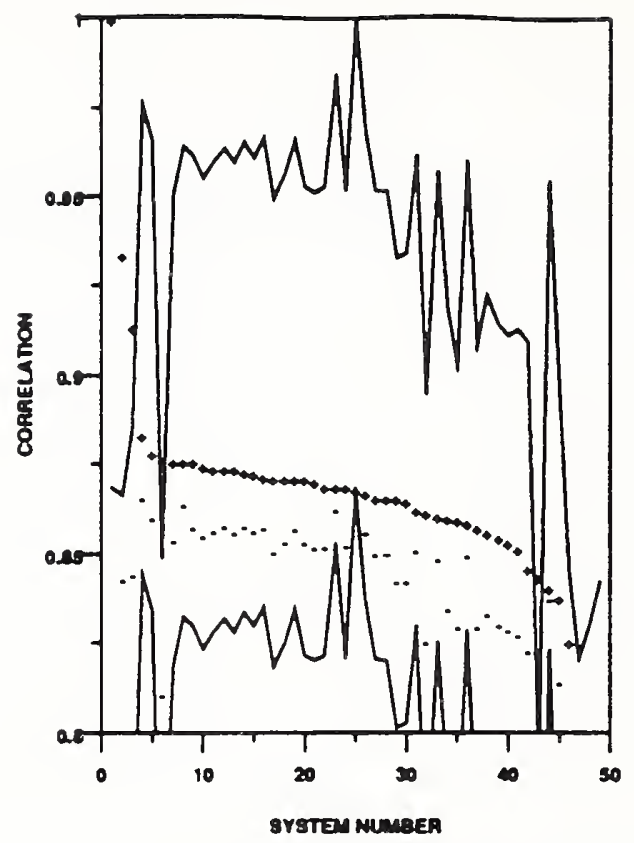

Figure 133: KAMAN_3 - digit correlation

\begin{tabular}{|c|c|c|c|}
\hline System Number & System Name & Coffelation (all) & Correlation (corfect) \\
\hline $\mathrm{l}$ & KAMAN_3 & 1.0000 & 1.0000 \\
\hline 2 & KAMAN_2 & 0.9343 & 0.8436 \\
\hline 3 & KAMAN-1 & 0.9142 & 0.8448 \\
\hline 4 & VOTEM & 0.8837 & 0.8664 \\
\hline 3 & AEG & 0.8789 & 0.8608 \\
\hline 6 & KAMAN_S & 0.8770 & 0.8111 \\
\hline 7 & NIST-4 & 0.8766 & 0.8544 \\
\hline 8 & VOTEP & 0.8765 & 0.8643 \\
\hline 8 & ERIM-1 & 0.8764 & 0.8583 \\
\hline 10 & NESTOR & 0.8753 & 0.8589 \\
\hline 11 & ATT 2 & 0.8747 & 0.8587 \\
\hline 12 & KODAK 2 & 0.8747 & 0.8572 \\
\hline 13 & A TT 4 & 0.8746 & 0.8566 \\
\hline 14 & IBM & 0.8737 & 0.8884 \\
\hline 13 & ERIM 2 & 0.8733 & 0.8572 \\
\hline 16 & ELSAGBA & 0.8723 & 0.8582 \\
\hline 17 & ELSAGB2 & 0.8720 & 0.8579 \\
\hline 18 & UBOL & 08720 & 0.8542 \\
\hline 19 & ELSAGB-1 & 0.8720 & 0.8513 \\
\hline 20 & KODAK」 & 0.8718 & 0.8538 \\
\hline 21 & THINK-1 & 0.8710 & 0.8323 \\
\hline 22 & SYMBUS & 0.8698 & 0.8528 \\
\hline 23 & OCRSYS & 0.8697 & 0.8633 \\
\hline 24 & ATT 3 & 0.8696 & 0.8530 \\
\hline 23 & REFERENCE & 08687 & 0.8687 \\
\hline 26 & ATT 1 & 0.8679 & 0.8569 \\
\hline 27 & HUGHES -1 & 0.8664 & 0.8508 \\
\hline 28 & HUGHES_2 & 0.8663 & 0.8507 \\
\hline 29 & $\operatorname{GTESS}_{2}$ & 0.8662 & 0.8432 \\
\hline 30 & GTESS_1 & 0.8653 & 0.8432 \\
\hline 31 & THINK 2 & 0.8630 & 0.8518 \\
\hline 32 & RISO & 0.8625 & 0.8238 \\
\hline 33 & NYNEX & 0.8614 & 0.8496 \\
\hline 34 & GMD_3 & 0.8610 & 0.8331 \\
\hline 33 & NIST 3 & 0.8604 & 0.8303 \\
\hline 36 & REI & 08596 & 0.8503 \\
\hline 37 & NIST 2 & 0.8580 & 0.8303 \\
\hline 38 & NIST_1 & 0.8870 & 0.8340 \\
\hline 39 & MIME & 0.8555 & 0.8306 \\
\hline 40 & ASOL & 0.8541 & 0.8291 \\
\hline 41 & GMD_-1 & 0.8520 & 0.8280 \\
\hline 42 & UPENN & 0.8466 & 0.8232 \\
\hline 43 & KAMAN_4 & 0.8443 & 0.7683 \\
\hline 44 & COMCOM & 0.8410 & 0.8378 \\
\hline 43 & GMD_4 & 0.8384 & 0.8147 \\
\hline 46 & GMD -2 & 0.8258 & 0.7887 \\
\hline 47 & VALEN_L & 0.7926 & 0.7384 \\
\hline 48 & IFAX & 0.7878 & 0.7603 \\
\hline 19 & VALEN 2 & 0.7847 & 0.7670 \\
\hline
\end{tabular}

Table 83: KAMAN_3 correlation graph key for digits. 


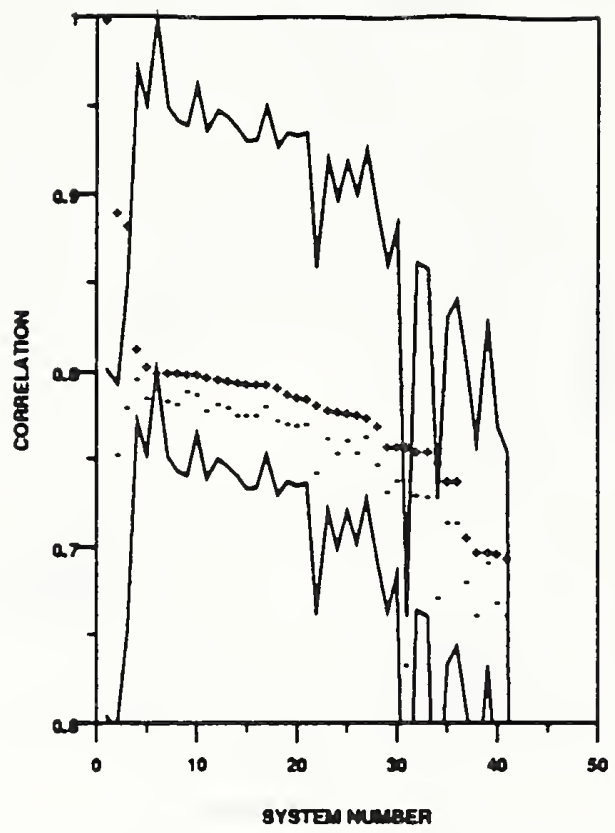

Figure 134: KAMAN_3 - upper case correlation

\begin{tabular}{|c|c|c|c|}
\hline Syatem Number & System Name & Correlation (all) & Coprelation (correct) \\
\hline $\mathrm{I}$ & KAMAN_3 & 1.0000 & 1.0000 \\
\hline 2 & KAMAN 2 & 0.8915 & 0.7346 \\
\hline 3 & $K_{A} M_{A N}$ & 08844 & 0.7818 \\
\hline 4 & VOTE_M & 0.8151 & 0.7983 \\
\hline 3 & A TT 4 & 0.8053 & 0.7873 \\
\hline 6 & REFERENCE & 0.8022 & 0.8022 \\
\hline 7 & UMICH-I & 08018 & 0.7849 \\
\hline 8 & NESTOR & 0.8013 & 0.7837 \\
\hline $\boldsymbol{9}$ & VOTEP & 0.8012 & 0.7910 \\
\hline 10 & AEG & 0.8007 & 0.7892 \\
\hline 11 & IBM & 0.7088 & 07801 \\
\hline 12 & ERIM_l & 0.7983 & 0.7833 \\
\hline 13 & ATT 2 & 0.7973 & 0.7813 \\
\hline 14 & UBOL & 07968 & 0.7774 \\
\hline 13 & KODAK」 & 0.7952 & 0.7769 \\
\hline 16 & ATT 3 & 0.7951 & 0.7767 \\
\hline 17 & NYNEX & 0.7949 & 0.7827 \\
\hline 18 & SYMBUS & 0.7934 & 0.7741 \\
\hline 19 & HUGHES -1 & 0.7894 & 0.7728 \\
\hline 20 & HUGHES -2 & 0.7881 & 0.7714 \\
\hline 21 & ATT $\perp$ & 07872 & 0.7723 \\
\hline 22 & RISO & 0.7835 & 0.7419 \\
\hline 23 & GTESS_L & 0.7807 & 0.7843 \\
\hline 24 & NIST 4 & 0.7797 & 0.7538 \\
\hline 23 & GTESS 2 & 0.7792 & 0.7633 \\
\hline 26 & MIME & 0.7782 & 0.7360 \\
\hline 27 & OCRSYS & 0.7756 & 0.7646 \\
\hline 28 & ASOL & 0.7711 & 0.7495 \\
\hline 29 & REI & 0.7597 & 07399 \\
\hline 30 & GMD . 1 & 07507 & 07339 \\
\hline 31 & KAMAN_3 & 0.7594 & 06348 \\
\hline 32 & NIST 1 & 0.7570 & 0.7313 \\
\hline 33 & GMD_3 & 0.7564 & 0.7313 \\
\hline 34 & KAMAN_4 & 07499 & 06728 \\
\hline 33 & GMD - 4 & 0.7408 & 0.7166 \\
\hline 36 & נב & 0.7408 & 0.7184 \\
\hline 37 & IFAX & 0.7081 & 0.6824 \\
\hline 38 & VALEN_I & 0.6998 & 0.6628 \\
\hline 39 & COMCOM & 0.6997 & 0.6937 \\
\hline 40 & NIST 2 & 0.6990 & 0.6708 \\
\hline 41 & GMD_2 & 0.6963 & 0.6633 \\
\hline 42 & $\mathrm{UMICH}_{2}$ & 00718 & 0.0137 \\
\hline
\end{tabular}

Table 84: KAMAN_3 correlation graph key for uppers. 


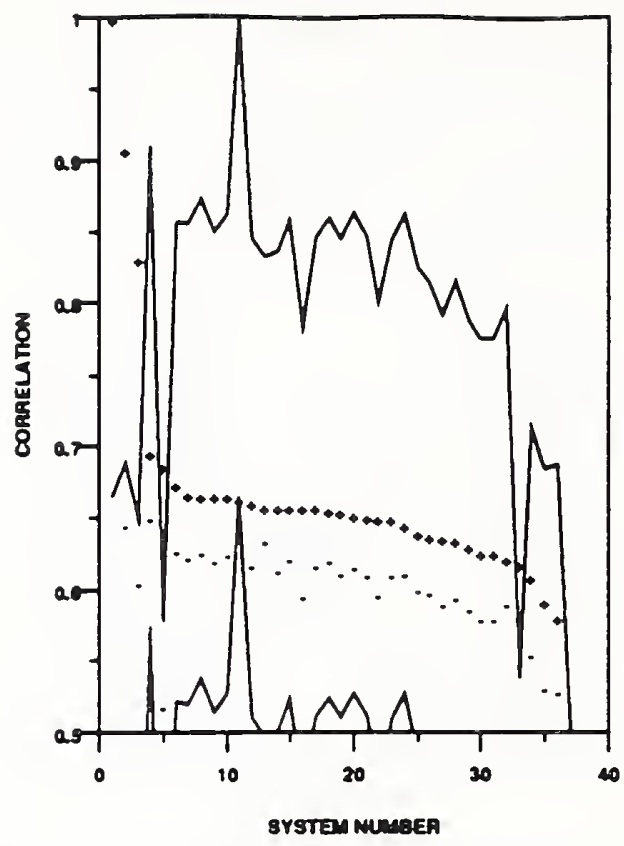

Figure 135: KAMAN_3 - lower case correlation

\begin{tabular}{|c|c|c|c|}
\hline Syotem Number & System Name & Correlation (sil) & Correlstion (correct) \\
\hline 1 & KAMAN_J & 1.0000 & 1.0000 \\
\hline 2 & KAMAN-1 & 09088 & 0.6464 \\
\hline 3 & KAMAN-2 & 08320 & 0.6050 \\
\hline 4 & VOTE_M & 0.6968 & 0.6808 \\
\hline 3 & KAMAN S & 0.8873 & 0.5186 \\
\hline 6 & ATT_A & 0.8730 & 0.6283 \\
\hline 7 & KODAKA & 06885 & 0.8230 \\
\hline 8 & $A E G$ & 0.6677 & 0.6266 \\
\hline 9 & UMICH_I & 0.6670 & 0.6216 \\
\hline 10 & ERIM-I & 06668 & 0.6262 \\
\hline 11 & REFERENCE & 0.6845 & 0.6645 \\
\hline 12 & IBM & 0.6624 & 0.8177 \\
\hline 13 & VOTE_P & 0.6897 & 0.6345 \\
\hline 14 & ATTS & 0.6597 & 0.6148 \\
\hline is & ATT 2 & 0.8898 & 0.6227 \\
\hline 16 & NESTOR & 0.6593 & 0.8179 \\
\hline 17 & RISO & 08393 & 0.5964 \\
\hline 18 & NYNEX & 0.8566 & 06209 \\
\hline 18 & UBOL & 0.8562 & 0.6116 \\
\hline 20 & OCRSYS & 0.6329 & 0.6169 \\
\hline 21 & HUGHES-1 & 0.6323 & 0.6112 \\
\hline 22 & NIST_4 & 0.8516 & 0.5969 \\
\hline 23 & HUGHES.2 & 0.6515 & 0.8109 \\
\hline 24 & ATT 1 & 0.6461 & 0.6118 \\
\hline 23 & GTESS $_{-1}$ & 0.6413 & 0.8007 \\
\hline 26 & NIST_l & 0.6388 & 0.5978 \\
\hline 27 & GMD_3 & 0.6373 & 0.5907 \\
\hline 28 & GTESS 2 & 0.6363 & 0.5949 \\
\hline 29 & ASOL & 0.6314 & 0.3863 \\
\hline 30 & GMD_4 & 0.6270 & 0.5793 \\
\hline 31 & GMD_I & 0.8270 & 0.5798 \\
\hline 32 & NIST_3 & 0.6222 & 0.3898 \\
\hline 33 & KAMAN-4 & 0.6184 & 0.4792 \\
\hline 34 & GMD _2 & 0.6093 & 0.5548 \\
\hline 33 & VALEN_1 & 0.5921 & 0.5312 \\
\hline 36 & NIST 2 & 05806 & 0.5297 \\
\hline 37 & СOMCOM & 04097 & 0.3981 \\
\hline 38 & $\mathrm{UMICH}_{2}$ & 0.1213 & 0.0336 \\
\hline
\end{tabular}

Table 85: KAMAN_3 correlation graph key for lowers. 


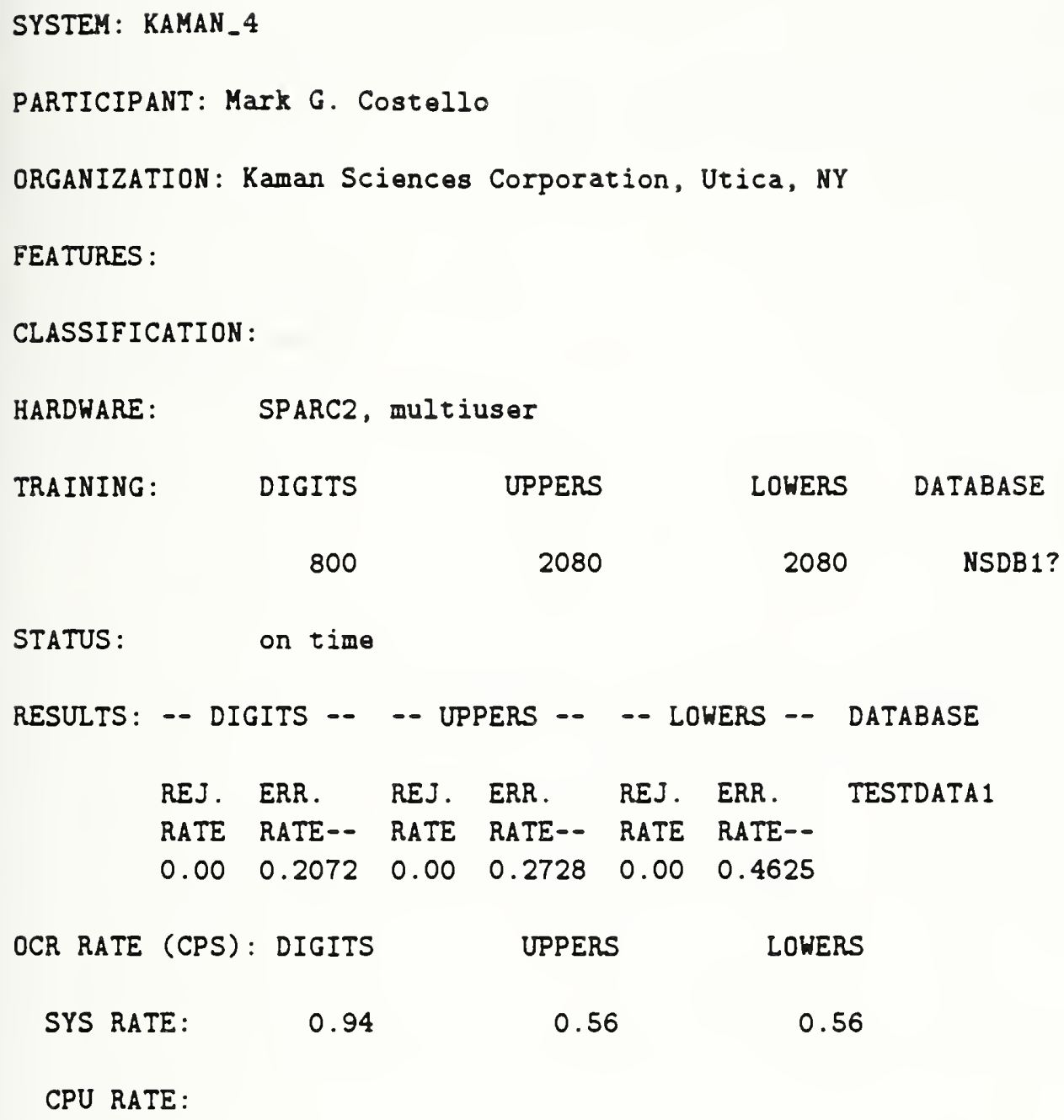


SYSTEM: KAMAN_4

BIBLIOGRAPHY:

The following references have been provided for this system: 


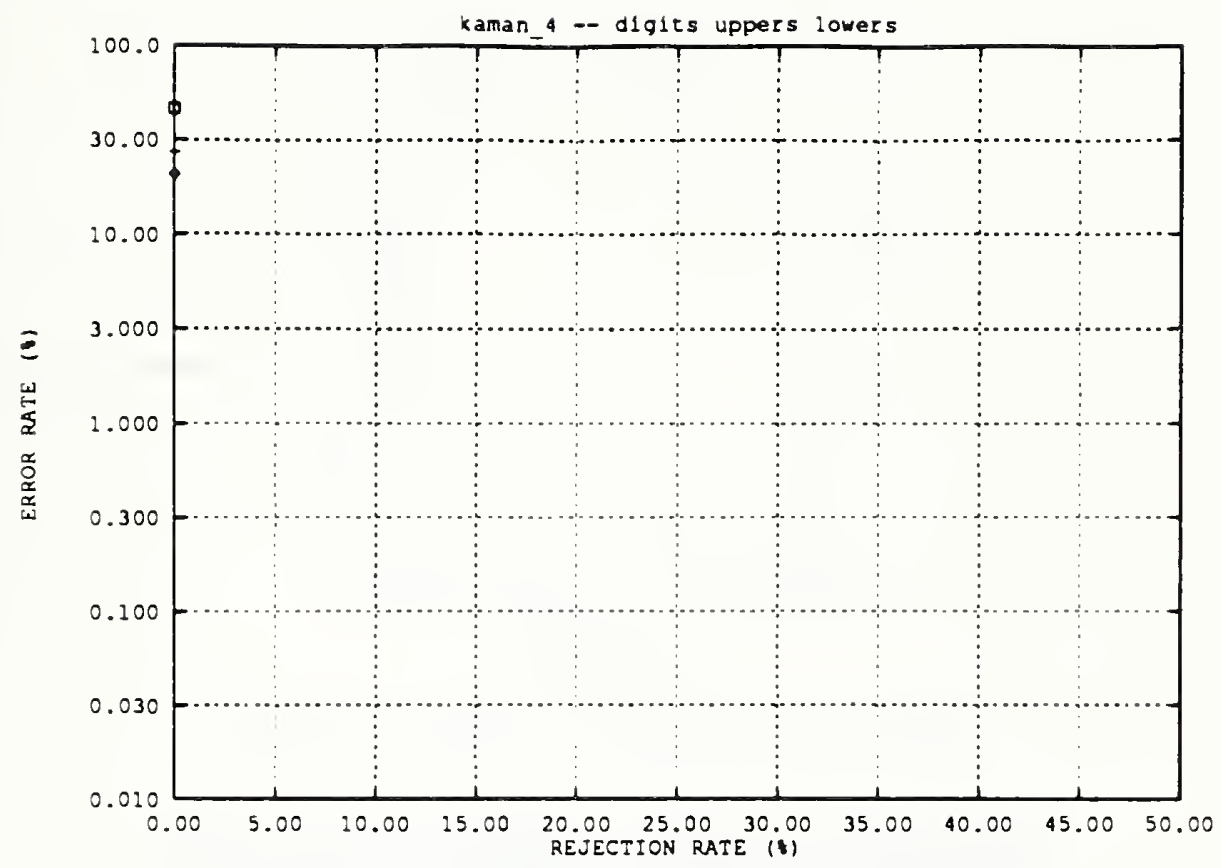

Figure 136: Error rate versus rejection rate for KAMAN_4

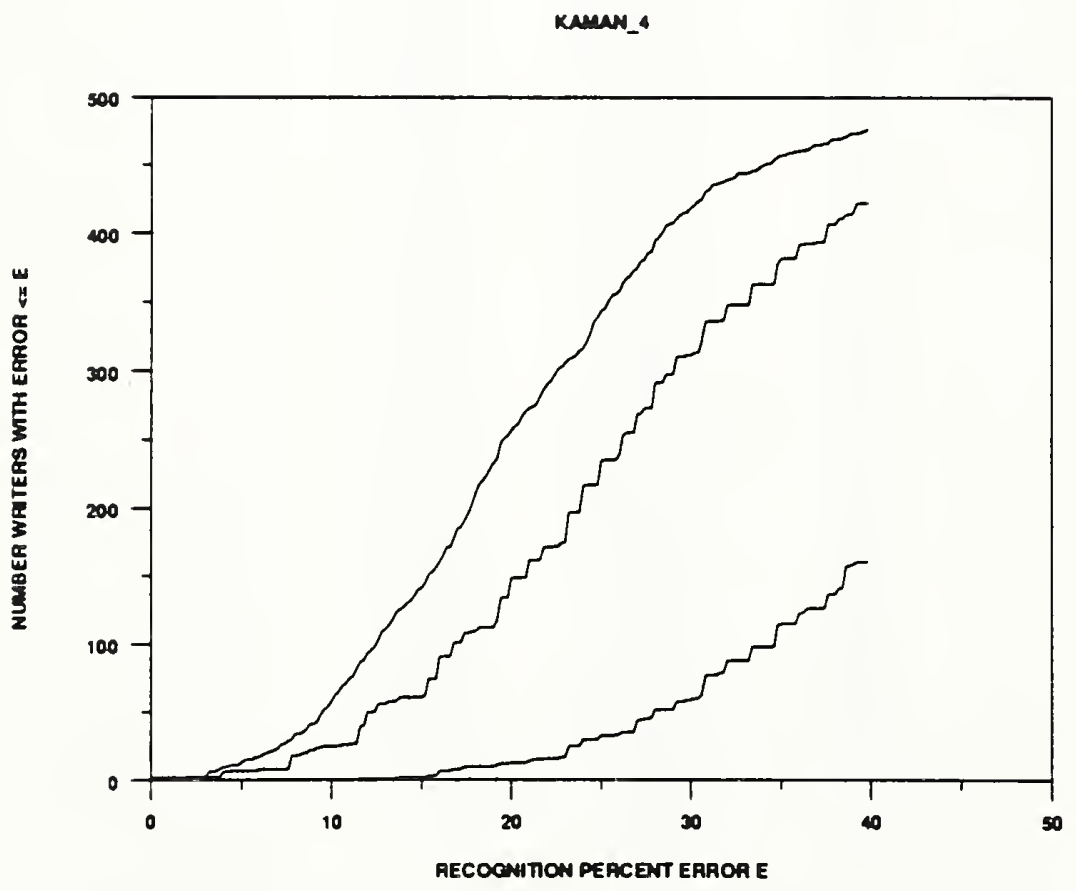

Figure 137: Error rate per writer of KAMAN_4 


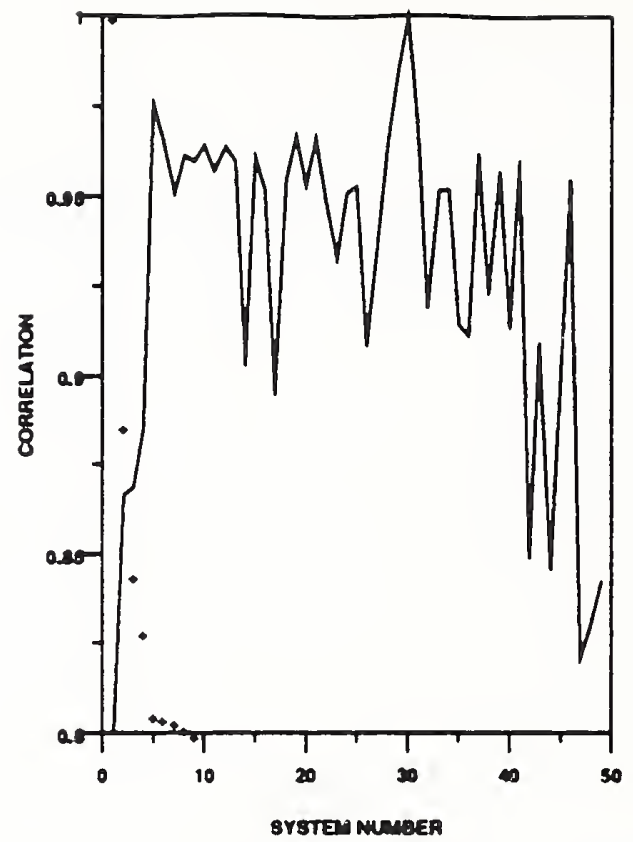

Figure 138: KAMAN_4 - digit correlation

\begin{tabular}{|c|c|c|c|}
\hline System Number & System Name & Corpelation (aII) & Correlation (correct) \\
\hline 1 & KAMAN_4 & 1.0000 & 1.0000 \\
\hline 2 & KAMAN_2 & 0.8864 & 0.7807 \\
\hline 3 & KAMAN_J & 0.8448 & 0.7683 \\
\hline 1 & KAMAN_1 & 08284 & 0.7696 \\
\hline 5 & VOTE_M & 0.8084 & 0.7903 \\
\hline 6 & $A E G$ & 0.8043 & 0.7867 \\
\hline 7 & NIST _4 & 0.8036 & 0.7813 \\
\hline 8 & ERIM_I & 0.8016 & 0.7844 \\
\hline 9 & ATT 4 & 0.8000 & 0.7826 \\
\hline 10 & VOTE_P & 0.7993 & $0.7 \mathrm{s8s}$ \\
\hline 11 & UBOL & 0.7990 & 0.7808 \\
\hline 12 & ATT 2 & 0.7983 & 0.7834 \\
\hline 13 & KODAKב 2 & 0.7978 & 07818 \\
\hline 14 & ERIM_2 & 0.7978 & 0.7825 \\
\hline 15 & NIST & 0.7976 & 0.7628 \\
\hline 16 & ATT 3 & 0.7978 & 0.7802 \\
\hline 17 & RISO & 0.7974 & 0.7582 \\
\hline 18 & NESTOR & 07971 & 0.7807 \\
\hline 19 & ELSAGBA & 0.7988 & 07834 \\
\hline 20 & ELSAGB 2 & 0.7965 & 07831 \\
\hline 21 & SYMBUS & 0.7965 & 07800 \\
\hline 22 & ELSAGB_I & 0.7984 & 07775 \\
\hline 23 & GTESS_2 & 0.7963 & 0.7715 \\
\hline 24 & THINK_I & 0.7962 & 0.7780 \\
\hline 25 & KODAK_ & 0.7955 & 0.7788 \\
\hline 26 & NIST 2 & 0.7980 & 0.7624 \\
\hline 27 & GTESS_1 & 0.7946 & 0.7713 \\
\hline 28 & IBM & 07245 & 0.7824 \\
\hline 29 & OCRSYS & 0.7933 & 0.7875 \\
\hline 30 & REFERENCE & 0.7928 & 0.7928 \\
\hline 31 & ATT_ $\perp$ & 0.7922 & 0.7821 \\
\hline 32 & GMD_3 & 0.7915 & 0.7646 \\
\hline 33 & HUGHES - 1 & 0.7894 & 0.7760 \\
\hline 34 & HUGHES_2 & 0.7891 & 0.7759 \\
\hline 35 & MIME & 0.7882 & 07608 \\
\hline 36 & THINK 2 & 07865 & 0.7766 \\
\hline 37 & ASOL & 0.7863 & 0.7592 \\
\hline 38 & $N_{1 S T}-1$ & 0.7863 & 0.7623 \\
\hline 38 & NYNEX & 0.7883 & 0.7750 \\
\hline 40 & REI & 0.7832 & 0.7755 \\
\hline 41 & GMD_I & 0.7832 & 0.7585 \\
\hline 42 & KAMAN .5 & 0.7763 & 0.7307 \\
\hline 43 & UPENN & 0.7740 & 0.7317 \\
\hline 14 & GMD _2 & 0.7727 & 0.7280 \\
\hline 45 & GMD_4 & 0.7713 & 0.7463 \\
\hline 46 & СОМСOM & 0.7676 & 0.7646 \\
\hline 17 & VALEN_I & 0.7310 & 0.6954 \\
\hline 48 & IFAX & 0.7267 & 0.6971 \\
\hline 49 & VALEN 2 & 0.7180 & 0.7006 \\
\hline
\end{tabular}

Table 86: KAMAN_4 correlation graph key for digits. 


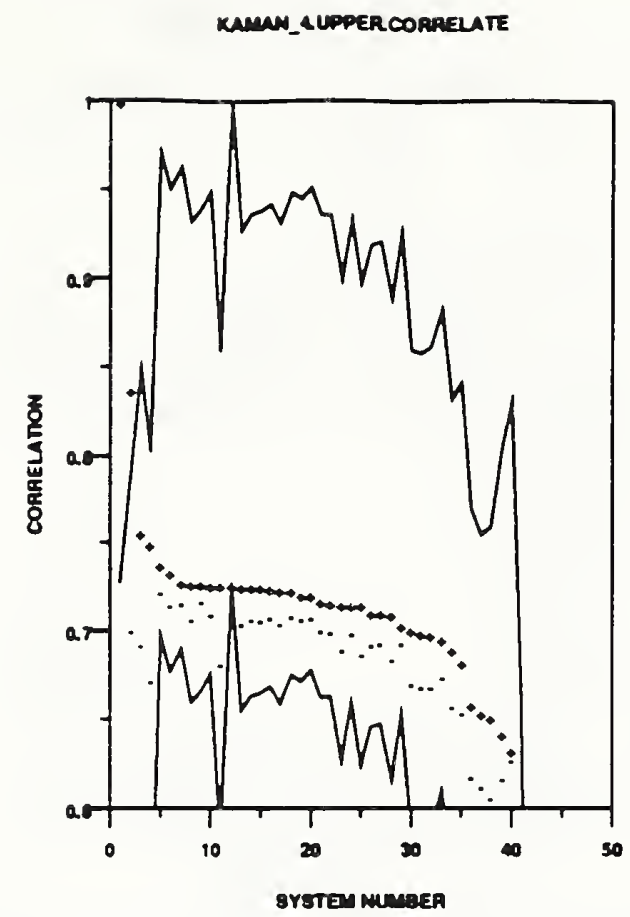

Figure 139: KAMAN_4 - upper case correlation

\begin{tabular}{|c|c|c|c|}
\hline System Number & System Name & Corretation (ail) & Corretation (correct) \\
\hline T & KAMAN.4 & 1.0000 & 1.0000 \\
\hline 2 & KAMAN_2 & 0.8382 & 0.7018 \\
\hline 3 & KAMAN_1 & 0.7366 & 0.6932 \\
\hline 4 & KAMAN_3 & 0.7499 & 0.6728 \\
\hline 3 & VOTE_M & 0.7397 & 0.7232 \\
\hline$B$ & ATT_4 & 07331 & 0.7162 \\
\hline 7 & $A E G$ & 0.7290 & 0.7169 \\
\hline 8 & ATT 3 & 0.7286 & 0.7078 \\
\hline 9 & VOTEP & 0.7278 & 0.7177 \\
\hline 10 & UMICH-1 & 0.7273 & 0.7108 \\
\hline 11 & REFERENCE & 0.7272 & 0.7272 \\
\hline 12 & RISO & 0.7272 & 0.6826 \\
\hline 13 & SYMBUS & 0.7267 & 0.7036 \\
\hline 14 & IBM & 0.7264 & 0.7076 \\
\hline 15 & UBOL & 0.7262 & 0.7070 \\
\hline 16 & NESTOR & 0.7238 & 0.7091 \\
\hline 17 & KODAK」 & 0.7249 & 0.7054 \\
\hline 18 & ERIM -1 & 0.7248 & 0.7097 \\
\hline 19 & ATT2 2 & 0.7222 & 0.7077 \\
\hline 20 & NYNEX & 0.7215 & 0.7094 \\
\hline 21 & ATTد & 0.7178 & 0.7012 \\
\hline 22 & HUGHES_I & 0.7174 & 0.7010 \\
\hline 23 & MIME & 0.7163 & 0.6904 \\
\hline 24 & HUGHES.2 & 0.7162 & 0.6997 \\
\hline 25 & NIST_4 & 0.7139 & 0.6880 \\
\hline 28 & GTESS 2 & 0.7117 & 0.6933 \\
\hline 27 & GTESS_l & 0.7116 & 0.6839 \\
\hline 28 & ASOL & 0.7107 & 0.8835 \\
\hline 29 & OCRSYS & 0.7047 & 0.6943 \\
\hline 30 & GMD_1 & 0.7012 & 0.6710 \\
\hline 31 & GMD_3 & 0.6998 & 0.6696 \\
\hline 32 & NIST_L & 0.8986 & 0.6691 \\
\hline 33 & REI & 0.6958 & 0.6750 \\
\hline 34 & NIST_3 & 0.6910 & 0.8383 \\
\hline 33 & GMD_4 & 06836 & 0.6350 \\
\hline 36 & NIST_2 & 0.8392 & 0.6189 \\
\hline 37 & GMD_2 & 0.6543 & 0.6134 \\
\hline 38 & VALEN_I & 0.6319 & 0.6074 \\
\hline 39 & IFAX & 0.6431 & 0.6176 \\
\hline 40 & COMCOM & 0.8333 & 0.6282 \\
\hline 41 & KAMAN_S & 0.5948 & 0.5491 \\
\hline 12 & $\mathrm{UMICH}_{2}$ & 0.0853 & 0.0126 \\
\hline
\end{tabular}

Table 87: KAMAN_4 correlation graph key for uppers. 


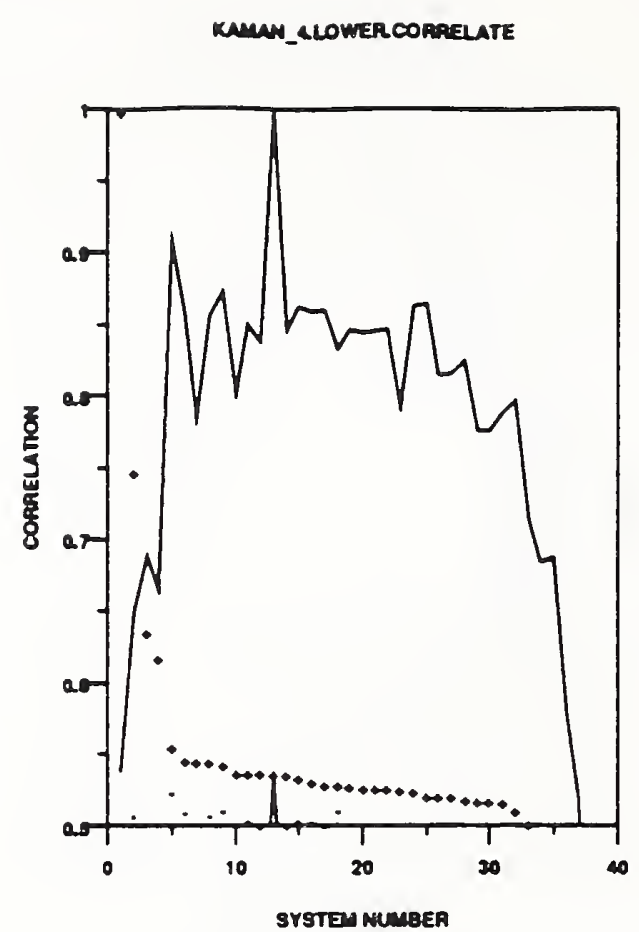

Figure 140: KAMAN_4 - lower case correlation

\begin{tabular}{|c|c|c|c|}
\hline Syotem Number & Syttem Name & Correistion (II) & Correlstion (correct) \\
\hline 1 & KAMAN-1 & 1.0000 & 1.0000 \\
\hline 2 & KAMAN_2 & 0.7484 & 0.3091 \\
\hline 3 & $K A M A N_{-1}$ & 0.6373 & 0.1952 \\
\hline 4 & $\mathrm{KAMAN}_{3}$ & 0.6184 & 0.4792 \\
\hline 3 & VOTE_M & 0.5372 & 0.3249 \\
\hline 6 & ATT-1 & 0.5477 & 0.3107 \\
\hline 7 & RISO & 0.3463 & 0.4889 \\
\hline 8 & KODAK 1 & 0.5461 & 0.3091 \\
\hline 9 & $A E G$ & 0.3438 & 0.3117 \\
\hline 10 & NIST-4 & 0.3388 & 0.4908 \\
\hline 11 & UMICH-I & 0.5384 & 0.3032 \\
\hline 12 & ATT & 0.5381 & 0.3007 \\
\hline 13 & REFERENCE & 0.5373 & 0.3373 \\
\hline 14 & UBOL & 0.5372 & 0.3010 \\
\hline 15 & ERIM_1 & 0.5356 & 0.3038 \\
\hline 16 & ATT 2 & 0.3334 & 0.3045 \\
\hline 17 & NYNEX & 0.5311 & 0.3022 \\
\hline 18 & VOTEP & 0.5306 & 0.5124 \\
\hline 19 & HUGHES_- & 0.5293 & 0.4966 \\
\hline 20 & HUGHES -2 & 0.5288 & 0.4958 \\
\hline 21 & IBM & 0.3284 & 0.4976 \\
\hline 22 & NESTOR & 0.5280 & 0.4996 \\
\hline 23 & GMD_3 & 0.5268 & 0.4839 \\
\hline 24 & ATT 1 & 0.5234 & 0.4979 \\
\hline 23 & OCRSYS & 0.5228 & 0.4985 \\
\hline 26 & NIST_-1 & 0.5223 & 0.4866 \\
\hline 27 & GTESS 2 & 0.5224 & 0.4880 \\
\hline 28 & GTESS-1 & 0.3203 & 0.4888 \\
\hline 29 & GMD - 4 & 0.5192 & 0.4737 \\
\hline 30 & GMD_1 & 0.3192 & 0.4757 \\
\hline 31 & ASOL & 0.3184 & 0.4772 \\
\hline 32 & NIST & 0.5127 & 04796 \\
\hline 33 & GMD_2 & 0.5033 & 0.4527 \\
\hline 34 & VALEN-1 & 0.4939 & 0.4405 \\
\hline 33 & NIST 2 & 0.4865 & 0.4370 \\
\hline 36 & KAMAN -5 & 0.4469 & 0.3871 \\
\hline 37 & COMCOM & 0.3302 & 0.3223 \\
\hline 38 & $\mathrm{UMICH}_{2}$ & 0.1213 & 0.0243 \\
\hline
\end{tabular}

Table 88: KAMAN_4 correlation graph key for lowers. 
SYSTEM: KAMAN_5

PARTICIPANT: Mark G. Costello

ORGANIZATION: Kaman Sciences Corporation, Utica, NY

FEATURES :

CLASSIFICATION :

HARDHARE: $\quad$ SPARC2, multiuser

TRAINING: DIGITS UPPERS LOWERS DATABASE

$8002080 \quad 2080 \quad$ NSDB1?

STATUS: $\quad$ on time

RESULTS: -- DIGITS -- -- UPPERS -- -- LOHERS -- DATABASE

REJ. ERR. REJ. ERR. REJ. ERR. TESTDATA1

RATE RATE-- RATE RATE-- RATE RATE--

$\begin{array}{lllllll}0.00 & 0.1513 & 0.00 & 0.3395 & 0.00 & 0.4220\end{array}$

OCR RATE (CPS): DIGITS

UPPERS

LOWERS

SYS RATE:

6.57

3.74

3.74

CPU RATE: 
SYSTEM: KAMAN_5

BIBLIOGRAPHY:

The following references have been provided for this system: 


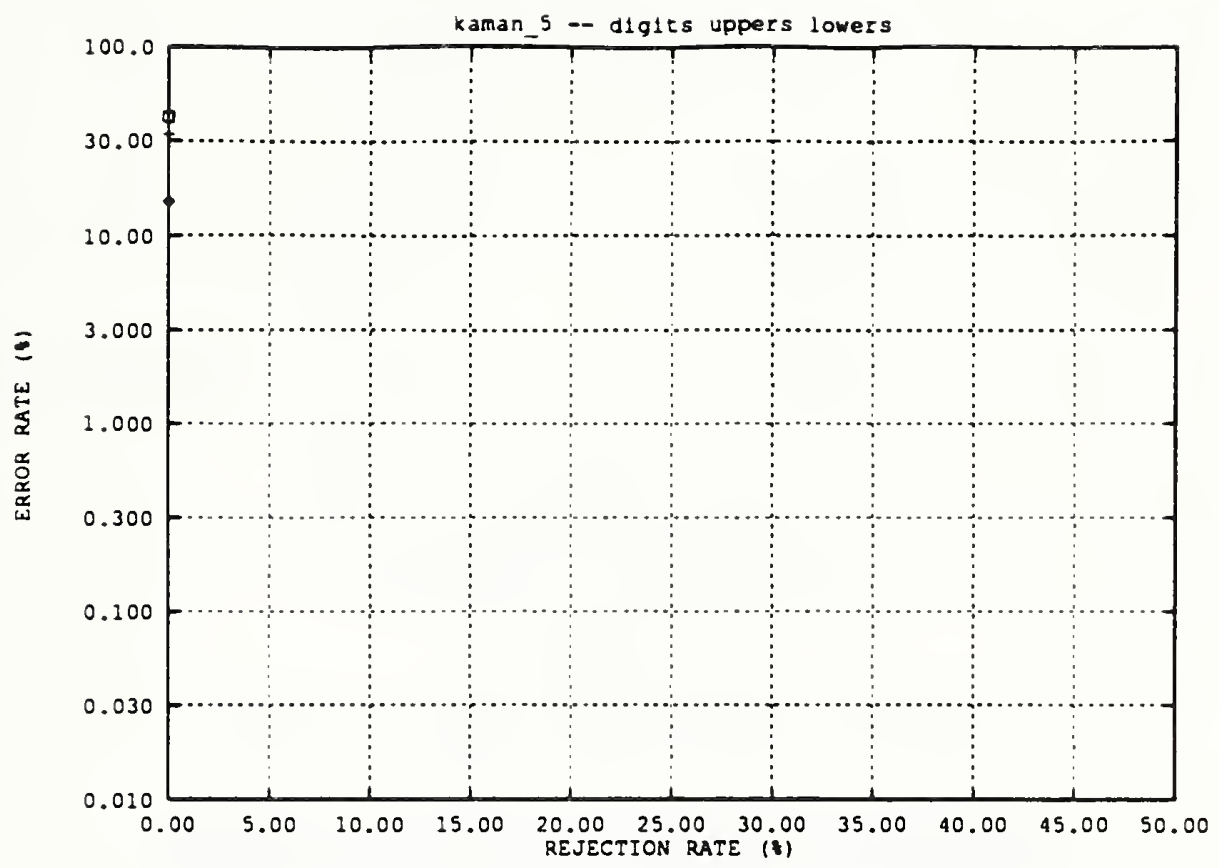

Figure 141: Error rate versus rejection rate for KAMAN_5

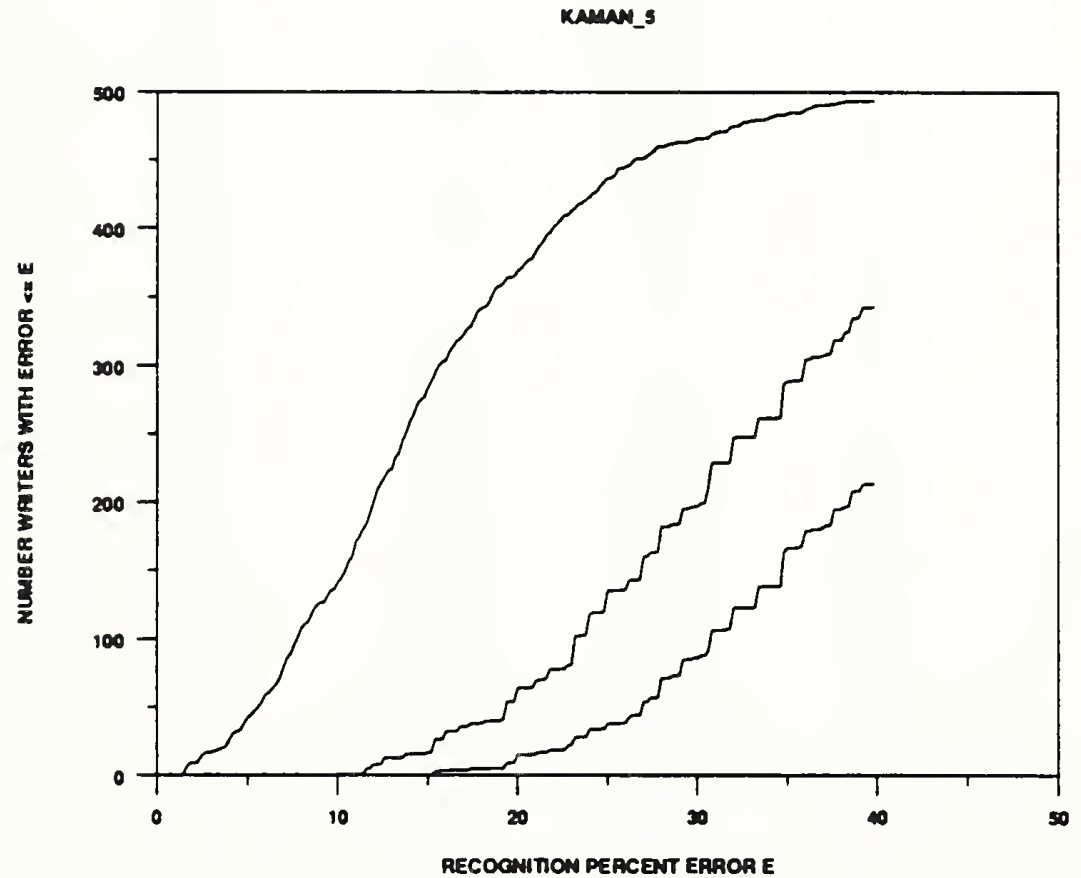

Figure 142: Error rate per writer of KAMAN_5 


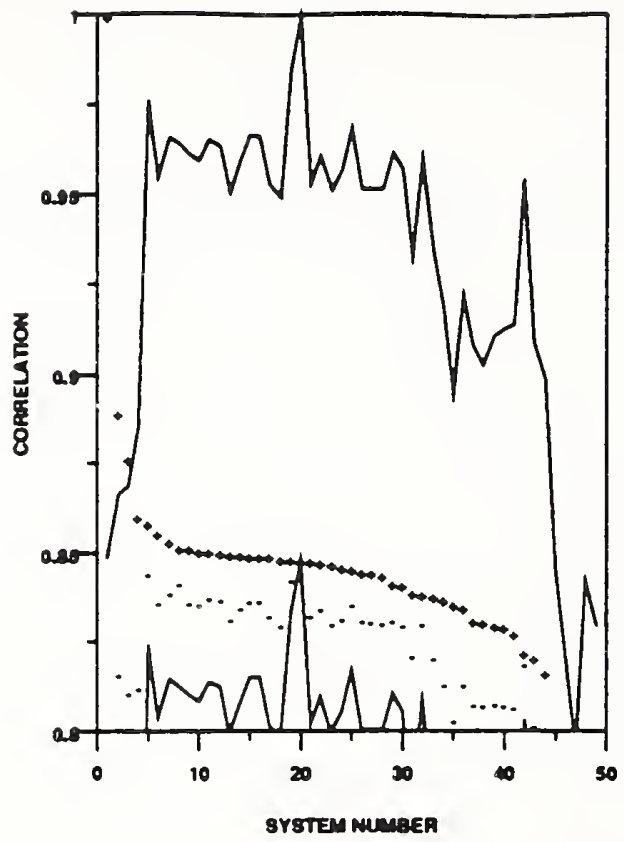

Figure 143: KAMAN_j - digit correlation

\begin{tabular}{|c|c|c|c|}
\hline System Number & System Name & Correlation (all) & Coprelation (coprect) \\
\hline $\mathrm{l}$ & KAMAN 3 & 1.0000 & 1.0000 \\
\hline 2 & $\mathrm{KAMAN}_{-2}$ & 0.8899 & 0.8162 \\
\hline 3 & KAMAN 3 & 08770 & 0.8111 \\
\hline 4 & KAMAN_1 & 0.8610 & 0.8127 \\
\hline 5 & VOTEM M & 08820 & 0.8448 \\
\hline$B$ & NESTOR & 0.8861 & 0.8364 \\
\hline 7 & AEG & 0.8540 & 08391 \\
\hline 8 & VOTE $P$ & 0.8822 & 08423 \\
\hline 8 & ERIM_I & 0.8521 & 0.8367 \\
\hline 10 & KODAK2 & 0.8814 & 0.8361 \\
\hline 11 & IBM & 0.8813 & 0.8380 \\
\hline 12 & ATT 2 & 0.8508 & 0.8375 \\
\hline 13 & ATT_4 & 0.8803 & 0.8352 \\
\hline 14 & NIST -4 & 0.8503 & 0.8319 \\
\hline 15 & ELSAGB_ & 08501 & 08371 \\
\hline 16 & ELSAGB2 & 0.8499 & 0.8369 \\
\hline 17 & KODAK_ & 08497 & 0.8330 \\
\hline 18 & ELSAGB_l & 08491 & 0.8303 \\
\hline 19 & OCRSYS & 08489 & 0.8431 \\
\hline 20 & REFERENCE & 0.8487 & 0.8487 \\
\hline 21 & SYMBUS & 0.8484 & 0.8327 \\
\hline 22 & ERIM 2 & 0.8482 & 0.8349 \\
\hline 23 & THINK_l & 0.8474 & 0.8307 \\
\hline 24 & UBOL & 0.8467 & 08321 \\
\hline 25 & ATT $\perp$ & 0.8463 & 0.8363 \\
\hline 26 & ATTS & 08485 & 0.8314 \\
\hline 27 & HUGHES_2 & 0.8452 & 0.8309 \\
\hline 28 & HUGHES_l & 0.8443 & 0.8304 \\
\hline 28 & THINK 2 & 0.8420 & 0.8317 \\
\hline 30 & NYNEX & 0.8414 & 0.8303 \\
\hline 31 & GTESS 2 & 0.8392 & 0.8212 \\
\hline 32 & REI & 0.8389 & 0.8305 \\
\hline 33 & GTESS _l & 0.8382 & 0.8211 \\
\hline 34 & GMD 3 & 0.8374 & 0.8138 \\
\hline 35 & RISO & 0.8358 & 0.8035 \\
\hline 36 & NIST 1 & 0.8350 & 0.8138 \\
\hline 37 & NIST 2 & 0.8313 & 08080 \\
\hline 38 & NISTA & 0.8310 & 0.8074 \\
\hline 39 & ASOL & 0.8302 & 0.8080 \\
\hline 40 & GMD_1 & 0.8297 & 0.8074 \\
\hline 41 & MIME & 0.8278 & 0.8071 \\
\hline 42 & СOMCOM & 0.8223 & 0.8189 \\
\hline 43 & UPENN & 0.8211 & 0.8022 \\
\hline 44 & GMD_4 & 0.8170 & 0.7846 \\
\hline 45 & GMD_2 & 0.7975 & 0.7664 \\
\hline 46 & VALEN_L & 0.7828 & 07440 \\
\hline 47 & KAMAN_4 & 07763 & 0.7307 \\
\hline 48 & VALEN 2 & 0.7695 & 0.7506 \\
\hline 49 & IFAX & 0.7853 & 0.7412 \\
\hline
\end{tabular}

Table 89: KAMAN_5 correlation graph key for digits. 


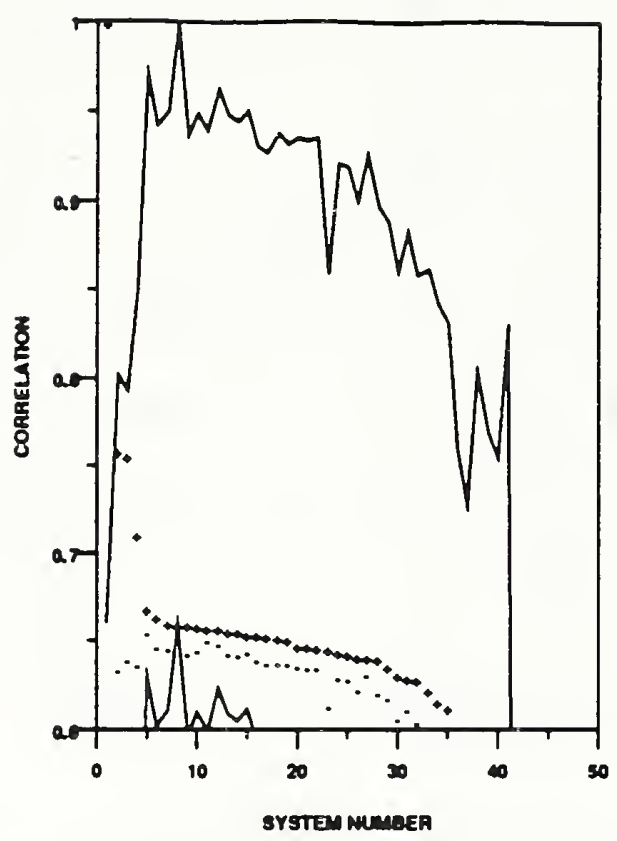

Figure 144: KAMAN_5 - upper case correlation

\begin{tabular}{|c|c|c|c|}
\hline Syotem Number & Syslem Name & Correlation (all) & Correlation (correcs) \\
\hline 1 & KAMANS & 1.0000 & 1.0000 \\
\hline 2 & KAMAN_ & 0.7594 & 0.6348 \\
\hline 3 & KAMAN 2 & 0.7566 & 0.6400 \\
\hline 4 & KAMAN_1 & 0.7117 & 0.6372 \\
\hline $\mathbf{3}$ & VOTE_M & 06690 & 0.6560 \\
\hline 6 & NEST OR & 0.6648 & 0.6473 \\
\hline 7 & A T T _4 & 0.6816 & 0.6469 \\
\hline 8 & REFERENCE & $0.660 \mathrm{~s}$ & $0.660 \mathrm{~s}$ \\
\hline 9 & IBM & 0.6803 & 0.6435 \\
\hline 10 & UMICH_I & 06392 & 0.6458 \\
\hline 11 & VOTEP & 06887 & 0.6508 \\
\hline 12 & $A E G$ & $0.858 \mathrm{~s}$ & 0.6491 \\
\hline 13 & ERIM-1 & 0.6568 & 0.6441 \\
\hline 14 & ATT 2 & 0.6562 & 0.6429 \\
\hline 15 & NYNEX & 0.8551 & 0.6450 \\
\hline 16 & KODAK」 & 0.6846 & 0.6398 \\
\hline 17 & SYMBUS & 0.8542 & 0.6381 \\
\hline 18 & UBOL & 0.6531 & 0.6386 \\
\hline 19 & ATT 3 & 0.8518 & 0.6384 \\
\hline 20 & HUGHES.I & 0.6481 & 0.6360 \\
\hline 21 & HUGHES - 2 & 0.6478 & 0.6351 \\
\hline 22 & $\mathrm{ATT} \perp$ & 0.8478 & 0.6356 \\
\hline $2 \mathrm{~J}$ & RISO & 0.8484 & 0.6143 \\
\hline 24 & GTESS_1 & 0.8443 & 0.6303 \\
\hline 23 & GTESS_2 & 06434 & 0.6284 \\
\hline 26 & MIME & 0.6422 & 0.8234 \\
\hline 27 & OCRSYS & 0.8417 & 0.6314 \\
\hline 28 & NIST_t & 0.6414 & 0.6215 \\
\hline 29 & ASOL & 0.6360 & 0.8189 \\
\hline 30 & GMD_1 & 0.6319 & 0.6073 \\
\hline 31 & REI & 0.6303 & 0.6121 \\
\hline 32 & GMD_J & 0.6286 & 0.6048 \\
\hline 33 & NIST 1 & 0.6239 & 0.6022 \\
\hline 34 & GMD_4 & 06171 & 0.3928 \\
\hline 35 & NIST & 0.6135 & 0.3922 \\
\hline 36 & VALEN_I & 0.3987 & 0.3595 \\
\hline 37 & KAMAN-4 & 0.5948 & 0.3491 \\
\hline 38 & IFAX & 0.3874 & 0.5646 \\
\hline 39 & NIST 2 & 0.3829 & 0.3580 \\
\hline 40 & GMD_2 & 0.5801 & 0.3490 \\
\hline 41 & СОМСOM & 0.5788 & 0.5718 \\
\hline 12 & $\mathrm{UMICH}_{2}$ & 0.0927 & 0.0113 \\
\hline
\end{tabular}

Table 90: KAMAN_5 correlation graph key for uppers. 


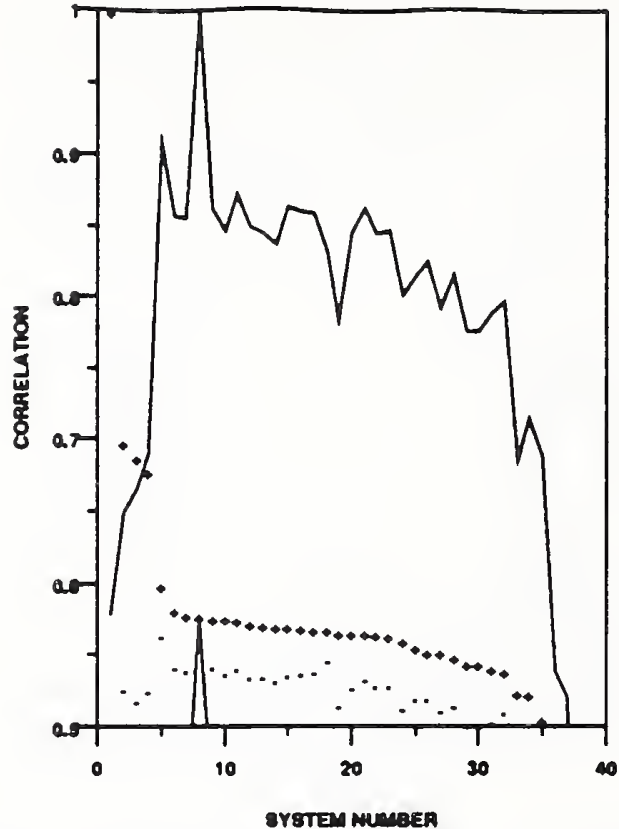

Figure 145: KAMAN_5 - lower case correlation

\begin{tabular}{|c|c|c|c|}
\hline Syatem Number & Syatem Name & Correlation (all) & Correlation (correet) \\
\hline 1 & KAMAN_S & 1.0000 & 1.0000 \\
\hline 2 & $\mathrm{KAMAN}_{2}$ & 0.6985 & 0.5268 \\
\hline 3 & KAMAN_3 & 0.6873 & 0.5186 \\
\hline 4 & KAMAN-1 & 0.6787 & 0.3261 \\
\hline 5 & VOTE_M & 0.6000 & 0.3641 \\
\hline 6 & ATT_4 & 0.3823 & 0.5420 \\
\hline 7 & KODAK」 & 0.5787 & 0.5397 \\
\hline 8 & REFERENCE & 0.5780 & 0.5780 \\
\hline 9 & ERIM-1 & 0.5763 & 0.5423 \\
\hline 10 & NESTOR & 0.3761 & 0.5374 \\
\hline 11 & AEG & 0.5755 & 0.5407 \\
\hline 12 & UMICH_I & 0.3727 & 0.5353 \\
\hline 13 & IBM & 0.5724 & 0.3352 \\
\hline 14 & ATT 3 & 0.5709 & 0.5332 \\
\hline 15 & OCRSYS & 0.5707 & 0.5363 \\
\hline 16 & NYNEX & 0.5693 & 0.5378 \\
\hline 17 & $\mathrm{ATT}_{2}$ & 0.3680 & 0.5383 \\
\hline 18 & VOTEP & 0.5679 & 0.5470 \\
\hline 19 & UBOL & 0.5687 & 0.3284 \\
\hline 20 & RISO & 0.5667 & 0.3138 \\
\hline 21 & ATT $\perp$ & 0.5658 & 0.3338 \\
\hline 22 & HUGHES_2 & 0.5648 & 0.3293 \\
\hline 23 & HUGHES_I & 0.5642 & 0.5293 \\
\hline 24 & NIST_t & 0.5602 & 0.3135 \\
\hline 25 & Nist_l & 0.5562 & 0.3202 \\
\hline 26 & GTESS_1 & 0.5527 & 0.3203 \\
\hline 27 & GMD_3 & 0.5522 & 0.3116 \\
\hline 28 & GTESS 2 & 0.5490 & 0.3156 \\
\hline 29 & GMD_4 & 0.5438 & 0.5020 \\
\hline 30 & GMD_I & 0.3438 & 0.3020 \\
\hline 31 & ASOL & 0.5112 & 0.3042 \\
\hline 32 & הIST & 0.5383 & 0.3111 \\
\hline 33 & VALEN_I & 0.5253 & 0.4665 \\
\hline 34 & GMD_2 & 0.3239 & 0.4779 \\
\hline 35 & NIST 2 & 0.5059 & 0.4800 \\
\hline 36 & KAMAN_4 & 0.1469 & 0.3871 \\
\hline 37 & COMCOM & 0.3382 & 0.3483 \\
\hline 38 & $\mathrm{UMICH}_{2}$ & 0.1278 & 0.0333 \\
\hline
\end{tabular}

Table 91: KAMAN 5 correlation graph key for lowers. 


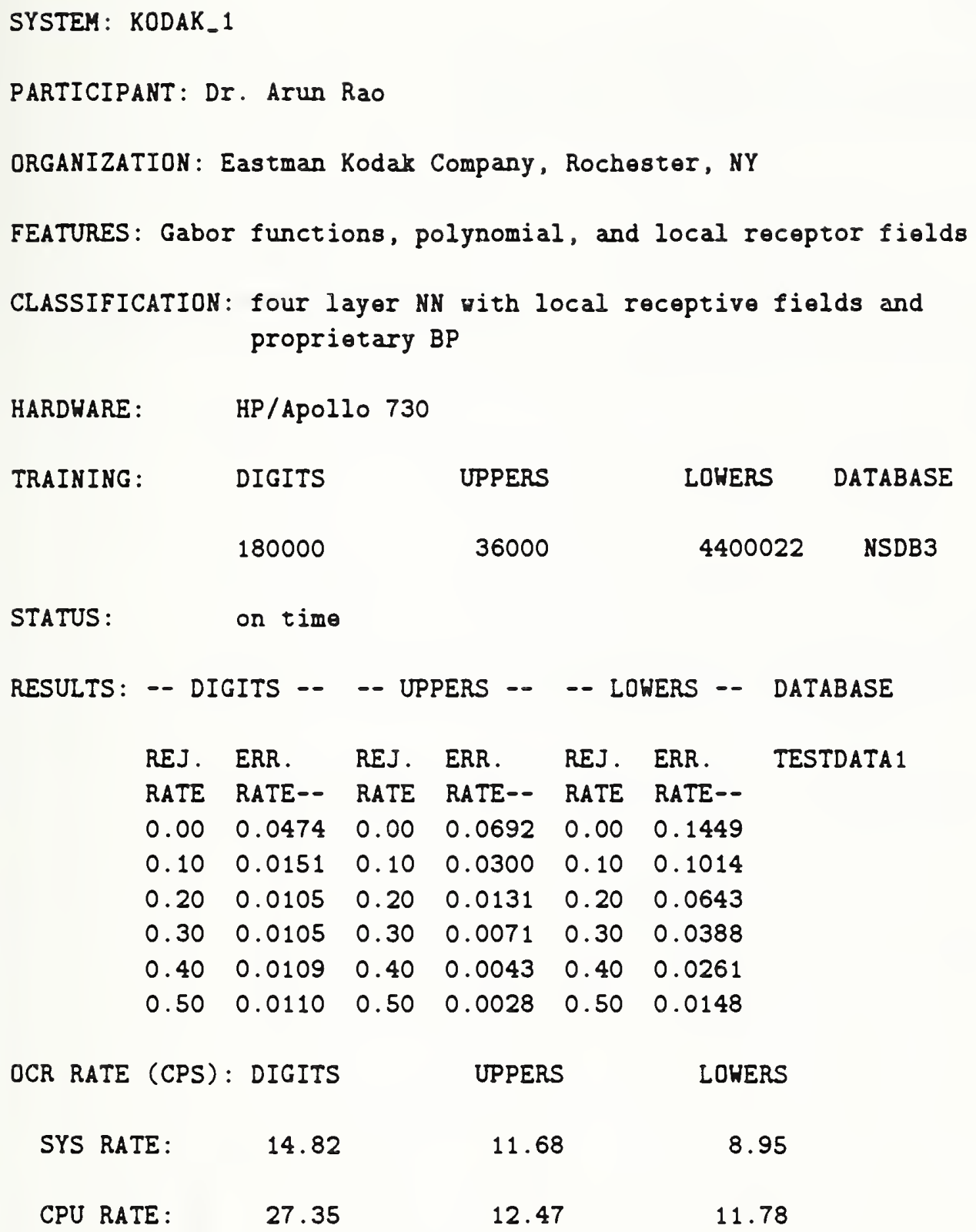

NOTE: Some upper case characters were added for training lowers. 
SYSTEM: KODAK_

BIBLIOGRAPHY:

The following references have been provided for this system:

$[5][27][28]$ 


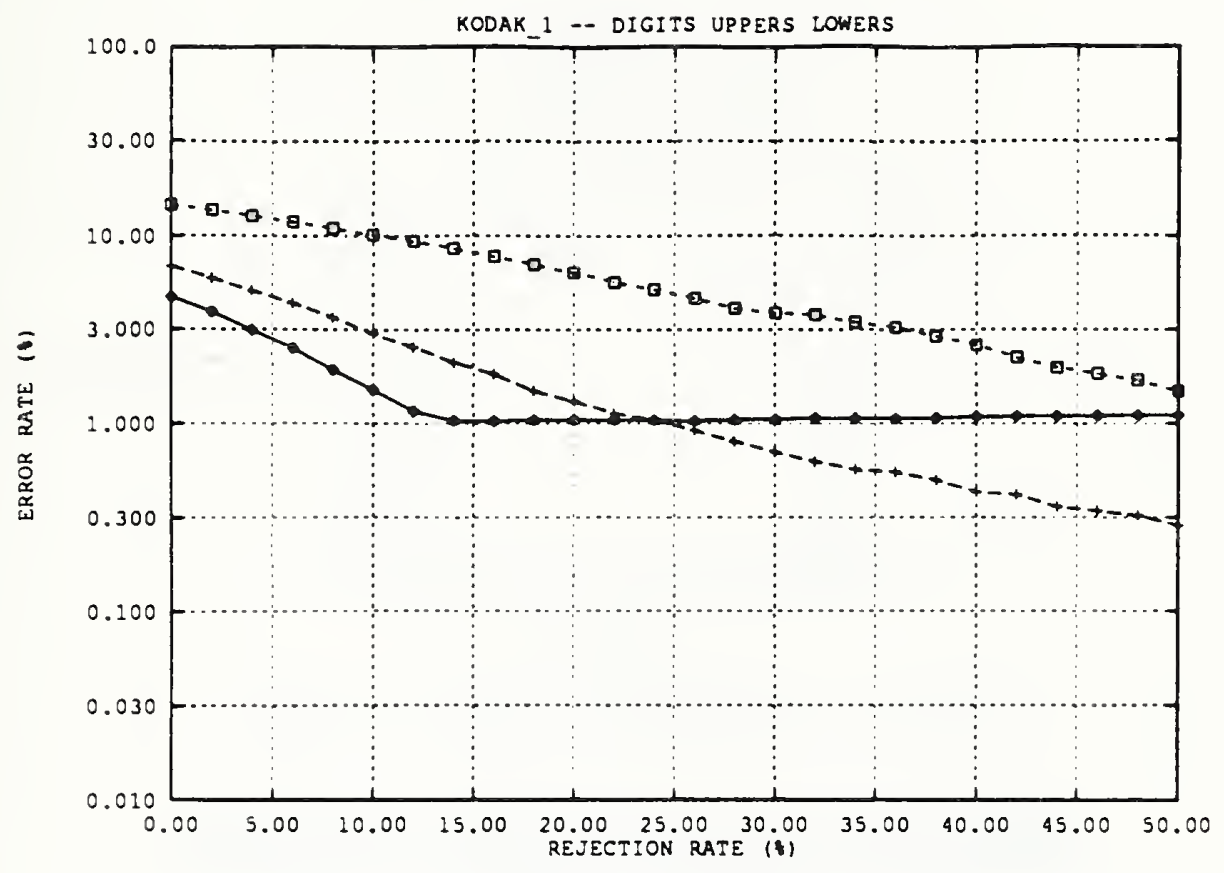

Figure 146: Error rate versus rejection rate for KODAK_1

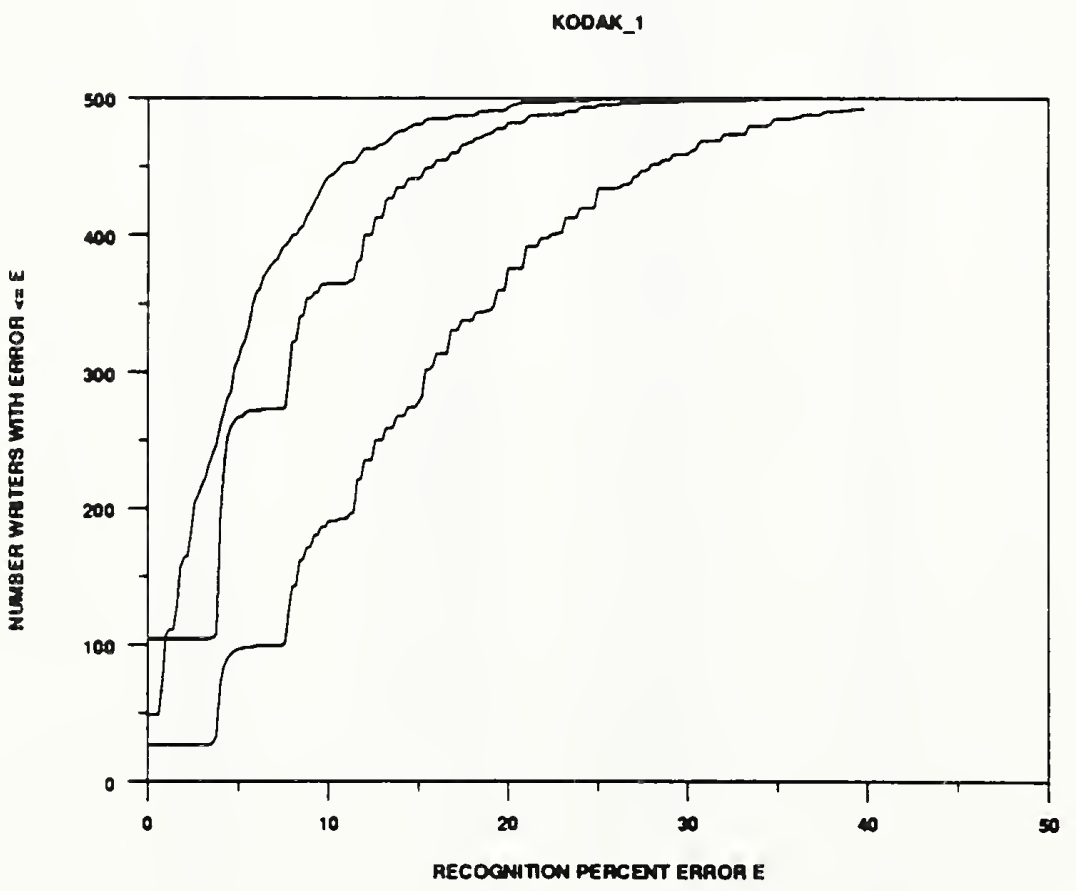

Figure 147: Error rate per writer of KODAK_1 


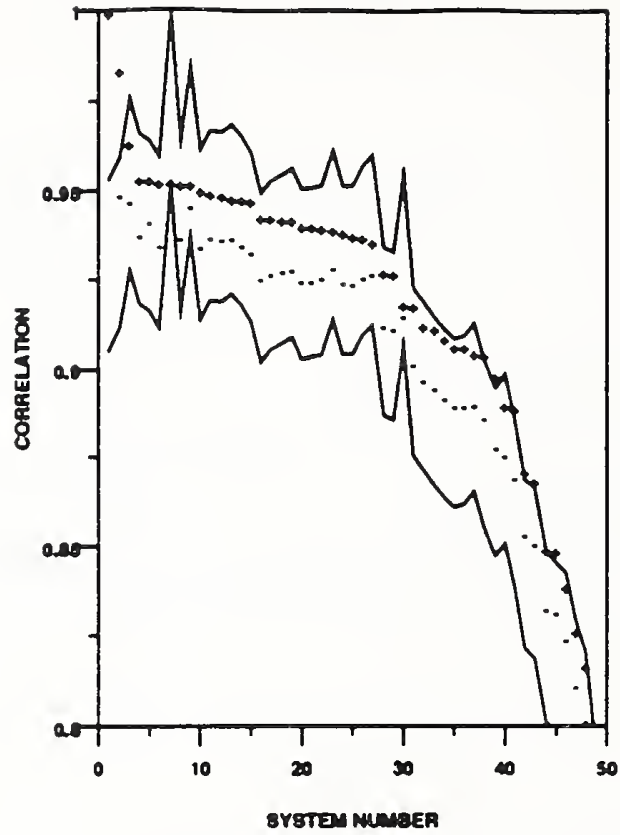

Figure 148: KODAK_1 - digit correlation

\begin{tabular}{|c|c|c|c|}
\hline System Number & System Name & Correlation (ail) & Correlation (correct) \\
\hline 1 & KODAK_ & 1.0000 & 1.0000 \\
\hline 2 & KODAK_ 2 & 0.9841 & 0.9489 \\
\hline 3 & VOTE_M & 0.9637 & 0.9474 \\
\hline 4 & AEG & 0.9336 & 0.8383 \\
\hline 3 & VOTEP & 0.9335 & 0.8418 \\
\hline$B$ & REFERENCE & 09526 & 0.8526 \\
\hline 7 & A TT -4 & 0.9328 & 0.9351 \\
\hline 8 & ATT 2 & 0.9523 & 0.9371 \\
\hline 9 & OCRSYS & 0.9522 & 0.9458 \\
\hline 10 & ERIM-I & 0.8504 & 0.9349 \\
\hline 11 & ELSAGBA & 0.9497 & 0.9371 \\
\hline 12 & ELSAGB_2 & 0.9492 & 0.9368 \\
\hline 13 & ATTد $\perp$ & 0.9484 & 0.9371 \\
\hline 14 & IBM & 0.9480 & 0.9354 \\
\hline 13 & ERIM_2 & 0.9476 & 0.9333 \\
\hline 16 & ELSAGB-1 & 0.9431 & 0.9280 \\
\hline 17 & SYMBUS & 0.9430 & 0.8273 \\
\hline 18 & NESTOR & 09427 & 0.9286 \\
\hline 19 & UBOL & 0.9423 & 0.9290 \\
\hline 20 & THINK-I & 0.9409 & 0.9233 \\
\hline 21 & NIST_4 & 09409 & 0.9235 \\
\hline 22 & ATT 3 & 0.9403 & 0.9287 \\
\hline 23 & THINK_2 & 0.9397 & 0.9293 \\
\hline 24 & HUGHES -1 & 0.9388 & 0.9251 \\
\hline 25 & HUGHES_2 & 0.9379 & 0.9248 \\
\hline 28 & NYNEX & 09374 & 0.9265 \\
\hline 27 & REI & 0.9363 & 0.9278 \\
\hline 28 & GTESS_1 & 0.9279 & 0.9126 \\
\hline 29 & GTESS 2 & 0.9273 & 0.8119 \\
\hline 30 & СОМСОМ & 0.9186 & 0.9158 \\
\hline 31 & NIST $_{-1}$ & 0.8182 & 0.9016 \\
\hline 32 & GMD_3 & 09130 & 0.8973 \\
\hline 33 & MIME & 0.9119 & 0.8948 \\
\hline 34 & ASOL & 09093 & 0.8921 \\
\hline 35 & NIST_2 & 09070 & 0.8899 \\
\hline 36 & UPENN & 0.9070 & 0.8897 \\
\hline 37 & GMD_I & 0.9032 & 0.8904 \\
\hline 38 & ב ב NIST & 0.9046 & 0.8868 \\
\hline 39 & RISO & 0.8983 & 0.8783 \\
\hline 40 & GMD_4 & 0.8904 & 0.8760 \\
\hline 41 & KAMAN_d & 08893 & 0.8699 \\
\hline 42 & KAMAN 3 & 0.8718 & 0.8838 \\
\hline 43 & KAMAN_2 & 0.8692 & 0.8512 \\
\hline 44 & KAMAN 3 & 0.8497 & 0.8330 \\
\hline 43 & GMD_2 & 0.8484 & 0.8318 \\
\hline 48 & VALEN_2 & 0.8393 & 0.8247 \\
\hline 47 & IFAX & 0.8271 & 0.8117 \\
\hline 48 & VALEN_I & 0.8172 & 0.8020 \\
\hline 49 & KAMAN_4 & 0.7955 & 0.7788 \\
\hline
\end{tabular}

Table 92: KODAK_1 correlation graph key for digits. 


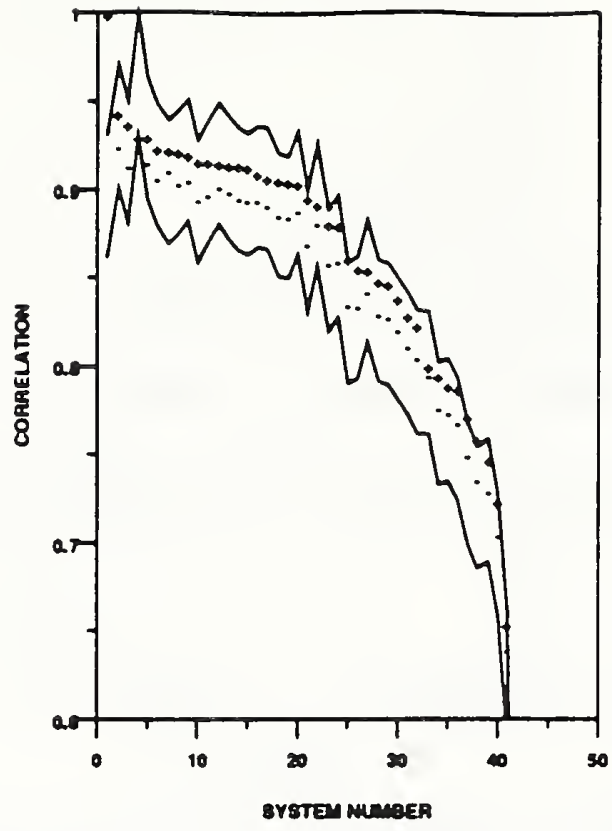

Figure 149: KODAK 1 - upper case correlation

\begin{tabular}{|c|c|c|c|}
\hline Syorem Number & System Name & Corretation (All) & Correlation (correct) \\
\hline 1 & KODAK & 1.0000 & 1.0000 \\
\hline 2 & VOTE_M & 0.9439 & 0.2249 \\
\hline 3 & ATT 4 & 0.9374 & 0.8140 \\
\hline 4 & REFERENCE & 0.9308 & 0.8308 \\
\hline s & AEG & 0.9302 & 0.2138 \\
\hline 6 & ERIM-1 & 0.9237 & 0.8066 \\
\hline 7 & VOTEP & 0.9228 & 0.2108 \\
\hline 8 & ATT 2 & 0.2225 & 0.8038 \\
\hline $\boldsymbol{\theta}$ & NYNEX & 0.9203 & 0.9060 \\
\hline 10 & SYMBUS & 0.9170 & 0.8841 \\
\hline 11 & UBOL & 0.9168 & 0.8977 \\
\hline 12 & UMICH_I & 0.8160 & 0.9022 \\
\hline 13 & NESTOR & 0.9144 & 0.8992 \\
\hline 14 & ATT 1 & 0.9143 & 08957 \\
\hline 13 & ATT 3 & 0.9141 & 0.8946 \\
\hline 16 & IBM & 0.9101 & 0.8947 \\
\hline 17 & HUGHES_- 1 & 0.9073 & 0.8917 \\
\hline 18 & GTESS_l & 0.9084 & 0.8864 \\
\hline 18 & GTESS 2 & 0.2080 & 0.8834 \\
\hline 20 & HUGHES_2 & 0.2047 & 0.8891 \\
\hline 21 & MIME & 0.8963 & 0.8711 \\
\hline 22 & OCRSYS & 0.8923 & 0.8320 \\
\hline 23 & ASOL & 0.8818 & 0.8592 \\
\hline 24 & NIST -4 & 0.8807 & 0.8608 \\
\hline 23 & RISO & 0.8623 & 0.8338 \\
\hline 26 & NIST_l & 0.8363 & 0.8344 \\
\hline 27 & REI & 0.8335 & 08428 \\
\hline 28 & GMD_1 & 0.8491 & 08304 \\
\hline 29 & GMD_3 & 0.8478 & 0.8287 \\
\hline 30 & KAMAN_I & 0.8393 & 0.8209 \\
\hline 31 & GMD_4 & 0.8282 & 0.8118 \\
\hline 32 & NIST 3 & 0.8241 & 0.8036 \\
\hline 33 & СОМСOM & 0.8007 & 0.7948 \\
\hline 34 & KAMAN3 & 0.7982 & 0.7768 \\
\hline 33 & IFAX & 0.7901 & 0.7738 \\
\hline 36 & KAMAN_2 & 0.7884 & 0.7683 \\
\hline 37 & NIST 2 & 0.7720 & 0.7300 \\
\hline 38 & GMD_2 & 0.7591 & 0.7364 \\
\hline 39 & VALEN-1 & 0.7484 & 0.7303 \\
\hline 40 & KAMAN_4 & 0.7249 & 0.7034 \\
\hline 41 & KAMAN S & 0.6346 & 0.6398 \\
\hline 12 & UMICH 2 & 0.0431 & 0.0222 \\
\hline
\end{tabular}

Table 93: KODAK_1 correlation graph key for uppers. 


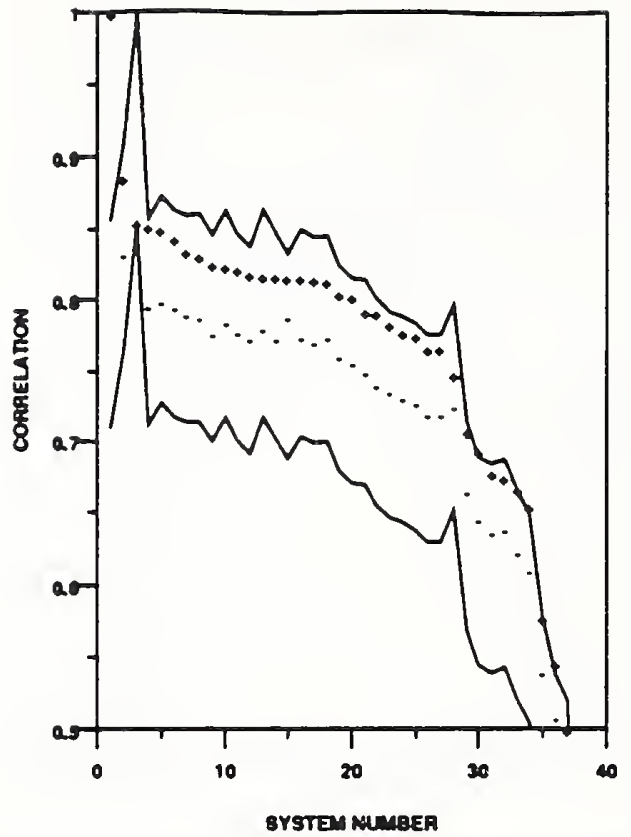

Figure 150: KODAK_1 - lower case correlation

\begin{tabular}{|c|c|c|c|}
\hline System Number & System Name & Correiation (all) & Correlalion (corpeci) \\
\hline$T$ & KODAK」 & 1.0000 & 1.0000 \\
\hline 2 & VOTE_M & 0.8866 & 0.8336 \\
\hline 3 & REFERENCE & 0.8351 & 0.8531 \\
\hline 4 & ATT 4 & 0.8529 & 0.7972 \\
\hline$s$ & AEG & 0.8808 & 0.8006 \\
\hline 6 & ERIM-1 & 0.8448 & 0.7961 \\
\hline 7 & ATT2 2 & 0.8357 & 0.7911 \\
\hline 8 & NYNEX & 0.8320 & 0.7888 \\
\hline 9 & UBOL & 0.8267 & 0.7778 \\
\hline 10 & ATT 1 & 0.8261 & 0.7833 \\
\hline 11 & NESTOR & 0.8238 & 07791 \\
\hline 12 & ATT & 0.8199 & 0.7735 \\
\hline 13 & OCRSYS & 0.8184 & 0.7811 \\
\hline 14 & HUGHES -1 & 0.8183 & 0.7738 \\
\hline 15 & VOTEP & 0.8177 & 0.7884 \\
\hline 16 & UMICH_L & 0.8174 & 0.7755 \\
\hline 17 & HUGHES_2 & 0.8163 & 0.7719 \\
\hline 18 & IBM & 0.8180 & 0.7749 \\
\hline 19 & GTESS_1 & 08038 & 0.7612 \\
\hline 20 & GTESS 2 & 0.8040 & 0.7573 \\
\hline 21 & NIST_1 & 0.7934 & 0.7503 \\
\hline 22 & NIST-4 & 07921 & 0.7408 \\
\hline 23 & GMD 3 & 0.7843 & 0.7362 \\
\hline 24 & ASOL & 0.7788 & 0.7320 \\
\hline 25 & flso & 0.7761 & 07279 \\
\hline 26 & GMD_-4 & 0.7673 & 0.7199 \\
\hline 27 & GMD_1 & 0.7673 & 0.7199 \\
\hline 28 & NIST_3 & 07485 & 0.7257 \\
\hline 29 & GMD_2 & 0.7081 & 0.6662 \\
\hline 30 & $\mathrm{KAMAN}_{-1}$ & 0.6947 & 0.6460 \\
\hline 31 & VALEN_I & 0.6798 & 06371 \\
\hline 32 & $\mathrm{NIST}_{2}$ & 0.6765 & 0.6399 \\
\hline 33 & KAMAN $\bar{N}_{-3}$ & 0.6685 & 06230 \\
\hline 34 & $K A M A N-2$ & 06556 & 0.6112 \\
\hline 35 & KAMAN_S & 0.5787 & 0.5397 \\
\hline 36 & KAMAN_4 & 0.5461 & 0.5091 \\
\hline 37 & COMCOM & 0.5007 & 0.4876 \\
\hline 38 & $\mathrm{UMICH}_{2}$ & 0.1031 & 00591 \\
\hline
\end{tabular}

Table 94: KODAK_1 correlation graph key for lowers. 
SYSTEM: KODAK_2

PARTICIPANT: Dr. ATUM Rao

ORGANIZATION: Eastman Kodak Company, Rochester, NY

FEATURES: Gabor functions, polynomial, and local receptor fields

CLASSIFICATION: four layer NN with local receptive fields and proprietary BP

HARDHARE: HP/APOIIO 730

TRAINING: DIGITS UPPERS LOWERS DATABASE

$180000 \quad$ NA $N A \quad$ NSDB3

2310 sevens with crosses INTERNAL

STATUS: $\quad$ on time

RESULTS: -- DIGITS -- -- UPPERS -- -- LOHERS -- DATABASE

REJ. ERR. REJ. ERR. REJ. ERR. TESTDATA 1

RATE RATE-- RATE RATE-- RATE RATE--

$0.00 \quad 0.0408$

$0.10 \quad 0.0117$

$0.20 \quad 0.0037$

$0.30 \quad 0.0021$

$0.40 \quad 0.0015$

$0.50 \quad 0.0016$

OCR RATE (CPS): DIGITS

UPPERS LOWERS

$\begin{array}{llll}\text { SYS RATE: } & 8.01 & \text { NA }\end{array}$

CPU RATE:

NOTE: Crossed sevens were added to training set after determining need for them from results of KODAK_1. 
SYSTEM: KODAK 2

BIBLIOGRAPHY:

The following references have been provided for this system:

[5][27][28] 


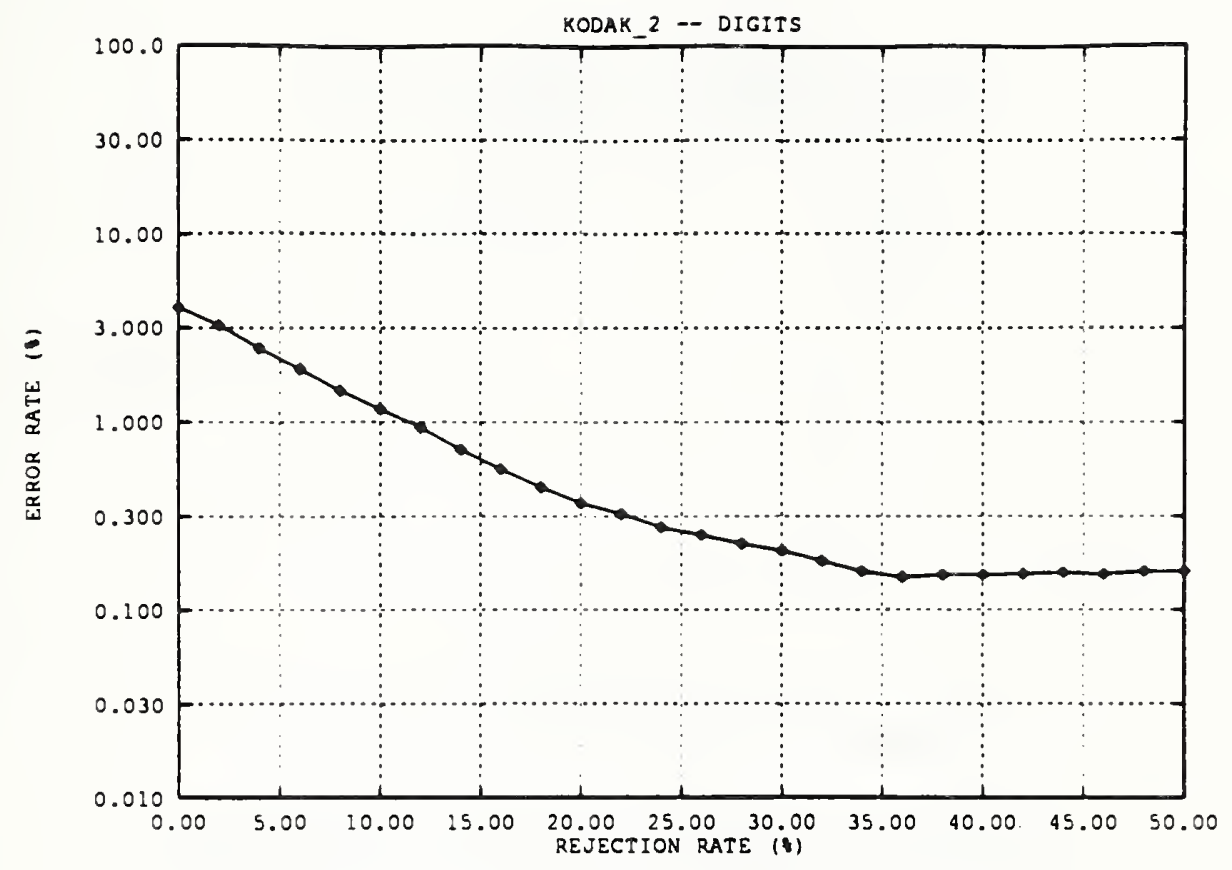

Figure 151: Error rate versus rejection rate for KODAK 2

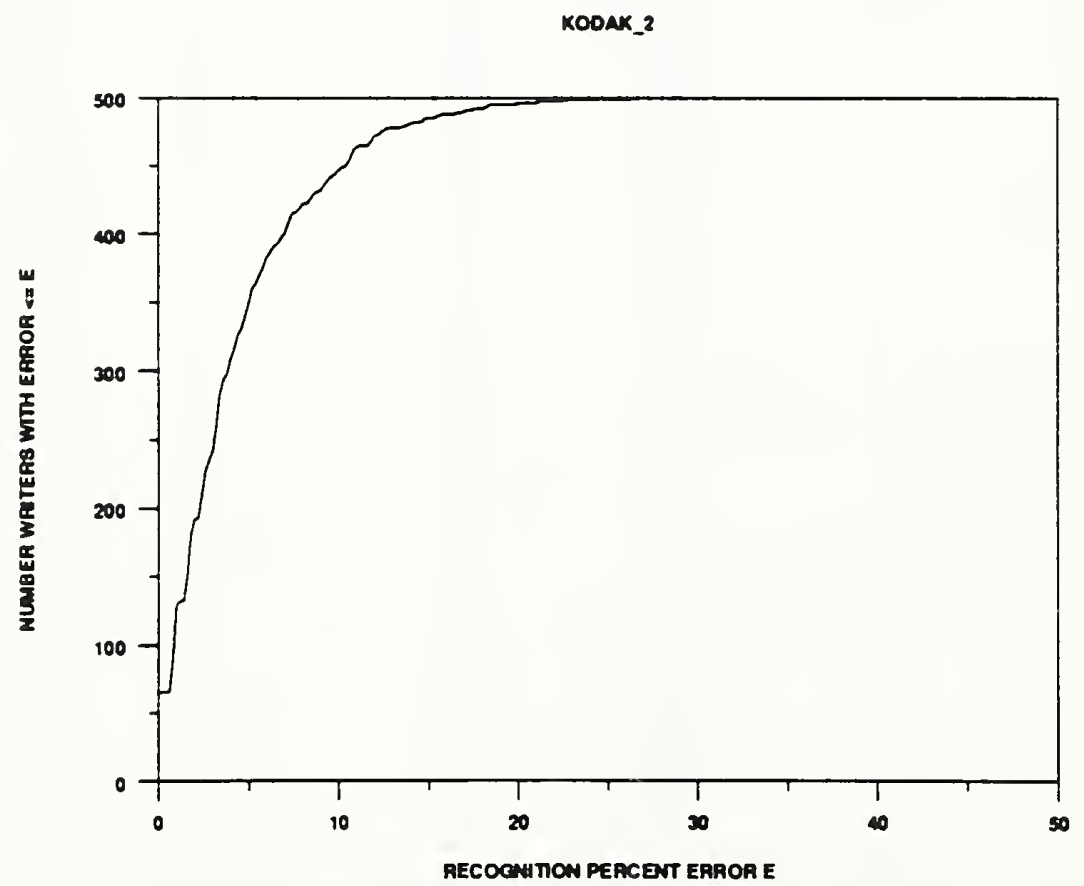

Figure 152: Error rate per writer of KODAK_2 


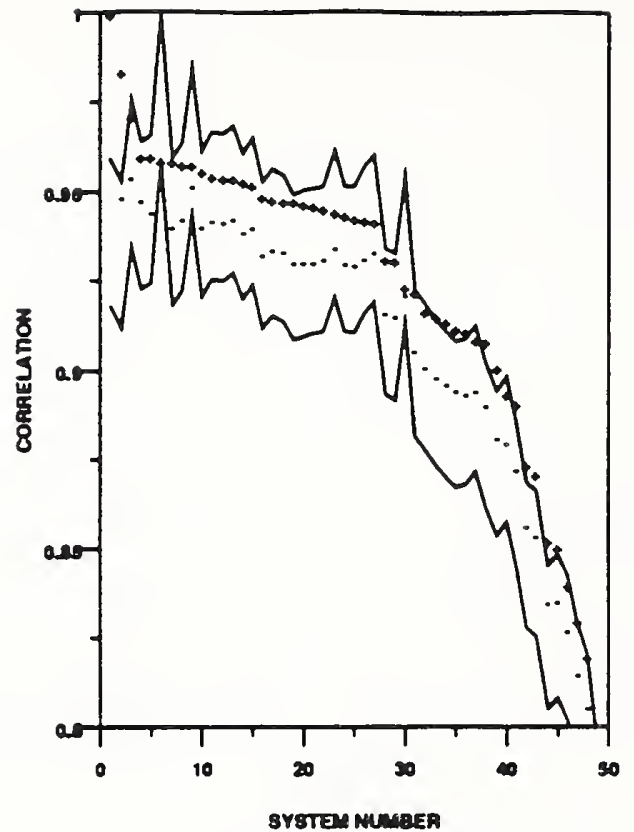

Figure 153: KODAK2 - digit correlation

\begin{tabular}{|c|c|c|c|}
\hline Syotem Number & System Name & Correlation (ail) & Correlation (correct) \\
\hline 1 & KODAK2 & 1.0000 & 1.0000 \\
\hline 2 & KODAK_ & 0.8841 & 0.9489 \\
\hline $\mathbf{3}$ & VOTEM M & 09716 & 0.9848 \\
\hline 4 & VOTEP & 0.2808 & 0.9482 \\
\hline 3 & $A E G$ & 0.9608 & 0.9448 \\
\hline 6 & REFERENCE & 0.8592 & 0.8892 \\
\hline 7 & ATT_4 & 0.8891 & 0.9410 \\
\hline e & ATT 2 & 0.9888 & 0.9431 \\
\hline 9 & OCRSYS & 0.8884 & 0.2521 \\
\hline 10 & ERIM_- & 0.8868 & 0.2410 \\
\hline 11 & ELSAGBA & 0.8860 & 0.9427 \\
\hline 12 & ELSAGB2 & 0.8648 & 0.2423 \\
\hline 13 & $\mathrm{ATT}_{\perp}$ & 0.9544 & 0.9431 \\
\hline 14 & ERIM 2 & 0.9830 & 0.9396 \\
\hline 15 & IBM & 0.9529 & 0.8408 \\
\hline 16 & SYMBUS & 0.9494 & 0.2337 \\
\hline 17 & UBOL & 0.8488 & 0.9349 \\
\hline 18 & NESTOR & 0.8481 & 0.9343 \\
\hline 19 & ELSAGB-1 & 0.9480 & 0.2313 \\
\hline 20 & NIST_4 & 0.8471 & 0.2314 \\
\hline 21 & THINK_1 & 0.9468 & 0.9312 \\
\hline 22 & ATT J & 0.8460 & 0.9323 \\
\hline 23 & THINK 2 & 0.8450 & 0.9332 \\
\hline 24 & HUGHES_- & 0.8442 & 0.8306 \\
\hline 25 & HUGHES_2 & 0.8432 & 0.8301 \\
\hline 26 & NYNEX & 0.9428 & 0.9323 \\
\hline 27 & REI & 0.8424 & 0.9338 \\
\hline 28 & GTESS_- & 0.9322 & 0.8170 \\
\hline 28 & GTESS 2 & 0.9318 & 0.2162 \\
\hline 30 & COMCOM & 0.2244 & 0.2218 \\
\hline 31 & NIST_1 & 0.9227 & 0.2063 \\
\hline 32 & GMD_3 & 0.2172 & 0.9018 \\
\hline 33 & MIME & 0.2160 & 0.8992 \\
\hline 34 & ASOL & 0.9148 & 0.8973 \\
\hline 35 & $\mathrm{NIST}_{2}$ & 0.8127 & 0.8932 \\
\hline 36 & UPENN & 0.2118 & 0.8948 \\
\hline 37 & GMD_1 & 09096 & 0.8983 \\
\hline 38 & NIST & 0.2093 & 0.8912 \\
\hline 39 & RISO & 0.9017 & 0.8821 \\
\hline 40 & GMD_4 & 0.8946 & 0.8807 \\
\hline 41 & KAMAN_l & 0.8218 & 0.8733 \\
\hline 42 & KAMAN_3 & 0.8747 & 0.8672 \\
\hline 43 & KAMAN_2 & 0.8718 & 0.8546 \\
\hline 44 & GMD_2 & 0.8530 & 0.8356 \\
\hline 45 & KAMANS & 0.8514 & 0.8361 \\
\hline 46 & VALEN 2 & 0.8409 & 0.8279 \\
\hline 47 & IFAX & 0.8304 & 0.8155 \\
\hline 48 & VALEN_L & 0.8207 & 0.8061 \\
\hline 49 & KAMAN_4 & 0.7978 & 0.7819 \\
\hline
\end{tabular}

Table 95: KODAK 2 correlation graph key for digits. 


\section{No Data Available}

Figure 154: KODAK 2 - upper case correlation

There was no date for this evaluation

Table 96: KODAK 2 correlation graph key for uppers. 


\section{No Data Available}

Figure 155: KODAK 2 - lower case correlation

There wad no data for this evaluation.

Table 97: KODAK 2 correlation graph key for lowers. 


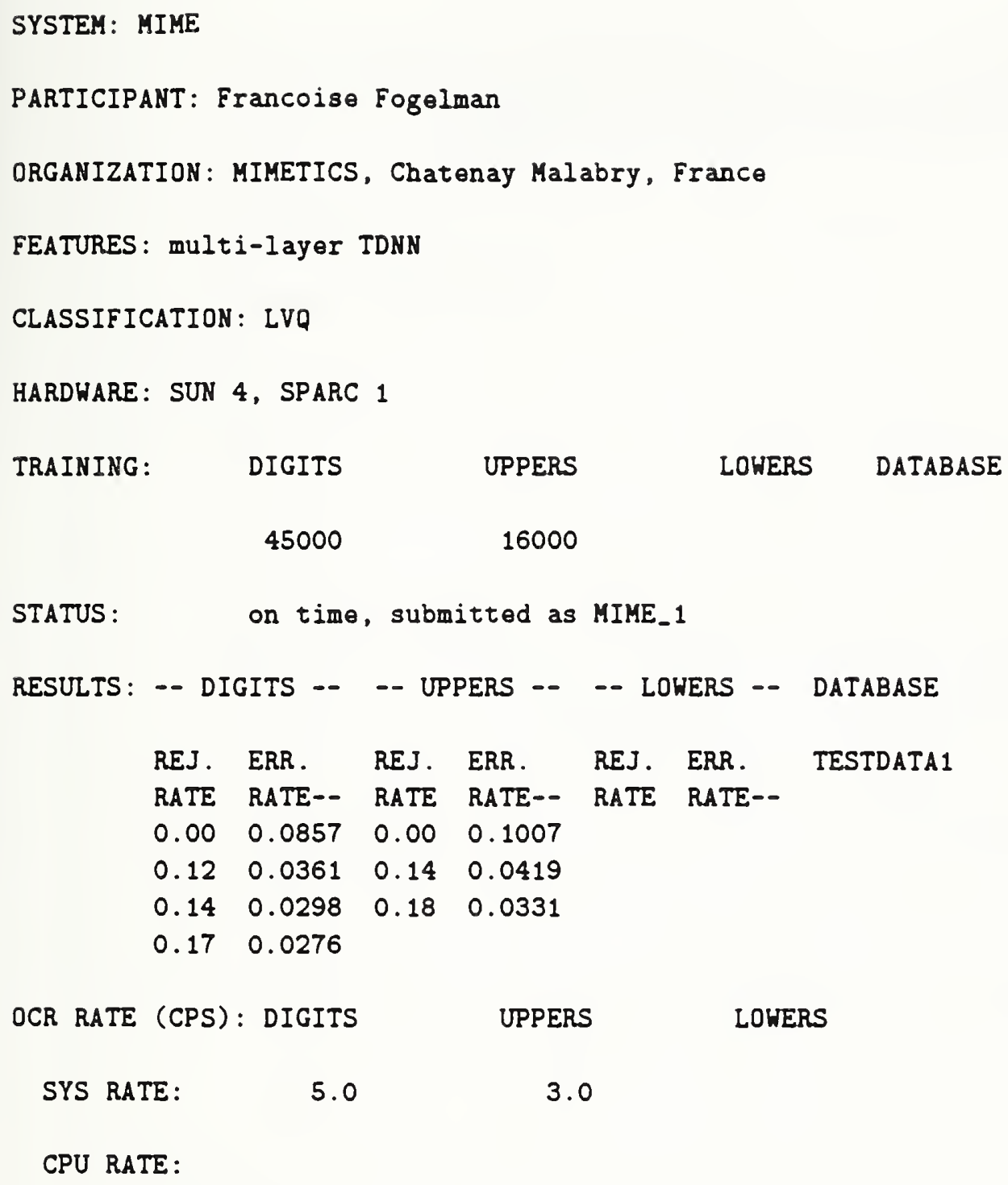

NOTE: classification is effectively nearest-neighbor 
SYSTEM: MIME

BIBLIOGRAPHY:

The following references have been provided for this system:

[29][30] 


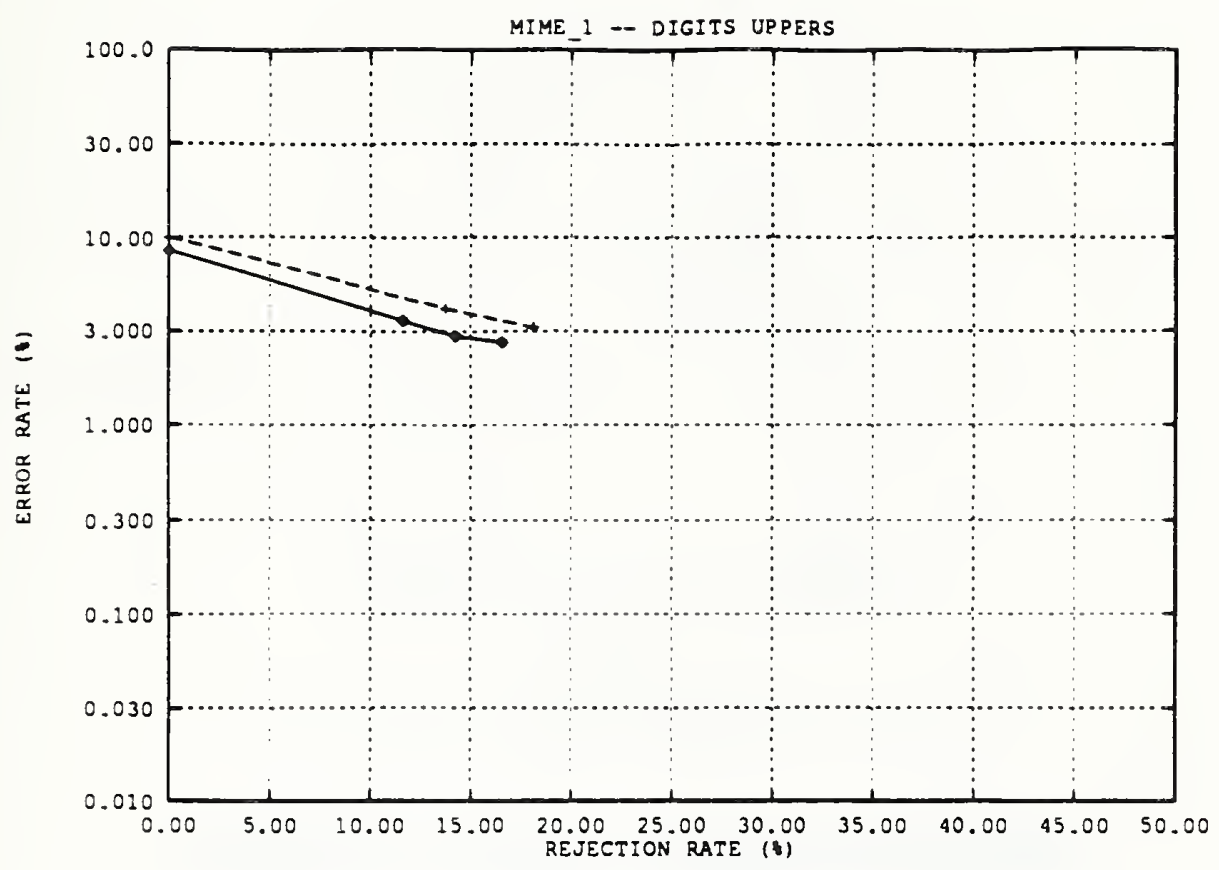

Figure 156: Error rate versus rejection rate for MIME

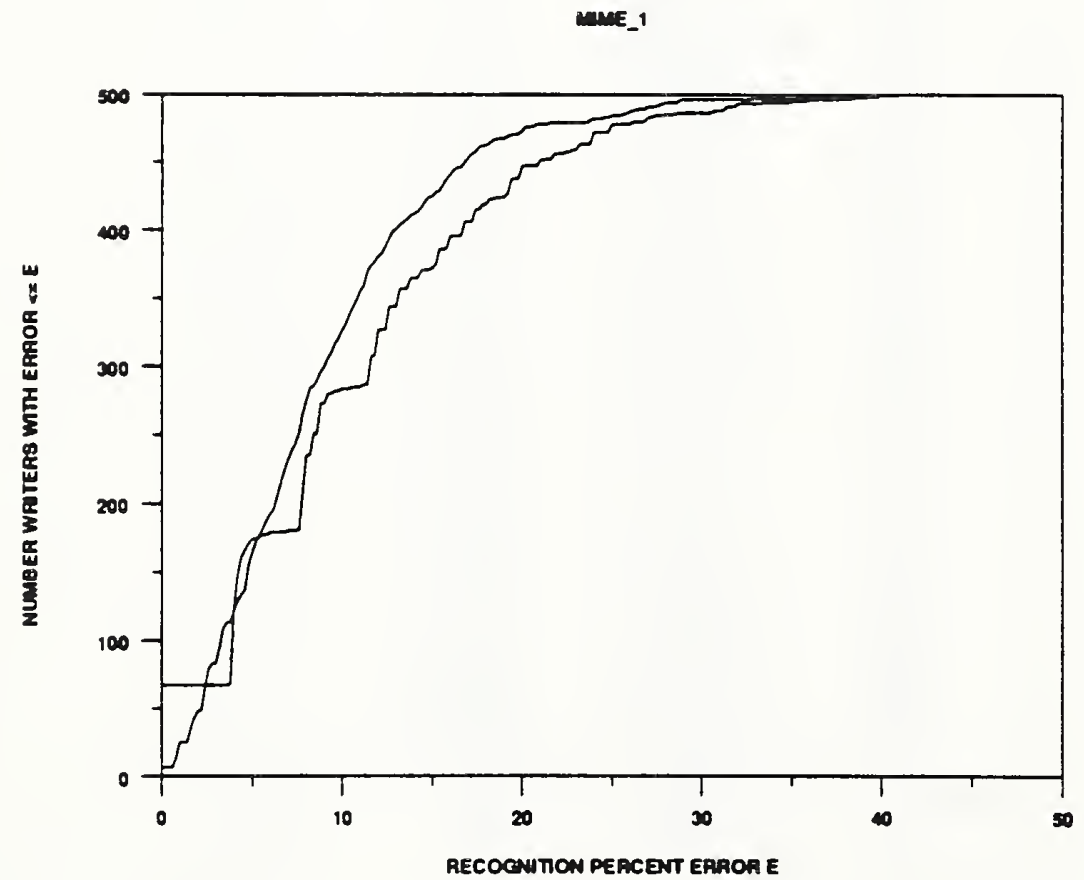

Figure 157: Error rate per writer of MIME 


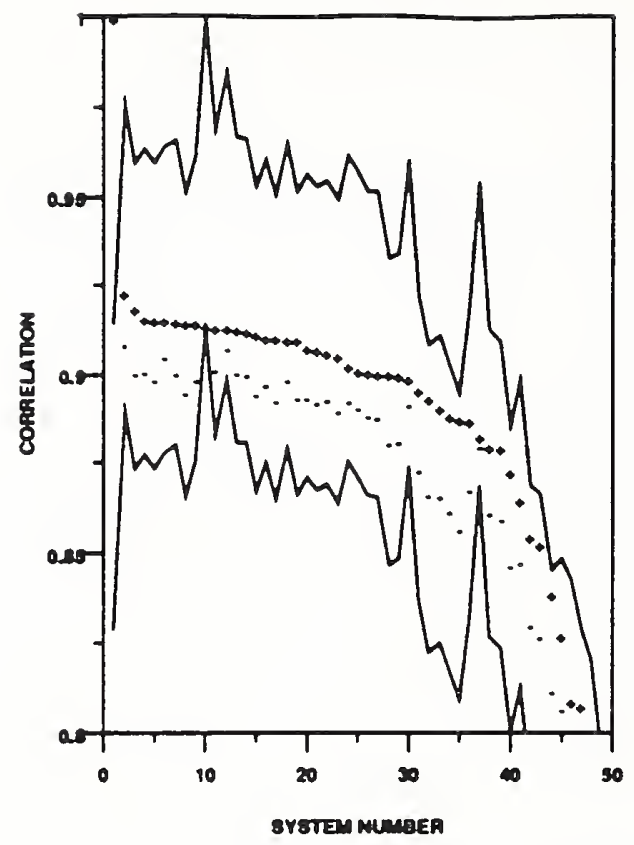

Figure 158: MIME - digit correlation

\begin{tabular}{|c|c|c|c|}
\hline System Number & System Name & Corretation (all) & Corretation (coprect) \\
\hline 1 & MIME & 1.0000 & 1.0000 \\
\hline 2 & VOTE_M & 0.2239 & 0.9093 \\
\hline 3 & A TT 44 & 0.9193 & 0.9007 \\
\hline 4 & ATT 2 & 0.9163 & 0.8013 \\
\hline 3 & KODAK2 2 & 0.9160 & 0.8992 \\
\hline 6 & VOTE_P & 0.9159 & 0.9054 \\
\hline 7 & AEG & 0.9153 & 0.2011 \\
\hline 8 & ERIM_1 & 0.9180 & 0.8992 \\
\hline 9 & THINK-1 & 0.9180 & 0.8982 \\
\hline 10 & REFERENCE & 0.2143 & 0.9143 \\
\hline 11 & ATT 1 & 0.9139 & 0.9017 \\
\hline 12 & OCRSYS & 0.9138 & 09079 \\
\hline 13 & ELSAGBA & 0.9132 & 0.9010 \\
\hline 14 & ELSAGB2 & 0.9128 & 0.2006 \\
\hline 15 & KODAK」 & 0.9119 & 0.8948 \\
\hline 16 & ERIM 2 & 0.9111 & 0.8978 \\
\hline 17 & NIST_4 & 0.9110 & 0.8930 \\
\hline 18 & IB M & 0.9105 & 0.8989 \\
\hline 19 & ATT & 0.9103 & 0.8942 \\
\hline 20 & UBOL & 09081 & 0.8941 \\
\hline 21 & SYMBUS & 0.9079 & 0.8927 \\
\hline 22 & NESTOR & 0.9069 & 0.8938 \\
\hline 23 & ELSAGB_1 & 0.8039 & 0.8902 \\
\hline 24 & THINK 2 & 0.9030 & 0.8932 \\
\hline 23 & NYNEX & 0.9020 & 0.8914 \\
\hline 26 & HUGHES_1 & 0.9014 & 0.8890 \\
\hline 27 & HUGHES_2 & 0.9008 & 0.8887 \\
\hline 28 & GTESS 2 & 0.9007 & 0.8809 \\
\hline 28 & GTESS_L & 0.9004 & 0.8814 \\
\hline 30 & REI & 0.8997 & 0.8920 \\
\hline $3 t$ & $N_{1 S T}$ & 0.8962 & 0.8739 \\
\hline 32 & NIST 2 & 0.8940 & 0.8670 \\
\hline 33 & ASOL & 0.8913 & 0.8664 \\
\hline 34 & NIST & 0.8889 & 0.8624 \\
\hline 33 & Riso & 0.8882 & 0.8371 \\
\hline 36 & GMD_3 & 0.8874 & 0.8683 \\
\hline 37 & COMCOM & 0.8831 & 0.8800 \\
\hline 38 & GMD -1 & 0.8800 & 0.8620 \\
\hline 39 & UPENN & 0.8797 & 0.8599 \\
\hline 40 & KAMAN_l & 0.8733 & 0.8469 \\
\hline 41 & GMD_4 & 0.8635 & 0.8480 \\
\hline 42 & KAMAN_J & 0.8585 & 0.8306 \\
\hline 43 & KAMAN_2 & 0.8830 & 0.8276 \\
\hline 44 & GMD_2 & 0.8391 & 0.8124 \\
\hline 45 & KAMAN_S & 0.8279 & 0.8071 \\
\hline 46 & VALEN 2 & 0.8096 & 0.7980 \\
\hline 47 & IFAX & 0.8082 & 0.7881 \\
\hline 48 & VALEN_1 & 0.7997 & 0.7789 \\
\hline 49 & KAMAN_4 & 0.7882 & 0.7606 \\
\hline
\end{tabular}

Table 98: MIME correlation graph key for digits. 


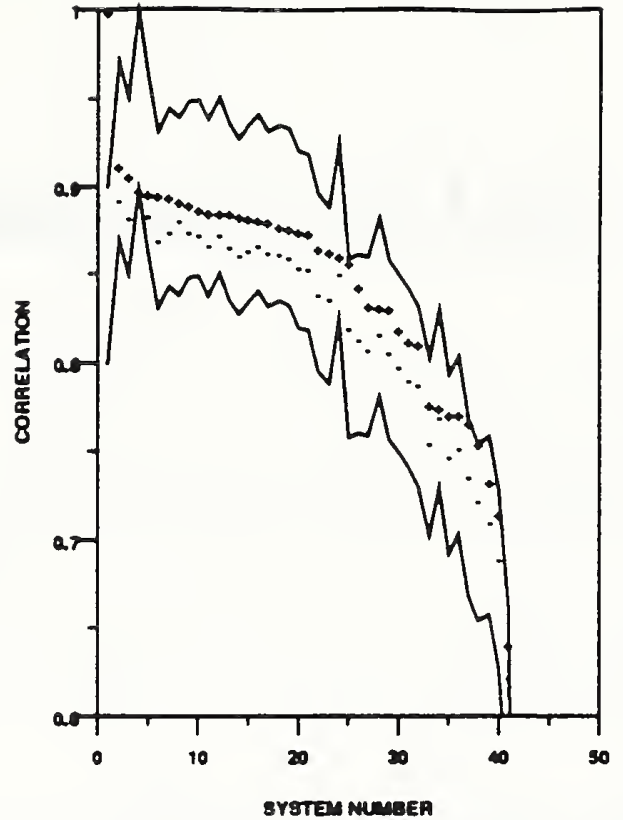

Figure 159: MIME - upper case correlation

\begin{tabular}{|c|c|c|c|}
\hline Syolem Number & System Name & Correlation (ali) & Correlacion (correct) \\
\hline 1 & MIME & 1.0000 & 1.0000 \\
\hline 2 & VOTE-M & 0.2131 & 08939 \\
\hline 3 & ATT 4 & 0.9077 & 0.8838 \\
\hline 4 & REFERENCE & 0.8993 & 0.8993 \\
\hline 5 & $A E G$ & 0.8977 & 0.8841 \\
\hline$B$ & KODAK」 & 08963 & 0.8711 \\
\hline 7 & ATT 2 & 0.8082 & 0.8758 \\
\hline 8 & VOTEP & 0.8931 & 08818 \\
\hline 9 & ERIM_] & 0.8911 & 0.8780 \\
\hline 10 & UMICH_i & 0.8878 & 0.8731 \\
\hline 11 & UBOL & 0.8867 & 0.8681 \\
\hline 12 & NYNEX & 08864 & 0.8734 \\
\hline 13 & IBM & 08860 & 0.8673 \\
\hline 14 & SYMBUS & 0.8843 & 0.8622 \\
\hline 15 & ATT $\perp$ & 0.8838 & 0.8653 \\
\hline 16 & NESTOR & 0.8828 & 0.8683 \\
\hline 17 & ATT 3 & 0.8813 & 0.8643 \\
\hline 18 & HUGHES-I & 0.8793 & 0.8631 \\
\hline 18 & HUGHES -2 & 0.8778 & 0.8612 \\
\hline 20 & GTESS -1 & 0.8784 & 0.8558 \\
\hline 21 & GTESS 2 & 0.8731 & 0.8346 \\
\hline 22 & NIST -4 & 0.8688 & 0.8401 \\
\hline 23 & ASOL & 0.8648 & 0.8372 \\
\hline 24 & OCRSYS & 0.8827 & 0.8321 \\
\hline 25 & RISO & 0.8585 & 0.8210 \\
\hline 26 & NIST_l & 0.8453 & 0.8149 \\
\hline 27 & GMD_1 & 0.8341 & 0.8093 \\
\hline 28 & REI & 08332 & 0.8176 \\
\hline 29 & GMD_3 & 0.8323 & 0.8076 \\
\hline 30 & KAMAN_1 & 0.8204 & 0.7987 \\
\hline 31 & GMD_4 & 0.8140 & 0.7913 \\
\hline 32 & NIST 3 & 0.8122 & 0.7886 \\
\hline 33 & KAMAN.3 & 0.7782 & 0.7560 \\
\hline 34 & COMCOM & 0.7764 & 0.7709 \\
\hline 35 & KAMAN_2 & 0.7728 & 0.7488 \\
\hline 36 & IFAX & 0.7726 & 0.7329 \\
\hline 37 & $\mathrm{NIST}_{2}$ & 0.7673 & 0.7375 \\
\hline 38 & GMD_2 & 0.7561 & 0.7236 \\
\hline 39 & VALEN_1 & 07348 & 0.7113 \\
\hline 40 & KAMAN_4 & 0.7163 & 0.6204 \\
\hline 41 & KAMAN_3 & 0.6422 & 0.6234 \\
\hline 42 & $\mathrm{UMICH}_{2}$ & 0.0532 & 0.0193 \\
\hline
\end{tabular}

Table 99: MIME correlation graph key for uppers. 


\section{No Data Available}

Figure 160: MIME - lower case correlation

There was no das for this evaluation

Table 100: MIME correlation graph key for lowers. 
SYSTEM: NESTOR

PARTICIPANT: Christopher L. Scofield

ORGANIZATION: Nestor, Inc., Providence, RI

FEATURES: neocognitron - convolution, 120 dimensional feature vector.

CLASSIFICATION: MLP. Two nets used in parallel, outputs averaged to generate overall confidence value. Training with gradient descent.

HARDWARE: IBM RS6000 Model 320H development environment

TRAINING: DIGITS UPPERS LOWERS DATABASE

$\begin{array}{rrrc}40000 & 20000 & 20000 & \text { NSDB1 } \\ 1800 & 1800 & 1800 & \text { writers } \\ 200000 & 40000 & 40000 & \text { NSDB1 } \\ 1800 & 1800 & 1800 & \text { writers } \\ & & 0 & \text { INTERNAL } \\ 15000 & 0 & 0 & \text { writers }\end{array}$

STATUS: $\quad$ on time

RESULTS: -- DIGITS -- -- UPPERS -- -- LOWERS -- DATABASE

REJ. ERR. REJ. ERR. REJ. ERR. TESTDATA1

RATE RATE-- RATE RATE-- RATE RATE--

$\begin{array}{lllllll}0.00 & 0.0453 & 0.00 & 0.0590 & 0.00 & 0.1539\end{array}$

$\begin{array}{lllllll}0.10 & 0.0129 & 0.10 & 0.0240 & 0.10 & 0.1074\end{array}$

$\begin{array}{lllllll}0.20 & 0.0050 & 0.20 & 0.0117 & 0.20 & 0.0704\end{array}$

$\begin{array}{lllllll}0.30 & 0.0029 & 0.30 & 0.0068 & 0.30 & 0.0469\end{array}$

$\begin{array}{lllllll}0.40 & 0.0016 & 0.40 & 0.0039 & 0.40 & 0.0325\end{array}$

$\begin{array}{lllllll}0.50 & 0.0011 & 0.50 & 0.0025 & 0.50 & 0.0213\end{array}$

OCR RATE (CPS): DIGITS UPPERS LOHERS

$\begin{array}{llll}\text { SYS RATE: } & 14.10 & 16.80 & 13.10\end{array}$

CPU RATE: 
SYSTEM: NESTOR

BIBLIOGRAPHY:

The following references have been provided for this system:

[19][31][34][32] [36][37][10] [33][34][35][38] 


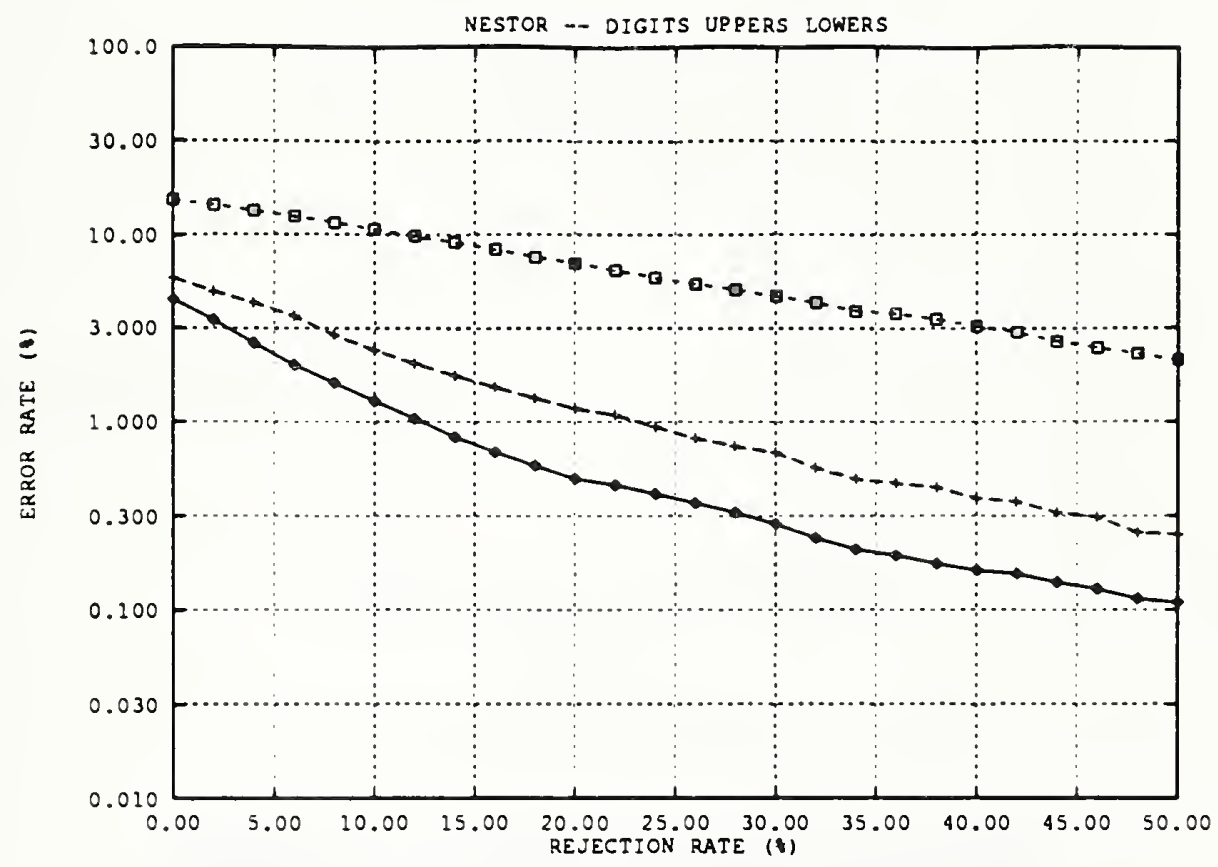

Figure 161: Error rate versus rejection rate for NESTOR

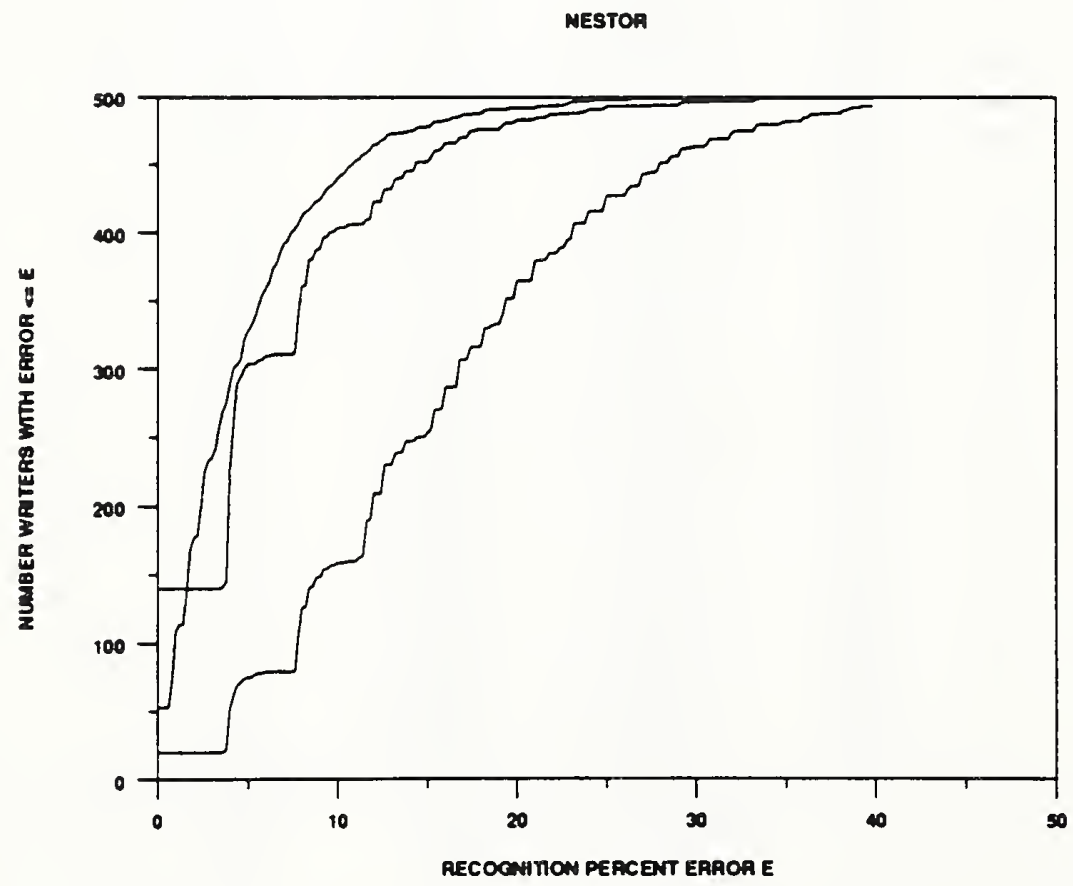

Figure 162: Error rate per writer of NESTOR 


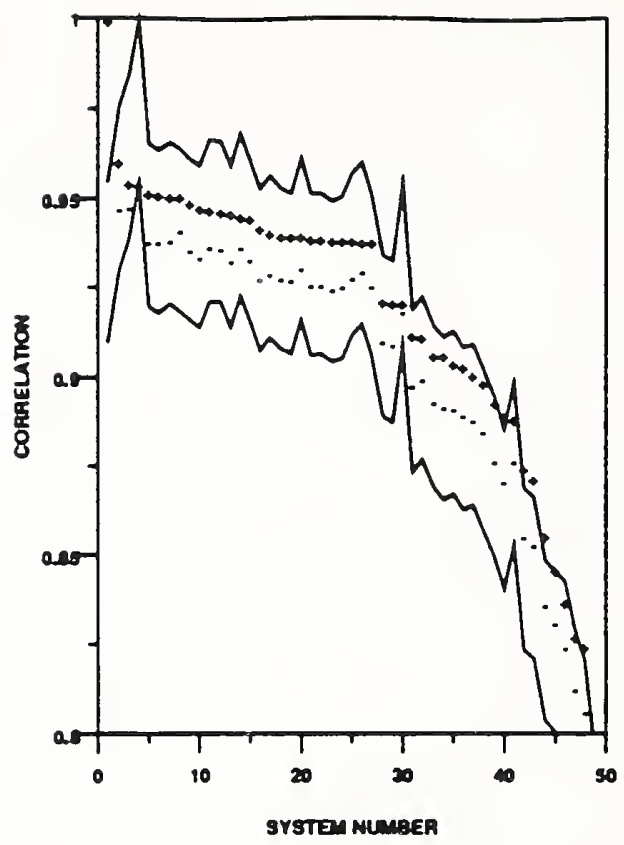

Figure 163: NESTOR - digit correlation

\begin{tabular}{|c|c|c|c|}
\hline System Number & System Name & Correlation (all) & Correlation (correct) \\
\hline $\mathrm{I}$ & NESTOK & 1.0000 & 10000 \\
\hline 2 & VOTEM & 0.2608 & 0.9478 \\
\hline $\mathbf{3}$ & OCRSYS & 0.9552 & 0.9483 \\
\hline 4 & REFERENCE & 0.2547 & 0.9547 \\
\hline 5 & IBM & 0.8523 & 0.9386 \\
\hline B & ATT2 2 & 0.0818 & 0.9384 \\
\hline 7 & AEG & 0.2816 & 0.8389 \\
\hline 8 & VOTEP & 0.2515 & 0.2418 \\
\hline 9 & ERIM-1 $_{-1}$ & 0.8497 & 0.8361 \\
\hline 10 & KODAK2 & 0.2481 & 0.2343 \\
\hline 11 & ELSAGBA & 0.9477 & 0.9373 \\
\hline 12 & ELSAGB 2 & 0.2471 & 0.9368 \\
\hline 13 & ATT 4 & 0.9466 & 0.9334 \\
\hline 14 & ATT」 & 0.9489 & 0.2372 \\
\hline 15 & ERIMZ & 0.2458 & 0.9339 \\
\hline 16 & KODAK_ & 0.9427 & $0.228 B$ \\
\hline 17 & UBOL & 0.2415 & 02289 \\
\hline 18 & SYMBUS & 0.9406 & 0.9283 \\
\hline 19 & THINK 2 & 0.2404 & 0.9311 \\
\hline 20 & ATT 3 & 0.8404 & 09282 \\
\hline 21 & HUGHES -2 & 0.2398 & 0.9268 \\
\hline 22 & HUGHES-1 & 0.9393 & 0.9268 \\
\hline 23 & NIST -4 & 0.2392 & 0.9260 \\
\hline 24 & $\operatorname{ELSAGB}_{-1}$ & 0.9392 & 0.9254 \\
\hline 25 & NYNEX & 0.2388 & 0.2286 \\
\hline 26 & REI & 0.9386 & 0.8303 \\
\hline 27 & THINK_- & 0.9384 & 0.9261 \\
\hline 28 & GTESS -1 & 0.9222 & 0.2107 \\
\hline 29 & COMCOM & 0.9218 & 0.8186 \\
\hline 30 & GTESS 2 & 0.2215 & 0.2096 \\
\hline 31 & GMD_3 & 0.9122 & 0.8982 \\
\hline 32 & NIST_l & 0.2117 & 0.8998 \\
\hline 33 & MIME & 0.2069 & 0.8935 \\
\hline 34 & ASOL & 0.2067 & 0.8221 \\
\hline 35 & GMD_I & 0.9044 & 0.8916 \\
\hline 36 & NIST 2 & 0.2035 & 0.8897 \\
\hline 37 & UPENN & 0.9013 & 08884 \\
\hline 38 & NIST & 0.8992 & 0.8853 \\
\hline 39 & RISO & 0.8935 & 0.8772 \\
\hline 40 & $K_{A M A N} 1$ & 0.8898 & $0.871 \mathrm{~J}$ \\
\hline 41 & GMD - 4 & 0.8891 & 0.8769 \\
\hline 42 & KAMAN_3 & 0.8753 & 0.8559 \\
\hline 43 & KAMAN_2 & 0.8726 & 0.8537 \\
\hline 44 & KAMAN_S & 0.8581 & 0.8364 \\
\hline 45 & GMD_2 & 0.8466 & 0.8316 \\
\hline 46 & VALEN? & 0.8375 & 0.8245 \\
\hline 47 & IFAX & 0.8278 & 0.8131 \\
\hline 48 & VALEN_L & 0.8251 & 0.8068 \\
\hline 49 & KAMAN_4 & 0.7971 & 0.7807 \\
\hline
\end{tabular}

Table 101: NESTOR correlation graph key for digits. 


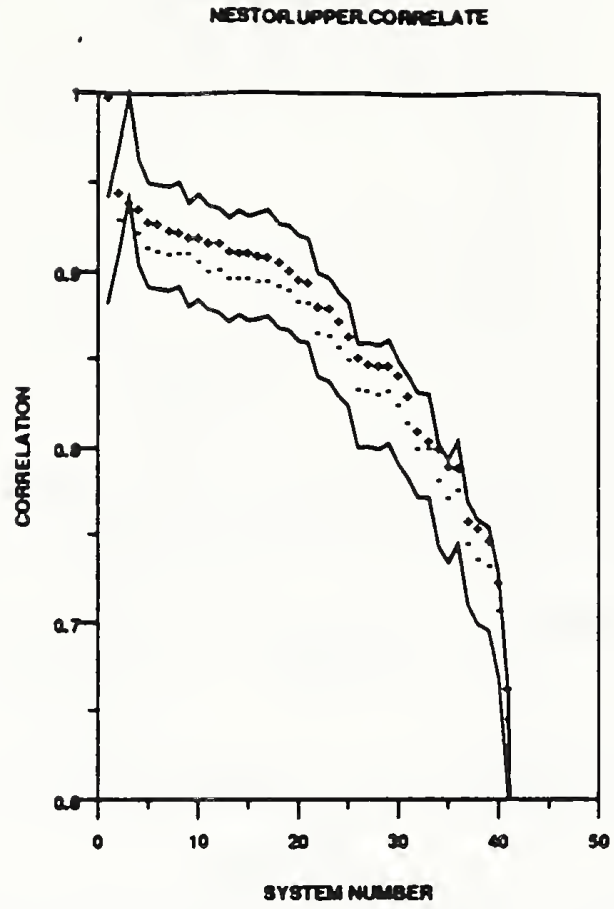

Figure 164: NESTOR - upper case correlation

\begin{tabular}{|c|c|c|c|}
\hline System Number & System Nome & Correlation (all) & Correlation (correct) \\
\hline$T$ & NESTOR & 1.0000 & 1.0000 \\
\hline 2 & VOTE_M & 0.9466 & 0.9310 \\
\hline 3 & REFERENCE & 0.9410 & 0.9410 \\
\hline 4 & AEG & 0.9374 & 0.9238 \\
\hline 5 & ATT_A & 0.9308 & 0.9135 \\
\hline 6 & UMICH.L & 0.9297 & 0.2141 \\
\hline 7 & ERIM-1 & 0.9255 & 0.9117 \\
\hline 8 & NYNEX & 0.8252 & 0.9126 \\
\hline 9 & VOTE_P & 0.9225 & 0.9132 \\
\hline 10 & ATT 2 & 0.9224 & 0.9080 \\
\hline 11 & UBOL & 0.9194 & 0.8030 \\
\hline 12 & IBM & 0.9191 & 0.9038 \\
\hline 13 & KODAK_ & 0.9144 & 0.8992 \\
\hline 14 & HUGHES-I & 0.9140 & 0.8991 \\
\hline 15 & ATT & 0.9139 & 0.8891 \\
\hline 16 & HUGHES_2 & 0.9121 & 0.8970 \\
\hline 17 & ATT $\lrcorner$ & 0.9111 & 0.8977 \\
\hline 18 & SYMBUS & 0.2082 & 0.8943 \\
\hline 10 & OCRSYS & 0.9036 & 0.8917 \\
\hline 20 & GTESS_1 & 0.8085 & 0.8855 \\
\hline 21 & GTESS_2 & 0.8968 & 0.8840 \\
\hline 22 & MIME & 0.8828 & 0.8683 \\
\hline 23 & NIST_4 & 0.8815 & 0.8658 \\
\hline 24 & ASOL & 0.8747 & 0.8599 \\
\hline 25 & REI & 0.8665 & 0.8827 \\
\hline 26 & RISO & 0.8345 & 0.8361 \\
\hline 27 & GMD_1 & 0.8308 & 0.8330 \\
\hline 28 & GMD_3 & 0.8497 & 0.8333 \\
\hline 29 & NIST 1 & 0.8492 & 0.8344 \\
\hline 30 & KAMAN_1 & 0.8436 & 0.8268 \\
\hline 31 & GMD_1 & 0.8318 & 0.8166 \\
\hline 32 & NIST & 0.8122 & 0.8020 \\
\hline 33 & COMCOM & 0.8065 & 0.8014 \\
\hline 34 & KAMAN_3 & 0.8013 & 0.7837 \\
\hline 35 & KAMAN_2 & 0.7916 & 0.7736 \\
\hline 36 & IFAX & 0.7909 & 0.7773 \\
\hline 37 & NIST 2 & 0.7604 & 0.7473 \\
\hline 38 & VALEN_I & 0.7835 & 0.7385 \\
\hline 39 & GMD_2 & 0.7494 & 0.7343 \\
\hline 40 & KAMAN-1 & 0.7258 & 0.7091 \\
\hline 41 & KAMAN_S & 0.6648 & 0.6473 \\
\hline 42 & $\mathrm{UMICH}_{2} 2$ & 0.0389 & 0.0203 \\
\hline
\end{tabular}

Table 102: NESTOR correlation graph key for uppers. 


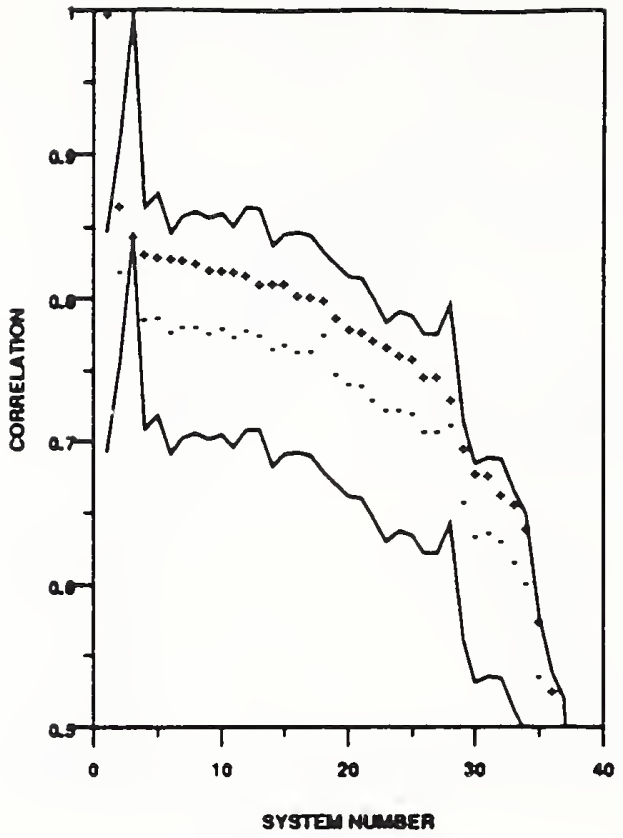

Figure 165: NESTOR - lower case correlation

\begin{tabular}{|c|c|c|c|}
\hline System Number & Syblem Name & Correlation (ali) & Correlation (correct) \\
\hline $\mathrm{I}$ & NESTOR & 1.0000 & 1.0000 \\
\hline 2 & VOTE_M & 0.8688 & 08225 \\
\hline 3 & REFERENCE & 0.8461 & 08461 \\
\hline 4 & ERIM_l & 08381 & 0.7889 \\
\hline 5 & $A E G$ & 0.8331 & 0.7900 \\
\hline 6 & IBM & 0.8311 & 0.7797 \\
\hline 7 & AT T -4 & 08301 & 0.7837 \\
\hline 8 & NYNEX & 0.8276 & 0.7837 \\
\hline 9 & ATT 2 & 08233 & 0.7818 \\
\hline 10 & KODAK」 & 08235 & 0.7791 \\
\hline 11 & UMICH_I & 0.8221 & 0.7761 \\
\hline 12 & OCRSYS & 08203 & 0.7809 \\
\hline 13 & ATT $\perp$ & 0.8142 & 0.7780 \\
\hline 14 & ATT 3 & 0.8141 & 0.7881 \\
\hline 15 & UBOL & 0.8137 & 0.7701 \\
\hline 16 & HUGHES.1 & 0.8062 & 0.7684 \\
\hline 17 & HUGHES - 2 & 0.8052 & 0.7654 \\
\hline 18 & VOTEP & 0.8030 & 0.7779 \\
\hline 19 & GTESS - 1 & 0.7898 & 0.7498 \\
\hline 20 & GTESS 2 & 0.7822 & 0.7429 \\
\hline 21 & NIST AI & 0.7803 & 0.7423 \\
\hline 22 & NIST -4 & 0.7744 & 0.7317 \\
\hline 23 & RISO & 0.7694 & 0.7243 \\
\hline 24 & GMD_3 & 0.7642 & 0.7247 \\
\hline 25 & ASOL & 0.7817 & 0.7222 \\
\hline 26 & GMD_4 & 0.7488 & 0.7095 \\
\hline 27 & GMD_I & 0.7483 & 0.7095 \\
\hline 28 & NIST 3 & 0.7322 & 0.7143 \\
\hline 29 & GMD_2 & 0.8977 & 0.6598 \\
\hline 30 & VALEN_L & 0.6812 & 0.6363 \\
\hline 31 & KAMAN_1 & 0.6800 & 0.6382 \\
\hline 32 & NIST 2 & 0.6837 & 0.6322 \\
\hline 33 & KAMAN_J & 0.6593 & 0.6179 \\
\hline 34 & KAMAN.2 & 0.6418 & 0.6028 \\
\hline 35 & KAMAN_S & 0.5761 & 0.5374 \\
\hline 36 & KAMAN_4 & 0.5280 & 0.4996 \\
\hline 37 & COMCOM & 04933 & 0.4822 \\
\hline 38 & $\mathrm{UMICH}_{2}$ & 0.0962 & 0.0331 \\
\hline
\end{tabular}

Table 103: NESTOR correlation graph key for lowers. 
SYSTEM: NIST_1

PARTICIPANT: Patrick J. Grother

ORGANIZATION: NIST, Gaithersburg, MD

PREPROCESSING: Size (preserving aspect ratio), Slant Normalization. Subtraction from binary image of mean of training images.

FEATURES: Projection onto principal components of training set. 32 leading elements of KL transform.

CLASSIFICATION: $K$ Nearest Neighbour. K not fixed; Distance veighted voting among prototypes vithin 1.1 * distance of the closest prototype.

HARDWARE: AMT 510C Array (32×32) Processor yith Sparc IO host.

$\begin{array}{lrrrr}\text { TRAINING : } & \text { DIGITS } & \text { UPPERS } & \text { LOWERS } & \text { DATABASE } \\ & -22000 & -22000 & -22000 & \text { NSDB3 } \\ & 2100 & 2100 & 2100 & \text { HRITERS }\end{array}$

STATUS: On time

RESULTS: -- DIGITS -- -- UPPERS -- -- LOHERS -- DATABASE

REJ. ERR. REJ. ERR. REJ. ERR. TESTDATA1

RATE RATE-- RATE RATE-- RATE RATE--

$\begin{array}{lllllll}0.00 & 0.0774 & 0.00 & 0.1385 & 0.00 & 0.1858\end{array}$

OCR RATE (CPS): DIGITS UPPERS LOHERS

OCR RATE:

$\begin{array}{llll}\text { CPU RATE: } & 4.8 & 4.8 & 4.8\end{array}$ 
SYSTEM: NIST_1

\section{BIBLIOGRAPHY:}

The following references have been provided for this system:

[39]

COMMENTS: NIST_1

See Cross Validation Section on Inadequacies of NIST Special Database 3 for the classification of NIST Test Data 1.

The late system NIST_4 outperforms this system on digits on the basis of further preprocessing, a larger training set, and more $\mathrm{KL}$ coefficients.

Very Slow Classification.

No exemplar pruning or aggregation.

Does not suffer from "minority" problems of perceptrons (e.g. crossed sevens). 


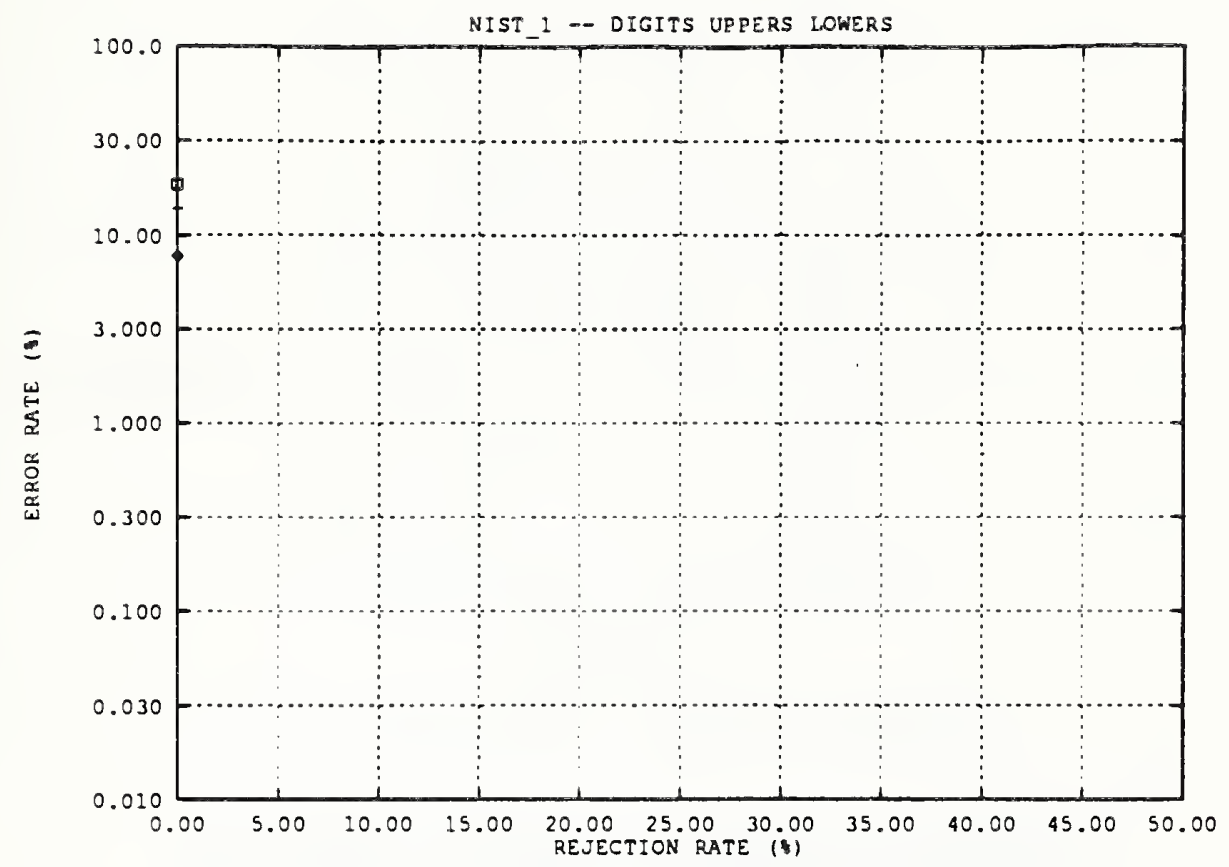

Figure 166: Error rate versus rejection rate for NIST_1

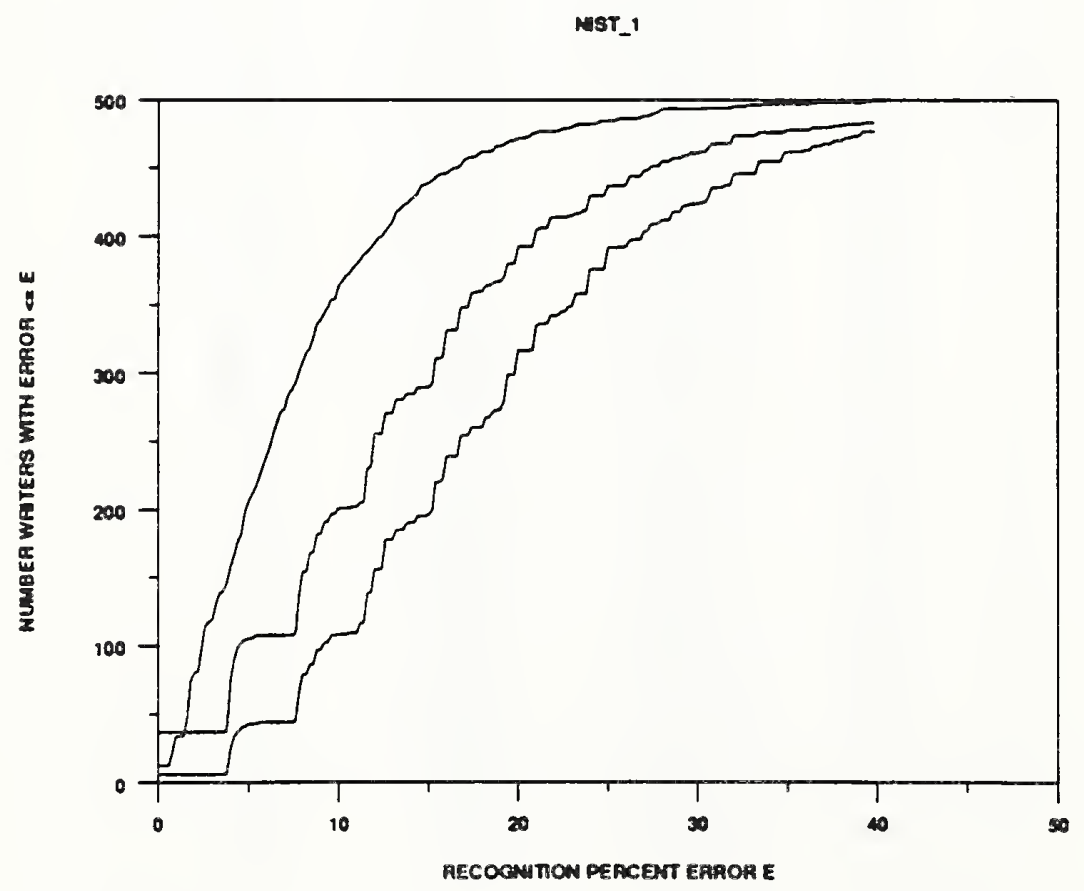

Figure 167: Error rate per writer of NIST_1 


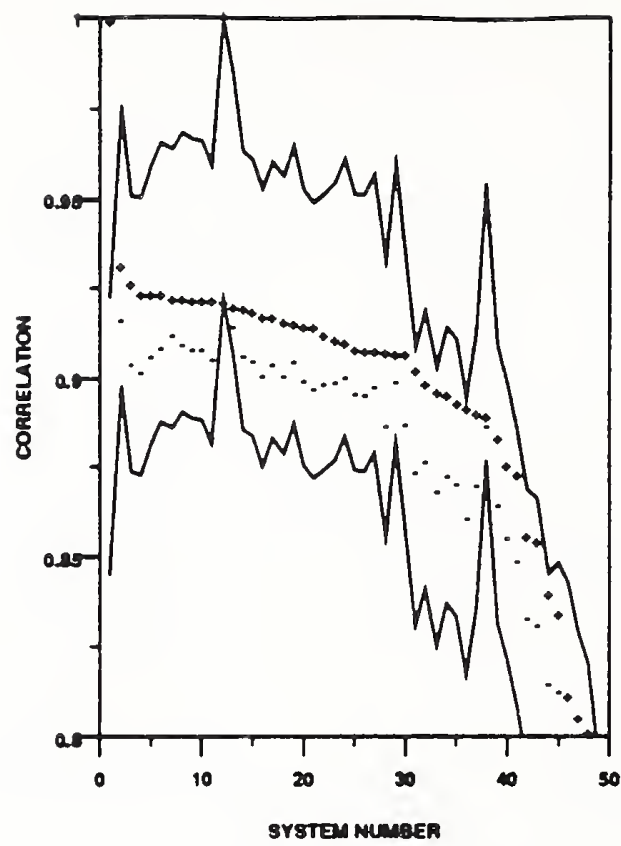

Figure 168: NIST_1 - digit correlation

\begin{tabular}{|c|c|c|c|}
\hline System Number & System Name & Corretation (ail) & Correlation (correct) \\
\hline 1 & NIST-1 & 1.0000 & 1.0000 \\
\hline 2 & VOTE_M & $0.932 \mathrm{~s}$ & 0.8174 \\
\hline 3 & THINK-I & 0.9277 & 0.9048 \\
\hline 4 & $A E G$ & 0.9247 & 0.8098 \\
\hline 3 & ATT_4 & 0.9247 & 0.9072 \\
\hline 6 & NIST 4 & 0.9247 & 0.8028 \\
\hline 7 & VOTEP & 0.9236 & 0.9131 \\
\hline 8 & $\mathrm{ATT} \perp$ & 0.9238 & 0.8108 \\
\hline $\boldsymbol{8}$ & ELSAGBA & 0.9231 & 0.9092 \\
\hline 10 & ELSAGB 2 & 0.9230 & 0.9090 \\
\hline 11 & KODAK2 & 0.2227 & 0.8063 \\
\hline 12 & REFERENCE & 0.9225 & 0.8225 \\
\hline 13 & OCRSYS & 0.9211 & 0.8136 \\
\hline 14 & АTT 2 & 0.9204 & 0.9072 \\
\hline 13 & ERIM_l & 0.8189 & 0.2057 \\
\hline 16 & ERIM 2 & 0.2182 & 0.8048 \\
\hline 17 & KODAK」 & 0.9182 & 0.8018 \\
\hline 18 & UBOL & 0.9170 & 0.9018 \\
\hline 19 & IBM & 0.9163 & 0.9058 \\
\hline 20 & SYMBUS & 0.8157 & 0.8006 \\
\hline 21 & ELSAGB-1 & 0.2138 & 0.8981 \\
\hline 22 & ATT 3 & 0.9132 & 0.8993 \\
\hline 23 & NESTOR & 0.9117 & 0.8898 \\
\hline 24 & THINK 2 & 0.9108 & 0.9012 \\
\hline 23 & HUGHES -1 & 0.8091 & 08986 \\
\hline 26 & HUGHES_2 & 0.9089 & 0.8963 \\
\hline 27 & NYNEX & 0.9087 & 0.8988 \\
\hline 28 & GTESS 2 & 0.8080 & 0.8876 \\
\hline 29 & REI & 0.9078 & 0.8999 \\
\hline 30 & GTESS_L & 0.9077 & 0.8882 \\
\hline 31 & $N_{1 S T} 2$ & 0.9032 & 08748 \\
\hline 32 & GMD_3 & 0.8993 & 08773 \\
\hline 33 & נה TIST & 08974 & 08691 \\
\hline 34 & MIME & 08962 & 0.8739 \\
\hline 35 & ASOL & 0.8942 & 0.8717 \\
\hline 36 & RISO & 08926 & 0.8618 \\
\hline 37 & GMD_l & 0.8913 & 0.8709 \\
\hline 38 & СОМСОM & 0.8903 & 0.8878 \\
\hline 39 & UPENN & 0.8843 & 0.8654 \\
\hline 40 & GMD - 4 & 0.8763 & 0.8564 \\
\hline 41 & KAMAN 1 & 0.8741 & 0.8498 \\
\hline 42 & KAMAN] & 0.8570 & 0.8340 \\
\hline 43 & $K A M A N_{-2}$ & 0.8538 & 0.8318 \\
\hline 44 & GMD _2 & 0.8406 & 0.8153 \\
\hline 45 & KAMAN_S & 0.8350 & 0.8138 \\
\hline 46 & VALEN 2 & 0.8121 & 0.7997 \\
\hline 47 & IFAX & 0.8062 & 0.7898 \\
\hline 48 & VALEN_I & 0.8018 & 07826 \\
\hline 49 & KAMAN_4 & 0.7863 & 0.7623 \\
\hline
\end{tabular}

Table 104: NIST_1 correlation graph key for digits. 


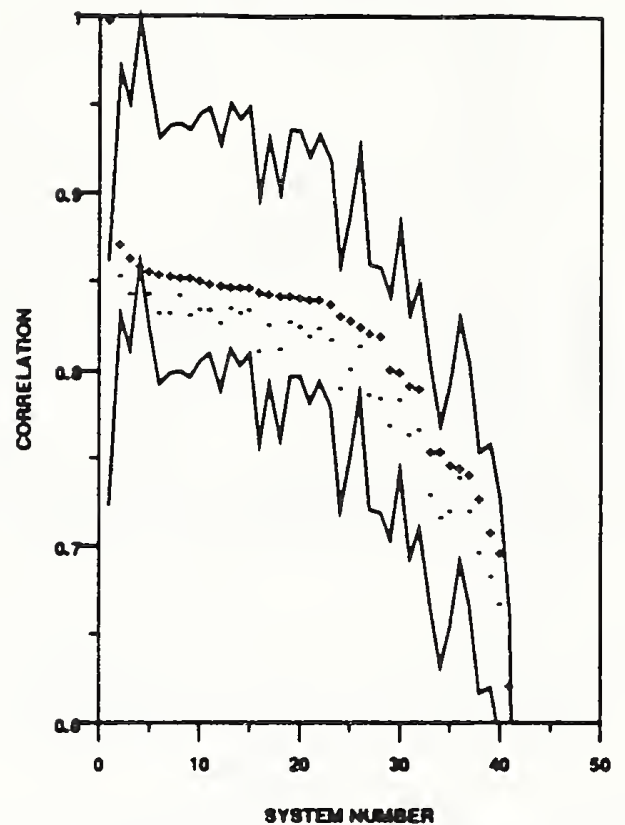

Figure 169: NIST_1 - upper case correlation

\begin{tabular}{|c|c|c|c|}
\hline System Number & System Name & Corretation (all) & Correlation (correct) \\
\hline 1 & NI5T-1 & 1.0000 & 1.0000 \\
\hline 2 & VOTEAM & 0.8730 & 0.8555 \\
\hline 3 & ATT -1 & 08684 & 0.8460 \\
\hline 4 & REFERENCE & 0.8615 & 0.8615 \\
\hline 3 & $A E G$ & 0.8585 & 0.8457 \\
\hline 6 & KODAK」 & 0.8565 & 0.8344 \\
\hline 7 & UBOL & 0.8557 & 0.8346 \\
\hline 8 & VOTEP & 0.8551 & 0.8453 \\
\hline $\boldsymbol{9}$ & ATT $\perp$ & 0.8550 & 0.8338 \\
\hline 10 & ATT2 & 0.8529 & 0.8364 \\
\hline 11 & ERIM.I & 0.8516 & 0.8371 \\
\hline 12 & SYMBUS & 0.8504 & 0.8291 \\
\hline 13 & NYNEX & 08498 & 0.8373 \\
\hline 14 & NESTOR & 0.8492 & 0.8344 \\
\hline 16 & UMICH-1 & 0.8491 & 08362 \\
\hline 18 & NIST-1 & 0.8467 & 0.8135 \\
\hline 17 & ATT 3 & 08463 & 0.8284 \\
\hline 18 & MIME & 0.6453 & 0.8149 \\
\hline 19 & IBM & 0.8449 & 0.8302 \\
\hline 20 & HUGHES_I & 0.8439 & 0.8275 \\
\hline 21 & GTESS_L & 0.8428 & 0.8217 \\
\hline 22 & HUGHES.2 & 0.8427 & 0.8261 \\
\hline 23 & GTESS 2 & 0.8404 & 0.8203 \\
\hline 24 & RISO & 0.8338 & $0.792 \mathrm{~s}$ \\
\hline 23 & ASOL & 0.8308 & 0.8036 \\
\hline 26 & OCRSYS & 0.8277 & 0.8186 \\
\hline 27 & GMD_-1 & 0.239 & 0.7892 \\
\hline 28 & GMD.3 & 0.8213 & 0.7870 \\
\hline 29 & GMD_4 & 0.8035 & 0.7711 \\
\hline 30 & REI & 0.8013 & 0.7860 \\
\hline 31 & ב-3 NIST & 0.7943 & 0.7658 \\
\hline 32 & KAMAN_1 & 0.7921 & 0.7689 \\
\hline 33 & KAMAN_J & 0.7570 & 0.7315 \\
\hline 34 & NIST? & 0.7589 & 0.7193 \\
\hline 35 & $\mathrm{KAMAN}_{-2}$ & 0.7494 & 0.7229 \\
\hline 36 & СомСоM & 0.7474 & 0.7421 \\
\hline 37 & IFAX & 0.7436 & 0.7231 \\
\hline 38 & GMD_2 & 0.7304 & 0.6986 \\
\hline 39 & $V_{A L E N-1}$ & 0.7111 & 0.6850 \\
\hline 40 & KAMAN-4 & 0.6986 & 0.6691 \\
\hline 41 & KAMAN_S & 0.6239 & 0.6022 \\
\hline 42 & UMICH 2 & 0.0616 & 0.0185 \\
\hline
\end{tabular}

Table 105: NIST_1 correlation graph key for uppers. 


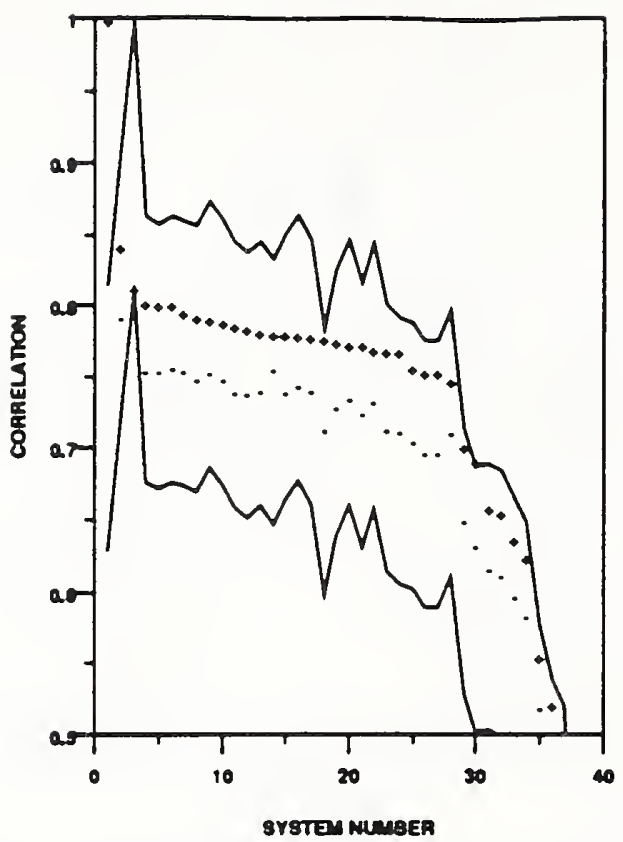

Figure 170: NIST_1 - lower case correlation

\begin{tabular}{|c|c|c|c|}
\hline System Number & System Name & Correlation (sil) & Correlation (correct) \\
\hline 1 & NIST_-1 & 1.0000 & 1.0000 \\
\hline 2 & VOTE_M & 0.8428 & 0.7937 \\
\hline $\mathbf{3}$ & REFERENCE & 0.8142 & 0.8142 \\
\hline 4 & ATT」 & 0.8038 & 0.7334 \\
\hline 5 & ERIM-1 & 0.8030 & 0.7384 \\
\hline 6 & A TT -4 & 0.8030 & 0.73389 \\
\hline 7 & ATT 2 & 0.7974 & 0.7332 \\
\hline $\mathbf{8}$ & KODAK_ & 0.7934 & 0.7503 \\
\hline 9 & $A E G$ & 0.7924 & 0.7542 \\
\hline 10 & NYNEX & 0.7887 & 0.7498 \\
\hline 11 & UBOL & 0.7883 & 0.7409 \\
\hline 12 & ATT 3 & 0.7837 & 0.7390 \\
\hline 13 & IBM & 0.7828 & 0.7413 \\
\hline 14 & VOTEP & 0.7822 & 0.7573 \\
\hline 15 & $\mathrm{UMICH}_{-1}$ & 0.7820 & 0.7408 \\
\hline 16 & OCRSYS & 0.7809 & 0.7448 \\
\hline 17 & NESTOR & 0.7803 & 0.7423 \\
\hline 18 & RISO & 0.7784 & 0.7148 \\
\hline 18 & GTESS_l & 0.7763 & 0.7307 \\
\hline 20 & HUGHES - 1 & 0.7744 & 0.7364 \\
\hline 21 & GTESS 2 & 0.7740 & 0.7282 \\
\hline 22 & HUGHES_2 & 07707 & 0.7337 \\
\hline 23 & NIST -4 & 0.7699 & 0.7114 \\
\hline 24 & GMD_3 & 0.7692 & 0.7132 \\
\hline 25 & ASOL & 0.7381 & 0.7065 \\
\hline 26 & GMD_4 & 0.7542 & 0.8981 \\
\hline 27 & GMD.1 & 0.7542 & 0.6981 \\
\hline 28 & NIST 3 & 0.7493 & 0.7122 \\
\hline 29 & GMD 2 & 0.7031 & 0.6513 \\
\hline so & NIST 2 & 0.6919 & 0.6340 \\
\hline 31 & KAMAN -1 & 0.6604 & 0.6181 \\
\hline 32 & VALEN_1 & 0.6572 & 0.6127 \\
\hline 33 & KAMAN_3 & 0.6388 & 0.8978 \\
\hline 34 & KAMAN_2 & 0.6255 & 0.3847 \\
\hline 35 & KAMAN_S & 0.5362 & 0.3202 \\
\hline 36 & KAMAN-4 & 0.5225 & 0.4866 \\
\hline 37 & COMCOM & 0.4782 & 0.4678 \\
\hline 38 & $\mathrm{UMICH}_{2}$ & 0.1046 & 0.0334 \\
\hline
\end{tabular}

Table 106: NIST_1 correlation graph key for lowers. 
SYSTEM: NIST_2

PARTICIPANT: Patrick J. Grother

ORGANIZATION: NIST, Gaithersburg, MD

PREPROCESSING: Size (preserving aspect ratio), Slant Normalization.

FEATURES: Projection onto 4 quadrant gabor wavelets. One frequency, two phases, four angles gives 32 element "Gabor Transform". Least squares fitting.

CLASSIFICATION: Scaled conjugate gradient trained $32: 48:\{10,26\}$ perceptron.

HARDHARE: AMT 510C Array (32×32) Processor uith Sparc IO host.

TRAINING :

$\begin{array}{rrrr}\text { DIGITS } & \text { UPPERS } & \text { LOWERS } & \text { DATABASE } \\ -102000 & -45000 & -46000 & \text { NSDB3 } \\ 2100 & 2100 & 2100 & \text { WRITERS }\end{array}$

STATUS: On time

RESULTS: -- DIGITS -- -- UPPERS -- -- LOWERS -- DATABASE

REJ. ERR. REJ. ERR. REJ. ERR. TESTDATA1

RATE RATE-- RATE RATE-- RATE RATE--

$\begin{array}{llllll}0.00 & 0.0919 & 0.00 & 0.2310 & 0.00 & 0.3120\end{array}$

$\begin{array}{lllllll}0.10 & 0.0519 & 0.10 & 0.1793 & 0.10 & 0.2657\end{array}$

$\begin{array}{lllllll}0.20 & 0.0285 & 0.20 & 0.1384 & 0.20 & 0.2220\end{array}$

$\begin{array}{lllllll}0.30 & 0.0150 & 0.30 & 0.1028 & 0.30 & 0.1805\end{array}$

$\begin{array}{lllllll}0.40 & 0.0092 & 0.40 & 0.0728 & 0.40 & 0.1440\end{array}$

$\begin{array}{lllllll}0.50 & 0.0060 & 0.50 & 0.0524 & 0.50 & 0.1078\end{array}$

OCR RATE (CPS): DIGITS UPPERS LOWERS

OCR RATE:

$\begin{array}{llll}\text { CPU RATE: } & 81.0 & 81.0 & 81.0\end{array}$ 


\section{SYSTEM: NIST 2}

\section{BIBLIOGRAPHY:}

The following references have been provided for this system:

[40]

COMMENTS: NIST_2

See Cross Validation Section on Inadequacies of NIST Special Database 3 for the classification of NIST Test Data 1.

Insufficient / Inappropriate Gabor Bases. 


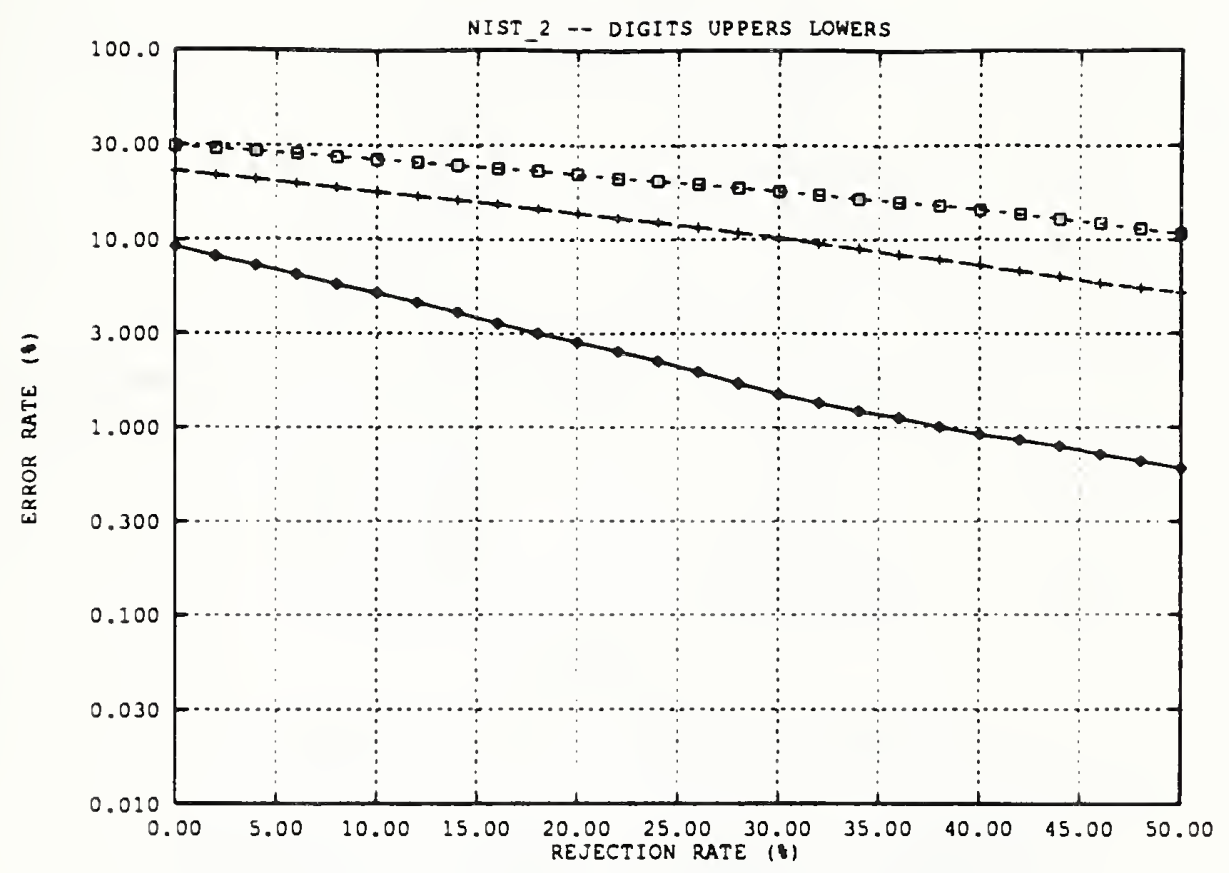

Figure 171: Error rate versus rejection rate for NIST_2

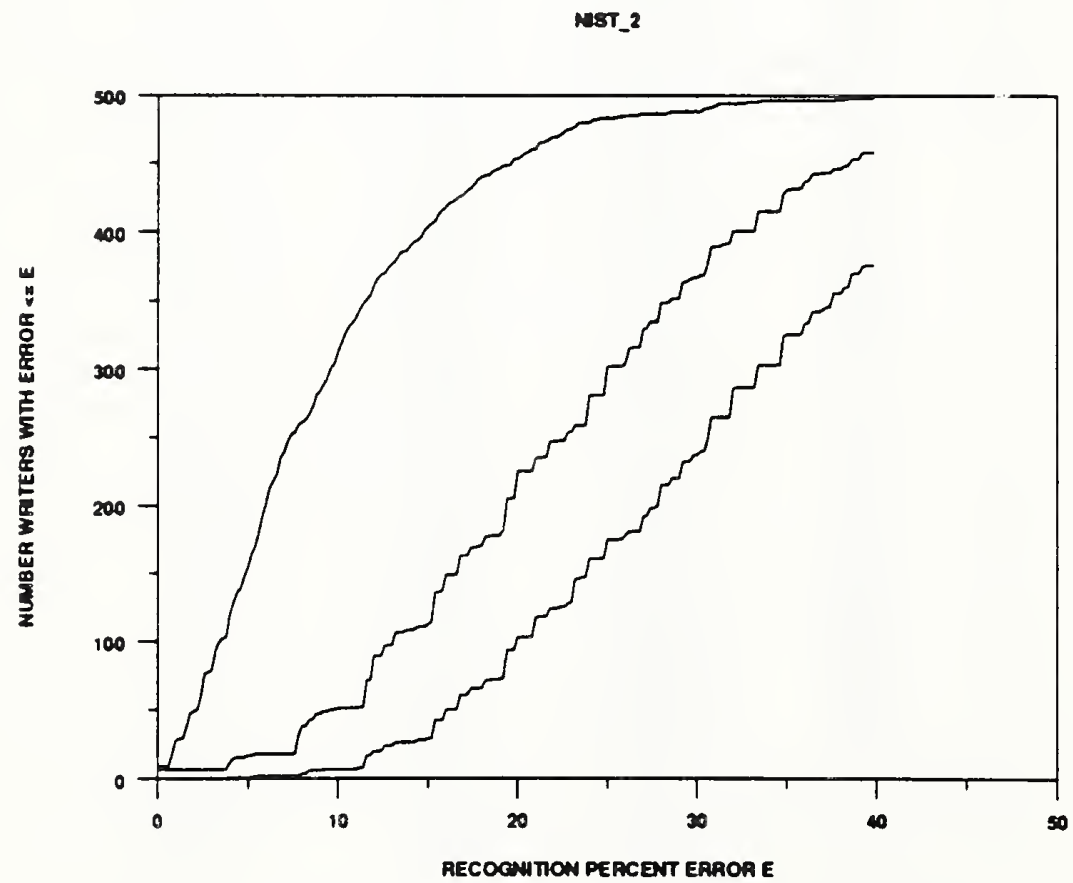

Figure 172: Error rate per writer of NIST_2 


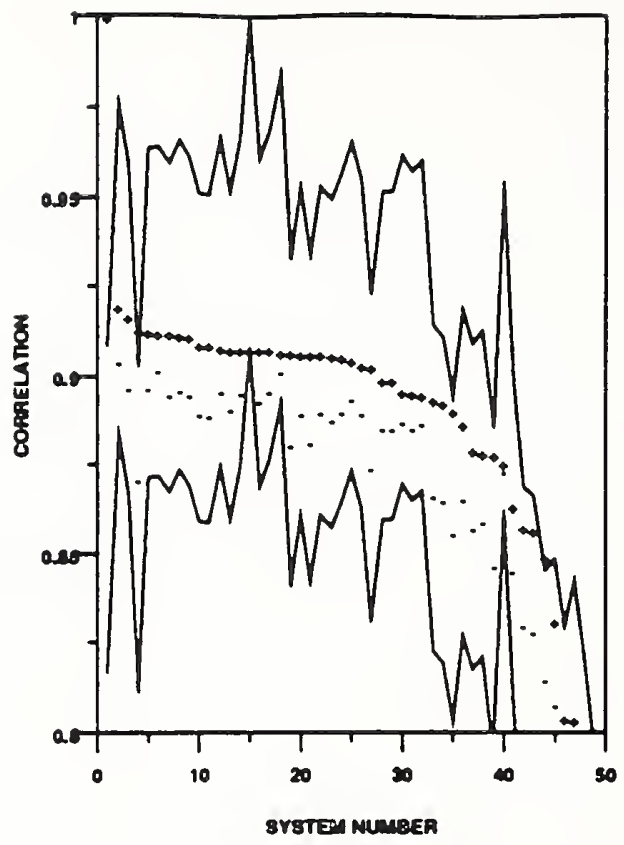

Figure 173: NIST 2 - digit correlation

\begin{tabular}{|c|c|c|c|}
\hline System Number & System Name & Correlation (ali) & Correistion (correct) \\
\hline 1 & NIST 2 & 1.0000 & 1.0000 \\
\hline 2 & VOTE_M & 0.8201 & 0.2046 \\
\hline 3 & ATT_4 & 0.2172 & 0.8973 \\
\hline 4 & NIST 3 & 0.8138 & 0.8715 \\
\hline $\mathbf{s}$ & ATT 2 & 0.8134 & 08874 \\
\hline 6 & VOTEP & 0.8130 & 0.8021 \\
\hline 7 & KODAK 2 & 0.8127 & 0.3952 \\
\hline 8 & AEG & 0.8124 & 0.8968 \\
\hline 9 & ERIM-1 & 0.2117 & 0.8934 \\
\hline 10 & THINK_I & 0.9097 & 0.8900 \\
\hline 11 & NIST_A & 0.8094 & 0.8896 \\
\hline 12 & ELSAGBA & 0.8087 & 0.8962 \\
\hline 13 & ATT 3 & 0.8084 & 0.8911 \\
\hline 14 & ELSAGB 2 & 0.8083 & 0.8938 \\
\hline 15 & REFERENCE & 0.9081 & 0.9081 \\
\hline 16 & ATT」 & 0.9080 & 0.8961 \\
\hline 17 & ERIM 2 & 0.9080 & 08936 \\
\hline 18 & OCRSYS & 0.8074 & 0.9016 \\
\hline 18 & GTESS 2 & 0.9071 & 0.8810 \\
\hline 20 & KODAK_ & 0.8070 & 08898 \\
\hline 21 & SYMBUS & 0.9068 & 0.8902 \\
\hline 22 & GTESS_- & 0.8068 & 0.8816 \\
\hline 23 & ELSAGB-I & 0.2065 & 08881 \\
\hline 24 & UBOL & 0.9058 & 0.8902 \\
\hline 25 & IBM & 0.8031 & 0.8939 \\
\hline 26 & NESTOR & 0.8035 & 0.8897 \\
\hline 27 & NIST_l & 0.9032 & 0.8748 \\
\hline 28 & HUGHES_2 & 08986 & 0.8857 \\
\hline 29 & HUGHES_l & 0.8985 & 0.8857 \\
\hline 30 & THINK 2 & 0.8964 & 0.8876 \\
\hline 31 & NYNEX & 0.8986 & 0.8858 \\
\hline 32 & REI & 0.8952 & 0.8870 \\
\hline 33 & MIME & 0.8940 & 0.8670 \\
\hline 34 & ASOL & 0.8928 & 0.8655 \\
\hline 35 & RIso & 0.8909 & 0.8561 \\
\hline 36 & GMD_3 & 0.8870 & 0.8661 \\
\hline 37 & UPENN & 0.8797 & 08576 \\
\hline 38 & GMD_l & 0.8786 & 0.8586 \\
\hline 39 & KAMAN_1 & 0.8785 & 0.8470 \\
\hline 40 & COMCOM & 0.8762 & 0.8739 \\
\hline 41 & GMD -4 & 0.8643 & 0.8456 \\
\hline 42 & KAMAN_3 & 0.8580 & 0.8303 \\
\hline 43 & KAMAN_2 & 0.8571 & 0.8283 \\
\hline 44 & GMD_2 & 0.8497 & 0.8152 \\
\hline is & KAMAN_5 & 0.8313 & 0.8080 \\
\hline 46 & IFAX & 0.8044 & 0.7843 \\
\hline 47 & VALEN 2 & 0.8038 & 0.7909 \\
\hline 48 & VALEN-1 & 0.7972 & 0.7766 \\
\hline 49 & KAMAN-4 & 0.7950 & 0.7624 \\
\hline
\end{tabular}

Table 107: NIST 2 correlation graph key for digits. 


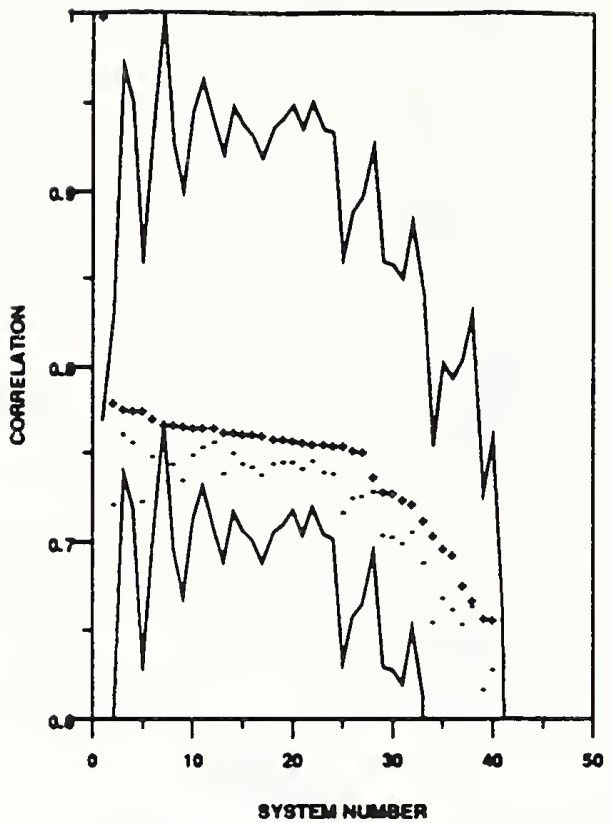

Figure 174: NIST_2 - upper case correlation

\begin{tabular}{|c|c|c|c|}
\hline System Number & Sydiem Name & Correlation (ali) & Correlation (correct) \\
\hline 1 & NIST 2 & 1.0000 & 1.0000 \\
\hline 2 & נ_ NIST & 0.7818 & 0.7235 \\
\hline $\mathbf{3}$ & VOTE_M & 0.7780 & 0.7634 \\
\hline 4 & ATT_A & 07770 & 0.7386 \\
\hline 3 & RISO & 0.7768 & 0.7253 \\
\hline 6 & KODAK_ & 0.7720 & 0.7300 \\
\hline 7 & REFERENCE & 0.7680 & 0.7690 \\
\hline 8 & SYMBUS & 0.7688 & 0.7463 \\
\hline 9 & MIME & 0.7673 & 0.7375 \\
\hline 10 & ATT 2 & 0.7668 & 0.7811 \\
\hline 11 & $A E G$ & 0.7667 & 0.7361 \\
\hline 12 & VOTEP & 0.7664 & 0.7382 \\
\hline 13 & GTESS_L & 0.7643 & 0.7414 \\
\hline 14 & ERIM-1 & 0.7840 & 0.7818 \\
\hline 13 & UBOL & 0.7633 & 0.7463 \\
\hline 16 & ATT 3 & 0.7631 & 0.7452 \\
\hline 17 & GTESS 2 & 0.7625 & 0.7406 \\
\hline 18 & IBM & 0.7607 & 0.7463 \\
\hline 19 & NESTOR & 0.7604 & 0.7473 \\
\hline 20 & UMICH_I & 07898 & 0.7478 \\
\hline 21 & ATT $\lrcorner$ & 0.7386 & 07441 \\
\hline 22 & NYNEX & 0.7378 & 0.7483 \\
\hline 23 & HUGHES_1 & 0.7373 & 0.7421 \\
\hline 24 & HUGHES_2 & 0.7371 & 0.7409 \\
\hline 23 & NIST_l & 0.7368 & 0.7193 \\
\hline 26 & ASOL & 0.7340 & 0.7270 \\
\hline 27 & NIST_A & 0.7338 & 0.7278 \\
\hline 28 & OCRSYS & 0.7396 & 0.7311 \\
\hline 28 & GMD_1 & 0.7313 & 0.7063 \\
\hline 30 & GMD_3 & 0.7302 & 0.7030 \\
\hline 31 & KAMAN_1 & 0.7268 & 0.7012 \\
\hline 32 & REI & 07232 & 0.7083 \\
\hline 33 & GMD-4 & 0.7142 & 0.6904 \\
\hline 34 & GMD_2 & 0.7063 & 0.6369 \\
\hline 35 & KAMAN_a & 0.6990 & 0.6706 \\
\hline 36 & KAMAN_2 & 0.6953 & 0.6840 \\
\hline 37 & IFAX & 0.6773 & 0.6336 \\
\hline 38 & COMCOM & 0.6687 & 0.6860 \\
\hline 38 & KAMAN_A & 0.6892 & 0.6189 \\
\hline 40 & VALEN_I & 0.6381 & 0.6298 \\
\hline 41 & KAMAN_5 & 0.3828 & 0.3580 \\
\hline 42 & $\mathrm{UMICH}_{2}$ & 0.0672 & 0.0187 \\
\hline
\end{tabular}

Table 108: NIST 2 correlation graph key for uppers. 


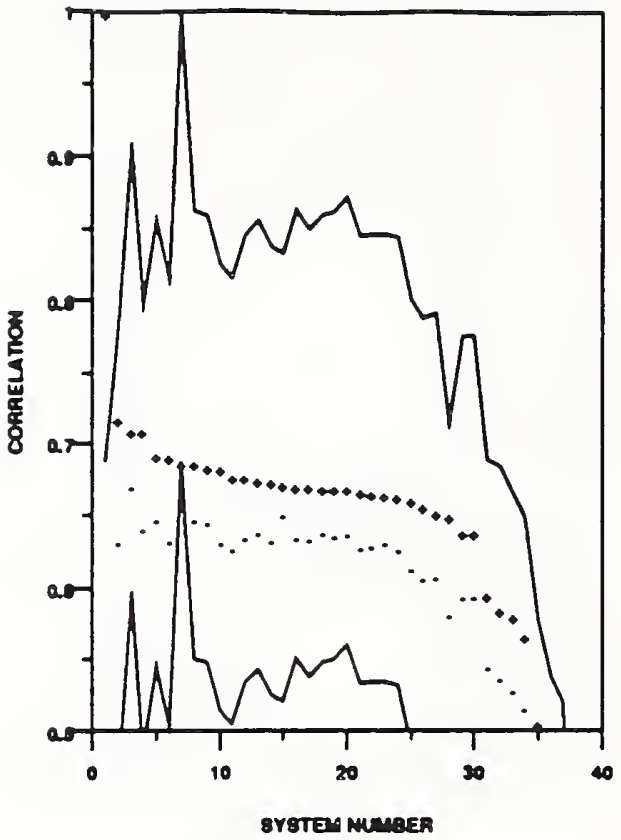

Figure 175: NIST_2 - lower case correlation

\begin{tabular}{|c|c|c|c|}
\hline System Number & Sysiem Name & Correlation (all) & Correlasion (correct) \\
\hline 1 & NIST 2 & 1.0000 & 1.0000 \\
\hline 2 & RISO & 0.7183 & 0.6332 \\
\hline 3 & VOTE M & 0.7112 & $0.671 \mathrm{~s}$ \\
\hline 4 & NIST & 0.7107 & 0.8418 \\
\hline 5 & ATT 44 & 0.6933 & 0.6484 \\
\hline 6 & NIST -1 & 0.6919 & 0.6340 \\
\hline 7 & REFERENCE & 0.6880 & 0.8880 \\
\hline s & ER1M-1 & 0.6877 & 0.8488 \\
\hline $\boldsymbol{\theta}$ & ATT 2 & 0.6860 & 0.6463 \\
\hline 10 & GTESS_-1 & 0.6843 & 0.6331 \\
\hline 11 & IBM & 0.6783 & 0.6357 \\
\hline 12 & GTESS 2 & 0.6783 & 0.6279 \\
\hline 13 & KODAK_ & 0.6768 & 0.6399 \\
\hline 14 & ATT & 0.6756 & 0.6342 \\
\hline 15 & VOTE_P & 0.6733 & 0.6321 \\
\hline 16 & OCRSYS & 0.6722 & 0.6364 \\
\hline 17 & UMICH-I & 0.6718 & 0.6334 \\
\hline 18 & NYNEX & 06707 & 0.6381 \\
\hline 19 & ATT 1 & 06703 & 0.6374 \\
\hline 20 & $A E G$ & 0.6702 & 0.6384 \\
\hline 21 & UBOL & 0.6684 & 0.6281 \\
\hline 22 & HUGHES_I & 0.6678 & 0.6302 \\
\hline 23 & NESTOR & 0.6657 & 0.6322 \\
\hline 24 & HUGHES_2 & 0.6853 & 0.6284 \\
\hline 23 & NIST_4 & 0.6631 & 0.6140 \\
\hline 26 & ASOL & 0.6381 & 0.6076 \\
\hline 27 & GMD_3 & 0.6838 & 0.6083 \\
\hline 28 & GMD 2 & 0.6518 & 0.5823 \\
\hline 29 & GMD_4 & 0.6398 & 0.3884 \\
\hline 30 & GMD_1 & 0.6398 & 0.3854 \\
\hline 31 & KAMAN-1 & 0.5861 & 0.3460 \\
\hline 32 & VALEN_I & 0.3858 & 0.3372 \\
\hline 33 & KAMAN_3 & 0.3808 & 0.3287 \\
\hline 34 & KAMAN_2 & 0.5673 & 0.3173 \\
\hline 35 & KAMAN_5 & 0.5059 & 0.4600 \\
\hline 36 & $K A M A N_{-4}$ & 04868 & 0.4370 \\
\hline 37 & COMCOM & 0.4168 & 0.4073 \\
\hline 38 & $\mathrm{UMICH}_{2}$ & 0.1052 & 0.0382 \\
\hline
\end{tabular}

Table 109: NIST 2 correlation graph key for lowers. 
SYSTEM: NIST_3

PARTICIPANT: Patrick J. Grother

ORGANIZATION: NIST, Gaithersburg, MD

PREPROCESSING: Size (preserving aspect ratio), Slant Normalization.

Subtraction from binary image of mean of training images.

FEATURES: Projection onto principal components of training set.

32 leading elements of KL transform.

CLASSIFICATION: Scaled conjugate gradient trained $32: 48:\{10,26\}$ perceptron.

HARDWARE: AMT 510C Array (32×32) Processor with Sparc Io host.

TRAINING :

$\begin{array}{rr}\text { DIGITS } & \text { UPPERS } \\ -77000 & -26000 \\ 2100 & 2100\end{array}$

LOWERS DATABASE

$-26000 \quad$ NSDB3

2100 WRITERS

STATUS: On time, submitted as NIST_O

\begin{tabular}{|c|c|c|c|c|c|c|c|}
\hline \multirow[t]{2}{*}{ RESULTS: } & \multicolumn{2}{|c|}{-- DIGITS -- } & \multicolumn{2}{|c|}{-- UPPERS -- } & \multicolumn{2}{|c|}{-- LOHERS -- } & DATABASE \\
\hline & REJ. & ERR . & REJ. & ERR. & REJ. & ERR. & TESTDATA 1 \\
\hline & RATE & RATE-- & RATE & RATE-- & RATE & RATE-- & \\
\hline & 0.00 & 0.0973 & 0.00 & 0.1693 & 0.00 & 0.2029 & \\
\hline & 0.10 & 0.0529 & 0.10 & 0.1172 & 0.10 & 0.1521 & \\
\hline & 0.20 & 0.0286 & 0.20 & 0.0757 & 0.20 & 0.1122 & \\
\hline & 0.30 & 0.0160 & 0.30 & 0.0520 & 0.30 & 0.0853 & \\
\hline & 0.40 & 0.0103 & 0.40 & 0.0331 & 0.40 & 0.0629 & \\
\hline & 0.50 & 0.0070 & 0.50 & 0.0184 & 0.50 & 0.0458 & \\
\hline
\end{tabular}

OCR RATE (CPS): DIGITS UPPERS LOHERS

OCR RATE:

$\begin{array}{llll}\text { CPU RATE: } & 142.6 & 64.3 & 64.3\end{array}$ 
SYSTEM: NIST_3

\section{BIBLIOGRAPHY:}

The following references have been provided for this system:

[41]

COMMENTS: NIST_3

See Cross Validation Section on Inadequacies of NIST Special Database 3 for the classification of NIST Test Data 1.

Small training set. Small number of KL coefficients. KL basis and first MLP layer both perform linear affine transformation. Therefore premultiply them. Algorithmic complexity is low: dominated by two matrix multiplies. Very fast. 


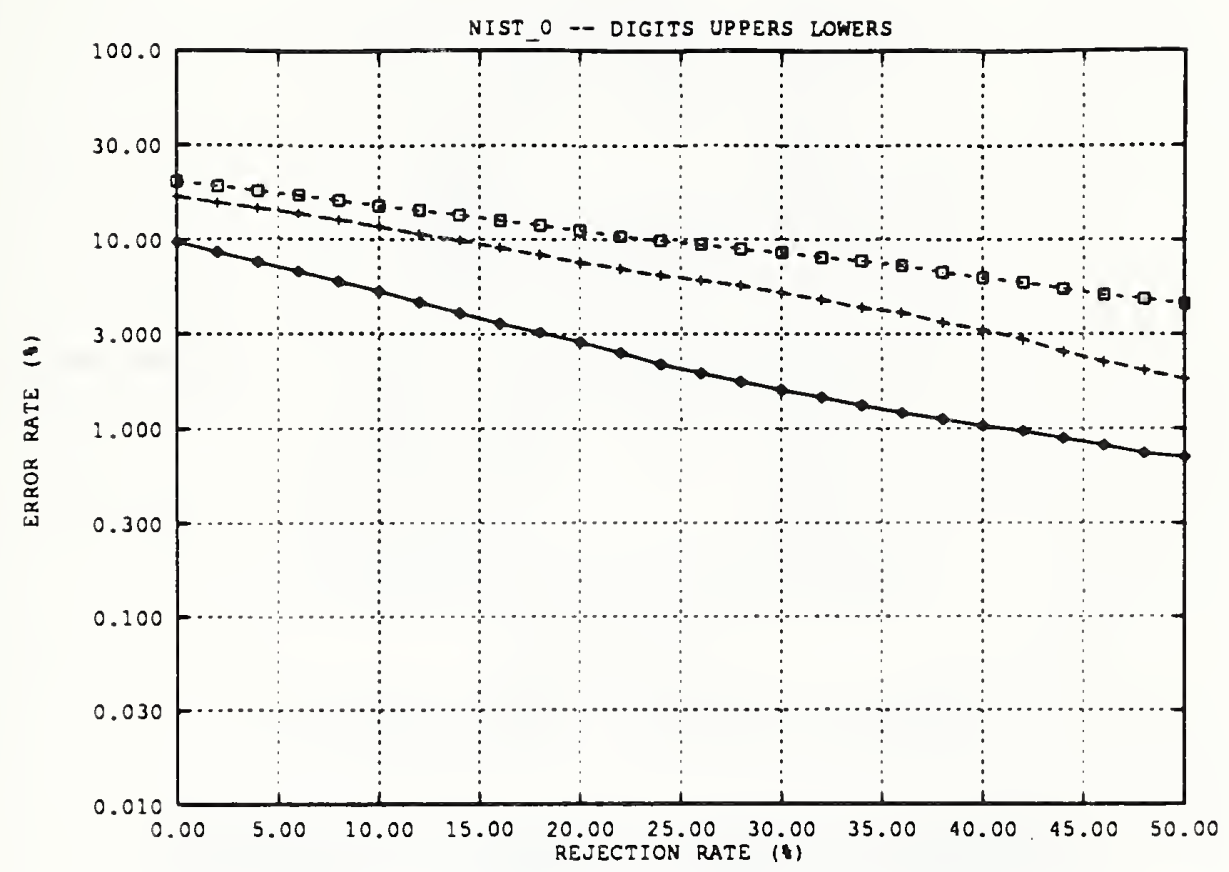

Figure 176: Error rate versus rejection rate for NIST_3

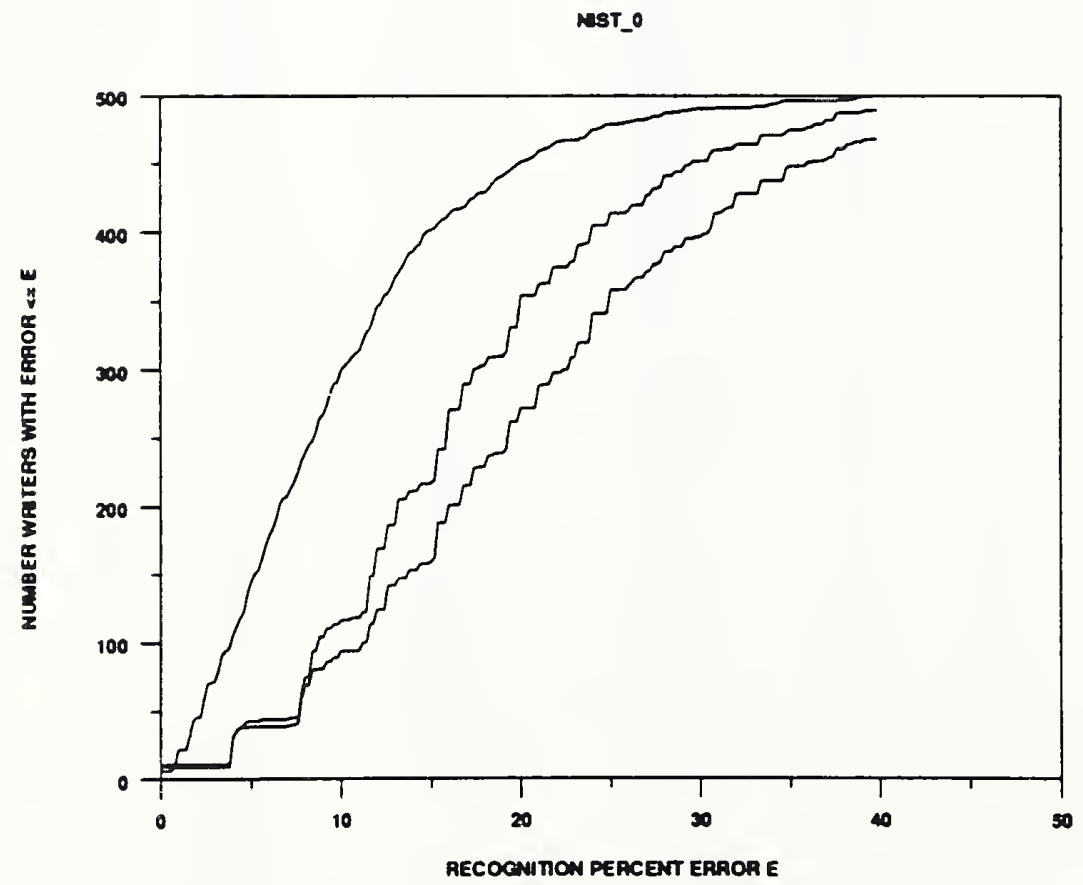

Figure 177: Error rate per writer of NIST_3 


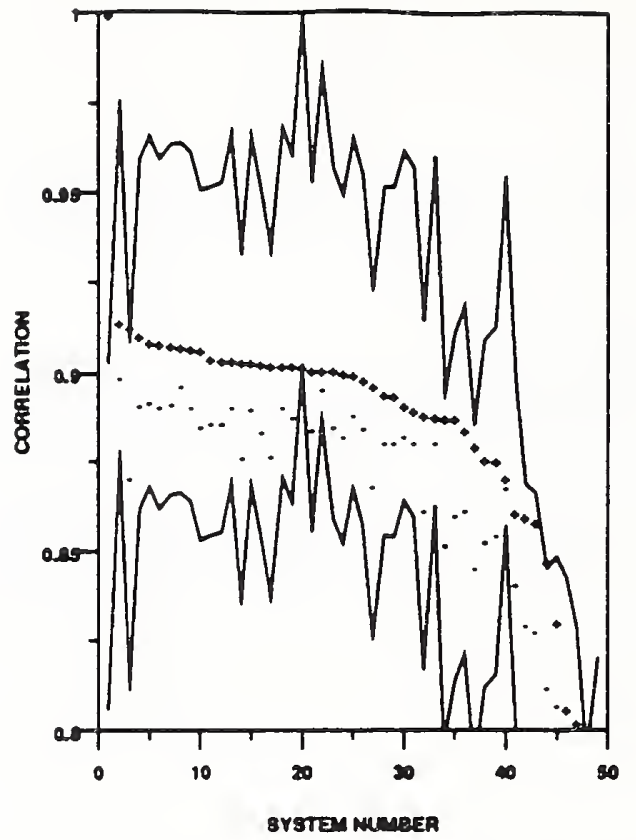

Figure 178: NIST_3 - digit correlation

\begin{tabular}{|c|c|c|c|}
\hline System Number & Syblem Nome & Correlation (s) & Correlation (correct) \\
\hline 1 & NIST3 & 1.0000 & 1.0000 \\
\hline 2 & VOTE_M & 0.9151 & 0.8994 \\
\hline 3 & NIST 2 & 0.8138 & 0.8715 \\
\hline 4 & ATT-4 & 0.9114 & 0.8915 \\
\hline 5 & $A E G$ & 0.8096 & 0.8928 \\
\hline 6 & KODAK2 & 0.9093 & 0.2912 \\
\hline 7 & ATT 2 & 0.9089 & 0.8923 \\
\hline 8 & VOTEP & 0.9081 & 0.8971 \\
\hline 9 & ERIM-1 & 0.9078 & 0.8910 \\
\hline 10 & NIST _4 & 0.9071 & 0.8858 \\
\hline 11 & ATT 3 & 0.9048 & 0.8866 \\
\hline 12 & KODAK_ & 0.9046 & 0.8868 \\
\hline 13 & ELSAGBA & 0.9044 & $0.891 \mathrm{~J}$ \\
\hline 14 & GTESS_2 & 0.9043 & 0.8772 \\
\hline 15 & ELSAGB2 & 0.8040 & 0.8909 \\
\hline 16 & THINK-1 & 0.9037 & 0.8845 \\
\hline 17 & ATT $\perp$ & 0.9032 & 0.8912 \\
\hline 18 & GTESS_I & 0.9032 & 0.8773 \\
\hline 19 & ERIM_2 & 0.8031 & 0.8883 \\
\hline 20 & REFERENCE & 0.8027 & 0.9027 \\
\hline 21 & OCRSYS & 0.9017 & 0.8963 \\
\hline 22 & SYMBUS & 0.9017 & 0.8850 \\
\hline 23 & UBOL & 0.9016 & 0.8857 \\
\hline 24 & ELSAGB-1 & 0.8010 & 0.8830 \\
\hline 23 & IBM & 0.8003 & 0.8889 \\
\hline 26 & NESTOR & 0.8282 & 0.8853 \\
\hline 27 & NIST AI & 0.8974 & 0.8691 \\
\hline 28 & HUGHES -1 & 0.8949 & 0.8811 \\
\hline 29 & HUGHES 2 & 0.8945 & 0.8809 \\
\hline 30 & THINK 2 & 0.8916 & 0.8828 \\
\hline 31 & NYNEX & 0.8905 & 0.8809 \\
\hline 32 & MIME & 0.8889 & 0.8624 \\
\hline 33 & REI & 0.8887 & 0.8812 \\
\hline 34 & RISO & 0.8882 & 0.8526 \\
\hline 33 & ASOL & 0.8881 & 0.8610 \\
\hline 36 & GMD_J & 0.8848 & 0.8624 \\
\hline 37 & KAMAN-1 & 0.8801 & 0.8463 \\
\hline 38 & UPENN & 0.8763 & 0.8537 \\
\hline 39 & GMD -1 & 0.8739 & 0.8556 \\
\hline 40 & COMCOM & 0.8713 & 0.8689 \\
\hline 41 & GMD_4 & 0.8617 & 0.8417 \\
\hline 42 & KAMAN_3 & 0.8604 & 0.8303 \\
\hline 43 & KAMAN-2 & 0.8591 & 0.8284 \\
\hline 44 & GMD_2 & 08482 & 0.8126 \\
\hline 43 & KAMAN 3 & 0.8310 & 0.8074 \\
\hline 46 & VALEN 2 & 0.8086 & 0.7922 \\
\hline 47 & IFAX & 0.8028 & 0.7825 \\
\hline 48 & KAMAN-4 & 0.7976 & 0.7628 \\
\hline 49 & VALEN_1 & 0.7929 & 0.7729 \\
\hline
\end{tabular}

Table 110: NIST_3 correlation graph key for digits. 


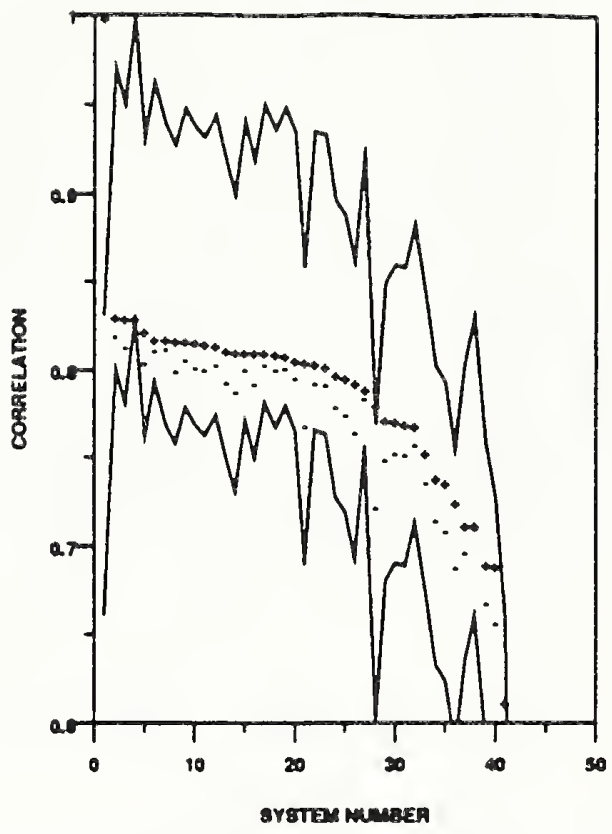

Figure 179: NIST_3 - upper case correlation

\begin{tabular}{|c|c|c|c|}
\hline System Number & System Name & Correlation (All) & Corretstion (correct) \\
\hline 1 & NIST J & 1.0000 & 1.0000 \\
\hline 2 & VOTE_M & 0.8318 & 0.8210 \\
\hline 3 & REFERENCE & 0.8307 & 0.8307 \\
\hline 4 & ATT -4 & 0.8307 & 0.8148 \\
\hline $\mathbf{5}$ & KODAK_ & 0.8241 & 0.8056 \\
\hline$e$ & AEG & 0.8196 & 0.8123 \\
\hline 7 & VOTEP & 0.8188 & 0.8137 \\
\hline 8 & ERIM_1 & 0.8179 & 0.8072 \\
\hline 9 & SYMBUS & 0.8178 & $0.800 \mathrm{~s}$ \\
\hline 10 & UBOL & 0.8172 & 0.8028 \\
\hline 11 & ATT 3 & 0.8168 & 0.8015 \\
\hline 12 & ATT2 2 & 0.8159 & 0.8049 \\
\hline 13 & GTESS_1 & 0.8123 & 0.7944 \\
\hline 14 & NESTOR & 0.8122 & 0.8020 \\
\hline 15 & MIME & 0.8122 & 0.7886 \\
\hline 16 & GTESS_2 & 0.8117 & 0.7934 \\
\hline 17 & NYNEX & 0.8116 & 0.8048 \\
\hline 18 & IBM & 0.8112 & 0.8013 \\
\hline 10 & UMICH_I & 0.8100 & 0.8024 \\
\hline 20 & ATr 1 & 0.8076 & 0.7972 \\
\hline 21 & RISO & 0.8062 & 0.7692 \\
\hline 22 & HUGHES - 1 & 0.8031 & 0.7947 \\
\hline 23 & HUGHES_2 & 0.8034 & 0.7931 \\
\hline 24 & NIST_4 & 0.7991 & 0.7803 \\
\hline 25 & ASOL & 0.7972 & 0.7761 \\
\hline 26 & NIST 1 & 0.7943 & 0.7858 \\
\hline 27 & OCRSYS & 0.7908 & 0.7853 \\
\hline 28 & NIST 2 & 0.7818 & 0.7235 \\
\hline 29 & KAMAN-L & 0.7729 & 0.7499 \\
\hline 30 & GMD.1 & 0.7719 & 0.7537 \\
\hline 31 & GMD_3 & 0.7703 & 0.7526 \\
\hline 32 & REI & 0.7694 & 0.7583 \\
\hline 33 & GMD - 1 & 0.7542 & 0.7370 \\
\hline 34 & KAMAN.3 & 0.7406 & 0.7164 \\
\hline 33 & KAMAN_2 & 0.7374 & 0.7100 \\
\hline 36 & GMD_2 & 07266 & 0.6895 \\
\hline 37 & IFAX & 0.7140 & 0.6981 \\
\hline 38 & СOMCOM & 0.7133 & 0.7119 \\
\hline 39 & VALEN-L & 0.6918 & 0.6891 \\
\hline 40 & KAMAN-4 & 0.6210 & 0.6583 \\
\hline 41 & KAMAN_S & 0.6135 & 0.8922 \\
\hline 42 & $\mathrm{UMICH}_{2}$ & 0.0601 & 0.0204 \\
\hline
\end{tabular}

Table 111: NIST_3 correlation graph key for uppers. 


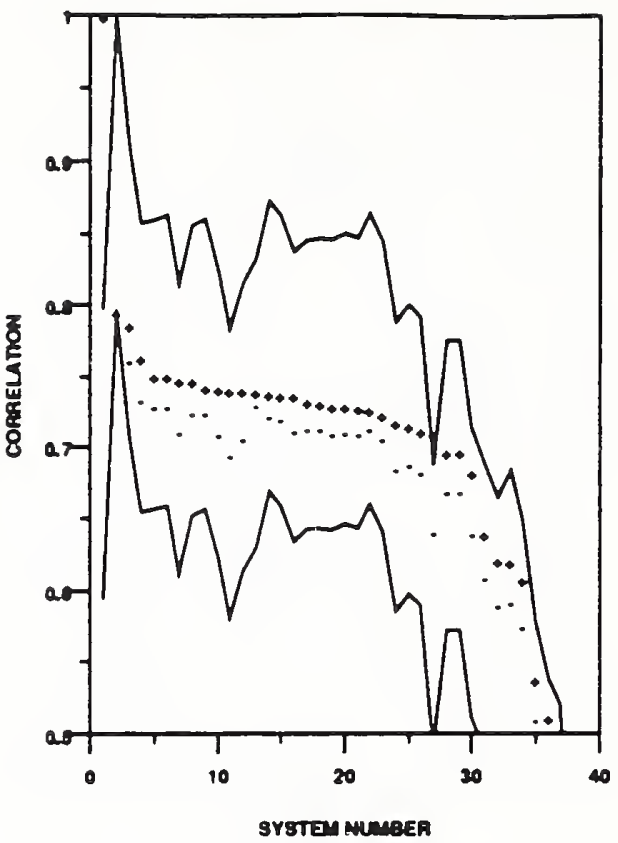

Figure 180: NIST_3 - lower case correlation

\begin{tabular}{|c|c|c|c|}
\hline Sybtem Number & System Name & Correlation (all) & Corpelation (eorrect) \\
\hline 1 & NISTA & 1.0000 & 1.0000 \\
\hline 2 & REFERENCE & 0.7971 & 0.7971 \\
\hline 3 & VOTE_M & 0.7874 & 0.7628 \\
\hline 4 & ATT 44 & 0.7650 & 0.7349 \\
\hline 3 & ATT 2 & 0.7527 & 07306 \\
\hline 6 & ERIM -I & 0.7521 & 0.7307 \\
\hline 7 & NIST.I & 07493 & 0.7122 \\
\hline 8 & KODAK」 & 07485 & 0.7237 \\
\hline 9 & NYNEX & 0.7443 & 0.7258 \\
\hline 10 & GTESS_1 & 0.7429 & 0.7107 \\
\hline 11 & Riso & 0.7418 & 0.6964 \\
\hline 12 & GTESS 2 & 0.7413 & 0.7079 \\
\hline 13 & VOTEP & 0.7412 & 0.7312 \\
\hline 14 & $A E G$ & 0.7394 & 0.7235 \\
\hline 15 & ATT」 & 0.7383 & 0.7210 \\
\hline 16 & ATT 3 & 0.7382 & 0.7129 \\
\hline 17 & IBM & 0.7337 & 0.7142 \\
\hline 18 & NEST OR & 0.7322 & 0.7143 \\
\hline 19 & UBOL & 0.7305 & 0.7105 \\
\hline 20 & UMICH.I & 0.7301 & 0.7120 \\
\hline 21 & HUGHES_I & 0.7293 & 0.7103 \\
\hline 22 & OCRSYS & 0.7278 & 0.7138 \\
\hline 23 & HUGHES.2 & 0.7251 & 0.7074 \\
\hline 24 & ASOL & 0.7187 & 0.6867 \\
\hline 23 & NIST_4 & 0.7171 & 0.6888 \\
\hline 26 & GMD_3 & 0.7129 & 08849 \\
\hline 27 & NIST 2 & 0.7107 & 0.6415 \\
\hline 28 & GMD_4 & 0.6983 & 0.6709 \\
\hline 29 & GMD_l & 0.6983 & 0.6709 \\
\hline 30 & GMD_2 & 0.6830 & 0.8412 \\
\hline 31 & KAMAN_1 & 0.6411 & 0.6094 \\
\hline 32 & KAMAN_3 & 0.6222 & 0.3898 \\
\hline 33 & VALEN_I & 0.6208 & 0.3924 \\
\hline 34 & KAMAN_2 & 0.6083 & 0.3735 \\
\hline 33 & KAMAN_S & 0.3383 & 0.3111 \\
\hline 36 & KAMAN_A & 0.5127 & 0.4796 \\
\hline 37 & СомСом & 0.4353 & 0.4523 \\
\hline 38 & UMICH_2 & 0.1038 & 0.0629 \\
\hline
\end{tabular}

Table 112: NIST_3 correlation graph key for lowers. 
SYSTEM : NYNEX

PARTICIPANT: Atul Chhabra

ORGANIZATION: Nynex Sciences Technology, Inc., White Plains, NY

FEATURES: model or stroke, automatic feature selection. A large number of pre-segmentation points are first generated. The algorithm effectively chooses a subset of thom that provide the most confident recognition.

CLASSIFICATION: MLP

HARDWARE: SPARC2 with 40 Mbyte ram, coded in C.

TRAINING: DIGITS UPPERS LOWERS DATABASE

$40000 \quad 45000 \quad$ INTERNAL

$\begin{array}{llll}0 & 35000 & \text { NSDB3 }\end{array}$

STATUS: $\quad$ on time

RESULTS: -- DIGITS -- -- UPPERS -- -- LOWERS -- DATABASE

REJ. ERR. REJ. ERR. REJ. ERR. TESTDATA1

RATE RATE-- RATE RATE-- RATE RATE--

$\begin{array}{lllllll}0.00 & 0.0432 & 0.00 & 0.0491 & 0.00 & 0.1403\end{array}$

$\begin{array}{lllllll}0.10 & 0.0128 & 0.10 & 0.0175 & 0.10 & 0.0994\end{array}$

$\begin{array}{lllllll}0.20 & 0.0052 & 0.20 & 0.0092 & 0.20 & 0.0646\end{array}$

$\begin{array}{lllllll}0.30 & 0.0029 & 0.30 & 0.0065 & 0.30 & 0.0433\end{array}$

$\begin{array}{lllllll}0.40 & 0.0032 & 0.40 & 0.0050 & 0.40 & 0.0283\end{array}$

$\begin{array}{llllll}0.50 & 0.0034 & 0.50 & 0.0050 & 0.50 & 0.0215\end{array}$

OCR RATE (CPS): DIGITS UPPERS LOWERS
SYS RATE :
22.00
12.00
12.00

CPU RATE:

NOTE: Internal database includes digits and upper case letters from NSDB1. NOTE: Suggested that NIST be involved in proctoring future tests. 
SYSTEM: NYNEX

\section{BIBLIOGRAPHY:}

The following references have been provided for this system:

none 


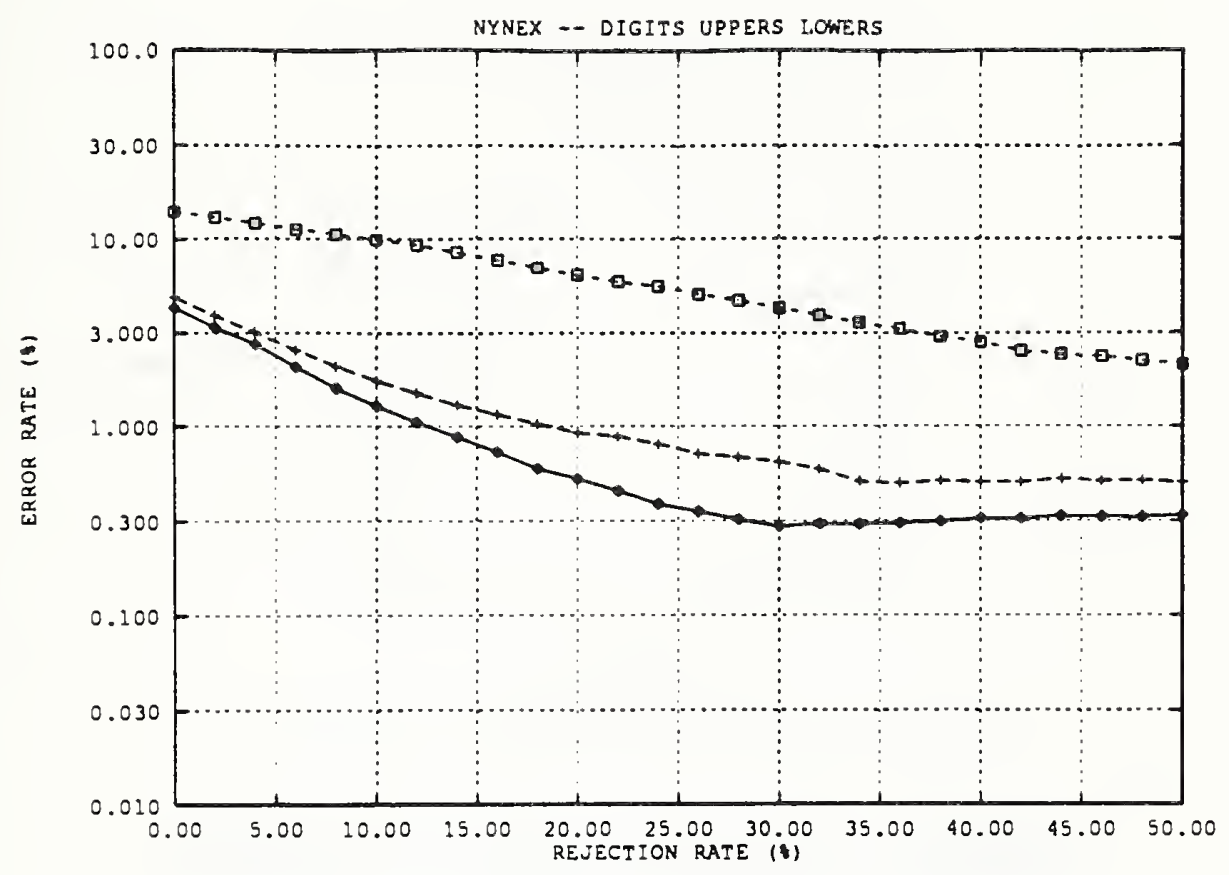

Figure 181: Error rate versus rejection rate for NYNEX

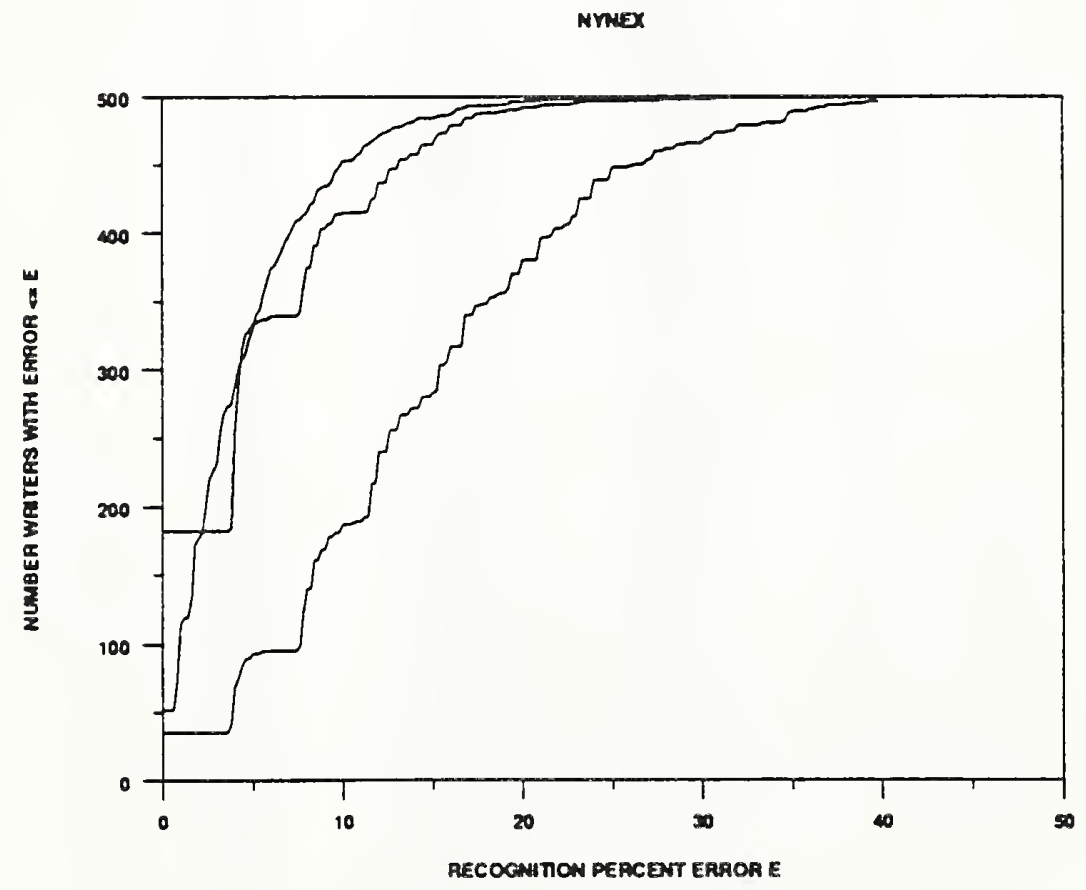

Figure 182: Error rate per writer of NYNEX 


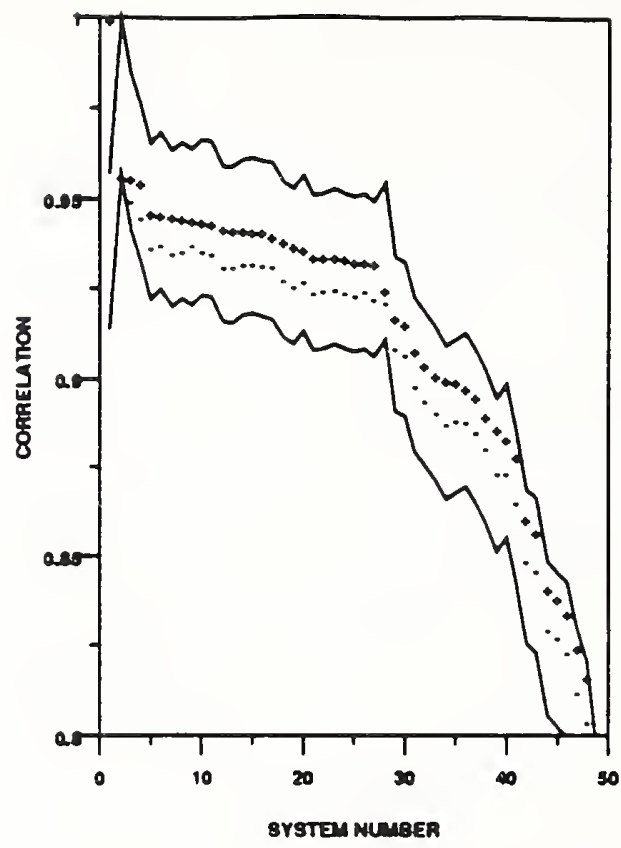

Figure 183: NYNEX - digit correlation

\begin{tabular}{|c|c|c|c|}
\hline Syssem Number & System Name & Correlation (all) & Correlation (correct) \\
\hline 1 & NYNEX & 1.0000 & 1.0000 \\
\hline 2 & REFERENCE & 0.9568 & 0.9568 \\
\hline j & OCRSYS & 0.8564 & 0.9501 \\
\hline 4 & VOTE_M & 0.9850 & 0.9456 \\
\hline 5 & IB M & 0.9470 & 0.9371 \\
\hline 6 & ATT $\perp$ & 0.8462 & 0.8380 \\
\hline 7 & ATT2 & 0.9439 & 0.8360 \\
\hline 8 & AEG & 0.9484 & 0.8363 \\
\hline 8 & VOTE.P & 0.8450 & 0.9379 \\
\hline 10 & ELSAGB & 0.9443 & 0.9363 \\
\hline 11 & ELSAGB 2 & 0.8440 & 0.9360 \\
\hline 12 & KODAK2 2 & 0.9428 & 0.9323 \\
\hline 13 & ATT 4 & 0.8422 & 0.9321 \\
\hline 14 & ERIM_I & 0.9421 & 0.8331 \\
\hline 13 & THINK 2 & 0.9419 & 0.9328 \\
\hline 16 & ERIM 2 & 0.9417 & 0.9325 \\
\hline 17 & REI & 08406 & 0.9323 \\
\hline 18 & NESTOR & 0.9388 & 0.9286 \\
\hline 19 & KODAK_ & 0.9374 & 0.9265 \\
\hline 20 & UBOL & 0.9366 & 0.9282 \\
\hline 21 & THINK-I & 0.8350 & 0.9247 \\
\hline 22 & HUGHES -1 & 0.9340 & 0.9250 \\
\hline 23 & SYMBUS & 0.8348 & 0.9256 \\
\hline 24 & HUGHES_2 & 0.9344 & 0.9247 \\
\hline 25 & NIST_4 & 0.9337 & 0.9238 \\
\hline 26 & ATT 3 & 0.9336 & 0.9251 \\
\hline 27 & ELSAGB_1 & 0.9329 & 0.9228 \\
\hline 28 & COMCOM & 0.9255 & 0.9219 \\
\hline 29 & GTESS_l & 0.8179 & 0.8093 \\
\hline so & GTESS 2 & 0.9159 & 0.9074 \\
\hline ग1 & NIST_l & 0.8087 & 0.8288 \\
\hline 32 & GMD_J & 0.9047 & 0.8944 \\
\hline נ3 & MIME & 0.8020 & 0.8914 \\
\hline 34 & UPENN & 0.9002 & 0.8880 \\
\hline 35 & ASOL & 0.8000 & 0.8889 \\
\hline 96 & GMD_1 & 0.8982 & 0.8883 \\
\hline 37 & NIST 2 & 0.8956 & 0.8859 \\
\hline 38 & $\mathrm{NIST}_{3}$ & 0.8905 & 0.8809 \\
\hline 39 & RISO & 0.8867 & 0.8741 \\
\hline 40 & GMD_4 & 0.8840 & 0.8743 \\
\hline 41 & KAMAN_1 & 0.8786 & 0.8660 \\
\hline 42 & KAMAN_3 & 0.8614 & 0.8496 \\
\hline 43 & KAMAN_2 & 0.8576 & 0.8468 \\
\hline 44 & KAMAN_S & 0.8414 & 0.8303 \\
\hline 45 & GMD_2 & 08389 & 0.8277 \\
\hline 46 & VALEN_2 & 0.8345 & 0.8237 \\
\hline 47 & IFAX & 0.8252 & 0.8127 \\
\hline 48 & VALEN_L & 0.8170 & 0.8042 \\
\hline 49 & KAMAN_4 & 0.7853 & 0.7750 \\
\hline
\end{tabular}

Table 113: NYNEX correlation graph key for digits. 


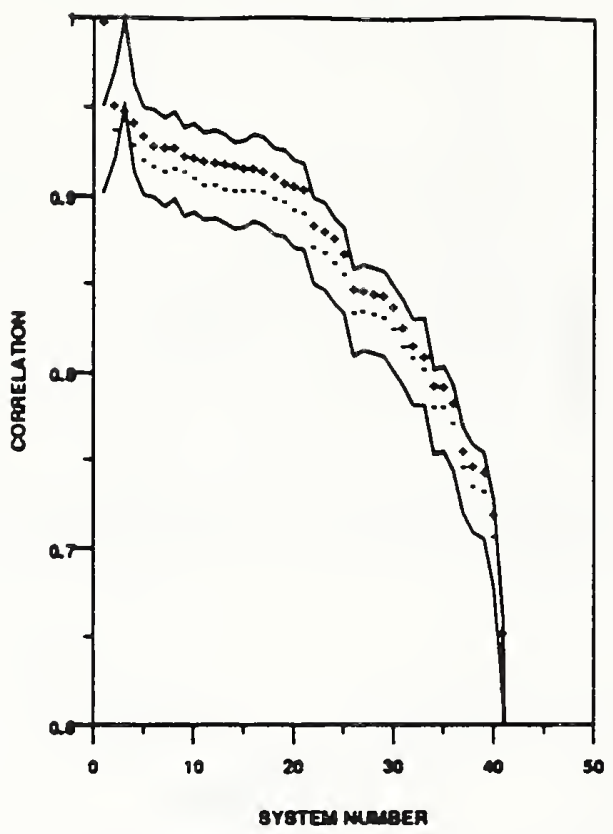

Figure 184: NYNEX - upper case correlation

\begin{tabular}{|c|c|c|c|}
\hline System Number & System Name & Corselation (all) & Corselation (correct) \\
\hline 1 & NYNEX & 1.0000 & 1.0000 \\
\hline 2 & VOTE_M & 0.9533 & 0.8393 \\
\hline 3 & REFERENCE & 0.9509 & 0.9309 \\
\hline 4 & $A E G$ & 0.8440 & 0.9312 \\
\hline 3 & ATT_4 & 0.9371 & 0.9227 \\
\hline$B$ & UMICH_I & 0.8311 & 0.2190 \\
\hline 7 & ATT 2 & 0.8307 & 0.8165 \\
\hline 8 & ERIM L & 0.8305 & 0.9182 \\
\hline 8 & VOTE $P$ & 0.2254 & 0.8163 \\
\hline 10 & NESTOR & 0.9232 & 0.2126 \\
\hline 11 & IBM & 0.8231 & 0.8094 \\
\hline 12 & UBOL & 0.2225 & 0.9091 \\
\hline 13 & ATT 2 & 0.9209 & 0.9069 \\
\hline 14 & KODAK 1 & 0.9203 & 0.9060 \\
\hline 15 & ATT 3 & 0.8185 & 0.8032 \\
\hline 16 & HUGHES_I & 0.9182 & 0.2060 \\
\hline 17 & HUGHES-2 & 0.9168 & 09042 \\
\hline 18 & SYMBUS & 0.9137 & 0.9008 \\
\hline 19 & OCRSYS & 0.9104 & 0.8989 \\
\hline 20 & GTESS _- & 0.8078 & 0.8942 \\
\hline 21 & GTESS 2 & 0.9065 & 0.8928 \\
\hline 22 & MIME & 0.8864 & 0.8734 \\
\hline 23 & NIST_4 & 0.8828 & 0.8704 \\
\hline 24 & ASOL & 0.8787 & 0.8647 \\
\hline 25 & REI & 0.8704 & 0.8586 \\
\hline 26 & Riso & 0.8503 & 0.8369 \\
\hline 27 & NIST_1 & 0.8498 & 0.8373 \\
\hline 28 & GMD_1 & 0.8478 & 0.8359 \\
\hline 29 & GMD_3 & 0.8467 & 0.8343 \\
\hline 30 & KAMAN-1 & 0.8400 & 0.8272 \\
\hline 31 & GMD.4 & 0.8288 & 0.8174 \\
\hline 32 & COMCOM & 0.8180 & 0.8113 \\
\hline 33 & NIST 3 & 0.8116 & 0.8048 \\
\hline 34 & KAMAN_S & 0.7948 & 0.7827 \\
\hline 35 & IFAX & 0.7947 & 0.7828 \\
\hline 36 & $\mathrm{KAMAN}_{-2}$ & 0.7856 & 0.7731 \\
\hline 37 & NIST 2 & 0.7378 & 0.7485 \\
\hline 38 & VALEN_L & 0.7495 & 0.7375 \\
\hline 39 & GMD .2 & 0.7437 & 0.7344 \\
\hline 40 & KAMAN_4 & 0.7213 & 0.7094 \\
\hline 41 & KAMAN_S & 0.6551 & 0.6450 \\
\hline 42 & UMICH 2 & 0.0407 & 0.0237 \\
\hline
\end{tabular}

Table 114: NYNEX correlation graph key for uppers. 


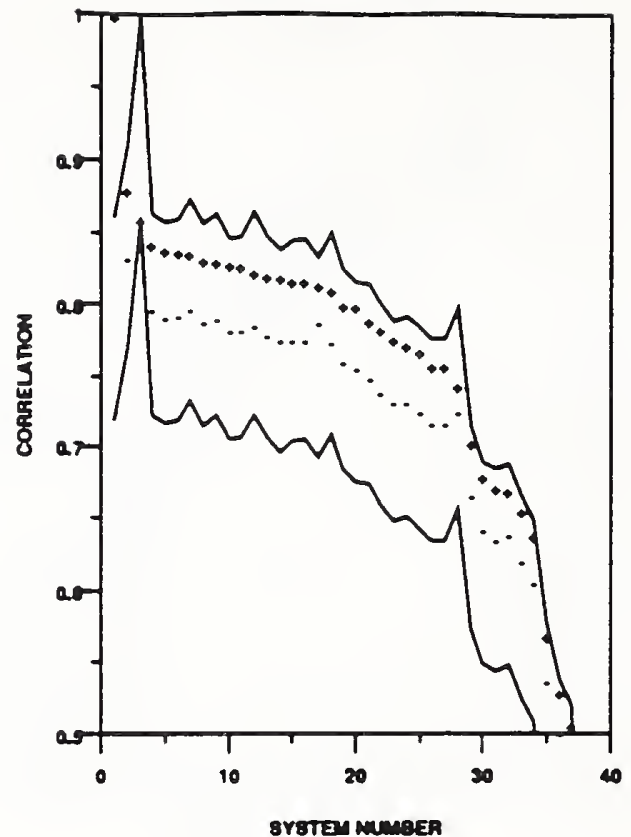

Figure 185: NYNEX - lower case correlation

\begin{tabular}{|c|c|c|c|}
\hline System Number & Sydtem Name & Correlation (all) & Correlation (correct) \\
\hline 1 & NYNEX & 1.0000 & 1.0000 \\
\hline 2 & VOTE_M & 0.8808 & 0.8339 \\
\hline 3 & REFERENCE & 0.8897 & 0.8897 \\
\hline 4 & ERIM_1 & 0.8429 & 0.7988 \\
\hline 3 & ATT_4 & 0.8395 & 0.7928 \\
\hline 6 & ATT 2 & 0.8378 & 0.7939 \\
\hline 7 & $A E G$ & 0.8373 & 0.7978 \\
\hline 8 & KODAK_1 & 0.8320 & 0.7888 \\
\hline 9 & ATT $\perp$ & 0.8318 & 0.7912 \\
\hline 10 & IBM & 0.8296 & 0.7837 \\
\hline 11 & NESTOR & 0.8276 & 0.7837 \\
\hline 12 & OCRSYS & 0.8232 & 0.7868 \\
\hline 13 & HUGHES_1 & 0.8209 & 0.7798 \\
\hline 14 & ATT 3 & 0.8197 & 0.7768 \\
\hline 13 & HUGHES-2 & 0.8172 & 0.7768 \\
\hline 16 & UBOL & 0.8172 & 0.7761 \\
\hline 17 & VOTEP & 0.8152 & 0.7887 \\
\hline 18 & UMICH_l & 0.8121 & 0.7737 \\
\hline 19 & GTESS-1 & 0.8018 & 0.7619 \\
\hline 20 & GTESS2 & 0.8000 & 0.7870 \\
\hline 21 & $N_{1 S T} T_{1}$ & 0.7897 & 0.7498 \\
\hline 22 & NIST-4 & 0.7843 & 0.7396 \\
\hline 23 & ASOL & 0.7773 & 0.7328 \\
\hline 24 & GMD_3 & 0.7728 & 0.7327 \\
\hline 23 & RISO & 0.7677 & 0.7288 \\
\hline 26 & GMD_4 & 0.7882 & 0.7182 \\
\hline 27 & GMD_1 & 0.7382 & 0.7182 \\
\hline 28 & NIST 3 & 0.7443 & 0.7258 \\
\hline 29 & GMD_2 & 0.7038 & 0.6671 \\
\hline 30 & KAMAN_-1 & 0.6809 & 0.6431 \\
\hline 31 & VALEN_1 & 0.6728 & 0.6339 \\
\hline 32 & $\mathrm{NIST}_{2}$ & 0.6707 & 0.6391 \\
\hline 33 & KAMAN & 0.6366 & 0.6209 \\
\hline 34 & KAMAN_2 & 0.6398 & 0.6059 \\
\hline 35 & KAMAN 3 & 0.3693 & 0.3378 \\
\hline 36 & KAMAN_4 & 0.8311 & 0.8022 \\
\hline 37 & COMCOM & 0.3074 & 0.4937 \\
\hline 38 & $\mathrm{UMICH}_{2}$ & 0.1028 & 0.0606 \\
\hline
\end{tabular}

Table 115: NYNEX correlation graph key for lowers. 
SYSTEM: OCRSYS

PARTICIPANT: Harry S. Gierhart

ORGANIZATION: OCR Systems, Inc., Huntingdon Valley, PA

FEATURES: convolution with hand-coded filters

CLASSIFICATION: MLP, top three choices and confidence value that discriminates betreen them are calculated.

HARDWARE :

TRAINING: DIGITS UPPERS LOWERS DATABASE

-.-- number used is proprietary -.- INTERNAL

STATUS: on time

RESULTS: -- DIGITS -- -- UPPERS -- -- LOHERS -- DATABASE

$\begin{array}{lllllll}\text { REJ. } & \text { ERR. } & \text { REJ. } & \text { ERR. } & \text { REJ. } & \text { ERR. } & \text { TESTDATA1 } \\ \text { RATE } & \text { RATE-- } & \text { RATE } & \text { RATE-- } & \text { RATE } & \text { RATE-- } & \\ 0.00 & 0.0156 & 0.00 & 0.0573 & 0.00 & 0.1370 & \\ 0.10 & 0.0150 & 0.10 & 0.0224 & 0.10 & 0.1042 & \\ 0.20 & 0.0166 & 0.20 & 0.0144 & 0.20 & 0.0800 & \\ 0.30 & 0.0188 & 0.30 & 0.0123 & 0.30 & 0.0636 & \\ 0.40 & 0.0219 & 0.40 & 0.0106 & 0.40 & 0.0586 & \\ 0.50 & 0.0262 & 0.50 & 0.0080 & 0.50 & 0.0528 & \end{array}$

OCR RATE (CPS): DIGITS UPPERS LOWERS

SYS RATE:

CPU RATE: $\quad 300.00 \quad 220.00 \quad 220.00$

NOTE: Internal database is very large.

NOTE: HYP files for upper case letters included letters classified as lower case letters. These were scored as incorrect for Conference giving a zero rejection rate score of 0.0738 . The score given above for UPPERS is case insensitive.

NOTE: Used a beta test version of an off-the-shelf system for this submission.

NOTE: Recently purchased by Adobe Systems. 
SYSTEM: OCRSYS

BIBLIOGRAPHY:

The following references have been provided for this system:

none 


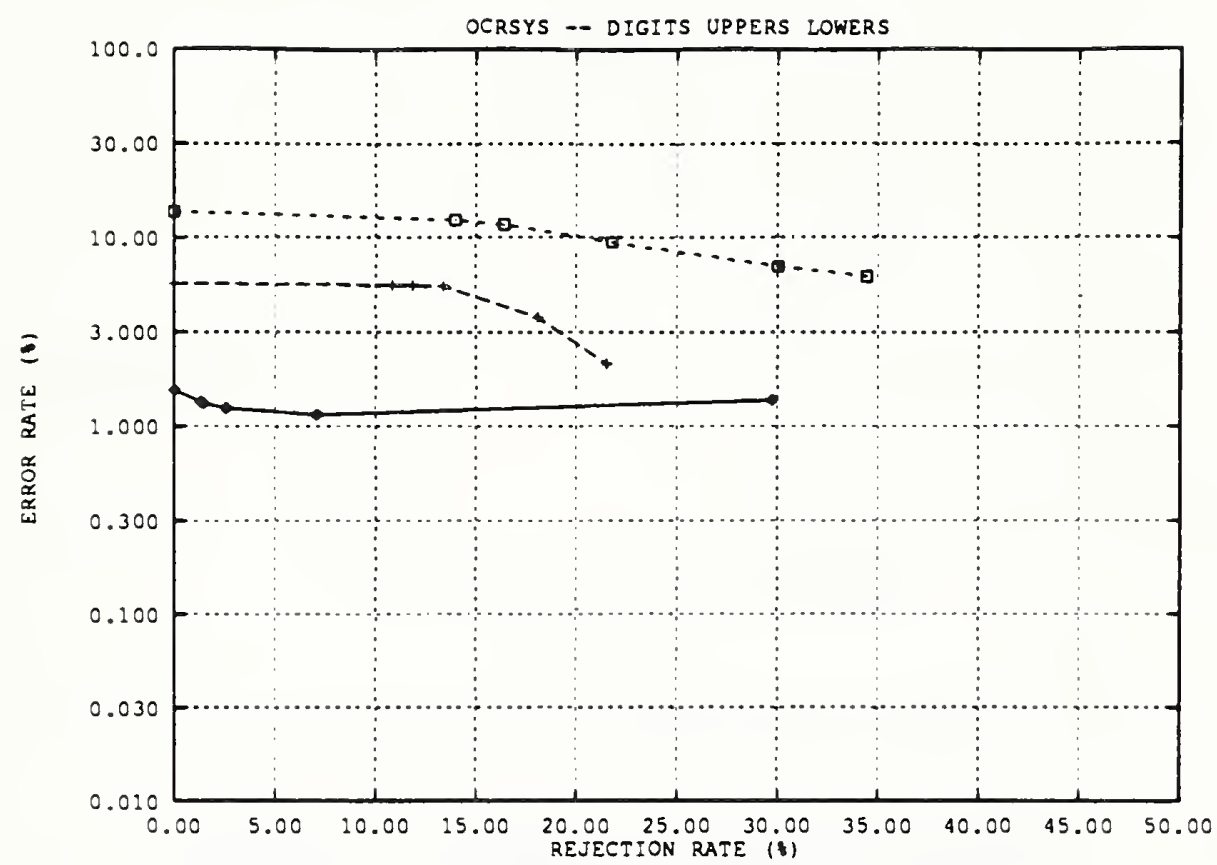

Figure 186: Error rate versus rejection rate for OCRSYS

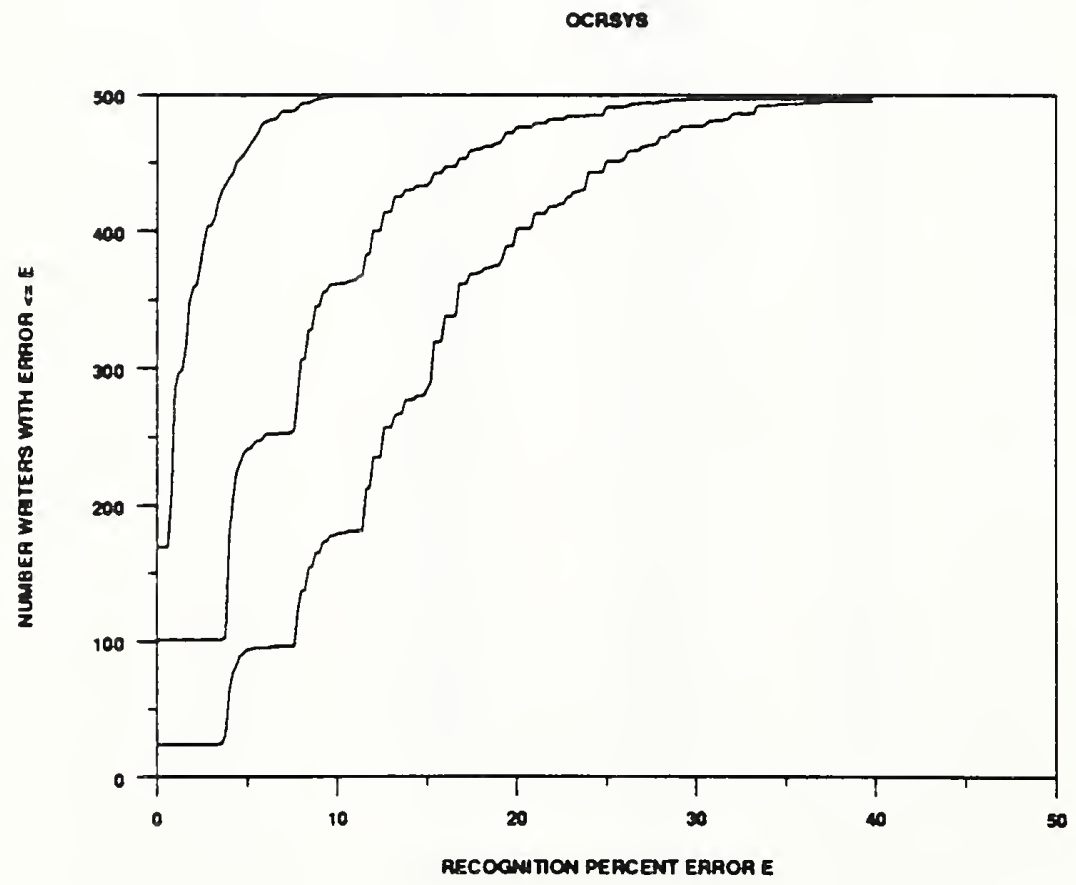

Figure 187: Error rate per writer of OCRSYS 


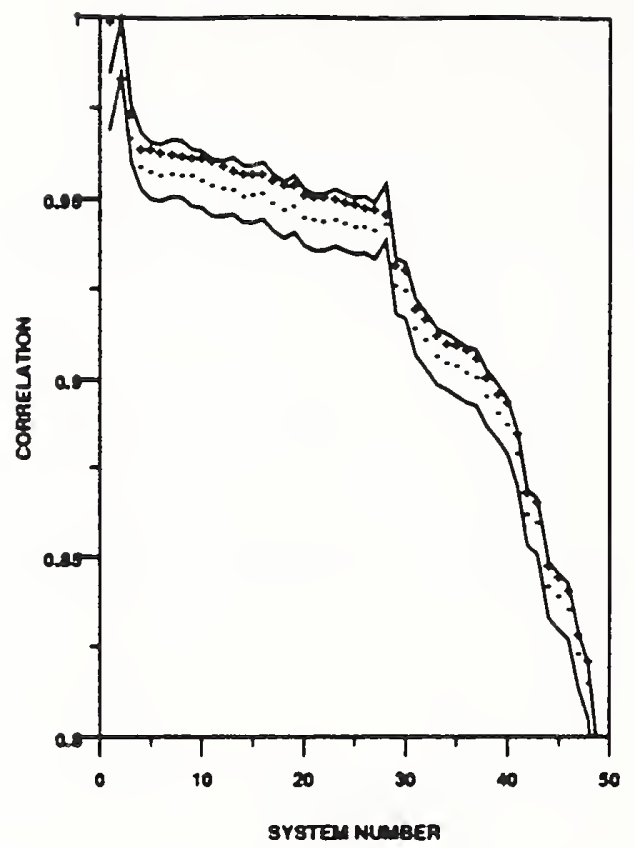

Figure 188: OCRSYS - digit correlation

\begin{tabular}{|c|c|c|c|}
\hline System Numbet & System Nome & Correlation (all) & Correlalion (coprect) \\
\hline 1 & OCRSYS & 1.0000 & 1.0000 \\
\hline 2 & REFERENCE & 0.9844 & 0.8844 \\
\hline $\mathbf{3}$ & VOTE_M & 0.9746 & 0.9681 \\
\hline 4 & ATT 1 & 0.9683 & 0.9601 \\
\hline 3 & AEG & 0.9682 & 0.9586 \\
\hline 6 & IBM & 0.9641 & 0.9577 \\
\hline 7 & ELSAGB 3 & 0.9636 & 0.9583 \\
\hline 8 & ELSAGB 2 & 0.8632 & 0.9579 \\
\hline$\theta$ & VOTE_P & 0.9629 & 0.9579 \\
\hline 10 & ATT 2 & 0.9627 & 0.9563 \\
\hline 11 & ERIM-I & 0.9619 & 0.9550 \\
\hline 12 & ERIM 2 & 0.9608 & 0.9542 \\
\hline 13 & THINK 2 & 0.9591 & 0.9537 \\
\hline 14 & A T T 44 & 0.9588 & 0.9520 \\
\hline 15 & KODAK_2 & 0.9584 & 0.9521 \\
\hline 16 & REI & 0.9881 & 09528 \\
\hline 17 & NYNEX & 0.9564 & 0.9501 \\
\hline 18 & UBOL & 0.9562 & 0.9492 \\
\hline 19 & NESTOR & 0.9562 & 0.9483 \\
\hline 20 & KODAK」 & 0.9522 & 0.9458 \\
\hline 21 & HUGHES-I & 0.9530 & 0.9453 \\
\hline 22 & HUGHES_2 & 0.9819 & 0.8451 \\
\hline 23 & SYMBUS & 0.9512 & 0.9456 \\
\hline 24 & ATT 3 & 0.9506 & 0.9447 \\
\hline 25 & NIST_4 & 0.9499 & 0.9435 \\
\hline 26 & THINK_-1 & 0.9492 & 0.9435 \\
\hline 27 & ELSAGB-1 & 0.9485 & 0.9425 \\
\hline 28 & СОMCOM & 0.9471 & 0.9445 \\
\hline 29 & GTESS_l & 0.9338 & 0.9277 \\
\hline 30 & GTESS 2 & 0.9320 & 0.8261 \\
\hline 31 & NIST 1 & 0.9211 & 0.9136 \\
\hline 32 & GMD_3 & 0.9181 & 0.9122 \\
\hline 33 & MIME & 0.9138 & 0.8079 \\
\hline 34 & GMD_I & 0.9114 & 0.9060 \\
\hline 33 & ASOL & 0.9109 & 0.9050 \\
\hline 36 & UPENN & 0.9094 & 0.9031 \\
\hline 37 & NIST 2 & 0.9074 & 0.9016 \\
\hline 38 & ה A NIST & 0.9017 & 0.8963 \\
\hline 39 & GMD_4 & 0.8971 & 0.8918 \\
\hline 40 & RISO & 0.8948 & 0.8884 \\
\hline 41 & KAMAN-1 & 0.8864 & 0.8800 \\
\hline 42 & KAMAN_3 & 0.8697 & 0.8633 \\
\hline 43 & KAMAN_2 & 0.8671 & 0.8608 \\
\hline 44 & KAMAN_S & 0.8489 & 0.8431 \\
\hline 45 & GMD 2 & 0.8437 & 0.8402 \\
\hline 46 & VALEN 2 & 0.8419 & 0.8366 \\
\hline 47 & IFAX & 0.8298 & 0.8241 \\
\hline 48 & VALEN_1 & 0.8222 & 0.8159 \\
\hline 49 & KAMAN-4 & 0.7933 & 0.7875 \\
\hline
\end{tabular}

Table 116: OCRSYS correlation graph key for digits. 


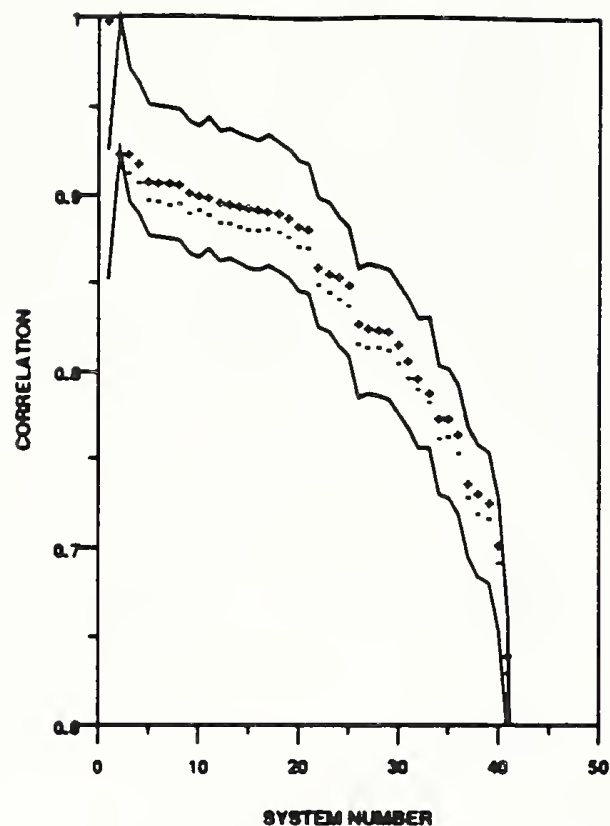

Figure 189: OCRSYS - upper case correlation

\begin{tabular}{|c|c|c|c|}
\hline System Number & System Name & Corretation (ail) & Correlation (correct) \\
\hline$I$ & OCRSYS & 1.0000 & 1.0000 \\
\hline 2 & REFERENCE & 0.9262 & 0.9262 \\
\hline 3 & VOTE_M & 0.2239 & 0.8147 \\
\hline 4 & AEG & 0.9208 & 0.8091 \\
\hline 3 & NYNEX & 0.8104 & 0.8989 \\
\hline 6 & ATT_4 & 0.9091 & 0.8983 \\
\hline 7 & UMICH_L & 0.8088 & 0.8988 \\
\hline 8 & ERIM_l & 0.9080 & 0.8972 \\
\hline 9 & NESTOR & 0.9036 & 0.8917 \\
\hline 10 & VOTE_P & 0.9019 & 0.8940 \\
\hline 11 & ATT 2 & 0.9010 & 0.8908 \\
\hline 12 & IBM & 0.8982 & 0.8868 \\
\hline 13 & UBOL & 0.8972 & 0.8861 \\
\hline 14 & HUGHES - 1 & 0.8967 & 0.8848 \\
\hline 15 & HUGHES.2 & 0.8932 & 0.8829 \\
\hline 16 & ATT 3 & 0.6943 & 0.8827 \\
\hline 17 & ATT $\perp$ & 0.8938 & 0.8832 \\
\hline 18 & KODAK」 & 0.8925 & 0.8820 \\
\hline 19 & SYMBUS & $0 \$ 801$ & 0.8792 \\
\hline 20 & GTESS.1 & 0.8858 & 0.8737 \\
\hline 21 & GTESSב & 0.8839 & 0.8723 \\
\hline 22 & MIME & 0.8627 & 0.8521 \\
\hline 23 & NIST_A & 0.8587 & 0.8476 \\
\hline 24 & ASOL & 0.8365 & 0.8441 \\
\hline 23 & REI & 0.8523 & 0.8404 \\
\hline 26 & RISO & 0.8298 & 0.8179 \\
\hline 27 & NIST_1 & 0.8277 & 0.8166 \\
\hline 28 & GMD_1 & 0.8267 & 0.8163 \\
\hline 29 & GMD_3 & 0.8239 & 0.8160 \\
\hline so & KAMAN_L & 0.8180 & 0.8070 \\
\hline 31 & GMD.4 & 0.8090 & 0.7988 \\
\hline 32 & СОМСOM & 0.7987 & 0.7927 \\
\hline 33 & נה & 0.7808 & 0.7853 \\
\hline 34 & IFAX & 0.7738 & 0.7642 \\
\hline 35 & KAMAN_3 & 0.7736 & 0.7646 \\
\hline 36 & KAMAN 2 & 0.7872 & 0.7535 \\
\hline 37 & $\mathrm{NIST}_{2}$ & 0.7396 & 0.7311 \\
\hline 38 & VALEN-1 & 0.7334 & 0.7216 \\
\hline 39 & GMD_2 & 0.7287 & 0.7187 \\
\hline 40 & KAMAN_4 & 0.7047 & 0.6948 \\
\hline 41 & KAMAN_S & 0.6417 & 0.6314 \\
\hline 42 & $\mathrm{UMICH}_{2}$ & 0.0338 & 0.0217 \\
\hline
\end{tabular}

Table 117: OCRSYS correlation graph key for uppers. 


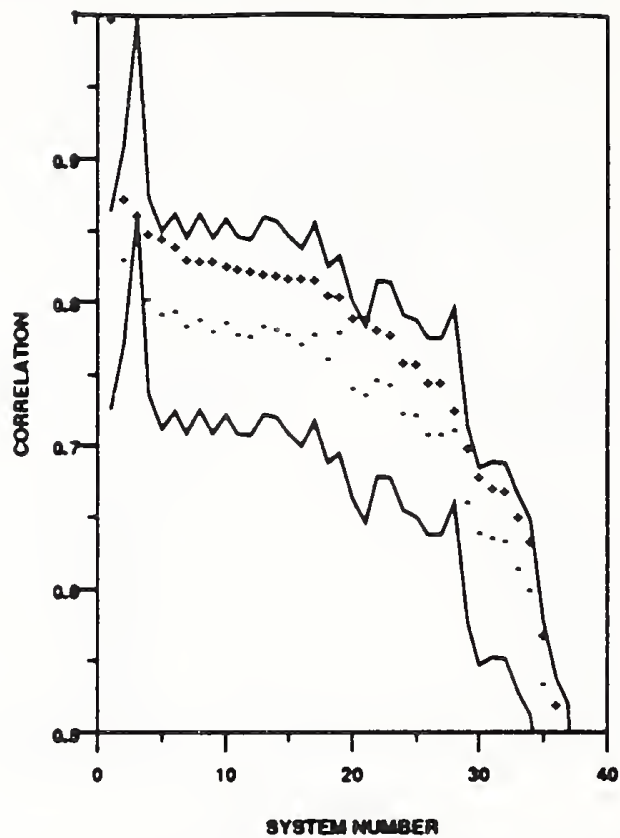

Figure 190: OCRSYS - lower case correlation

\begin{tabular}{|c|c|c|c|}
\hline System Number & System Name & Correlation (all) & Correlation (correcs) \\
\hline 1 & OCRSYS & 1.0000 & 1.0000 \\
\hline 2 & VOTE_M & 0.8748 & 0.8327 \\
\hline $\mathbf{3}$ & REFERENCE & 0.8630 & 0.8630 \\
\hline 4 & AEG & 0.8608 & 08046 \\
\hline $\mathbf{5}$ & $\mathrm{UMICH}_{-1}$ & 0.8472 & 0.7941 \\
\hline 6 & ERIM-1 & 0.8417 & 0.7968 \\
\hline 7 & IBM & 0.8342 & 0.7863 \\
\hline 8 & ATT_ & 0.8328 & 0.7908 \\
\hline $\boldsymbol{g}$ & UBOL & 0.8320 & 0.7828 \\
\hline 10 & ATT 2 & 0.8291 & 0.7893 \\
\hline 11 & HUGHES.I & 0.8267 & 0.7810 \\
\hline 12 & HUGHES_2 & 0.8252 & 0.7794 \\
\hline 13 & NYNEX & 0.8232 & 0.7868 \\
\hline 14 & ATT 4 & 0.8219 & 0.7849 \\
\hline 15 & NESTOR & 0.8208 & 0.7809 \\
\hline 16 & ATT & 0.8194 & 0.7744 \\
\hline 17 & KODAK」 & 0.8184 & 0.7811 \\
\hline 18 & GTESS.I & 0.8083 & 0.7641 \\
\hline 19 & VOTE_P & 0.8068 & 0.7822 \\
\hline 20 & NIST 4 & 0.7928 & 0.7431 \\
\hline 21 & RISO & 0.7908 & 0.7379 \\
\hline 22 & GTESS 2 & 0.7843 & 0.7483 \\
\hline 23 & NIST 1 & 0.7809 & 0.7448 \\
\hline 24 & GMD_3 & 0.7613 & 0.7256 \\
\hline 25 & A SOL & 0.7800 & 0.7242 \\
\hline 26 & GMD_4 & 0.7473 & 0.7110 \\
\hline 27 & GMD_L & 0.7473 & 0.7110 \\
\hline 28 & NIST J & 0.7278 & 0.7138 \\
\hline 29 & GMD_2 & 0.7021 & 0.6842 \\
\hline 30 & VALEN_I & 0.6817 & 0.6418 \\
\hline 31 & KAMAN_l & 0.6744 & 0.6381 \\
\hline 32 & NIST 2 & 0.6722 & 0.6364 \\
\hline 33 & KAMAN 3 & 0.6529 & 0.8169 \\
\hline 34 & $K A M A N_{2} 2$ & 0.6362 & 0.6022 \\
\hline 35 & KAMAN_S & 0.5707 & 0.5385 \\
\hline 36 & KAMAN-4 & 0.5228 & 0.4985 \\
\hline 37 & СОМСOM & 0.4980 & 0.4882 \\
\hline 38 & $\mathrm{UMICH}_{2}$ & 0.0827 & 0.0451 \\
\hline
\end{tabular}

Table 118: OCRSYS correlation graph key for lowers. 
SYSTEM: REI

PARTICIPANT: David L. Cauthron

ORGANIZATION: Recognition Equipment Inc. (REI),

FEATURES : model-based

CLASSIFICATION : MLP

HARDWARE: VAX simulation of 386 with coprocessor boards ICR handprint recognizer

TRAINING: DIGITS UPPERS LOHERS DATABASE

$245000 \quad 100000 \quad$ NA INTERNAL

STATUS: $\quad$ on time

RESULTS: -- DIGITS -- -- UPPERS -- -- LOWERS -- DATABASE

REJ. ERR. REJ. ERR. REJ. ERR. TESTDATA 1

RATE RATE-- RATE RATE-- RATE RATE--

$0.00 \quad 0.0401 \quad 0.00 \quad 0.1174$

$\begin{array}{llll}0.14 & 0.0055 & 0.57 & 0.0117\end{array}$

$\begin{array}{llll}0.10 & 0.0088 & 0.40 & 0.0244\end{array}$

$0.07 \quad 0.0139 \quad 0.24 \quad 0.0379$

$\begin{array}{llll}0.04 & 0.0194 & 0.15 & 0.0582\end{array}$

OCR RATE (CPS): DIGITS UPPERS LOWERS
SYS RATE:
1.97
2.06

CPU RATE:

NOTE: Internal database contains approximately 245000 digits and 100000 upper case letters.

NOTE: Few details of system description provided. Did not train on NIST data. 
SYSTEM: REI

BIBLIOGRAPHY:

The following references have been provided for this system:

none 


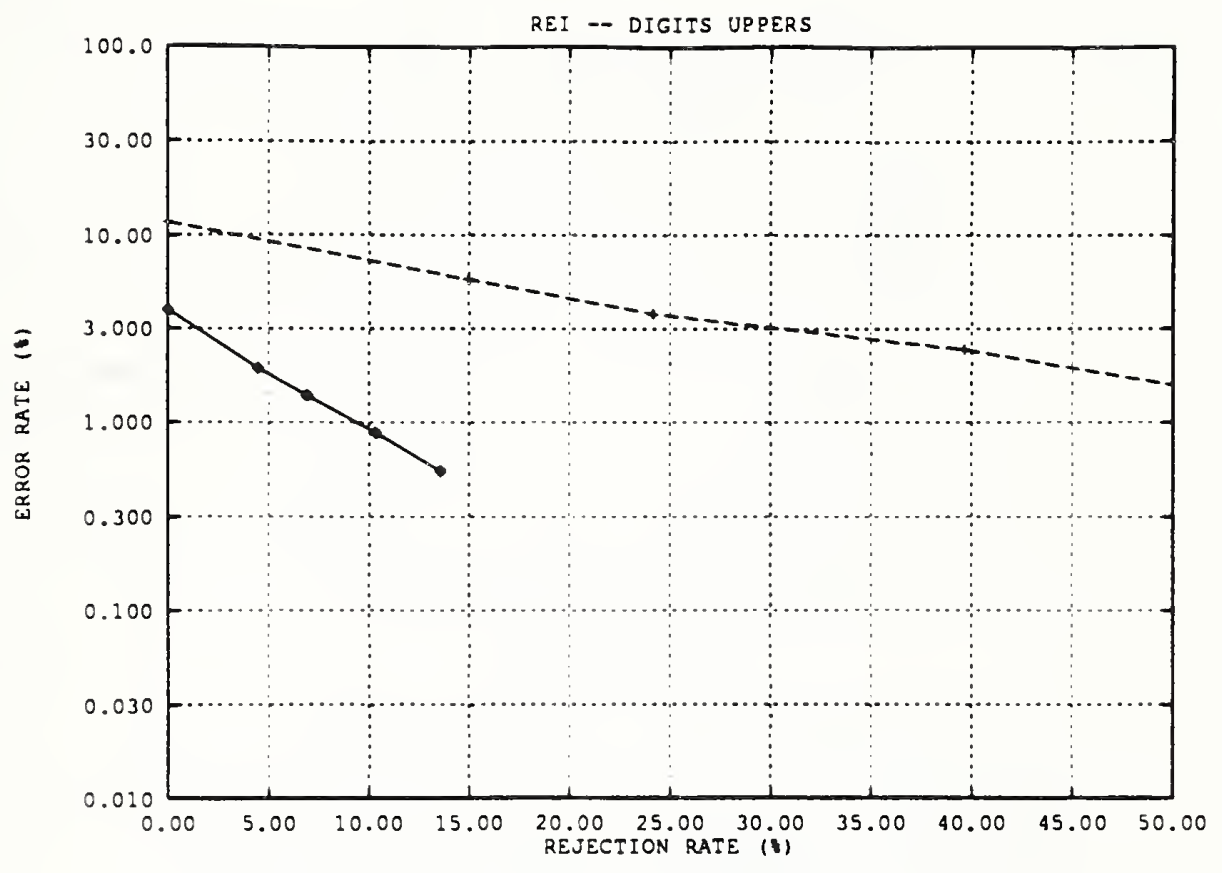

Figure 191: Error rate versus rejection rate for REI

คอ

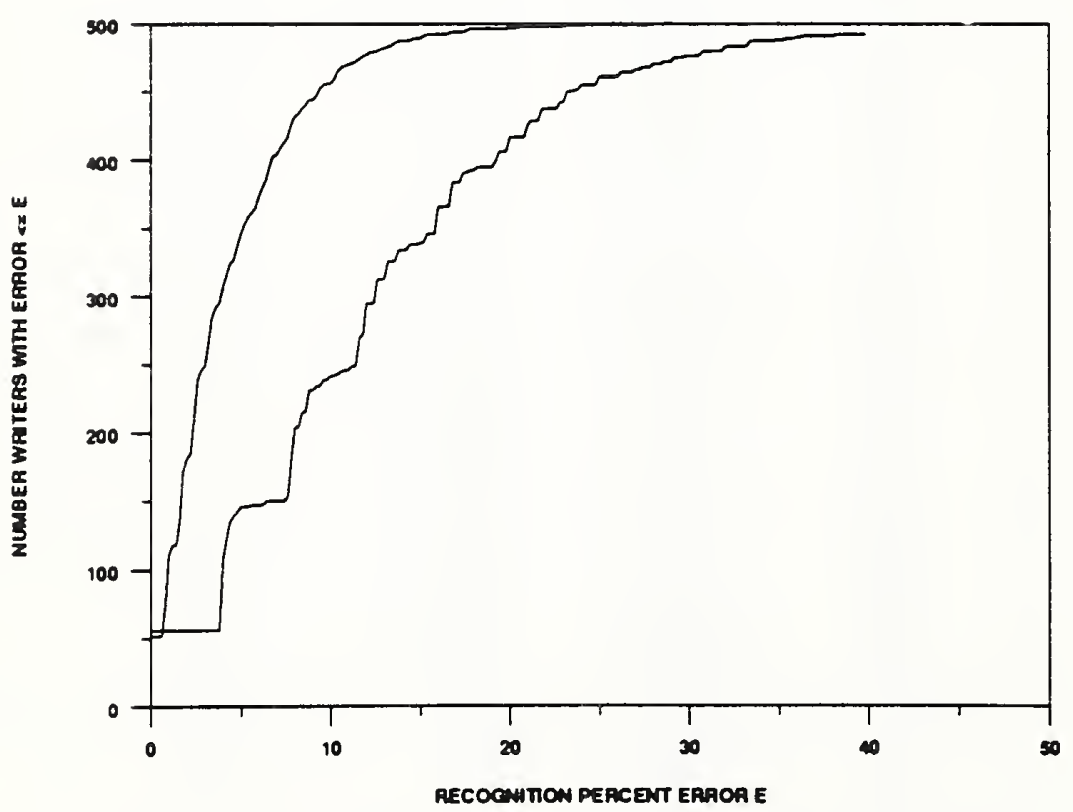

Figure 192: Error rate per writer of REI 


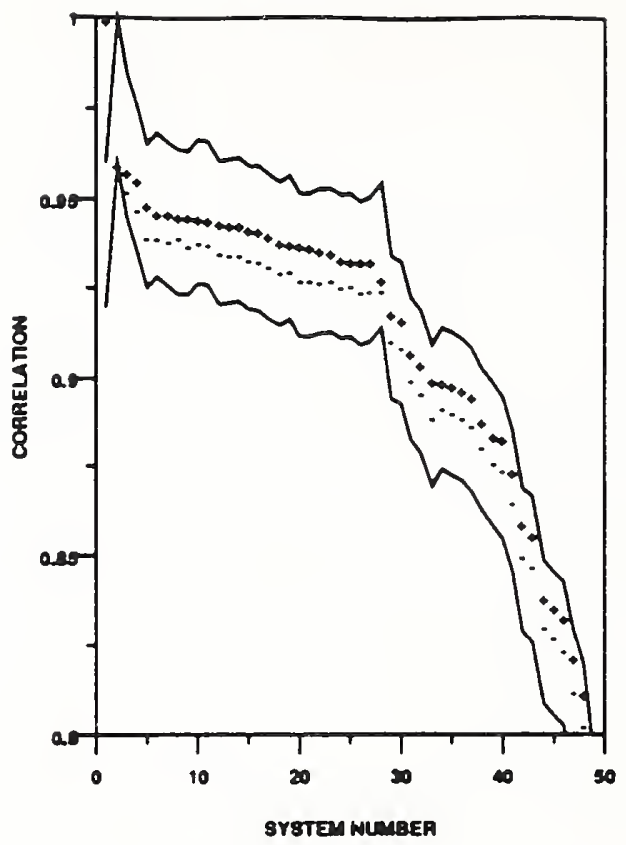

Figure 193: REI - digit correlation

\begin{tabular}{|c|c|c|c|}
\hline System Number & Syslem Name & Correlation (all) & Correlation (eorrect) \\
\hline $\mathrm{T}$ & REI & 1.0000 & 1.0000 \\
\hline 2 & REFERENCE & 0.8898 & 0.8590 \\
\hline 3 & OCRSYS & 0.9581 & 0.2528 \\
\hline 4 & VOTE_M & 0.9561 & 0.9479 \\
\hline 5 & IB M & 0.8488 & 0.9400 \\
\hline 6 & ATT」 & 0.9467 & 0.9400 \\
\hline 7 & AEG & 0.9466 & 0.9388 \\
\hline 8 & VOTE_P & 0.8460 & 0.9401 \\
\hline 9 & ATT 2 & 0.9459 & 09377 \\
\hline 10 & ELSAGBA & 0.8455 & 0.2387 \\
\hline 11 & ELSAGB? & 0.9481 & 0.9383 \\
\hline 12 & ERIM_2 & 0.9439 & 0.9387 \\
\hline 13 & ERIM_l & 0.9436 & $0.935 \mathrm{~s}$ \\
\hline 14 & THINK 2 & 0.8434 & 0.9385 \\
\hline 15 & KODAK2 & 0.9424 & 0.9338 \\
\hline 16 & ATT_4 & 0.9417 & 0.9335 \\
\hline 17 & NYNEX & 0.8406 & 0.8323 \\
\hline 18 & NESTOR & 0.9386 & 0.9303 \\
\hline 18 & UBOL & 09379 & 0.2307 \\
\hline 20 & HUGHES_l & 0.8377 & 0.9282 \\
\hline 21 & HUGHES_2 & 0.9372 & 0.8278 \\
\hline 22 & KODAK」 & 0.9363 & 0.9276 \\
\hline 23 & SYMBUS & 0.9357 & 0.9280 \\
\hline 24 & THINK.I & 08339 & 0.8263 \\
\hline 25 & ATT 3 & 0.9337 & 09268 \\
\hline 26 & NIST_A & 0.9334 & 0.8253 \\
\hline 27 & ELSAGB-1 & 0.8394 & 0.9248 \\
\hline 28 & COMCOM & 09288 & 0.8251 \\
\hline 29 & GTESS_l & 0.9188 & 0.8108 \\
\hline 30 & GTESS 2 & 0.9168 & 0.9092 \\
\hline 3I & NIST_I & 0.9078 & 0.8999 \\
\hline 32 & GMD_3 & 0.9046 & 0.8965 \\
\hline 3s & UPENN & 0.8998 & 0.8896 \\
\hline 34 & MIME & 0.8997 & 0.8920 \\
\hline 35 & GMD_1 & 0.8984 & 0.8906 \\
\hline 36 & ASOL & 0.8972 & 0.8893 \\
\hline 37 & NIST? & 0.8952 & 0.8870 \\
\hline 38 & NIST 3 & 0.8887 & 0.8812 \\
\hline 39 & GMD-4 & 0.8044 & 0.8766 \\
\hline 40 & RISO & 0.8836 & 0.8748 \\
\hline 11 & KAMAN_1 & 0.8743 & 0.8655 \\
\hline 42 & KAMAN_3 & 0.8596 & 0.8503 \\
\hline 43 & KAMAN_2 & 0.8564 & 0.8476 \\
\hline 14 & KAMAN-S & 0.8389 & 0.8305 \\
\hline 45 & GMD_2 & 0.8362 & 0.8280 \\
\hline 46 & VALEN_2 & 0.8332 & 0.8241 \\
\hline 17 & IFAX & 0.8221 & 0.8128 \\
\hline 48 & VALEN_I & 0.8122 & 0.8032 \\
\hline 48 & KAMAN-4 & 0.7832 & 07755 \\
\hline
\end{tabular}

Table 119: REI correlation graph key for digits. 


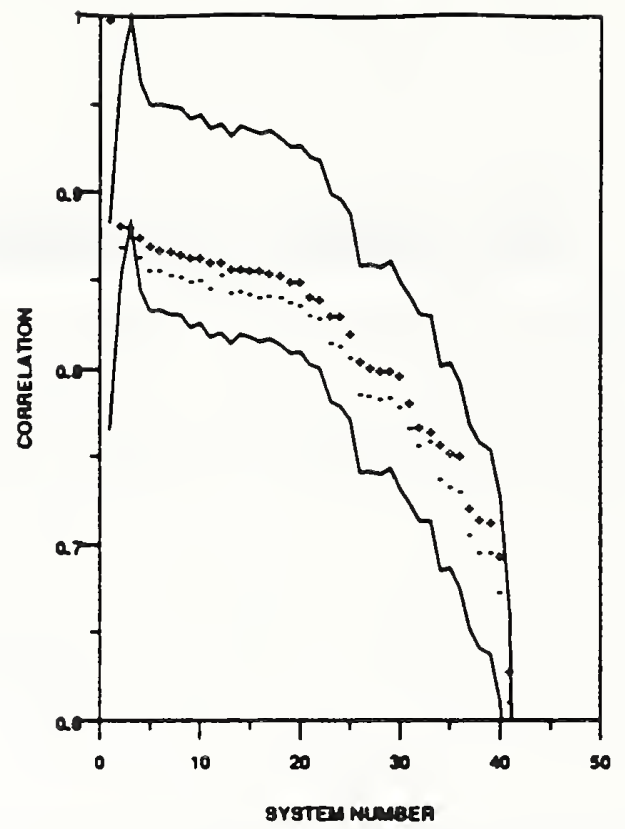

Figure 194: REI - upper case correlation

\begin{tabular}{|c|c|c|c|}
\hline Sydtem Number & System Name & Corretation (sil) & Correlation (correct) \\
\hline 1 & REI & 1.0000 & 1.0000 \\
\hline 2 & VOTE_M & 0.8836 & 0.8715 \\
\hline 3 & REFERENCE & 0.8826 & 0.8826 \\
\hline 4 & AEG & 0.8771 & 0.8658 \\
\hline 5 & ATT -4 & 0.8726 & 0.8589 \\
\hline 6 & NYNEX & 08704 & 0.8586 \\
\hline 7 & $\mathrm{UMICH}_{-1}$ & 0.8694 & 0.8581 \\
\hline 8 & ERIM_1 & 08675 & 0.8552 \\
\hline 9 & NESTOR & 0.8665 & 08527 \\
\hline 10 & ATT 2 & 0.8658 & 0.8531 \\
\hline 11 & VOTEP & 08634 & 08556 \\
\hline 12 & IBM & 08634 & 0.8490 \\
\hline 13 & UBOL & 0.8596 & 0.8468 \\
\hline 14 & ATT_3 & 0.8598 & 0.8456 \\
\hline 15 & HUGHES-1 & 0.8891 & 0.8447 \\
\hline 16 & HUGHES.2 & 0.8387 & 08433 \\
\hline 17 & ATT $T_{\perp}$ & 0.8571 & 0.8442 \\
\hline 18 & KODAK_ & 0.8535 & 0.8428 \\
\hline 19 & OCRSYS & 0.8523 & 0.8404 \\
\hline 20 & SYMBUS & 0.8519 & 08388 \\
\hline 21 & GTESS_L & 0.8442 & 0.8327 \\
\hline 22 & $\operatorname{GTESS} 2$ & 08426 & 0.8312 \\
\hline 23 & MIME & 0.8332 & 0.8176 \\
\hline 24 & NIST_4 & 0.8328 & 0.8153 \\
\hline 25 & ASOL & 08225 & 08092 \\
\hline 26 & RISO & 0.8073 & 0.7876 \\
\hline 27 & GMD _1 & 0.8037 & 0.7870 \\
\hline 28 & GMD_3 & 0.8013 & 0.7851 \\
\hline 29 & NIST.1 & 0.8013 & 07860 \\
\hline 30 & KAMAN_1 & 07991 & 0.7803 \\
\hline 31 & GMD.4 & 0.7834 & 0.7688 \\
\hline 32 & NIST.3 & 0.7694 & 0.7583 \\
\hline 33 & СOMCOM & 0.7668 & 0.7612 \\
\hline 34 & KAMAN_3 & 0.7597 & 0.7399 \\
\hline 35 & IFAX & 07549 & 0.7357 \\
\hline 36 & KAMAN_2 & 0.7533 & 0.7328 \\
\hline 37 & NIST 2 & 0.7232 & 0.7083 \\
\hline 38 & VALEN-1 & 0.7169 & 0.6983 \\
\hline 39 & GMD_2 & 0.7154 & 0.6973 \\
\hline 40 & KAMAN-4 & 0.6938 & 0.8730 \\
\hline 41 & KAMAN_S & 0.8303 & 0.6121 \\
\hline 42 & $\mathrm{UMICH}_{2}$ & 0.0550 & 0.0204 \\
\hline
\end{tabular}

Table 120: REI correlation graph key for uppers. 


\section{No Data Available}

Figure 195: REI - lower case correlation

There was no dala for this eveluation.

Table 121: REI correlation graph key for lowers. 
SYSTEM : RISO

PARTICIPANT: Christian Liisberg

ORGANIZATION: Riso National Laboratories, Roskilde, Denmark

PREPROCESSING: size normalization to $16 \times 16$, no deskering.

The normalized image is directly input to the neural net.

FEATURES: receptor field (LVT)

CLASSIFICATION: self-organizing geometric, ensembles of look-up table networks used.

HARDWARE: $\quad 33 \mathrm{MHz} 486$ with 16 Mbyte RAM

TRAINING: DIGITS UPPERS LOWERS DATABASE

$210000 \quad 40000 \quad 40000 \quad$ NSDB3

1 to 2 ambiguous characters removed by hand

STATUS: $\quad$ on time

RESULTS: -- DIGITS -- -- UPPERS -- -- LOWERS -- DATABASE

REJ. ERR. REJ. ERR. REJ. ERR. TESTDATA1

RATE RATE-- RATE RATE-- RATE RATE--

$\begin{array}{lllllll}0.00 & 0.1055 & 0.00 & 0.1414 & 0.00 & 0.2172\end{array}$

$\begin{array}{lllllll}0.02 & 0.0975 & 0.03 & 0.1244 & 0.04 & 0.1979\end{array}$

$\begin{array}{lllllll}0.06 & 0.0759 & 0.10 & 0.0943 & 0.13 & 0.1580\end{array}$

$\begin{array}{lllllll}0.10 & 0.0594 & 0.16 & 0.0694 & 0.21 & 0.1273\end{array}$

$\begin{array}{lllllll}0.14 & 0.0460 & 0.22 & 0.0504 & 0.28 & 0.1013\end{array}$

$\begin{array}{lllllll}0.18 & 0.0345 & 0.29 & 0.0347 & 0.36 & 0.0790\end{array}$

$\begin{array}{lllllll}0.23 & 0.0241 & 0.38 & 0.0221 & 0.45 & 0.0578\end{array}$

$\begin{array}{lllllll}0.30 & 0.0141 & 0.55 & 0.0105 & 0.61 & 0.0288\end{array}$

OCR RATE (CPS): DIGITS UPPERS LOWERS

$\begin{array}{llll}\text { SYS RATE: } & 4.67 & 2.00 & ? \\ \text { CPU RATE: } & 6.79 & 2.31\end{array}$


SYSTEM: RISO

PARTICIPANT: Christian Liisberg

BIBLIOGRAPHY:

The following references have been provided for this system: 


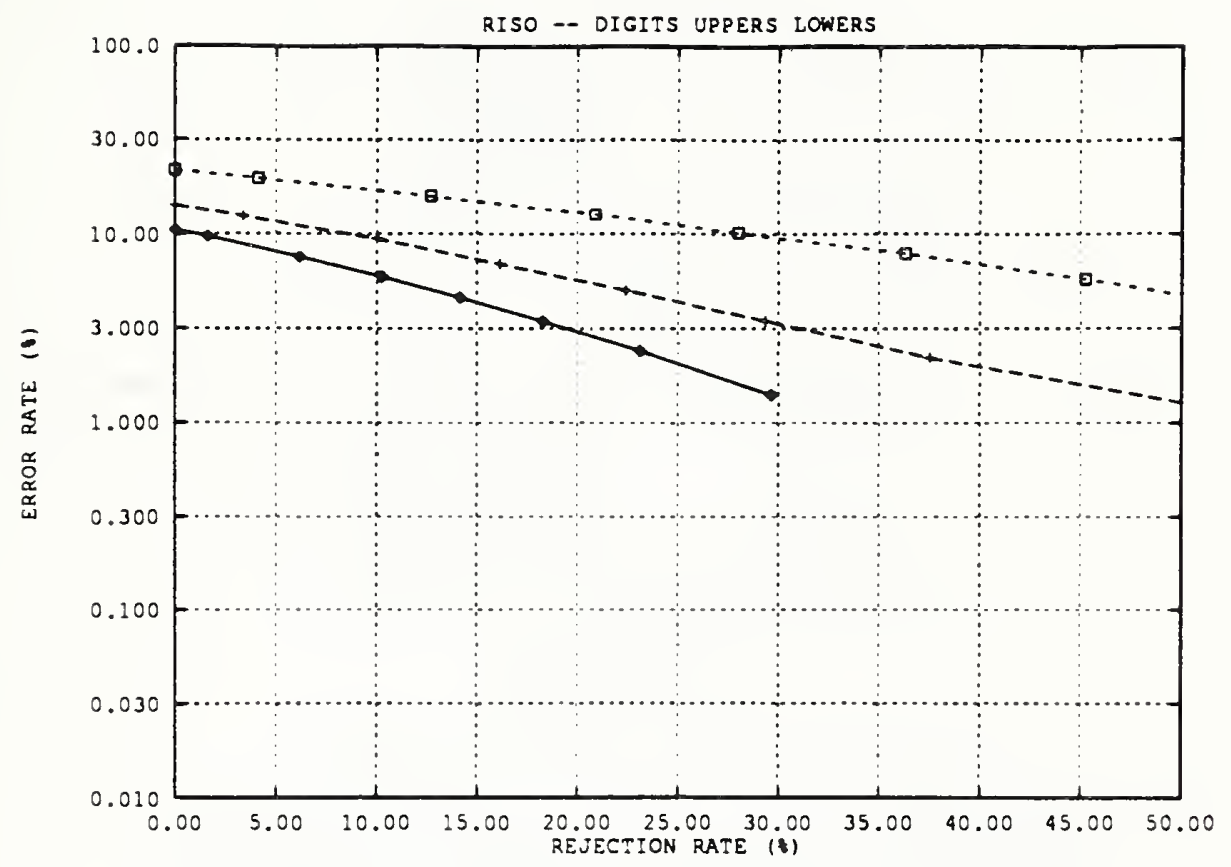

Figure 196: Error rate versus rejection rate for RISO

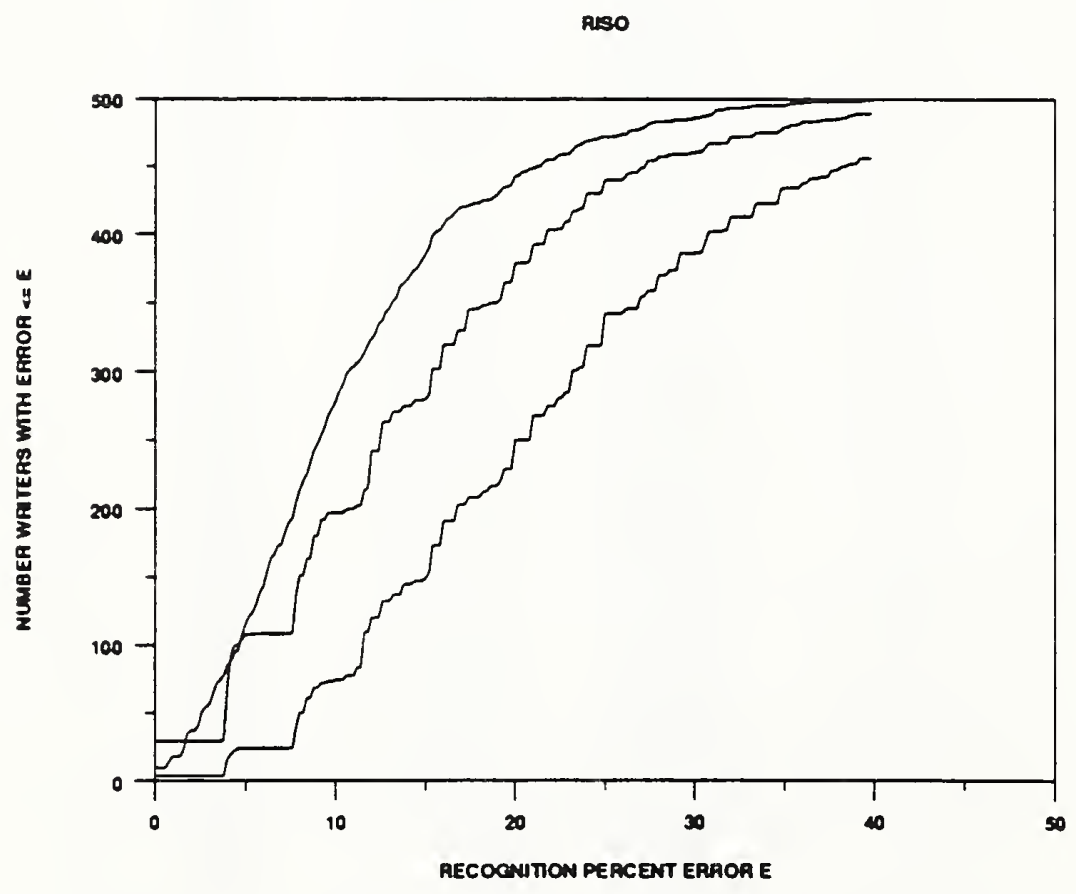

Figure 197: Error rate per writer of RISO 


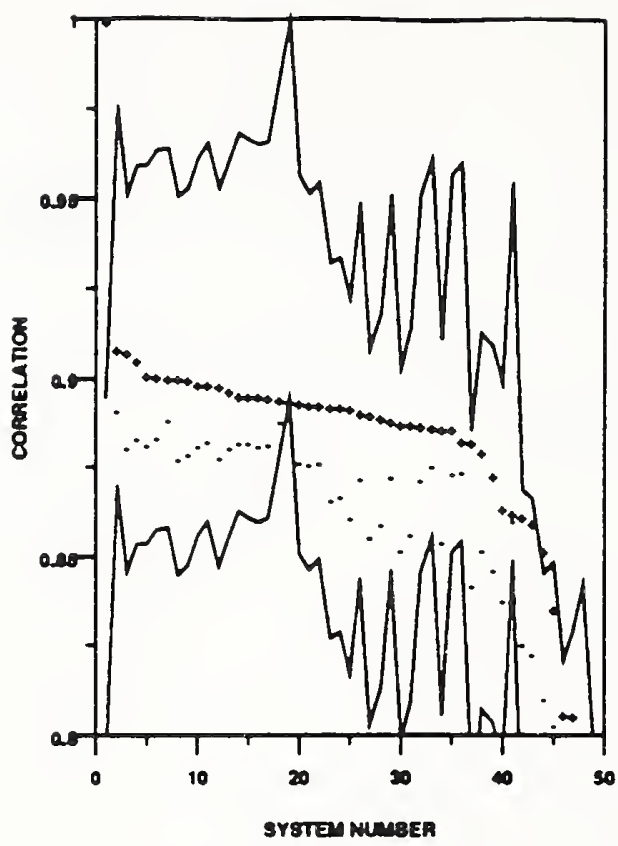

Figure 198: RISO - digit correlation

\begin{tabular}{|c|c|c|c|}
\hline System Number & System Name & Corretation (all) & Corpelation (correct) \\
\hline$T$ & RISO & 1.0000 & 1.0000 \\
\hline 2 & VOTE_M & 0.0001 & 0.8916 \\
\hline 3 & THINK_I & 0.9080 & 0.8812 \\
\hline 4 & ATT 4 & 0.9060 & 0.8838 \\
\hline 3 & KODAK 2 & 0.9017 & 0.8821 \\
\hline 6 & ATT 2 & 0.9013 & 0.8839 \\
\hline 7 & VOTE_P & 0.9011 & 0.8889 \\
\hline 8 & NIST_4 & 0.9008 & 0.8777 \\
\hline 9 & SYMBUS & 0.9002 & 0.8794 \\
\hline 10 & AEG & 0.8990 & 08828 \\
\hline 11 & ERIM_1 & 0.8990 & 0.8818 \\
\hline 12 & KODAK_ & 0.8983 & 0.8785 \\
\hline 13 & ERIM_2 & 0.8974 & 0.8812 \\
\hline 14 & ATT 1 & 0.8959 & 0.8827 \\
\hline 15 & ELSAGBA & 0.8988 & 0.8823 \\
\hline 16 & IBM & 08937 & 0.8816 \\
\hline 17 & ELSAGB2 & 0.8953 & 0.8820 \\
\hline 18 & OCRSYS & 0.8948 & 08884 \\
\hline 19 & REFERENCE & 0.8945 & 08943 \\
\hline 20 & UBOL & 0.8941 & 0.8771 \\
\hline 21 & ATT 3 & 0.8936 & 0.6763 \\
\hline 22 & NESTOR & 0.8935 & 0.8772 \\
\hline 23 & GTESS 2 & 08930 & 0.8669 \\
\hline 24 & GTESS_l & 0.8929 & 0.8677 \\
\hline 25 & NIST_l & 0.8926 & 0.8618 \\
\hline 26 & ELSAGB_1 & 0.8912 & 0.8730 \\
\hline 27 & $\mathrm{NIST}_{2}$ & 0.8909 & 0.8561 \\
\hline 28 & GMD_3 & 0.8899 & 0.8599 \\
\hline 29 & HUGHES.1 & 0.8890 & 0.8732 \\
\hline 30 & MIME & 0.8882 & 0.6371 \\
\hline 31 & ב_ NIST & 0.8882 & 0.8526 \\
\hline 32 & HUGHES_2 & 0.8875 & 0.8725 \\
\hline 39 & THINK 2 & 0.8869 & 0.8739 \\
\hline 34 & NYNEX & 0.8867 & 0.8741 \\
\hline 35 & ASOL & 0.8867 & 0.8551 \\
\hline 36 & REI & 0.8836 & 0.8743 \\
\hline 37 & KAMAN_l & 0.8828 & 0.8426 \\
\hline 38 & GMD_I & 0.8800 & 0.8326 \\
\hline 39 & UPENN & 0.8739 & 0.8470 \\
\hline 10 & GMD_4 & 0.8648 & 0.8382 \\
\hline 41 & COMCOM & 0.8633 & 0.8607 \\
\hline 42 & KAMAN_3 & 0.8625 & 0.8258 \\
\hline 43 & $K A M A N_{-2}$ & 0.8605 & 0.8233 \\
\hline 14 & GMD 2 & 0.8525 & 0.8110 \\
\hline 43 & KAMAN_S & 0.8339 & 0.8033 \\
\hline 46 & VALEN_I & 0.8068 & 0.7739 \\
\hline 47 & IFAX & 0.8064 & 0.7802 \\
\hline 48 & VALEN 2 & 0.7984 & 0.7832 \\
\hline 49 & KAMAN-4 & 0.7974 & 0.7582 \\
\hline
\end{tabular}

Table 122: RISO correlation graph key for digits. 


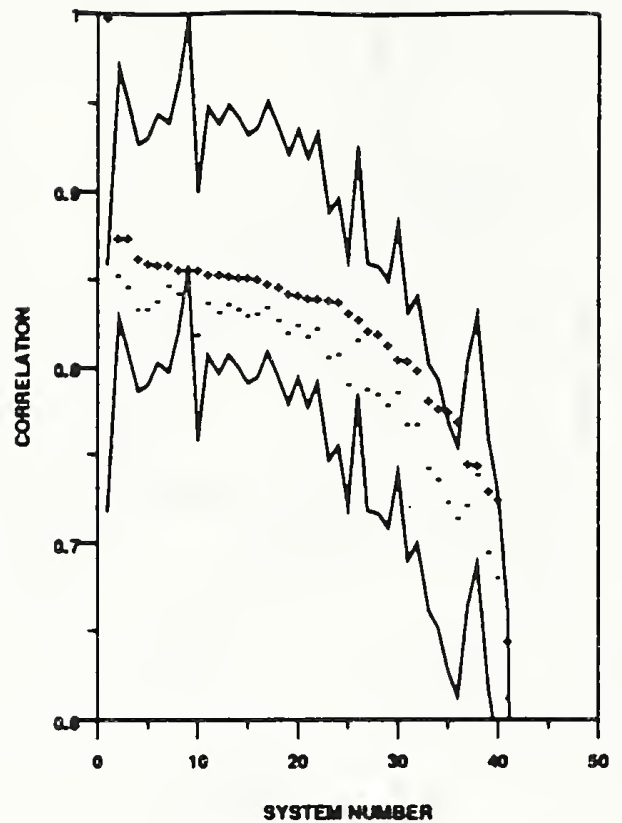

Figure 199: RISO - upper case correlation

\begin{tabular}{|c|c|c|c|}
\hline System Number & System Name & Cortelation (ali) & Correlation (correct) \\
\hline 1 & RISO & 1.0000 & 1.0000 \\
\hline 2 & VOTE_M & 0.8761 & 0.8554 \\
\hline 3 & ATT_4 & 0.8760 & 0.8488 \\
\hline 4 & SYMBUS & 0.8633 & 0.8353 \\
\hline 5 & KODAK 1 & 0.8625 & 0.8338 \\
\hline 6 & ATT 2 & 0.8618 & 0.8406 \\
\hline 7 & VOTE_P & 0.8814 & 0.8496 \\
\hline 8 & $A E G$ & 0.8587 & 0.8446 \\
\hline 9 & REFERENCE & 0.8586 & 0.8586 \\
\hline 10 & MIME & 0.8585 & 0.8210 \\
\hline 11 & ERIM_1 & 0.8562 & 0.8380 \\
\hline 12 & UBOL & 0.8357 & 0.8337 \\
\hline 13 & UMICH_L & 0.8552 & 0.8383 \\
\hline 14 & NESTOR & 0.8545 & 0.8361 \\
\hline 15 & ATTA & 0.8537 & 0.8321 \\
\hline 16 & IBM & 0.8528 & 0.8331 \\
\hline 17 & NYNEX & 0.8503 & 0.8368 \\
\hline 18 & ATT $\perp$ & 0.8484 & 0.8296 \\
\hline 18 & GTESS_l & 0.8449 & 0.8219 \\
\hline 20 & HUGHES.1 & 0.8443 & 0.8263 \\
\hline 21 & HUGHES_2 & 0.8425 & 0.8245 \\
\hline 22 & GTESS 2 & 0.8425 & 0.8204 \\
\hline 23 & ASOL & 0.8409 & 0.8085 \\
\hline 24 & NIST_A & 0.8405 & 0.8096 \\
\hline 25 & NIST_I & 0.8338 & 0.7925 \\
\hline 26 & OCRSYS & 0.8298 & 0.8179 \\
\hline 27 & GMD.1 & 0.8241 & 0.7899 \\
\hline 28 & GMD_3 & 0.8220 & 0.7875 \\
\hline 29 & KAMAN_1 & 08133 & 07803 \\
\hline 30 & REI & 0.8073 & 0.7876 \\
\hline 31 & ב_ NIST & 0.8062 & 0.7682 \\
\hline 32 & GMD_4 & 0.8009 & 0.7697 \\
\hline 33 & KAMAN_J & 0.7833 & 0.7449 \\
\hline 34 & KAMAN_2 & 0.7792 & 0.7381 \\
\hline 35 & NIST 2 & 0.7763 & 0.7253 \\
\hline 36 & GMD .2 & 0.7710 & 07165 \\
\hline 37 & IFAX & 0.7479 & 0.7241 \\
\hline 38 & СОМСОМ & 0.7468 & 0.7409 \\
\hline 39 & VALEN_1 & 0.7320 & 0.6968 \\
\hline 40 & $K A M A N-4$ & 0.7272 & 0.6826 \\
\hline 41 & KAMAN_S & 0.6464 & 0.8143 \\
\hline 42 & $\mathrm{UMICH}_{2}$ & 0.0841 & 0.0161 \\
\hline
\end{tabular}

Table 123: RISO correlation graph key for uppers. 


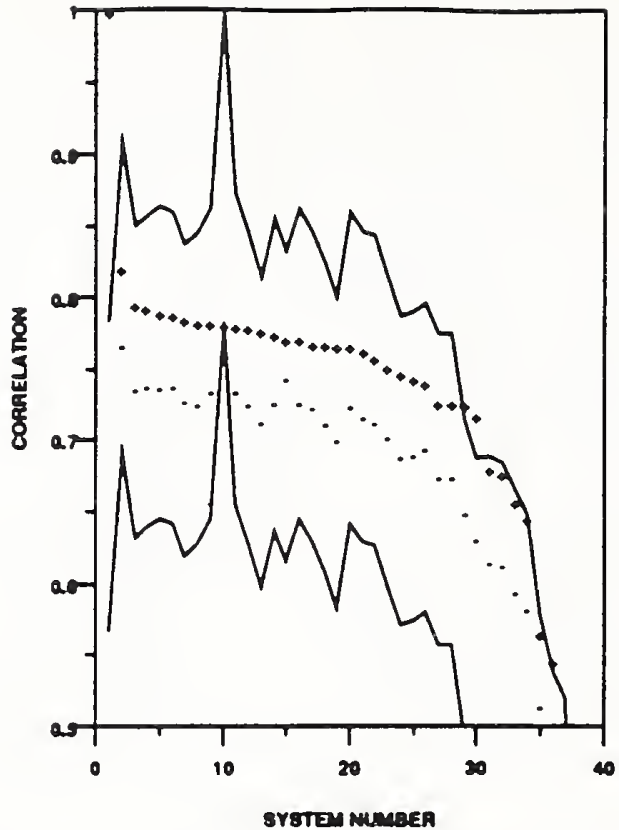

Figure 200: RISO - lower case correlation

\begin{tabular}{|c|c|c|c|}
\hline Syolem Numbet & System Name & Corretation (ail) & Coprelation (corpect) \\
\hline $\mathrm{I}$ & RISO & 1.0000 & 1.0000 \\
\hline 2 & VOTE_M & 0.8227 & 0.7683 \\
\hline 3 & UMICH_I & 0.7968 & 0.7369 \\
\hline 4 & ATT & 0.7947 & 0.7391 \\
\hline 5 & OCRSYS & 0.7908 & 0.7379 \\
\hline 6 & ATT2 2 & 0.7902 & 0.7393 \\
\hline 7 & ATT 3 & 0.7869 & 0.7289 \\
\hline 8 & UBOL & 0.7843 & 0.7269 \\
\hline 9 & ERIM_1 & 0.7838 & 0.7388 \\
\hline 10 & REFERENCE & 0.7828 & 0.7828 \\
\hline 11 & $A E G$ & 0.7817 & 0.7360 \\
\hline 12 & IBM & 0.7811 & 0.7272 \\
\hline 13 & NisT 1 & 0.7784 & 0.7145 \\
\hline 14 & KODAK_ & 0.7761 & 0.7279 \\
\hline 15 & VOTEP & 0.7732 & 07450 \\
\hline 16 & ATT $\perp$ & 0.7724 & 0.7281 \\
\hline 17 & NESTOR & 0.7694 & 0.7243 \\
\hline 18 & GTESS_1 & 0.7690 & 0.7131 \\
\hline 19 & NIST 4 & 0.7678 & 0.7017 \\
\hline 20 & NYNEX & 0.7677 & 0.7258 \\
\hline 21 & HUGHES-I & 0.7647 & 0.7173 \\
\hline 22 & HUGHES_2 & 0.7602 & 07145 \\
\hline 23 & GTESS_2 & 0.7538 & 0.7039 \\
\hline 24 & ASOL & 0.7493 & 0.6898 \\
\hline 25 & GMD_3 & 0.7449 & 0.6917 \\
\hline 26 & NIST & 0.7418 & 0.6964 \\
\hline 27 & GMD_4 & 0.7285 & 0.6765 \\
\hline 28 & GMD_l & 0.7285 & 0.6765 \\
\hline 29 & GMD.2 & 0.7268 & 0.6507 \\
\hline 30 & NIST 2 & 0.7193 & 0.6332 \\
\hline 31 & KAMAN 1 & 0.6821 & 0.8172 \\
\hline 32 & VALEN_I & 0.6790 & 0.6148 \\
\hline 33 & KAMAN_3 & 0.6593 & 0.5964 \\
\hline 34 & KAMAN 2 & 0.6172 & 0.5833 \\
\hline 35 & KAMAN_S & 0.5667 & 0.5138 \\
\hline 36 & KAMAN.A & 0.5463 & 0.1889 \\
\hline 37 & COMCOM & 0.4627 & 0.4540 \\
\hline 38 & $\mathrm{UMICH}_{2}$ & 0.0906 & 0.0335 \\
\hline
\end{tabular}

Table 124: RISO correlation graph key for lowers. 
SYSTEM: SYMBUS

PARTICIPANT: Jerry Fisher

ORGANIZATION: Symbus Technology, Brookline, MA

FEATURES: output of preprocessing

CLASSIFICATION: cascaded self-organizing NNs

HARDWARE :

TRAINING: DIGITS UPPERS LOHERS DATABASE

number used is proprietary NA INTERNAL

STATUS: on time, three RJX files missing

RESULTS: -- DIGITS -- -- UPPERS -- -- LOWERS -- DATABASE

REJ. ERR. REJ. ERR. REJ. ERR. TESTDATA1

RATE RATE-- RATE RATE-- RATE RATE--

$\begin{array}{lllll}0.00 & 0.0471 & 0.00 & 0.0729\end{array}$

$\begin{array}{lllll}0.00 & 0.0470 & 0.00 & 0.0727\end{array}$

$\begin{array}{lllll}0.02 & 0.0397 & 0.07 & 0.0462\end{array}$

$\begin{array}{llll}0.04 & 0.0327 & 0.15 & 0.0289\end{array}$

$\begin{array}{llll}0.11 & 0.0194 & 0.28 & 0.0151\end{array}$

0.190 .0111

OCR RATE (CPS): DIGITS UPPERS LOWERS

SYS RATE: NA NA NA

CPU RATE:

NOTE: Some of the HYP files contained tildes to indicate that no classification was attempted. Every classification in the whole file, rather than just the tilde was inadvertently converted to a question mark at NIST before scoring for the Conference. This gave zero rejection rate error rates of $7.0 \%$ and $12.0 \%$ for digits and uppers, respectively. The scores above reflect the correction of this NIST error.

NOTE: Few if any details provided about features or recognition algorithm.

NOTE: Internal database includes NSDB1. 


\section{SYSTEM: SYMBUS}

\section{BIBLIOGRAPHY:}

The following references have been provided for this system: 


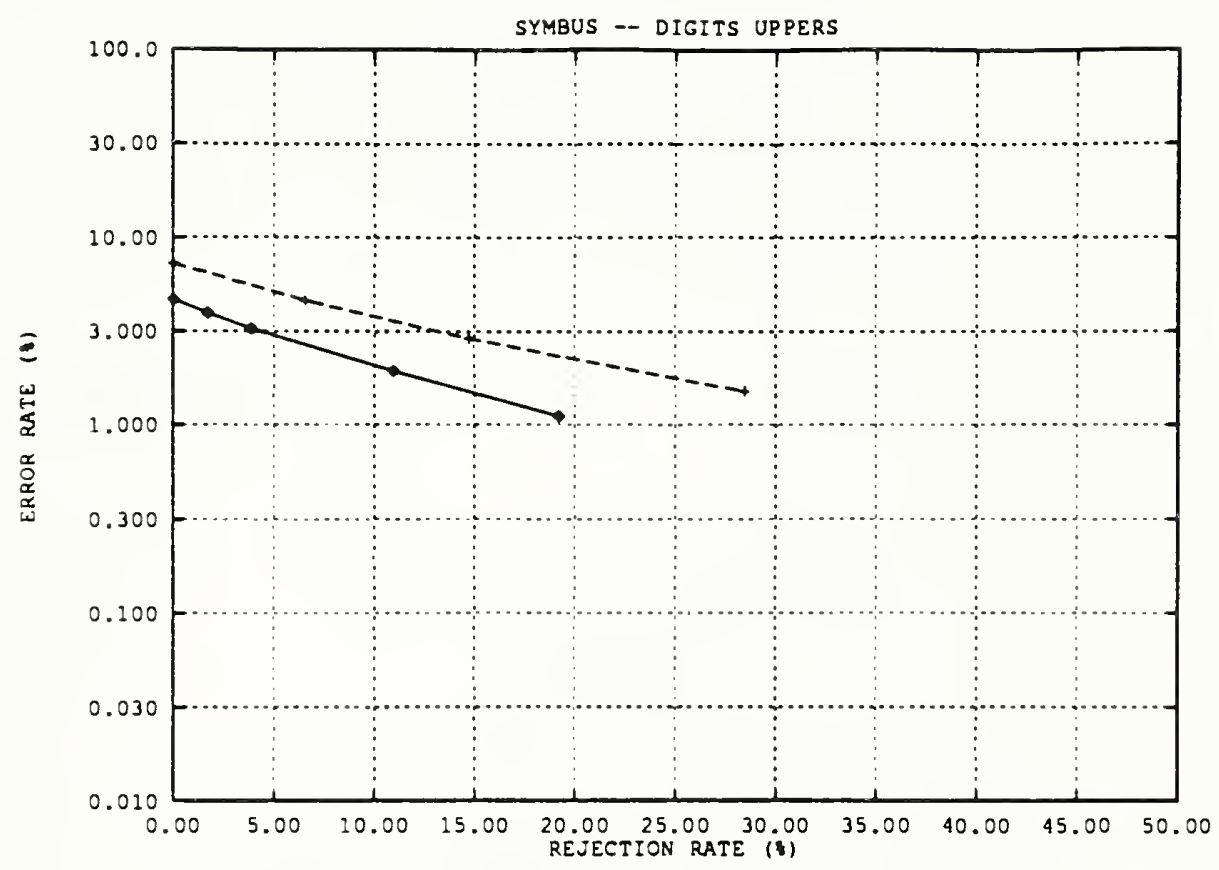

Figure 201: Error rate versus rejection rate for SYMBUS

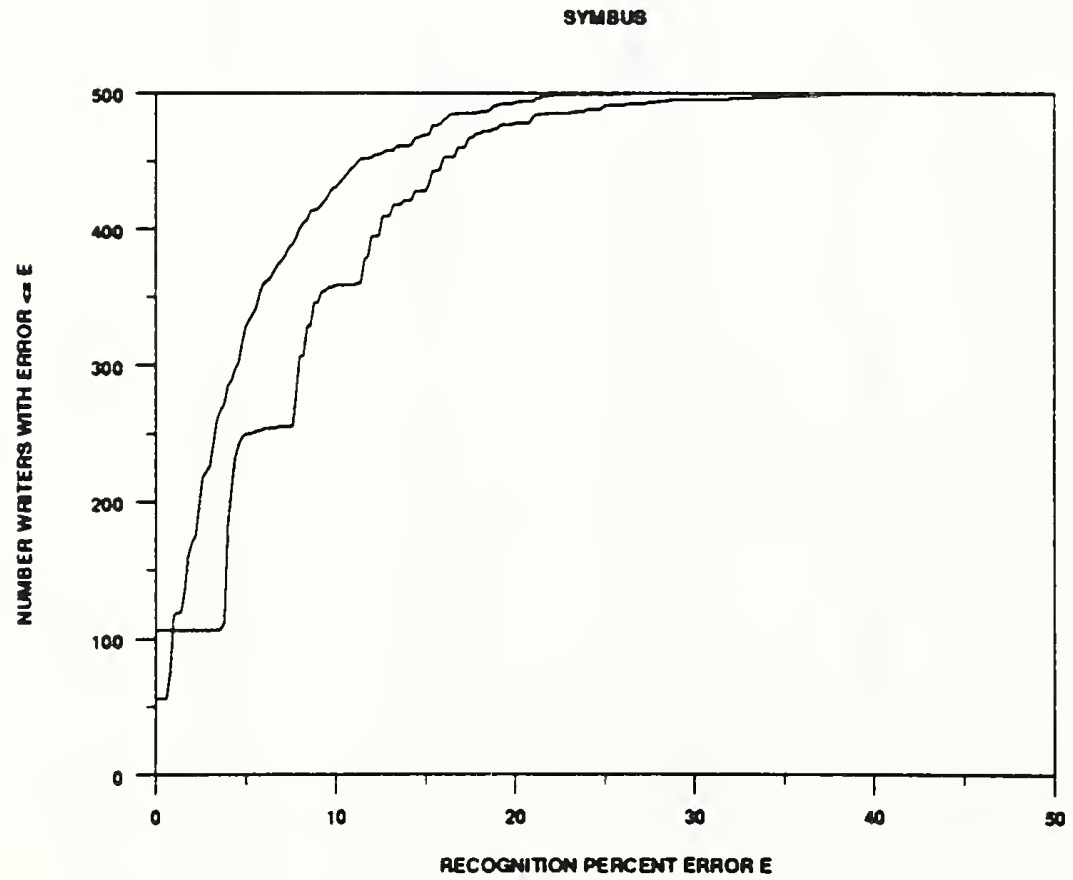

Figure 202: Error rate per writer of SYMBUS 


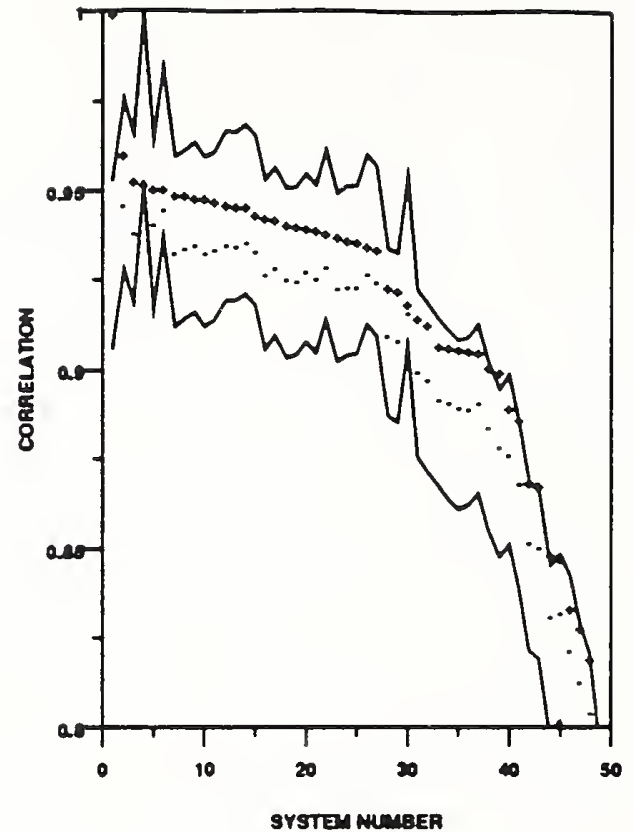

Figure 203: SYMBUS - digit correlation

\begin{tabular}{|c|c|c|c|}
\hline System Number & System Name & Correlation (all) & Correlation (eorrect) \\
\hline$T$ & SYMBUS & 1.0000 & 1.0000 \\
\hline 2 & VOTE_M & 0.9611 & 0.9468 \\
\hline 3 & AEG & 0.8336 & 0.9388 \\
\hline 4 & REFERENCE & 0.9529 & 0.9829 \\
\hline 5 & OCRSYS & 0.8512 & 0.9486 \\
\hline 6 & VOTEA & 0.2512 & 0.9411 \\
\hline 7 & ERIM_l & 0.9494 & 0.9330 \\
\hline 8 & KODAK 2 & 0.8494 & 0.9337 \\
\hline 9 & ATT 2 & 0.9488 & 0.9356 \\
\hline 10 & ATT_4 & 0.8486 & 0.8333 \\
\hline 11 & ERIM_2 & 0.9479 & 0.8345 \\
\hline 12 & ELSAGBد & 0.9467 & 0.9356 \\
\hline 13 & ATT_ & 0.8462 & 0.9361 \\
\hline 14 & ELSAGB2 & 0.9462 & 0.8332 \\
\hline 15 & IBM & 0.9412 & 0.9341 \\
\hline 16 & KODAK_ & 0.8430 & 0.8273 \\
\hline 17 & UBOL & 0.9428 & 0.8294 \\
\hline 18 & NIST_4 & 0.9411 & 0.9260 \\
\hline 18 & THINK_I & 0.9407 & 0.9259 \\
\hline 20 & NESTOR & 0.9406 & 0.9283 \\
\hline 21 & ATT3 & 0.9399 & 0.9268 \\
\hline 22 & THINK 2 & 0.9389 & 0.9297 \\
\hline 23 & ELSAGB_1 & 0.9379 & 0.9240 \\
\hline 24 & HUGHES_2 & 0.8370 & 0.8244 \\
\hline 25 & HUGHES 1 & 0.8366 & 0.9242 \\
\hline 26 & REI & 0.9357 & 0.9280 \\
\hline 27 & NYNEX & 0.9348 & 0.9236 \\
\hline 28 & GTESS.1 & 0.9243 & 0.9108 \\
\hline 29 & GTESS_2 & 0.9234 & 0.9093 \\
\hline 30 & СOMCOM & 0.9197 & 0.8169 \\
\hline 31 & NIST_ & 0.9157 & 0.8006 \\
\hline 32 & GMD_3 & 0.9136 & 0.8981 \\
\hline 33 & MIME & 0.9079 & 0.8927 \\
\hline 34 & ASOL & $0.907 \mathrm{~s}$ & 0.8915 \\
\hline 35 & $\mathrm{NIST}_{2}$ & 0.9068 & 0.8902 \\
\hline 36 & UPENN & 0.9065 & 0.8900 \\
\hline 37 & GMD_l & 0.9088 & 0.8918 \\
\hline 38 & NIST $_{3}$ & 0.9017 & 0.8850 \\
\hline 39 & Riso & 0.9002 & 0.8794 \\
\hline 40 & GMD_4 & 0.8904 & 0.8768 \\
\hline 41 & KAMAN_1 & 0.8873 & 0.8693 \\
\hline 42 & KAMAN_-3 & 0.8698 & 0.8528 \\
\hline 43 & $\mathrm{KAMAN}_{-2}$ & 0.8685 & 0.8512 \\
\hline 44 & GMD_2 & 0.8495 & 0.8320 \\
\hline 45 & KAMAN_s & 0.8484 & 0.8327 \\
\hline 46 & VALEN 2 & 0.8341 & 0.8223 \\
\hline 47 & IFAX & 0.8286 & 0.8134 \\
\hline 48 & VALEN_1 & 0.8201 & 0.8048 \\
\hline 19 & KAMAN-4 & 0.7965 & 0.7800 \\
\hline
\end{tabular}

Table 125: SYMBUS correlation graph key for digits. 


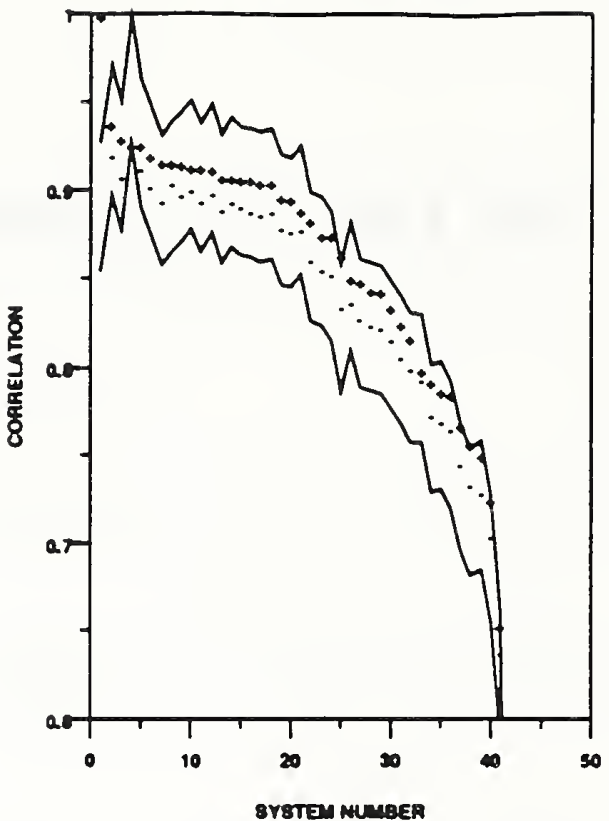

Figure 204: SYMBUS - upper case correlation

\begin{tabular}{|c|c|c|c|}
\hline System Number & System Name & Correlation (sil) & Correlation (correct) \\
\hline 1 & SYMBUS & 1.0000 & 1.0000 \\
\hline 2 & VOTE_M & 0.8382 & 0.8204 \\
\hline 3 & ATT -4 & 0.9308 & 0.9081 \\
\hline 4 & REFERENCE & 0.9271 & 0.9271 \\
\hline 3 & $A E G$ & 0.9268 & 0.8126 \\
\hline 6 & ERIM -1 & 0.9200 & 0.9029 \\
\hline 7 & KODAK_ & 0.9170 & 0.8941 \\
\hline 8 & VOTEP & 0.2168 & 0.2049 \\
\hline 9 & ATT 2 & 0.9188 & 0.8985 \\
\hline 10 & NYNEX & 0.9137 & 0.9008 \\
\hline 11 & UBOL & 09136 & 0.8942 \\
\hline 12 & UMICH_I & 0.2133 & 0.8993 \\
\hline 13 & NESTOA & 0.9082 & 0.8943 \\
\hline 14 & ATT3 & 0.9082 & 0.8897 \\
\hline 13 & IBM & 0.9071 & 0.8913 \\
\hline 16 & HUGHES - 1 & 0.9070 & 0.8893 \\
\hline 17 & HUGHES-2 & 0.9084 & 0.8872 \\
\hline 18 & ATT 1 & 0.9051 & 0.8887 \\
\hline 19 & GTESS-1 & 0.8977 & 0.8794 \\
\hline 20 & GTESS 2 & 0.8963 & 0.8781 \\
\hline 21 & OCRSYS & 0.8801 & 0.8792 \\
\hline 22 & MIME & 0.8843 & 0.8622 \\
\hline 23 & NIST_4 & 0.8764 & 0.8387 \\
\hline 24 & ASOL & 0.8761 & 0.8543 \\
\hline 23 & RISO & 0.8853 & 0.8353 \\
\hline 26 & REI & 0.8318 & 0.8388 \\
\hline 27 & NIST_1 & 08504 & 0.8291 \\
\hline 28 & GMD_1 & 0.8480 & 0.8253 \\
\hline 29 & GMD_3 & 0.8483 & 0.8240 \\
\hline 30 & KAMAN_1 & 0.8361 & 0.8173 \\
\hline 31 & GMD_4 & 0.8270 & 0.8070 \\
\hline 32 & NIST3 & 0.8179 & 0.8003 \\
\hline 33 & COMCOM & 0.7993 & 0.7941 \\
\hline 34 & KAMAN_J & 0.7934 & 0.7741 \\
\hline 35 & IFAX & 0.7877 & 0.7706 \\
\hline 36 & KAMAN_2 & 0.7862 & 0.7858 \\
\hline 37 & NIST 2 & 0.7688 & 0.73 \\
\hline 38 & GMD_2 & 0.7579 & 0.7344 \\
\hline 39 & VALEN_1 & 0.7309 & 0.7301 \\
\hline 40 & KAMAN_4 & 0.7267 & 0.7056 \\
\hline 41 & KAMAN_5 & 0.8542 & 0.6381 \\
\hline 42 & $\mathrm{UMICH}_{2}$ & 0.0424 & 0.0204 \\
\hline
\end{tabular}

Table 126: SYMBUS correlation graph key for uppers. 


\section{No Data Available}

Figure 205: SYMBUS - lower case correlation

There was no dats for this evaluation.

Table 127: SYMBUS correlation graph key for lowers. 
SYSTEM: THINK -1

PARTICIPANT: Stephen Smith

ORGANIZATION: Thinking Machines Corporation, Cambridge, MA

PREPROCESSING: size normalization

FEATURES: template, model, including arcs extracted from a $32 \times 32$ image after normalization.

CLASSIFICATION: distance maps, modified nearest neighbor, modified Haming distance used where each pixel is represented by its distance to the nearest matching pixel.

HARDWARE: $\quad 32,768$ processor CM2 with SUN front end

TRAINING: DIGITS UPPERS LOWERS DATABASE

al1 NA NA NSDB3

STATUS: $\quad$ on time

RESULTS: -- DIGITS -- -- UPPERS -- -- LOWERS -- DATABASE

REJ. ERR. REJ. ERR. REJ. ERR. TESTDATA1

RATE RATE-- RATE RATE-- RATE RATE--

$0.00 \quad 0.0489$

$0.10 \quad 0.0152$

$\begin{array}{lll}0.20 & 0.0059\end{array}$

$0.30 \quad 0.0027$

$0.40 \quad 0.0014$

$0.50 \quad 0.0006$

OCR RATE (CPS): DIGITS UPPERS LOWERS

SYS RATE: $\quad 0.67$

CPU RATE: 
SYSTEM: THINK_1

BIBLIOGRAPHY:

The following references have been provided for this system:

none 


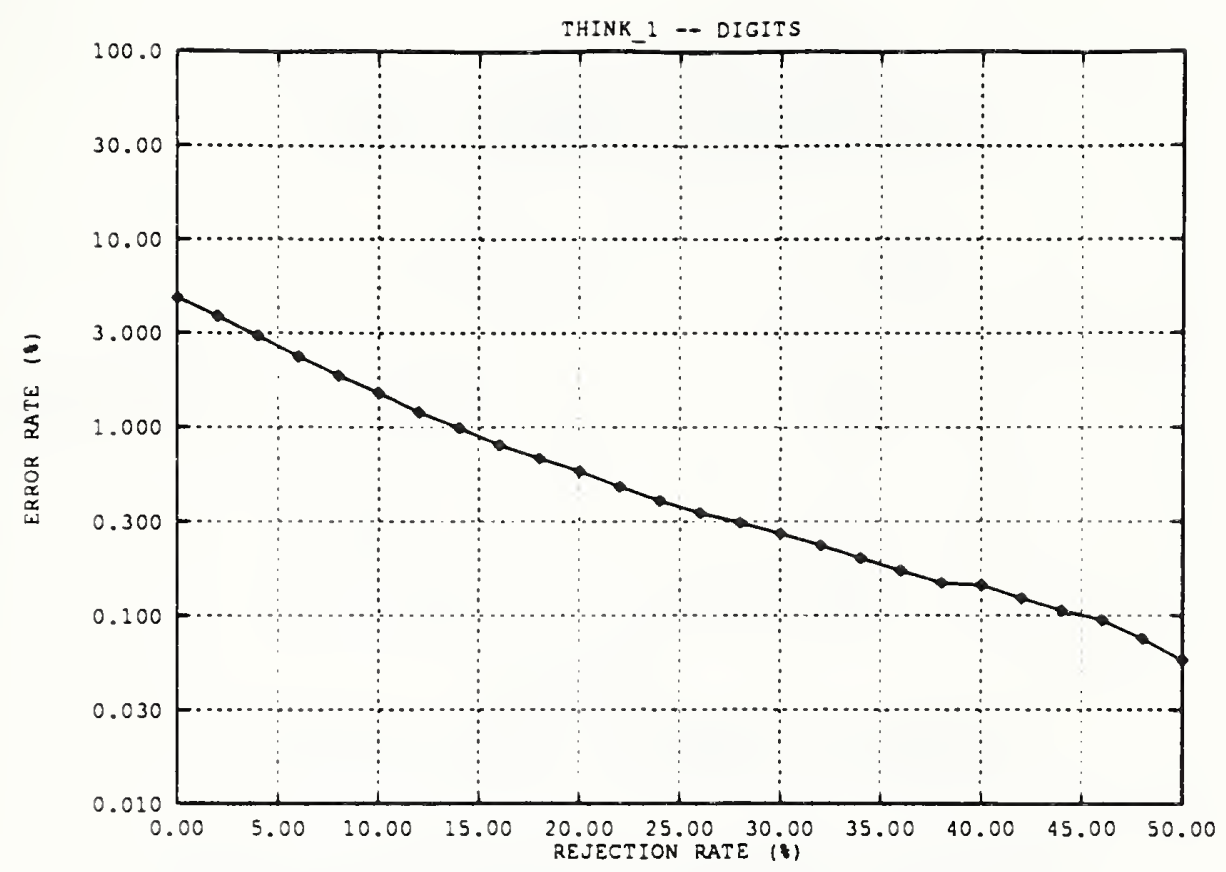

Figure 206: Error rate versus rejection rate for THINK_1

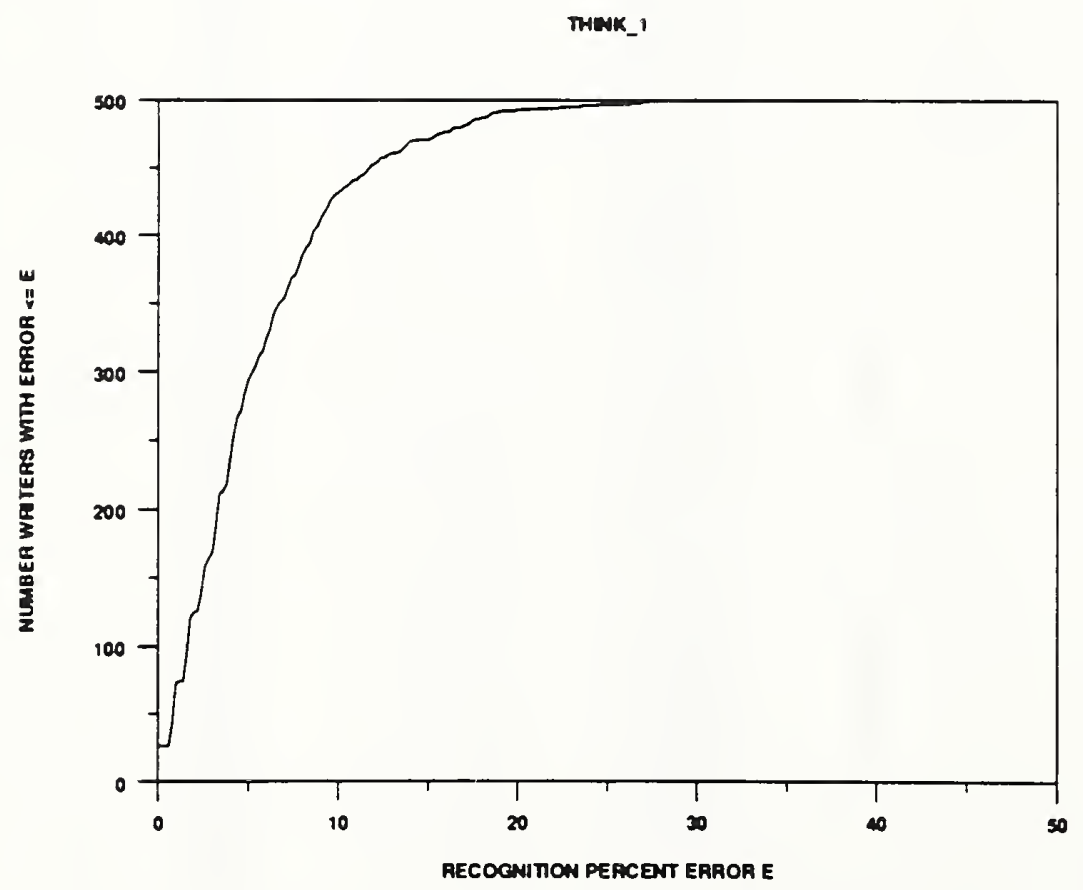

Figure 207: Error rate per writer of THINK_1 


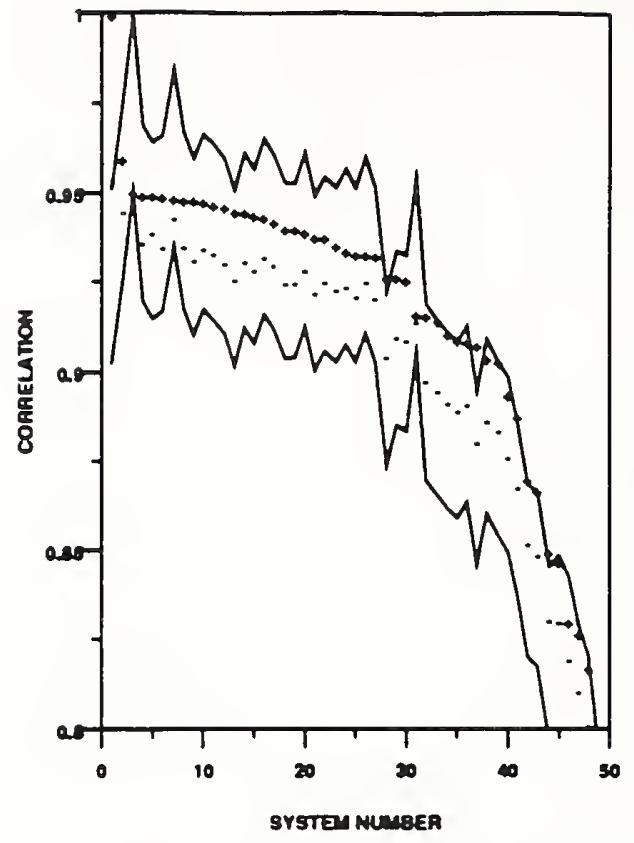

Figure 208: THINK_1 - digit correlation

\begin{tabular}{|c|c|c|c|}
\hline System Number & System Name & Correlation (all) & Correlation (correct) \\
\hline $\mathrm{l}$ & THINK-1 & 10000 & 1.0000 \\
\hline 2 & VOTE_M & 0.8603 & 0.9452 \\
\hline 3 & REFERENCE & 0.2511 & 0.9511 \\
\hline 4 & ATT 1 & 0.8500 & 0.9369 \\
\hline s & VOTEP & 0.9499 & 0.8394 \\
\hline 6 & AEG & 0.9498 & 0.9356 \\
\hline 7 & OCRSYS & 0.8492 & 0.9435 \\
\hline 8 & ELSAGB_3 & 0.8486 & 0.9356 \\
\hline 9 & ATT 4 & 0.9485 & 0.9322 \\
\hline 10 & ELSAGB2 & 0.9482 & 0.9352 \\
\hline 11 & ATT2 & 0.9474 & 0.8341 \\
\hline 12 & KODAK2 & 0.2466 & 0.9312 \\
\hline 13 & NIST_A & 0.9434 & 0.9265 \\
\hline 14 & ERIM_1 & 0.9453 & 0.9316 \\
\hline 15 & UBOL & 0.9445 & 0.9292 \\
\hline 16 & IBM & 0.8440 & 0.9328 \\
\hline 17 & ERIM_2 & 0.9429 & 0.9306 \\
\hline 18 & KODAK」 & 0.8402 & 0.9285 \\
\hline 19 & SYMBUS & 0.9407 & 0.9239 \\
\hline 20 & THINK 2 & 0.8401 & 0.9293 \\
\hline 21 & ELSAGB-1 & 0.9386 & 0.9228 \\
\hline 22 & NESTOR & 0.9384 & 0.9261 \\
\hline 23 & ATT 3 & 0.9364 & 09240 \\
\hline 24 & NYNEX & 0.9350 & 0.9247 \\
\hline 25 & HUGHES_1 & 0.9341 & 0.9220 \\
\hline 26 & REI & 0.9339 & 0.9263 \\
\hline 27 & HUGHES_2 & 0.9336 & 0.9217 \\
\hline 28 & NIST_1 & 0.9277 & 0.9048 \\
\hline 29 & GTESS_-1 & 0.9273 & 0.9107 \\
\hline 30 & GTESS 2 & 0.9268 & 0.9098 \\
\hline 31 & COMCOM & 0.9171 & 0.9145 \\
\hline 32 & GMD_J & 0.9167 & 0.8979 \\
\hline 33 & MIME & 0.9150 & 08952 \\
\hline 34 & ASOL & 0.9115 & 0.8922 \\
\hline 35 & NIST 2 & 0.9097 & 0.8900 \\
\hline 36 & GMD_1 & 0.9093 & 0.8916 \\
\hline 37 & RISO & 0.9080 & 0.8812 \\
\hline 38 & UPENN & 0.9047 & 0.8873 \\
\hline 39 & NIST_3 & 0.9037 & 0.8845 \\
\hline 40 & GMD_4 & 0.8944 & 0.8771 \\
\hline 41 & KAMAN-1 & 0.8887 & 0.8687 \\
\hline 42 & KAMAN_J & 0.8710 & 0.8525 \\
\hline 43 & KAMAN_2 & 0.8678 & 0.8494 \\
\hline 44 & GMD 2 & 0.8802 & 0.8312 \\
\hline 45 & KAMAN_5 & 0.8474 & 0.8307 \\
\hline 46 & VALEN 2 & 0.8308 & 0.8201 \\
\hline 47 & IFAX & 0.8273 & 0.8114 \\
\hline 48 & VALEN-1 & 0.8178 & 0.8015 \\
\hline 49 & KAMAN-4 & 0.7962 & 0.7780 \\
\hline
\end{tabular}

Table 128: THINK_1 correlation graph key for digits. 


\section{No Data Available}

Figure 209: THINK_1 - upper case correlation

There was no data for this evaluation.

Table 129: THINK_1 correlation graph key for uppers. 


\section{No Data Available}

Figure 210: THINK_1 - lower case correlation

There was no data for this evaluation

Table 130: THINK_1 correlation graph key for lowers. 
SYSTEM : UBOL

PARTICIPANT: Dr. Zsolt M. Kovacs-V.

ORGANIZATION: University of Bologna, Bologna, Italy

PREPROCESSING: noise removal, slant normalization, thinning, and size normalization to $32 \times 32$. Then a distance transform is performed on the background and a further reduction is performed to $8 \times 8$. This provides

a 64-dimensional feature vector.

FEATURES: rule-based distance transform

CLASSIFICATION: KNN with novel metric

HARDWARE: simulation of CM2 with $64 \mathrm{~K}$ processors on SPARC

TRAINING : $\quad$ DIGITS

UPPERS LOWERS DATABASE

$\begin{array}{llll}72000 & \text { all all NSDB3 }\end{array}$

STATUS: $\quad$ on time

RESULTS: -- DIGITS -- -- UPPERS -- -- LOWERS -- DATABASE

REJ. ERR. REJ. ERR. REJ. ERR. TESTDATA1

RATE RATE-- RATE RATE-- RATE RATE--

$\begin{array}{lllllll}0.00 & 0.0435 & 0.00 & 0.0624 & 0.00 & 0.1548\end{array}$

$\begin{array}{lllllll}0.04 & 0.0271 & 0.03 & 0.0506 & 0.04 & 0.1365\end{array}$

$\begin{array}{lllllll}0.06 & 0.0215 & 0.06 & 0.0390 & 0.11 & 0.1107\end{array}$

$\begin{array}{lllllll}0.07 & 0.0184 & 0.09 & 0.0334 & 0.17 & 0.0909\end{array}$

$\begin{array}{lllllll}0.09 & 0.0148 & 0.11 & 0.0282 & 0.22 & 0.0745\end{array}$

$\begin{array}{lllllll}0.11 & 0.0122 & 0.15 & 0.0221 & 0.25 & 0.0655\end{array}$

$\begin{array}{lllllll}0.13 & 0.0108 & 0.18 & 0.0197 & 0.28 & 0.0564\end{array}$

$\begin{array}{lllllll}0.15 & 0.0096 & 0.20 & 0.0171 & 0.33 & 0.0436\end{array}$

$\begin{array}{lllllll}0.17 & 0.0086 & 0.25 & 0.0130 & 0.37 & 0.0379\end{array}$

$\begin{array}{lllllll}0.19 & 0.0079 & 0.31 & 0.0105 & 0.42 & 0.0287\end{array}$

OCR RATE (CPS): DIGITS UPPERS LOWERS
SYS RATE:
0.06
0.09
0.04

CPU RATE:

0.08

0.10

0.05 
SYSTEM: UBOL

BIBLIOGRAPHY:

The following references have been provided for this system:

$[43][44][45][46][47][12][6][48][49]$ 


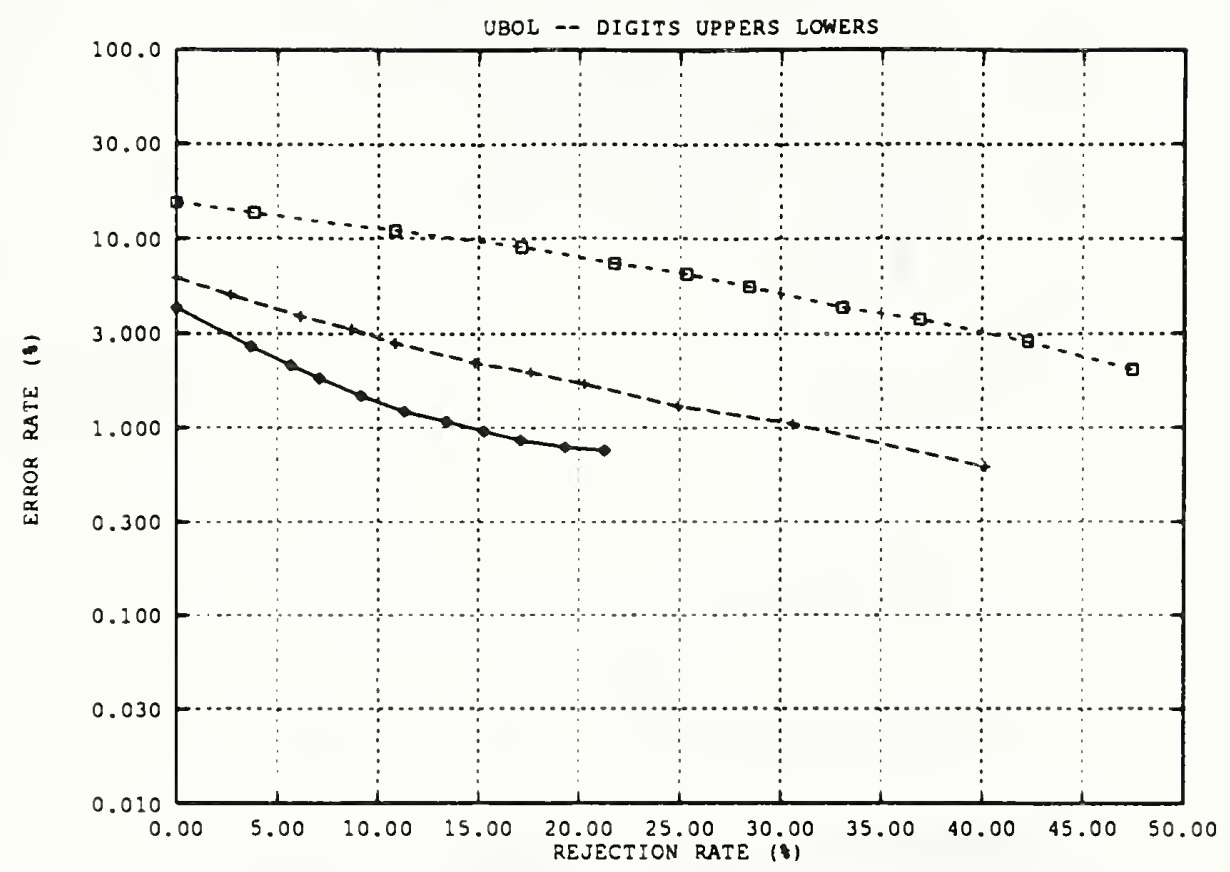

Figure 211: Error rate versus rejection rate for UBOL

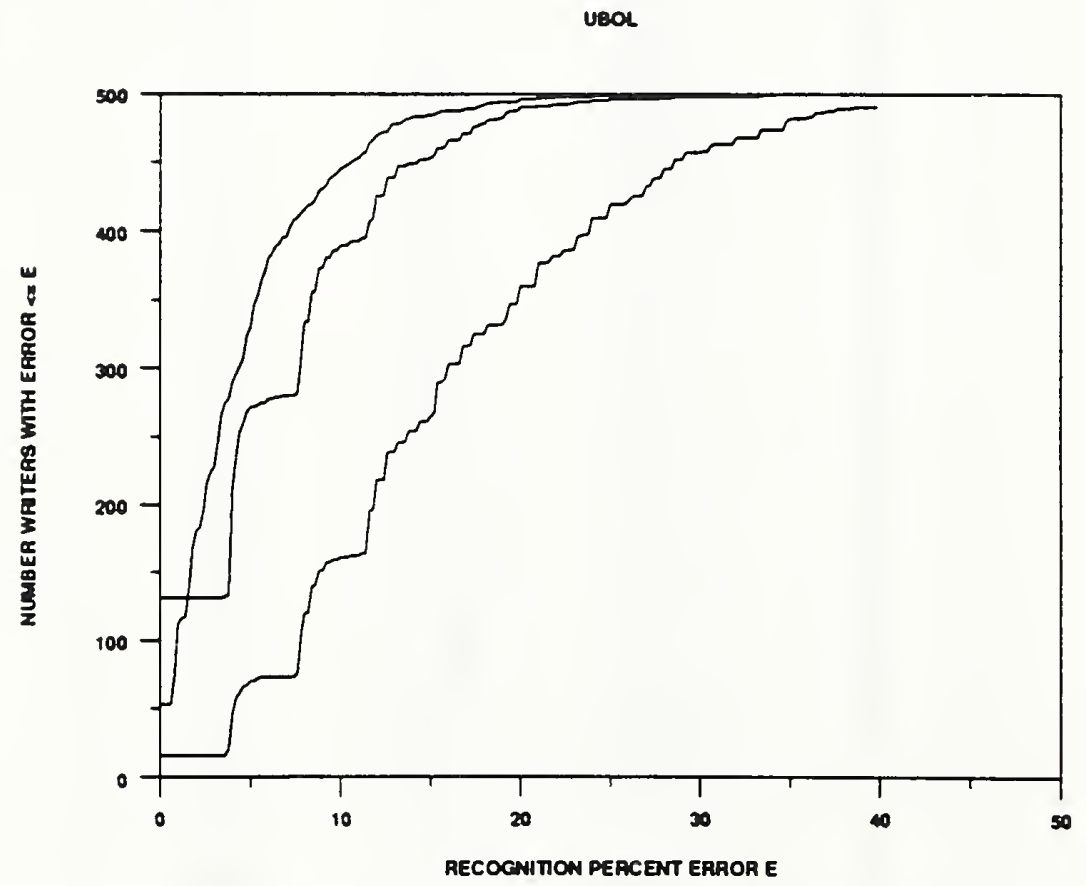

Figure 212: Error rate per writer of UBOL 


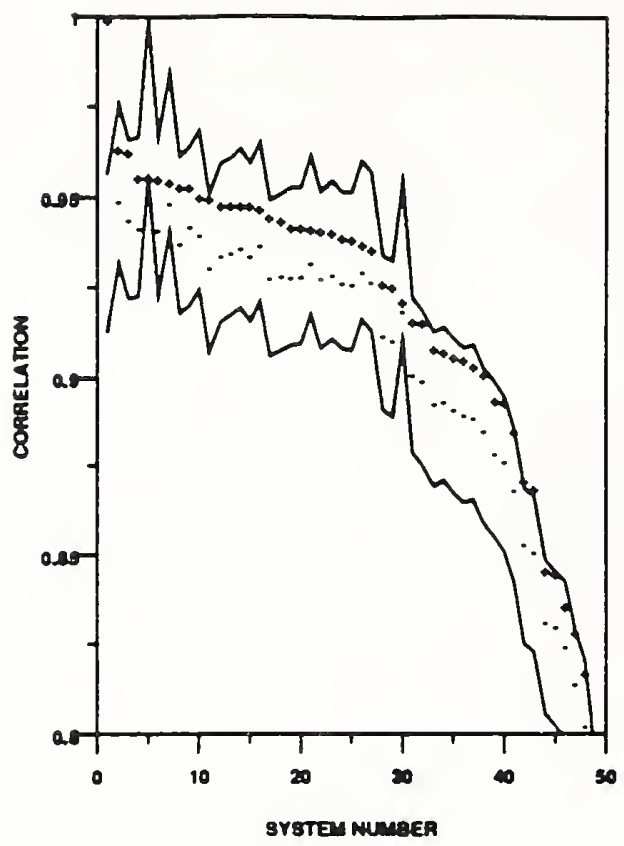

Figure 213: UBOL - digit correlation

\begin{tabular}{|c|c|c|c|}
\hline System Number & System Name & Correlasion (all) & Correlasion (correct) \\
\hline 1 & UEOL & 1.0000 & 1.0000 \\
\hline 2 & VOTE_M & 0.8644 & 0.9496 \\
\hline 3 & $A E G$ & 0.8632 & 0.8446 \\
\hline 4 & ELSAGB & 0.2866 & 0.8420 \\
\hline 3 & REFERENCE & 0.8368 & 0.8568 \\
\hline 6 & ELSAGB 2 & 0.0561 & 0.8416 \\
\hline 7 & OCRSYS & 0.8552 & 0.9492 \\
\hline 8 & ERIM-1 & 0.2536 & 0.8382 \\
\hline 9 & VOTEP & 0.8535 & 0.9428 \\
\hline 10 & ATT_ & 0.8511 & 0.8403 \\
\hline 11 & NIST -4 & 0.2808 & 0.8316 \\
\hline 12 & ATT -4 & 0.8488 & 0.8348 \\
\hline 13 & ERIM 2 & 0.8487 & 0.9389 \\
\hline 14 & ATT 2 & 0.8486 & 0.8368 \\
\hline 15 & KODAK2 & 0.8485 & 0.8349 \\
\hline 16 & IBM & 0.9477 & 0.9374 \\
\hline 17 & ELSAGB-A & 0.2455 & 0.9287 \\
\hline 18 & THINK-1 & 0.8445 & 0.9292 \\
\hline 18 & SYMBUS & 0.2425 & 0.8294 \\
\hline 20 & KODAK_ & 0.9425 & 0.8280 \\
\hline 21 & THINK 2 & 0.2423 & 0.9328 \\
\hline 22 & ATT 3 & 0.8417 & 0.9289 \\
\hline 23 & NESTOR & 0.2415 & 0.8298 \\
\hline 24 & HUGHES_- 1 & 0.8401 & 0.8275 \\
\hline 25 & HUGHES 2 & 0.9396 & 0.8272 \\
\hline 26 & REI & 0.8379 & 0.9307 \\
\hline 27 & NYNEX & 0.8366 & 0.8282 \\
\hline 28 & GTESS 1 & 0.2276 & 0.8128 \\
\hline 28 & GTESS 2 & 0.8263 & 0.8116 \\
\hline 30 & COMCOM & 0.8224 & 0.8198 \\
\hline 31 & NIST_A & 0.8170 & 0.2018 \\
\hline 32 & GMD_3 & 0.8165 & 0.8989 \\
\hline 33 & GMD - 1 & 0.8091 & 0.8235 \\
\hline 34 & MIME & 0.8081 & 0.8941 \\
\hline 35 & ASOL & 0.8067 & 0.8918 \\
\hline 36 & NIST 2 & 0.8059 & 0.8902 \\
\hline 37 & UPENN & 0.2041 & 0.8898 \\
\hline 38 & NIST & 0.8016 & 0.8857 \\
\hline 39 & GMD_4 & 0.8944 & 0.8792 \\
\hline 40 & RISO & 0.8941 & 0.8771 \\
\hline 41 & KAMAN-1 & 0.8857 & 0.8691 \\
\hline 42 & KAMAN_3 & 0.8720 & 0.8842 \\
\hline 43 & KAMAN.2 & 0.8698 & 0.8318 \\
\hline 44 & KAMAN 5 & 0.8467 & 0.8321 \\
\hline 45 & GMD _2 & 0.8458 & 0.8307 \\
\hline 48 & VALEN 2 & 0.8365 & $0.82 \mathrm{BL}$ \\
\hline 47 & IFAX & 0.8293 & 0.8143 \\
\hline 48 & VALEN_I & 0.8177 & $0.803 \mathrm{~L}$ \\
\hline 49 & KAMAN_4 & 0.7890 & 0.7809 \\
\hline
\end{tabular}

Table 131: UBOL correlation graph key for digits. 


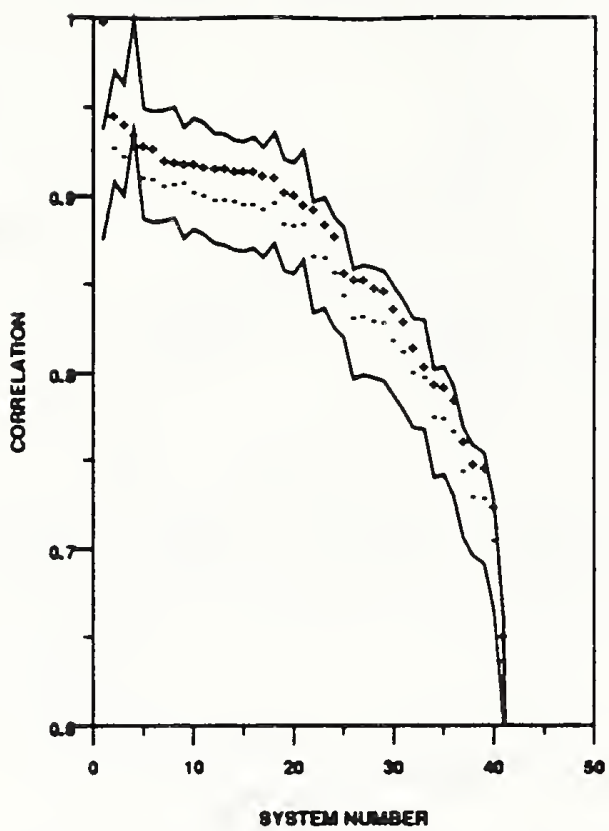

Figure 214: UBOL - upper case correlation

\begin{tabular}{|c|c|c|c|}
\hline Syalem Number & System Name & Correlation (all) & Coprelation (correct) \\
\hline $\mathrm{I}$ & UBOL & 1.0000 & 1.0000 \\
\hline 2 & VOTEM & 0.9481 & 0.9299 \\
\hline 3 & AEG & 0.9433 & 0.9246 \\
\hline 4 & REFERENCE & 0.9376 & 0.9376 \\
\hline 3 & ATT 4 & 0.9313 & 0.9132 \\
\hline 6 & ERIM-I & 0.9290 & 0.9116 \\
\hline 7 & UMICH-I & 0.9231 & 0.9080 \\
\hline 8 & NYNEX & $0.922 \mathrm{~s}$ & 0.9091 \\
\hline 9 & VOTEP & 0.9215 & 0.9104 \\
\hline 10 & ATT 2 & 0.9210 & 0.9049 \\
\hline 11 & NESTOR & 0.8194 & 0.9030 \\
\hline 12 & HUGHES-I & 0.9188 & 0.8997 \\
\hline 13 & ATT $\perp$ & 0.9186 & 0.8998 \\
\hline 14 & ATT 3 & 0.9169 & 0.8989 \\
\hline 15 & KODAK_ & 0.9168 & 0.8977 \\
\hline 16 & HUGHES_2 & 0.9168 & $0.897 \mathrm{~s}$ \\
\hline 17 & SYMBUS & 0.9136 & 0.8942 \\
\hline 18 & IBM & 0.9126 & 0.8979 \\
\hline 19 & GTESS_l & 0.8044 & 0.8865 \\
\hline 20 & GTESS 2 & 0.9031 & 0.8840 \\
\hline 21 & OCRSYS & 0.8972 & 0.8861 \\
\hline 22 & NIST _4 & 0.8941 & 0.8692 \\
\hline 23 & MIME & 0.8867 & 0.8681 \\
\hline 24 & ASOL & 0.8797 & 0.8598 \\
\hline 25 & REI & 0.8596 & 0.8463 \\
\hline 26 & NIST 1 & 0.8537 & 0.8346 \\
\hline 27 & RISO & 0.8537 & 0.8337 \\
\hline 28 & GMD_1 & 0.8510 & 0.8324 \\
\hline 29 & GMD_3 & 0.8496 & 0.8308 \\
\hline 30 & KAMAN_1 & 0.8395 & 0.8213 \\
\hline 31 & GMD_4 & 0.8323 & 0.8144 \\
\hline 32 & הIST & 0.8172 & 0.8028 \\
\hline 33 & СОMСOM & 0.8060 & 0.7999 \\
\hline 34 & KAMAN_3 & 0.7958 & 0.7774 \\
\hline 35 & IFAX & 0.7943 & 0.7762 \\
\hline 36 & KAMAN_2 & 0.7870 & 0.7684 \\
\hline 37 & NIST 2 & 0.7633 & 0.7468 \\
\hline 38 & VALEN_I & 0.7503 & 0.7315 \\
\hline 39 & GMD -2 & 0.7488 & 0.7311 \\
\hline 40 & KAMAN_A & 0.7262 & 0.7070 \\
\hline 11 & KAMAN_S & 0.6831 & 0.8386 \\
\hline 12 & UMICH 2 & 0.0448 & 0.0211 \\
\hline
\end{tabular}

Table 132: UBOL correlation graph key for uppers. 


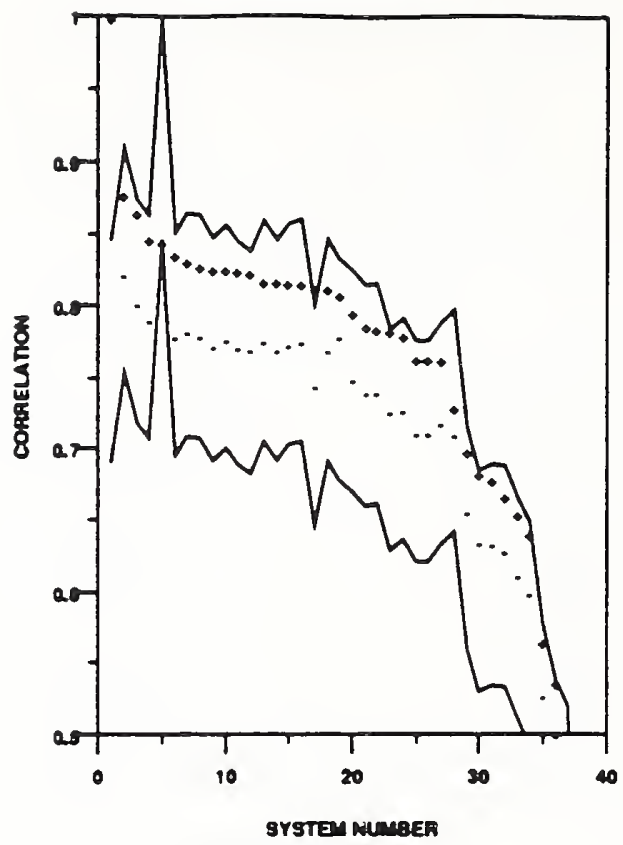

Figure 215: UBOL - lower case correlation

\begin{tabular}{|c|c|c|c|}
\hline System Number & System Name & Correlation (ali) & Correlation (correct) \\
\hline 1 & UBOL & 1.0000 & 1.0000 \\
\hline 2 & VOTE_M & 0.8784 & 0.8238 \\
\hline 3 & $A E G$ & 0.8663 & 0.8026 \\
\hline 4 & ERIM_1 & 0.8471 & 0.7914 \\
\hline 5 & REFERENCE & 0.8452 & 0.8452 \\
\hline 6 & UMICHAI & 0.8373 & 0.7800 \\
\hline 7 & OCRSYS & 0.8320 & 0.7828 \\
\hline 8 & ATT & 0.8288 & 0.7796 \\
\hline 9 & HUGHES - 1 & 0.8273 & 0.7732 \\
\hline 10 & KODAK」 & 0.8267 & 0.7778 \\
\hline 11 & HUGHES -2 & 0.8257 & 0.7715 \\
\hline 12 & ATT 3 & 0.8248 & 0.7704 \\
\hline 13 & ATT 2 & 0.8188 & 0.7763 \\
\hline 14 & IBM & 0.8184 & 0.7706 \\
\hline 15 & ATT_A & 0.8173 & 0.7740 \\
\hline 16 & NYNEX & 0.8172 & 0.7761 \\
\hline 17 & NIST_4 & 0.8115 & 0.7449 \\
\hline 18 & NESTOR & 0.8137 & 0.7701 \\
\hline 19 & VOTEP & 0.8097 & 0.7793 \\
\hline 20 & GTESS-1 & 0.7965 & 0.7504 \\
\hline 21 & NIST_l & 0.7883 & 0.7409 \\
\hline 22 & GTESS2 & 0.7851 & 0.7410 \\
\hline 23 & RISO & 0.7843 & 0.7269 \\
\hline 24 & GMD.3 & 0.7809 & 0.7283 \\
\hline 28 & GMD_-4 & 07844 & 0.7122 \\
\hline 26 & GMD.1 & 0.7644 & 0.7122 \\
\hline 27 & ASOL & 0.7634 & 0.7189 \\
\hline 28 & NIST & 0.7305 & 0.7105 \\
\hline 29 & GMD_2 & 0.6998 & 0.6568 \\
\hline 30 & VALEN_L & 0.6848 & 0.6347 \\
\hline 31 & KAMAN-1 & 0.6802 & 0.6338 \\
\hline 32 & NIST 2 & 0.6884 & 0.6291 \\
\hline 33 & KAMAN_J & 0.6362 & 0.6116 \\
\hline 34 & KAMAN_2 & 0.6417 & 0.5992 \\
\hline 35 & KAMAN 3 & 0.5667 & 0.3284 \\
\hline 36 & $K A M A N-4$ & 0.5372 & 0.3010 \\
\hline 37 & COMCOM & 0.4919 & 0.4798 \\
\hline 38 & $\mathrm{UMICH}_{2}$ & 0.0966 & 0.0467 \\
\hline
\end{tabular}

Table 133: UBOL correlation graph key for lowers. 
SYSTEM: UPENN

PARTICIPANT: Thomas Fontaine

ORGANIZATION: University of Pennsylvania, Philadelphia, PA

FEATURES: local receptor fields

CLASSIFICATION: Spatio-temporal connectionist model. Learning using a gradient-based technique. Shift invariance is achieved along temporalized directions.

HARDWARE: IBM RS/6000

TRAINING: DIGITS UPPERS LOWERS DATABASE $5400 \quad$ USPS

STATUS: $\quad$ on time

RESULTS: -- DIGITS -- -- UPPERS -- -- LOWERS -- DATABASE

REJ. ERR. REJ. ERR. REJ. ERR. TESTDATA1

RATE RATE-- RATE RATE-- RATE RATE--

$0.00 \quad 0.0908$

$0.10 \quad 0.0517$

$0.20 \quad 0.0277$

$0.30 \quad 0.0169$

$0.40 \quad 0.0122$

$0.50 \quad 0.0102$

OCR RATE (CPS): DIGITS

UPPERS LOWERS

SYS RATE:

0.50

NA

NA

CPU RATE: 
SYSTEM: UPENN

\section{BIBLIOGRAPHY:}

The following references have been provided for this system:

$[50][51][37][56][57][52][5][53][54][55]$ 


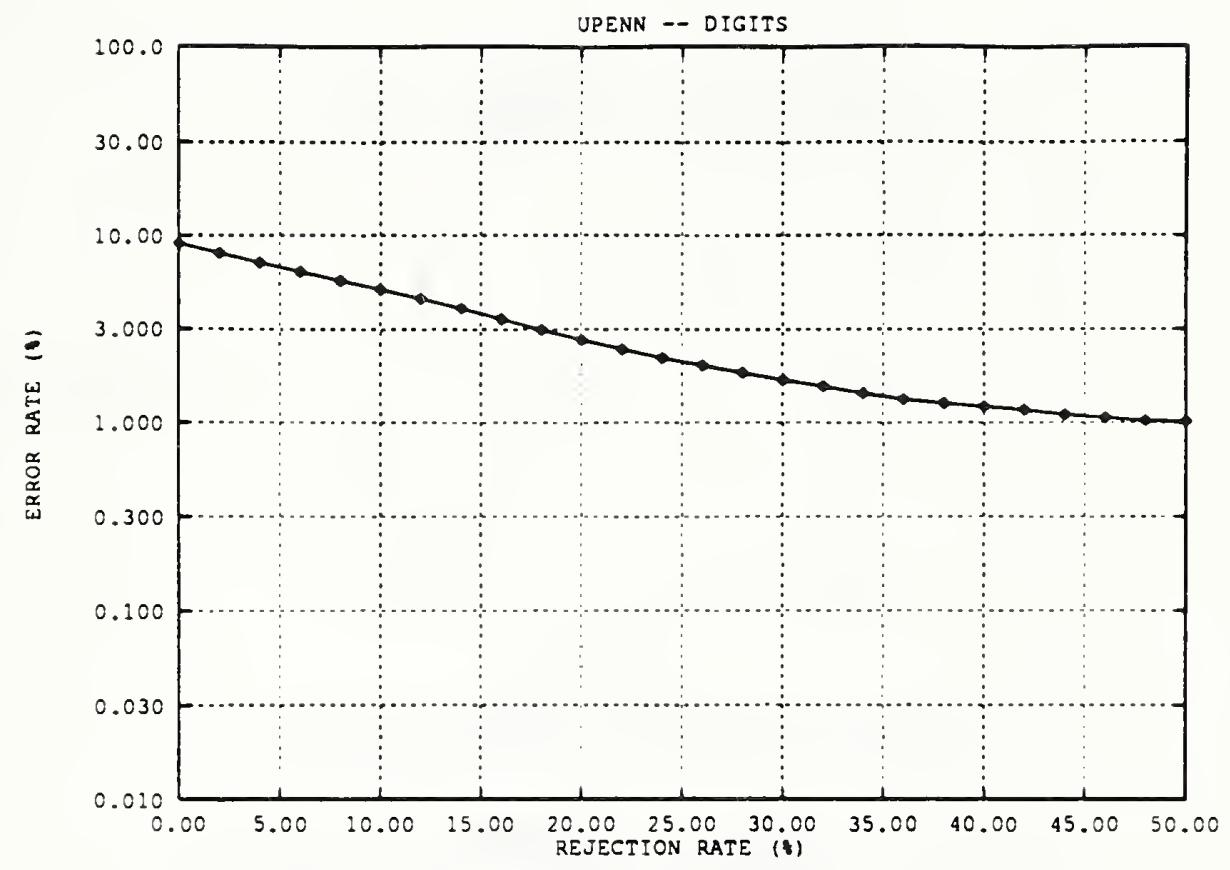

Figure 216: Error rate versus rejection rate for UPENN

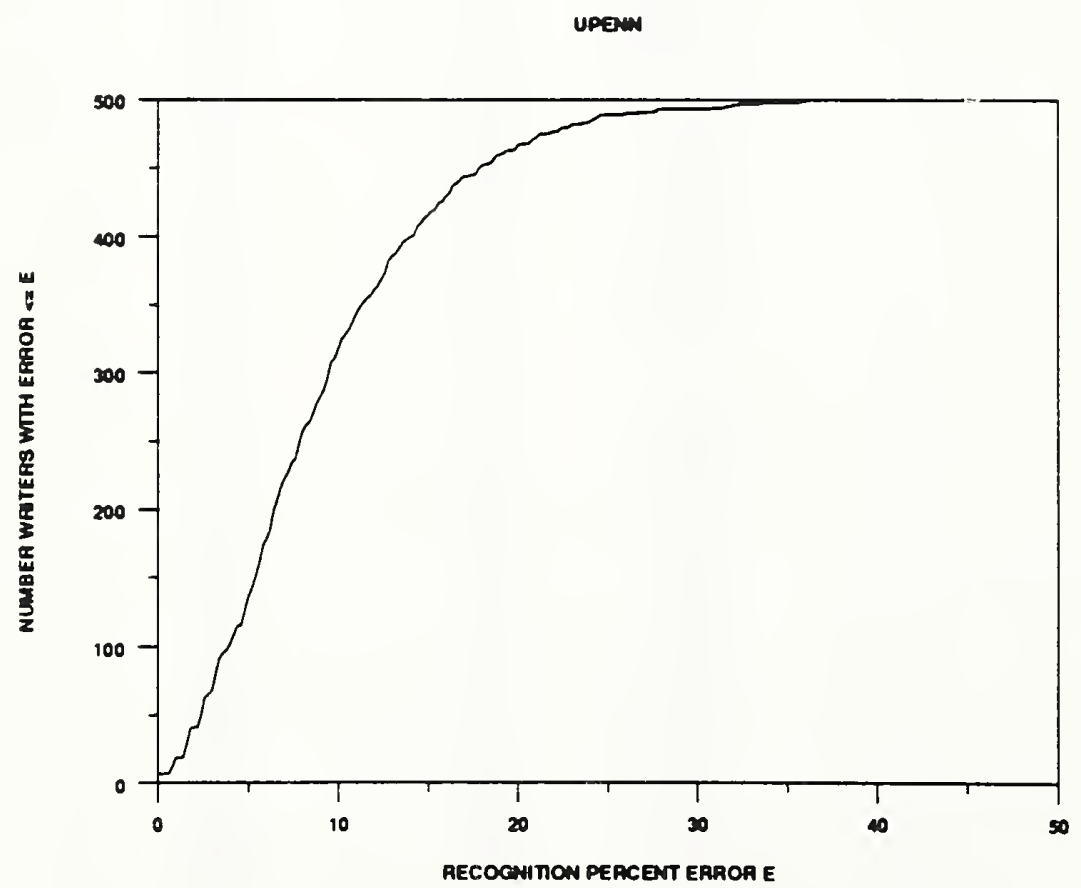

Figure 217: Error rate per writer of UPENN 


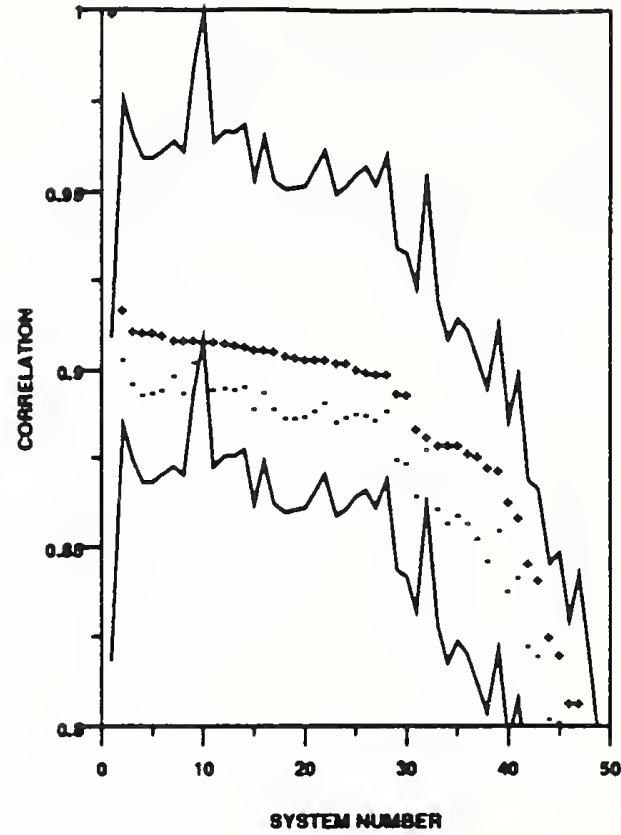

Figure 218: UPENN - digit correlation

\begin{tabular}{|c|c|c|c|}
\hline System Number & System Name & Correlation (ali) & Correlation (correct) \\
\hline 1 & UPENN & 1.0000 & 1.0000 \\
\hline 2 & VOTE_M & 0.8183 & 0.8041 \\
\hline 3 & AEG & 0.9124 & 0.8074 \\
\hline 4 & A T T $T_{-4}$ & 0.9120 & 08942 \\
\hline 5 & KODAK 2 & 0.8119 & 0.8945 \\
\hline 6 & ERIM_-1 & 0.9110 & 0.8952 \\
\hline 7 & VOTEP & 0.9096 & 0.8995 \\
\hline 8 & OCRSYS & 0.9094 & 0.8031 \\
\hline 8 & ERIM_2 & 0.8094 & 0.8945 \\
\hline 10 & REFERENCE & 0.9092 & 0.8082 \\
\hline 11 & ATT 2 & 0.8081 & 0.8853 \\
\hline 12 & ELSAGB3 & 0.2086 & 0.8958 \\
\hline 13 & ELSAGB2 & 0.8080 & 0.8853 \\
\hline 14 & ATT $\lrcorner$ & 0.9079 & 0.8081 \\
\hline 15 & KODAK_ & 0.8070 & 0.8807 \\
\hline 16 & IBM & 0.8069 & 0.8945 \\
\hline 17 & SYMBUS & 0.8065 & 0.8900 \\
\hline 18 & NIST-4 & 0.8031 & 0.8873 \\
\hline 19 & THINK_I & 0.8047 & 0.8873 \\
\hline 20 & HUGHES.1 & 0.8043 & 0.8877 \\
\hline 21 & THINK 2 & 0.9041 & 0.8015 \\
\hline 22 & UBOL & 0.8041 & 0.8896 \\
\hline 23 & ELSAGB-1 & 0.9033 & 0.8861 \\
\hline 24 & HUGHES_2 & 0.8031 & 0.8874 \\
\hline 23 & NESTOR & 0.8013 & 0.8884 \\
\hline 26 & NYNEX & 0.2002 & 0.8880 \\
\hline 27 & REI & 0.8988 & 0.8896 \\
\hline 28 & ATTT & 0.8998 & 0.8867 \\
\hline 28 & GTESS_1 & 0.8945 & 0.8754 \\
\hline 30 & GTESS 2 & 0.8941 & 0.8748 \\
\hline 31 & NIST_I & 0.8845 & 0.8654 \\
\hline 32 & СOMCOM & 0.8818 & 0.8782 \\
\hline 33 & GMD_3 & 0.8798 & 0.8620 \\
\hline 34 & MIME & 0.8797 & 0.8589 \\
\hline 35 & $\mathrm{NIST}_{2}$ & 08787 & 0.8578 \\
\hline 36 & ASOL & 0.8773 & 0.8577 \\
\hline 37 & NIST & 0.8763 & 0.8537 \\
\hline 38 & Riso & 0.8739 & 0.8470 \\
\hline 39 & GMD_1 & 0.8727 & 0.8558 \\
\hline 40 & KAMAN-1 & 0.8840 & 0.8387 \\
\hline 41 & GMD_4 & 0.8584 & 0.8425 \\
\hline 42 & KAMAN_3 & 0.8466 & 0.8232 \\
\hline 43 & KAMAN_2 & 0.8423 & 0.8203 \\
\hline 44 & GMD 2 & 0.8258 & 0.8031 \\
\hline 45 & KAMAN_S & 0.8211 & 0.8022 \\
\hline 46 & IFAX & 0.8078 & 0.7862 \\
\hline 47 & VALEN 2 & 0.8076 & 0.7928 \\
\hline 48 & VALEN-1 & 0.7836 & 0.7739 \\
\hline 49 & KAMAN_4 & 0.7740 & 0.7517 \\
\hline
\end{tabular}

Table 134: UPENN correlation graph key for digits. 


\section{No Data Available}

Figure 219: UPENN - upper case correlation

There was no dala for this evaluation.

Table 135: UPENN correlation graph key for uppers. 


\section{No Data Available}

Figure 220: UPENN - lower case correlation

There was no data for this evaluation.

Table 136: UPENN correlation graph key for lowers. 
SYSTEM: VALEN_. 1

PARTICIPANT: Enrique Vidal

ORGANIZATION: Universidad Politecnica de Valencia, Valencia, Spain

FEATURES: line fit features

CLASSIFICATION: KNN Or NN with BP

HARDWARE: $\quad$ model 380 HP-9000

TRAINING: DIGITS UPPERS LOWERS DATABASE

STATUS: on time

RESULTS: -- DIGITS -- -- UPPERS -- -- LOWERS -- DATABASE

REJ. ERR. REJ. ERR. REJ. ERR. TESTDATA1

RATE RATE-- RATE RATE-- RATE RATE--

$\begin{array}{llllll}0.00 & 0.1795 & 0.00 & 0.2418 & 0.00 & 0.3160\end{array}$

$\begin{array}{lllllll}0.10 & 0.1358 & 0.10 & 0.2023 & 0.10 & 0.2813\end{array}$

$\begin{array}{lllllll}0.20 & 0.0971 & 0.20 & 0.1633 & 0.20 & 0.2460\end{array}$

$\begin{array}{lllllll}0.30 & 0.0647 & 0.30 & 0.1331 & 0.30 & 0.2096\end{array}$

$\begin{array}{lllllll}0.40 & 0.0422 & 0.40 & 0.1048 & 0.40 & 0.1786\end{array}$

$\begin{array}{lllllll}0.50 & 0.0275 & 0.50 & 0.0799 & 0.50 & 0.1468\end{array}$

OCR RATE (CPS): DIGITS UPPERS LOWERS

$\begin{array}{lrrr}\text { SYS RATE: } & 5.15 & 3.14 & 3.14 \\ \text { CPU RATE: } & 18.18 & 5.58 & 5.58\end{array}$


SYSTEM: VALEN_1

The following references have been provided for this system:

[58] 


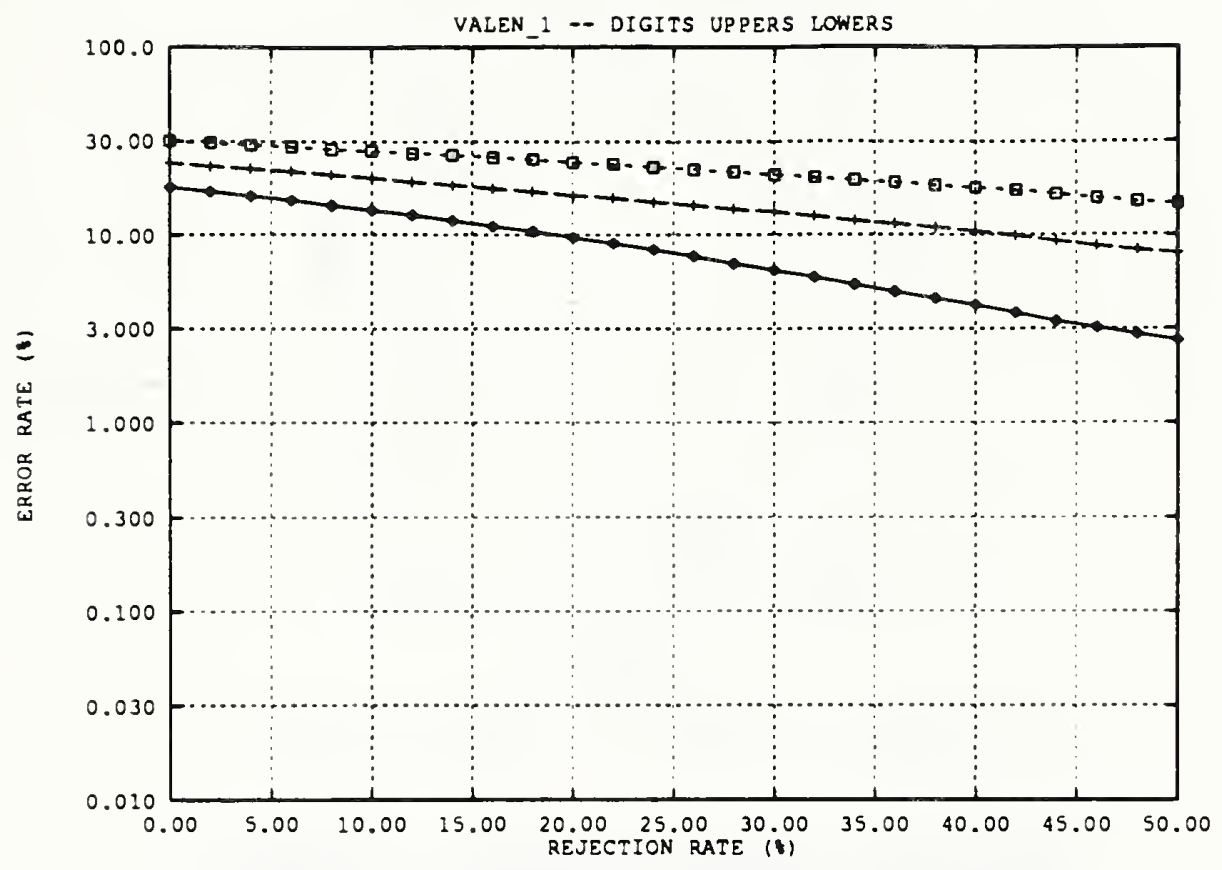

Figure 221: Error rate versus rejection rate for VALEN 1

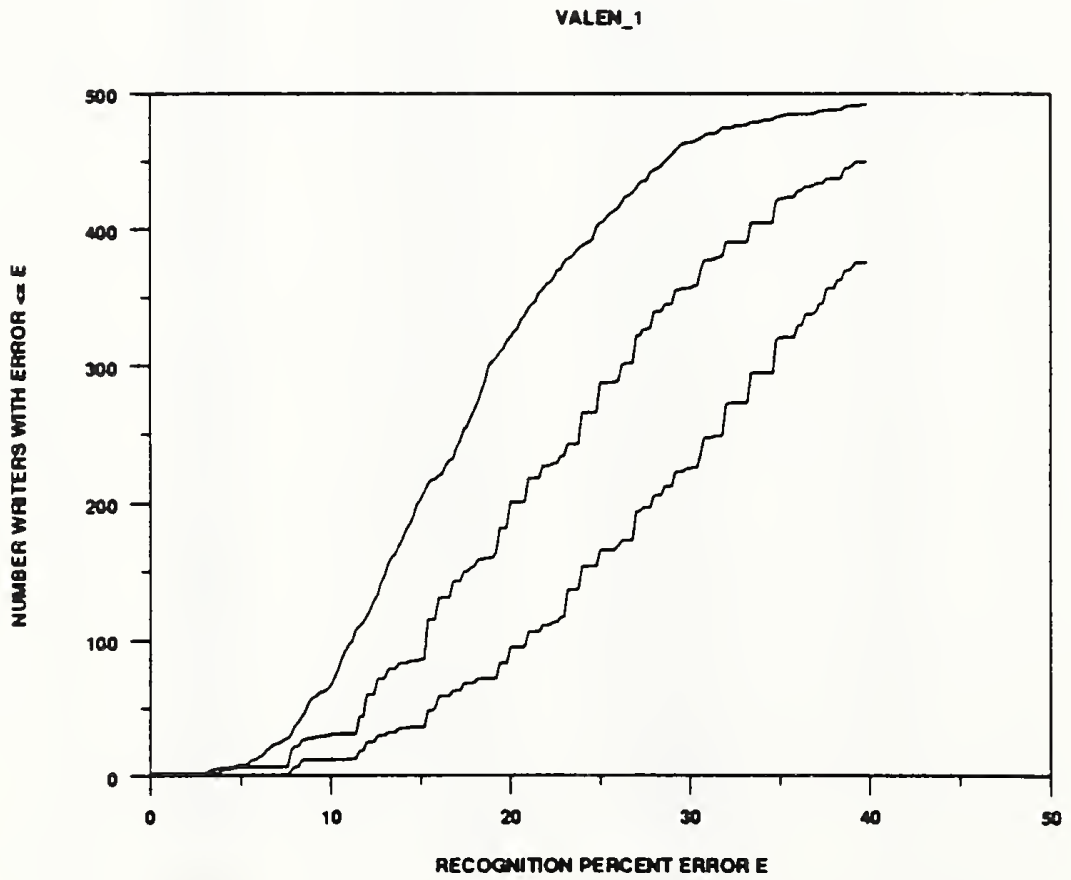

Figure 222: Error rate per writer of VALEN 1 


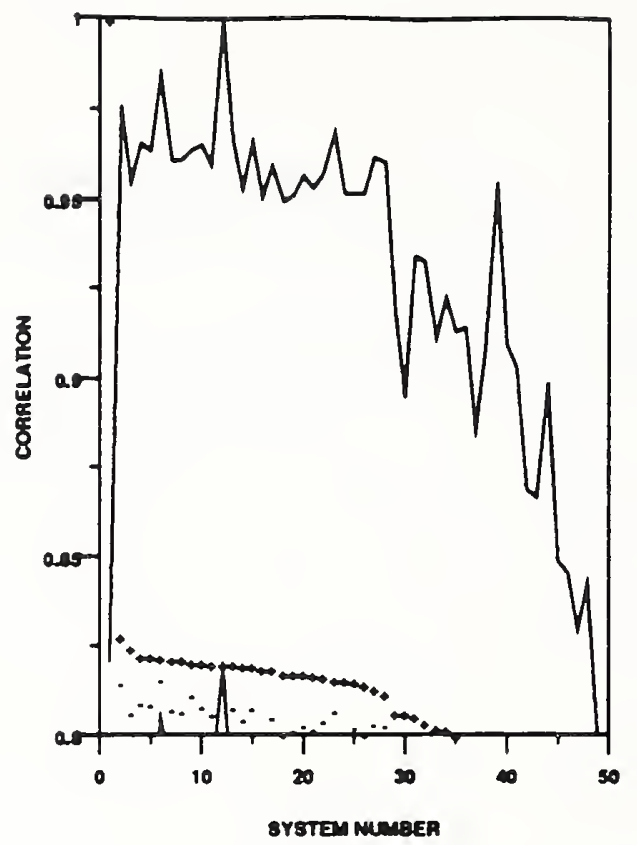

Figure 223: VALEN_1 - digit correlation

\begin{tabular}{|c|c|c|c|}
\hline System Number & Syolem Name & Correlation (all) & Corretalion (correct) \\
\hline$T$ & VALEN-1 & 1.0000 & 1.0000 \\
\hline 2 & VOTE_M & 0.8281 & 0.8152 \\
\hline $\mathbf{3}$ & NESTOR & 0.8251 & 0.8068 \\
\hline 4 & AEG & 0.8229 & 0.8093 \\
\hline 5 & ATT 2 & 0.8228 & 0.8091 \\
\hline 6 & OCRSYS & 0.8222 & 0.8159 \\
\hline 7 & ERIM 2 & 0.8218 & 0.8077 \\
\hline 8 & ERIM-1 & 0.8217 & 0.8073 \\
\hline 9 & VOTEP & 0.8210 & 0.8118 \\
\hline 10 & IBM & 0.8209 & 0.8086 \\
\hline 11 & KODAK2 & 0.8207 & 0.8081 \\
\hline 12 & REFERENCE & 0.8208 & 0.8208 \\
\hline 13 & ELSA GB & 0.8204 & 0.8082 \\
\hline 14 & ELSAGB 2 & 0.8201 & 0.8079 \\
\hline 15 & SYMBUS & 0.8201 & 0.8048 \\
\hline 16 & NIST_4 & 0.8192 & 0.8014 \\
\hline 17 & ATT-4 & 0.8191 & 0.8082 \\
\hline 18 & THINK-1 & 0.8178 & 0.8015 \\
\hline 19 & ELSAGB_1 & 0.8178 & 0.8003 \\
\hline 20 & UBOL & 0.8177 & 0.8031 \\
\hline 21 & KODAK_ & 0.8172 & 0.8020 \\
\hline 22 & NYNEX & 0.8170 & 0.8042 \\
\hline 23 & ATT $\lrcorner$ & 0.8161 & 0.8071 \\
\hline 24 & HUGHES_2 & 0.8157 & 0.8010 \\
\hline 23 & ATT 3 & 08156 & 0.8026 \\
\hline 28 & HUGHES - 1 & 0.8144 & 0.8003 \\
\hline 27 & THINK 2 & 0.8138 & 0.8033 \\
\hline 28 & REI & 0.8122 & 0.8032 \\
\hline 29 & GMD_3 & 0.8069 & 0.7839 \\
\hline 30 & RISO & 0.8068 & 0.7739 \\
\hline 31 & GTESS & 0.8058 & 0.7899 \\
\hline 32 & GTESS 2 & 0.8040 & 0.7884 \\
\hline 33 & ASOL & 0.8028 & 0.7802 \\
\hline 34 & NIST_l & 0.8019 & 0.7826 \\
\hline 35 & GMD -1 & 0.8002 & 0.7782 \\
\hline 36 & MIME & 0.7997 & 0.7788 \\
\hline 37 & KAMAN-1 & 0.7973 & 0.7878 \\
\hline 38 & $\mathrm{NIST}_{2}$ & 0.7972 & 0.7766 \\
\hline 39 & COMCOM & 0.7946 & 0.7914 \\
\hline 40 & UPENN & 0.7936 & 0.7739 \\
\hline 41 & NIST 3 & 0.7829 & 0.7729 \\
\hline 42 & KAMAN & 0.7926 & 0.7384 \\
\hline 43 & KAMAN 2 & 0.7921 & 0.7867 \\
\hline 44 & GMD_4 & 0.7888 & 0.7682 \\
\hline 48 & KAMAN 5 & 0.7828 & 0.7440 \\
\hline 46 & GMD_2 & 0.7659 & 0.7377 \\
\hline 47 & IFAX & 0.7479 & 0.7196 \\
\hline 48 & VALEN 2 & 0.7348 & 0.7178 \\
\hline 49 & KAMAN-4 & 0.7310 & 0.6954 \\
\hline
\end{tabular}

Table 137: VALEN_l correlation graph key for digits. 


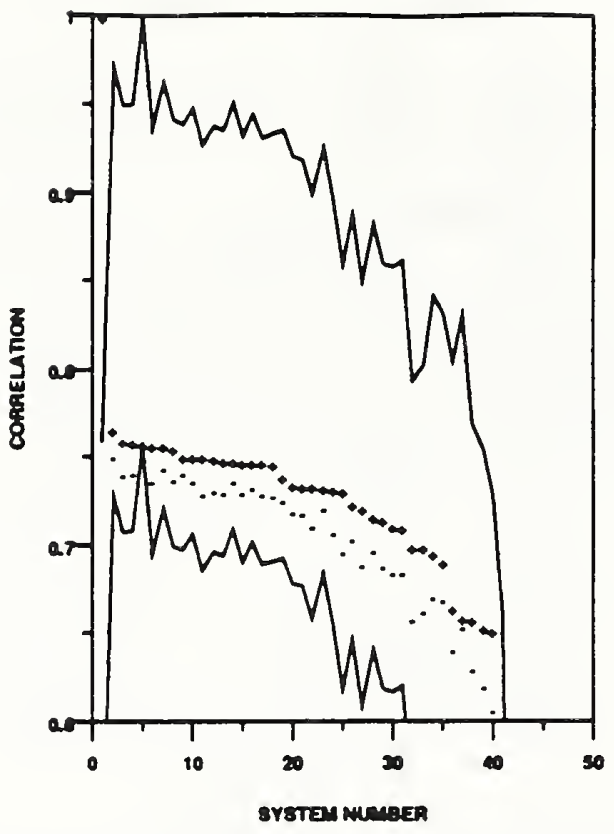

Figure 224: VALEN_1 - upper case correlation

\begin{tabular}{|c|c|c|c|}
\hline Syotem Number & System Name & Correlation (all) & Correlation (correct) \\
\hline 1 & VALEN-I & 1.0000 & 1.0000 \\
\hline 2 & VOTE_M & 0.7664 & 0.7512 \\
\hline 3 & UMICH_L & 0.7608 & 0.7413 \\
\hline 4 & ATT_A & 0.7396 & 0.7416 \\
\hline 3 & REFEFENCE & 0.7882 & 0.7582 \\
\hline 6 & IBM & 0.7574 & 0.7378 \\
\hline 7 & AEG & 0.7572 & 0.7480 \\
\hline B & NESTOR & 0.7858 & 0.7388 \\
\hline 9 & VOTEP & 0.7813 & 0.7421 \\
\hline 10 & ERIM_ & 0.7513 & 0.7372 \\
\hline 11 & SYMBUS & 0.7800 & 0.7301 \\
\hline 12 & UBOL & 0.7803 & 0.7316 \\
\hline 13 & HUGHES_I & 0.7496 & 0.7300 \\
\hline 14 & NYNEX & 0.7495 & $0.737 \mathrm{~b}$ \\
\hline 16 & ATTS & 0.7489 & 0.7308 \\
\hline 16 & ATT 2 & 0.7487 & 0.7342 \\
\hline 17 & KODAK_- & 0.7484 & 0.7303 \\
\hline 18 & HUGHES_? & 0.7479 & 0.7393 \\
\hline 19 & ATT $\perp$ & 0.7404 & 0.7262 \\
\hline 20 & GTESS_I & 0.7357 & 0.7196 \\
\hline 21 & GTESS2 & 0.7380 & 0.7187 \\
\hline 22 & MIME & 0.7349 & 0.7113 \\
\hline 23 & OCRSYS & 0.7334 & 0.7216 \\
\hline 24 & NIST_4 & 0.7329 & 0.7076 \\
\hline 28 & RISO & 0.7320 & 0.6968 \\
\hline 26 & ASOL & 0.7249 & 0.7043 \\
\hline 27 & KAMAN_1 & 0.7219 & 0.6001 \\
\hline 28 & REI & 0.7169 & 0.6883 \\
\hline 29 & GMD_1 & 0.7152 & 0.6886 \\
\hline 30 & GMD_3 & 0.7117 & 0.6858 \\
\hline 31 & NIST_1 & 0.7111 & 0.6850 \\
\hline 32 & KAMAN_S & 0.6898 & 0.6828 \\
\hline 33 & KAMAN_2 & 0.6998 & 0.6588 \\
\hline 34 & GMD_4 & 0.6965 & 0.6716 \\
\hline 35 & NIST 3 & 0.6918 & 0.6691 \\
\hline 36 & IFAX & 0.6650 & 0.6406 \\
\hline 37 & СОМСОМ & 0.6898 & 0.6540 \\
\hline 38 & NIST 2 & 0.6881 & 0.6298 \\
\hline 39 & GMD 2 & 0.6840 & 0.6208 \\
\hline 40 & $K_{A M A N}-4$ & 0.6819 & 0.6074 \\
\hline 41 & KAMAN 3 & 0.5987 & 0.8898 \\
\hline 42 & UMICH_2 & 0.0987 & 0.0134 \\
\hline
\end{tabular}

Table 138: VALEN 1 correlation graph key for uppers. 


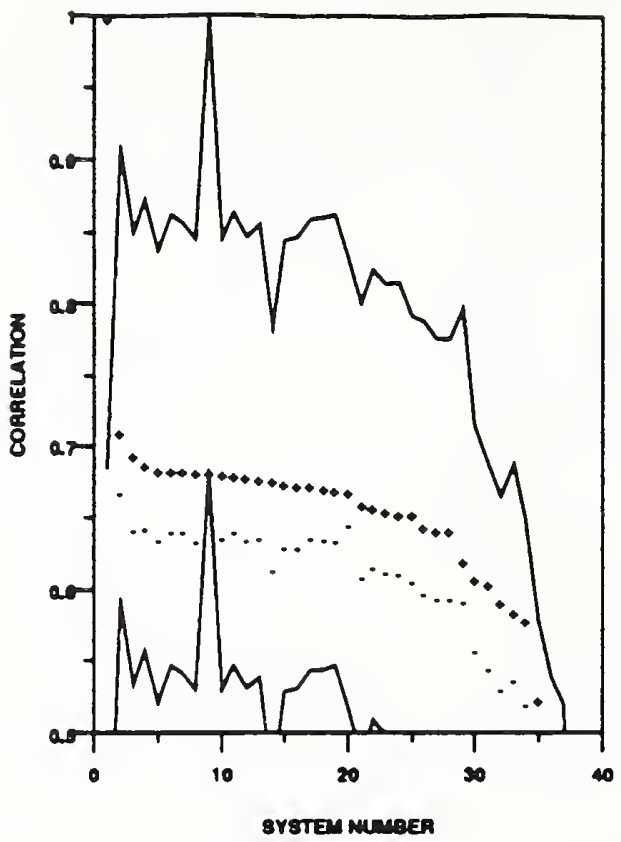

Figure 225: VALEN_1 - lower case correlation

\begin{tabular}{|c|c|c|c|}
\hline System Number & Syatem Nome & Correlation (all) & Correlation (coprect) \\
\hline 1 & VALEN_I & 1.0000 & 1.0000 \\
\hline 2 & VOTE_M & 0.7122 & 0.6683 \\
\hline 3 & UMICH.1 & 0.6957 & 0.6426 \\
\hline 4 & $A E G$ & 0.6888 & 0.6446 \\
\hline 3 & ATT 3 & 0.8861 & 0.6339 \\
\hline 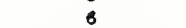 & ERIM-I & 0.6839 & 0.6423 \\
\hline 7 & ATT 4 & 0.6851 & 0.6418 \\
\hline 8 & UBOL & 0.6848 & 0.6347 \\
\hline 9 & REFERENCE & 0.6840 & 0.6840 \\
\hline 10 & IBM & 0.6837 & 0.6368 \\
\hline 11 & OCRSYS & 0.6817 & 0.6418 \\
\hline 12 & NESTOR & 0.8812 & 0.8363 \\
\hline 13 & KODAK_ & 0.6798 & 0.6371 \\
\hline 14 & RISO & 0.6790 & 0.6148 \\
\hline 15 & HUGHES_2 & 0.6769 & 0.6308 \\
\hline 16 & HUGHES -1 & 0.6737 & 0.6309 \\
\hline 17 & ATT 2 & 0.6747 & 0.6376 \\
\hline 18 & NYNEX & 06728 & 0.6339 \\
\hline 19 & ATT 1 & 0.6718 & 0.6346 \\
\hline 20 & VOTEP & 0.6701 & 0.6461 \\
\hline 21 & NIST_4 & 0.6611 & 0.6096 \\
\hline 22 & GTESS 1 & 0.8587 & 0.6168 \\
\hline 23 & NIST_I & 0.6572 & 0.6127 \\
\hline 24 & GTESS 2 & 0.6550 & 0.8117 \\
\hline 25 & GMD_3 & 0.8542 & 0.6062 \\
\hline 26 & ASOL & 0.6450 & 0.5987 \\
\hline 27 & GMD_4 & 0.8428 & $0.394 \mathrm{~s}$ \\
\hline 28 & GMD .1 & 0.6428 & 0.3943 \\
\hline 29 & NIST 3 & 0.6208 & 0.5924 \\
\hline 30 & GMD_2 & 0.8081 & 0.5585 \\
\hline 31 & KAMAN_I & 0.6057 & 0.5458 \\
\hline 32 & KAMAN_3 & 0.3921 & 0.5312 \\
\hline 33 & $\mathrm{NIST}_{2}$ & 0.5888 & 0.5372 \\
\hline 34 & KAMAN_2 & 0.3794 & 0.5218 \\
\hline 35 & KAMAN_S & 0.5253 & 0.4663 \\
\hline 36 & KAMAN_4 & 0.4238 & 0.4403 \\
\hline 37 & COMCOM & 0.4204 & 0.4100 \\
\hline 38 & $\mathrm{UMICH}_{2}$ & 0.1188 & 0.0321 \\
\hline
\end{tabular}

Table 139: VALEN_1 correlation graph key for lowers. 


\section{F System Summaries For Late Submitted Results}

Jon Geist, Jonathan J. Hull, Stanley Janet, R. Allen Wilkinson, and Charles L. Wilson

This appendix contains summaries for most systems whose HYP files were received late. Some results that were received late were not included because they would not add anything to the report even though in some cases the results are interesting. In such cases the results are mentioned in the body of the report. The summary format is exactly the same as that used for the summaries in Appendix E. 
SYSTEM: NIST_ 4

PARTICIPANT: Patrick J. Grother

ORGANIZATION: NIST, Gaithersburg, MD

PREPROCESSING: Size (both height and width), Slant, Stroke Width, Normalization. Subtraction from binary image of mean of training images.

FEATURES: Projection onto principal components of training set.

48 leading elements of "KL" transform. Digits

96 leading elements of "KL" transform. Uppers

96 leading elements of "KL" transform. Lovers

CLASSIFICATION: PNN: Gaussian distance veighted voting among all prototypes. Equivalent to KNN algorithm of NIST_1.

HARDWARE: Sparc 2 running optimized C code.

$\begin{array}{lrrrr}\text { TRA INING : } & \text { DIGITS } & \text { UPPERS } & \text { LOWERS } & \text { DATABASE } \\ & -56000 & -11000 & -11000 & \text { NSDB3 } \\ & 500 & 500 & 500 & \text { WRITERS }\end{array}$

STATUS: submitted after Conference

\begin{tabular}{|c|c|c|c|c|c|c|c|}
\hline \multirow[t]{2}{*}{ RESULTS: } & \multicolumn{2}{|c|}{-- DIGITS -- } & \multicolumn{2}{|c|}{-- UPPERS -- } & \multicolumn{2}{|c|}{-- LOWERS -- } & DATABASE \\
\hline & $\begin{array}{l}\text { REJ. } \\
\text { RATE }\end{array}$ & $\begin{array}{l}\text { ERR. } \\
\text { RATE-- }\end{array}$ & $\begin{array}{l}\text { REJ. } \\
\text { RATE }\end{array}$ & $\begin{array}{l}\text { ERR. } \\
\text { RATE-- }\end{array}$ & $\begin{array}{l}\text { REJ. } \\
\text { RATE }\end{array}$ & $\begin{array}{l}\text { ERR. } \\
\text { RATE-- }\end{array}$ & TESTDATA 1 \\
\hline & 0.00 & 0.0497 & 0.00 & 0.1037 & 0.00 & 0.2001 & \\
\hline & 0.10 & 0.0105 & 0.10 & 0.0614 & 0.10 & 0.1570 & \\
\hline & 0.20 & 0.0064 & 0.20 & 0.0346 & 0.20 & 0.1199 & \\
\hline & 0.30 & 0.0035 & 0.30 & 0.0214 & 0.30 & 0.0889 & \\
\hline & 0.40 & 0.0021 & 0.40 & 0.0141 & 0.40 & 0.0610 & \\
\hline & 0.50 & 0.0014 & 0.50 & 0.0092 & 0.50 & 0.0420 & \\
\hline
\end{tabular}

OCR RATE (CPS): DIGITS UPPERS LOWERS

OCR RATE:

CPU RATE: 


\section{SYSTEM: NIST_4}

The following references have been provided for this system:

[42]

COMMENTS: NIST_4

See Cross Validation Section on Inadequacies of NIST Special Database 3 for the classification of NIST Test Data 1.

Very Slow Classification. No exemplar pruning or aggregation. Does not suffer from "minority" problems of perceptrons (e.g. crossed sevens).

Size normalization enforces 32 pixel height 24 pixel width, does not preserve aspect ratio. Dilation / erosion used to normalize stroke widths. Significant recognition gains over NIST_1. 


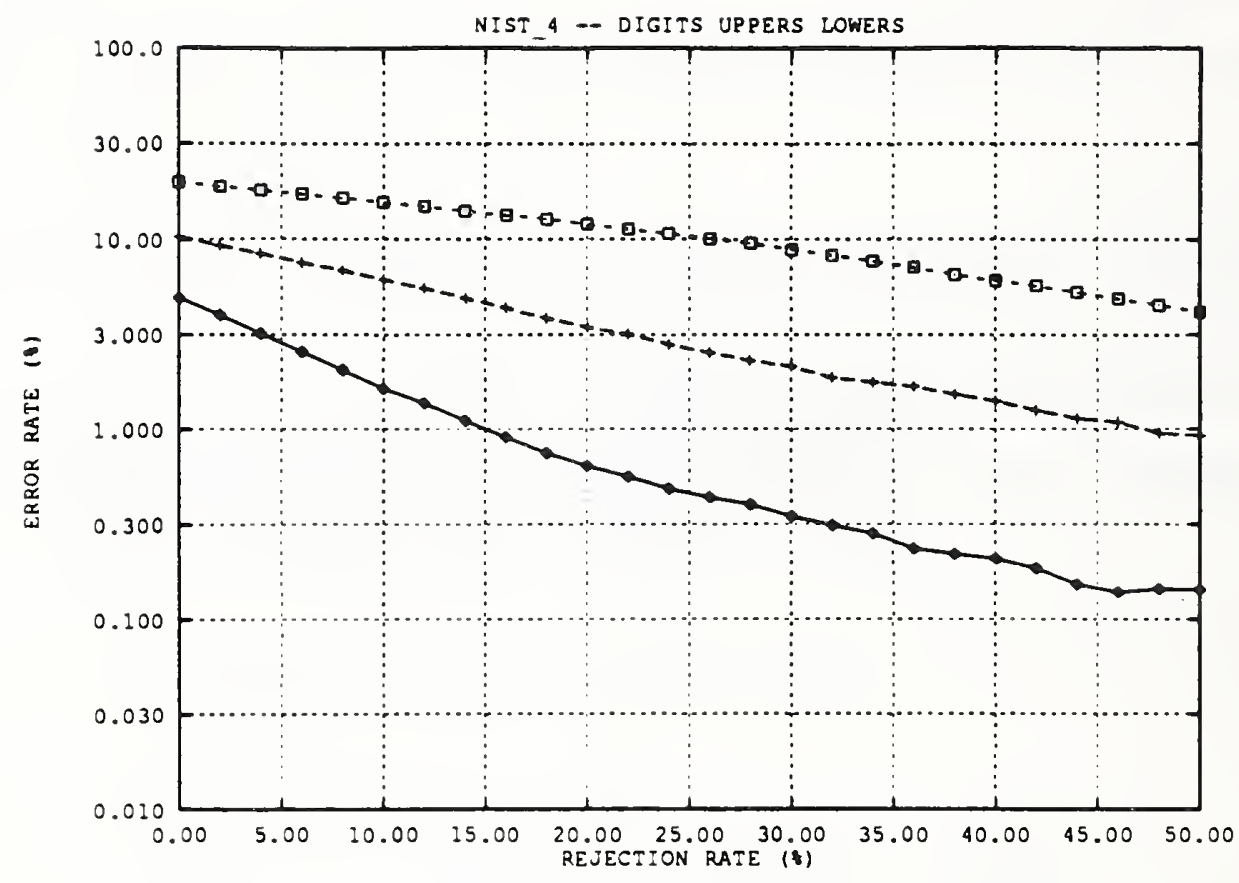

Figure 226: Error rate versus rejection rate for NIST_4

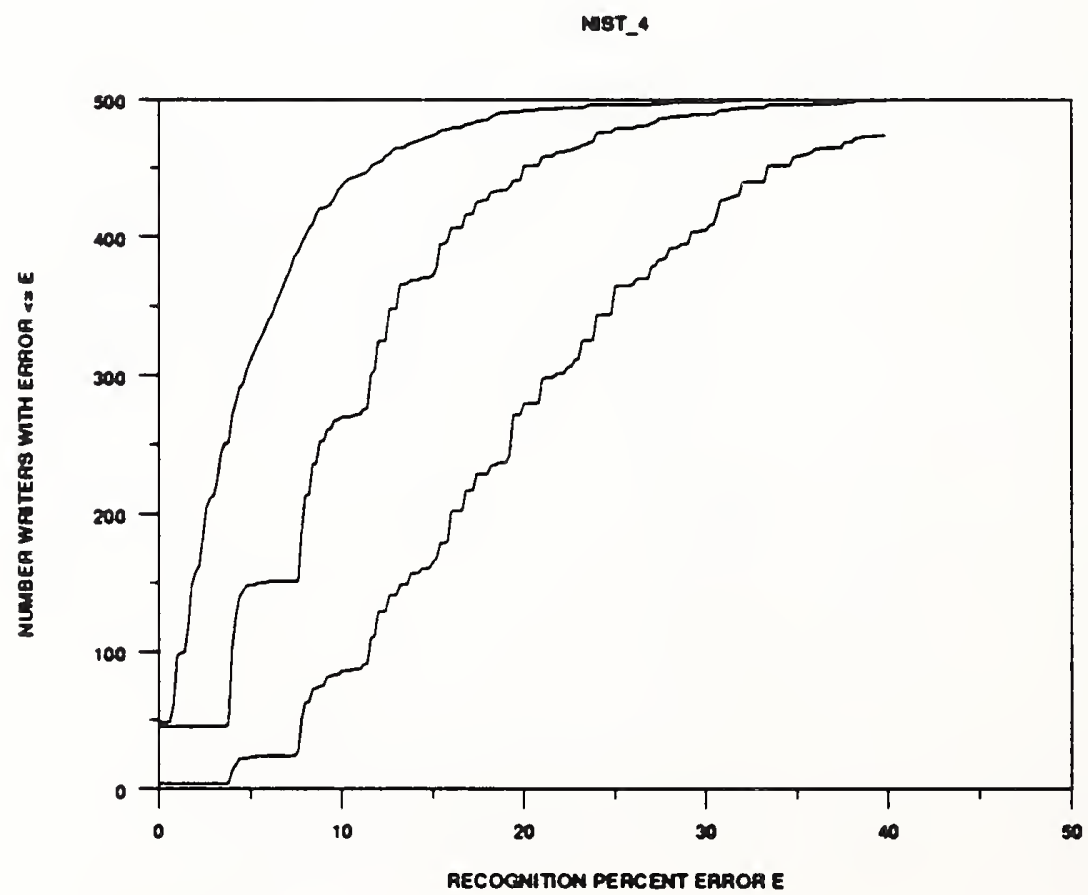

Figure 227: Error rate per writer of NIST_4 


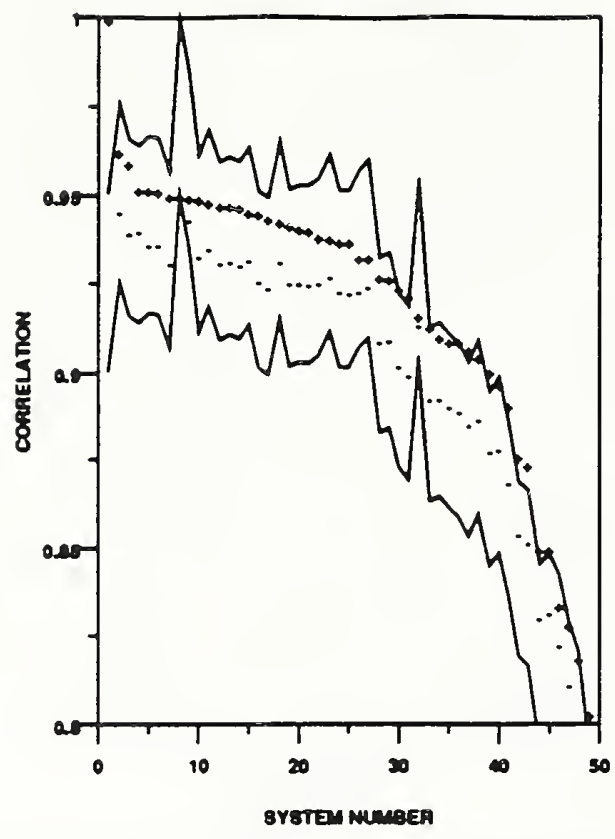

Figure 228: NIST_4 - digit correlation

\begin{tabular}{|c|c|c|c|}
\hline System Number & Sydlem Name & Correlation (sll) & Correlation (correct) \\
\hline 1 & NIST-4 & 1.0000 & 1.0000 \\
\hline 2 & VOTE_M & 0.8630 & 08457 \\
\hline 3 & AEG & 09898 & 0.9397 \\
\hline 4 & VOTE_P & 0.8524 & 0.8403 \\
\hline s & נה ELSAGB & 0.9522 & 0.8369 \\
\hline 6 & ELSAGB-2 & 0.8517 & 0.9365 \\
\hline 7 & UBOL & 0.8306 & 0.9316 \\
\hline 8 & REFERENCE & 0.8503 & 0.9503 \\
\hline $\boldsymbol{\theta}$ & OCRSYS & 0.8499 & 0.9435 \\
\hline 10 & ERIM-I & 0.8496 & 0.9334 \\
\hline 11 & ATT $\perp$ & 0.8485 & 0.9357 \\
\hline 12 & ERIM 2 & 0.9479 & 0.9323 \\
\hline 13 & ATT 4 & 0.8478 & 0.9315 \\
\hline 14 & KODAK.2 & 0.9471 & 0.9314 \\
\hline 15 & ATT 2 & 0.9460 & 0.8327 \\
\hline 16 & THINK-1 & 0.9464 & 0.9265 \\
\hline 17 & ELSA GB -1 & 0.9439 & 0.8249 \\
\hline 18 & IBM & 0.2433 & 0.9323 \\
\hline 19 & ATT 3 & 0.8420 & 0.9262 \\
\hline 20 & SYMBUS & 0.8411 & 0.9260 \\
\hline 21 & KODAK」 & 0.8409 & 0.8255 \\
\hline 22 & NESTOR & 0.9392 & 0.8260 \\
\hline 23 & THINK_2 & 0.8385 & 0.8280 \\
\hline 24 & HUGHES -1 & 0.8377 & 0.9237 \\
\hline 23 & HUGHES_2 & 0.9374 & 0.9235 \\
\hline 26 & NYNEX & 0.9337 & 0.9238 \\
\hline 27 & REI & 0.8334 & 0.9253 \\
\hline 28 & GTESS_ 2 & 0.8280 & 0.9096 \\
\hline 28 & GTESS_l & 0.9277 & 0.9101 \\
\hline 30 & NIST_I & 0.8247 & 0.9028 \\
\hline 31 & GMD_3 & 0.8223 & 0.8001 \\
\hline 32 & COMCOM & 0.8170 & 08141 \\
\hline 33 & GMD-1 & 0.8138 & 0.8932 \\
\hline 34 & MIME & 0.9110 & 0.8930 \\
\hline 35 & ASOL & 0.9097 & 0.8907 \\
\hline 36 & NIST 2 & 0.8094 & 0.8896 \\
\hline 37 & NIST & 0.8071 & 0.8858 \\
\hline 38 & UPENN & 0.9031 & 0.8873 \\
\hline 39 & RISO & 0.8008 & 0.8777 \\
\hline 40 & GMD_A & 0.8977 & 0.8783 \\
\hline 41 & KAMAN-1 & 0.8914 & 0.8694 \\
\hline 12 & KAMAN_3 & 0.8766 & 0.8544 \\
\hline 43 & KAMAN_2 & 0.8742 & 0.8520 \\
\hline 44 & GMD_2 & 0.8304 & 0.8305 \\
\hline 45 & KAMAN_S & 0.8503 & 0.8319 \\
\hline 46 & VALEN_2 & 08343 & 0.8227 \\
\hline 47 & IFAX & 0.8288 & 0.8118 \\
\hline 18 & VALEN -1 & 0.8182 & 0.8014 \\
\hline 10 & KAMAN-4 & 08036 & 0.7813 \\
\hline
\end{tabular}

Table 140: NIST_4 correlation graph key for digits. 


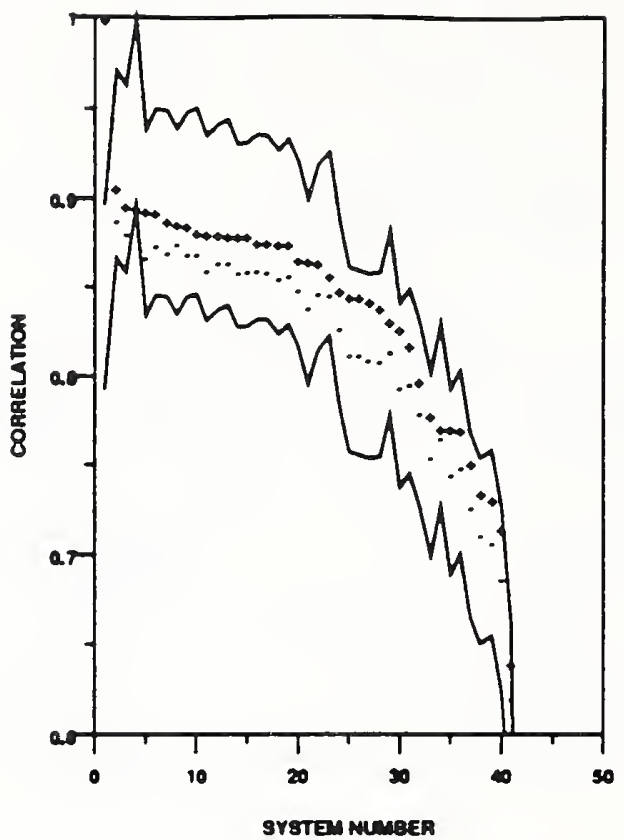

Figure 229: NIST_4 - upper case correlation

\begin{tabular}{|c|c|c|c|}
\hline System Number & Sysiem Name & Correlation (all) & Correlation (correct) \\
\hline $\mathrm{I}$ & NIST-4 & 1.0000 & 1.0000 \\
\hline 2 & VOTE_M & 0.0070 & 0.8893 \\
\hline 3 & AEG & 0.8972 & 0.8820 \\
\hline 4 & REFERENCE & 0.8963 & 0.8063 \\
\hline s & UBOL & 0.8941 & 0.8692 \\
\hline 6 & ATT 4 & 0.8937 & 0.8786 \\
\hline 7 & UMICH_I & 0.8886 & 0.8714 \\
\hline 8 & VOTEP & 0.8871 & 0.8763 \\
\hline 9 & ERIM-1 & 0.8865 & 0.8705 \\
\hline 10 & NYNEX & 0.8829 & 0.8704 \\
\hline 11 & ATT $\perp$ & 0.8818 & 0.8617 \\
\hline 12 & NESTOR & 0.8815 & 0.8658 \\
\hline 13 & ATT 2 & 0.8812 & 0.8658 \\
\hline 14 & ATT & 0.8807 & 0.8618 \\
\hline 15 & KODAK」 & 0.8807 & 0.8608 \\
\hline 16 & IBM & 0.8775 & 0.8617 \\
\hline 17 & HUGHES_I & 0.8774 & 0.8602 \\
\hline 18 & SYMBUS & 0.8764 & 0.8567 \\
\hline 18 & HUGHES_2 & 0.8759 & 0.8884 \\
\hline 20 & GTESS-1 & 0.8683 & 0.8601 \\
\hline 21 & MIME & 0.8668 & 0.8401 \\
\hline 22 & GTESS 2 & 0.8663 & 0.8488 \\
\hline 23 & OCRSYS & 0.8587 & 0.8476 \\
\hline 24 & ASOL & 0.8508 & 0.8283 \\
\hline 25 & NIST & 0.8467 & 0.8135 \\
\hline 26 & GMD_1 & 0.8464 & 0.8133 \\
\hline 27 & GMD_3 & 0.8442 & 0.8113 \\
\hline 28 & RISO & 0.8405 & 0.8096 \\
\hline 29 & REI & 0.8328 & 0.8183 \\
\hline 30 & GMD_4 & 0.8282 & 0.7857 \\
\hline 31 & KAMAN_1 & 0.8189 & 0.7967 \\
\hline 32 & NIST_3 & 0.7991 & 0.7803 \\
\hline 33 & KAMAN_3 & 0.7797 & 0.7558 \\
\hline 34 & COMCOM & 0.7728 & 0.7668 \\
\hline 35 & KAMAN_2 & 0.7726 & 0.7470 \\
\hline 36 & IFAX & 0.7715 & 0.7307 \\
\hline 37 & $\mathrm{NIST}_{2}$ & 0.7535 & 0.7278 \\
\hline 38 & GMD 2 & 0.7363 & 0.7123 \\
\hline 39 & VALEN_I & 0.7329 & 0.7076 \\
\hline 40 & KAMAN_4 & 0.7159 & 0.6880 \\
\hline 41 & KAMAN_S & 0.6414 & 0.6215 \\
\hline 42 & $\mathrm{UMICH}_{2}$ & 0.0547 & 0.0177 \\
\hline
\end{tabular}

Table 141: NIST_4 correlation graph key for uppers. 


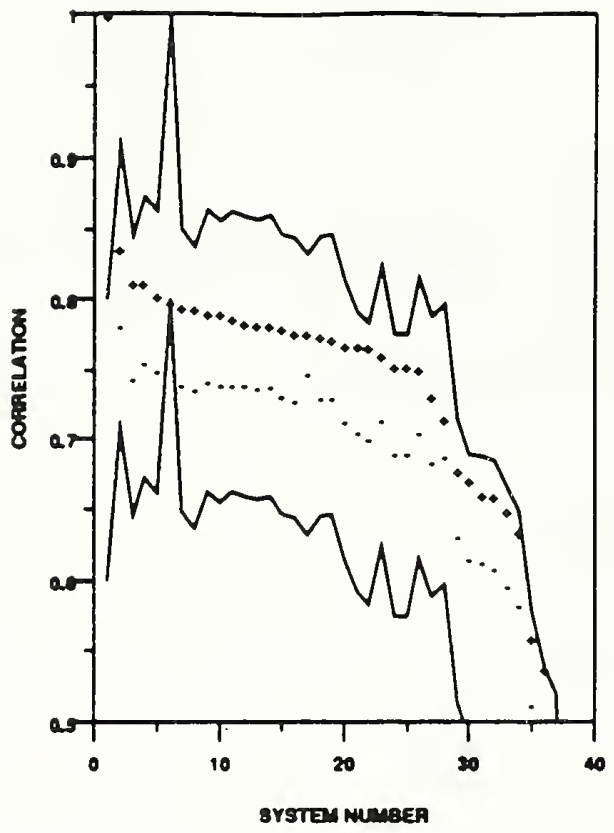

Figure 230: NIST_4 - lower case correlation

\begin{tabular}{|c|c|c|c|}
\hline Syalem Number & System Name & Correlation (ali) & Corretation (correct) \\
\hline 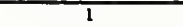 & NIST.4 & 1.0000 & 1.0000 \\
\hline 2 & VOTE_M & 0.8378 & 0.7830 \\
\hline $\mathbf{3}$ & UBOL & 0.8145 & 0.7449 \\
\hline 4 & $A E G$ & 0.8144 & 0.7563 \\
\hline 3 & ERIM-I & 0.8082 & 0.7508 \\
\hline 6 & REFERENCE & 0.7899 & 0.7998 \\
\hline 7 & UMICH-I & 0.7971 & 0.7402 \\
\hline 8 & ATT 3 & 0.7960 & 0.7376 \\
\hline 8 & OCRSYS & 0.7928 & 0.7431 \\
\hline 10 & KODAK」 & 0.7921 & 0.7408 \\
\hline 11 & ATT $\perp$ & 0.7885 & 0.7403 \\
\hline 12 & А T T 2 & 0.7851 & 0.7402 \\
\hline 13 & AT T -4 & 0.7847 & 0.7387 \\
\hline 14 & NYNEX & 0.7843 & 0.7396 \\
\hline 15 & HUGHES_L & 0.7817 & $0.7 \mathrm{~J} 2 \mathrm{~J}$ \\
\hline 16 & HUGHES_2 & 0.7782 & 0.7293 \\
\hline 17 & VOTEP & 0.7781 & 07492 \\
\hline 18 & IBM & 0.7762 & 0.7312 \\
\hline 18 & NESTOR & 0.7744 & 0.7317 \\
\hline 20 & NIST_L & 07629 & 0.7144 \\
\hline 21 & GMD_J & 0.7698 & 0.7067 \\
\hline 22 & RISO & 0.7678 & 0.7017 \\
\hline 23 & GTESS_l & 0.7628 & 0.7133 \\
\hline 24 & GMD_4 & 0.7341 & 0.6915 \\
\hline 25 & GMD_l & 0.7541 & $0.691 \mathrm{~s}$ \\
\hline 26 & GTESS 2 & $0.732 \mathrm{3}$ & 0.7067 \\
\hline 27 & ASOL & 0.7323 & 0.6857 \\
\hline 28 & הIST & 0.7171 & 0.6888 \\
\hline 29 & GMD_2 & 0.6788 & 0.6330 \\
\hline so & $K_{A} M_{A N} N_{-1}$ & 0.6733 & 0.6166 \\
\hline 31 & NIST 2 & 0.6631 & 0.6140 \\
\hline 32 & VALEN_L & 0.6811 & 0.6096 \\
\hline 33 & KAMAN 3 & 0.6516 & 0.3969 \\
\hline 34 & KAMAN_2 & 0.6360 & 0.5839 \\
\hline 35 & KAMAN_S & 0.5602 & 0.3135 \\
\hline J6 & KAMAN-4 & 0.5388 & 0.4908 \\
\hline 37 & СОМСOM & 04688 & 0.4364 \\
\hline 38 & UMICH 2 & 0.1091 & 0.0434 \\
\hline
\end{tabular}

Table 142: NIST_4 correlation graph key for lowers. 
SYSTEM: THINK_2

PARTICIPANT: Stephen Smith

ORGANIZATION: Thinking Machines Corporation, Cambridge, MA

PREPROCESSING: Thinning and normalization.

FEATURES: contour model of are

CLASSIFICATION: KNN for variable length vectors

HARDWARE: $\quad 32,768$ processor CM2 1 ith SUN front ond

TRAINING: DIGITS UPPERS LOWERS DATABASE

$\begin{array}{llll}\text { al1 NA } & \text { NA } & \text { NSDB3 }\end{array}$

STATUS: $\quad$ one day late

RESULTS: -- DIGITS -- -- UPPERS -- -- LOWERS -- DATABASE

REJ. ERR. REJ. ERR. REJ. ERR. TESTDATA1

RATE RATE-- RATE RATE-- RATE RATE--

$0.00 \quad 0.0385$

$0.10 \quad 0.0086$

$0.20 \quad 0.0036$

$0.30 \quad 0.0019$

$0.40 \quad 0.0012$

$0.50 \quad 0.0008$

OCR RATE (CPS): DIGITS

UPPERS

LOHERS

SYS RATE: $\quad 0.67$

CPU RATE: 
SYSTEM: THINK_2

BIBLIOGRAPHY:

The following references have been provided for this system:

none 


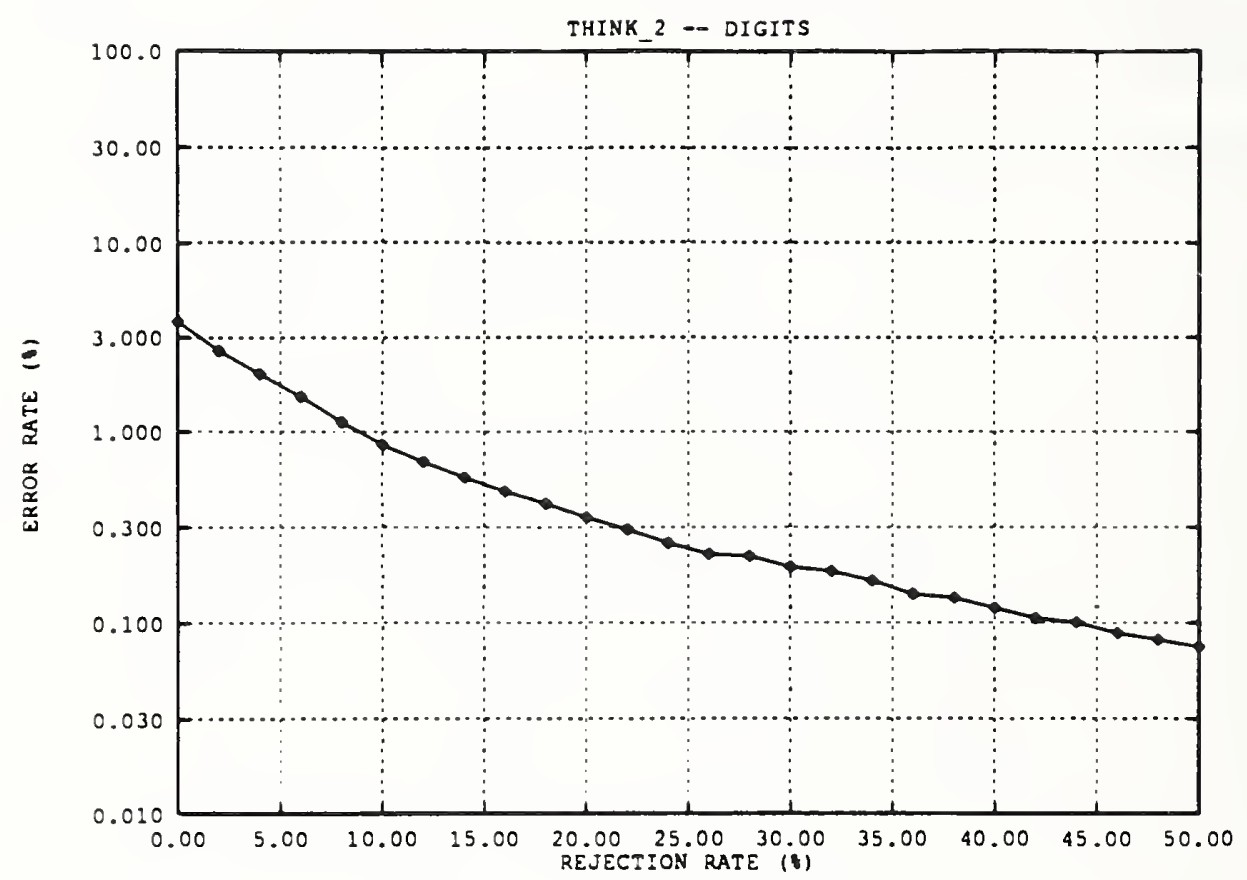

Figure 231: Error rate versus rejection rate for THINK_2

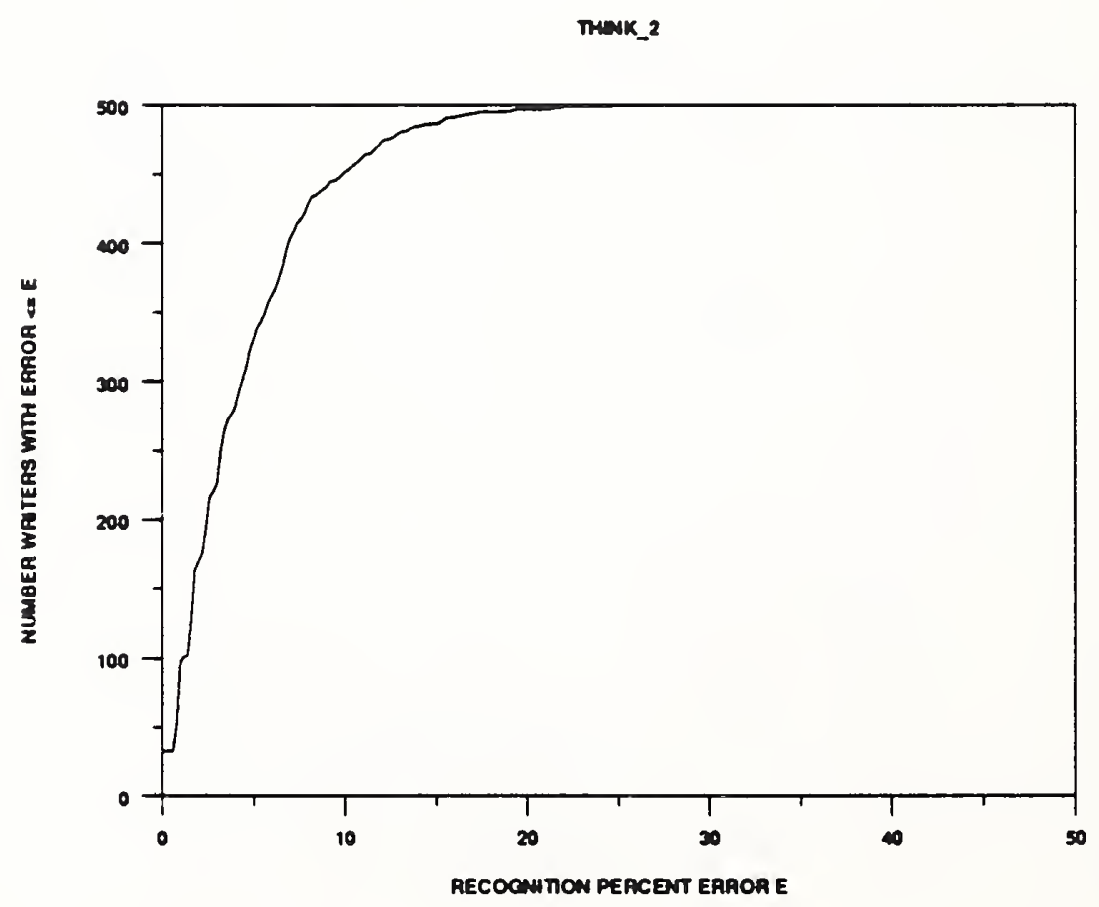

Figure 232: Error rate per writer of THINK_2 


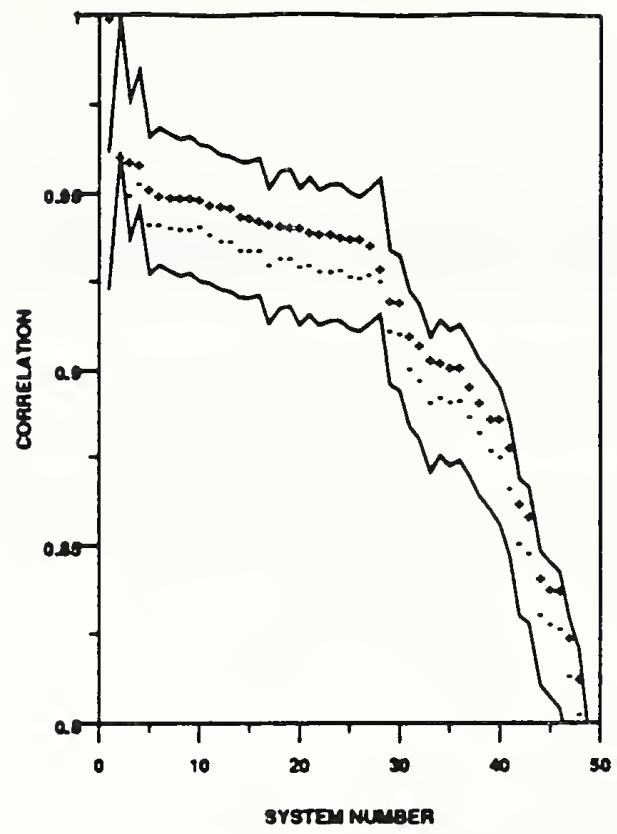

Figure 233: THINK 2 - digit correlation

\begin{tabular}{|c|c|c|c|}
\hline System Number & System Name & Copretation (ali) & Correlation (correct) \\
\hline 1 & THINK 2 & 1.0000 & 1.0000 \\
\hline 2 & REFERENCE & $0.961 \mathrm{~s}$ & $0.861 \mathrm{~s}$ \\
\hline 3 & VOTE_M & 0.9600 & 09504 \\
\hline 4 & OCRSYS & 0.2891 & 0.9537 \\
\hline 5 & AEG & 0.9523 & 0.9420 \\
\hline 6 & ATT 1 & 0.9806 & 0.9422 \\
\hline 7 & ELSAGBA & 0.9502 & 0.9413 \\
\hline 8 & IBM & 0.9501 & 0.9407 \\
\hline 9 & ELSAGB 2 & 0.9498 & 0.9410 \\
\hline 10 & VOTEP & 09494 & 0.9419 \\
\hline 11 & ATT2 & 0.9484 & 0.9394 \\
\hline 12 & ERIM-1 & 0.9477 & 0.9376 \\
\hline 13 & ERIM 2 & 0.9474 & 0.9375 \\
\hline 14 & KODAK 2 & 0.9430 & 0.9382 \\
\hline 15 & ATT.4 & 0.9443 & 0.9331 \\
\hline 16 & REI & 0.9434 & 09355 \\
\hline 17 & HUGHES - 1 & 0.9426 & 0.9311 \\
\hline 18 & UBOL & 0.2423 & 0.9328 \\
\hline 18 & NYNEX & 0.9418 & 0.9328 \\
\hline 20 & HUGHES.2 & 0.9416 & 0.9306 \\
\hline 21 & NESTOR & 09404 & 0.8311 \\
\hline 22 & THINK-1 & 0.9401 & 0.9293 \\
\hline 23 & KODAK」 & 09397 & 0.9293 \\
\hline 24 & SYMBUS & 0.9389 & 0.9297 \\
\hline 25 & NIST $T_{-4}$ & 0.9383 & 0.9280 \\
\hline 26 & ELSAGB-1 & 0.9384 & 0.9277 \\
\hline 27 & ATt 3 & 0.9368 & 0.9284 \\
\hline 28 & COMCOM & 0.9302 & 0.9264 \\
\hline 29 & GTESS -1 & 0.9213 & 0.9124 \\
\hline 30 & GTESS 2 & 0.9206 & 0.9113 \\
\hline 31 & NIST_I & 0.9108 & 0.9012 \\
\hline 32 & GMD_3 & 0.9081 & 0.8980 \\
\hline 33 & UPENN & 0.9041 & 0.8915 \\
\hline 34 & MIME & 0.9030 & 0.8932 \\
\hline 35 & GMD_1 & 0.9018 & 0.8920 \\
\hline 36 & ASOL & 0.8018 & 08917 \\
\hline 37 & NIST 2 & 0.8964 & 08876 \\
\hline 38 & NIST 3 & 0.8916 & 0.8828 \\
\hline 39 & GMD_4 & 08873 & 0.8778 \\
\hline 10 & RISO & 0.8869 & 0.8759 \\
\hline 41 & $K A M A N_{-1}$ & 0.8786 & 0.8674 \\
\hline 42 & KAMAN_3 & 0.8630 & 0.8518 \\
\hline 43 & $K A M A N \_2$ & 0.8395 & 0.8489 \\
\hline 44 & $\mathrm{KAMAN} S$ & 0.8420 & 0.8317 \\
\hline 43 & GMD -2 & 0.8387 & 0.8289 \\
\hline 46 & VALEN_2 & 0.8385 & 08272 \\
\hline 47 & IFAX & 0.8248 & 0.8139 \\
\hline 48 & VALEN_1 & 0.8138 & 0.8033 \\
\hline 49 & $K_{A M A N_{-4}}$ & 0.7865 & 0.7766 \\
\hline
\end{tabular}

Table 143: THINK 2 correlation graph key for digits. 


\section{No Data Available}

Figure 234: THINK 2 - upper case correlation

There was no data for this evaluation.

Table 144: THINK_2 correlation graph key for uppers. 


\section{No Data Available}

Figure 235: THINK 2 - lower case correlation

There wes no dats for this evaluation.

Table 145: THINK_2 correlation graph key for lowers. 
SYSTEM: UMICH_1

PARTICIPANT: M. Shridhar

ORGANIZATION: University of Michigan, Dearborn, MI

PREPROCESSING: size normalization.

FEATURES: rule-based features of all sorts, histogram of direction vectors (4 directions) evaluated in 16 zones. Provides a 64 dimensional feature vector.

CLASSIFICATION: hybrid statistical, structural, and NN. Used modified quadratic discriminant function.

HARDWARE:

TRAINING :

DIGITS

UPPERS

LOWERS DATABASE

600

600

600 NSDB3?

STATUS: five days late

RESULTS: DIGITS UPPERS LOWERS DATABASE

RESULTS: -- DIGITS -- -- UPPERS -- -- LOWERS -- DATABASE

REJ. ERR. REJ. ERR. REJ. ERR. TESTDATA1

RATE RATE-- RATE RATE-- RATE RATE--

$0.00 \quad 0.0511 \quad 0.00 \quad 0.1508$

$\begin{array}{lllll}0.10 & 0.0337 & 0.10 & 0.1198\end{array}$

$\begin{array}{lllll}0.20 & 0.0256 & 0.20 & 0.1012\end{array}$

$\begin{array}{lllll}0.30 & 0.0207 & 0.30 & 0.0912\end{array}$

$\begin{array}{lllll}0.40 & 0.0179 & 0.40 & 0.0811\end{array}$

$\begin{array}{llll}0.50 & 0.0172 & 0.50 & 0.0720\end{array}$

OCR RATE (CPS): DIGITS UPPERS LOWERS

SYS RATE:

CPU RATE: 
SYSTEM: UMICH_1

The following references have been provided for this system: 


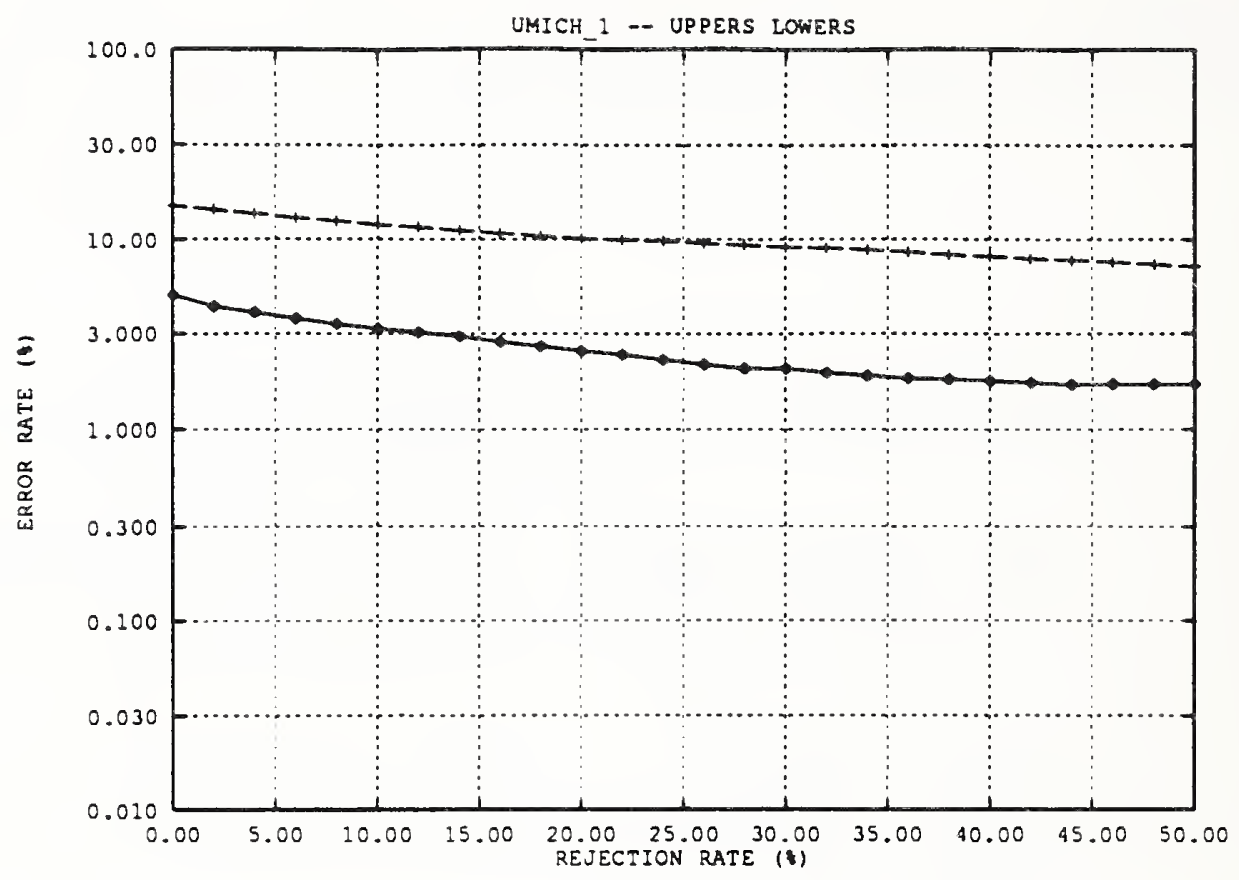

Figure 236: Error rate versus rejection rate for UMICH_1

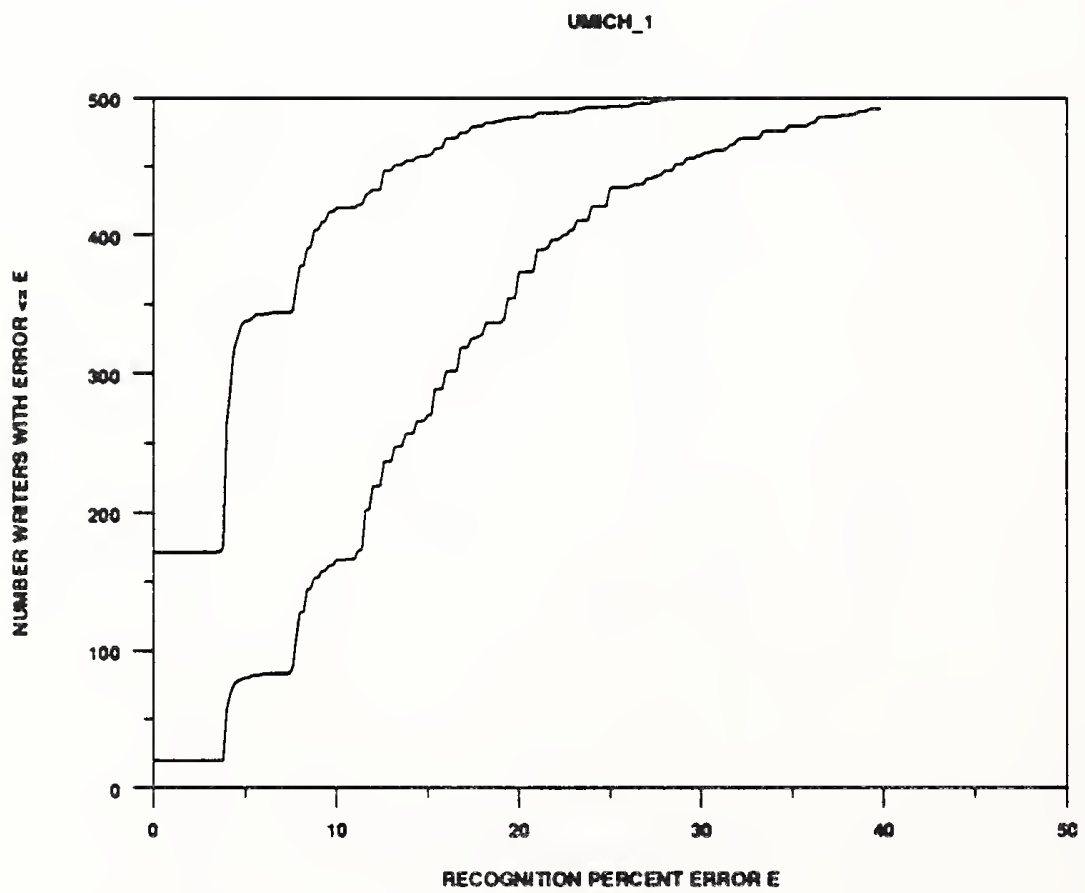

Figure 237: Error rate per writer of UMICH_1 


\section{No Data Available}

Figure 238: UMICH_1 - digit correlation

There was no dats for this evaluation.

Table 146: UMICH_1 correlation graph key for digits. 


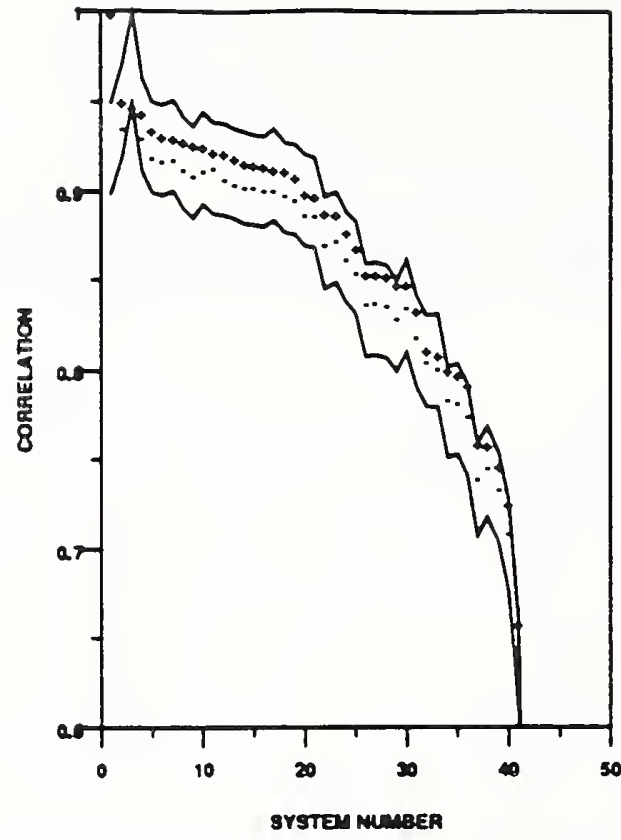

Figure 239: UMICH_1 - upper case correlation

\begin{tabular}{|c|c|c|c|}
\hline System Number & System Nome & Copreiation (sil) & Correlation (correct) \\
\hline$T$ & UMICH_1 & 1.0000 & 1.0000 \\
\hline 2 & VOTE_M & 0.9313 & 0.9369 \\
\hline 3 & REFERENCE & 0.9489 & 0.9489 \\
\hline 4 & AEG & 0.8450 & 0.9313 \\
\hline 5 & ATT_4 & 0.9361 & 0.9206 \\
\hline 8 & ERIM_I & 0.2323 & 0.9181 \\
\hline 8 & NYNEX & 0.8311 & 0.8190 \\
\hline 8 & NESTOR & 0.8297 & 0.9141 \\
\hline 9 & IBM & 0.9274 & 0.9106 \\
\hline 10 & ATT 2 & 0.8265 & 0.9131 \\
\hline 11 & VOTEP & 0.8240 & 0.9149 \\
\hline 12 & UBOL & 0.2231 & 0.9080 \\
\hline 13 & HUGHES_I & 0.9199 & 0.9036 \\
\hline 14 & HUGHES-2 & 0.8178 & 0.9034 \\
\hline 15 & ATT 3 & 0.9166 & 0.9034 \\
\hline 16 & KODAK」 & 0.9160 & 0.9022 \\
\hline 17 & ATT $\perp$ & 0.9140 & 0.9022 \\
\hline 18 & SYMBUS & 0.2133 & 08993 \\
\hline 19 & OCRSYS & 0.9088 & 0.8968 \\
\hline 20 & GTESS-I & 0.8997 & 0.8883 \\
\hline 21 & GTESS 2 & 0.8986 & 0.8869 \\
\hline 22 & NIST -4 & 0.8886 & 0.8714 \\
\hline 23 & MIME & 0.8879 & 0.8731 \\
\hline 24 & ASOL & 0.8781 & 0.8633 \\
\hline 25 & REI & 0.8694 & 0.8561 \\
\hline 26 & RISO & 0.8852 & 0.8383 \\
\hline 27 & GMD_l & 0.8551 & 0.8390 \\
\hline 28 & GMD_J & 0.8539 & 0.8372 \\
\hline 29 & NIST $T_{-l}$ & 0.8491 & 0.8362 \\
\hline 30 & KAMAN_L & 0.8491 & 0.8306 \\
\hline 31 & GMD_4 & 0.8344 & 0.8197 \\
\hline 32 & СOMCOM & 0.8132 & 0.8068 \\
\hline 33 & NIST & 0.8100 & 0.8024 \\
\hline 34 & KAMAN」 & 0.8018 & 0.7849 \\
\hline 23 & IFAX & 0.7992 & 0.7834 \\
\hline 36 & KAMAN-2 & 0.7931 & 0.7756 \\
\hline 37 & VALEN_L & $0.760 \mathrm{~s}$ & 0.7413 \\
\hline 38 & NIST 2 & 0.7598 & 0.7478 \\
\hline 39 & GMD .2 & 0.7488 & 0.7356 \\
\hline 40 & KAMAN_4 & 0.7278 & 0.7108 \\
\hline 41 & KAMAN_5 & 0.6392 & 0.6458 \\
\hline 42 & $\mathrm{UMICH}_{2}$ & 0.0000 & 0.0000 \\
\hline
\end{tabular}

Table 147: UMICH_1 correlation graph key for uppers. 


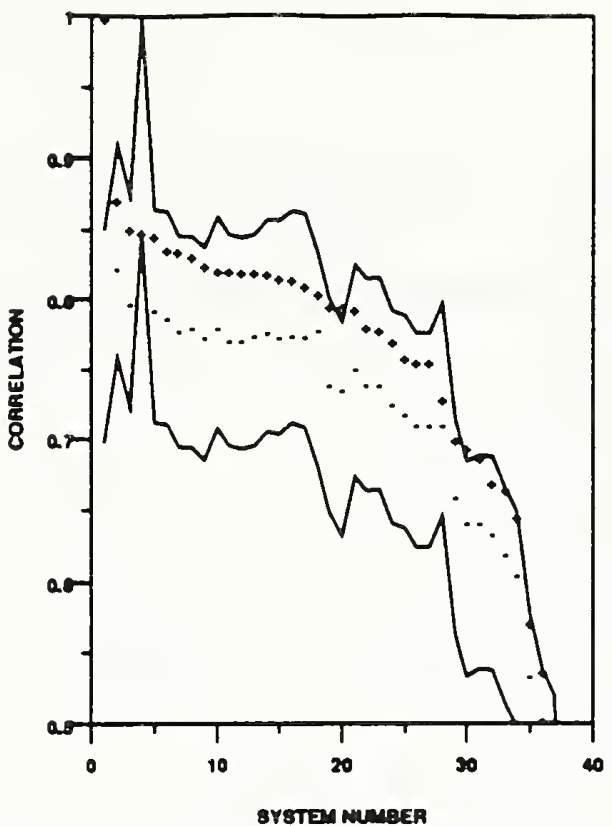

Figure 240: UMICH_1 - lower case correlation

\begin{tabular}{|c|c|c|c|}
\hline Syolem Number & System Name & Correlation (all) & Correlation (correct) \\
\hline 1 & UMICH.I & 1.0000 & 1.0000 \\
\hline 2 & VOTE_M & 08728 & 0.8245 \\
\hline 3 & $A E G$ & 0.8321 & 0.7987 \\
\hline 4 & REFERENCE & 0.8493 & 08493 \\
\hline 3 & OCRSYS & 0.8472 & 0.7941 \\
\hline 6 & ERIM-1 & 0.8378 & 0.7888 \\
\hline 7 & UBOL & 0.8373 & 0.7800 \\
\hline 8 & IBM & 0.8339 & 0.7817 \\
\hline 9 & ATT 3 & 0.8273 & 0.7747 \\
\hline 10 & ATT 2 & 0.8239 & 0.7818 \\
\hline 11 & HUGHES. 1 & 0.8232 & 0.7732 \\
\hline 12 & HUGHES_2 & 0.8227 & 0.7723 \\
\hline 13 & NESTOR & 0.8221 & 0.7781 \\
\hline 14 & ATT_4 & 0.8210 & 0.7791 \\
\hline 15 & KODAKA & 0.8174 & 0.7735 \\
\hline 16 & ATT $\lrcorner$ & 0.8168 & 0.7767 \\
\hline 17 & NYNEX & 0.8121 & 0.7757 \\
\hline 18 & VOTEP & 0.8062 & 0.7803 \\
\hline 18 & NIST_4 & 0.7971 & 0.7402 \\
\hline 20 & RISO & 0.7968 & 0.7369 \\
\hline 21 & GTESS 1 & 0.7943 & 0.7317 \\
\hline 22 & NIST.I & 0.7820 & 0.7408 \\
\hline 23 & GTESS_2 & 0.7799 & 0.7407 \\
\hline 24 & GMD_3 & 0.7713 & 0.7263 \\
\hline 23 & ASOL & 0.7603 & 0.7198 \\
\hline 26 & GMD_4 & 0.7371 & 0.7117 \\
\hline 27 & GMD_- 1 & 0.7571 & 0.7117 \\
\hline 28 & הב הIST & 0.7301 & 0.7120 \\
\hline 29 & GMD_2 & 0.7022 & 0.8610 \\
\hline 30 & VALEN_1 & 0.6937 & 0.6426 \\
\hline$\$ 1$ & KAMAN_1 & 0.8890 & 0.6428 \\
\hline 32 & $\mathrm{NIST}_{2}$ & 0.6718 & 0.6354 \\
\hline 33 & KAMAN_3 & 0.6670 & 0.6216 \\
\hline 34 & KAMAN_2 & 0.6476 & 0.6062 \\
\hline 35 & KAMAN_S & 0.5727 & 0.5353 \\
\hline 36 & KAMAN_A & 0.3384 & 0.5052 \\
\hline 37 & COMCOM & 0.4923 & 0.4823 \\
\hline 38 & UMICH 2 & 0.0000 & 0.0000 \\
\hline
\end{tabular}

Table 148: UMICH_1 correlation graph key for lowers. 
SYSTEM: VALEN_2

PARTICIPANT: Enrique Vidal

ORGANIZATION: Universidad Politecnica de Valencia, Valencia, Spain

FEATURES: line fit features

CLASSIFICATION: $\mathrm{k}-\mathrm{NN}$ or NN with BP

HARDWARE: model $380 \mathrm{HP}-9000$

TRAINING: DIGITS UPPERS LOWERS DATABASE

STATUS: seven days late

RESULTS: -- DIGITS -- -- UPPERS -- -- LOWERS -- DATABASE

REJ. ERR. REJ. ERR. REJ. ERR. TESTDATA 1

RATE RATE-- RATE RATE-- RATE RATE--

$0.00 \quad 0.1575$

$0.10 \quad 0.1144$

$0.20 \quad 0.0756$

$0.30 \quad 0.0488$

$0.40 \quad 0.0307$

$0.50 \quad 0.0192$

OCR RATE (CPS): DIGITS

UPPERS

LOWERS

SYS RATE :

CPU RATE: 


\section{SYSTEM: VALEN 2}

The following references have been provided for this system:

[59] 


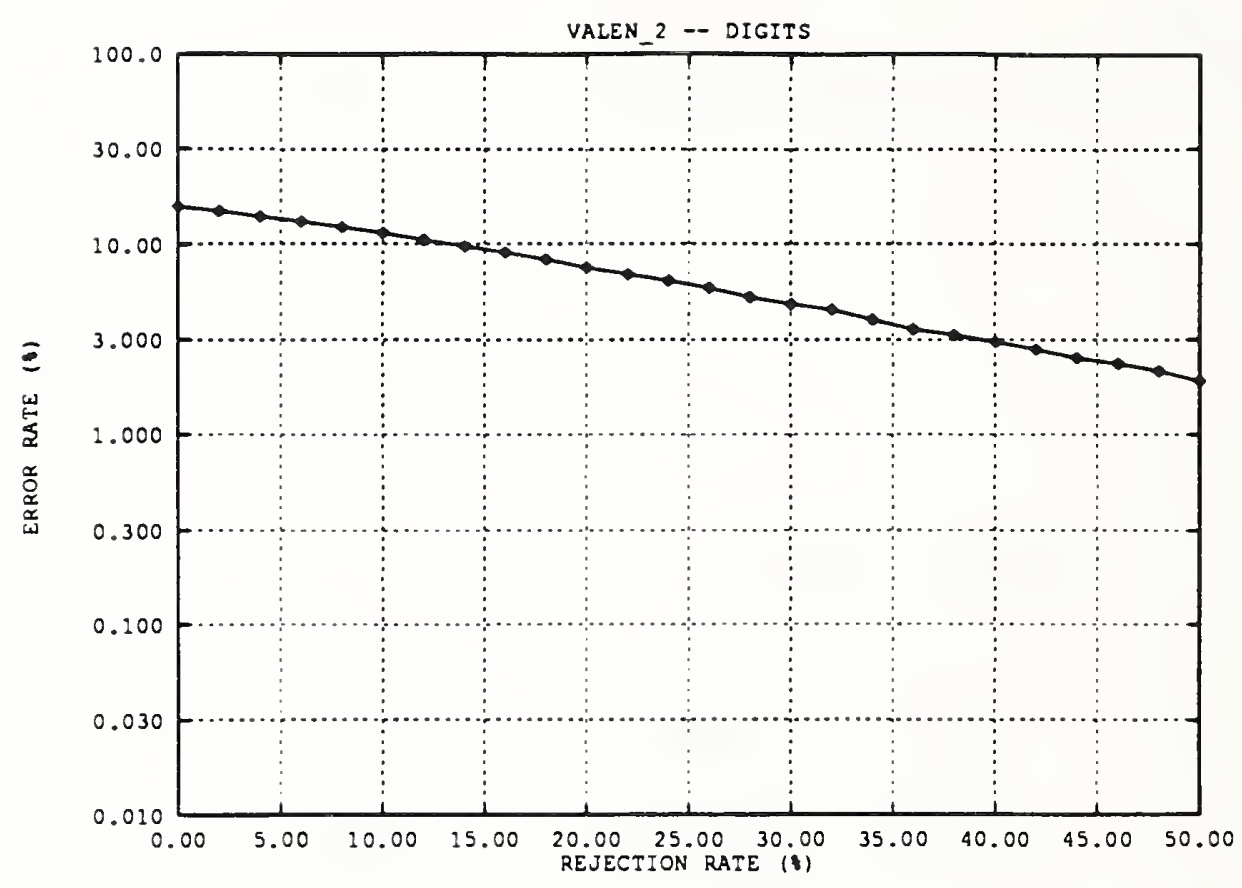

Figure 241: Error rate versus rejection rate for VALEN 2

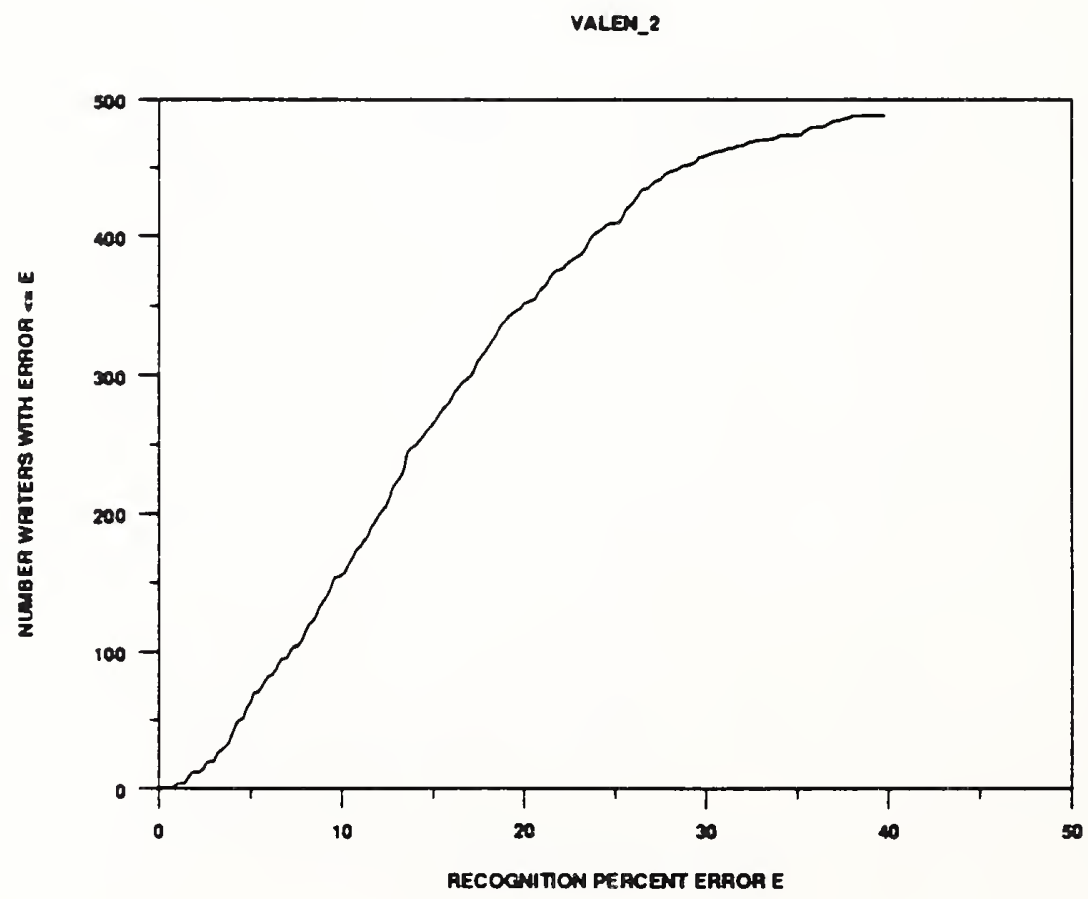

Figure 242: Error rate per writer of VALEN_2 


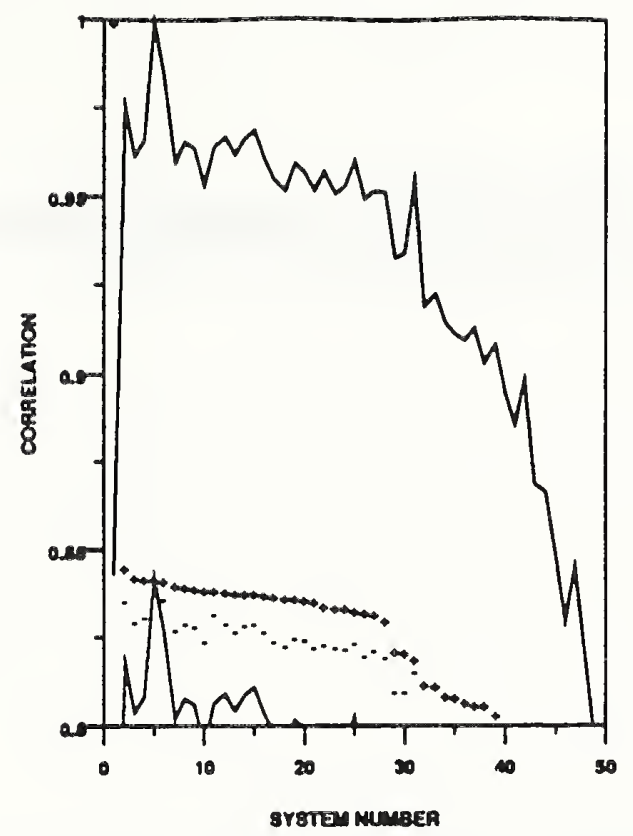

Figure 243: VALEN2 - digit correlation

\begin{tabular}{|c|c|c|c|}
\hline Syotem Number & System Narne & Corretation (ali) & Correlation (correct) \\
\hline 1 & VALENב & 1.0000 & 1.0000 \\
\hline 2 & VOTE_M & 0.8457 & 0.8362 \\
\hline 3 & ERIM_1 & 0.8429 & 0.8301 \\
\hline 4 & AEG & 0.8427 & 0.8315 \\
\hline 8 & REFERENCE & 0.8425 & 0.8425 \\
\hline 6 & OCRSYS & 0.8419 & 0.8266 \\
\hline 7 & KODAK2 & 0.8409 & 0.8278 \\
\hline 8 & IBM & 0.8403 & 0.8296 \\
\hline $\boldsymbol{\theta}$ & ATT 2 & 0.8396 & 0.8288 \\
\hline 10 & KODAK_ & 0.8393 & 0.8247 \\
\hline 11 & VOTEP & 0.8392 & 0.8323 \\
\hline 12 & ELSAGBS & 0.8387 & 0.8296 \\
\hline 13 & THINK 2 & 0.8385 & 0.8272 \\
\hline 14 & ELSAGB 2 & 0.8384 & 0.8293 \\
\hline 15 & ATT $\perp$ & 0.8382 & 0.8298 \\
\hline 16 & ERIM 2 & 0.8379 & 0.8275 \\
\hline 17 & NESTOR & 0.8375 & 0.8243 \\
\hline 18 & HUGHES_1 & 0.8371 & 0.8234 \\
\hline 19 & ATT -4 & 0.8370 & 0.8237 \\
\hline 20 & UBOL & 0.8368 & 0.8251 \\
\hline 21 & HUGHES_2 & 0.8360 & 0.8228 \\
\hline 22 & NYNEX & 0.8345 & 0.8237 \\
\hline 23 & NIST_4 & 0.8343 & 0.8227 \\
\hline 24 & SYMBUS & 0.8341 & 0.8223 \\
\hline 25 & REI & 0.8332 & 0.8241 \\
\hline 26 & ELSA GB_1 & 0.8327 & 0.8203 \\
\hline 27 & ATT $\mathbf{3}$ & 0.8325 & 0.8218 \\
\hline 28 & THINK_I & 0.8308 & 0.8201 \\
\hline 29 & GTESS 2 & 0.8220 & 0.8102 \\
\hline 30 & GTESS_1 & 0.8212 & 0.8102 \\
\hline 31 & СомСом & 0.8196 & 0.8139 \\
\hline 32 & GMD_3 & 0.8128 & 0.7990 \\
\hline 33 & NIST 1 & 0.8121 & 0.7997 \\
\hline 34 & MIME & 0.8096 & 0.7950 \\
\hline 35 & ASOL & 0.8092 & 0.7844 \\
\hline 36 & UPENN & 0.8076 & 0.7929 \\
\hline 37 & GMD_1 & 0.8069 & 0.7834 \\
\hline 38 & NIST 3 & 0.8060 & 0.7922 \\
\hline 39 & NIST 2 & 0.8038 & 0.7809 \\
\hline 40 & RISO & 0.7904 & 0.7832 \\
\hline 41 & KAMAN_1 & 0.7961 & 0.7782 \\
\hline 42 & GMD_4 & 0.7944 & 0.7807 \\
\hline 43 & KAMAN_3 & 0.7847 & 0.7670 \\
\hline 44 & KAMAN_2 & 0.7834 & 0.7656 \\
\hline 45 & KAMAN_S & 0.7898 & 0.7506 \\
\hline 46 & IFAX & 0.7623 & 0.7391 \\
\hline 47 & GMD_2 & 0.7394 & 0.7438 \\
\hline 48 & VALEN_I & 0.7348 & 0.7179 \\
\hline 49 & KAMAN_4 & 0.7180 & 0.7006 \\
\hline
\end{tabular}

Table 149: VALEN_2 correlation graph key for digits. 


\section{No Data Available}

Figure 244: VALEN 2 - upper case correlation

There was no data for this evaluation.

Table 150: VALEN 2 correlation graph key for uppers. 


\section{No Data Available}

Figure 245: VALEN 2 - lower case correlation

There was no data for this evalustion.

Table 151: VALEN 2 correlation graph key for lowers. 

NIST-114A
(REV. 3-90)

BIBLIOGRAPHIC DATA SHEET
1. PUBLCATION OA REPORT MUMBER

NISTIR 4912

2 PEAFOAMINO ORGANIZATION REPORT NUMBEA

3. PUBLCATION DATE

4. TITLE AMD SUBTITLE

The First Census Optical Character Recognition Systems Conference

5. AUTHOR(S)

R. Allen Wilkinson, Jon Geist, Stanley Janet, Christopher J. C. Burges, Robert Creecy, Bob Hammond, Jonathan J. Hull, Norman W. Larsen. Thomas P. Vogl, and Charles $L_{\text {. Wilson }}$

6. PERFORMINO ORCAMIZATION (IF JOINT OR OTMER THAN NIST, SEE INSTRUCTIONS)

U.S. DEPARTMEMT OF COMMERCE

MATIONAL INSTITUTE OF STANDAADS AND TECHMOLOQY

GATTERSBUAQ, MD 2089

7. CONTRACT/OAANT NUMBEA

8. TYPE OF REPOAT AMD PERIOD COVERED

9. SPONSOAINO DRGANIZATION MAME AND COMPLETE ADDRESS (STREET, CITY, STATE, ZIP)

U. S. Bureau of the Census

Washington, DC 20233

10. SUPPLEMENTARY MOTES

11. ABSTAACT (A 200-WOAD OR LESS FACTUAL SUMMAAY OF MOST SIOMIFICANT IMFORMATION. IF DOCUMENT INCLUDES A SIONIFICAMT BIBLOORAPHY OR UTERATURE SUAVEY, MENTION IT MERE)

The First Census Optical Character Recognition (OCR) Systems Conference tested a number of systems developed by different commercial, educational, and government organizations in the OCR of segmented hand-printed digits, upper case letters, and lower case letters. This report discusses the results, conclusions, and open questions of the Conference.

12 KEY WORDS (6 TO 12 ENTRIES; ALPHABETICAL OADER; CAPTTALZE ONLY PROPER MAMES; AND SEPARATE KEY WORDS BY SEMICOLONS)

digits; hand print; lower case; OCR; optical character recognition; upper case.

FOR OFFCLLL DISTAIBUTION. DO NOT RELEASE TO MATIOMAL TECHMICAL INFOAMATION SERVICE (NTSS).

OMDER FROM SUPERINTENDENT OF DOCUMENTS, U.S. OOVERMLENT PRINTING OFFICE, WASHIMGTON, DC 20402

$\mathrm{X}$ OROER FROM MATIONAL TECMMICAL INFORMATION SERVICE (MTIS), SPRINOFIED, VA 22161.

373

15. PRice

A16 


